\title{
Some Algebraic and Logical Aspects of $\mathcal{C}^{\infty}$-Rings
}

\author{
Jean Cerqueira Berni
}

\author{
TESE APRESENTADA \\ Instituto de Matemática e Estatística \\ DA \\ Universidade DE SÃo PAUlo \\ PARA \\ OBTENÇÂO DO TÍTULO \\ DOUTOR EM CIÊNCIAS
}

Programa: Pós-Graduação em Matemática do IME-USP

Orientador: Prof. Dr. Hugo Luiz Mariano

Durante o desenvolvimento deste trabalho o autor recebeu auxílio financeiro da CAPES

São Paulo, novembro de 2018 


\section{Some Algebraic and Logical Aspects of $\mathcal{C}^{\infty}$-Rings}

Esta versão da tese contém as correções e alterações sugeridas pela Comissão Julgadora durante a defesa da versão original do trabalho, realizada em 09/11/2018. Uma cópia da versão original está disponível no

Instituto de Matemática e Estatística da Universidade de São Paulo.

Comissão Julgadora:

- Prof. Dr. Hugo Luiz Mariano (orientador) - IME-USP

- Prof. Dr. Ricardo Bianconi - IME-USP

- Prof. Dr. Marcelo Esteban Coniglio - UNICAMP

- Prof. Dr. Vinicius Cifú Lopes - UFABC

- Prof. Dr. Peter Arndt - Universität Düsseldorf 


\section{Agradecimentos}

Agradeço a todos que, direta ou indiretamente contribuíram para a finalização deste trabalho. A Deus, provedor primário de todo o conhecimento e poder criativo, aos santos e orixás, como Santa Rita de Cássia, Nossa Senhora, mãe de Jesus, Oxóssi e Ogum, que me deram, juntamente com os demais mentores espirituais, sustentação emocional e espiritual desde o começo.

Agradeço imensamente ao professor Hugo Luiz Mariano, que desde o começo me acolheu e me orientou de modo paciente, amigo e com extrema competência, me guiando por esta que me era, ao começo deste projeto, a "terra incognita" da Teoria das Categorias e da Lógica. O professor Hugo é, sem dúvida, um exemplo que procurarei seguir de perto enquanto pessoa e enquanto matemático.

Sou imensamente grato ao Prof. Odilon Otávio Luciano, pelas inúmeras conversas produtivas a respeito deste projeto (e da cultura matemática, em geral) - bem como por sua ajuda em compreender vários conceitos - e por sua amizade e apoio. Obrigado, também, pela revisão cuidadosa da bibliografia deste trabalho, feita com muito primor.

Os membros da banca trouxeram inúmeros pontos que enriqueceram este trabalho por meio de suas sugestões valiosíssimas, de modo que devo um agradecimento especial aos professores Peter Arndt, Marcelo Esteban Coniglio, Vinicius Cifú Lopes e Ricardo Bianconi. Obrigado pelas cuidadosas observações, correções e sugestões.

Agradeço aos competentes funcionários da secretaria de pós-graduação do IME-USP, em especial à Ana Paula Sanches Veloso, por sua presteza e imensa boa vontade em me ajudar, sempre.

Àqueles que me serviram como inspiração e modelo desde o início da jornada, como a professora Marta Cilene Gadotti, a professora Elíris Cristina Rizziolli e a professora Suzi Marconatto, só tenho a agradecer e a dizer que parte do matemático que me tornei provém vocês. Minha eterna gratidão, admiração e respeito.

Aos meus familiares - especialmente minha mãe, minha tia Lila, minha tia Higa, meu pai e meus avós sou eterno credor pelo apoio e incentivo. Aos amigos Caio, Dimi, Luan, Hugo, Kaique, Renan, Priscila, Ana Luíza e Laís, obrigado pelos momentos de apoio, amizade e cooperação. Ao Jonas, obrigado por fazer parte da minha história e da minha vida.

Finalmente, agradeço à CAPES pela bolsa (condição sine qua non para a execução deste projeto) e à USP pelo apoio financeiro que possibilitou minha participação nos eventos científicos sediados fora do estado de São Paulo. 


\section{Resumo}

BERNI J.C. Alguns aspectos algébricos e lógicos dos $\mathcal{C}^{\infty}$-Anéis. 2018. 283+viii f. Tese (Doutorado) - Instituto de Matemática e Estatística, Universidade de São Paulo, São Paulo, 2018.

Conforme observado por I. Moerdijk e G. Reyes em [63], os anéis $\mathcal{C}^{\infty}$ têm sido estudados especialmente tendo em vista suas aplicações em Teoria de Singularidades e para construir toposes que sirvam de modelos para a Geometria Diferencial Sintética. Neste trabalho, seguimos um caminho complementar, aprofundando nosso conhecimento sobre eles por um viés mais puro, fazendo uso da Teoria das Categorias e os analisando a partir de pontos de vistas algébrico e lógico-categorial. Iniciamos o trabalho apresentando uma sistematização abrangente dos fatos fundamentais da teoria (equacional) dos anéis $\mathcal{C}^{\infty}$, distribuídos aqui e ali na literatura atual - a maioria sem demonstrações - mas que servem de base para a teoria. Na sequência, desenvolvemos alguns tópicos do que denominamos Álgebra Comutativa $\mathcal{C}^{\infty}$, expandindo resultados parciais de [66] e [67]. Realizamos um estudo sistemático dos anéis $\mathcal{C}^{\infty}$ von Neumann-regulares - na linha do estudo algébrico realizado em [2]- e apresentamos alguns resultados interessantes a seu respeito, juntamente com sua relação (funtorial) com os espaços booleanos. Estudamos algumas noções pertinentes à Teoria de Feixes para anéis $\mathcal{C}^{\infty}$, tais como espaços (localmente) $\mathcal{C}^{\infty}$-anelados e o sítio de Zariski liso. Finalmente, descrevemos toposes classificantes para a teoria (algébrica) dos anéis $\mathcal{C}^{\infty}$, a teoria (coerente) dos anéis locais $\mathcal{C}^{\infty}$ e a teoria (algébrica) dos anéis $\mathcal{C}^{\infty}$ von Neumann regulares.

Palavras-chave: $\mathcal{C}^{\infty}$-Anéis; Álgebra Comutativa $\mathcal{C}^{\infty}$; Feixes e Lógica. 


\section{Abstract}

BERNI J.C. Some Algebraic and Logical Aspects of $\mathcal{C}^{\infty}$-Rings. 2018. 283+viii pp. PhD thesisInstituto de Matemática e Estatística, Universidade de São Paulo, São Paulo, 2018.

As pointed out by I. Moerdijk and G. Reyes in [63], $\mathcal{C}^{\infty}$-rings have been studied specially for their use in Singularity Theory and in order to construct topos models for Synthetic Differential Geometry. In this work, we follow a complementary trail, deepening our knowledge about them through a more pure bias, making use of Category Theory and accounting them from a logical-categorial viewpoint. We begin by giving a comprehensive systematization of the fundamental facts of the (equational) theory of $\mathcal{C}^{\infty}$-rings, widespread here and there in the current literature - mostly without proof - which underly the theory of $\mathcal{C}^{\infty}$-rings. Next we develop some topics of what we call a $\mathcal{C}^{\infty}$-Commutative Algebra, expanding some partial results of [66] and 67]. We make a systematic study of von Neumann-regular $\mathcal{C}^{\infty}$-rings (following [2]) and we present some interesting results about them, together with their (functorial) relationship with Boolean spaces. We study some sheaf theoretic notions on $\mathcal{C}^{\infty}$-rings, such as $\mathcal{C}^{\infty}$-(locally)-ringed spaces and the smooth Zariski site. Finally we describe classifying toposes for the (algebraic) theory of $\mathcal{C}^{\infty}$-rings, the (coherent) theory of local $\mathcal{C}^{\infty}$-rings and the (algebraic) theory of von Neumann regular $\mathcal{C}^{\infty}$-rings.

Keywords: $\mathcal{C}^{\infty}$-Rings; Smooth Commutative Algebra; Sheaves and Logic. 


\section{Contents}

$\begin{array}{ll}\text { Introduction } & 1\end{array}$

\begin{tabular}{lll}
\hline $\mathbf{1}$ & $\mathcal{C}^{\infty}$-Rings & $\mathbf{3}$
\end{tabular}

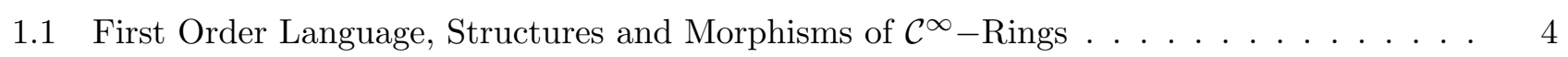

1.2 Birkhoff's HSP Theorem for $\mathcal{C}^{\infty}$-rings . . . . . . . . . . . . . . . . . . . 8

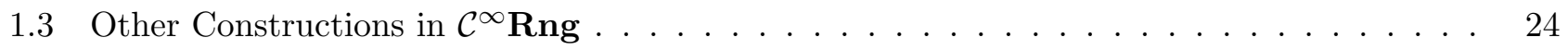

$1.4 \quad$ Finitely Generated and Finitely Presented $\mathcal{C}^{\infty}-$ rings $\ldots \ldots \ldots \ldots$. . . . . . . . . 57

1.5 The Free $\mathcal{C}^{\infty}$-Ring Associated with a Ring $\ldots \ldots \ldots \ldots$. . . . . . . . . . . 69

1.6 Appendix on Free $\mathcal{C}^{\infty}-$ Rings $\ldots \ldots \ldots \ldots \ldots \ldots \ldots \ldots$

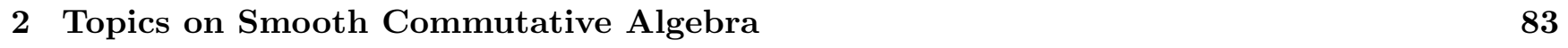

2.1 Smooth Ring of Fractions $\ldots \ldots \ldots \ldots \ldots \ldots$

2.1 .1 Smooth Saturation . . . . . . . . . . . . . . . . . . . . 87

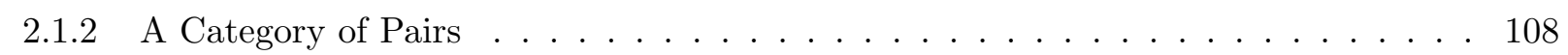

2.2 Distinguished Classes of $\mathcal{C}^{\infty}$-rings $\ldots \ldots \ldots \ldots \ldots \ldots \ldots \ldots \ldots$

2.3 Order Theory of $\mathcal{C}^{\infty}$-rings $\ldots \ldots \ldots \ldots \ldots$

$2.4 \quad$ Algebraic Concepts versus Smooth Algebraic Concepts . . . . . . . . . . . . . . . . . 180

\begin{tabular}{|lll}
3 & Sheaves of $\mathcal{C}^{\infty}$-Rings and Applications & 183
\end{tabular}

$3.1 \quad$ Some topological features of $\operatorname{Spec}^{\infty}(A) \ldots \ldots \ldots \ldots \ldots$. . . . . . . . . . . 184

$3.2 \quad C^{\infty}$-rings and $C^{\infty}$-locally ringed spaces $\ldots \ldots \ldots \ldots \ldots \ldots$

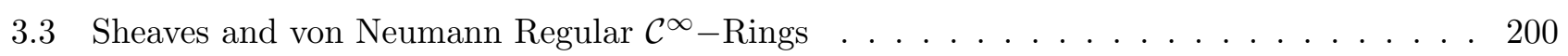

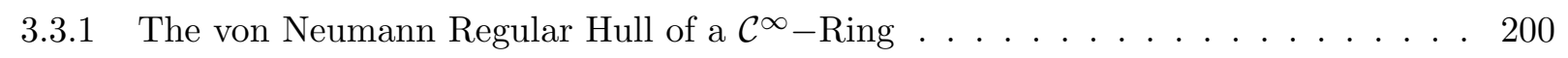

$3.3 .2 \quad$ Sheaves for the von Neumann regular hull of $\mathcal{C}^{\infty}$-ring . . . . . . . . . . . . . . 212

$3.4 \quad$ Von Neumann-regular $\mathcal{C}^{\infty}-$ Rings and Boolean Algebras . . . . . . . . . . . . . . . . . . 231

4 Some Logical Aspects of the $\mathcal{C}^{\infty}$-rings $\quad 249$

4.1 Categorial Logic and classifying topoi $\ldots \ldots \ldots$. . . . . . . . . . . . . 249

$4.2 \quad$ A Classifying Topos for the Theory of $\mathcal{C}^{\infty}$-rings $\ldots \ldots \ldots \ldots \ldots \ldots$

$4.3 \quad$ A Classifying Topos for the Theory of Local $\mathcal{C}^{\infty}$-rings $\ldots \ldots \ldots$. . . . . . . . . . . 260

4.4 A Classifying Topos for the Theory of the von Neumann-regular $\mathcal{C}^{\infty}$-rings $\ldots . . . .268$

\begin{tabular}{|ll}
5 Conclusions and future works & 271
\end{tabular} 


\section{Introduction}

In this work, we study a general class of rings of smooth functions, the class of $\mathcal{C}^{\infty}$-rings, frequently used in virtue of their applications to Singularity Theory (rings of germs of smooth functions at a point, jet bundles and others) and in order to construct topos-models for Synthetic Differential Geometry ${ }^{1}$ (cf. [17), such as the "Dubuc topos".

Synthetic Differential Geometry deals with infinitely small entities - the so-called "infinitesimals" - so their model topoi must have an internal language that is capable to provide room for them 2 . These topoi, thus, legitimate the reasoning with infinitesimals, 'allowing us to instantiate arguments and constructions "à la Cartan". However, the presence of nilpotent elements, for example, is not compatible with classical logic, but rather with a weaker one, the intuitionistic logic, which allows fewer deductions.

The category of $\mathcal{C}^{\infty}$-rings has many features which give us many advantages for a more intuitive treatment of Differential Geometry than the category of all finite-dimensional smooth manifolds and smooth functions, Man. This occurs partly because the category of $\mathcal{C}^{\infty}$-rings is a variety of algebras (i.e., it is the class of models of an equational theory), and it includes, as a full subcategory, the (dual of the) category Man. Also, using the category of $\mathcal{C}^{\infty}$-rings, one constructs topoi in which we can, for example, describe "the tangent bundle" of a manifold as a representable functor. The Cartesian closedness of such categories enormously simplifies the exposition of the theory.

Despite being a very useful tool for many branches of Mathematics, it is difficult to find a detailed and systematic presentation of the fundamental facts about $\mathcal{C}^{\infty}$-rings (the outstanding book of I. Moerdijk and G. Reyes, [62], brings an excellent account on the main facts about $\mathcal{C}^{\infty}$-rings, but from a more sophisticated viewpoint). In this work we dedicate an entire chapter (the first one) to describe, in an universal-algebraic fashion, the (algebraic) category of $\mathcal{C}^{\infty}$-rings, $\mathcal{C}^{\infty} \mathbf{R n g}$, and some of the constructions one can perform in it. We state and prove many (almost folkloric) results about $\mathcal{C}^{\infty}$-rings, whose demonstrations could not be found, up to this point, anywhere in the literature - and in some cases, not even a sketch of the proof was pointed out. The purpose of this chapter is twofold: to present the main constructions that we need in the subsequent chapters (that contain new results) and to provide technical support for a beginner in this subject.

Along this work, we follow a complementary trail to the ones pursued so far (either for its use in Synthetic Differential Geometry or for its use in Singularity Theory), deepening our knowledge about $\mathcal{C}^{\infty}$-rings by analyzing them by themselves - that is, looking at them as an interesting theory, independently of their main applications in the current Mathematics. In order to do so, we make use of many mathematical tools, such as categories, topoi, sheaves, categorial logic and others.

Algebraic Geometry and Commutative Algebra have a strong interplay, to the extent that commutative

\footnotetext{
${ }^{1}$ A first step in this direction was given by F. W. Lawvere, in a series of lectures given in 1967 (cf. 48])

${ }^{2}$ Even Alexander Grothendieck, as observed by R. Lavendhomme in 46], insisted on not excluding nilpotent elements in Algebraic Geometry
} 
rings and algebraic varieties hold many useful and important relations. The same is true for Differential Geometry and a smooth version of Commutative Algebra - the commutative algebra in the context of $\mathcal{C}^{\infty}$-rings, that we call "Smooth Commutative Algebra". In Chapter 2, we expand and generalize results on "Smooth Commutative Algebra", presented in [66] and 67]: we analyze and study the analogous "smooth" notions to those of "radical ideals" $\left(\mathcal{C}^{\infty}\right.$-radical ideal $)$, "saturation of a multiplicative set" (smooth saturation, given in Definition 2.1.7), "ring of fractions" $\left(\mathcal{C}^{\infty}\right.$-ring of fractions with respect to some subset of a $\mathcal{C}^{\infty}$-ring, given in Theorem 2.1.24, generalizing the definition given by I. Moerdijk and G. Reyes in Theorem 1.4 of [66]), "reduced rings" $\left(\mathcal{C}^{\infty}\right.$-reduced $\mathcal{C}^{\infty}$-rings), "fields" $\left(\mathcal{C}^{\infty}\right.$-fields), "von Neumann regular rings" (von Neumann regular $C^{\infty}$-rings, given in Definition 2.2.61), "separa-

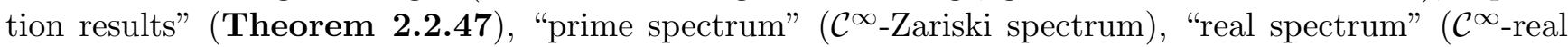
spectrum, given in Definition 2.3.16), among others. We prove several results regarding preservation properties of some features of $\mathcal{C}^{\infty}$-rings under limits and colimits (e.g., Proposition 2.2.35). We have even found an interesting result that establishes a spectral bijection between the $\mathcal{C}^{\infty}$-spectrum and the $\mathcal{C}^{\infty}$-analog of the real spectrum of a $\mathcal{C}^{\infty}$-ring (Theorem 2.3.22).

The central notion of a $\mathcal{C}^{\infty}$-spectrum of a $\mathcal{C}^{\infty}$-ring was introduced by I. Moerdijk and G. Reyes in [63, about which they make a more detailed exposition in later papers ([67] and [65]). We present some contributions to the study of that spectrum in Section 3.1. giving an explicit description of the smooth Zariski topology together with a proof that the $\mathcal{C}^{\infty}$-spectrum is a spectral space (Theorem 3.1.16. The $\mathcal{C}^{\infty}$-spectrum of a $\mathcal{C}^{\infty}$-ring has some additional topological properties when compared to the usual Zariski spectrum of a ring. In fact, the $\mathcal{C}^{\infty}$-spectra of von Neumann regular $\mathcal{C}^{\infty}$-rings classify Boolean spaces: a precise and even stronger result holds (Theorem 3.4.8), which has no analog in the purely algebraic setting of von Neumann regular rings (see for instance [2]).

The notion of a von Neumann regular $\mathcal{C}^{\infty}$-ring and some of their properties is introduced in Chapter $\mathbf{3}$, together with a categorial and logical treatment for them. In particular, the subcategory of $\mathcal{C}^{\infty} \mathbf{R n g}$ consisting of all von Neumann regular $\mathcal{C}^{\infty}$-rings is characterized as the closure under small limits of the category of $\mathcal{C}^{\infty}$-fields, i.e., it is the smallest subcategory of $\mathcal{C}^{\infty} \mathbf{R n g}$ which contain all $\mathcal{C}^{\infty}$-fields and is closed under small limits (Proposition 2.2.80). It is proven, by two different methods (Theorem 3.3.3 and Theorem 3.3.12 ), that the subcategory of von Neumann regular $\mathcal{C}^{\infty}$-rings is reflective in the category of all $\mathcal{C}^{\infty}$-rings.

In Chapter 4 we develop first results on the categorial-logic aspects of the theory of $\mathcal{C}^{\infty}$-rings: we describe in details the classifying toposes for the theories of $\mathcal{C}^{\infty}$-rings ( Theorem 4.2.9), adapting a proof of the analogous construction for commutative rings given in [50], local $\mathcal{C}^{\infty}$-rings (Theorem 4.3.7), both were just stated without proofs in [62], and the classifying topos for the theory of von Neumann regular $\mathcal{C}^{\infty}$-rings (Theorem 4.4.8).

Chapter 5 contains possible developments - mainly on model-theoretic and order-theoretic aspects of the theory of $\mathcal{C}^{\infty}$-rings - that, due to the lack of time, were not developed in this thesis - but that we intend to address in the future.

We finish this work by providing a compendium with the main results on Smooth Analysis, for the reader's benefit and in order to provide internal references used along the text. The list of references we give is long due to one of the purposes of this work, namely to provide a systematic and comprehensive exposition of the theory of $\mathcal{C}^{\infty}$-rings.

For the sake of clarity, this work has a great number of pages, a large part of it is rich in commutative diagrams - what makes, we expect, the understanding of the reader of this work much easier. 


\section{Chapter 1}

\section{$\mathcal{C}^{\infty}$-Rings}

In this chapter we present some fundamental results and concepts upon which this thesis' corpus is based, the theory of $\mathcal{C}^{\infty}$-rings qua algebraic theory. This chapter, except for section 1.5, is devoted to state and prove some known results used in the literature about $\mathcal{C}^{\infty}$-rings and their applications, whose proofs either could not be found or were merely sketched. We present, in detail, the main constructions one can perform within this category, such as limits and directed colimits, providing a "propaedeutic exposition" for the reader's benefit.

Perhaps the most natural way to think about a $\mathcal{C}^{\infty}$-ring is to regard it as an $\mathbb{R}$-algebra in which one can evaluate not only polynomials with real coefficients, but every $\mathcal{C}^{\infty}$-function (cf. [17]). Recall that an $\mathbb{R}$-algebra $A$ can be regarded as a finite product preserving functor from the category Pol, whose objects are Cartesian powers of $\mathbb{R}$, that is:

$$
\operatorname{Obj}(\mathbf{P o l})=\left\{\mathbb{R}^{n} \mid n \in \mathbb{N}\right\}
$$

and whose morphisms are all polynomial functions - that is, $\operatorname{Pol}\left(\mathbb{R}^{m}, \mathbb{R}^{n}\right)=\left\{p: \mathbb{R}^{m} \rightarrow \mathbb{R}^{n} \mid p\right.$ is polynomial $\}$, to the category of sets,

$$
A: \mathbf{P o l} \rightarrow \text { Set }
$$

such that $A(\mathbb{R})=A$, its underlying set. Every polynomial map, $p: \mathbb{R}^{m} \rightarrow \mathbb{R}^{n}$, given by an $n$-tuple of polynomials with real coefficients, $\left(p_{1}, \cdots, p_{n}\right)$, can be interpreted as a map $A(p): A^{m} \rightarrow A^{n}$.

Analogously, a $\mathcal{C}^{\infty}$-ring $A$ can be thought of as a finite product preserving functor from $\mathcal{C}^{\infty}$, the category whose objects are the Euclidean spaces, that is:

$$
\operatorname{Obj}\left(\mathcal{C}^{\infty}\right)=\left\{\mathbb{R}^{n} \mid n \in \mathbb{N}\right\}
$$

and whose morphisms are all smooth functions - that is, $\mathcal{C}^{\infty}\left(\mathbb{R}^{m}, \mathbb{R}^{n}\right)=\mathcal{C}^{\infty}\left(\mathbb{R}^{m}, \mathbb{R}^{n}\right)$, to Set (cf. [62]).

Every smooth function $f: \mathbb{R}^{m} \rightarrow \mathbb{R}^{n}$, given by an $n$-tuple of $\mathcal{C}^{\infty}$-functions, $\left(f_{1}, \cdots, f_{n}\right)$, can be interpreted as a map $A(f): A^{m} \rightarrow A^{n}$. In a broader context, a $\mathcal{C}^{\infty}$-ring in a topos $\mathcal{E}$ is defined as a finite product preserving functor (cf. 63]):

$$
A: \mathcal{C}^{\infty} \rightarrow \mathcal{E}
$$

In this context, the category $\mathcal{C}^{\infty}$ is a Lawvere theory (or a finite-product theory), whose generic object is $\mathbb{R}$. When dealing with classifying topoi for some theories based on $\mathcal{C}^{\infty}$-rings, this definition will be 
proved to be very useful.

This chapter presents $\mathcal{C}^{\infty}$-rings both briefly as a first order theory, formulated within an explicit first order language, and as a universal algebra whose functional symbols are smooth functions from Cartesian powers of $\mathbb{R}$ to $\mathbb{R}$. Such an approach has the advantage of giving us explicitly many constructions, such as products, coproducts, directed colimits and so on, and more simple proofs of the main results.

Our idea of describing $\mathcal{C}^{\infty}$-rings from a universal-algebraic point of view right at the beginning was mainly due to the clear presentation of $\mathcal{C}^{\infty}$-rings made in 29 - which we found very enlightening.

\subsection{First Order Language, Structures and Morphisms of $\mathcal{C}^{\infty}$-Rings}

In order to present and deal with the concept of $\mathcal{C}^{\infty}$-ring, we now present a 1st. order language, $\mathbb{L}$, in which these rings can be described.

The logical symbols are:

(i) A denumerable set of individual variables, $\operatorname{Var}(\mathbb{L})=\left\{x_{1}, x_{2}, \cdots, x_{n}, \cdots\right\}$;

(ii) The propositional connectives $\neg$ ("negation"),$\wedge$ (the conjunction "and", sometimes written \&), $\vee$ ("or"),$\rightarrow$ (implies, sometimes also denoted by $\Rightarrow$ ) and $\leftrightarrow$ ("if, and only if", sometimes also denoted by $\Longleftrightarrow$ )

(iii) The universal and the existential quantifiers, $\forall$ and $\exists$.

(iv) The delimiters ( (left parenthesis), ) (right parenthesis).

(v) The equality symbol, $=$.

The non-logical symbols consist only of function symbols, described as follows:

For each $n \in \mathbb{N}$, the $n$-ary function symbols of the set $\mathcal{C}^{\infty}\left(\mathbb{R}^{n}\right)$, i.e., $\mathcal{F}_{(n)}=\left\{f^{(n)} \mid f \in \mathcal{C}^{\infty}\left(\mathbb{R}^{n}\right)\right\}$. So the set of function symbols of our language is given by:

$$
\mathcal{F}=\bigcup_{n \in \mathbb{N}} \mathcal{F}_{(n)}=\bigcup_{n \in \mathbb{N}} \mathcal{C}^{\infty}\left(\mathbb{R}^{n}\right)
$$

Note that our set of constants is identified with the set of all 0 -ary function symbols, i.e., $\mathcal{C}=\mathcal{F}_{(0)}=$ $\mathcal{C}^{\infty}\left(\mathbb{R}^{0}\right) \cong \mathcal{C}^{\infty}(\{*\}) \cong \mathbb{R}$

The set of terms of our language, $\mathbb{T}$, is recursively defined as follows:

(i') Every variable and every constant symbol is a term;

(ii') If $t_{1}, \cdots, t_{n}$ are terms and $f^{(n)} \in \mathcal{F}_{n}=\mathcal{C}^{\infty}\left(\mathbb{R}^{n}\right)$, then $f^{(n)}\left(t_{1}, \cdots, t_{n}\right)$ is a term;

(iii') There are no terms but those which can be obtained by (i') and (ii').

Since we do not include any relational symbol in our language, the set of the atomic formulas, AF, is given simply by the equality between terms, that is

$$
\mathbf{A F}=\left\{t_{1}=t_{2} \mid t_{1}, t_{2} \in \mathbb{T}\right\}
$$

Finally, the well formed formulas, WFF are those formulas that can be constructed, by finitely many steps, according to the following formation rules: 
(1) Every atomic formula is a well formed formula, i.e., $\mathbf{A F} \subseteq \mathbf{W F F}$;

(2) Whenever $\varphi$ and $\psi$ are well formed formulas, $\neg \varphi,(\varphi \& \psi),(\varphi \vee \psi)$ and $(\varphi \rightarrow \psi)$ are well formed formulas;

(3) If $\varphi$ is a well formed formula and $v \in \operatorname{Var}(\mathbb{L})$, then both $(\exists v) \varphi$ and $(\forall v) \varphi$ are well formed formulas;

(4) There are no other well formed formulas but those described by (1), (2) and (3).

One possible set of axioms for the theory of the $\mathcal{C}^{\infty}$-rings can be given by the following two sets of equations:

(E1) For each $n \in \mathbb{N}$ and for every $k \leq n$, denoting the $k$-th projection by $p_{k}: \mathbb{R}^{n} \rightarrow \mathbb{R}$, the equations:

$$
\mathbf{E q}_{(1)}^{n, k}=\left\{\left(\forall x_{1}\right) \cdots\left(\forall x_{n}\right)\left(p_{k}\left(x_{1}, \cdots, x_{n}\right)=x_{k}\right)\right\}
$$

(E2) for every $k, n \in \mathbb{N}$ and for every $(n+2)$-tuple of function symbols, $\left(f, g_{1}, \cdots, g_{n}, h\right)$ such that $f \in \mathcal{F}_{(n)}, g_{1}, \cdots, g_{n}, h \in \mathcal{F}_{(k)}$ and $h=f \circ\left(g_{1}, \cdots, g_{n}\right)$, the equations:

$$
\mathbf{E q}_{(2)}^{n, k}=\left\{\left(\forall x_{1}\right) \cdots\left(\forall x_{k}\right)\left(h\left(x_{1}, \cdots, x_{k}\right)=f\left(g_{1}\left(x_{1}, \cdots, x_{k}\right), \cdots, g_{n}\left(x_{1}, \cdots, x_{k}\right)\right)\right)\right\}
$$

Now we present an alternative description of a $\mathcal{C}^{\infty}$-rings making use of universal algebraic concepts, given, for instance, by [29].

We first define the notion of a $\mathcal{C}^{\infty}$-structure:

Definition 1.1.1. $A \mathcal{C}^{\infty}$-structure on a set $A$ is a pair $(A, \Phi)$, where:

$$
\begin{aligned}
\Phi: \bigcup_{n \in \mathbb{N}} \mathcal{C}^{\infty}\left(\mathbb{R}^{n}, \mathbb{R}\right) & \rightarrow \bigcup_{n \in \mathbb{N}} \operatorname{Func}\left(A^{n} ; A\right) \\
\left(f: \mathbb{R}^{n} \stackrel{\mathcal{C}^{\infty}}{\rightarrow} \mathbb{R}\right) & \mapsto \Phi(f):=\left(f^{A}: A^{n} \rightarrow A\right)
\end{aligned}
$$

that is, $\Phi$ is a function that interprets the symbols ${ }^{1}$ of all smooth real functions of $n$ variables as $n$-ary function symbols on $A$.

Definition 1.1.2. Let $(A, \Phi)$ and $(B, \Psi)$ be two $\mathcal{C}^{\infty}$-structures. A function $\varphi: A \rightarrow B$ is called a morphism of $\mathcal{C}^{\infty}$-structures if for any $n \in \mathbb{N}$ and any $f \in \mathcal{C}^{\infty}\left(\mathbb{R}^{n}, \mathbb{R}\right)$ the following diagram commutes:

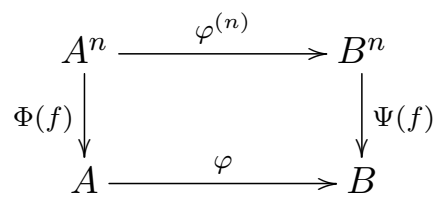

i.e., $\Psi(f) \circ \varphi^{(n)}=\varphi \circ \Phi(f)$.

Theorem 1.1.3. Let $(A, \Phi),(B, \Psi),(C, \Omega)$ be any $\mathcal{C}^{\infty}$-structures, and let $\varphi:(A, \Phi) \rightarrow(B, \Psi)$ and $\psi:(B, \Psi) \rightarrow(C, \Omega)$ be two morphisms of $\mathcal{C}^{\infty}$-structures. We have:

(1) $\operatorname{id}_{A}:(A, \Phi) \rightarrow(A, \Phi)$ is a morphism of $\mathcal{C}^{\infty}$-structures;

(2) $\psi \circ \varphi:(A, \Phi) \rightarrow(C, \Omega)$ is a morphism of $\mathcal{C}^{\infty}$-structures.

\footnotetext{
${ }^{1}$ here considered simply as syntactic symbols rather than functions.
} 
Proof. Ad (1): Given any $n \in \mathbb{N}$ and any $f \in \mathcal{C}^{\infty}\left(\mathbb{R}^{n}, \mathbb{R}\right)$, since $\operatorname{id}_{\mathrm{A}}{ }^{(n)}=\operatorname{id}_{A^{n}}$, we have:

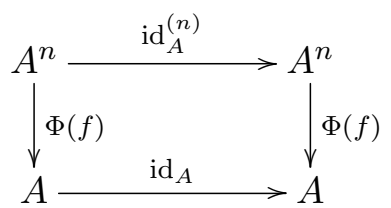

so $\Phi(f) \circ \operatorname{id}_{A}^{(n)}=\Phi(f) \circ \operatorname{id}_{A^{n}}=\Phi(f)=\operatorname{id}_{A} \circ \Phi(f)$, thus $\operatorname{id}_{A}:(A, \Phi) \rightarrow(A, \Phi)$ is a morphism of $\mathcal{C}^{\infty}$-structures.

Ad (2): Suppose that $\varphi:(A, \Phi) \rightarrow(B, \Psi)$ and $\psi:(B, \Psi) \rightarrow(C, \Omega)$ are two morphisms of $\mathcal{C}^{\infty}$-structures, so given any $m \in \mathbb{N}$ and any $f \in \mathcal{C}^{\infty}\left(\mathbb{R}^{m}, \mathbb{R}\right)$ the following diagrams commute:
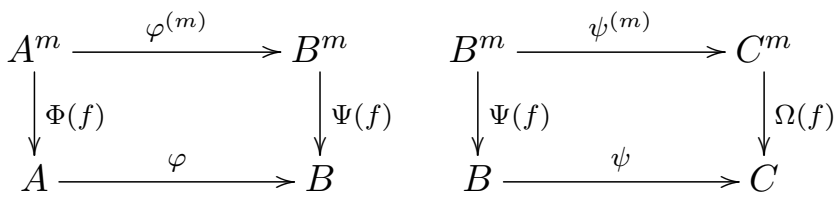

Since $(\psi \circ \varphi)^{(m)}=\psi^{(m)} \circ \varphi^{(m)}$, the following diagram commutes:

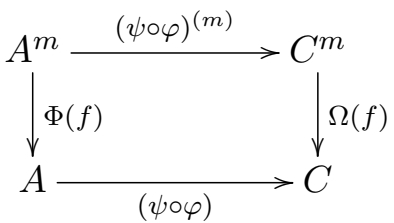

so $\psi \circ \varphi:(A, \Phi) \rightarrow(C, \Omega)$ is a morphism of $\mathcal{C}^{\infty}$-structures.

Theorem 1.1.4. Let $(A, \Phi),(B, \Psi),(C, \Omega),(D, \Gamma)$ be any $\mathcal{C}^{\infty}$-structures, and let $\varphi:(A, \Phi) \rightarrow(B, \Psi)$, $\psi:(B, \Psi) \rightarrow(C, \Omega)$ and $\nu:(C, \Omega) \rightarrow(D, \Gamma)$ be morphisms of $\mathcal{C}^{\infty}$-structures. We have the following equations between pairs of morphisms of $\mathcal{C}^{\infty}$-structures:

$$
\begin{gathered}
\nu \circ(\psi \circ \varphi)=(\nu \circ \psi) \circ \varphi ; \\
\varphi \circ \operatorname{id}_{A}=\operatorname{id}_{B} \circ \varphi .
\end{gathered}
$$

Proof. Since, as functions, we have:

$$
\nu \circ(\psi \circ \varphi)=(\nu \circ \psi) \circ \varphi
$$

and

$$
\varphi \circ \operatorname{id}_{A}=\operatorname{id}_{B} \circ \varphi
$$

and the composition of morphisms of $\mathcal{C}^{\infty}$-structures is again a morphism of $\mathcal{C}^{\infty}$-structures, it follows that the equality between morphisms of $\mathcal{C}^{\infty}$-structures holds.

Definition 1.1.5. We are going to denote by $\mathcal{C}^{\infty} \mathbf{S t r}$ the category whose objects are the $\mathcal{C}^{\infty}$-structures and whose morphisms are the morphisms of $\mathcal{C}^{\infty}$-structures.

As a full subcategory of $\mathcal{C}^{\infty} \operatorname{Str}$ we have the category of $\mathcal{C}^{\infty}$-rings, defined as follows:

Definition 1.1.6. Let $(A, \Phi)$ be a $\mathcal{C}^{\infty}$-structure. We say that $(A, \Phi)$ is a $\mathcal{C}^{\infty}$-ring if the following two conditions are fulfilled: 
(1) For each $n \in \mathbb{N}$ and for every $k \leq n$, denoting the $k$-th projection by $p_{k}: \mathbb{R}^{n} \rightarrow \mathbb{R}$, we have:

$$
\Phi\left(p_{k}\right)=\pi_{k}: A^{n} \rightarrow A,
$$

that is, each projection is interpreted as the canonical projection on the $k$-th coordinate, $\pi_{k}: A^{n} \rightarrow$ A

(2) for every $k, n \in \mathbb{N}$ and for every $(n+2)$-tuple of smooth functions, $\left(f, g_{1}, \cdots, g_{n}, h\right)$ such that $f \in \mathcal{C}^{\infty}\left(\mathbb{R}^{n}, \mathbb{R}\right), g_{1}, \cdots, g_{n}, h \in \mathcal{C}^{\infty}\left(\mathbb{R}^{k}, \mathbb{R}\right)$, we have:

$$
h=f \circ\left(g_{1}, \cdots, g_{n}\right) \Rightarrow \Phi(h)=\Phi(f) \circ\left(\Phi\left(g_{1}\right), \cdots, \Phi\left(g_{n}\right)\right)
$$

Given two $\mathcal{C}^{\infty}$-rings $(A, \Phi)$ and $(B, \Psi)$, a morphism of $\mathcal{C}^{\infty}$-rings from $(A, \Phi)$ to $(B, \Psi)$, or a $\mathcal{C}^{\infty}$-homomorphism from $(A, \Phi)$ to $(B, \Psi)$, is simply a morphism of $\mathcal{C}^{\infty}$-structures from $(A, \Phi)$ to $(B, \Psi)$. We will denote the category of $\mathcal{C}^{\infty}$-rings and $\mathcal{C}^{\infty}$-homomorphisms by $\mathcal{C}^{\infty} \mathbf{R n g}$.

Thus, in the context of first order theories, a model of a $\mathcal{C}^{\infty}$-ring is a $\mathcal{C}^{\infty}$-structure, $\mathfrak{A}=(A, \Phi)$ such that:

- For every $n \in \mathbb{N}, k \leq n$, denoting the projection on the $k$-th coordinate by $p_{k}: \mathbb{R}^{n} \rightarrow \mathbb{R}$ :

$$
\mathfrak{A}=\left(\forall x_{1}\right) \cdots\left(\forall x_{n}\right)\left(p_{k}\left(x_{1}, \cdots, x_{n}\right)=x_{k}\right)
$$

that is, $\Phi\left(p_{k}\right)=\pi_{k}: A^{n} \rightarrow A$;

- For every $n, k \in \mathbb{N}, f \in \mathcal{C}^{\infty}\left(\mathbb{R}^{n}, \mathbb{R}\right), h, g_{1}, \cdots, g_{n} \in \mathcal{C}^{\infty}\left(\mathbb{R}^{k}, \mathbb{R}\right)$ such that $h=f\left(g_{1}, \cdots, g_{n}\right)$ :

$$
\mathfrak{A}=\left(\forall x_{1}\right) \cdots\left(\forall x_{k}\right)\left(h\left(x_{1}, \cdots, x_{k}\right)=f\left(g_{1}\left(x_{1}, \cdots, x_{k}\right), \cdots, g_{n}\left(x_{1}, \cdots, x_{k}\right)\right)\right.
$$

that is, $\Phi(h)=\Phi(f)\left(\Phi\left(g_{1}\right), \cdots, \Phi\left(g_{n}\right)\right)$.

As we shall see, the category of $\mathcal{C}^{\infty}$-rings and its morphisms has many constructions, such as arbitrary products, coproducts, directed colimits, quotients, rings of fractions, localizations and many others. It also "extends" the category of commutative unital rings, CRing, in the following sense:

Remark 1.1.7. Since the sum $+: \mathbb{R}^{2} \rightarrow \mathbb{R}$, the opposite, $-: \mathbb{R} \rightarrow \mathbb{R}, \cdot: \mathbb{R}^{2} \rightarrow \mathbb{R}$ and the constant functions $0: \mathbb{R} \rightarrow \mathbb{R}$ and $1: \mathbb{R} \rightarrow \mathbb{R}$ are particular cases of $\mathcal{C}^{\infty}$-functions, any $\mathcal{C}^{\infty}$-ring $(A, \Phi)$ may be regarded as a commutative ring $(A, \Phi(+), \Phi(\cdot), \Phi(-), \Phi(0), \Phi(1))$, where:

$$
\begin{aligned}
& \Phi(+): A \times A \rightarrow \quad A \\
& \left(a_{1}, a_{2}\right) \mapsto \Phi(+)\left(a_{1}, a_{2}\right)=a_{1}+a_{2} \\
& \Phi(-): A \rightarrow \quad A \\
& a \mapsto \Phi(-)(a)=-a \\
& \Phi(0): \quad A^{0} \rightarrow A \\
& * \quad \mapsto \Phi(0) \\
& \Phi(1): A^{0} \rightarrow A \\
& * \quad \mapsto \Phi(1)
\end{aligned}
$$

where $A^{0}=\{*\}$, and: 


$$
\begin{array}{cccc}
\Phi(\cdot): & A \times A & \rightarrow & A \\
\left(a_{1}, a_{2}\right) & \mapsto & \Phi(\cdot)\left(a_{1}, a_{2}\right)=a_{1} \cdot a_{2}
\end{array}
$$

Thus, we have a forgetful functor:

$$
\begin{array}{ccc}
\widetilde{U}: \begin{array}{c}
\mathcal{C}^{\infty} \text { Rng } \\
(A \Phi)
\end{array} & \rightarrow & (A, \Phi(+), \Phi(\cdot), \Phi(-), \Phi(0), \Phi(1)) \\
\downarrow \varphi & & \downarrow_{\varphi} \\
(B, \Psi) & & (B, \Psi(+), \Psi(\cdot), \Psi(-), \Psi(0), \Psi(1)) \\
& & \widetilde{U}: \mathcal{C}^{\infty} \mathbf{R n g} \rightarrow \text { CRing }
\end{array}
$$

Analogously, we can define a forgetful functor from the category of $\mathcal{C}^{\infty}$-rings and $\mathcal{C}^{\infty}$-homomorphisms into the category of commutative $\mathbb{R}$-algebras with unity,

$$
\widehat{U}: \mathcal{C}^{\infty} \mathbf{R n g} \rightarrow \mathbb{R}-\mathbf{A l g}
$$

\subsection{Birkhoff's HSP Theorem for $\mathcal{C}^{\infty}$-rings}

Since the theory of $\mathcal{C}^{\infty}$-rings is equational, the class $\mathcal{C}^{\infty} \mathbf{R n g}$ is closed in $\mathcal{C}^{\infty} \mathbf{S t r}$ under many algebraic constructions, such as substructures, products, quotients, directed colimits and others. In this section we give explicit descriptions of some of these constructions.

We begin defining what we mean by a $\mathcal{C}^{\infty}$-subring.

Definition 1.2.1. Let $(A, \Phi)$ be a $\mathcal{C}^{\infty}$-ring and let $B \subseteq A$. Under these circumstances, we say that $\left(B, \Phi^{\prime}\right)$ is a $\mathcal{C}^{\infty}$-subring of $(A, \Phi)$ if, and only if, for any $n \in \mathbb{N}, f \in \mathcal{C}^{\infty}\left(\mathbb{R}^{n}, \mathbb{R}\right)$ and any $\left(b_{1}, \cdots, b_{n}\right) \in B^{n}$ we have:

$$
\Phi(f)\left(b_{1}, \cdots, b_{n}\right) \in B
$$

That is to say that $B$ is closed under any $\mathcal{C}^{\infty}$-function n-ary symbol. Note that the $\mathcal{C}^{\infty}$-structure of $B$ is virtually the same as the $\mathcal{C}^{\infty}$-structure of $(A, \Phi)$, since they interpret every smooth function in the same way. However $\Phi^{\prime}$ has a different codomain, as:

$$
\begin{aligned}
\Phi^{\prime}: \bigcup_{n \in \mathbb{N}} \mathcal{C}^{\infty}\left(\mathbb{R}^{n}, \mathbb{R}\right) & \rightarrow \bigcup_{n \in \mathbb{N}} \operatorname{Func}\left(B^{n}, B\right) \\
\left(\mathbb{R}^{n} \stackrel{f}{\rightarrow} \mathbb{R}\right) & \mapsto\left(\Phi(f)\left\lceil_{B^{n}}: B^{n} \rightarrow B\right)\right.
\end{aligned}
$$

We observe that $\Phi^{\prime}$ is the unique $\mathcal{C}^{\infty}$-structure such that the inclusion map:

$$
\iota_{B}^{A}: B \hookrightarrow A
$$

is a $\mathcal{C}^{\infty}$-homomorphism.

We are going to denote the class of all $\mathcal{C}^{\infty}$-subrings of a given $\mathcal{C}^{\infty}$-ring $(A, \Phi)$ by $\operatorname{Sub}(A, \Phi)$.

Next we prove that the intersection of any family of $\mathcal{C}^{\infty}$-subrings of a given $\mathcal{C}^{\infty}$-ring is, again, a $\mathcal{C}^{\infty}$-subring. 
Proposition 1.2.2. Let $\left\{\left(A_{\alpha}, \Phi_{\alpha}\right) \mid \alpha \in \Lambda\right\}$ be a family of $\mathcal{C}^{\infty}$-subrings of $(A, \Phi)$, so

$$
(\forall \alpha \in \Lambda)(\forall n \in \mathbb{N})\left(\forall f \in \mathcal{C}^{\infty}\left(\mathbb{R}^{n}, \mathbb{R}\right)\right)\left(\Phi_{\alpha}(f)=\Phi(f)\left\lceil A_{A_{\alpha}}{ }^{n}: A_{\alpha}{ }^{n} \rightarrow A_{\alpha}\right)\right.
$$

We have that:

$$
\left(\bigcap_{\alpha \in \Lambda} A_{\alpha}, \Phi^{\prime}\right)
$$

where:

$$
\begin{aligned}
\Phi^{\prime}: \bigcup_{n \in \mathbb{N}} \mathcal{C}^{\infty}\left(\mathbb{R}^{n}, \mathbb{R}\right) & \rightarrow \quad \bigcup_{n \in \mathbb{N}} \text { Func }\left(\left(\bigcap_{\alpha \in \Lambda} A_{\alpha}\right)^{n}, \bigcap_{\alpha \in \Lambda} A_{\alpha}\right) \\
\left(\mathbb{R}^{n} \stackrel{f}{\rightarrow} \mathbb{R}\right) & \mapsto \Phi(f) \uparrow_{\left(\bigcap_{\alpha \in \Lambda} A_{\alpha}\right)^{n}:\left(\bigcap_{\alpha \in \Lambda} A_{\alpha}\right)^{n} \rightarrow \bigcap_{\alpha \in \Lambda} A_{\alpha}}
\end{aligned}
$$

is a $\mathcal{C}^{\infty}$-subring of $(A, \Phi)$.

Proof. Let $n \in \mathbb{N}, f \in \mathcal{C}^{\infty}\left(\mathbb{R}^{n}, \mathbb{R}\right)$ and $\left(a_{1}, \cdots, a_{n}\right) \in\left(\bigcap_{\alpha \in \Lambda} A_{\alpha}\right)^{n}$.

Since for every $i \in\{1, \cdots, n\}$ we have $a_{i} \in \bigcap_{\alpha \in \Lambda} A_{\alpha}$, we have, for every $\alpha \in \Lambda, a_{i} \in A_{\alpha}$ for every $i \in\{1, \cdots, n\}$.

Since every $\left(A_{\alpha}, \Phi_{\alpha}\right)$ is a $\mathcal{C}^{\infty}$-subring of $(A, \Phi)$, it follows that for every $\alpha \in \Lambda$ we have:

$$
\Phi_{\alpha}(f)\left(a_{1}, \cdots, a_{n}\right):=\Phi(f) \uparrow_{\alpha}^{n}\left(a_{1}, \cdots, a_{n}\right) \in A_{\alpha}
$$

thus

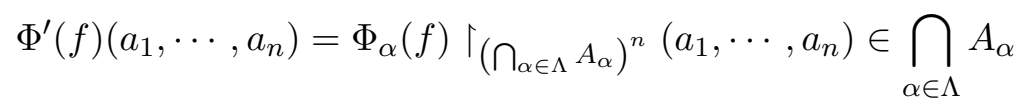

so the result follows.

As an application of the previous result, we can define the $\mathcal{C}^{\infty}$-subring generated by a subset of the carrier of a $\mathcal{C}^{\infty}$-ring:

Definition 1.2.3. Let $(A, \Phi)$ be a $\mathcal{C}^{\infty}$-ring and $X \subseteq A$. The $\mathcal{C}^{\infty}$-subring of $(A, \Phi)$ generated by $X$ is given by:

$$
\langle X\rangle=\bigcap_{\substack{X \subseteq A_{i} \\\left(A_{i}, \Phi_{i}\right) \preceq(A, \Phi)}}\left(A_{i}, \Phi_{i}\right),
$$

where $\left(A_{i}, \Phi_{i}\right) \preceq(A, \Phi)$ means that $\left(A_{i}, \Phi_{i}\right)$ is a $\mathcal{C}^{\infty}$-subring of $(A, \Phi)$

together with the $\mathcal{C}^{\infty}$-structure given in Proposition 1.2.2.

We note that, given any $\mathcal{C}^{\infty}$-ring $(A, \Phi)$, the map of partially ordered sets given by:

$$
\begin{array}{ccc}
\sigma: \quad(\wp(A), \subseteq) & \rightarrow & (\operatorname{Sub}(A), \subseteq) \\
X & \mapsto & \langle X\rangle
\end{array}
$$

satisfies the axioms of a closure operation. 
In general, given an arbitrary family of $\mathcal{C}^{\infty}$-subrings, $\left(A_{\alpha}, \Phi_{\alpha}\right)_{\alpha \in \Lambda}$ of a given $\mathcal{C}^{\infty}$-ring $(A, \Phi)$, its union, $\bigcup_{\alpha \in \Lambda} A_{\alpha}$, together with $\Phi\left\lceil\cup_{\alpha \in \Lambda} A_{\alpha}\right.$, needs not to be a $\mathcal{C}^{\infty}$-subring of $(A, \Phi)$. However, there is an important case in which the union of a family of $\mathcal{C}^{\infty}$-subrings of a $\mathcal{C}^{\infty}$-ring $(A, \Phi)$ is again a $\mathcal{C}^{\infty}$-ring. This case is discussed in the following:

Proposition 1.2.4. Let $(A, \Phi)$ be a $\mathcal{C}^{\infty}$-ring and let $\left\{\left(A_{\alpha}, \Phi_{\alpha}\right) \mid \alpha \in \Lambda\right\}, \Lambda \neq \varnothing$, be a directed family of $\mathcal{C}^{\infty}$-subrings of $(A, \Phi)$, that is, a family such that for every pair $(\alpha, \beta) \in \Lambda \times \Lambda$ there is some $\gamma \in \Lambda$ such that:

$$
A_{\alpha} \subseteq A_{\gamma}
$$

and

$$
A_{\beta} \subseteq A_{\gamma}
$$

We have that:

$$
\left(\bigcup_{\alpha \in \Lambda} A_{\alpha}, \Phi^{\prime}\right)
$$

where:

$$
\begin{aligned}
\Phi^{\prime}: \bigcup_{n \in \mathbb{N}} \mathcal{C}^{\infty}\left(\mathbb{R}^{n}, \mathbb{R}\right) & \rightarrow \bigcup_{n \in \mathbb{N}} \text { Func }\left(\left(\bigcup_{\alpha \in \Lambda} A_{\alpha}\right)^{n}, \bigcup_{\alpha \in \Lambda} A_{\alpha}\right) \\
\left(\mathbb{R}^{n} \stackrel{f}{\rightarrow} \mathbb{R}\right) & \mapsto \Phi(f) \Gamma_{\left(\bigcup_{\alpha \in \Lambda} A_{\alpha}\right)^{n}:\left(\bigcup_{\alpha \in \Lambda} A_{\alpha}\right)^{n} \rightarrow \bigcup_{\alpha \in \Lambda} A_{\alpha}}
\end{aligned}
$$

is a $\mathcal{C}^{\infty}$-subring of $(A, \Phi)$.

Proof. First we note that since $\Lambda$ is directed, we have:

$$
\bigcup_{\alpha \in \Lambda}\left(A_{\alpha}\right)^{n}=\left(\bigcup_{\alpha \in \Lambda} A_{\alpha}\right)^{n}
$$

Let $n \in \mathbb{N}, f \in \mathcal{C}^{\infty}\left(\mathbb{R}^{n}, \mathbb{R}\right)$ and $\left(a_{1}, \cdots, a_{n}\right) \in\left(\bigcup_{\alpha \in \Lambda} A_{\alpha}\right)^{n}$. By definition of the union $\bigcup_{\alpha \in \Lambda} A_{\alpha}$, for every $i \in\{1, \cdots, n\}$ there is some $\alpha_{i} \in \Lambda$ such that:

$$
a_{i} \in A_{\alpha_{i}}
$$

Since $\left\{\left(A_{\alpha}, \Phi_{\alpha}\right) \mid \alpha \in \Lambda\right\}$ is directed, given $\left(A_{\alpha_{1}}, \Phi_{\alpha_{1}}\right), \cdots,\left(A_{\alpha_{n}}, \Phi_{\alpha_{n}}\right)$, there is some $\gamma \in \Lambda$ such that for every $i \in\{1, \cdots, n\}$ we have:

$$
A_{\alpha_{i}} \subseteq A_{\gamma}
$$

Thus, $\left(a_{1}, \cdots, a_{n}\right) \in A_{\gamma}^{n}$, and since $\left(A_{\gamma}, \Phi_{\gamma}\right)$ is a $\mathcal{C}^{\infty}$-subring of $(A, \Phi)$, it follows that:

$$
\Phi_{\gamma}(f)\left(a_{1}, \cdots, a_{n}\right):=\Phi(f)\left\lceil_{A_{\gamma}{ }^{n}}\left(a_{1}, \cdots, a_{n}\right) \in A_{\gamma} \subseteq \bigcup_{\alpha \in \Lambda} A_{\alpha}\right.
$$

Since $\Lambda$ is directed and $\left(A_{\gamma}\right)^{n} \subseteq\left(\bigcup_{\alpha \in \lambda} A_{\alpha}\right)^{n}$, we have:

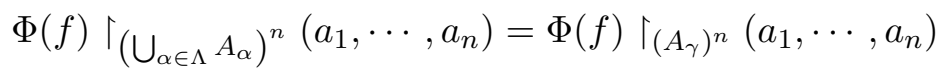

so:

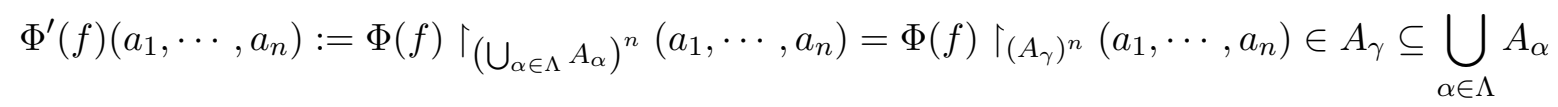


Thus, for every $\left(a_{1}, \cdots, a_{n}\right) \in\left(\bigcup_{\alpha \in \Lambda} A_{\alpha}\right)^{n}$, we have $\Phi^{\prime}(f)\left(a_{1}, \cdots, a_{n}\right) \in \bigcup_{\alpha \in \Lambda} A_{\alpha}$.

This proves that $\left(\bigcup_{\alpha \in \Lambda} A_{\alpha}, \Phi^{\prime}\right)$ is a $\mathcal{C}^{\infty}$-subring of $(A, \Phi)$.

Next we describe the products in the category $\mathcal{C}^{\infty} \mathbf{R n g}$, that is, products of arbitrary families of $\mathcal{C}^{\infty}$-rings.

Definition 1.2.5. Let $\left\{\left(A_{\alpha}, \Phi_{\alpha}\right) \mid \alpha \in \Lambda\right\}$ be a family of $\mathcal{C}^{\infty}$-rings. The product of this family is the pair:

$$
\left(\prod_{\alpha \in \Lambda} A_{\alpha}, \Phi^{(\Lambda)}\right)
$$

where $\Phi$ is given by:

$$
\begin{aligned}
& \Phi^{(\Lambda)}: \bigcup_{n \in \mathbb{N}} \mathcal{C}^{\infty}\left(\mathbb{R}^{n}, \mathbb{R}\right) \rightarrow \quad \bigcup_{n \in \mathbb{N}} \operatorname{Func}\left(\left(\prod_{\alpha \in \Lambda} A_{\alpha}\right)^{n}, \prod_{\alpha \in \Lambda} A_{\alpha}\right) \\
& \left(f: \mathbb{R}^{n} \stackrel{\mathcal{C}^{\infty}}{\rightarrow} \mathbb{R}\right) \quad \mapsto \quad \Phi^{(\Lambda)}(f): \quad\left(\prod_{\alpha \in \Lambda} A_{\alpha}\right)^{n} \quad \rightarrow \quad \prod_{\alpha \in \Lambda} A_{\alpha} \\
& \left(\left(x_{\alpha}^{1}\right)_{\alpha \in \Lambda}, \cdots,\left(x_{\alpha}^{n}\right)_{\alpha \in \Lambda}\right) \mapsto\left(\Phi_{\alpha}(f)\left(x_{\alpha}^{1}, \cdots, x_{\alpha}^{n}\right)\right)_{\alpha \in \Lambda}
\end{aligned}
$$

Remark 1.2.6. In particular, given a $\mathcal{C}^{\infty}$-ring $(A, \Phi)$, we have the product $\mathcal{C}^{\infty}$-ring:

$$
\left(A \times A, \Phi^{(2)}\right)
$$

where:

$$
\begin{aligned}
\Phi^{(2)}: \bigcup_{n \in \mathbb{N}} \mathcal{C}^{\infty}\left(\mathbb{R}^{n}, \mathbb{R}\right) & \rightarrow \bigcup_{n \in \mathbb{N}} \text { Func }\left((A \times A)^{n}, A \times A\right) \\
\left(f: \mathbb{R}^{n} \stackrel{\mathcal{C}^{\infty}}{\rightarrow} \mathbb{R}\right) & \mapsto(\Phi \times \Phi)(f):(A \times A)^{n} \rightarrow A \times A
\end{aligned}
$$

and:

$$
\begin{aligned}
& \Phi^{(2)}(f): \quad(A \times A)^{n} \quad \rightarrow \quad A \times A \\
& \left(\left(x_{1}, y_{1}\right), \cdots,\left(x_{n}, y_{n}\right)\right) \mapsto\left(\Phi(f)\left(x_{1}, \cdots, x_{n}\right), \Phi(f)\left(y_{1}, \cdots, y_{n}\right)\right)
\end{aligned}
$$

We turn now to the definition of congruence relations in $\mathcal{C}^{\infty}$-rings. As we shall see later on, the congruences of a $\mathcal{C}^{\infty}$-rings will be classified by their ring-theoretic ideals.

Definition 1.2.7. Let $(A, \Phi)$ be a $\mathcal{C}^{\infty}$-ring. $A \mathcal{C}^{\infty}$-congruence is an equivalence relation $R \subseteq A \times A$ such that for every $n \in \mathbb{N}$ and $f \in \mathcal{C}^{\infty}\left(\mathbb{R}^{n}, \mathbb{R}\right)$ we have:

$$
\left(x_{1}, y_{1}\right), \cdots,\left(x_{n}, y_{n}\right) \in R \Rightarrow \Phi^{(2)}(f)\left(\left(x_{1}, y_{1}\right), \cdots,\left(x_{n}, y_{n}\right)\right) \in R
$$

In other words, a $\mathcal{C}^{\infty}$-congruence is an equivalence relations that preserves $\mathcal{C}^{\infty}$-function symbols.

A characterization of a $\mathcal{C}^{\infty}$-congruence can be given using the product $\mathcal{C}^{\infty}$-structure, as we see in the following:

Proposition 1.2.8. Let $(A, \Phi)$ be a $\mathcal{C}^{\infty}$-ring and let $R \subseteq A \times A$ be an equivalence relation. Under these circumstances, $R$ is a $\mathcal{C}^{\infty}$-congruence on $(A, \Phi)$ if, and only if, $\left(R \times R, \Phi^{(2)^{\prime}}\right)$, where: 


$$
\begin{aligned}
\Phi^{(2)^{\prime}}: \bigcup_{n \in \mathbb{N}} \mathcal{C}^{\infty}\left(\mathbb{R}^{n}, \mathbb{R}\right) & \rightarrow \bigcup_{n \in \mathbb{N}} \operatorname{Func}\left(R^{n}, R\right) \\
\left(\mathbb{R}^{n} \stackrel{f}{\rightarrow} \mathbb{R}\right) & \mapsto \Phi^{(2)}(f) \uparrow_{R^{n}}: R^{n} \rightarrow R
\end{aligned}
$$

is a $\mathcal{C}^{\infty}$-subring of $\left(A \times A, \Phi^{(2)}\right)$, with the structure described in the Remark 1.2.6.

Proof. Given any $n \in \mathbb{N}, f \in \mathcal{C}^{\infty}\left(\mathbb{R}^{n}, \mathbb{R}\right),\left(\left(x_{1}, y_{1}\right), \cdots,\left(x_{n}, y_{n}\right)\right) \in R^{n}$ we have, by definition:

$$
\Phi^{(2)}(f)\left(\left(x_{1}, y_{1}\right) \cdots,\left(x_{n}, y_{n}\right)\right):=\left(\Phi(f)\left(x_{1}, \cdots, x_{n}\right), \Phi(f)\left(y_{1}, \cdots, y_{n}\right)\right)
$$

and since $R$ is a $\mathcal{C}^{\infty}$-congruence, we have:

$$
\left(\left(x_{1}, y_{1}\right), \cdots,\left(x_{n}, y_{n}\right)\right) \in R^{n} \Rightarrow \Phi^{(2)}(f)\left(\left(x_{1}, y_{1}\right), \cdots,\left(x_{n}, y_{n}\right)\right) \in R
$$

Since:

$$
\Phi^{(2)}(f)\left(\left(x_{1}, y_{1}\right), \cdots,\left(x_{n}, y_{n}\right)\right)=\left(\Phi(f)\left(x_{1}, \cdots, x_{n}\right), \Phi(f)\left(y_{1}, \cdots, y_{n}\right)\right)
$$

it follows that

$$
\left(\left(x_{1}, y_{1}\right), \cdots,\left(x_{n}, y_{n}\right)\right) \in R^{n} \Rightarrow\left(\Phi(f)\left(x_{1}, \cdots, x_{n}\right), \Phi(f)\left(y_{1}, \cdots, y_{n}\right)\right) \in R
$$

Also, since $R^{n} \subseteq(A \times A)^{n}$,

$$
\Phi^{(2)}(f) \uparrow_{R^{n}}\left(\left(x_{1}, y_{1}\right), \cdots,\left(x_{n}, y_{n}\right)\right)=\Phi^{(2)}(f)\left(\left(x_{1}, y_{1}\right), \cdots,\left(x_{n}, y_{n}\right)\right)
$$

so

$$
\begin{aligned}
& \Phi^{(2)^{\prime}}(f)\left(\left(x_{1}, y_{1}\right), \cdots,\left(x_{n}, y_{n}\right)\right)=\Phi^{(2)}(f)\left\lceil R^{n}\left(\left(x_{1}, y_{1}\right), \cdots,\left(x_{n}, y_{n}\right)\right)=\right. \\
& =\Phi^{(2)}(f)\left(\left(x_{1}, y_{1}\right), \cdots,\left(x_{n}, y_{n}\right)\right)=\left(\Phi(f)\left(x_{1}, \cdots, x_{n}\right), \Phi(f)\left(y_{1}, \cdots, y_{n}\right)\right) \in R
\end{aligned}
$$

Thus $\left(R, \Phi^{(2)^{\prime}}\right)$ is a $\mathcal{C}^{\infty}$-subring of $\left(A \times A, \Phi^{(2)}\right)$.

Conversely, let $R$ be an equivalence relation on $A$ such that $\left(R, \Phi^{(2)^{\prime}}\right)$ is a $\mathcal{C}^{\infty}$-subring of $\left(A \times A, \Phi^{(2)}\right)$.

Given any $n \in \mathbb{N}, f \in \mathcal{C}^{\infty}\left(\mathbb{R}^{n}, \mathbb{R}\right)$ and $\left(x_{1}, y_{1}\right), \cdots,\left(x_{n}, y_{n}\right) \in R$, since $\left(R, \Phi^{(2)^{\prime}}\right)$ is a $\mathcal{C}^{\infty}$-subring of $\left(A \times A, \Phi^{(2)}\right)$, we have $\Phi^{(2)^{\prime}}(f)\left(\left(x_{1}, y_{1}\right), \cdots,\left(x_{n}, y_{n}\right)\right) \in R$.

By definition,

$$
\Phi^{(2)^{\prime}}(f)\left(\left(x_{1}, y_{1}\right), \cdots,\left(x_{n}, y_{n}\right)\right)=\Phi^{(2)}(f)\left\lceil R^{n}\left(\left(x_{1}, y_{1}\right), \cdots,\left(x_{n}, y_{n}\right)\right)\right.
$$

and since $R^{n} \subseteq(A \times A)^{n}$, we have:

$$
\Phi^{(2)}(f) \uparrow_{R^{n}}\left(\left(x_{1}, y_{1}\right), \cdots,\left(x_{n}, y_{n}\right)\right)=\Phi^{(2)}(f)\left(\left(x_{1}, y_{1}\right), \cdots,\left(x_{n}, y_{n}\right)\right)
$$

and

$$
\Phi^{(2)}(f)\left(\left(x_{1}, y_{1}\right), \cdots,\left(x_{n}, y_{n}\right)\right)=\left(\Phi(f)\left(x_{1}, \cdots, x_{n}\right), \Phi(f)\left(y_{1}, \cdots, y_{n}\right)\right)
$$


Thus,

$$
\left(x_{1}, y_{1}\right), \cdots,\left(x_{n}, y_{n}\right) \in R \Rightarrow\left(\Phi(f)\left(x_{1}, \cdots, x_{n}\right), \Phi(f)\left(y_{1}, \cdots, y_{n}\right)\right) \in R
$$

and $R$ is a $\mathcal{C}^{\infty}$-congruence.

Remark 1.2.9. Given a $\mathcal{C}^{\infty}$-ring $(A, \Phi)$ and a $\mathcal{C}^{\infty}$-congruence $R \subseteq A \times A$, let:

$$
\frac{A}{R}=\{\bar{a} \mid a \in A\}
$$

be the quotient set. Given any $n \in \mathbb{N}, f \in \mathcal{C}^{\infty}\left(\mathbb{R}^{n}, \mathbb{R}\right)$ and $\left(\overline{a_{1}}, \cdots, \overline{a_{n}}\right) \in\left(\frac{A}{R}\right)^{n}$ we define:

$$
\begin{aligned}
\bar{\Phi}: \bigcup_{n \in \mathbb{N}} \mathcal{C}^{\infty}\left(\mathbb{R}^{n}, \mathbb{R}\right) & \rightarrow \bigcup_{n \in \mathbb{N}} \operatorname{Func}\left(\left(\frac{A}{R}\right)^{n}, \frac{A}{R}\right) \\
\left(f: \mathbb{R}^{n} \stackrel{\mathcal{C}^{\infty}}{\rightarrow} \mathbb{R}\right) & \mapsto\left(\bar{\Phi}(f):\left(\frac{A}{R}\right)^{n} \rightarrow \frac{A}{R}\right)
\end{aligned}
$$

where:

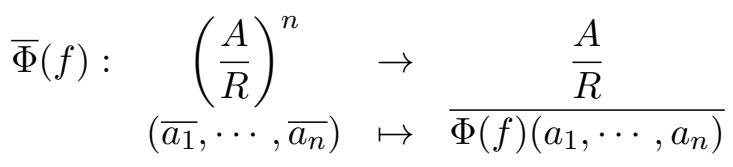

Note that the interpretation above is indeed a function, that is, its value does not depend on any particular choice of the representing element. This means that given $\left(\overline{a_{1}}, \cdots, \overline{a_{n}}\right),\left(\overline{a_{1}^{\prime}}, \cdots, \overline{a_{n}^{\prime}}\right) \in\left(\frac{A}{R}\right)^{n}$ such that $\left(a_{1}, a_{1}^{\prime}\right), \cdots,\left(a_{n}, a_{n}^{\prime}\right) \in R$, we have:

$$
\bar{\Phi}(f)\left(\overline{a_{1}}, \cdots, \overline{a_{n}}\right)=\overline{\Phi(f)\left(a_{1}, \cdots, a_{n}\right)}
$$

and since $R$ is a $\mathcal{C}^{\infty}$-congruence,

$$
\left(a_{1}, a_{1}^{\prime}\right), \cdots,\left(a_{n}, a_{n}^{\prime}\right) \in R \Rightarrow\left(\Phi(f)\left(a_{1}, \cdots, a_{n}\right), \Phi(f)\left(a_{1}^{\prime}, \cdots, a_{n}^{\prime}\right)\right) \in R
$$

so:

$$
\bar{\Phi}\left(\overline{a_{1}}, \cdots, \overline{a_{n}}\right)=\overline{\Phi(f)\left(a_{1}, \cdots, a_{n}\right)}=\overline{\Phi(f)\left(a_{1}^{\prime}, \cdots, a_{n}^{\prime}\right)}=\bar{\Phi}(f)\left(\overline{a_{1}^{\prime}}, \cdots, \overline{a_{n}^{\prime}}\right)
$$

The above construction leads directly to the following:

Definition 1.2.10. Let $(A, \Phi)$ be a $\mathcal{C}^{\infty}$-ring and let $R \subseteq A \times A$ be a $\mathcal{C}^{\infty}$-congruence. The quotient $\mathcal{C}^{\infty}$-ring of $A$ by $R$ is the ordered pair:

$$
\left(\frac{A}{R}, \bar{\Phi}\right)
$$

where:

$$
\frac{A}{R}=\{\bar{a} \mid a \in A\}
$$

and 


$$
\begin{aligned}
\bar{\Phi}: \bigcup_{n \in \mathbb{N}} \mathcal{C}^{\infty}\left(\mathbb{R}^{n}, \mathbb{R}\right) & \rightarrow \bigcup_{n \in \mathbb{N}} \operatorname{Func}\left(\left(\frac{A}{R}\right)^{n}, \frac{A}{R}\right) \\
\left(f: \mathbb{R}^{n} \stackrel{\mathcal{C}^{\infty}}{\rightarrow} \mathbb{R}\right) & \mapsto\left(\bar{\Phi}(f):\left(\frac{A}{R}\right)^{n} \rightarrow \frac{A}{R}\right)
\end{aligned}
$$

where $\bar{\Phi}(f)$ is described in Remark 1.2.9.

The following result shows that the canonical quotient map is, again, a $\mathcal{C}^{\infty}$-homomorphism.

Proposition 1.2.11. Let $(A, \Phi)$ be a $\mathcal{C}^{\infty}$-ring and let $R \subseteq A \times A$ be a $\mathcal{C}^{\infty}$-congruence. The function:

$$
\begin{array}{rlc}
q: \quad(A, \Phi) & \rightarrow & \left(\frac{A}{R}, \bar{\Phi}\right) \\
a & \mapsto & \bar{a}
\end{array}
$$

is a $\mathcal{C}^{\infty}$-homomorphism.

Proof. Let $n \in \mathbb{N}$ and $f \in \mathcal{C}^{\infty}\left(\mathbb{R}^{n}, \mathbb{R}\right)$. We are going to show that the following diagram commutes:

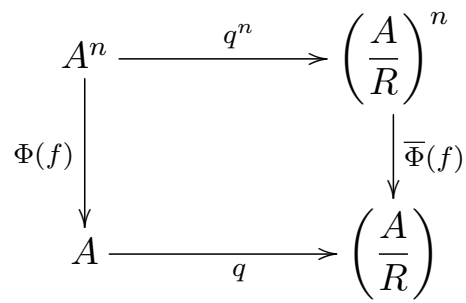

Given $\left(a_{1}, \cdots, a_{n}\right) \in A^{n}$, we have on the one hand:

$$
\bar{\Phi}(f) \circ q^{n}\left(a_{1}, \cdots, a_{n}\right)=\bar{\Phi}(f)\left(q\left(a_{1}\right), \cdots, q\left(a_{n}\right)\right)=\bar{\Phi}(f)\left(\overline{a_{1}}, \cdots, \overline{a_{n}}\right):=\overline{\Phi(f)\left(a_{1}, \cdots, a_{n}\right)}
$$

On the other hand,

$$
q \circ \Phi(f)\left(a_{1}, \cdots, a_{n}\right)=\overline{\Phi(f)\left(a_{1}, \cdots, a_{n}\right)}
$$

so $\bar{\Phi} \circ q^{n}=q \circ \Phi(f)$, and $q$ is a $\mathcal{C}^{\infty}$-homomorphism.

We remark that the structure given above is the unique $\mathcal{C}^{\infty}$-structure such that the quotient map is a $\mathcal{C}^{\infty}$-homomorphism.

Proposition 1.2.12. Let $(A, \Phi)$ and $(B, \Psi)$ be two $\mathcal{C}^{\infty}$ _rings and let $\varphi:(A, \Phi) \rightarrow(B, \Psi)$ be a $\mathcal{C}^{\infty}$-homomorphism. The set:

$$
\operatorname{ker}(\varphi)=\left\{\left(a, a^{\prime}\right) \in A \times A \mid \varphi(a)=\varphi\left(a^{\prime}\right)\right\}
$$

is a $\mathcal{C}^{\infty}$-congruence on $(A, \Phi)$.

Proof. It is easy to check that $\operatorname{ker}(\varphi)$ is an equivalence relation on $A$.

Let $n \in \mathbb{N}, f \in \mathcal{C}^{\infty}\left(\mathbb{R}^{n}, \mathbb{R}\right)$ and $\left(a_{1}, a_{1}^{\prime}\right), \cdots,\left(a_{n}, a_{n}^{\prime}\right) \in \operatorname{ker}(\varphi)$, that is:

$$
(\forall i \in\{1, \cdots, n\})\left(\varphi\left(a_{i}\right)=\varphi\left(a_{i}^{\prime}\right)\right)
$$


We are going to show that $\left(\Phi(f)\left(a_{1}, \cdots, a_{n}\right), \Phi(f)\left(a_{1}^{\prime}, \cdots, a_{n}^{\prime}\right)\right) \in \operatorname{ker}(\varphi)$.

Since $\varphi$ is a $\mathcal{C}^{\infty}$-homomorphism, we have the following commutative diagram:

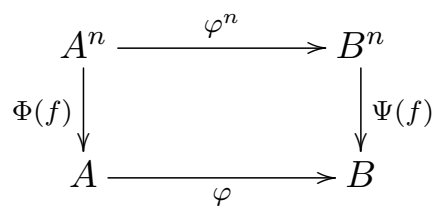

thus, we have:

$$
\varphi\left(\Phi(f)\left(a_{1}, \cdots, a_{n}\right)\right)=\Psi(f)\left(\varphi\left(a_{1}\right), \cdots, \varphi\left(a_{n}\right)\right)=\Psi(f)\left(\varphi\left(a_{1}^{\prime}\right), \cdots, \varphi\left(a_{n}^{\prime}\right)\right)=\varphi\left(\Phi(f)\left(a_{1}^{\prime}, \cdots, a_{n}^{\prime}\right)\right)
$$

and $\left(\Phi(f)\left(a_{1}, \cdots, a_{n}\right), \Phi(f)\left(a_{1}^{\prime}, \cdots, a_{n}^{\prime}\right)\right) \in \operatorname{ker}(\varphi)$.

Corollary 1.2.13. Let $(A, \Phi)$ and $(B, \Psi)$ be two $\mathcal{C}^{\infty}$-rings and let $\varphi:(A, \Phi) \rightarrow(B, \Psi)$ be a $\mathcal{C}^{\infty}$-homomorphism. Then $\left(\operatorname{ker}(\varphi), \Phi^{(2)^{\prime}}\right)$ is a $\mathcal{C}^{\infty}$-subring of $\left(A \times A, \Phi^{(2)}\right)$.

Proposition 1.2.14. For every $\mathcal{C}^{\infty}$-congruence $R \subseteq A \times A$ in $(A, \Phi)$, there are some $\mathcal{C}^{\infty}$-ring $(B, \Psi)$ and some $\mathcal{C}^{\infty}$-homomorphism $\varphi:(A, \Phi) \rightarrow(B, \Psi)$ such that $R=\operatorname{ker}(\varphi)$.

Proof. It suffices to take $(B, \Psi)=\left(\frac{A}{R}, \bar{\Phi}\right)$ and $\varphi=q_{R}:(A, \Phi) \rightarrow\left(\frac{A}{R}, \bar{\Phi}\right)$

Theorem 1.2.15. Fundamental Theorem of the $\mathcal{C}^{\infty}$-Homomorphism Let $(A, \Phi)$ be a $\mathcal{C}^{\infty}$-ring and $R \subseteq A \times A$ be a $\mathcal{C}^{\infty}$-congruence. For every $\mathcal{C}^{\infty}$-ring $(B, \Psi)$ and for every $\mathcal{C}^{\infty}$-homomorphism $\varphi:(A, \Phi) \rightarrow(B, \Psi)$ such that $R \subseteq \operatorname{ker}(\varphi)$, that is, such that:

$$
\left(a, a^{\prime}\right) \in R \Rightarrow \varphi(a)=\varphi\left(a^{\prime}\right),
$$

there is a unique $\mathcal{C}^{\infty}$-homomorphism:

$$
\widetilde{\varphi}:\left(\frac{A}{R}, \bar{\Phi}\right) \rightarrow(B, \Psi)
$$

such that the following diagram commutes:

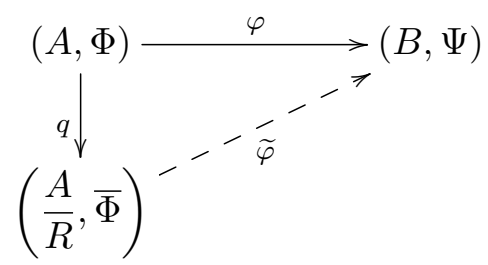

that is, such that $\widetilde{\varphi} \circ q=\varphi$, where $\bar{\Phi}$ is the canonical $\mathcal{C}^{\infty}$-structure induced on the quotient $\frac{A}{R}$

Proof. Define:

$$
\begin{aligned}
\widetilde{\varphi}: \frac{A}{R} & \rightarrow B \\
\bar{a} & \mapsto \varphi(a)
\end{aligned}
$$


and note that $\widetilde{\varphi}$ defines a function, since given $\left(a, a^{\prime}\right) \in R$, i.e., such that $\bar{a}=\overline{a^{\prime}}$, we have $\varphi(a)=\varphi\left(a^{\prime}\right)$, so $\widetilde{\varphi}(\bar{a})=\varphi(a)=\varphi\left(a^{\prime}\right)=\widetilde{\varphi}\left(\overline{a^{\prime}}\right)$.

As functions, we have $\widetilde{\varphi} \circ q=\varphi$.

Given $n \in \mathbb{N}$ and $f \in \mathcal{C}^{\infty}\left(\mathbb{R}^{n}, \mathbb{R}\right)$, the following diagram commutes,

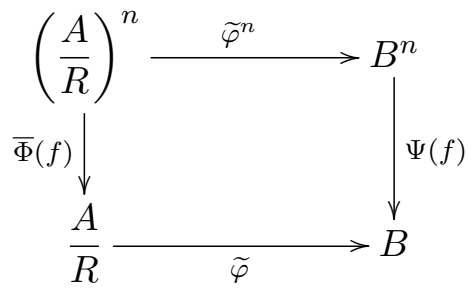

since for any $\left(\overline{a_{1}}, \cdots, \overline{a_{n}}\right) \in\left(\frac{A}{R}\right)^{n}$ we have:

$$
\widetilde{\varphi} \circ \bar{\Phi}(f)\left(\overline{a_{1}}, \cdots, \overline{a_{n}}\right)=\widetilde{\varphi}\left(\overline{\Phi(f)\left(a_{1}, \cdots, a_{n}\right)}\right):=\varphi\left(\Phi(f)\left(a_{1}, \cdots, a_{n}\right)\right)
$$

and since $\varphi$ is a $\mathcal{C}^{\infty}$-homomorphism, we have:

$$
\varphi\left(\Phi(f)\left(a_{1}, \cdots, a_{n}\right)\right)=\Psi(f)\left(\varphi\left(a_{1}\right), \cdots, \varphi\left(a_{n}\right)\right)
$$

thus:

$$
\widetilde{\varphi} \circ \bar{\Phi}(f)\left(\overline{a_{1}}, \cdots, \overline{a_{n}}\right)=\Psi(f)\left(\varphi\left(a_{1}\right), \cdots, \varphi\left(a_{n}\right)\right) .
$$

On the other hand, we have:

$$
\Psi(f) \circ \widetilde{\varphi}^{n}\left(\overline{a_{1}}, \cdots, \overline{a_{n}}\right)=\Psi(f)\left(\widetilde{\varphi}\left(\overline{a_{1}}\right), \cdots, \widetilde{\varphi}\left(\overline{a_{n}}\right)\right)=\Psi(f)\left(\varphi\left(a_{1}\right), \cdots, \varphi\left(a_{n}\right)\right)
$$

so

$$
\left(\forall\left(\overline{a_{1}}, \cdots, \overline{a_{n}}\right) \in\left(\frac{A}{R}\right)^{n}\right)\left(\widetilde{\varphi} \circ \bar{\Phi}(f)\left(\overline{a_{1}}, \cdots, \overline{a_{n}}\right)=\Psi(f) \circ \widetilde{\varphi}^{n}\left(\overline{a_{1}}, \cdots, \overline{a_{n}}\right)\right),
$$

and $\widetilde{\varphi}$ is a $\mathcal{C}^{\infty}$-homomorphism.

Thus we have the following equation of $\mathcal{C}^{\infty}$-homomorphisms:

$$
\widetilde{\varphi} \circ q=\varphi
$$

Since $q$ is surjective, it follows that $\widetilde{\varphi}$ is unique.

The following result is straightforward:

Proposition 1.2.16. Let $(A, \Phi)$ and $(B, \Psi)$ be two $\mathcal{C}^{\infty}$ _rings and let $\varphi:(A, \Phi) \rightarrow(B, \Psi)$ be a $\mathcal{C}^{\infty}$-homomorphism. The ordered pair:

$$
\left(\varphi[A], \Psi^{\prime}\right)
$$

where: 


$$
\begin{aligned}
& \Psi^{\prime}: \bigcup_{n \in \mathbb{N}} \mathcal{C}^{\infty}\left(\mathbb{R}^{n}, \mathbb{R}\right) \rightarrow \bigcup_{n \in \mathbb{N}} \text { Func }\left(\varphi[A]^{n}, \varphi[A]\right) \\
& \left(\mathbb{R}^{n} \stackrel{f}{\rightarrow} \mathbb{R}\right) \quad \mapsto \Psi(f) \uparrow_{\varphi[A]^{n}}: \varphi[A]^{n} \rightarrow \varphi[A]
\end{aligned}
$$

is a $\mathcal{C}^{\infty}$-subring of $(B, \Psi)$, called the homomorphic image of $A$ by $\varphi$.

Corollary 1.2.17. Let $(A, \Phi)$ and $(B, \Psi)$ be two $\mathcal{C}^{\infty}$-rings and let $\varphi:(A, \Phi) \rightarrow(B, \Psi)$ be a $\mathcal{C}^{\infty}$-homomorphism. As we have noticed in Proposition 1.2.16, $\left(\varphi[A], \Psi^{\prime}\right)$ is a $\mathcal{C}^{\infty}-\operatorname{subring}$ of $(B, \Psi)$.

Under these circumstances, there is a unique $\mathcal{C}^{\infty}$-isomorphism:

$$
\widetilde{\varphi}:\left(\frac{A}{\operatorname{ker}(\varphi)}, \bar{\Phi}\right) \rightarrow\left(\varphi[A], \Psi^{\prime}\right)
$$

such that the following diagram commutes:

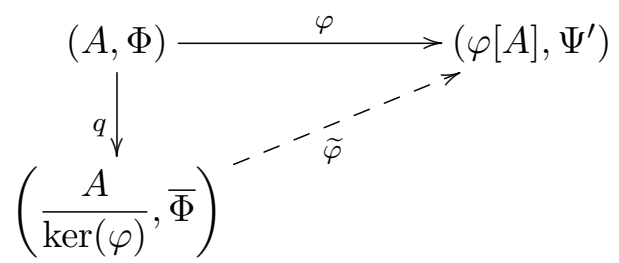

that is, such that $\widetilde{\varphi} \circ q=\varphi$, where $\bar{\Phi}$ is the canonical $\mathcal{C}^{\infty}$-structure induced on the quotient $\frac{A}{\operatorname{ker}(\varphi)}$

Proof. Applying the previous result to $R=\operatorname{ker}(\varphi)$ yields the existence of a unique $\mathcal{C}^{\infty}$-homomorphism such that the diagram commutes. We need only to prove that $\widetilde{\varphi}$ is bijective.

Given $\bar{a}, \overline{a^{\prime}}$ such that $\widetilde{\varphi}(\bar{a})=\widetilde{\varphi}\left(\overline{a^{\prime}}\right)$, by definition we have $\varphi(a)=\varphi\left(a^{\prime}\right)$, so $\left(a, a^{\prime}\right) \in \operatorname{ker}(\varphi)$ and $\bar{a}=\overline{a^{\prime}}$.

Also, given any $y \in \varphi[A]$, there is some $a \in A$ such that $\varphi(a)=y$. Thus, $\widetilde{\varphi}(\bar{a})=\varphi(a)=y$, so $\varphi$ is surjective.

The following result is going to be used to construct directed colimits of $\mathcal{C}^{\infty}$-rings.

Lemma 1.2.18. Let $(A, \Phi)$ be a $\mathcal{C}^{\infty}$-ring. The ordered pair:

$$
\left(A \times\{\alpha\}, \Phi \times \operatorname{id}_{\alpha}\right)
$$

where:

$$
\begin{aligned}
& \Phi \times \operatorname{id}_{\alpha}: \bigcup_{n \in \mathbb{N}} \mathcal{C}^{\infty}\left(\mathbb{R}^{n}, \mathbb{R}\right) \rightarrow \bigcup_{n \in \mathbb{N}} \text { Func }\left((A \times\{\alpha\})^{n}, A \times\{\alpha\}\right)
\end{aligned}
$$

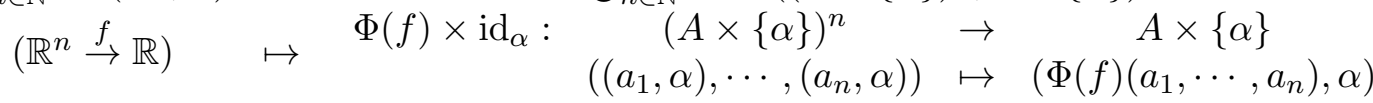

is a $\mathcal{C}^{\infty}$-ring and:

$$
\begin{aligned}
\pi_{1}: A \times\{\alpha\} & \rightarrow A \\
(a, \alpha) & \mapsto a
\end{aligned}
$$

is a $\mathcal{C}^{\infty}$-isomorphism, that is:

$$
(A, \Phi) \cong_{\pi_{1}}\left(A \times\{\alpha\}, \Phi \times \operatorname{id}_{\alpha}\right)
$$


Proof. It is clear that $\pi_{1}$ is a bijection, so it suffices to prove it is a $\mathcal{C}^{\infty}$-homomorphism such that its inverse:

$$
\begin{aligned}
\iota_{1}: A & \rightarrow A \times\{\alpha\} \\
a & \mapsto(a, \alpha)
\end{aligned}
$$

is a $\mathcal{C}^{\infty}$-homomorphism.

Let $n \in \mathbb{N}$ and $f \in \mathcal{C}^{\infty}\left(\mathbb{R}^{n}, \mathbb{R}\right)$. Given $\left(\left(a_{1}, \alpha\right), \cdots,\left(a_{n}, \alpha\right)\right) \in(A \times\{\alpha\})^{n}$, we have:

$$
\pi_{1} \circ\left(\left(\Phi \times \operatorname{id}_{\alpha}\right)(f)\right)\left(\left(a_{1}, \alpha\right), \cdots,\left(a_{n}, \alpha\right)\right)=\pi_{1}\left(\Phi(f)\left(a_{1}, \cdots, a_{n}\right), \alpha\right)=\Phi(f)\left(a_{1}, \cdots, a_{n}\right)
$$

and

$$
\Phi(f) \circ \pi_{1}^{n}\left(\left(a_{1}, \alpha\right), \cdots,\left(a_{n}, \alpha\right)\right)=\Phi(f)\left(\pi_{1}\left(a_{1}, \alpha\right), \cdots, \pi_{1}\left(a_{n}, \alpha\right)\right)=\Phi(f)\left(a_{1}, \cdots, a_{n}\right) .
$$

Thus, the following diagram commutes:

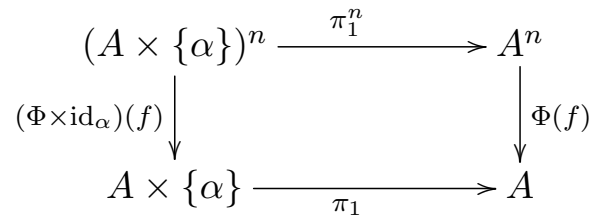

and $\pi_{1}$ is a $\mathcal{C}^{\infty}$-homomorphism.

Also, for any $\left(a_{1}, \cdots, a_{n}\right) \in A^{n}$ we have:

$$
\left(\Phi \times \operatorname{id}_{\alpha}\right) \circ \iota^{n}\left(a_{1}, \cdots, a_{n}\right)=\left(\Phi \times \operatorname{id}_{\alpha}\right)\left(\left(a_{1}, \alpha\right), \cdots,\left(a_{n}, \alpha\right)\right)=\left(\Phi(f)\left(a_{1}, \cdots, a_{n}\right), \alpha\right)
$$

and

$$
\iota \circ \Phi(f)\left(a_{1}, \cdots, a_{n}\right)=\left(\Phi(f)\left(a_{1}, \cdots, a_{n}\right), \alpha\right)
$$

so the following diagram commutes:

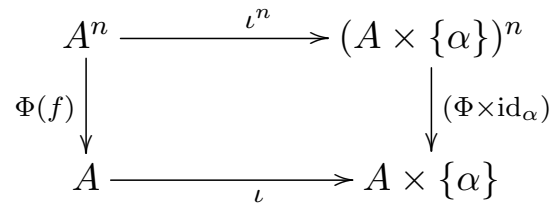

and $\iota$ is a $\mathcal{C}^{\infty}$-homomorphism, inverse to $\pi_{1}$.

Now we are going to describe the construction of directed colimits of directed families of $\mathcal{C}^{\infty}$-rings.

Theorem 1.2.19. Let $(I, \leq)$ be a directed set and let $\left(\left(A_{\alpha}, \Phi_{\alpha}\right), \mu_{\alpha \beta}\right)_{\alpha, \beta \in I}$ be a directed system. There is an object $(A, \Phi)$ in $\mathcal{C}^{\infty} \mathbf{R n g}$ such that:

$$
(A, \Phi) \cong \underset{\alpha \in I}{\lim }\left(A_{\alpha}, \Phi_{\alpha}\right)
$$


Proof. Let:

$$
A^{\prime}:=\bigcup_{\alpha \in I} A_{\alpha} \times\{\alpha\}
$$

and consider the following equivalence relation:

$$
R=\left\{((a, \alpha),(b, \beta)) \in A^{\prime} \times A^{\prime} \mid(\exists \gamma \in I)(\gamma \geq \alpha, \beta)\left(\mu_{\alpha \gamma}(a)=\mu_{\beta \gamma}(b)\right)\right\}
$$

Take $A=\frac{A^{\prime}}{R}$ and define the following $\mathcal{C}^{\infty}$-structure on $A$ :

$$
\begin{array}{rlc}
\Phi: \bigcup_{n \in \mathbb{N}} \mathcal{C}^{\infty}\left(\mathbb{R}^{n}, \mathbb{R}\right) & \rightarrow & \bigcup_{n \in \mathbb{N}} \operatorname{Func}\left(A^{n}, A\right) \\
\left(\mathbb{R}^{n} \stackrel{f}{\rightarrow} \mathbb{R}\right) & \mapsto & \left(A^{n} \stackrel{\Phi(f)}{\rightarrow} A\right)
\end{array}
$$

where $\Phi(f): A^{n} \rightarrow A$ is defined as follows: given $\left(\overline{\left(a_{1}, \alpha_{1}\right)}, \cdots, \overline{\left(a_{n}, \alpha_{n}\right)}\right) \in A^{n}$, we have $\left\{\alpha_{1}, \cdots, \alpha_{n}\right\} \subseteq$ $I$, and since $(I, \leq)$ is directed, there is some $\alpha \in I$ such that:

$$
(\forall i \in\{1, \cdots, n\})\left(\alpha_{i} \leq \alpha\right)
$$

We define:

$$
\Phi(f)\left(\overline{\left(a_{1}, \alpha_{1}\right)}, \cdots, \overline{\left(a_{n}, \alpha_{n}\right)}\right):=\overline{\left(\Phi_{\alpha}(f)\left(\mu_{\alpha_{1} \alpha}\left(a_{1}\right), \cdots, \mu_{\alpha_{n} \alpha}\left(a_{n}\right)\right), \alpha\right)}
$$

We are going to show that this definition is independent of the choice of $\alpha$.

Let $\beta \geq \alpha_{1}, \cdots, \alpha_{n}$ so we have:

$$
\Phi(f)\left(\overline{\left(a_{1}, \alpha_{1}\right)}, \cdots, \overline{\left(a_{n}, \alpha_{n}\right)}\right)=\overline{\left(\Phi_{\beta}(f)\left(\mu_{\alpha_{1} \beta}\left(a_{1}\right), \cdots, \mu_{\alpha_{n} \beta}\left(a_{n}\right)\right), \beta\right)}
$$

We need to show that there is some $\gamma \in I$ such that $\gamma \geq \alpha, \beta$ and:

$$
\left.\left.\mu_{\alpha \gamma}\left(\Phi_{\alpha}(f)\left(\mu_{\alpha_{1} \alpha}\left(a_{i}\right)\right), \cdots, \mu_{\alpha_{n} \alpha}\left(a_{n}\right)\right)\right)=\mu_{\beta \gamma}\left(\Phi_{\beta}(f)\left(\mu_{\alpha_{1} \beta}\left(a_{i}\right)\right), \cdots, \mu_{\alpha_{n} \beta}\left(a_{n}\right)\right)\right)
$$

Choose $\gamma \geq \alpha, \beta$. Then:

$$
(\forall i \in\{1, \cdots, n\})\left(\mu_{\beta \gamma} \circ \mu_{\alpha_{i} \beta}=\mu_{\alpha \beta} \circ \mu_{\alpha_{i} \alpha}\right)
$$

Since $\mu_{\alpha \gamma}:\left(A_{\alpha}, \Phi_{\alpha}\right) \rightarrow\left(A_{\gamma}, \Phi_{\gamma}\right)$ is a $\mathcal{C}^{\infty}$-homomorphism, we have the following commutative diagram:

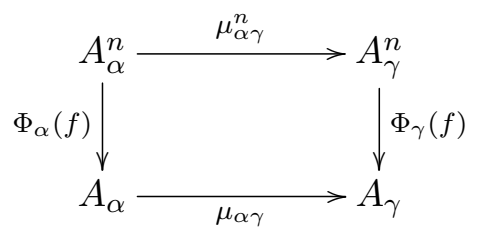

$\mathrm{SO}$

$$
\mu_{\alpha \gamma}\left(\Phi_{\alpha}(f)\left(\mu_{\alpha_{1} \alpha}\left(a_{1}\right)\right), \cdots, \Phi_{\alpha}(f)\left(\mu_{\alpha_{n} \alpha}\left(a_{n}\right)\right)\right)=\Phi_{\gamma}(f)\left(\mu_{\alpha \gamma}\left(\mu_{\alpha_{1} \alpha}\left(a_{1}\right)\right), \cdots, \mu_{\alpha \gamma}\left(\mu_{\alpha_{n} \alpha}\left(a_{n}\right)\right)\right)
$$

and since:

$$
(\forall i \in\{1, \cdots, n\})\left(\mu_{\alpha \gamma} \circ \mu_{\alpha_{i} \alpha}=\mu_{\alpha_{i} \gamma}\right)
$$


we have:

$$
\mu_{\alpha \gamma}\left(\Phi_{\alpha}(f)\left(\mu_{\alpha_{1} \alpha}\left(a_{1}\right)\right), \cdots, \Phi_{\alpha}(f)\left(\mu_{\alpha_{n} \alpha}\left(a_{n}\right)\right)\right)=\Phi_{\gamma}(f)\left(\mu_{\alpha_{1} \gamma}\left(a_{1}\right), \cdots, \mu_{\alpha_{n} \gamma}\left(a_{n}\right)\right)
$$

Also, since $\mu_{\beta \gamma}:\left(A_{\beta}, \Phi_{\beta}\right) \rightarrow\left(A_{\gamma}, \Phi_{\gamma}\right)$ is a $\mathcal{C}^{\infty}$-homomorphism, we have the following commutative diagram:

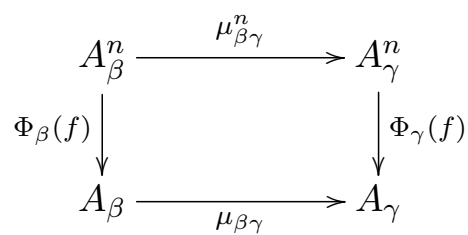

so

$$
\mu_{\beta \gamma}\left(\Phi_{\beta}(f)\left(\mu_{\alpha_{1} \beta}\left(a_{1}\right)\right), \cdots, \Phi_{\beta}(f)\left(\mu_{\alpha_{n} \beta}\left(a_{n}\right)\right)\right)=\Phi_{\gamma}(f)\left(\mu_{\beta \gamma}\left(\mu_{\alpha_{1} \beta}\left(a_{1}\right)\right), \cdots, \mu_{\beta \gamma}\left(\mu_{\alpha_{n} \beta}\left(a_{n}\right)\right)\right)
$$

and since:

$$
(\forall i \in\{1, \cdots, n\})\left(\mu_{\beta \gamma} \circ \mu_{\alpha_{i} \beta}=\mu_{\alpha_{i} \gamma}\right)
$$

we have:

$$
\mu_{\beta \gamma}\left(\Phi_{\beta}(f)\left(\mu_{\alpha_{1} \beta}\left(a_{1}\right)\right), \cdots, \Phi_{\beta}(f)\left(\mu_{\alpha_{n} \beta}\left(a_{n}\right)\right)\right)=\Phi_{\gamma}(f)\left(\mu_{\alpha_{1} \gamma}\left(a_{1}\right), \cdots, \mu_{\alpha_{n} \gamma}\left(a_{n}\right)\right)
$$

Comparing (1.1) and (1.2), we get:

$$
\mu_{\alpha \gamma}\left(\Phi_{\alpha}(f)\left(\mu_{\alpha_{1} \alpha}\left(a_{1}\right)\right), \cdots, \Phi_{\alpha}(f)\left(\mu_{\alpha_{n} \alpha}\left(a_{n}\right)\right)\right)=\mu_{\beta \gamma}\left(\Phi_{\beta}(f)\left(\mu_{\alpha_{1} \beta}\left(a_{1}\right)\right), \cdots, \Phi_{\beta}(f)\left(\mu_{\alpha_{n} \beta}\left(a_{n}\right)\right)\right)
$$

so

$$
\left(\overline{\Phi_{\alpha}\left(\mu_{\alpha_{1} \alpha}\left(a_{1}\right), \cdots, \mu_{\alpha_{n} \alpha}\left(a_{n}\right)\right)}, \alpha\right)=\overline{\left(\Phi_{\beta}\left(\mu_{\alpha_{1} \beta}\left(a_{1}\right), \cdots, \mu_{\alpha_{n} \beta}\left(a_{n}\right)\right), \beta\right)}
$$

and $\Phi(f)$ does not depend on the choice of the index. The definition of $\Phi(f)$ does not depend on the choice of the representing elements, $\left(a_{i}, \alpha_{i}\right)+R$, either ${ }^{2}$

Hence $\Phi$ is a $\mathcal{C}^{\infty}$-structure on $A$ and this shows that $(A, \Phi)$ is a $\mathcal{C}^{\infty}$-ring.

For each $\alpha \in I$ we define:

$$
\begin{aligned}
\eta_{\alpha}: A_{\alpha} \times\{\alpha\} & \rightarrow \frac{A}{(a, \alpha)} \\
(a, \alpha) & \mapsto \frac{1}{(a, \alpha)}
\end{aligned}
$$

Claim: $\eta_{\alpha}: A_{\alpha} \times\{\alpha\} \rightarrow A$ is a $\mathcal{C}^{\infty}$-homomorphism between $\left(A_{\alpha} \times\{\alpha\}, \Phi_{\alpha} \times \operatorname{id}_{\alpha}\right)$ and $(A, \Phi)$.

Let $n \in \mathbb{N}$ and $f \in \mathcal{C}^{\infty}\left(\mathbb{R}^{n}, \mathbb{R}\right)$. Given $\left(\left(a_{1}, \alpha\right), \cdots,\left(a_{n}, \alpha\right)\right) \in\left(A_{\alpha} \times\{\alpha\}\right)^{n}$. We have:

$$
\Phi(f) \circ \eta_{\alpha}^{n}\left(\left(a_{1}, \alpha\right), \cdots,\left(a_{n}, \alpha\right)\right):=\left(\Phi_{\alpha} \times \operatorname{id}_{\alpha}\right)(f)\left(\eta_{\alpha}\left(a_{1}, \alpha\right), \cdots, \eta_{\alpha}\left(a_{n}, \alpha\right)\right)=\overline{\left(\Phi_{\alpha}(f)\left(a_{1}, \cdots, a_{n}\right), \alpha\right)}
$$

\footnotetext{
${ }^{2}$ the proof of this fact is analogous to the one we just made.
} 
and

$$
\eta_{\alpha} \circ\left(\Phi_{\alpha} \times \operatorname{id}_{\alpha}\right)(f)\left(\left(a_{1}, \alpha\right), \cdots,\left(a_{n}, \alpha\right)\right)=\eta_{\alpha}\left(\Phi_{\alpha}(f)\left(a_{1}, \cdots, a_{n}\right), \alpha\right)=\overline{\left(\Phi_{\alpha}(f)\left(a_{1}, \cdots, a_{n}\right), \alpha\right)}
$$

so the following diagram commutes:

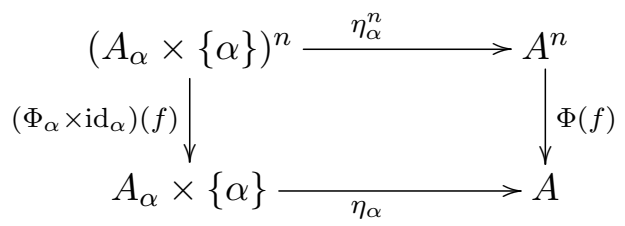

Now we take, for each $\alpha \in I$ the following $\mathcal{C}^{\infty}$-homomorphism:

$$
\lambda_{\alpha}:=\eta_{\alpha} \circ \iota_{\alpha}: A_{\alpha} \rightarrow A
$$

where $\iota_{\alpha}: A_{\alpha} \rightarrow A_{\alpha} \times\{\alpha\}$ is the $\mathcal{C}^{\infty}$-isomorphism described in Lemma 1.2.18,

Claim: $(\forall \alpha \in I)(\forall \beta \in I)\left(\alpha \leq \beta \rightarrow \lambda_{\beta} \circ \mu_{\alpha \beta}=\lambda_{\alpha}\right)$, that is, the following diagram commutes:

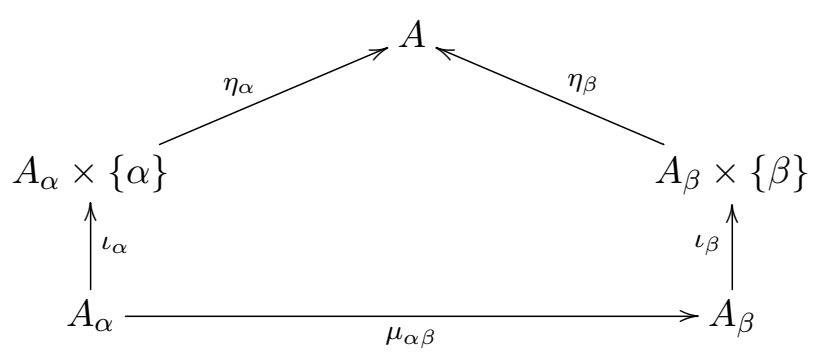

Indeed, given $a \in A_{\alpha}$, we have:

$$
\lambda_{\beta} \circ \mu_{\alpha \beta}(a)=\eta_{\beta} \circ \iota_{\beta}\left(\mu_{\alpha \beta}(a)\right)=\eta_{\beta}\left(\mu_{\alpha \beta}(a), \beta\right)=\overline{\left(\mu_{\alpha \beta}(a), \beta\right)}
$$

and

$$
\lambda_{\alpha}(a)=\eta_{\alpha}\left(\iota_{\alpha}(a)\right)=\eta_{\alpha}(a, \alpha)=\overline{(a, \alpha)}
$$

Given any $\gamma \geq \alpha, \beta$, we have $\mu_{\alpha \gamma}=\mu_{\beta \gamma} \circ \mu_{\alpha \beta}$, so:

$$
\mu_{\alpha \gamma}(a)=\mu_{\beta \gamma}\left(\mu_{\alpha \beta}(a)\right)
$$

and $\left((a, \alpha),\left(\mu_{\alpha \beta}(a), \beta\right)\right) \in R$, hence $\lambda_{\beta} \circ \mu_{\alpha \beta}(a)=\lambda_{\alpha}(a)$, and the diagram commutes.

Now we need only to show that $\left((A, \Phi), \lambda_{\alpha}\right)$ has the universal property of the colimit.

Let $(B, \Psi)$ be a $\mathcal{C}^{\infty}$-ring and let $\zeta_{\alpha}: A_{\alpha} \rightarrow B$ be a family of $\mathcal{C}^{\infty}$-homomorphisms such that for every $\alpha, \beta \in I$ with $\alpha \leq \beta$ we have $\zeta_{\beta} \circ \mu_{\alpha \beta}=\zeta_{\alpha}$, that is, the diagram:

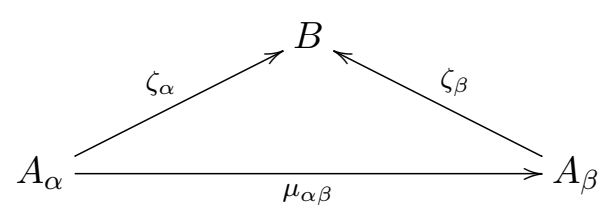


commutes. We are going to show that there is a unique $\mathcal{C}^{\infty}$-homomorphism $\theta: A \rightarrow B$ such that the following diagram commutes:

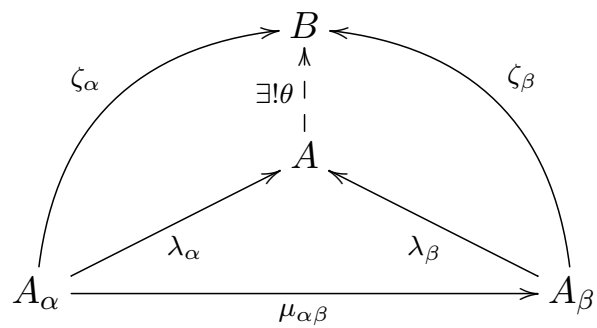

Indeed, given any $\overline{(a, \alpha)} \in A$, we have $(a, \alpha)+R=\lambda_{\alpha}(a)=\lambda_{\beta}\left(\mu_{\alpha \beta}(a)\right)$, so we define:

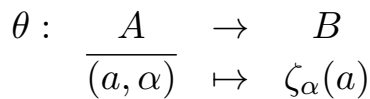

which is a function, since $\left(\zeta_{\alpha}\right)_{\alpha \in I}$ is a commutative co-cone.

Claim: $\theta:(A, \Phi) \rightarrow(B, \Psi)$ is a $\mathcal{C}^{\infty}$-homomorphism.

Let $n \in \mathbb{N}$ and $f \in \mathcal{C}^{\infty}\left(\mathbb{R}^{n}, \mathbb{R}\right)$. Given $\left(\overline{\left(a_{1}, \alpha_{1}\right)}, \cdots, \overline{\left(a_{n}, \alpha_{n}\right)}\right) \in A^{n}$, we have, by definition,

$$
\Phi(f)\left(\overline{\left(a_{1}, \alpha_{1}\right)}, \cdots, \overline{\left(a_{n}, \alpha_{n}\right)}\right)=\overline{\left(\Phi_{\alpha}(f)\left(a_{1}, \cdots, a_{n}\right), \alpha\right)}
$$

for every $\alpha \in I$ such that $\alpha \geq \alpha_{i}$, for every $i \in\{1, \cdots, n\}$. Thus:

$$
\begin{array}{r}
\theta \circ \Phi(f)\left(\overline{\left(a_{1}, \alpha_{1}\right)}, \cdots, \overline{\left(a_{n}, \alpha_{n}\right)}\right)=\theta\left(\overline{\left(\Phi_{\alpha}(f)\left(\mu_{\alpha_{1} \alpha}\left(a_{1}\right), \cdots, \mu_{\alpha_{n} \alpha}\left(a_{n}\right)\right), \alpha\right)}\right)= \\
=\zeta_{\alpha}\left(\Phi_{\alpha}(f)\left(\mu_{\alpha_{1} \alpha}\left(a_{1}\right), \cdots, \mu_{\alpha_{n} \alpha}\left(a_{n}\right)\right)\right)
\end{array}
$$

On the other hand,

$$
\begin{aligned}
\Psi(f) \circ \theta^{n}\left(\left(a_{1}, \alpha_{1}\right)+R, \cdots,\left(a_{n}, \alpha_{n}\right)+R\right)=\Psi(f)\left(\zeta_{\alpha_{1}}\left(a_{1}\right),\right. & \left.\cdots, \zeta_{\alpha_{n}}\left(a_{n}\right)\right)= \\
& =\Psi(f)\left(\zeta_{\alpha}\left(\mu_{\alpha_{1} \alpha}\left(a_{1}\right)\right), \cdots, \zeta_{\alpha}\left(\mu_{\alpha_{n} \alpha}\left(a_{n}\right)\right)\right)
\end{aligned}
$$

Since for every $\alpha \in I, \zeta_{\alpha}: A_{\alpha} \rightarrow B$ is a $\mathcal{C}^{\infty}$-homomorphism, it follows that:

$$
\zeta_{\alpha}\left(\Phi_{\alpha}(f)\left(\mu_{\alpha_{1} \alpha}\left(a_{1}\right), \cdots, \mu_{\alpha_{n} \alpha}\left(a_{n}\right)\right)\right)=\Psi(f)\left(\zeta_{\alpha}\left(\mu_{\alpha_{1} \alpha}\left(a_{1}\right)\right), \cdots, \zeta_{\alpha}\left(\mu_{\alpha_{n} \alpha}\left(a_{n}\right)\right)\right)
$$

so $\theta$ is a $\mathcal{C}^{\infty}$-homomorphism.

Now, given $a \in A_{\alpha}$, we have:

$$
\theta\left(\lambda_{\alpha}(a)\right)=\theta(\overline{(a, \alpha)})=\zeta_{\alpha}(a)
$$

so the required diagram commutes.

Since $A=\bigcup_{\alpha \in I} \lambda_{\alpha}\left[A_{\alpha}\right], \theta$ is uniquely determined, and the result follows. 
Theorem 1.2.20. Given any small category $\mathcal{J}$ and any diagram:

$$
\begin{array}{llcc}
D: & \mathcal{J} & \rightarrow & \mathcal{C}^{\infty} \mathbf{R n g} \\
(\alpha \stackrel{h}{\rightarrow} \beta) & \mapsto & \left(A_{\alpha}, \Phi_{\alpha}\right) \stackrel{D(h)}{\rightarrow}\left(A_{\beta}, \Phi_{\beta}\right)
\end{array}
$$

there is a $\mathcal{C}^{\infty}-\operatorname{ring}(A, \Phi)$ such that:

$$
(A, \Phi) \cong \lim _{\alpha \in I} D(\alpha)
$$

Proof. Consider the product $\mathcal{C}^{\infty}$-ring:

$$
\left(\prod_{i \in I} A_{i}, \Phi^{(I)}\right)
$$

and take:

$$
A=\left\{\left(a_{\alpha}\right)_{\alpha \in I} \in \prod_{\alpha \in I} A_{\alpha} \mid(\forall \alpha, \beta \in I)(\forall \alpha \stackrel{h}{\rightarrow} \beta)\left(D(h)\left(a_{\alpha}\right)=a_{\beta}\right)\right\} \subseteq \prod_{\alpha \in I} A_{\alpha}
$$

together with the $\mathcal{C}^{\infty}$-subring structure $\Phi^{\prime}$ of $\Phi^{(I)}$.

We have $\left(\left(A, \Phi^{\prime}\right), \pi_{\alpha} \Gamma_{A}: A \rightarrow A_{\alpha}\right) \cong \lim _{\alpha \in I}\left(A_{\alpha}, \Phi_{\alpha}\right)$

Remark 1.2.21. Let $\Sigma=\bigcup_{n \in \mathbb{N}} \mathcal{C}^{\infty}\left(\mathbb{R}^{n}, \mathbb{R}\right)$ and let $X=\left\{x_{1}, x_{2}, \cdots, x_{n}, \cdots\right\}$ be a denumerable set of variables, so $F(\Sigma, X)$ will denote the algebra of terms of this language $\Sigma$. A class of ordered pairs will be simply a subset $S \subseteq F(\Sigma, X) \times F(\Sigma, X)$. In our case, these pairs are given by the axioms, so $S$ consists of the following:

- For any $n \in \mathbb{R}, i \leq n$ and a (smooth) projection map $p_{i}: \mathbb{R}^{n} \rightarrow \mathbb{R}$ we have:

$$
\left(p_{i}\left(x_{1}, \cdots, x_{i}, \cdots, x_{n}\right), x_{i}\right) \in S
$$

- for every $f, g_{1}, \cdots, g_{n} \in \mathcal{C}^{\infty}\left(\mathbb{R}^{m}, \mathbb{R}\right)$ and $h \in \mathcal{C}^{\infty}\left(\mathbb{R}^{n}, \mathbb{R}\right)$ such that $f=h \circ\left(g_{1}, \cdots, g_{n}\right)$, we have

$$
\left(h\left(g_{1}\left(x_{1}, \cdots, x_{m}\right), \cdots, g_{n}\left(x_{1}, \cdots, x_{m}\right)\right), f\left(x_{1}, \cdots, x_{m}\right)\right) \in S
$$

Noting that the category of $\mathcal{C}^{\infty}$-rings is closed under products, homomorphic images and subalgebras, by the HSP Birkhoff's Theorem, $\mathcal{C}^{\infty} \mathbf{R n g}$ is a variety, and by the previous remark, $\mathcal{C}^{\infty} \mathbf{R n g}=V(S)$, the variety of algebras defined by $S$. In particular, we have some classical results. We list some of them:

- for every set $X$ there is a free $\mathcal{C}^{\infty}$-ring determined by $X$;

- any $\mathcal{C}^{\infty}$-ring is a homomorphic image of some free $\mathcal{C}^{\infty}$-ring;

- a $\mathcal{C}^{\infty}$-homomorphism is monic if, and only if, it is an injective map;

- any indexed set of $\mathcal{C}^{\infty}$-rings, $\left\{\left(A_{\alpha}, \Phi_{\alpha}\right) \mid \alpha \in I\right\}$, has a coproduct in $\mathcal{C}^{\infty} \mathbf{R n g}$. 


\subsection{Other Constructions in $\mathcal{C}^{\infty}$ Rng}

The results given in the previous section assure the existence of some constructions within the category of $\mathcal{C}^{\infty}$-rings, such as quotients, products, coproducts and so on.

As we shall see, the category of $\mathcal{C}^{\infty}$-rings holds a strong relation with rings of the form $\mathcal{C}^{\infty}\left(\mathbb{R}^{n}\right)$.

In this section we are going to describe concretely such constructions in $\mathcal{C}^{\infty} \mathbf{R n g}$.

Our definition of $\mathcal{C}^{\infty}$-ring yields a forgetful functor:

$$
\begin{array}{rccc}
U: & \mathcal{C}^{\infty} \text { Rng } & \rightarrow & \text { Set } \\
(A, \Phi) & \mapsto & A \\
((A, \Phi) \stackrel{\varphi}{\rightarrow}(B, \Psi)) & \mapsto & (A \stackrel{U(\varphi)}{\rightarrow} B)
\end{array}
$$

We are going to show that this functor has a left adjoint, the "free $\mathcal{C}^{\infty}$-ring", that we shall denote by $L$ : Set $\rightarrow \mathcal{C}^{\infty}$ Rng. Before we do it, we need the following:

Remark 1.3.1. Given any $m \in \mathbb{N}$, we note that the set $\mathcal{C}^{\infty}\left(\mathbb{R}^{n}\right)$ may be endowed with a $\mathcal{C}^{\infty}$-structure:

$$
\begin{aligned}
& \Omega: \bigcup_{n \in \mathbb{N}} \mathcal{C}^{\infty}\left(\mathbb{R}^{n}, \mathbb{R}\right) \rightarrow \bigcup_{n \in \mathbb{N}} \operatorname{Func}\left(\mathcal{C}^{\infty}\left(\mathbb{R}^{m}\right)^{n}, \mathcal{C}^{\infty}\left(\mathbb{R}^{m}\right)\right) \\
& \left(\mathbb{R}^{n} \stackrel{f}{\rightarrow} \mathbb{R}\right) \quad \mapsto \quad \Omega(f)=f \circ-: \begin{array}{ccc}
\mathcal{C}^{\infty}\left(\mathbb{R}^{m}\right)^{n} & \rightarrow & \mathcal{C}^{\infty}\left(\mathbb{R}^{m}\right) \\
\left(h_{1}, \cdots, h_{n}\right) & \mapsto & f \circ\left(h_{1}, \cdots, h_{n}\right)
\end{array}
\end{aligned}
$$

so it is easy to see that it can be made into a $\mathcal{C}^{\infty}$-ring $\left(\mathcal{C}^{\infty}\left(\mathbb{R}^{m}\right), \Omega\right)$. From now, when dealing with this "canonical" $\mathcal{C}^{\infty}$-structure, we shall omit the symbol $\Omega$, writting $\mathcal{C}^{\infty}\left(\mathbb{R}^{m}\right)$ instead of $\left(\mathcal{C}^{\infty}\left(\mathbb{R}^{m}\right), \Omega\right)$.

In fact, we are going to show that for finite sets $X$ with $\sharp X=m$, for instance, the $\mathcal{C}^{\infty}$-ring given in the previous remark is (up to isomorphism) the free $\mathcal{C}^{\infty}$-ring on $m$ generators.

Indeed, given any finite set $X$ with $\sharp X=m \in \mathbb{N}$, we define:

$$
L(X):=\mathcal{C}^{\infty}\left(\mathbb{R}^{m}\right)
$$

together with the canonical $\mathcal{C}^{\infty}$-structure $\Omega$ given in Remark 1.3.1.

We have the following:

Proposition 1.3.2. Let $U: \mathcal{C}^{\infty} \mathbf{R n g} \rightarrow$ Set,$(A, \Phi) \mapsto A$, be the forgetful functor. The pair $\left(\jmath_{n},\left(\mathcal{C}^{\infty}\left(\mathbb{R}^{n}\right), \Omega\right)\right)$, where:

$$
\begin{aligned}
& \jmath_{n}:\{1, \cdots, n\} \rightarrow U\left(\mathcal{C}^{\infty}\left(\mathbb{R}^{n}\right), \Omega\right) \\
& i \quad \mapsto \quad \pi_{i}: \mathbb{R}^{n} \rightarrow \mathbb{R}
\end{aligned}
$$

is the free $\mathcal{C}^{\infty}$-ring with $n$ generators, which are the projections:

$$
\begin{aligned}
& \pi_{i}: \quad \mathbb{R}^{n} \quad \rightarrow \mathbb{R} \\
& \left(x_{1}, \cdots, x_{i}, \cdots, x_{n}\right) \mapsto x_{i}
\end{aligned}
$$


Proof. (Cf. Proposition 1.1 of $[59]$.

Given any $\mathcal{C}^{\infty}$-ring $(A, \Phi)$ and any function $\alpha:\{1,2, \cdots, n\} \rightarrow U(A, \Phi)$, we are going to show that there is a unique $\mathcal{C}^{\infty}$-homomorphism $\widetilde{\alpha}:\left(\mathcal{C}^{\infty}\left(\mathbb{R}^{n}\right), \Omega\right) \rightarrow(A, \Phi)$ such that the following diagram commutes:

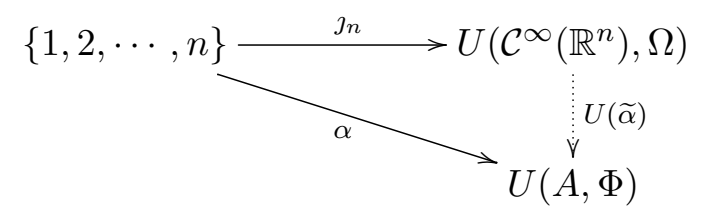

Given $\alpha(1), \alpha(2), \cdots, \alpha(n) \in A$, define

$$
\begin{array}{ccc}
\widetilde{\alpha}: \mathcal{C}^{\infty}\left(\mathbb{R}^{n}\right) & \rightarrow & A \\
f & \mapsto & \Phi(f)(\alpha(1), \alpha(2), \cdots, \alpha(n))
\end{array}
$$

Note that such a function satisfies $(\forall i \in\{1,2, \cdots, n\})\left(\widetilde{\alpha}\left(\jmath_{n}(i)\right)=\alpha(i)\right)$, since for every $i \in\{1,2, \cdots, n\}$ :

$$
\widetilde{\alpha}\left(\jmath_{n}(i)\right)=\widetilde{\alpha}\left(\pi_{i}\right)=\Phi\left(\pi_{i}\right)(\alpha(1), \cdots, \alpha(i), \cdots, \alpha(n))=\pi_{i}(\alpha(1), \cdots, \alpha(i), \cdots, \alpha(n))=\alpha(i) .
$$

Next thing we show is that $\widetilde{\alpha}$ is a $\mathcal{C}^{\infty}$-homomorphism.

Let $f: \mathbb{R}^{m} \rightarrow \mathbb{R}$ be any smooth function. We claim that the following diagram commutes:

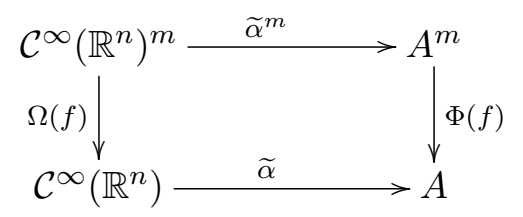

Let $\left(\varphi_{1}, \cdots, \varphi_{m}\right) \in \mathcal{C}^{\infty}\left(\mathbb{R}^{n}\right)^{m}$. On the one hand we have:

$$
\begin{aligned}
& \Phi(f) \circ \widetilde{\alpha}^{m}\left(\varphi_{1}, \cdots, \varphi_{m}\right)=\Phi(f) \circ\left(\widetilde{\alpha}\left(\varphi_{1}\right), \cdots, \widetilde{\alpha}\left(\varphi_{m}\right)\right)= \\
& \Phi(f)\left(\Phi\left(\varphi_{1}\right)(\alpha(1), \alpha(2), \cdots, \alpha(n)), \cdots, \Phi\left(\varphi_{m}\right)(\alpha(1), \alpha(2), \cdots, \alpha(n))\right)
\end{aligned}
$$

On the other hand we have:

$$
\widetilde{\alpha} \circ \mathcal{C}^{\infty}\left(\mathbb{R}^{n}\right)(f)\left(\varphi_{1}, \cdots, \varphi_{m}\right)=\widetilde{\alpha}\left(f \circ\left(\varphi_{1}, \cdots, \varphi_{m}\right)\right)=\Phi\left(f \circ\left(\varphi_{1}, \cdots, \varphi_{m}\right)\right)(\alpha(1), \alpha(2), \cdots, \alpha(n))
$$

Since $(A, \Phi)$ is a $\mathcal{C}^{\infty}$-ring, we have also:

$$
\Phi\left(f \circ\left(\varphi_{1}, \cdots, \varphi_{m}\right)\right)=\Phi(f) \circ\left(\Phi\left(\varphi_{1}\right), \cdots, \Phi\left(\varphi_{m}\right)\right),
$$

hence:

$$
\Phi(f) \circ \widetilde{\alpha}^{m}\left(\varphi_{1}, \cdots, \varphi_{m}\right)=\widetilde{\alpha} \circ \Omega(f)\left(\varphi_{1}, \cdots, \varphi_{m}\right),
$$

and $\widetilde{\alpha}$ is indeed a $\mathcal{C}^{\infty}$-homomorphism.

For the uniqueness of $\widetilde{\alpha}$, suppose $\Psi:\left(\mathcal{C}^{\infty}\left(\mathbb{R}^{n}\right), \Omega\right) \rightarrow(A, \Phi)$ is a $\mathcal{C}^{\infty}$-homomorphism such that $(\forall i \in\{1,2, \cdots, n\})\left(\Psi\left(\jmath_{n}(i)\right)=\alpha(i)\right)$. Since $\Psi$ is a $\mathcal{C}^{\infty}$-homomorphism, in particular the following diagram commutes: 


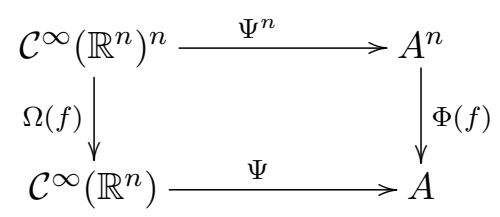

Note that for any $f \in \mathcal{C}^{\infty}\left(\mathbb{R}^{n}\right), f \circ\left(\pi_{1}, \cdots, \pi_{n}\right)=f$, and since the diagram above commutes, we have:

$$
\Phi(f)\left(\Psi\left(\pi_{1}\right), \cdots, \Psi\left(\pi_{n}\right)\right)=\Psi\left(f \circ\left(\pi_{1}, \cdots, \pi_{n}\right)\right)=\Psi(f)
$$

so

$$
\Psi(f)=\Phi(f)\left(\Psi\left(\pi_{1}\right), \cdots, \Psi\left(\pi_{n}\right)\right)=\Phi(f)\left(\Psi\left(\jmath_{n}(1)\right), \cdots, \Psi\left(\jmath_{n}(n)\right)\right)=\Phi(f)(\alpha(1), \cdots, \alpha(n))=\widetilde{\alpha}(f) .
$$

Thus it is proved that $\left(\mathcal{C}^{\infty}\left(\mathbb{R}^{n}\right), \Omega\right.$ ) is (up to isomorphism) the free $\mathcal{C}^{\infty}$-ring on $n$ generators.

Given a finite set $X$ with $\sharp X=n$, consider a bijection $\omega:\{1, \cdots, n\} \rightarrow X$ and denote, for any $i \in\{1, \cdots, n\}, x_{i}=\omega(i)$.

We define:

$$
\mathbb{R}^{X}=\{f: X \rightarrow \mathbb{R} \mid f \text { is a function }\} .
$$

Due to the bijection $\omega$, any smooth function $g \in \mathcal{C}^{\infty}\left(\mathbb{R}^{n}, \mathbb{R}\right)$ can be interpreted as:

$$
\begin{array}{cccc}
\widehat{g}: & \mathbb{R}^{X} & \rightarrow & \mathbb{R} \\
(X \stackrel{f}{\rightarrow} \mathbb{R}) & \mapsto & g\left(f\left(x_{1}\right), \cdots, f\left(x_{n}\right)\right)
\end{array}
$$

so we define:

$$
\mathcal{C}^{\infty}\left(\mathbb{R}^{X}\right):=\left\{\widehat{g}: \mathbb{R}^{X} \rightarrow \mathbb{R} \mid g \in \mathcal{C}^{\infty}\left(\mathbb{R}^{n}, \mathbb{R}\right)\right\}
$$

Note that $\mathcal{C}^{\infty}\left(\mathbb{R}^{X}\right)$ has a natural $\mathcal{C}^{\infty}$-structure given by:

$$
\begin{array}{cc}
\Phi_{X}: \bigcup_{n \in \mathbb{N}} \mathcal{C}^{\infty}\left(\mathbb{R}^{n}, \mathbb{R}\right) & \rightarrow \bigcup_{n \in \mathbb{N}} \operatorname{Func}\left(\mathcal{C}^{\infty}\left(\mathbb{R}^{X}\right)^{n}, \mathcal{C}^{\infty}\left(\mathbb{R}^{X}\right)\right) \\
\left(\mathbb{R}^{n} \stackrel{f}{\rightarrow} \mathbb{R}\right) & \mapsto \Phi_{X}(f): \mathcal{C}^{\infty}\left(\mathbb{R}^{X}\right)^{n} \rightarrow \mathcal{C}^{\infty}\left(\mathbb{R}^{X}\right)
\end{array}
$$

where:

$$
\begin{aligned}
& \Phi_{X}(f): \quad \mathcal{C}^{\infty}\left(\mathbb{R}^{X}\right)^{n} \quad \rightarrow \quad \mathcal{C}^{\infty}\left(\mathbb{R}^{X}\right) \\
& \left(\widehat{g_{1}}, \cdots, \widehat{g_{n}}\right) \mapsto f \circ\left(\widehat{g_{1}, \cdots}, g_{n}\right): \mathbb{R}^{X} \rightarrow \mathbb{R}
\end{aligned}
$$

which is well-defined since the map $g \mapsto \widehat{g}$ is a bijection for a fixed $\omega$.

Thus we have the following $\mathcal{C}^{\infty}$-ring:

$$
\left(\mathcal{C}^{\infty}\left(\mathbb{R}^{X}\right), \Phi_{X}\right)
$$

Note that given any $x \in X$, the evaluation map: 


$$
\begin{aligned}
\mathrm{ev}_{x}: \mathbb{R}^{X} & \rightarrow \mathbb{R} \\
f & \mapsto f(x)
\end{aligned}
$$

belongs to $\mathcal{C}^{\infty}\left(\mathbb{R}^{X}\right)$. In fact, given $x \in X=\left\{x_{1}, \cdots, x_{n}\right\}$, there is $i \in\{1, \cdots, n\}$ such that $x=x_{i}$.

Claim: $\mathrm{ev}_{x_{i}}=\widehat{\pi}_{i}$, where $\pi_{i}: \mathbb{R}^{n} \rightarrow \mathbb{R}$ is the (smooth) projection on the $i$-th coordinate.

We have, in fact, for any $f \in \mathbb{R}^{X}$, $\mathrm{ev}_{x_{i}}(f)=f\left(x_{i}\right)=\pi_{i}\left(f\left(x_{1}\right), \cdots, f\left(x_{i}\right), \cdots, f\left(x_{n}\right)\right)=\widehat{\pi}_{i}(f)$, so $\mathrm{ev}_{x} \in \mathcal{C}^{\infty}\left(\mathbb{R}^{X}\right)$.

We have, thus, the following function:

$$
\begin{array}{rlrrrr}
\jmath_{X}: \quad X & \rightarrow & U\left(\mathcal{C}^{\infty}\left(\mathbb{R}^{X}\right), \Phi_{X}\right) \\
x & \mapsto & \operatorname{ev}_{x}: & \mathbb{R}^{X} & \rightarrow & \mathbb{R} \\
& f & \mapsto f(x)
\end{array}
$$

Proposition 1.3.3. Let $X$ be a finite set with $\sharp X=n$ and consider:

$$
\mathbb{R}^{X}=\{f: X \rightarrow \mathbb{R} \mid f \text { is a function }\}
$$

together with some bijection $\omega:\{1, \cdots, n\} \rightarrow X, \omega(i)=x_{i} \in X$.

Under those circumstances, the following function is a $\mathcal{C}^{\infty}$-isomorphism:

$$
\begin{array}{rlll}
\widehat{\omega}: & \left(\mathcal{C}^{\infty}\left(\mathbb{R}^{X}\right), \Phi_{X}\right) & \rightarrow & \left(\mathcal{C}^{\infty}\left(\mathbb{R}^{n}\right), \Omega\right) \\
\widehat{g}: \mathbb{R}^{X} & \rightarrow \mathbb{R} & \mapsto g: \mathbb{R}^{n} \rightarrow \mathbb{R} \\
f & \mapsto \widehat{g}(f) & &
\end{array}
$$

Proof. First we show that $\widehat{\omega}$ is a $\mathcal{C}^{\infty}$-homomorphism.

Let $m \in \mathbb{N}$ and $h \in \mathcal{C}^{\infty}\left(\mathbb{R}^{m}, \mathbb{R}\right)$. We claim that the following diagram commutes:

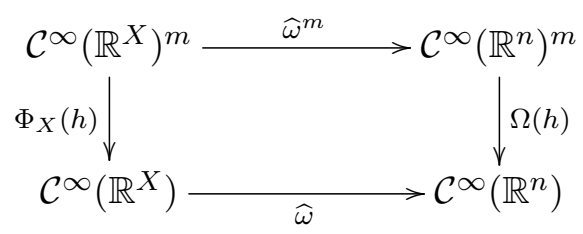

Given $\left(\widehat{g_{1}}, \cdots, \widehat{g_{m}}\right) \in \mathcal{C}^{\infty}\left(\mathbb{R}^{X}\right)^{m}$, we have:

$$
\widehat{\omega}\left(\Phi_{X}(h)\left(\widehat{g_{1}}, \cdots, \widehat{g_{m}}\right)\right)=\widehat{\omega}\left(h \circ\left(\widehat{g_{1}, \cdots}, g_{m}\right)\right)=h \circ\left(g_{1}, \cdots, g_{m}\right)
$$

and

$$
\Omega(h)\left(\widehat{\omega}\left(\widehat{g_{1}}\right), \cdots, \widehat{\omega}\left(\widehat{g_{m}}\right)\right)=\Omega(h) \circ\left(g_{1}, \cdots, g_{m}\right)=h \circ\left(g_{1}, \cdots, g_{m}\right)
$$

so $\widehat{\omega}$ is a $\mathcal{C}^{\infty}$-homomorphism.

We claim that: 


$$
\begin{aligned}
& \widehat{\delta}: \quad\left(\mathcal{C}^{\infty}\left(\mathbb{R}^{m}\right), \Omega\right) \quad \mapsto \quad\left(\mathcal{C}^{\infty}\left(\mathbb{R}^{X}\right), \Phi_{X}\right)
\end{aligned}
$$

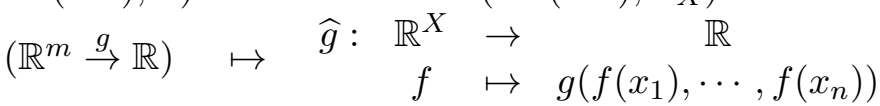

is a $\mathcal{C}^{\infty}$-homomorphism. In fact, for every $n \in \mathbb{N}, h \in \mathcal{C}^{\infty}\left(\mathbb{R}^{n}, \mathbb{R}\right)$ the following diagram commutes:

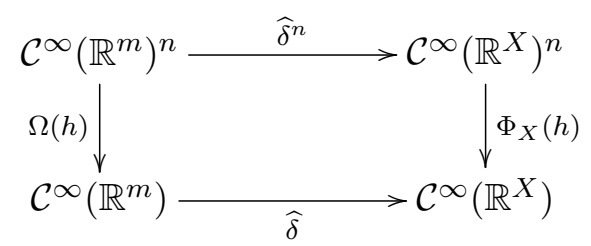

since given $\left(g_{1}, \cdots, g_{n}\right) \in \mathcal{C}^{\infty}\left(\mathbb{R}^{m}\right)^{n}$ we have:

$$
\Phi_{X}(h)\left(\widehat{\delta}\left(g_{1}\right), \cdots, \widehat{\delta}\left(g_{m}\right)\right)=\Phi_{X}(h) \circ\left(\widehat{g_{1}}, \cdots, \widehat{g_{n}}\right)=h \circ\left(\widehat{g_{1}, \cdots}, g_{n}\right)
$$

and

$$
\widehat{\delta}\left(\Omega(h)\left(g_{1}, \cdots, g_{n}\right)\right)=\widehat{\delta}\left(h \circ\left(g_{1}, \cdots, g_{n}\right)\right)=h \circ\left(\widehat{g_{1}, \cdots}, g_{n}\right),
$$

so $\widehat{\delta}$ is a $\mathcal{C}^{\infty}$-homomorphism.

Given $g \in \mathcal{C}^{\infty}\left(\mathbb{R}^{m}\right)$, we have:

$$
\widehat{\omega}(\widehat{\delta}(g))=\widehat{\omega}(\widehat{g})=g
$$

so

$$
\widehat{\omega} \circ \widehat{\delta}=\operatorname{id}_{\mathcal{C} \infty\left(\mathbb{R}^{m}\right)}
$$

Also, given $\widehat{g} \in \mathcal{C}^{\infty}\left(\mathbb{R}^{X}\right)$, we have:

$$
\widehat{\delta}(\widehat{\omega}(\widehat{g}))=\widehat{\delta}(g)=\widehat{g},
$$

so

$$
\widehat{\delta} \circ \widehat{\omega}=\operatorname{id}_{\mathcal{C}^{\infty}\left(\mathbb{R}^{X}\right)}
$$

As an immediate consequence of Propositions 1.3.3 and 1.3.2, we have:

Corollary 1.3.4. For any finite set $X,\left(\jmath_{X},\left(\mathcal{C}^{\infty}\left(\mathbb{R}^{X}\right), \Phi_{X}\right)\right)$ is the free $\mathcal{C}^{\infty}$-ring defined by $X$.

Proof. Let $\omega:\{1, \cdots, n\} \rightarrow X$ be a bijection, $\widehat{\omega}:\left(\mathcal{C}^{\infty}\left(\mathbb{R}^{X}\right), \Phi_{X}\right) \rightarrow\left(\mathcal{C}^{\infty}\left(\mathbb{R}^{n}\right), \Omega\right)$ be the $\mathcal{C}^{\infty}$-isomorphism induced by $\omega$, as given in Proposition 1.3.3. $(A, \Phi)$ be any $\mathcal{C}^{\infty}$-ring and let $f: X \rightarrow U(A, \Phi)$ be any function.

Note that the following diagram commutes: 


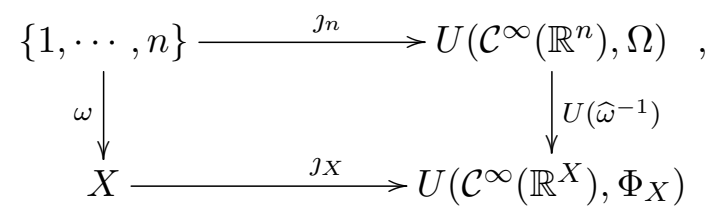

since given any $i \in\{1, \cdots, n\}, \widehat{\omega}^{-1}\left(\jmath_{n}\right)(i)=\widehat{\omega}^{-1}\left(\pi_{i}\right)=\widehat{\pi}_{i}=\mathrm{ev}_{x_{i}}=\jmath_{X}\left(x_{i}\right)=\jmath_{X} \circ \omega(i)$.

We have the following diagram:

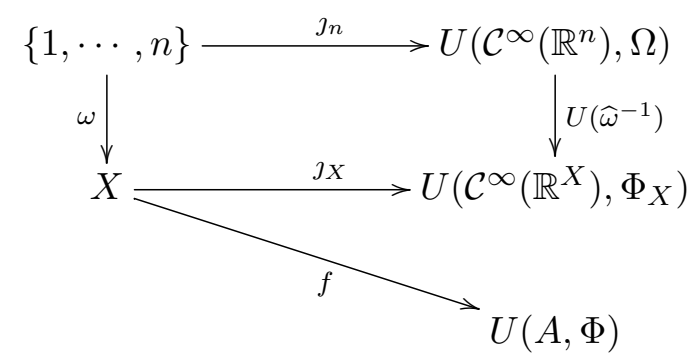

Since $\left(\jmath_{n},\left(\mathcal{C}^{\infty}\left(\mathbb{R}^{n}\right), \Omega\right)\right)$ is free, given the function $f \circ \omega:\{1, \cdots, n\} \rightarrow U(A, \Phi)$, there is a unique $\mathcal{C}^{\infty}$-homomorphism $\tilde{f \circ \omega}:\left(\mathcal{C}^{\infty}\left(\mathbb{R}^{n}\right), \Omega\right) \rightarrow(A, \Phi)$ such that the following diagram commutes:

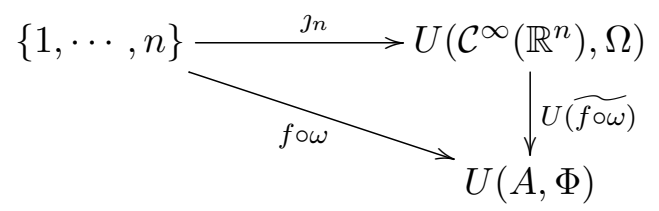

We have, thus, the following commutative diagram:

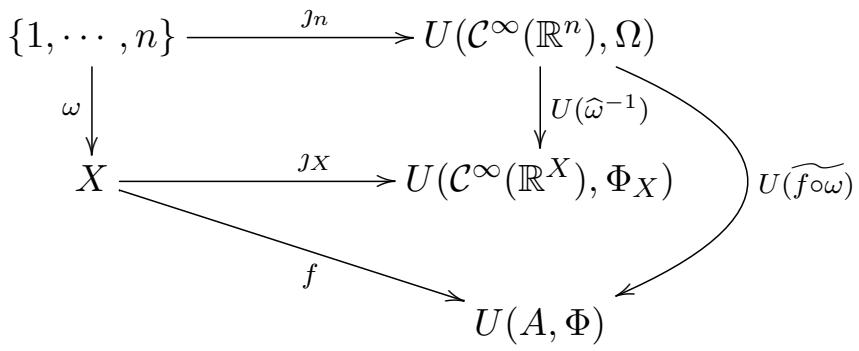

It now suffices to take the $\mathcal{C}^{\infty}$-homomorphism $\tilde{f}:=(\widetilde{f \circ \omega}) \circ \widehat{\omega}:\left(\mathcal{C}^{\infty}\left(\mathbb{R}^{X}\right), \Phi_{X}\right) \rightarrow(A, \Phi)$, and the following diagram commutes:

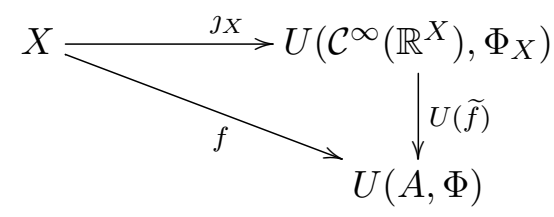

By construction such a $\tilde{f}$ is unique, so $\left(\jmath_{X},\left(\mathcal{C}^{\infty}\left(\mathbb{R}^{X}\right), \Phi_{X}\right)\right)$ is the free $\mathcal{C}^{\infty}$-ring determined by $X$.

Now we turn to the definition of the free $\mathcal{C}^{\infty}$-ring determined by an arbitrary set.

Let $E$ be any set, and consider: 


$$
\left(\mathcal{C}^{\infty}\left(\mathbb{R}^{E}\right), \Phi_{E}\right)=\underset{E^{\prime} \subseteq_{f} E}{\lim _{\longrightarrow}}\left(\mathcal{C}^{\infty}\left(\mathbb{R}^{E^{\prime}}\right), \Phi_{E^{\prime}}\right)
$$

where " $\subseteq$ " stands for "is a finite subset of", together with the unique arrow $\jmath_{E}: E=\bigcup_{E^{\prime} \subseteq_{f} E} E^{\prime} \rightarrow$ $\mathcal{C}^{\infty}\left(\mathbb{R}^{E}\right)$ such that for every $E^{\prime} \subseteq_{f} E$ the following diagram commutes:

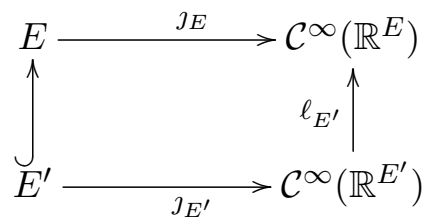

This arrow is described, in detail, in the Appendix on Free $\mathcal{C}^{\infty}$-Rings.

Proposition 1.3.5. Let $E$ be any set. The pair $\left(\jmath_{E},\left(\mathcal{C}^{\infty}\left(\mathbb{R}^{E}\right), \Phi_{E}\right)\right)$ is the free $\mathcal{C}^{\infty}$-ring determined by $E$.

Proof. Let $(A, \Phi)$ be any $\mathcal{C}^{\infty}$-ring and let $f: E \rightarrow A$ be any function. We decompose the set $E$ as

$$
E=\bigcup_{E^{\prime} \subseteq_{f} E} E^{\prime}={\underset{\lim ^{\prime} \subseteq f}{ } E}_{\subseteq_{f}} E
$$

and take the following cone:

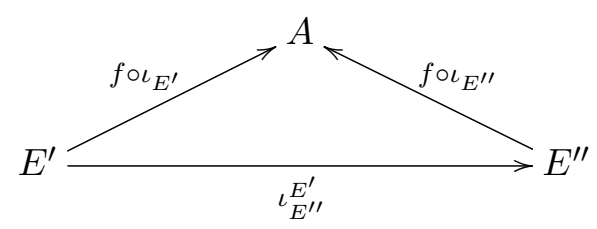

Since for every finite $E^{\prime} \subseteq E,\left(\jmath_{E^{\prime}}, \mathcal{C}^{\infty}\left(\mathbb{R}^{E^{\prime}}\right)\right)$ is the free $\mathcal{C}^{\infty}$-ring determined by $E^{\prime}$, given the function $f \circ \iota_{E^{\prime}}: E^{\prime} \rightarrow A$, there is a unique $\mathcal{C}^{\infty}$-homomorphism $\widehat{f_{E^{\prime}}}:\left(\mathcal{C}^{\infty}\left(\mathbb{R}^{E^{\prime}}\right), \Phi_{E^{\prime}}\right) \rightarrow(A, \Phi)$ such that the following diagram commutes:

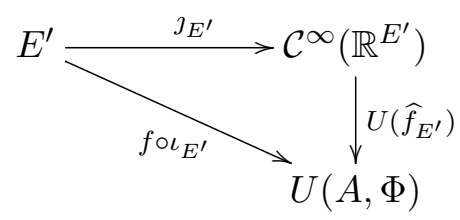

Note that the following diagram commutes:

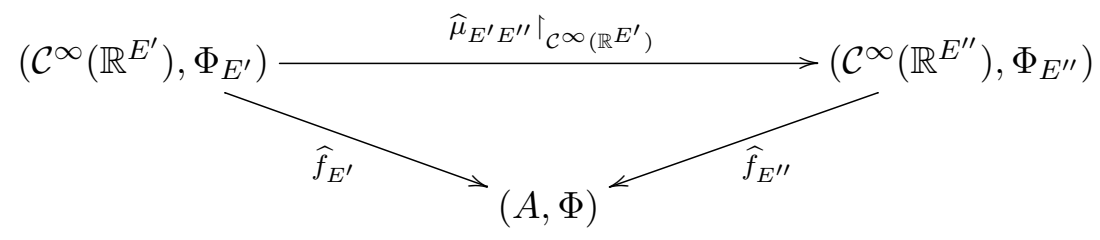

In fact, the following rectangle commutes: 


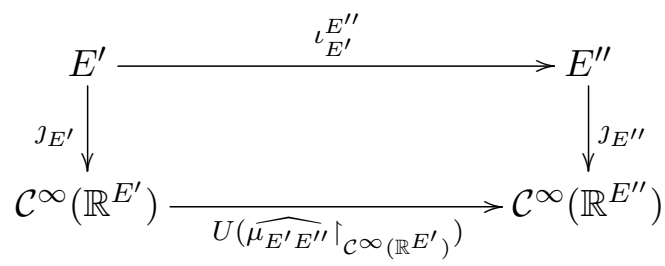

So:

$$
\jmath_{E^{\prime \prime}} \circ \iota_{E^{\prime}}^{E^{\prime \prime}}=U\left(\widehat{\mu}_{E^{\prime} E^{\prime \prime}} \uparrow_{\mathcal{C}^{\infty}\left(\mathbb{R}^{E^{\prime}}\right)}\right) \circ \jmath_{E^{\prime}}
$$

and $\widehat{f}_{E^{\prime}}:\left(\mathcal{C}^{\infty}\left(\mathbb{R}^{E^{\prime}}\right), \Phi_{E^{\prime}}\right) \rightarrow(A, \Phi)$ is the unique $\mathcal{C}^{\infty}$-homomorphism such that:

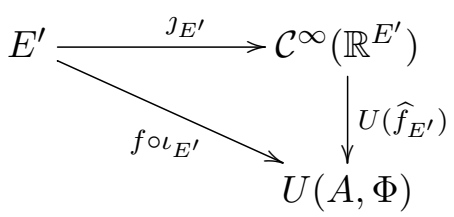

commutes, i.e.,

$$
U\left(\widehat{f}_{E^{\prime}}\right) \circ \jmath_{E^{\prime}}=f \circ \iota_{E^{\prime}}
$$

We are going to show that $\widehat{f}_{E^{\prime \prime}} \circ \widehat{\mu}_{E^{\prime} E^{\prime \prime}} \uparrow_{\mathcal{C}^{\infty}\left(\mathbb{R}^{E^{\prime}}\right)}:\left(\mathcal{C}^{\infty}\left(\mathbb{R}^{E^{\prime}}\right), \Phi_{E^{\prime}}\right) \rightarrow(A, \Phi)$ is such that:

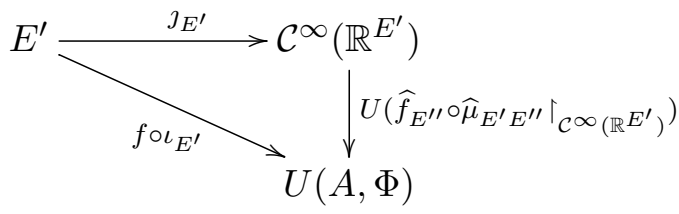

commutes. By uniqueness it will follow that:

$$
\widehat{f}_{E^{\prime \prime}} \circ \widehat{\mu}_{E^{\prime} E^{\prime \prime}}\left\lceil_{\mathcal{C}^{\infty}\left(\mathbb{R}^{E^{\prime}}\right)}=\widehat{f}_{E^{\prime}}\right.
$$

Composing $(1.3)$ with $U\left(\widehat{f}_{E^{\prime \prime}}\right)$ by the left yields:

$$
U\left(\widehat{f}_{E^{\prime \prime}}\right) \circ \jmath_{E^{\prime \prime}} \circ \iota_{E^{\prime}}^{E^{\prime \prime}}=U\left(\widehat{f}_{E^{\prime \prime}}\right) \circ U\left(\widehat{\mu}_{E^{\prime} E^{\prime \prime}} \uparrow_{\mathcal{C}^{\infty}\left(\mathbb{R}^{E^{\prime}}\right)}\right) \circ \jmath_{E^{\prime}}
$$

i.e.,

$$
U\left(\widehat{f}_{E^{\prime \prime}}\right) \circ \jmath_{E^{\prime \prime}} \circ \iota_{E^{\prime}}^{E^{\prime \prime}}=U\left(\widehat{f}_{E^{\prime \prime}} \circ \widehat{\mu}_{E^{\prime} E^{\prime \prime}} \uparrow_{\mathcal{C} \infty\left(\mathbb{R}^{E^{\prime}}\right)}\right) \circ \jmath_{E^{\prime}}
$$

Since $U\left(\widehat{f}_{E^{\prime \prime}}\right) \circ \jmath_{E^{\prime \prime}}=f \circ \iota_{E^{\prime \prime}}$, we have:

$$
\left(f \circ \iota_{E^{\prime \prime}}\right) \circ \iota_{E^{\prime}}^{E^{\prime \prime}}=U\left(\widehat{f}_{E^{\prime \prime}} \circ \widehat{\mu}_{E^{\prime} E^{\prime \prime}}\left\lceil_{\mathcal{C}^{\infty}\left(\mathbb{R}^{E^{\prime}}\right)}\right) \circ \jmath_{E^{\prime}}\right.
$$

We know that $\iota_{E^{\prime \prime}} \circ \iota_{E^{\prime}}^{E^{\prime \prime}}=\iota_{E^{\prime}}$, so we get:

$$
f \circ \iota_{E^{\prime}}=U\left(\widehat{f}_{E^{\prime \prime}} \circ \widehat{\mu}_{E^{\prime} E^{\prime \prime}} \uparrow_{\mathcal{C}^{\infty}\left(\mathbb{R}^{E^{\prime}}\right)}\right) \circ \jmath_{E^{\prime}},
$$


and the diagram commutes.

By the universal property of the colimit $\left(\mathcal{C}^{\infty}\left(\mathbb{R}^{E}\right), \Phi_{E}\right)$, there is a unique $\mathcal{C}^{\infty}$-homomorphism $\hat{f}$ : $\left(\mathcal{C}^{\infty}\left(\mathbb{R}^{E}\right), \Phi_{E}\right) \rightarrow(A, \Phi)$ such that:

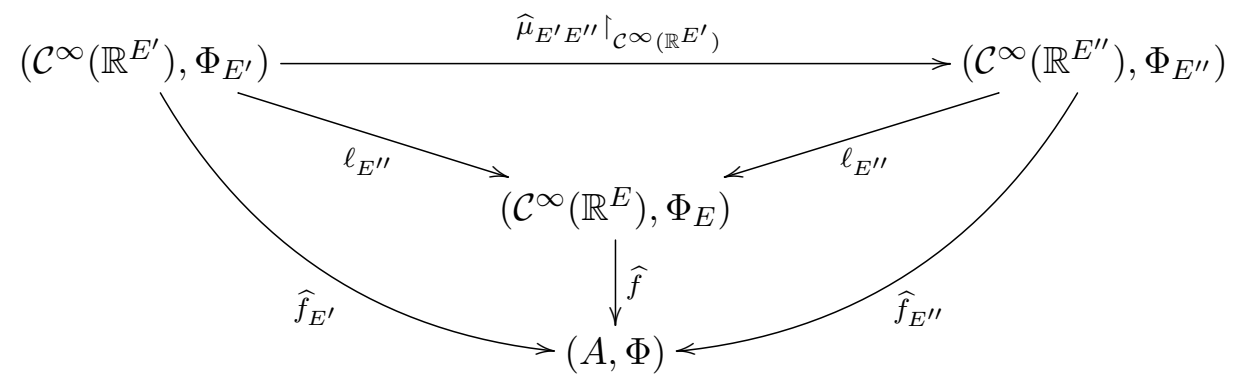

Now we need only to show that $U(\widehat{f}) \circ \jmath_{E}=f$.

Given any $x \in E$, there is some finite $E^{\prime} \subseteq_{f} E$ such that $x \in E^{\prime}$.

We have the following commutative diagram:

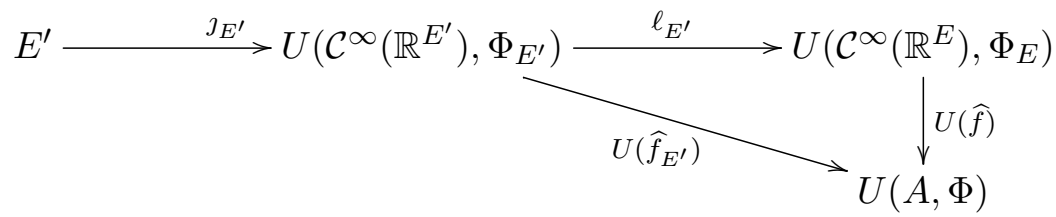

So:

$$
U(\widehat{f}) \circ \ell_{E^{\prime}} \circ \jmath_{E^{\prime}}=U\left(\widehat{f}_{E^{\prime}}\right) \circ \jmath_{E^{\prime}}
$$

Since $\ell_{E^{\prime}} \circ \jmath_{E^{\prime}}=\jmath_{E} \circ \iota_{E^{\prime}}$ and $U\left(\widehat{f}_{E^{\prime}}\right) \circ \jmath_{E^{\prime}}=f \circ \iota_{E^{\prime}}$, we get:

$$
U(\widehat{f}) \circ \jmath_{E} \circ \iota_{E^{\prime}}=f \circ \iota_{E^{\prime}}
$$

thus:

$$
U(\widehat{f}) \circ \jmath_{E}(x)=U(\widehat{f}) \circ \jmath_{E} \circ \iota_{E^{\prime}}(x)=f\left(\iota_{E^{\prime}}(x)\right)=f(x)
$$

and the diagram commutes.

The uniqueness of $\widehat{f}$ comes from its construction.

In the following proposition, we present a description of a left adjoint to the forgetful functor $U$ : $\mathcal{C}^{\infty}$ Rng $\rightarrow$ Set.

Proposition 1.3.6. The functions:

$$
\begin{aligned}
& L_{0}: \operatorname{Obj}(\text { Set }) \rightarrow \operatorname{Obj}\left(\mathcal{C}^{\infty} \mathbf{R n g}\right) \\
& X \quad \mapsto\left(\mathcal{C}^{\infty}\left(\mathbb{R}^{X}\right), \Phi_{X}\right)
\end{aligned}
$$

and 


$$
\begin{aligned}
& L_{1}: \operatorname{Mor}(\text { Set }) \rightarrow \quad \operatorname{Mor}\left(\mathcal{C}^{\infty} \text { Rng }\right) \\
& (X \stackrel{f}{\rightarrow} Y) \mapsto\left(\mathcal{C}^{\infty}\left(\mathbb{R}^{X}\right), \Phi_{X}\right) \stackrel{\tilde{f}}{\rightarrow}\left(\mathcal{C}^{\infty}\left(\mathbb{R}^{Y}\right), \Phi_{Y}\right)
\end{aligned}
$$

where $\tilde{f}: L_{0}(X) \rightarrow L_{0}(Y)$ is the unique $\mathcal{C}^{\infty}$-homomorphism given by the universal property of the free $\mathcal{C}^{\infty}$-ring $\jmath_{X}: X \rightarrow \mathcal{C}^{\infty}\left(\mathbb{R}^{X}\right)$ : given the function $\jmath_{Y} \circ f: X \rightarrow U\left(\mathcal{C}^{\infty}\left(\mathbb{R}^{Y}\right)\right)$, there is a unique $\mathcal{C}^{\infty}$-homomorphism such that the following diagram commutes:

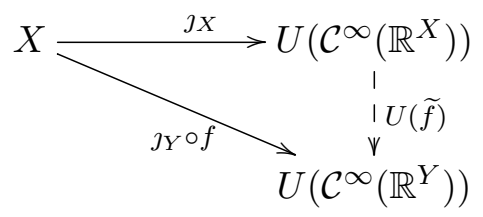

define a functor $L:$ Set $\rightarrow \mathcal{C}^{\infty} \mathbf{R n g}$ which is left adjoint to the forgetful functor $U: \mathcal{C}^{\infty} \mathbf{R n g} \rightarrow \mathbf{S e t}$.

Proof. We claim that $L_{1}\left(\operatorname{id}_{X}\right)=\operatorname{id}_{\left(\mathcal{C}^{\infty}\left(\mathbb{R}^{X}\right), \Phi_{X}\right)}$.

On the one hand, $\widetilde{\mathrm{id}_{X}}$ is the only $\mathcal{C}^{\infty}$-homomorphism such that:

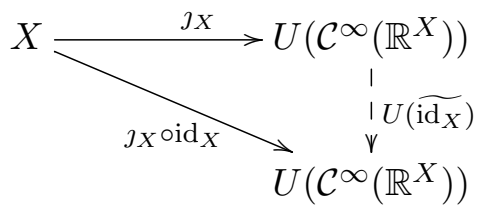

commutes.

On the other hand, since $\operatorname{id}_{\left(\mathcal{C}^{\infty}\left(\mathbb{R}^{X}\right), \Phi_{X}\right)}$ is a $\mathcal{C}^{\infty}$-homomorphism which makes the following diagram to commute:

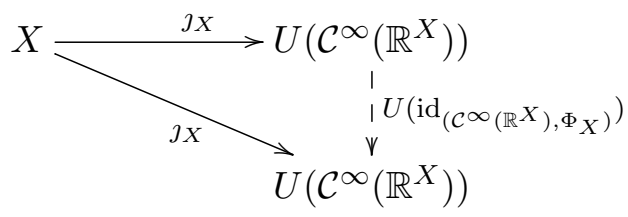

it follows that:

$$
L_{1}\left(\operatorname{id}_{X}\right)=\widetilde{\operatorname{id}_{X}}=\operatorname{id}_{\left(\mathcal{C}^{\infty}\left(\mathbb{R}^{X}\right), \Phi_{X}\right)}
$$

Now, let $X, Y, Z$ be sets and $f: X \rightarrow Y$ and $g: Y \rightarrow Z$ be functions. We claim that:

$$
L_{1}(g \circ f)=L_{1}(g) \circ L_{1}(f)
$$

By definition, $L_{1}(f)=\tilde{f}:\left(\mathcal{C}^{\infty}\left(\mathbb{R}^{X}\right), \Phi_{X}\right) \rightarrow\left(\mathcal{C}^{\infty}\left(\mathbb{R}^{Y}\right), \Phi_{Y}\right)$ is the only $\mathcal{C}^{\infty}$-homomorphism such that:

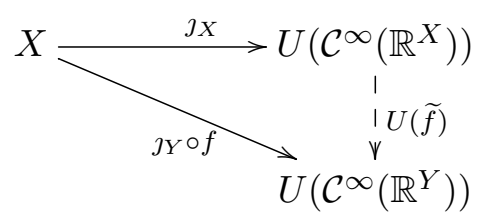


commutes, that is, such that $U(\widetilde{f}) \circ \jmath_{X}=\jmath_{Y} \circ f$. Also, by definition, $L_{1}(g)=\widetilde{g}:\left(\mathcal{C}^{\infty}\left(\mathbb{R}^{Y}\right), \Phi_{Y}\right) \rightarrow$ $\left(\mathcal{C}^{\infty}\left(\mathbb{R}^{Z}\right), \Phi_{Z}\right)$ is the only $\mathcal{C}^{\infty}$-homomorphism such that:

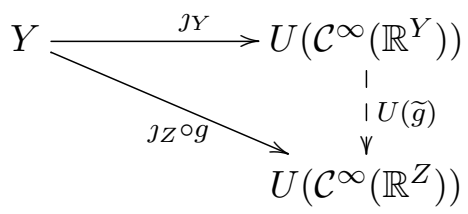

commutes, that is, such that $U(\widetilde{g}) \circ \jmath_{Y}=\jmath_{Z} \circ g$.

Finally, $\widetilde{g \circ f}:\left(\mathcal{C}^{\infty}\left(\mathbb{R}^{X}\right), \Phi_{X}\right) \rightarrow\left(\mathcal{C}^{\infty}\left(\mathbb{R}^{Z}\right), \Phi_{Z}\right)$ is the unique $\mathcal{C}^{\infty}$-homomorphism such that:

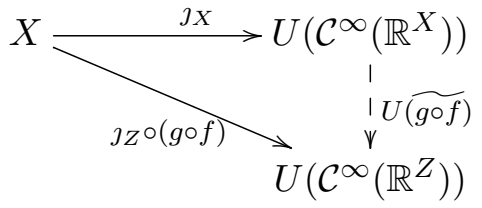

commutes, that is, such that $U(\widetilde{g \circ f}) \circ \jmath_{X}=\jmath_{Z} \circ(g \circ f)$.

We are going to show that $\widetilde{g} \circ \tilde{f}:\left(\mathcal{C}^{\infty}\left(\mathbb{R}^{X}\right), \Phi_{X}\right) \rightarrow\left(\mathcal{C}^{\infty}\left(\mathbb{R}^{Z}\right), \Phi_{Z}\right)$ also has this property.

In fact,

$U(\widetilde{f}) \circ \jmath_{X}=\jmath_{Y} \circ f \Rightarrow U(\widetilde{g}) \circ\left(U(\widetilde{f}) \circ \jmath_{X}\right)=\left(U(\widetilde{g}) \circ \jmath_{Y}\right) \circ f \Rightarrow$

$$
\Rightarrow(U(\widetilde{g}) \circ U(\widetilde{f})) \circ \jmath X=(\jmath Z \circ g) \circ f
$$

and thus

$$
U(\widetilde{g} \circ \widetilde{f}) \circ \jmath_{X}=\jmath_{Z} \circ(g \circ f) .
$$

By uniqueness it follows that:

$$
L_{1}(g \circ f)=L_{1}(g) \circ L_{1}(f),
$$

so $L=\left(L_{0}, L_{1}\right)$ defines a functor.

We claim that:

$$
\begin{array}{rlcc}
\phi_{X,(A, \Phi)}: & \operatorname{Hom}_{\text {Set }}(X, A) & \rightarrow & \operatorname{Hom}_{\mathcal{C}^{\infty} \mathbf{R n g}}\left(\left(L(X), \Phi_{X}\right),(A, \Phi)\right) \\
(X \stackrel{\varphi}{\rightarrow} A) & \mapsto & \left(L(X), \Phi_{X}\right) \stackrel{\widetilde{\varphi}}{\rightarrow}(A, \Phi)
\end{array}
$$

where $\widetilde{\varphi}$ is the unique $\mathcal{C}^{\infty}$-homomorphism such that $U(\widetilde{\varphi}) \circ \jmath_{X}=\varphi$

is a natural bijection, so $L \dashv U$.

$\phi_{X,(A, \Phi)}$ is surjective. Given any $\mathcal{C}^{\infty}$-homomorphism $\widetilde{\varphi}:\left(L(X), \Phi_{X}\right) \rightarrow(A, \Phi)$, taking $\varphi:=U(\widetilde{\varphi}) \circ \jmath_{X}:$ $X \rightarrow A$ we have $L(\varphi)=\widetilde{\varphi}$. 
Given $\varphi, \psi: X \rightarrow A$ such that $\phi_{X,(A, \Phi)}(\varphi)=\widetilde{\varphi}=\widetilde{\psi}=\phi_{X,(A, \Phi)}(\psi)$, we have:

$$
U(\widetilde{\varphi})=U(\widetilde{\psi})
$$

and

$$
\varphi=U(\widetilde{\varphi}) \circ \jmath_{X}=U(\widetilde{\psi}) \circ \jmath_{X}=\psi
$$

so $\phi_{X,(A, \Phi)}$ is a bijection.

Let $(A, \Phi)$ be a $\mathcal{C}^{\infty}$-ring and let $X \subseteq A$. Given $\iota_{X}^{A}: X \hookrightarrow A$, there is a unique $\mathcal{C}^{\infty}$-homomorphism $\widetilde{\iota_{X}^{A}}:\left(L(X), \Phi_{X}\right) \rightarrow(A, \Phi)$ such that the following diagram commutes:

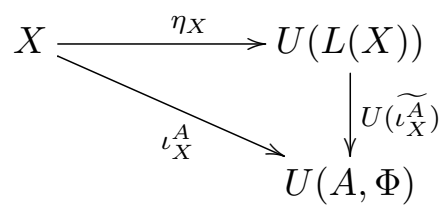

We claim that if $\langle X\rangle=A$, then $\widetilde{\iota_{X}^{A}}$ is surjective.

Indeed,

$$
X=\iota_{X}^{A}[X]=\operatorname{im}\left(\iota_{X}^{A}\right)
$$

and since $U\left(\widetilde{\iota_{X}^{A}}\right) \circ \eta_{X}=\iota_{X}^{A}$, we have:

$$
\operatorname{im}\left(\iota_{X}^{A}\right)=\operatorname{im}\left(U\left(\widetilde{\iota_{X}^{A}}\right) \circ \eta_{X}\right) .
$$

Since the diagram 1.5 commutes, on the other hand,

$$
\operatorname{im}\left(U\left(\widetilde{\iota_{X}^{A}}\right) \circ \eta_{X}\right) \subseteq \operatorname{im}\left(U\left(\widetilde{\iota_{X}^{A}}\right)\right)
$$

thus

$$
X \subseteq \operatorname{im}\left(U\left(\widetilde{\iota_{X}^{A}}\right)\right)
$$

and

$$
\langle X\rangle \subseteq\left\langle\operatorname{im}\left(U\left(\widetilde{\iota_{X}^{A}}\right)\right)\right\rangle=\widetilde{\iota_{X}^{A}}[A]
$$

Since $X$ generates $A$ and $\left\langle\operatorname{im}\left(U\left(\widetilde{\iota_{X}^{A}}\right)\right)\right\rangle=\operatorname{im}\left(\widetilde{\iota_{X}^{A}}\right)$ is a $\mathcal{C}^{\infty}$-subring of $(A, \Phi)$, it follows that::

$$
\langle X\rangle=A \subseteq \operatorname{im}\left(\widetilde{\iota_{X}^{A}}\right) \subseteq A
$$

so $\operatorname{im}\left(\widetilde{\iota_{X}^{A}}\right)=A$ and $\iota_{X}^{A}$ is surjective.

In particular, taking $X=A$ yields $\iota_{A}^{A}=\mathrm{id}_{A}$, and since $\varepsilon_{A}=\phi_{A,(A, \Phi)}\left(\mathrm{id}_{A}\right)=\widetilde{\mathrm{id}_{(A, \Phi)}}$, we have: 


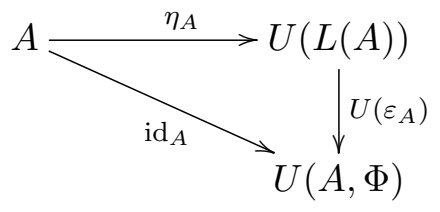

so $\operatorname{im}\left(\varepsilon_{A}\right)=(A, \Phi)$, and $\varepsilon_{A}$ is surjective. Now, given any $\mathcal{C}^{\infty}$-ring $(A, \Phi)$ we have the surjective morphism:

$$
\varepsilon_{A}: L(U(A, \Phi)) \rightarrow(A, \Phi) .
$$

We have seen that since $\varepsilon_{A}$ is a $\mathcal{C}^{\infty}$-homomorphism, $\operatorname{ker}\left(\varepsilon_{A}\right)$ is a $\mathcal{C}^{\infty}$-congruence. By the Fundamental Theorem of the $\mathcal{C}^{\infty}$-Isomorphism we have:

$$
(A, \Phi) \cong \frac{L(A)}{\operatorname{ker}\left(\varepsilon_{A}\right)}
$$

Our next goal is to classify the congruences of any $\mathcal{C}^{\infty}$-ring. We shall see that they are classified by their ring-theoretic ideals.

We begin with the finitely generated case (defined in Section 1.4).

An interesting result, which is a consequence of Hadamard's Lemma, Theorem 5.0.1, is the description of the ideals of $\mathcal{C}^{\infty}$-rings in terms of $\mathcal{C}^{\infty}$-congruences.

Proposition 1.3.7. Given a finitely generated $\mathcal{C}^{\infty}$-ring $(A, \Phi)$, considering the forgetful functor given in Remark 1.1.7, $\widetilde{U}: \mathcal{C}^{\infty} \mathbf{R n g} \rightarrow \mathbf{C R i n g}$, we have that if $I$ is a subset of $A$ that is an ideal (in the ordinary ring-theoretic sense) in $\widetilde{U}(A, \Phi)$, then $\widehat{I}=\{(a, b) \in A \times A \mid a-b \in I\}$ is a $\mathcal{C}^{\infty}$-congruence in $A$.

Proof. (Cf. p. 18 of 62 )

Let $n \in \mathbb{N}$ and $f \in \mathcal{C}^{\infty}\left(\mathbb{R}^{n}, \mathbb{R}\right)$.

We have to show that if for every $i \in\{1, \cdots, n\}$ we have $\left(a_{i}, b_{i}\right) \in \widehat{I}$, that is, $a_{i}-b_{i} \in I$, then:

$$
\Phi^{(2)}(f)\left(\left(a_{1}, b_{1}\right), \cdots,\left(a_{n}, b_{n}\right)\right)=\left(\Phi(f)\left(a_{1}, \cdots, a_{n}\right), \Phi(f)\left(b_{1}, \cdots, b_{n}\right)\right) \in \widehat{I},
$$

that is, $\Phi(f)\left(a_{1}, \cdots, a_{n}\right)-\Phi(f)\left(b_{1}, \cdots, b_{n}\right) \in I$.

Suppose that $\left(a_{1}, b_{1}\right), \cdots,\left(a_{n}, b_{n}\right) \in A \times A$ are such that $a_{i}-b_{i} \in I$ for every $i \in\{1, \cdots, n\}$. By Hadamard's Lemma, there are smooth functions $g_{1}, \cdots, g_{n}: \mathbb{R}^{n} \times \mathbb{R} \rightarrow \mathbb{R}$ such that for all $x=$ $\left(x_{1}, \cdots, x_{n}\right)$ and $y=\left(y_{1}, \cdots, y_{n}\right) \in \mathbb{R}^{n}$,

$$
f(x)-f(y)=\sum_{i=1}^{n}\left(x_{i}-y_{i}\right) \cdot g_{i}(x, y)
$$

$\Phi$ preserves this equation, i.e., 


$$
\Phi(f)\left(a_{1}, \cdots, a_{n}\right)-\Phi(f)\left(b_{1}, \cdots, b_{n}\right)=\sum_{i=1}^{n} \underbrace{\left(a_{i}-b_{i}\right)}_{\in I} \cdot \Phi\left(g_{i}\right)\left(a_{1}, \cdots, a_{n}, b_{1}, \cdots, b_{n}\right) \in I,
$$

and the result follows.

The following result tells us that the ideals of the $\mathcal{C}^{\infty}$-rings classify its congruences.

Proposition 1.3.8. Given any finitely generated $\mathcal{C}^{\infty}$-ring $(A, \Phi)$, let $\operatorname{Cong}(A, \Phi)$ denote the set of all the $\mathcal{C}^{\infty}$-congruences in $A$ and let $\Im(A, \Phi)$ denote the set of all ideals of $A$. The following map is a bijection:

$$
\begin{array}{clc}
\psi_{A}: \operatorname{Cong}(A, \Phi) & \rightarrow & \Im(A, \Phi) \\
R & \mapsto\{x \in A \mid(x, 0) \in R\}
\end{array}
$$

whose inverse is given by:

$$
\begin{array}{ccc}
\varphi_{A}: \quad \mathfrak{I}(A, \Phi) & \rightarrow & \operatorname{Cong}(A, \Phi) \\
I & \mapsto & \{(x, y) \in A \times A \mid x-y \in I\}
\end{array}
$$

Proof. Proposition 1.3.7 assures that $\varphi_{A}$ maps ideals of $(A, \Phi)$ to $\mathcal{C}^{\infty}$-congruences.

Also, given a $\mathcal{C}^{\infty}$-congruence $R$ in $A$, we have that $\psi_{A}(R)$ is an ideal.

Claim: $\{x \in A \mid(x, 0) \in R\}$ is closed under $\Phi(+)$ and $\Phi(-)$, since for any $x, y \in A$ such that $(x, 0),(y, 0) \in R$, we have $\Phi^{(2)}(+)((x, 0),(y, 0))=(\Phi(+)(x, y), \Phi(+)(0,0))=(x+y, 0) \in R$, for $R$ is a $\mathcal{C}^{\infty}$-congruence and $\left(R, \Phi^{(2)^{\prime}}\right)$ is a $\mathcal{C}^{\infty}$-subring of $\left(A \times A, \Phi^{(2)}\right)$.

Also, given $x \in A$ such that $(x, 0) \in R$, since $\left(R, \Phi^{(2)^{\prime}}\right)$ is a $\mathcal{C}^{\infty}$-subring of $\left(A \times A, \Phi^{(2)}\right)$, we have $\Phi^{(2)^{\prime}}(-)(x, 0)=(-x, 0) \in R$.

Finally, given $x \in A$ such that $(x, 0) \in R$ and any $y \in A$, we have both $(x, 0),(y, y) \in R$, and since $R$ is a $\mathcal{C}^{\infty}$-congruence, and thus $\left(R, \Phi^{(2)^{\prime}}\right)$ is a $\mathcal{C}^{\infty}$-subring of $\left(A \times A, \Phi^{(2)}\right)$, we have:

$$
\Phi^{(2)^{\prime}}(\cdot)((x, 0),(y, y))=(x \cdot y, y \cdot 0)=(x \cdot y, 0) \in R
$$

so $x \cdot y \in\{x \in A \mid(x, 0) \in R\}$. Hence $\{x \in A \mid(x, 0) \in R\}$ is an ideal in $(A, \Phi)$.

Now we are going to show that $\psi_{A}$ and $\varphi_{A}$ are inverse functions.

Given $R \in \operatorname{Cong}(A, \Phi)$, we have:

$$
\varphi_{A}\left(\psi_{A}(R)\right)=\varphi_{A}(\{x \in A \mid(x, 0) \in R\})=\{(x, y) \in A \times A \mid(x-y, 0) \in R\}=R
$$

so $\varphi_{A} \circ \psi_{A}=\operatorname{id}_{\operatorname{Cong}(A, \Phi)}$.

Given $I \in \mathfrak{I}(A)$, we have:

$$
\psi_{A}\left(\varphi_{A}(I)\right)=\psi_{A}(\{(x, y) \in A \times A \mid x-y \in I\})=\{z \in A \mid z-0 \in I\}=I,
$$

so $\psi_{A} \circ \varphi_{A}=\operatorname{id}_{\mathfrak{I}(A, \Phi)}$. 
For any $\mathcal{C}^{\infty}$-ring $(A, \Phi)$ we have the function:

$$
\begin{array}{ccc}
\psi_{A}: \operatorname{Cong}(A, \Phi) & \rightarrow & \Im(A, \Phi) \\
R & \mapsto\{a \in A \mid(a, \Phi(0)) \in R\}
\end{array}
$$

and whenever $(A, \Phi)$ is a finitely generated $\mathcal{C}^{\infty}$-ring, we have seen in Proposition 1.3 .8 that $\psi_{A}$ has $\varphi_{A}$ as inverse - which is a consequence of Hadammard's Lemma.

In order to show that $\psi_{A}$ is a bijection for any $\mathcal{C}^{\infty}$-ring $(A, \Phi)$, first we decompose it as a directed colimit of its finitely generated $\mathcal{C}^{\infty}$-subrings (cf. Theorem 1.3.9):

$$
(A, \Phi) \cong \lim _{\left(A_{i}, \Phi_{i}\right) \underset{\text { f.g. }}{\longrightarrow}(A, \Phi)}\left(A_{i}, \Phi \uparrow_{A_{i}}\right)
$$

and then we use Proposition 1.3.8 to obtain a bijection:

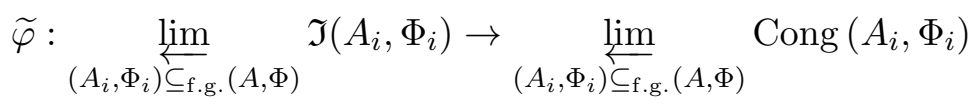

such that for every $\left(A_{i}, \Phi_{i}\right) \subseteq_{\text {f.g. }}(A, \Phi)$ the following diagram commutes:

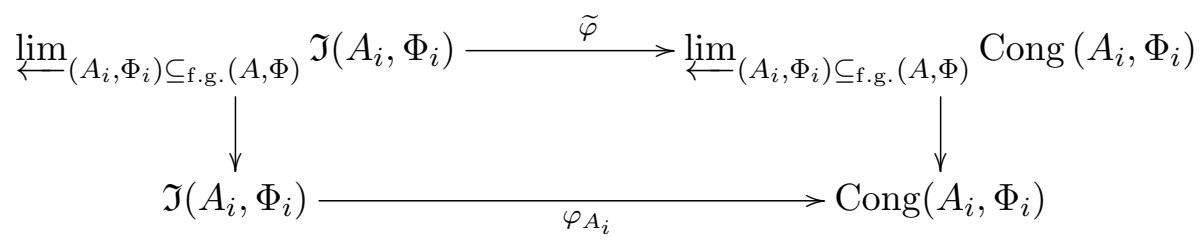

where the vertical downward arrows are the canonical arrows of the projective limit.

Finally we show that there is a bijective correspondence, $\alpha$, between $\lim _{\left(A_{i}, \Phi_{i}\right) \subseteq_{\text {f.g. }}(A, \Phi)} \mathfrak{I}\left(A_{i}, \Phi_{i}\right)$ and $\mathfrak{I}\left(\lim _{\left(A_{i}, \Phi_{i}\right) \subseteq_{\text {f.g. }}(A, \Phi)}\left(A_{i}, \Phi_{i}\right)\right)$, and a bijective correspondence, $\beta$, between $\varliminf_{\left(A_{i}, \Phi_{i}\right) \subseteq_{\text {f.g. }}(A, \Phi)} \operatorname{Cong}\left(A_{i}, \Phi_{i}\right)$ and Cong $\left(\underline{\lim }_{\left(A_{i}, \Phi_{i}\right) \underline{\subseteq}_{\text {f.g. }}(A, \Phi)}\left(A_{i}, \Phi_{i}\right)\right)$. In fact, the bijections $\alpha, \beta, \widetilde{\varphi}$ and $\psi$ are complete lattices isomorphisms.

By composing these bijections we prove that the congruences of $\left(\mathcal{C}^{\infty}\left(\mathbb{R}^{E}\right), \Phi_{E}\right)$ are classified by the ring-theoretic ideals of $\left(\mathcal{C}^{\infty}\left(\mathbb{R}^{E}\right), \Phi_{E}\right)$.

Theorem 1.3.9. Let $(A, \Phi)$ be any $\mathcal{C}^{\infty}$-ring. There is a directed system of $\mathcal{C}^{\infty}$-rings, $\left(\left(A_{i}, \Phi_{i}\right), \alpha_{i j}\right)$, where each $\left(A_{i}, \Phi_{i}\right)$ is a finitely generated $\mathcal{C}^{\infty}$-ring and each $\alpha_{i j}:\left(A_{i}, \Phi_{i}\right) \rightarrow\left(A_{j}, \Phi_{j}\right)$ is a monomorphism such that:

$$
(A, \Phi) \cong \lim _{\longrightarrow}\left(A_{i}, \Phi_{i}\right)
$$

Proof. Let $\mathcal{P}_{f}$ be the set of all finite subsets of $A$, on which we consider the partial order defined by inclusion, i.e., $(\forall i \in I)(\forall j \in I)(i \preceq j \leftrightarrow i \subseteq j)$.

Let $\left(A_{i}, \Phi_{i}\right):=\langle i\rangle$ denote the intersection of all $\mathcal{C}^{\infty}$-subrings $\left(B, \Phi_{B}\right)$ of $(A, \Phi)$ which contain $i$, i.e.,

$$
\left(A_{i}, \Phi_{i}\right)=\left(\langle i\rangle, \Phi_{i}\right)=\bigcap_{i \subset B}\left(B, \Phi^{\prime}\right)
$$

where $\Phi_{i}=\Phi^{\prime}$ is the $\mathcal{C}^{\infty}$-structure described in Proposition 1.2.2 
Note that $I=\left\{\langle i\rangle=\left(A_{i}, \Phi_{i}\right) \mid i \in \mathcal{P}_{f}\right\}$, partially ordered by the inclusion, is a directed set, since it is not empty and given any $\langle i\rangle$ and $\langle j\rangle$ we can take, for instance, $k=i \cup j \in \mathcal{P}_{f}$ such that $\langle i\rangle \subseteq\langle k\rangle$ and $\langle j\rangle \subseteq\langle k\rangle$.

By Proposition 1.2.4 and using its notation,

$$
\bigcup_{i \in \mathcal{P}_{f}}\left(\langle i\rangle, \Phi^{\prime}\right)
$$

is a $\mathcal{C}^{\infty}$-subring of $(A, \Phi)$. By construction, each $\left(A_{i}, \Phi_{i}\right)$ is a finitely generated $\mathcal{C}^{\infty}$-subring of $(A, \Phi)$.

We clearly have the following set-theoretic equality:

$$
A=U(A, \Phi)=U\left(\bigcup_{i \in \mathcal{P}_{f}}\left(\langle i\rangle, \Phi^{\prime}\right)\right)=\bigcup_{i \in \mathcal{P}_{f}}\langle i\rangle,
$$

hence the equality $\Phi^{\prime}=\Phi$, so:

$$
(A, \Phi)=\bigcup_{i \in \mathcal{P}_{f}}\left(\langle i\rangle, \Phi^{\prime}\right)
$$

For each $i \in I$ we define $A_{i}=\langle i\rangle$ and take $D(i):=\left(A_{i}, \Phi_{i}\right)$. Whenever $i \subseteq j$, we have the inclusion $D(i \subseteq j):=\alpha_{i j}:\left(\langle i\rangle, \Phi_{i}\right) \hookrightarrow\left(\langle j\rangle, \Phi_{j}\right)$ which makes the following triangle commute:

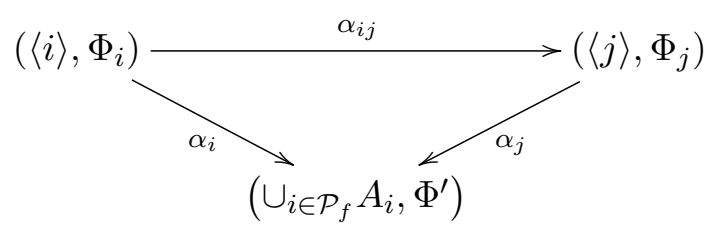

where $\alpha_{i}:\left(\langle i\rangle, \Phi_{i}\right) \rightarrow(A, \Phi)$ and $\alpha_{j}:\left(\langle j\rangle, \Phi_{j}\right) \rightarrow(A, \Phi)$ are the $\mathcal{C}^{\infty}$-homomorphisms of inclusion.

Consider the following diagram, where $\mathcal{P}_{f}$ is viewed as a category:

$$
\begin{array}{rlcc}
D: & \mathcal{P}_{f} & \rightarrow & \mathcal{C}^{\infty} \mathrm{Rng} \\
i \rightarrow j & \mapsto & \left(\langle i\rangle, \Phi_{i}\right) \stackrel{\alpha_{i j}}{\rightarrow}\left(\langle j\rangle, \Phi_{j}\right)
\end{array}
$$

We claim that:

$$
\lim _{i \in \mathcal{P}_{f}} D(i) \cong(A, \Phi)
$$

Let $(B, \Psi)$ be any $\mathcal{C}^{\infty}$-ring and $h_{i}:\left(\langle i\rangle, \Phi_{i}\right) \rightarrow(B, \Psi)$ and $h_{j}:\left(\langle j\rangle, \Phi_{j}\right) \rightarrow(B, \Psi)$ be any two $\mathcal{C}^{\infty}$-homomorphisms. We are going to show that there exists a unique $\mathcal{C}^{\infty}$-homomorphism $h:\left(\cup_{i \in \mathcal{P}_{f}} A_{i}, \Phi\right) \rightarrow$ $(B, \Psi)$ such that the following diagram commutes:

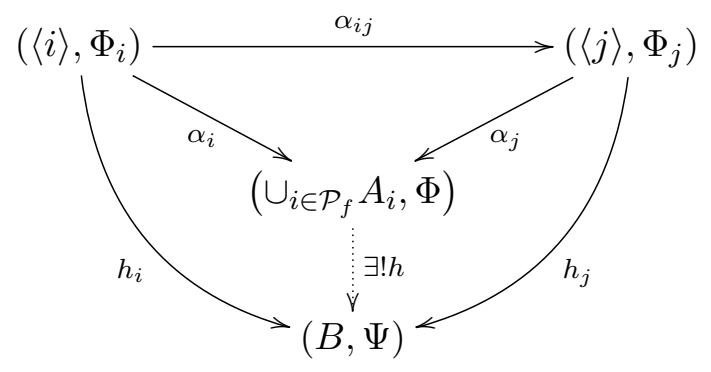


We now define the function which underlies $h$ in the following way: given $a \in \cup_{i \in \mathcal{P}_{f}} A_{i}$ there is some $i_{0} \in \mathcal{P}_{f}$ such that $a \in i_{0}$. For any $j_{0} \in \mathcal{P}_{f}$ such that $a \in j_{0}$, since $I$ is directed, there is some $k_{0} \in \mathcal{P}_{f}$ such that $k_{0} \supseteq i_{0}, j_{0}$, so we have $a \in k_{0}$ and the following commutative diagram:

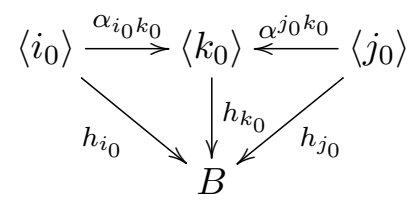

so:

$$
h_{i_{0}}(a)=h_{k_{0}}\left(\alpha_{i_{0} k_{0}}(a)\right)=h_{k_{0}}(a)=h_{k_{0}}\left(\alpha_{j_{0} k_{0}}(a)\right)=h_{j_{0}}(a) .
$$

Hence, given $a \in \cup_{i \in \mathcal{P}_{f}} A_{i}$, choose any $i_{0} \in \mathcal{P}_{f}$ such that $a \in i_{0}$ and define $h(a)=h_{i_{0}}(a)$ (up to here $h$ is merely a function, not a $\mathcal{C}^{\infty}$-homomorphism).

Now we prove that $h$ is a $\mathcal{C}^{\infty}$-homomorphism.

Let $f \in \mathcal{C}^{\infty}\left(\mathbb{R}^{n}, \mathbb{R}\right)$ be any $n$-ary function symbol. We must show that the following diagram commutes:

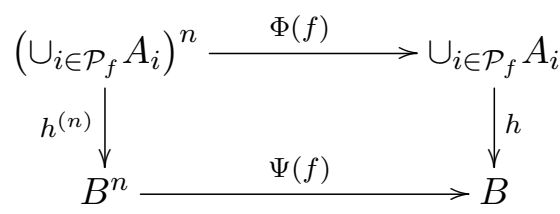

that is,

$$
\left(\forall a_{1}\right)\left(\forall a_{2}\right) \cdots\left(\forall a_{n}\right)\left(\Psi(f)\left(h\left(a_{1}\right), h\left(a_{2}\right), \cdots, h\left(a_{n}\right)\right)\right)=h\left(\Phi(f)\left(a_{1}, a_{2}, \cdots, a_{n}\right)\right) .
$$

Let $i_{1}, i_{2}, \cdots, i_{n} \in \mathcal{P}_{f}$ be such that $a_{1} \in\left\langle i_{1}\right\rangle, a_{2} \in\left\langle i_{2}\right\rangle, \cdots, a_{n} \in\left\langle i_{n}\right\rangle$, and take $j \in \mathcal{P}_{f}$ such that $j \supseteq i_{1}, \cdots, i_{n}$, so

$$
a_{1}, a_{2}, \cdots, a_{n} \in\langle j\rangle
$$

For any $\ell \in\{1,2, \cdots, n\}, h_{i}\left(a_{\ell}\right)=h_{j}\left(a_{\ell}\right)$, so $h\left(a_{1}\right)=h_{j}\left(a_{1}\right), h\left(a_{2}\right)=h_{j}\left(a_{2}\right), \cdots, h\left(a_{n}\right)=h_{j}\left(a_{n}\right)$.

We have:

$$
\begin{aligned}
\Psi(f)\left(h\left(a_{1}\right), h\left(a_{2}\right), \cdots, h\left(a_{n}\right)\right)=\Psi(f)\left(h_{j}\left(a_{1}\right), h_{j}\left(a_{2}\right), \cdots, h_{j}\left(a_{n}\right)\right) \stackrel{(1)}{=} \\
=h_{j}\left(\Phi_{j}(f)\left(a_{1}, a_{2}, \cdots, a_{n}\right)\right) \stackrel{(2)}{=} h_{j}\left(\Phi(f)\left(a_{1}, a_{2}, \cdots, a_{n}\right)\right) \stackrel{(3)}{=} h\left(\Phi_{i}(f)\left(a_{1}, a_{2}, \cdots, a_{n}\right)\right)
\end{aligned}
$$

where (1) is due to the fact that $h_{j}:\left(\langle i\rangle, \Phi_{i}\right) \rightarrow(B, \Psi)$ is a $\mathcal{C}^{\infty}$-homomorphism, (2) is due to the fact that $\left(\langle j\rangle, \Phi_{j}\right)$ is a $\mathcal{C}^{\infty}$-subring of $\left(\cup_{i \in \mathcal{P}_{f}} A_{i}, \Phi\right)$ and (3) occurs by the very definition of $h$. The uniqueness is granted by the property that $h$ must satisfy as a function.

Under those circumstances,

$$
\left(\bigcup_{i \in \mathcal{P}_{f}}\langle i\rangle, \Phi\right) \cong \underset{i \in I}{\lim _{i \in I}}\left(A_{i}, \Phi_{i}\right)
$$

so

$$
(A, \Phi)=\left(\bigcup_{i \in \mathcal{P}_{f}}\langle i\rangle, \Phi\right) \cong \lim _{i \in \mathcal{P}_{f}}\left(A_{i}, \Phi_{i}\right)
$$


Remark 1.3.10. Let $\left(\left(A_{i}, \Phi_{i}\right)_{i \in I}, \alpha_{i j}:\left(A_{i}, \Phi_{i}\right) \rightarrow\left(A_{j}, \Phi_{j}\right)\right)$ be an inductive directed system of $\mathcal{C}^{\infty}$-rings, and let $\left(J_{\ell}\right)_{\ell \in I} \in \lim _{\ell \in I} \Im\left(A_{\ell}, \Phi_{\ell}\right)$.

For every $i \in I$, we have the maps:

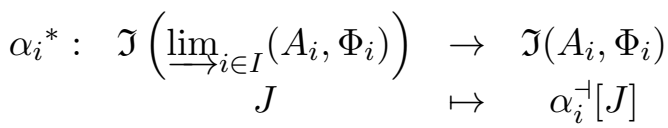

$$
\begin{aligned}
& \begin{array}{ccc}
\widehat{\alpha}: \quad \mathfrak{I}\left(\lim _{i \in I}\left(A_{i}, \Phi_{i}\right)\right) & \rightarrow & \Im\left(A_{i}, \Phi_{i}\right) \\
J & \mapsto & \left(\alpha_{i}^{\dashv}[J]\right)_{i \in I}
\end{array}
\end{aligned}
$$

and the following limit diagram:

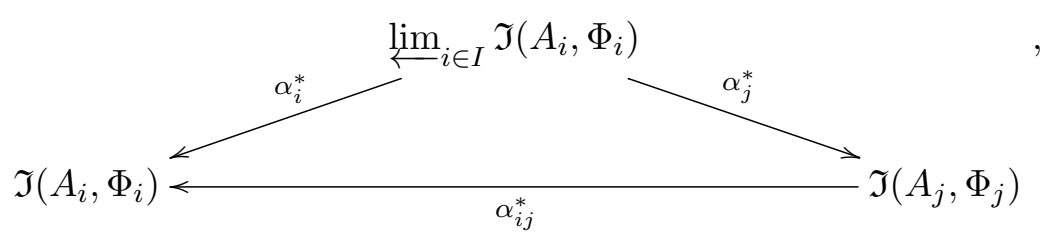

where:

$$
\begin{aligned}
& \alpha_{i j}{ }^{*}: \quad \Im\left(A_{j}, \Phi_{j}\right) \rightarrow \mathfrak{I}\left(A_{i}, \Phi_{i}\right) \\
& J_{j} \quad \mapsto \alpha_{i j}{ }^{-}\left[J_{j}\right]
\end{aligned}
$$

We note, first, that $\alpha$ maps ideals of $\varliminf_{i \in I}\left(A_{i}, \Phi_{i}\right)$ to an element of $\varliminf_{i \in I} \Im\left(A_{i}, \Phi_{i}\right)$.

In fact, given any ideal $J \in \mathfrak{I}\left(\lim _{i \in I}\left(A_{i}, \Phi_{i}\right)\right)$, since for every $i \in I, \alpha_{i}$ is a $\mathcal{C}^{\infty}$-homomorphism, it follows that for every $i \in I, \alpha_{i}^{*}(J)=\alpha_{i}{ }^{\dashv}[J]$ is an ideal of $\left(A_{i}, \Phi_{i}\right)$, so $\alpha(J)=\left(\alpha_{i}^{*}(J)\right)_{i \in I} \in \prod_{i \in I} \mathfrak{I}\left(A_{i}, \Phi_{i}\right)$. Moreover, the family $\left(\alpha_{i}^{*}(J)\right)_{i \in I}$ is compatible, since:

$$
(\forall i \in I)(\forall j \in I)(i \preceq j)\left(\alpha_{j} \circ \alpha_{i j}=\alpha_{i} \Rightarrow \alpha_{i}^{*}=\alpha_{i j}{ }^{*} \circ \alpha_{j}^{*}\right)
$$

and

$$
(\forall i \in I)(\forall j \in I)(i \preceq j)\left(\alpha_{i}^{*}(J)=\left(\alpha_{i j}{ }^{*} \circ \alpha_{j}^{*}\right)(J)=\alpha_{i j}{ }^{*}\left(\alpha_{j}^{*}(J)\right)\right.
$$

so

$$
\alpha(J)=\left(\alpha_{i}^{*}(J)\right)_{i \in I} \in{\underset{i}{i \in I}}_{\lim } \Im\left(A_{i}, \Phi_{i}\right) .
$$

Proposition 1.3.11. Let $(I, \preceq)$ be a directed partially ordered set and

$$
\left\{\left(A_{i}, \Phi_{i}\right), \alpha_{i j}:\left(A_{i}, \Phi_{i}\right) \rightarrow\left(A_{j}, \Phi_{j}\right)\right\}_{i, j \in I}
$$

be a directed inductive system of $\mathcal{C}^{\infty}$-rings and $\mathcal{C}^{\infty}$-homomorphisms. For every $i \in I$, we have the map:

$$
\begin{array}{ccc}
\alpha_{i}^{*}: \quad \mathfrak{I}\left({\underset{\lim }{\longrightarrow} i \in I}_{J}\left(A_{i}, \Phi_{i}\right)\right) & \rightarrow & \mathfrak{I}\left(A_{i}, \Phi_{i}\right) \\
J & \mapsto & \alpha_{i}^{\dashv}[J]
\end{array}
$$

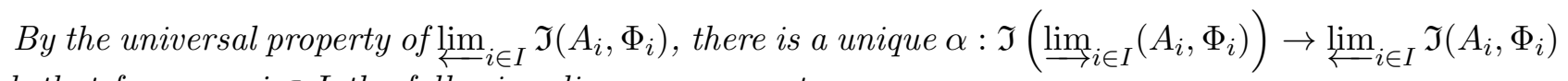
such that for every $i \in I$ the following diagram commutes: 


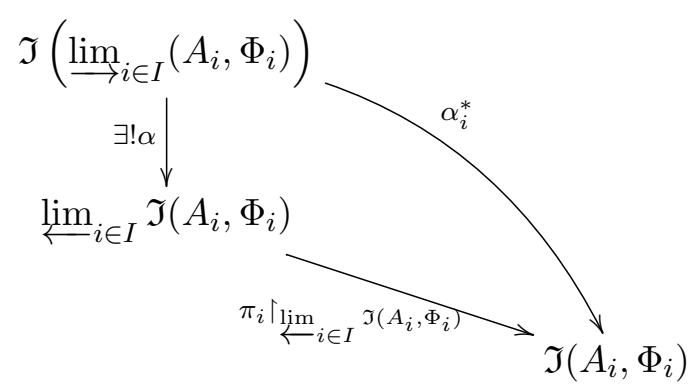

that is, such that $\alpha_{i}^{*}=\pi_{i} \uparrow_{\lim _{i \in I}} \Im\left(A_{i}, \Phi_{i}\right) \circ \alpha$.

We have, thus:

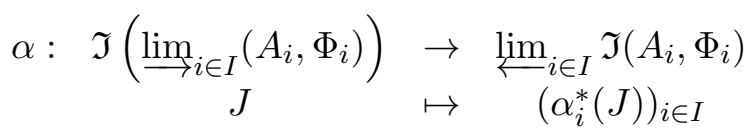

For any $\left(J_{i}\right)_{i \in I} \in \lim _{i \in I} \Im\left(A_{i}, \Phi_{i}\right), \bigcup_{i \in I} \alpha_{i}\left[J_{i}\right]$ is an ideal of $\lim _{i \in I}\left(A_{i}, \Phi_{i}\right)$ and the map:

$$
\begin{aligned}
\alpha^{\prime}: \lim _{i \in I} \mathfrak{I}\left(A_{i}, \Phi_{i}\right) & \rightarrow \mathfrak{I}\left({\underset{\lim }{\left(J_{i}\right)_{i \in I}}}_{\bigcup_{i \in I}} \alpha_{i}\left[A_{i}, \Phi_{i}\right]\right.
\end{aligned}
$$

is an inverse for $\alpha$, so $\alpha$ is a bijection.

Proof. In Set, we have:

$$
U\left(\lim _{i \in I}\left(A_{i}, \Phi_{i}\right)\right)=\frac{\bigcup_{i \in I} A_{i} \times\{i\}}{\sim}
$$

where:

$$
\left(a_{i}, i\right) \sim\left(a_{j}, j\right) \Longleftrightarrow(\exists k \in I)(i, j \preceq k)\left(\alpha_{i k}\left(a_{i}\right)=\alpha_{j k}\left(a_{j}\right)\right)
$$

and

$$
U\left(\lim _{\overleftarrow{i \in I}} \Im\left(A_{i}, \Phi_{i}\right)\right)=\left\{\left(J_{\ell}\right)_{\ell \in I} \in \prod_{i \in I} \Im\left(A_{i}, \Phi_{i}\right) \mid(\forall i, j \in I)(i \preceq j)\left(\alpha_{i j}^{*}\left(J_{j}\right)=J_{i}\right)\right\}
$$

Given the following colimit diagram:

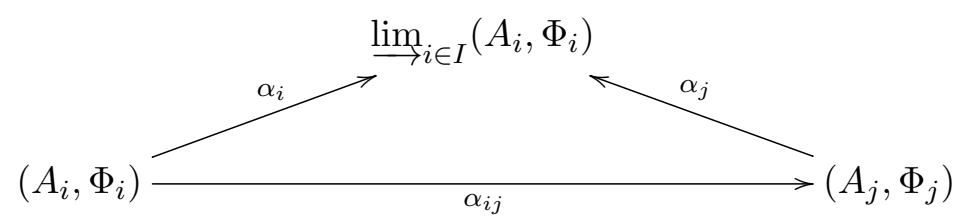

with:

$$
\begin{array}{rll}
\alpha_{i}:\left(A_{i}, \Phi_{i}\right) & \rightarrow & \lim _{i \in I}\left(A_{i}, \Phi_{i}\right) \\
a_{i} & \mapsto & {\left[\left(a_{i}, i\right)\right]}
\end{array}
$$

consider the following limit diagram: 


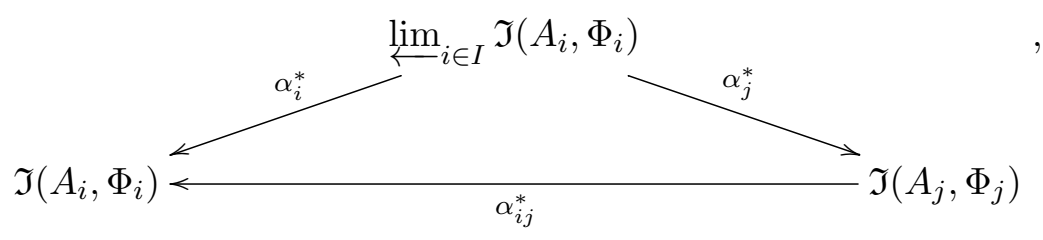

where:

$$
\begin{aligned}
& \alpha_{i j}{ }^{*}: \quad \mathfrak{I}\left(A_{j}, \Phi_{j}\right) \rightarrow \mathfrak{I}\left(A_{i}, \Phi_{i}\right) \\
& J_{j} \quad \mapsto \quad \alpha_{i j}{ }^{\dashv}\left[J_{j}\right]
\end{aligned}
$$

Given any $\left(J_{i}\right)_{i \in I}$, we are going to show that $\alpha^{\prime}\left(\left(J_{i}\right)_{i \in I}\right) \in \mathfrak{I}\left(\underline{\lim }_{i \in I}\left(A_{i}, \Phi_{i}\right)\right)$.

Since $\lim _{i \in I}\left(A_{i}, \Phi_{i}\right)$ is directed, given $\bar{x}, \bar{y} \in \bigcup_{i \in I} \alpha_{i}\left[J_{i}\right]$, there are $i, j \in I, x_{i} \in J_{i}$ and $y_{j} \in J_{j}$ such that $\alpha_{i}\left(x_{i}\right)=\left[\left(x_{i}, i\right)\right]=\bar{x}$ and $\alpha_{j}\left(y_{j}\right)=\left[\left(y_{j}, j\right)\right]=\bar{y}$. Also, since the colimit is directed, there is $k \in I$ with $i, j \leq k$ such that:

$$
\alpha_{i k}\left(x_{i}\right) \in \alpha_{i k}\left[J_{i}\right], \alpha_{j k}\left(y_{j}\right) \in \alpha_{j k}\left[J_{j}\right],
$$

so

$$
\alpha_{k}\left(\alpha_{i k}\left(x_{i}\right)\right), \alpha_{k}\left(\alpha_{j k}\left(y_{j}\right)\right) \in \alpha_{k}\left[J_{k}\right]
$$

Since $\alpha_{k}\left[J_{k}\right]$ is an ideal in $\alpha_{k}\left[A_{k}\right]$, it follows that:

$$
\alpha_{k}\left(\alpha_{i k}\left(x_{i}\right)\right)-\alpha_{k}\left(\alpha_{j k}\left(y_{j}\right)\right) \in \alpha_{k}\left[J_{k}\right]
$$

and

$$
\bar{x}-\bar{y}=\alpha_{k}\left(\alpha_{i k}\left(x_{i}\right)\right)-\alpha_{k}\left(\alpha_{j k}\left(y_{j}\right)\right) \in \alpha_{k}\left[J_{k}\right] \subseteq \bigcup_{i \in I} \alpha_{i}\left[J_{i}\right]
$$

Given $\bar{x} \in \lim _{i \in I} \alpha_{i}\left[J_{i}\right]$ there are some $i \in I, x_{i} \in J_{i}$ such that $\bar{x}=\alpha_{i}\left(x_{i}\right)=\left[\left(x_{i}, i\right)\right]$, and given $\bar{a} \in \underline{\lim }_{i \in I}\left(A_{i}, \overrightarrow{\left.\Phi_{i}\right)}\right.$, there are some $j \in I$ and some $a_{j} \in A_{j}$ such that $\bar{a}=\alpha_{j}\left(a_{j}\right)=\left[\left(a_{j}, j\right)\right]$.

Since the colimit is directed, there is some $k \in I$ with $i, j \leq k$ such that:

$$
\alpha_{i k}\left(x_{i}\right) \in \alpha_{i k}\left[J_{i}\right], \alpha_{j k}\left(a_{j}\right) \in \alpha_{j k}\left[A_{j}\right]
$$

so

$$
\alpha_{i}\left(x_{i}\right)=\alpha_{k}\left(\alpha_{i k}\left(x_{i}\right)\right) \in \alpha_{i}\left[\alpha_{i k}\left[J_{i}\right]\right]=\alpha_{k}\left[J_{k}\right]
$$

and

$$
\alpha_{j}\left(a_{j}\right)=\alpha_{k}\left(\alpha_{j k}\left(a_{j}\right)\right) \in \alpha_{j}\left[\alpha_{j k}\left[J_{j}\right]\right]=\alpha_{k}\left[J_{k}\right]
$$

Since $\alpha_{k}\left[J_{k}\right]$ is an ideal of $\alpha_{k}\left[A_{k}\right]$, it follows that:

$$
\alpha_{k}\left(\alpha_{i k}\left(x_{i}\right)\right) \cdot \alpha_{k}\left(\alpha_{j k}\left(a_{j}\right)\right) \in \alpha_{k}\left[J_{k}\right] .
$$

Thus, 
$\bar{x} \cdot \bar{a}=\alpha_{i}\left(x_{i}\right) \cdot \alpha_{j}\left(a_{j}\right)=\alpha_{k}\left(\alpha_{i k}\left(x_{i}\right)\right) \cdot \alpha_{k}\left(\alpha_{j k}\left(a_{j}\right)\right)=$

$$
=\alpha_{k}\left(\alpha_{i k}\left(x_{i}\right)\right) \cdot \alpha_{k}\left(\alpha_{j k}\left(a_{j}\right)\right) \in \alpha_{k}\left[J_{k}\right] \subseteq \bigcup_{i \in I} \alpha_{i}\left[J_{i}\right]
$$

so $\alpha^{\prime}$ maps elements of $\lim _{i \in I} \mathfrak{I}\left(A_{i}, \Phi_{i}\right)$ to ideals of $\lim _{i \in I}\left(A_{i}, \Phi_{i}\right)$.

Claim: $\alpha \circ \alpha^{\prime}=\operatorname{id}_{\lim _{i \in I}} \Im\left(A_{i}, \Phi_{i}\right)$.

Given $\left(J_{\ell}\right)_{\ell \in I} \in \lim _{i \in I} \Im\left(A_{i}, \Phi_{i}\right)$, we have:

$$
\alpha^{\prime}\left(\left(J_{\ell}\right)_{\ell \in I}\right):=\bigcup_{\ell \in I} \alpha_{\ell}\left[J_{\ell}\right]
$$

so

$$
\alpha\left(\alpha^{\prime}\left(\left(J_{\ell}\right)_{\ell \in I}\right)\right)=\alpha\left(\bigcup_{\ell \in I} \alpha_{\ell}\left[J_{\ell}\right]\right):=\left(\alpha_{i}{ }^{-}\left[\bigcup_{\ell \in I} \alpha_{\ell}\left[J_{\ell}\right]\right]\right)_{i \in I} .
$$

We are going to show that for every $i \in I$,

$$
\alpha_{i}^{*}\left(\alpha^{\prime}\left(\left(J_{\ell}\right)_{\ell \in I}\right)\right)=J_{i}
$$

Given $i \in I$, on the one hand we have:

$$
J_{i} \subseteq \alpha_{i}{ }^{\dashv}\left[\alpha_{i}\left[J_{i}\right]\right] \subseteq \alpha_{i}{ }^{\dashv}\left[\bigcup_{\ell \in I} \alpha_{\ell}\left[J_{\ell}\right]\right]=\alpha_{i}^{*}\left(\alpha^{\prime}\left(\left(J_{\ell}\right)_{\ell \in I}\right)\right)=\bigcup_{\ell \in I} \alpha_{i}{ }^{\dashv}\left[\alpha_{\ell}\left[J_{\ell}\right]\right]
$$

On the other hand, given $a_{i} \in \alpha_{i}{ }^{*}\left(\alpha^{\prime}\left(\left(J_{\ell}\right)_{\ell \in I}\right)\right)=\alpha_{i}{ }^{\dashv}\left[\bigcup_{\ell \in I} \alpha_{\ell}\left[J_{\ell}\right]\right]$, that is, $a_{i} \in A_{i}$ such that $\alpha_{i}\left(a_{i}\right) \in$ $\bigcup_{\ell \in I} \alpha_{\ell}\left[J_{\ell}\right]$, there is some $j \in I$ and some $b_{j} \in J_{j}$ such that:

$$
\alpha_{i}\left(a_{i}\right)=\alpha_{j}\left(b_{j}\right)
$$

Since the system is directed, there is some $k \in I, i, j \preceq k$ such that $\alpha_{i k}\left(a_{i}\right)=\alpha_{j k}\left(b_{j}\right)$.

By compatibility we have $\alpha_{j k}{ }^{-1}\left[J_{k}\right]=\alpha_{j k}{ }^{*}\left(J_{k}\right)=J_{j}$, so in particular $\alpha_{j k}^{\dashv}\left[J_{k}\right] \supseteq J_{j}$ and $\alpha_{j k}\left(b_{j}\right) \in$ $\alpha_{j k}\left[J_{j}\right] \subseteq \alpha_{j k}\left[\alpha_{j k}{ }^{-1}\left[J_{k}\right]\right] \subseteq J_{k}$

Thus, taking $c_{k}=\alpha_{j k}\left(b_{j}\right)$, we have:

$$
c_{k}=\alpha_{j k}\left(b_{j}\right) \in \alpha_{j k}^{\dashv}\left[J_{j}\right] \subseteq J_{k},
$$

that is,

$$
c_{k} \in J_{k} .
$$

For any $k^{\prime} \geq k$ we have:

$$
\alpha_{i k^{\prime}}\left(a_{i}\right)=\alpha_{k k^{\prime}}\left(c_{k}\right) \in J_{k^{\prime}}
$$


SO

$$
a_{i} \in \alpha_{i k^{\prime}}^{-1}\left[J_{k^{\prime}}\right]=J_{i}
$$

Hence:

$$
(\forall i \in I)\left(\bigcup_{\ell \in I} \alpha_{i}{ }^{\dashv}\left[\alpha_{\ell}\left[J_{\ell}\right]\right]=J_{i}\right)
$$

and

$$
\alpha\left(\alpha^{\prime}\left(\left(J_{i}\right)_{i \in I}\right)\right)=\left(J_{i}\right)_{i \in I}
$$

Since $\left(J_{i}\right)_{i \in I}$ is arbitrary, we have:

$$
\alpha \circ \alpha^{\prime}=\operatorname{id}_{\lim _{i \in I}} \Im\left(A_{i}, \Phi_{i}\right) .
$$

On the other hand, given $J \in \mathfrak{I}\left(\lim _{i \in I}\left(A_{i}, \Phi_{i}\right)\right)$, we have $\alpha(J)=\left(\alpha_{i}^{*}(J)\right)_{i \in I}$ and:

$$
\alpha^{\prime}(\alpha(J))=\alpha^{\prime}\left(\left(\alpha_{i}^{*}(J)\right)_{i \in I}\right)=\bigcup_{i \in I} \alpha_{i}\left[\alpha_{i}^{*}(J)\right] .
$$

On the one hand, since for every $i \in I, \alpha_{i}\left[\alpha_{i}{ }^{*}(J)\right]=\alpha_{i}\left[\alpha_{i}{ }^{-1}[J]\right] \subseteq J$, we have $\alpha^{\prime}(\alpha(J)) \subseteq J$.

Recall that given $J \in \mathfrak{I}\left(\lim _{i \in I}\left(A_{i}, \Phi_{i}\right)\right)$, we have:

$$
J \subseteq \underset{i \in I}{\lim }\left(A_{i}, \Phi_{i}\right)=\bigcup_{i \in I} \alpha_{i}\left[A_{i}\right]
$$

so given $\bar{x} \in J \subseteq \bigcup_{i \in I} \alpha_{i}\left[A_{i}\right]$, there are some $i_{0} \in I$ and some $x_{i_{0}} \in \alpha_{i_{0}}{ }^{*}(J)=\alpha_{i_{0}}{ }^{-1}[J]$ such that:

$$
\bar{x}=\left[\left(x_{i_{0}}, i_{0}\right)\right]
$$

and since $x_{i_{0}} \in \alpha_{i_{0}}^{*}(J)$, we have $\left[\left(x_{i_{0}}, i_{0}\right)\right] \in \alpha_{i_{0}}\left[\alpha_{i_{0}}{ }^{*}(J)\right] \subseteq \bigcup_{i \in I} \alpha_{i}\left[\alpha_{i}^{*}(J)\right]$, so

$$
\bar{x} \in \bigcup_{i \in I} \alpha_{i}\left[\alpha_{i}^{*}(J)\right]=\alpha^{\prime}\left(\left(\alpha_{i}^{*}(J)\right)_{i \in I}\right)=\alpha^{\prime}(\alpha(J))
$$

and:

$$
\alpha^{\prime}(\alpha(J))=J
$$

Since $J$ is an arbitrary ideal of $\lim _{i \in I} \Im\left(A_{i}, \Phi_{i}\right)$, it follows that:

$$
\alpha^{\prime} \circ \alpha=\operatorname{id}_{\mathfrak{I}}\left({\underset{\lim }{\rightarrow} i \in I}_{i}\left(A_{i}, \Phi_{i}\right)\right) .
$$

so $\alpha$ is a bijection whose inverse is $\alpha^{\prime}$.

The proof of the following proposition is similar to the proof we just made, so we are going to omit it. 
Proposition 1.3.12. Let $(I, \preceq)$ be a directed partially ordered set and $\left\{\left(A_{i}, \Phi_{i}\right), \alpha_{i j}:\left(A_{i}, \Phi_{i}\right) \rightarrow\left(A_{j}, \Phi_{j}\right)\right\}_{i, j \in I}$ be a directed inductive system of $\mathcal{C}^{\infty}$-rings and $\mathcal{C}^{\infty}$-homomorphisms. The following function is a bijection:

$$
\begin{aligned}
& \beta: \operatorname{Cong}\left({\underset{\lim }{\longrightarrow} i \in I}_{R}\left(A_{i}, \Phi_{i}\right)\right) \rightarrow \underbrace{}_{\left(\lim _{i \in I}\right.} \operatorname{Cong}\left(A_{i}, \Phi_{i}\right) \\
& R \quad \mapsto \quad\left(\left(\alpha_{i} \times \alpha_{i}\right)^{-}(R)\right)_{i \in I}
\end{aligned}
$$

whose inverse is given by:

$$
\begin{aligned}
& \beta^{\prime}: \lim _{i \in I} \operatorname{Cong}\left(A_{i}, \Phi_{i}\right) \rightarrow \operatorname{Cong}\left(\lim _{i \in I}\left(A_{i}, \Phi_{i}\right)\right) \\
& \left(R_{i}\right)_{i \in I} \quad \mapsto \quad \lim _{i \in I} R_{i}
\end{aligned}
$$

Lemma 1.3.13. The congruences of $\left(\mathcal{C}^{\infty}\left(\mathbb{R}^{E}\right), \Phi_{E}\right)$, the free $\mathcal{C}^{\infty}$-ring determined by the set $E$, are classified by their ring-theoretic ideals.

Proof. By Proposition 1.3.7, for any finite $E$ the result holds.

Suppose $E$ is any set (not necessarily finite). We have, by definition,

$$
\left(\mathcal{C}^{\infty}\left(\mathbb{R}^{E}\right), \Phi_{E}\right) \cong \underset{E^{\prime} \underline{\lim }_{E}}{\lim _{(}}\left(\mathcal{C}^{\infty}\left(\mathbb{R}^{E^{\prime}}, \mathbb{R}\right), \Phi_{E^{\prime}}\right)
$$

where $\Phi_{E}$ is the $\mathcal{C}^{\infty}$-structure of the colimit.

The family of bijections $\left\{\psi_{E^{\prime}}: \operatorname{Cong}\left(\mathcal{C}^{\infty}\left(\mathbb{R}^{E^{\prime}}\right), \Phi_{E^{\prime}}\right) \rightarrow \mathfrak{I}\left(\mathcal{C}^{\infty}\left(\mathbb{R}^{E^{\prime}}\right), \Phi_{E^{\prime}}\right)\right\}$ always exist, so it yields a coherent bijection:

$$
\widetilde{\psi}: \lim _{E^{\prime} \subseteq f} \operatorname{Cong}\left(\mathcal{C}^{\infty}\left(\mathbb{R}^{E^{\prime}}\right), \Phi_{E^{\prime}}\right) \rightarrow \lim _{E^{\prime} \subseteq_{f} E} \Im\left(\mathcal{C}^{\infty}\left(\mathbb{R}^{E^{\prime}}\right), \Phi_{E^{\prime}}\right)
$$

We have the following commutative diagram:

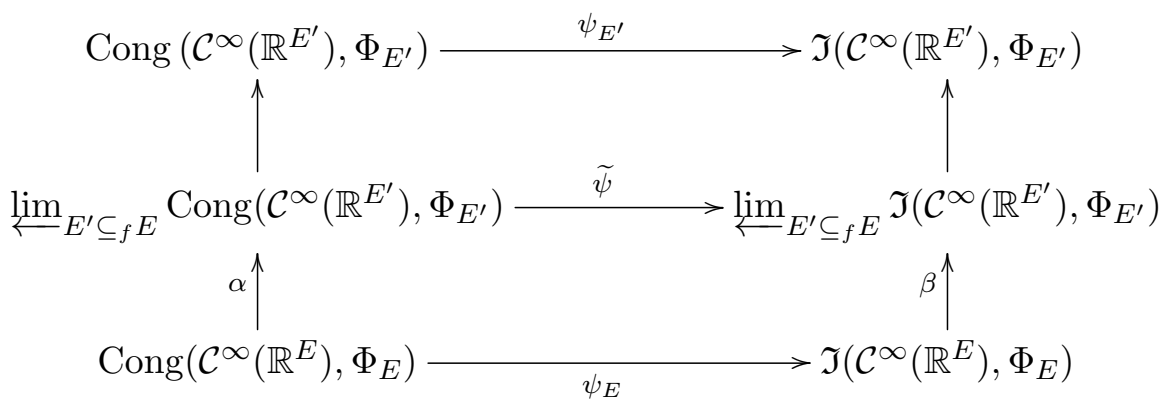

where the two upper vertical arrows are the canonical arrows of the projective limit, $\alpha$ is given in Proposition 1.3.11 and $\beta$ is given in Proposition 1.3.12.

Since $\alpha, \beta$ and $\widetilde{\psi}$ are bijections, it follows that $\psi_{E}: \operatorname{Cong}\left(\mathcal{C}^{\infty}\left(\mathbb{R}^{E}\right), \Phi_{E}\right) \rightarrow \Im\left(\mathcal{C}^{\infty}\left(\mathbb{R}^{E}\right), \Phi_{E}\right)$ is a bijection.

The following lemma is a well-known result of Universal Algebra applied to $\mathcal{C}^{\infty}$-rings: 
Lemma 1.3.14. Let $(A, \Phi)$ be a $\mathcal{C}^{\infty}$-ring and let $R \in \operatorname{Cong}(A, \Phi)$. Given the quotient $\mathcal{C}^{\infty}$-homomorphism:

$$
\begin{aligned}
q_{R}:(A, \Phi) & \rightarrow\left(\frac{A}{R}, \bar{\Phi}\right) \\
x & \mapsto \quad x+R
\end{aligned}
$$

we have the bijection:

$$
\begin{array}{clc}
\left(q_{R}\right)_{*}:\{S \in \operatorname{Cong}(A, \Phi) \mid R \subseteq S\} & \rightarrow & \operatorname{Cong}\left(\frac{A}{R}, \bar{\Phi}\right) \\
S & \mapsto \quad\left\{\left(q_{R}(s), q_{R}(t)\right) \in \frac{A}{R} \times \frac{A}{R} \mid(s, t) \in S\right\}
\end{array}
$$

whose inverse is given by:

$$
\begin{array}{ccc}
\left(q_{R}\right)^{*}: \quad \operatorname{Cong}\left(\frac{A}{R}, \bar{\Phi}\right) & \rightarrow & \{S \in \operatorname{Cong}(A, \Phi) \mid R \subseteq S\} \\
S^{\prime} & \mapsto & \left(q_{R} \times q_{R}\right)^{-1}\left[S^{\prime}\right]
\end{array}
$$

As a consequence of the above lemma, we have:

Lemma 1.3.15. Let $(A, \Phi)$ be a $\mathcal{C}^{\infty}$-ring and $R \in \operatorname{Cong}(A, \Phi)$, and suppose that $\psi_{A}$ : Cong $(A, \Phi) \rightarrow$ $\mathfrak{I}(A, \Phi)$ is a bijection with an inverse, $\varphi_{A}: \mathfrak{I}(A, \Phi) \rightarrow \operatorname{Cong}(A, \Phi)$. Under those circumstances, the quotient $\mathcal{C}^{\infty}$-homomorphism:

$$
q_{R}:(A, \Phi) \rightarrow\left(\frac{A}{R}, \bar{\Phi}\right)
$$

induces a pair of inverse bijections:

$$
\begin{aligned}
\left(q_{R}\right)_{+}: \quad \mathfrak{I}\left(\frac{A}{R}, \bar{\Phi}\right) & \rightarrow & \left\{I^{\prime} \in \mathfrak{I}(A, \Phi) \mid \psi_{A}(R) \subseteq I^{\prime}\right\} \\
J & \mapsto & q_{R}[J]
\end{aligned}
$$

and

$$
\begin{array}{cc}
\left(p_{R}\right)^{-}:\left\{I^{\prime} \in \mathfrak{I}(A, \Phi) \mid \psi_{A}(R) \subseteq I^{\prime}\right\} & \rightarrow \mathfrak{I}\left(\frac{A}{R}, \bar{\Phi}\right) \\
J^{\prime} & \mapsto\left(q_{R}\right)^{-1}\left[J^{\prime}\right]
\end{array}
$$

Proof. The proof is a direct consequence of Lemma 1.3.14 and a diagram chase.

Proposition 1.3.16. Let $(A, \Phi)$ and $(B, \Psi)$ be two $\mathcal{C}^{\infty}$-rings and let $h:(A, \Phi) \rightarrow(B, \Psi)$ be a surjective $\mathcal{C}^{\infty}$-homomorphism. The following functions are bijections:

$$
\begin{array}{cllc}
h^{*}: \operatorname{Cong}(B, \Psi) & \rightarrow & \{S \in \operatorname{Cong}(A, \Phi) \mid \operatorname{ker}(h) \subseteq S\} \\
R & \mapsto & (h \times h)^{-1}[R] \\
& & \\
h^{-}: \quad \mathfrak{I}(A, \Phi) & \rightarrow & \left\{I^{\prime} \in \mathfrak{I}(B, \Psi) \mid \varphi_{A}(\operatorname{ker}(h)) \subseteq I^{\prime}\right\} \\
J & \mapsto & h^{-1}[J]
\end{array}
$$

Proof. By the Theorem of the $\mathcal{C}^{\infty}$-Isomorphism, we have the $\mathcal{C}^{\infty}$-isomorphism: 


$$
\begin{aligned}
\bar{h}:\left(\frac{A}{\operatorname{ker}(h)}, \bar{\Phi}\right) & \rightarrow(B, \Psi) \\
a+\operatorname{ker}(h) & \mapsto h(a)
\end{aligned}
$$

so

$$
\begin{array}{rlc}
\alpha: \operatorname{Cong}\left(\frac{A}{\operatorname{ker}(h)}, \bar{\Phi}\right) & \rightarrow & \operatorname{Cong}(B, \Psi) \\
S^{\prime} & \mapsto\left\{\left(\bar{h}(s), \bar{h}\left(s^{\prime}\right)\right) \mid\left(s, s^{\prime}\right) \in S^{\prime}\right\}
\end{array}
$$

is a bijection whose inverse is given by:

$$
\begin{array}{clc}
\beta: \operatorname{Cong}(B, \Psi) & \rightarrow & \operatorname{Cong}\left(\frac{A}{\operatorname{ker}(h)}, \bar{\Phi}\right) \\
R & \mapsto\left\{\left(\bar{h}^{-1}(r), \bar{h}^{-1}\left(r^{\prime}\right)\right) \mid\left(r, r^{\prime}\right) \in R\right\}=\left\{\left(a+\operatorname{ker}(h), a^{\prime}+\operatorname{ker}(h)\right) \mid\left(h(a), h\left(a^{\prime}\right)\right) \in R\right\}
\end{array}
$$

Taking $R=\operatorname{ker}(h)$ in the Lemma 1.3 .14 yields a bijection $\left(q_{\operatorname{ker}(h)}\right)^{*}: \operatorname{Cong}\left(\frac{A}{\operatorname{ker}(h)}\right) \rightarrow\{S \in$ Cong $(A) \mid \operatorname{ker}(h) \subseteq S\}$.

Now it suffices to prove that $h^{*}=\left(q_{\operatorname{ker}(h)}\right)^{*} \circ \beta$.

Since $\bar{h}$ is an isomorphism, note that $\beta=\bar{h}^{*}$, and as $h=\bar{h} \circ q_{\operatorname{ker}(h)}$, we get $h^{*}=q_{\mathrm{ker}(h)}^{*} \circ \bar{h}^{*}$, as we claimed.

The proof for $h^{-}$is analogous, since $h^{-}=\left(q_{\operatorname{ker}(h)}\right)^{-} \circ \bar{h}^{-}$.

The following proposition gives us a description of $\mathcal{C}^{\infty}$-rings via generators and relations.

Proposition 1.3.17. Let $(A, \Phi)$ be any $\mathcal{C}^{\infty}$-ring. The $\mathcal{C}^{\infty}$-congruences of $(A, \Phi)$ are classified by the ring-theoretic ideals of $(A, \Phi)$.

Proof. Given any $\mathcal{C}^{\infty}-\operatorname{ring}(A, \Phi)$, we have the following isomorphism:

$$
\bar{\varepsilon}_{A}: \frac{\mathcal{C}^{\infty}\left(\mathbb{R}^{U(A)}\right)}{\operatorname{ker}\left(\varepsilon_{A}\right)} \cong A
$$

so we can write:

$$
A \cong \frac{L(A)}{\operatorname{ker}\left(\varepsilon_{A}\right)}=\frac{\mathcal{C}^{\infty}\left(\mathbb{R}^{A}\right)}{\operatorname{ker}\left(\varepsilon_{A}\right)}
$$

By Lemma 1.3.13, we have a bijection:

$$
\psi_{A}: \operatorname{Cong}\left(\mathcal{C}^{\infty}\left(\mathbb{R}^{A}\right), \Phi_{A}\right) \rightarrow \mathfrak{I}\left(\mathcal{C}^{\infty}\left(\mathbb{R}^{A}\right), \Phi_{A}\right)
$$

Note that given any $R \in \operatorname{Cong}\left(\mathcal{C}^{\infty}\left(\mathbb{R}^{A}\right), \Phi_{A}\right)$ such that $\operatorname{ker}\left(\varepsilon_{A}\right) \subseteq R$, we have $\psi_{A}\left(\operatorname{ker}\left(\varepsilon_{A}\right)\right)=\{g \in$ $\left.\mathcal{C}^{\infty}\left(\mathbb{R}^{A}\right) \mid(g, 0) \in \operatorname{ker}\left(\varepsilon_{A}\right)\right\} \subseteq\left\{g \in \mathcal{C}^{\infty}\left(\mathbb{R}^{A}\right) \mid(g, 0) \in R\right\}=\psi_{A}(R)$, so: 


$$
\psi_{A}\left[\left\{R \in \operatorname{Cong}\left(\mathcal{C}^{\infty}\left(\mathbb{R}^{A}\right), \Phi_{A}\right) \mid \operatorname{ker}\left(\varepsilon_{A}\right) \subseteq R\right\}\right] \subseteq\left\{I^{\prime} \in \mathfrak{I}\left(\mathcal{C}^{\infty}\left(\mathbb{R}^{A}\right), \Phi_{A}\right) \mid \psi_{A}\left(\operatorname{ker}\left(\varepsilon_{A}\right)\right) \subseteq I^{\prime}\right\}
$$

Also, let $I^{\prime} \in \mathfrak{I}\left(\mathcal{C}^{\infty}\left(\mathbb{R}^{A}\right), \Phi_{A}\right)$ be such that $\psi_{A}\left(\operatorname{ker}\left(\varepsilon_{A}\right)\right)=\left\{g \in \mathcal{C}^{\infty}\left(\mathbb{R}^{A}\right) \mid\left(g, \Phi_{A}(0)\right) \in \operatorname{ker}\left(\varepsilon_{A}\right)\right\} \subseteq I^{\prime}$. Given $\left(g_{1}, g_{2}\right) \in \operatorname{ker}\left(\varepsilon_{A}\right)$, we have $\left(g_{1}-g_{2}, \Phi_{A}(0)\right) \in \operatorname{ker}\left(\varepsilon_{A}\right)$, and since $\left\{g \in \mathcal{C}^{\infty}\left(\mathbb{R}^{A}\right) \mid\left(g, \Phi_{A}(0)\right) \in\right.$ $\left.\operatorname{ker}\left(\varepsilon_{A}\right)\right\} \subseteq I^{\prime}, g_{1}-g_{2} \in I^{\prime}$, so $\operatorname{ker}\left(\varepsilon_{A}\right) \subseteq \varphi_{A}\left(I^{\prime}\right)$ and :

$$
\varphi_{A}\left[\left\{I^{\prime} \in \Im\left(\mathcal{C}^{\infty}\left(\mathbb{R}^{A}\right), \Phi_{A}\right) \mid \psi_{A}\left(\operatorname{ker}\left(\varepsilon_{A}\right)\right) \subseteq I^{\prime}\right\}\right] \subseteq\left\{R \in \operatorname{Cong}\left(\mathcal{C}^{\infty}\left(\mathbb{R}^{A}\right), \Phi_{A}\right) \mid \operatorname{ker}\left(\varepsilon_{A}\right) \subseteq R\right\}
$$

We have, thus the functions:

$$
\begin{array}{cl}
\psi_{A}^{\prime}:\left\{R \in \operatorname{Cong}\left(\mathcal{C}^{\infty}\left(\mathbb{R}^{A}\right), \Phi_{A}\right) \mid \operatorname{ker}\left(\varepsilon_{A}\right) \subseteq R\right\} & \rightarrow \quad\left\{I^{\prime} \in \mathfrak{I}\left(\mathcal{C}^{\infty}\left(\mathbb{R}^{A}\right), \Phi_{A}\right) \mid \psi_{A}[R] \subseteq I^{\prime}\right\} \\
R & \mapsto \varphi_{A}(R)=\left\{g \in \mathcal{C}^{\infty}\left(\mathbb{R}^{A}\right) \mid\left(g, \phi_{A}(0)\right) \in R\right\}
\end{array}
$$

that is, $\psi_{A}^{\prime}=\psi_{A} \Upsilon_{\left\{R \in \operatorname{Cong}\left(\mathcal{C}^{\infty}\left(\mathbb{R}^{A}\right), \Phi_{A}\right) \mid \operatorname{ker}\left(\varepsilon_{A}\right) \subseteq R\right\}}$, and:

$$
\begin{aligned}
\varphi_{A}^{\prime}:\left\{I^{\prime} \in \Im\left(\mathcal{C}^{\infty}\left(\mathbb{R}^{A}\right), \Phi_{A}\right) \mid \psi_{A}[R] \subseteq I^{\prime}\right\} & \rightarrow \quad\left\{R \in \operatorname{Cong}\left(\mathcal{C}^{\infty}\left(\mathbb{R}^{A}\right), \Phi_{A}\right) \mid \operatorname{ker}\left(\varepsilon_{A}\right) \subseteq R\right\} \\
I^{\prime} & \mapsto \varphi_{A}\left(I^{\prime}\right)=\left\{\left(g_{1}, g_{2}\right) \in \mathcal{C}^{\infty}\left(\mathbb{R}^{A}\right) \times \mathcal{C}^{\infty}\left(\mathbb{R}^{A}\right) \mid g_{1}-g_{2} \in I^{\prime}\right\}
\end{aligned}
$$

where

$$
\varphi_{A}^{\prime}=\varphi_{A}\left\lceil_{\left\{I^{\prime} \in \mathcal{I}\left(\mathcal{C}^{\infty}\left(\mathbb{R}^{A}\right), \Phi_{A}\right) \mid \varphi_{A}[R] \subseteq I^{\prime}\right\}} .\right.
$$

Since $\varphi_{A}^{\prime}$ and $\psi_{A}^{\prime}$ are inverse bijections, we have the following commutative diagram:

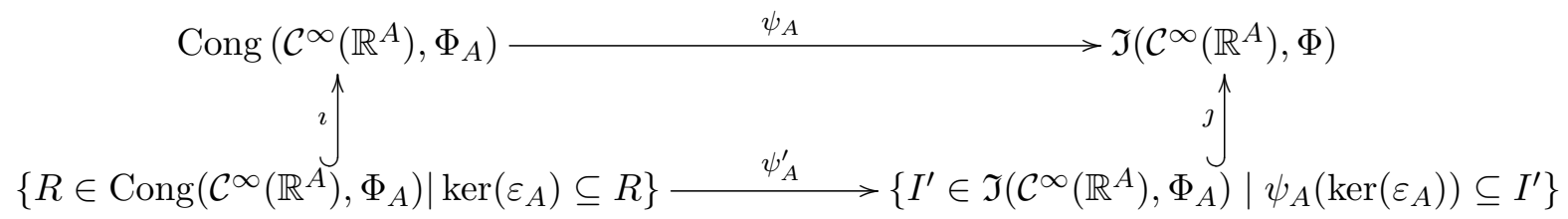

where $\imath$ and $\jmath$ are the ordinary inclusions.

We also have the bijection given in the Lemma $\mathbf{1 . 3 . 1 4}$

$$
\left(q_{\operatorname{ker}\left(\varepsilon_{A}\right)}\right)^{*}: \operatorname{Cong}\left(\frac{\mathcal{C}^{\infty}\left(\mathbb{R}^{A}\right)}{\operatorname{ker}\left(\varepsilon_{A}\right)}, \bar{\Phi}\right) \rightarrow\left\{R \in \operatorname{Cong}(A, \Phi) \mid \operatorname{ker}\left(\varepsilon_{A}\right) \subseteq R\right\}
$$

and the bijection given in lemmas Lemma 1.3.15 and 1.3.13.

$$
\left(q_{\operatorname{ker}\left(\varepsilon_{A}\right)}\right)^{-}: \mathfrak{I}\left(\frac{\mathcal{C}^{\infty}\left(\mathbb{R}^{A}\right)}{\operatorname{ker}\left(\varepsilon_{A}\right)}, \Phi\right) \rightarrow\left\{I^{\prime} \in \mathfrak{I}\left(\mathcal{C}^{\infty}\left(\mathbb{R}^{A}\right), \Phi\right) \mid \varphi_{A}\left(\operatorname{ker}\left(\varepsilon_{A}\right)\right) \subseteq I^{\prime}\right\}
$$

Hence, we have the following bijection:

$$
\psi_{\frac{\left.\mathcal{C}_{(\mathbb{R} A}\right)}{\operatorname{ker}\left(\varepsilon_{A}\right)}}: \operatorname{Cong}\left(\frac{\mathcal{C}^{\infty}\left(\mathbb{R}^{A}\right)}{\operatorname{ker}\left(\varepsilon_{A}\right)}, \bar{\Phi}\right) \rightarrow \mathfrak{I}\left(\frac{\mathcal{C}^{\infty}\left(\mathbb{R}^{A}\right)}{\operatorname{ker}\left(\varepsilon_{A}\right)}, \bar{\Phi}\right)
$$

since the following diagram commutes: 


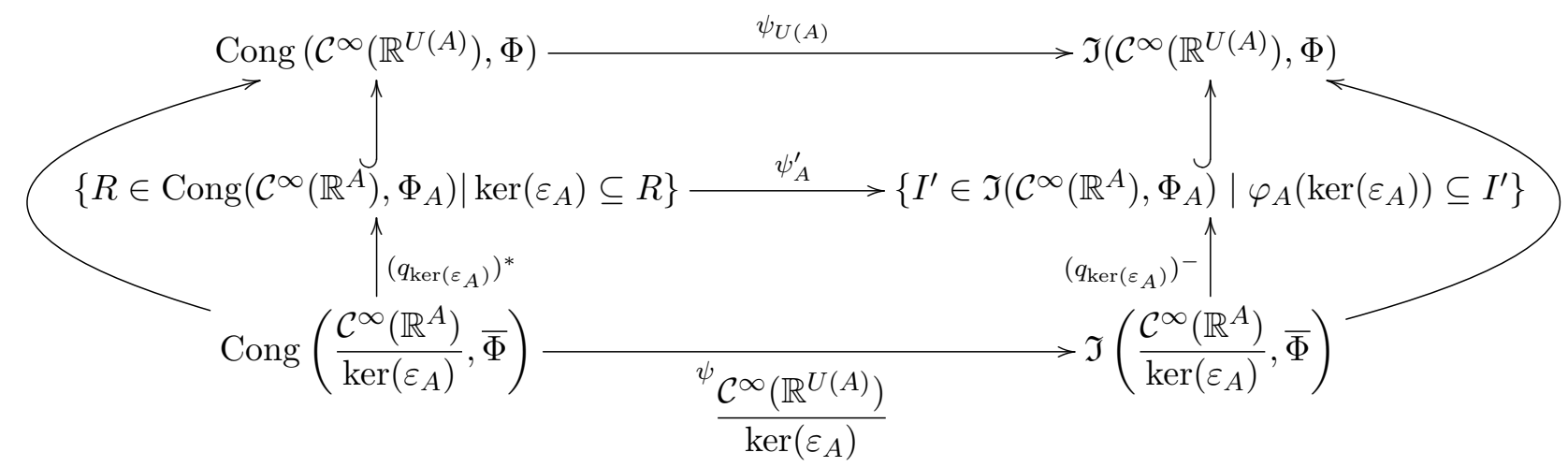

Since $\bar{\varepsilon}_{A}: \frac{\mathcal{C}^{\infty}\left(\mathbb{R}^{U(A)}\right)}{\operatorname{ker}\left(\varepsilon_{A}\right)} \cong A$ is an isomorphism, we have the bijections:

$$
\operatorname{Cong}(A, \Phi) \stackrel{\left(\bar{\varepsilon}_{A}\right)^{*}}{\rightarrow} \operatorname{Cong}\left(\frac{\mathcal{C}^{\infty}\left(\mathbb{R}^{A}\right)}{\operatorname{ker}\left(\varepsilon_{A}\right)}, \bar{\Phi}\right)
$$

and

$$
\mathfrak{I}(A, \Phi) \stackrel{\left(\bar{\varepsilon}_{A}\right)^{-}}{\rightarrow} \mathfrak{I}\left(\frac{\mathcal{C}^{\infty}\left(\mathbb{R}^{A}\right)}{\operatorname{ker}\left(\varepsilon_{A}\right)}, \bar{\Phi}\right)
$$

so we have the bijection:

$$
\psi_{(A, \Phi)}: \operatorname{Cong}(A, \Phi) \rightarrow \mathfrak{I}(A, \Phi)
$$

since the following diagram commutes:

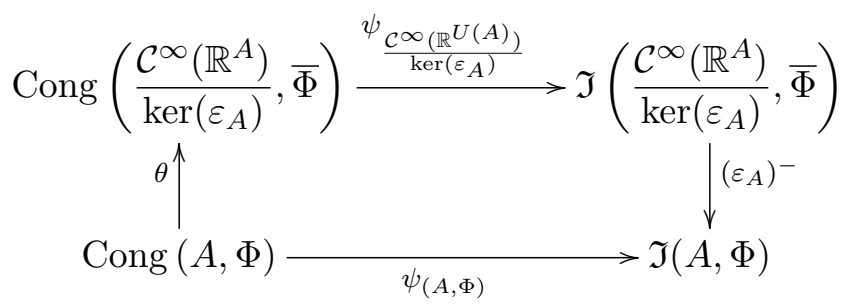

Given any $\mathcal{C}^{\infty}$-ring $(A, \Phi)$, there is a ring-theoretical ideal $I=\psi_{A}\left(\operatorname{ker}\left(\varepsilon_{A}\right)\right)$ such that:

$$
(A, \Phi) \cong\left(\frac{\mathcal{C}^{\infty}\left(\mathbb{R}^{A}\right)}{I}, \bar{\Phi}\right)
$$

that is, every $\mathcal{C}^{\infty}$-ring is the quotient of a free $\mathcal{C}^{\infty}$-ring by some of its ring-theoretic ideals. We say that any $\mathcal{C}^{\infty}$-ring is given by generators and relations.

Remark 1.3.18. Let $(A, \Phi)$ be a $\mathcal{C}^{\infty}$-ring. The set $\operatorname{Cong}(A, \Phi)$ is partially ordered by inclusion. Also, given $\left\{R_{i} \mid i \in I\right\} \subseteq \operatorname{Cong}(A, \Phi)$, we have:

$$
\bigcap_{i \in I} R_{i} \in \operatorname{Cong}(A, \Phi)
$$

so we can define: 


$$
\begin{array}{ccc}
\wedge: \mathcal{P}(\operatorname{Cong}(A, \Phi)) & \rightarrow & \text { Cong } \\
\left\{R_{i} \mid i \in I\right\} & \mapsto \bigcap_{i \in I}\left\{R_{i} \mid i \in I\right\}
\end{array}
$$

Also, given $\left\{R_{i} \mid i \in I\right\} \subseteq \operatorname{Cong}(A, \Phi)$, we define:

$$
\begin{aligned}
\bigvee: \mathcal{P}(\operatorname{Cong}(A, \Phi)) & \rightarrow \\
\left\{R_{i} \mid i \in I\right\} & \mapsto \bigcap\left\{R \in \operatorname{Cong}(A, \Phi) \mid \bigcup_{i \in I}\left\{R_{i} \mid i \in I\right\} \subseteq R\right\}
\end{aligned}
$$

so $(\operatorname{Cong}(A, \Phi), \bigwedge, \bigvee)$ is a complete lattice.

Note that $\mathfrak{I}(A, \Phi)$, partially ordered by inclusion, also has a structure of complete lattice, since it the set of ring-theoretic ideals of $(A, \Phi(+), \Phi(\cdot), \Phi(-), \Phi(0), \Phi(1))$.

We have constructed, in Proposition 1.3.17, a bijection:

$$
\begin{aligned}
& \varphi_{(A, \Phi)}: \operatorname{Cong}(A, \Phi) \rightarrow \Im(A, \Phi) . \\
& \varphi_{(A, \Phi)}: \operatorname{Cong}(A, \Phi) \rightarrow \quad \Im \quad(A, \Phi) \\
& R \quad \mapsto\{g \in A \mid(g, 0) \in R\}
\end{aligned}
$$

We claim that $\varphi_{(A, \Phi)}: \operatorname{Cong}(A, \Phi) \rightarrow \mathfrak{I}(A, \Phi)$ is an isomorphism of lattices.

Given $\left\{R_{i} \mid i \in I\right\} \subseteq \operatorname{Cong}(A, \Phi)$, it is easy to see that:

$$
\psi_{(A, \Phi)}\left(\bigwedge\left\{R_{i} \mid i \in I\right\}\right)=\bigwedge \psi_{(A, \Phi)}\left(R_{i}\right)
$$

so $\psi_{(A, \Phi)}$ is a homomorphism of lattices. Also, given $R, S \in \operatorname{Cong}(A, \Phi)$ such that $R \subseteq S$, we have:

$$
\psi_{(A, \Phi)}(R) \subseteq \psi_{(A, \Phi)}(S)
$$

Given $I^{\prime} \supseteq \psi_{(A, \Phi)}(R)$, since $\psi_{A}^{\prime}$ is surjective, there is some $S \in \operatorname{Cong}(A, \Phi)$ with $\psi_{A}^{\prime}(S)=I^{\prime}$. Also, since $\psi_{A}^{\prime}$ is injective, such an $S$ is unique.

Now,

$$
\psi_{(A, \Phi)}(R) \subseteq \psi_{(A, \Phi)}(S)
$$

so

$$
R=\psi_{(A, \Phi)}^{\dashv}\left[\psi_{(A, \Phi)}(R)\right] \subseteq \psi_{(A, \Phi)}^{\dashv}\left[\psi_{(A, \Phi)}(S)\right]=S
$$

Since $\psi_{(A, \Phi)}$ is bijective, it follows that $\psi_{(A, \Phi)}$ is an isomorphism of complete lattices. Moreover, both lattices are algebraic lattices, whose compact elements are the finitely generated congruences or ideals.

The following result relates the ideals of a product of $\mathcal{C}^{\infty}$-rings with the ideals of its factors.

Proposition 1.3.19. Let $A$ and $B$ be two $\mathcal{C}^{\infty}$-rings and let $\Im(A)$ be the set of all ideals of $A$ and $\Im(B)$ be the set of all ideals of $B$. Every ideal of the product $A \times B$ has the form $\mathfrak{a} \times \mathfrak{b}$, where $\mathfrak{a}$ is an ideal of $A$ and $\mathfrak{b}$ is an ideal of $B$, so we have the following bijection: 


$$
\begin{aligned}
& \Phi: \quad \mathfrak{I}(A) \times \mathfrak{I}(B) \quad \rightarrow \quad \mathfrak{I}(A \times B) \\
& (\mathfrak{a}, \mathfrak{b}) \quad \mapsto \quad \mathfrak{a} \times \mathfrak{b}
\end{aligned}
$$

Proof. Let $p_{1}: A \times B \rightarrow A$ and $p_{2}: A \times B \rightarrow B$ be the canonical projections of the product:

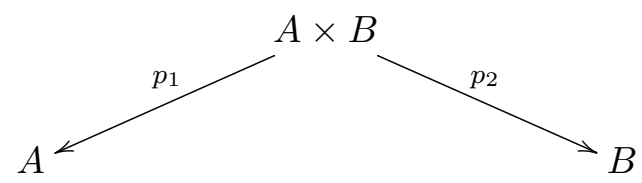

and let $I$ be any ideal of $A \times B$. We have:

$$
\begin{aligned}
& p_{1}[I]=\{a \in A \mid(\exists b \in B)((a, b) \in I)\}=\{a \in A \mid(a, 0) \in I\} \\
& p_{2}[I]=\{b \in B \mid(\exists a \in A)((a, b) \in I)\}=\{b \in B \mid(0, b) \in I\}
\end{aligned}
$$

We claim that $p_{1}[I] \times p_{2}[I]=I$.

Given $(a, b) \in I$, then $p_{1}(a, b)=a \in p_{1}[I]$ and $p_{2}(a, b)=b \in p_{2}[I]$, so $(a, b) \in p_{1}[I] \times p_{2}[I]$. Conversely, given $(a, b) \in p_{1}[I] \times p_{2}[I], a \in p_{1}[I]$ and $b \in p_{2}[I]$, so $(a, 0) \in I$ and $(0, b) \in I$, so $(a, b)=(a, 0)+(0, b) \in I$.

Since for every $\mathfrak{a}$, ideal of $A$ and for every $\mathfrak{b}$ ideal of $B, \mathfrak{a} \times \mathfrak{b}$ is an ideal of $A \times B$, the following map is a bijection:

$$
\begin{array}{ccc}
\Phi: \mathfrak{I}(A) \times \mathfrak{I}(B) & \rightarrow & \mathfrak{I}(A \times B) \\
(\mathfrak{a}, \mathfrak{b}) & \mapsto & \mathfrak{a} \times \mathfrak{b}
\end{array}
$$

In the following proposition we are going to describe how to calculate any limit and any directed colimit, making use of the forgetful functor $U: \mathcal{C}^{\infty} \mathbf{R n g} \rightarrow$ Set.

Proposition 1.3.20. In the category $\mathcal{C}^{\infty} \mathbf{R n g}$ of the $\mathcal{C}^{\infty}$-rings, all the limits and all filtered colimits exist and are created by the forgetful functor $U: \mathcal{C}^{\infty} \mathbf{R n g} \rightarrow$ Set.

Proof. Cf. p. 7 of 62

By a general argument, it can be shown that the category $\mathcal{C}^{\infty}$ Rng has all small colimits. In particular, coequalizers of pairs of $\mathcal{C}^{\infty}$-homomorphisms, $f, g:(A, \Phi) \rightarrow(B, \Psi)$, are given by quotients:

$$
(A, \Phi) \underset{g}{\stackrel{f}{\longrightarrow}}(B, \Psi) \stackrel{q_{I}}{\longrightarrow}\left(\frac{B}{I}, \bar{\Psi}\right)
$$

where $I=\langle\{(f(a), g(a)) \mid a \in A\}\rangle$.

In order to describe all small colimits, it is enough to construct coproducts, and since $\mathcal{C}^{\infty} \mathbf{R n g}$ has filtered colimits, it suffices to construct only finite coproducts. Also, since $\mathbb{R} \cong \mathcal{C}^{\infty}(\{*\})$ is the initial $\mathcal{C}^{\infty}$-ring, it is enough, by induction, to describe binary coproducts in $\mathcal{C}^{\infty} \mathbf{R n g}$. 
Definition 1.3.21. Let $(A, \Phi)$ and $(B, \Psi)$ be two $\mathcal{C}^{\infty}$-rings. We will denote the underlying set of the coproduct of $(A, \Phi)$ and $(B, \Psi)$ by $A \otimes_{\infty} B$, and its corresponding canonical arrows by $\iota_{A}$ and $\iota_{B}$ :

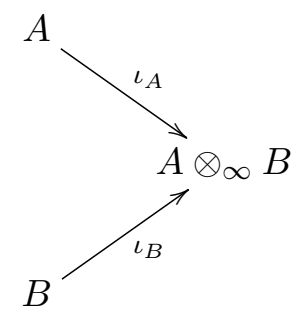

In order to describe concretely the coproduct in $\mathcal{C}^{\infty} \mathbf{R n g}$, first we compute the coproduct of two free $\mathcal{C}^{\infty}$-rings with $m$ and $n$ generators.

Since $m=\{0, \cdots, m-1\}, n=\{0, \cdots, n-1\}, m \sqcup n \cong m+n$ and the functor $L:$ Set $\rightarrow \mathcal{C}^{\infty} \mathbf{R n g}$ preserves coproducts (since it is a left adjoint), we have:

$$
\mathcal{C}^{\infty}\left(\mathbb{R}^{m}\right) \otimes_{\infty} \mathcal{C}^{\infty}\left(\mathbb{R}^{n}\right) \cong \mathcal{C}^{\infty}\left(\mathbb{R}^{m} \times \mathbb{R}^{n}\right) \cong \mathcal{C}^{\infty}\left(\mathbb{R}^{m+n}\right)
$$

Now, given ideals $I \subset \mathcal{C}^{\infty}\left(\mathbb{R}^{m}\right)$ and $J \subset \mathcal{C}^{\infty}\left(\mathbb{R}^{n}\right)$, then:

$$
\frac{\mathcal{C}^{\infty}\left(\mathbb{R}^{m}\right)}{I} \otimes_{\infty} \frac{\mathcal{C}^{\infty}\left(\mathbb{R}^{n}\right)}{J} \cong \frac{\mathcal{C}^{\infty}\left(\mathbb{R}^{m} \times \mathbb{R}^{n}\right)}{(I, J)}
$$

where $(I, J)=\left\langle f \circ \pi_{1}, g \circ \pi_{2} \mid(f \in I) \&(g \in J)\right\rangle$, where $\pi_{1}: \mathbb{R}^{m} \times \mathbb{R}^{n} \rightarrow \mathbb{R}^{m}$ and $\pi_{2}: \mathbb{R}^{m} \times \mathbb{R}^{n} \rightarrow \mathbb{R}^{n}$ are the projections on the first and the second coordinates.

Now, given any two $\mathcal{C}^{\infty}$-rings, $(A, \Phi)$ and $(B, \Psi)$, we describe concretely their coproduct.

First we write $(A, \Phi)$ and $(B, \Psi)$ as colimits of their finitely generated $\mathcal{C}^{\infty}$-subrings:

$$
(A, \Phi) \cong \underset{i \in I}{\lim _{i \in I}}\left(A_{i}, \Phi_{i}\right)
$$

and

$$
(B, \Psi) \cong{\underset{j \in I}{\longrightarrow}}_{\lim _{j}}\left(B_{j}, \Psi_{j}\right)
$$

Then, observing that colimits commute with coproducts, we have:

$$
A \otimes_{\infty} B \cong \lim _{\substack{i \in I \\ j \in J}} A_{i} \otimes_{\infty} B_{j}
$$

As an application of the construction given above, we can describe the process of "adding a set $S$ of variables to a $\mathcal{C}^{\infty}$-ring $(A, \Phi)$ ". The construction is given as follows:

Let $(A, \Phi)$ be any $\mathcal{C}^{\infty}$-ring and let $S$ be any set. Consider $L(S)=\mathcal{C}^{\infty}\left(\mathbb{R}^{S}\right)$, the free $\mathcal{C}^{\infty}$-ring on the set $S$ of generators, together with its canonical map, $\jmath_{S}: S \rightarrow \mathcal{C}^{\infty}\left(\mathbb{R}^{S}\right)$. If we denote by: 


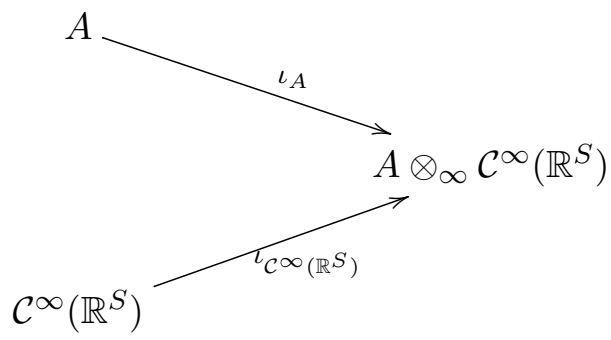

the coproduct of $A$ and $\mathcal{C}^{\infty}\left(\mathbb{R}^{S}\right)$, define:

$$
x_{s}:=\iota_{\mathcal{C}^{\infty}\left(\mathbb{R}^{S}\right)}\left(\jmath_{S}(s)\right) .
$$

We thus define:

$$
A\left\{x_{s} \mid s \in S\right\}:=A \otimes_{\infty} \mathcal{C}^{\infty}\left(\mathbb{R}^{S}\right) .
$$

Now we turn to a functorial approach of the algebraic theory of $\mathcal{C}^{\infty}$-rings. In order to introduce some motivation, we compare this theory with the theory of the $\mathbb{R}$-algebras.

Definition 1.3.22. Let $R$ be a commutative ring and $A$ be any ring. An $R$-algebra is a ring homomorphism $\alpha: R \rightarrow Z(A)$, where $Z(A)$ is the center of the ring $A$. When the ring homomorphism $\alpha$ is understood, it is usual to say that $A$ is an $R$-algebra.

Keeping in mind the above definition, it is a fact that, for $\mathbb{R}$-algebras every polynomial function:

$$
p: \begin{array}{ccc}
\mathbb{R}^{n} & \longrightarrow & \mathbb{R}^{m} \\
\left(x_{1}, \cdots, x_{n}\right) & \longmapsto & \left(p_{1}\left(x_{1}, \cdots, x_{n}\right), \cdots, p_{m}\left(x_{1}, \cdots, x_{n}\right)\right),
\end{array}
$$

given by an $m$-tuple of polynomial functions ( $p_{i}$ is polynomial for every $1 \leq i \leq m$ ) may be interpreted as a function:

$$
\begin{array}{ccc}
A(p): & A^{n} & \longrightarrow \\
\left(a_{1}, \cdots, a_{n}\right) & \longmapsto & A^{m} \\
& & \left(p_{1}\left(a_{1}, \cdots, a_{n}\right), \cdots, p_{m}\left(a_{1}, \cdots, a_{n}\right)\right)
\end{array}
$$

which consists of the evaluation of each polynomial function $p_{i}: \mathbb{R}^{n} \rightarrow \mathbb{R}$ at the $n$-tuple $\left(a_{1}, \cdots, a_{n}\right) \in$ $A^{n}$. This interpretation is possible because polynomial functions may be written solely in terms of the sum and the product, which are defined within the $\mathbb{R}$-algebra structure of $A$.

We also remark that this interpretation has a functorial feature, since it preserves composition of polynomial functions and identities in the following sense:

Proposition 1.3.23. Let

$$
\begin{aligned}
& p: \quad \mathbb{R}^{n} \quad \rightarrow \quad \mathbb{R}^{m} \\
& \left(x_{1}, \cdots, x_{n}\right) \mapsto\left(p_{1}\left(x_{1}, \cdots, x_{n}\right), \cdots, p_{m}\left(x_{1}, \cdots, x_{n}\right)\right)
\end{aligned}
$$

and

$$
q: \begin{array}{ccc}
\mathbb{R}^{m} & \rightarrow & \mathbb{R}^{k} \\
\left(y_{1}, \cdots, y_{m}\right) & \mapsto & \left(q_{1}\left(y_{1}, \cdots, y_{m}\right), \cdots, q_{k}\left(y_{1}, \cdots, y_{m}\right)\right)
\end{array}
$$

be two polynomial functions such that their composition is defined:

$$
q \circ p: \underset{\mathbb{R}^{n}}{\vec{x}=\left(x_{1}, \cdots, x_{n}\right)} \stackrel{\mathbb{R}^{k}}{\longrightarrow}\left(q_{1}\left(p_{1}(\vec{x}), \cdots, p_{m}(\vec{x})\right), \cdots, q_{k}\left(p_{1}(\vec{x}), \cdots, p_{m}(\vec{x})\right)\right) .
$$

If $A$ is an $\mathbb{R}$-algebra, then $A(q \circ p)=A(q) \circ A(p)$. 
Moreover, we have the following:

Proposition 1.3.24. Let $\operatorname{id}_{\mathbb{R}^{n}}: \mathbb{R}^{n} \rightarrow \mathbb{R}^{n}$ be the identity function of an $\mathbb{R}$-algebra $A$. Then $A\left(\operatorname{id}_{\mathbb{R}^{n}}\right)=$ $\operatorname{id}_{A^{n}}: A^{n} \rightarrow A^{n}$

Apart from these functorial properties, the interpretation within a $\mathbb{R}$-algebra $A$ also preserves finite products, as we shall see in the following:

Proposition 1.3.25. Let:

$$
\pi_{i}: \begin{gathered}
\mathbb{R} \times \mathbb{R} \times \cdots \times \mathbb{R} \times \cdots \times \mathbb{R} \\
\left(x_{1}, x_{2}, \cdots, x_{i}, \cdots, x_{n-1}, x_{n}\right)
\end{gathered}
$$

be the projection onto the ith coordinate. Then:

$$
\begin{aligned}
A\left(\pi_{i}\right): \quad A \times A \times \cdots \times A \times \cdots \times A \times A & \longrightarrow A \\
\left(a_{1}, a_{2}, \cdots, a_{i}, \cdots, a_{n-1}, a_{n}\right) & \longmapsto
\end{aligned}
$$

In view of the properties described by the propositions $1.3 .23,1.3 .24$ and 1.3 .25 , we can characterize a commutative $\mathbb{R}$-algebra with unity as a finite product preserving functor from the category Pol, whose objects are the spaces $\mathbb{R}^{n}, n \geq 0$ and whose morphisms are polynomial functions between them in the category Set.

$$
A: \text { Pol } \rightarrow \text { Set }
$$

We identify $A(\mathbb{R})$ with the underlying set of the $\mathbb{R}$-algebra and we convention that $A\left(\mathbb{R}^{0}\right)=A(\{*\})=$ $\{*\}$. The unity $1_{A}$ of $A(\mathbb{R})$ is given by $A(1:\{*\} \rightarrow \mathbb{R}):\{*\} \rightarrow A(\mathbb{R})$, and we exclude the trivial case $1_{A}=0_{A}$ from our analysis.

Analogously to this definition, we can describe a more general structure than that of an $\mathbb{R}$-algebra, which is capable of interpreting not only polynomial functions from $\mathbb{R}^{n}$ to $\mathbb{R}^{m}$, but every smooth function (i.e., $\mathcal{C}^{\infty}$-functions) from $\mathbb{R}^{n}$ to $\mathbb{R}^{m}$, most of which are not even definable within the language of rings.

Example 1.3.26. Let $\mathcal{C}^{\infty}$ be the category whose objects are all the finite dimensional real vector spaces $\mathbb{R}^{n}, n \geq 0$, and whose morphisms are all of the infinitely differentiable functions between them. Given a functor:

$$
A: \mathcal{C}^{\infty} \longrightarrow \text { Set }
$$

which preserves finite products, then $(A(\mathbb{R}), A)$ is an actual $\mathcal{C}^{\infty}$-ring, according to the universal algebraic definition.

In what follows we shall define the homomorphisms between $\mathcal{C}^{\infty}$-rings, qua finite product preserving functors.

Definition 1.3.27. Let $A, B: \mathcal{C}^{\infty} \rightarrow$ Set be two $\mathcal{C}^{\infty}$-rings. $A \mathcal{C}^{\infty}$-rings homomorphism is a natural transformation $\varphi: A \Rightarrow B$, i.e., for every $m, n \in \mathbb{N}$ and for any smooth function $f: A \rightarrow B$, the following diagram commutes:

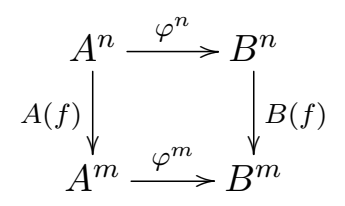

In particular, $\varphi_{\mathbb{R}}: A(\mathbb{R}) \rightarrow B(\mathbb{R})$ is a $\mathcal{C}^{\infty}$-homomorphism in the universal algebraic sense too. 
Keeping the above definitions in mind we can think of a real $\mathcal{C}^{\infty}$-ring as a commutative $\mathbb{R}$-algebra with unity, namely

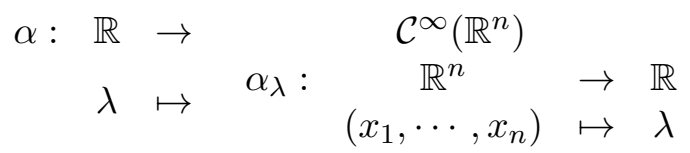

We present below a concrete example of a $\mathcal{C}^{\infty}$-ring, as given in [62]. It will play an important role in our work, since it is one of the models of the theory of $\mathcal{C}^{\infty}$-rings.

Example 1.3.28. For every subset $X \subset \mathbb{R}^{r}$, the ring of smooth functions from $X$ to $\mathbb{R}, \mathcal{C}^{\infty}(X, \mathbb{R})$ has a $\mathcal{C}^{\infty}$-ring structure, that we shall denote by $\mathcal{C}^{\infty}(X)$. The $\mathcal{C}^{\infty}$-structure is given by the functor:

$$
\begin{aligned}
& \mathcal{C}^{\infty}(X): \quad \mathcal{C}^{\infty} \quad \rightarrow \quad \text { Set }
\end{aligned}
$$

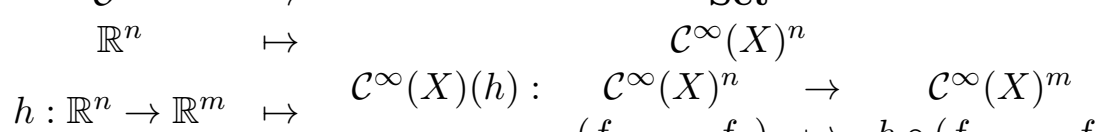

$$
\begin{aligned}
& \left(f_{1}, \cdots, f_{n}\right) \mapsto h \circ\left(f_{1}, \cdots, f_{n}\right)
\end{aligned}
$$

This is a functorial construction in $X$, i.e., if $X \subset \mathbb{R}^{n}, Y \subset \mathbb{R}^{m}$ and $\varphi: X \rightarrow Y$ is a smooth function, we obtain a natural transformation between the functors $\mathcal{C}^{\infty}(Y)$ e $\mathcal{C}^{\infty}(X)$, simply by composing it with $\varphi$.

Example 1.3.29. As we already know, for every $n \in \mathbb{N}, \mathcal{C}^{\infty}\left(\mathbb{R}^{n}\right)$ has a natural structure of $\mathcal{C}^{\infty}$-ring. Consider:

$$
\begin{array}{cccc}
\Phi: \bigcup_{m \geq 0} \mathcal{C}^{\infty}\left(\mathbb{R}^{m}, \mathbb{R}\right) & \rightarrow & \bigcup_{m \geq 0} \operatorname{Func}\left(\mathcal{C}^{\infty}\left(\mathbb{R}^{n}\right)^{m}, \mathcal{C}^{\infty}\left(\mathbb{R}^{n}\right)\right) \\
\mathbb{R}^{m} \stackrel{h}{\longrightarrow} \mathbb{R} & \mapsto & \mathcal{C}^{\infty}\left(\mathbb{R}^{n}\right)^{m} \stackrel{h^{*}}{\longrightarrow} \mathcal{C}^{\infty}\left(\mathbb{R}^{n}\right)
\end{array}
$$

where:

$$
\begin{aligned}
& h^{*}: \quad \mathcal{C}^{\infty}\left(\mathbb{R}^{n}\right)^{m} \quad \rightarrow \quad \mathcal{C}^{\infty}\left(\mathbb{R}^{n}\right) \\
& \left(f_{1}, \cdots, f_{m}\right) \mapsto h \circ\left(f_{1}, \cdots, f_{m}\right)
\end{aligned}
$$

We use to denote, for $h \in \mathcal{C}^{\infty}\left(\mathbb{R}^{m}, \mathbb{R}\right), \Phi(h) \doteq \mathcal{C}^{\infty}\left(\mathbb{R}^{n}\right)(h)=h^{*}$.

Note that the definitions $\mathbf{1 . 3 . 2 6}$ e 1.1 .1 give us exactly the same information. If we have a $\mathcal{C}^{\infty}$-ring $B$, according to Definition 1.3.26, we have a $\mathcal{C}^{\infty}$-ring in the sense of Definition 1.1.1. taking $\mathfrak{B}=C(\mathbb{R})$ and, for each $f: \mathbb{R}^{n} \rightarrow \mathbb{R}, \Phi_{f}=C(f)$, that is, $\left(\mathfrak{B}, \Phi_{f}\right)_{f: \mathbb{R}^{n} \rightarrow \mathbb{R}, n \geq 0}$, which moreover preserves projections, since the functor $B$, by its very definition, preserves finite products.

Conversely, if we have a $\mathcal{C}^{\infty}$-ring according to Definition 1.1.1. $\left(\mathfrak{C}, \Phi_{f}\right)_{f: \mathbb{R}^{n} \rightarrow \mathbb{R}, n \geq 0}$, we may define the functor of Definition $\mathbf{1 . 3 . 2 6}$ as following: $B(\mathbb{R})=\mathfrak{C}$ e $B\left(f: \mathbb{R}^{n} \rightarrow \mathbb{R}^{m}\right)=\Phi_{f}: \mathfrak{C}^{n} \rightarrow \mathfrak{C}^{m}$.

Remark 1.3.30. It can be shown that both definitions of $\mathcal{C}^{\infty}$-rings (the universal algebraic and the functorial version) are equivalent. More precisely, the categories described in both cases are equivalent categories.

We shall denote the category whose objects are all the functors in $\mathbf{S e t}^{\mathcal{C}^{\infty}}$ which preserve finite products and whose morphisms are the natural transformations between them by $\mathcal{C}^{\infty}-\mathbf{R n g}$, and we must call it the "category of the $\mathcal{C}^{\infty}$-rings". In the following section we will stress some properties of the category $\mathcal{C}^{\infty}-\mathbf{R n g}$, as well as some important constructions that are possible within it.

Proposition 1.3.31. Let $n \in \mathbb{N} \backslash\{0\}$. The ring $\mathcal{C}^{\infty}\left(\mathbb{R}^{n}\right)$ is neither Artinian nor Noetherian. 
Proof. Consider the following ideals of $\mathcal{C}^{\infty}\left(\mathbb{R}^{n}\right)$ :

$$
I_{m}=\left\{f \in \mathcal{C}^{\infty}\left(\mathbb{R}^{n}\right) \mid f \uparrow_{\mathbb{R}^{n} \backslash B(\overrightarrow{0}, m)} \equiv 0\right\},
$$

and note that:

$$
(\forall m \in \mathbb{N})\left(I_{m+1} \subsetneq I_{m}\right)
$$

so we have an infinite descending chain, so $\mathcal{C}^{\infty}\left(\mathbb{R}^{n}\right)$ is not Artinian.

On the other hand, for every $m \in \mathbb{N} \backslash\{0\}$ we have the ideal:

$$
J_{m}=\left\langle\left\{f_{1}(x)=e^{x}, f_{2}(x)=e^{x^{2}}, \cdots, f_{m}(x)=e^{x^{m}}\right\}\right\rangle
$$

which compose an ascending sequence of ideals such that $J_{m} \subsetneq J_{m+1}$, so $\mathcal{C}^{\infty}\left(\mathbb{R}^{n}\right)$ is not a Noetherian ring.

\subsection{Finitely Generated and Finitely Presented $\mathcal{C}^{\infty}$-rings}

In this section we describe a very important class of $\mathcal{C}^{\infty}$-rings, which occupies a central position in the theory of the $\mathcal{C}^{\infty}$-rings, namely the finitely generated and the finitely presented $\mathcal{C}^{\infty}$-rings.

As we shall see, both the categories of finitely generated and finitely presented $\mathcal{C}^{\infty}$-rings are closed under coproducts and coequalizers, thus are finitely co-complete categories. This feature is going to be essential to our construction of a classifying topos for the theory of $\mathcal{C}^{\infty}$-rings, and also for constructing a classifying topos for the theory of local $\mathcal{C}^{\infty}$-rings, on Chapter 4.

We begin with some definitions.

Definition 1.4.1. A finitely generated $\mathcal{C}^{\infty}$-ring is the quotient of a free $\mathcal{C}^{\infty}$-ring on a finite number of generators by some of its ideals, that is, a $\mathcal{C}^{\infty}$-ring of the form:

$$
\frac{\mathcal{C}^{\infty}\left(\mathbb{R}^{n}\right)}{I}
$$

for some I ideal of $\mathcal{C}^{\infty}\left(\mathbb{R}^{n}\right)$.

The category of all finitely generated $\mathcal{C}^{\infty}$-rings and their $\mathcal{C}^{\infty}$-homomorphisms is denoted by $\mathcal{C}^{\infty} \mathbf{R n g}_{\mathrm{fg}}$.

Among the finitely generated $\mathcal{C}^{\infty}$-rings, we have the finitely presented $\mathcal{C}^{\infty}$-rings.

Definition 1.4.2. A finitely presented $\mathcal{C}^{\infty}$-ring is the quotient of a free $\mathcal{C}^{\infty}$-ring on a finite number of generators by some of its finitely generated ideals, that is, a $\mathcal{C}^{\infty}$-ring of the form:

$$
\frac{\mathcal{C}^{\infty}\left(\mathbb{R}^{n}\right)}{\left\langle f_{1}, \cdots, f_{k}\right\rangle}
$$

for some $n, k \in \mathbb{N}$ and some $f_{1}, \cdots, f_{k} \in \mathcal{C}^{\infty}\left(\mathbb{R}^{n}\right)$.

The category of all finitely presented $\mathcal{C}^{\infty}$-rings and their $\mathcal{C}^{\infty}$-homomorphisms is denoted by $\mathcal{C}^{\infty} \mathbf{R n g}_{\mathrm{fp}}$.

As pointed out by I. Moerdijk and G. Reyes in [62], finitely generated $\mathcal{C}^{\infty}$-rings are convenient to deal with because the $\mathcal{C}^{\infty}$-homomorphisms between them can be described explicitly. In particular, finitely presented $\mathcal{C}^{\infty}$-rings also have this convenient feature, as we see in the following: 
Lemma 1.4.3. Given any $\mathcal{C}^{\infty}$-homomorphism between two free $\mathcal{C}^{\infty}$-rings on a finite number of generators, say:

$$
\Phi: \mathcal{C}^{\infty}\left(\mathbb{R}^{n}\right) \rightarrow \mathcal{C}^{\infty}\left(\mathbb{R}^{m}\right)
$$

there is a unique $\mathcal{C}^{\infty}$-function $\varphi: \mathbb{R}^{m} \rightarrow \mathbb{R}^{n}$ such that $\Phi=\varphi_{*}$ :

$$
\begin{aligned}
\varphi_{*}: \mathcal{C}^{\infty}\left(\mathbb{R}^{n}\right) & \rightarrow \mathcal{C}^{\infty}\left(\mathbb{R}^{m}\right) \\
f & \mapsto f \circ \varphi
\end{aligned}
$$

Proof. Given a $\mathcal{C}^{\infty}$-homomorphism $\Phi: \mathcal{C}^{\infty}\left(\mathbb{R}^{n}\right) \rightarrow \mathcal{C}^{\infty}\left(\mathbb{R}^{m}\right)$, for each generator of $\mathcal{C}^{\infty}\left(\mathbb{R}^{n}\right), \pi_{i}: \mathbb{R}^{n} \rightarrow \mathbb{R}$ we have a $\mathcal{C}^{\infty}$-function $\Phi\left(\pi_{i}\right) \in \mathcal{C}^{\infty}\left(\mathbb{R}^{m}\right)$.

Define $\varphi=\left(\Phi\left(\pi_{1}\right), \cdots, \Phi\left(\pi_{n}\right)\right): \mathbb{R}^{m} \rightarrow \mathbb{R}^{n}$, which is smooth since each of its coordinates is smooth.

\section{Claim: $\Phi=\varphi_{*}$}

Let $f \in \mathcal{C}^{\infty}\left(\mathbb{R}^{n}\right)$ be any $\mathcal{C}^{\infty}$-function.

Using the notation established on the Example 1.3.29, since $\Phi$ is a $\mathcal{C}^{\infty}$-homomorphism (i.e., $\Phi \in$ Func $\left.\left(\mathcal{C}^{\infty}\left(\mathbb{R}^{n}\right), \mathcal{C}^{\infty}\left(\mathbb{R}^{m}\right)\right)\right)$, in particular the following diagram commutes:

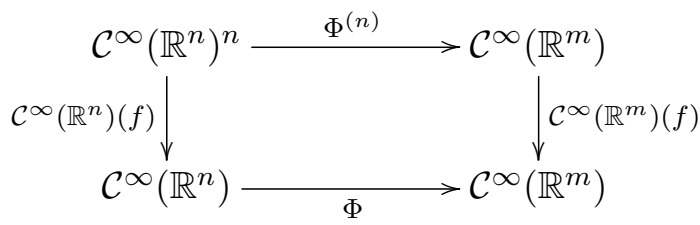

We have $\operatorname{id}_{\mathbb{R}^{n}}=\left(\pi_{1}, \cdots, \pi_{n}\right)$, and chasing $\left(\pi_{1}, \cdots, \pi_{n}\right)$ around the diagram above yields:

$$
\mathcal{C}^{\infty}\left(\mathbb{R}^{m}\right)(f) \circ \Phi^{(n)}\left(\pi_{1}, \cdots, \pi_{n}\right)=\mathcal{C}^{\infty}\left(\mathbb{R}^{m}\right)(f) \circ\left(\Phi\left(\pi_{1}\right), \cdots, \Phi\left(\pi_{n}\right)\right)=\mathcal{C}^{\infty}\left(\mathbb{R}^{m}\right)(f)(\varphi)=f \circ \varphi
$$

since $\mathcal{C}^{\infty}\left(\mathbb{R}^{m}\right)(f)=f \circ-$.

We also have:

$$
\Phi \circ \mathcal{C}^{\infty}\left(\mathbb{R}^{n}\right)(f)\left(\pi_{1}, \cdots, \pi_{n}\right)=\Phi \circ\left(f \circ\left(\pi_{1}, \cdots, \pi_{n}\right)\right)=\Phi\left(f \circ \operatorname{id}_{\mathbb{R}^{n}}\right)=\Phi(f),
$$

so it follows that $\Phi(f)=f \circ \varphi=\varphi_{*}(f)$.

The uniqueness of such a $\varphi$ is due to its construction - which made use of the generators of a free object: given a smooth function $\psi=\left(\psi_{1}, \cdots, \psi_{n}\right): \mathbb{R}^{m} \rightarrow \mathbb{R}^{n}$ such that $\Phi=\psi_{*}$, then for every $i \in\{1, \cdots, n\}$, $\pi_{i} \circ \varphi=\Phi\left(\pi_{i}\right)=\psi_{*}\left(\pi_{i}\right)=\pi_{i} \circ \psi=\psi_{i}$, hence $\varphi=\left(\varphi_{1}, \cdots, \varphi_{n}\right)=\left(\psi_{1}, \cdots, \psi_{n}\right)=\psi$.

We note that the converse of Lemma 1.4 .3 also holds, that is, given any $\mathcal{C}^{\infty}$-function $\varphi: \mathbb{R}^{n} \rightarrow \mathbb{R}^{m}$, there is a unique $\mathcal{C}^{\infty}$-homomorphism given by $\varphi_{*}: \mathcal{C}^{\infty}\left(\mathbb{R}^{m}\right) \rightarrow \mathcal{C}^{\infty}\left(\mathbb{R}^{n}\right)$. Thus we have:

Theorem 1.4.4. There is a one-to-one correspondence between smooth functions from $\mathbb{R}^{n}$ to $\mathbb{R}^{m}$ and $\mathcal{C}^{\infty}$-homomorphisms from $\mathcal{C}^{\infty}\left(\mathbb{R}^{m}\right)$ to $\mathcal{C}^{\infty}\left(\mathbb{R}^{n}\right)$ :

$$
\frac{\mathcal{C}^{\infty}\left(\mathbb{R}^{n}, \mathbb{R}^{m}\right)}{\mathcal{C}^{\infty} \mathbf{R n g}\left(\mathcal{C}^{\infty}\left(\mathbb{R}^{m}\right), \mathcal{C}^{\infty}\left(\mathbb{R}^{n}\right)\right)}
$$


The following result tells us that every $\mathcal{C}^{\infty}$-homomorphism between finitely generated $\mathcal{C}^{\infty}$ is induced by some $\mathcal{C}^{\infty}$-function.

Lemma 1.4.5. Let $\frac{\mathcal{C}^{\infty}\left(\mathbb{R}^{n}\right)}{I}$ and $\frac{\mathcal{C}^{\infty}\left(\mathbb{R}^{m}\right)}{J}$ be two finitely generated $\mathcal{C}^{\infty}$-rings and let:

$$
\Phi: \frac{\mathcal{C}^{\infty}\left(\mathbb{R}^{n}\right)}{I} \rightarrow \frac{\mathcal{C}^{\infty}\left(\mathbb{R}^{m}\right)}{J}
$$

be any $\mathcal{C}^{\infty}$-homomorphism between them.

Under those circumstances, there is some (not necessarily unique) $\mathcal{C}^{\infty}$-function, $\varphi: \mathbb{R}^{m} \rightarrow \mathbb{R}^{n}$ such that the following diagram commutes:

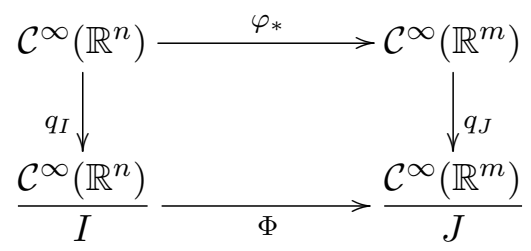

Moreover, $\varphi$ is such that $I \subseteq \varphi_{*}^{-1}[J]=\left\{f \in \mathcal{C}^{\infty}\left(\mathbb{R}^{n}\right) \mid f \circ \varphi \in J\right\}$.

Proof. Given any $\pi_{i} \in \mathcal{C}^{\infty}\left(\mathbb{R}^{n}\right)$ we have:

$$
\Phi\left(q_{I}\left(\pi_{i}\right)\right)=\Phi\left(\pi_{i}+I\right)=f_{i}+J \in \frac{\mathcal{C}^{\infty}\left(\mathbb{R}^{m}\right)}{J}
$$

For each $i \in\{1, \cdots, n\}$ choose some representative of $\Phi\left(\pi_{I}+I\right)=f_{i}+I, f_{i} \in \mathcal{C}^{\infty}\left(\mathbb{R}^{m}\right)$ and define:

$$
\begin{array}{rlc}
\varphi: \mathbb{R}^{m} & \rightarrow & \mathbb{R}^{n} \\
x & \mapsto & \left(f_{1}(x), \cdots, f_{n}(x)\right)
\end{array}
$$

which is smooth, since each of its coordinates is smooth.

Claim 1: The map $\varphi_{*}$, constructed from $\varphi$ above is such that the diagram 1.6 commutes.

Now we use the fact the $\mathcal{C}^{\infty}$-ring $\mathcal{C}^{\infty}\left(\mathbb{R}^{n}\right)$ is a free object on $n$ generators: if we prove that for every $i \in\{1, \cdots, n\}$ we have $\left(q_{J} \circ \varphi_{*}\right)\left(\pi_{i}\right)=\left(\Phi \circ q_{I}\right)\left(\pi_{i}\right)$ then $q_{J} \circ \varphi_{*}=\Phi \circ q_{I}$, and 1.6$)$ commutes.

On the one hand we have $q_{J} \circ \varphi_{*}\left(\pi_{i}\right)=q_{J}\left(\pi_{i} \circ \varphi\right)=q_{J}\left(\pi_{i} \circ\left(f_{1}, \cdots, f_{n}\right)\right)=q_{J}\left(f_{i}\right)=f_{i}+J$.

On the other hand we have $\Phi \circ q_{I}\left(\pi_{i}\right)=\Phi\left(\pi_{i}+I\right)=f_{i}+J$, so the equality $\left(q_{j} \circ \varphi_{i}\right)\left(\pi_{i}\right)=\left(\Phi \circ q_{I}\right)\left(\pi_{i}\right)$ holds for every $i \in\{1, \cdots, n\}$. Hence (1.6) commutes.

Claim 2: $I \subseteq \varphi_{*}^{-1}[J]=\left\{g \in \mathcal{C}^{\infty}\left(\mathbb{R}^{n}\right) \mid g \circ \varphi \in J\right\}$.

Given $g \in I$, we have $q_{I}(g)=0+I$, so $\left(\Phi \circ q_{I}\right)(g)=0+J$. Since (1.6) commutes, it follows that $\left(q_{J} \circ \varphi_{*}\right)(g)=0+J$, so $q_{J}(g \circ \varphi)=0+J$, that is, $g \circ \varphi+J=0+J$, thus $g \circ \varphi \in J$.

Given two finitely generated $\mathcal{C}^{\infty}$-rings, $\frac{\mathcal{C}^{\infty}\left(\mathbb{R}^{n}\right)}{I}$ and $\frac{\mathcal{C}^{\infty}\left(\mathbb{R}^{m}\right)}{J}$, we consider the following subset of $\mathcal{C}^{\infty}\left(\mathbb{R}^{m}, \mathbb{R}^{n}\right)$ : 


$$
\mathcal{F}=\left\{\varphi: \mathbb{R}^{m} \rightarrow \mathbb{R}^{n} \mid I \subseteq \varphi_{*}^{-}[J]\right\}
$$

An (almost) immediate consequence of Lemma 1.4.5 is the following:

Proposition 1.4.6. There is a surjective correspondence (function):

$$
\mu:\left\{\varphi: \mathbb{R}^{m} \rightarrow \mathbb{R}^{n} \mid I \subseteq \varphi_{*}^{\dashv}[J]\right\} \rightarrow \operatorname{Hom}_{\mathcal{C}^{\infty} \mathbf{R n g}_{\mathrm{fg}}}\left(\frac{\mathcal{C}^{\infty}\left(\mathbb{R}^{n}\right)}{I}, \frac{\mathcal{C}^{\infty}\left(\mathbb{R}^{m}\right)}{J}\right)
$$

Proof. Given $\left(\varphi: \mathbb{R}^{m} \rightarrow \mathbb{R}^{n}\right) \in \mathcal{F}$, we have the induced $\mathcal{C}^{\infty}$-homomorphism:

$$
\begin{aligned}
q_{J} \circ \varphi_{*}: \mathcal{C}^{\infty}\left(\mathbb{R}^{n}\right) & \rightarrow \frac{\mathcal{C}^{\infty}\left(\mathbb{R}^{m}\right)}{J} \\
f & \mapsto f \circ \varphi+J
\end{aligned}
$$

Since $I \subseteq \varphi_{*}^{\dashv}[J]$, for every $g \in I, g \circ \varphi \in J$, so $g \circ \varphi+J=J$. Thus $\left(q_{J} \circ \varphi_{*}\right)(g)=0+J$ and $I \subseteq \operatorname{ker}\left(q_{J} \circ \varphi_{*}\right)$.

It follows from the Theorem of Homomorphism that there is a unique $\widetilde{\Phi}: \frac{\mathcal{C}^{\infty}\left(\mathbb{R}^{n}\right)}{I} \rightarrow \mathcal{C}^{\infty}\left(\mathbb{R}^{m}\right)$ such that the following diagram commutes:

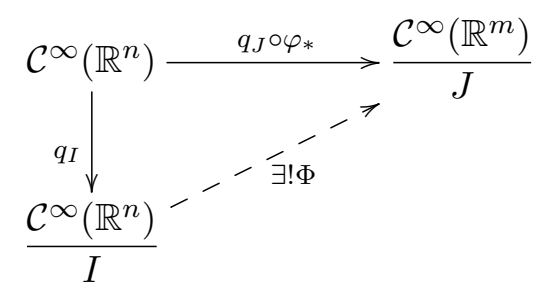

Thus we have the function:

$$
\begin{aligned}
& \mu:\left\{\varphi: \mathbb{R}^{m} \rightarrow \mathbb{R}^{n} \mid I \subseteq \varphi_{*}[J]\right\} \rightarrow \operatorname{Hom}_{\mathcal{C}^{\infty} \mathbf{R n g}_{\mathrm{fg}}}\left(\frac{\mathcal{C}^{\infty}\left(\mathbb{R}^{n}\right)}{I}, \frac{\mathcal{C}^{\infty}\left(\mathbb{R}^{m}\right)}{J}\right) \\
& \left(\mathbb{R}^{m} \stackrel{f}{\longrightarrow} \mathbb{R}^{n}\right) \quad \mapsto \quad \frac{\mathcal{C}^{\infty}\left(\mathbb{R}^{n}\right)}{I} \stackrel{\Phi}{\longrightarrow} \frac{\mathcal{C}^{\infty}\left(\mathbb{R}^{m}\right)}{J}
\end{aligned}
$$

We claim that $\mu$ is surjective.

Given any $\Phi: \frac{\mathcal{C}^{\infty}\left(\mathbb{R}^{n}\right)}{I} \rightarrow \frac{\mathcal{C}^{\infty}\left(\mathbb{R}^{m}\right)}{J}$, by Lemma 1.4.5 there is some $\varphi \in \mathcal{F}$ such that:

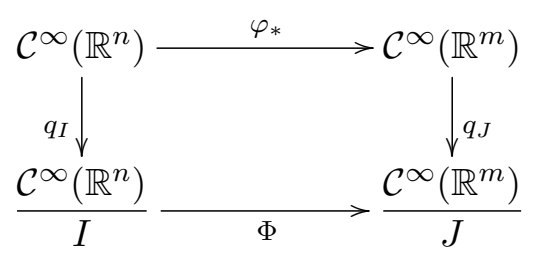

commutes, so the triangle:

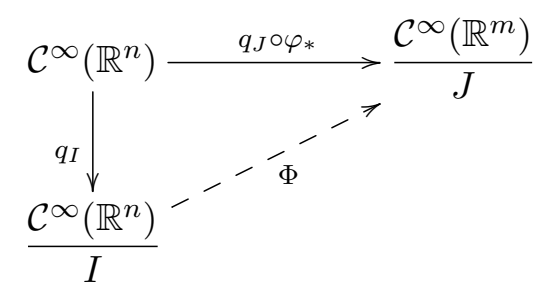


commutes. By construction, $\mu(\varphi)=\Phi$, so $\mu$ is surjective.

Proposition 1.4.7. Given the set $\mathcal{F}$ as above, the relation:

$$
\sim=\left\{\left(\varphi, \varphi^{\prime}\right) \in \mathcal{C}^{\infty}\left(\mathbb{R}^{m}, \mathbb{R}^{n}\right) \times \mathcal{C}^{\infty}\left(\mathbb{R}^{m}, \mathbb{R}^{n}\right) \mid(\forall i \in\{1, \cdots, n\})\left(\pi_{i} \circ \varphi-\pi_{i} \circ \varphi^{\prime} \in J\right)\right\}
$$

is an equivalence relation.

Proof. It is clear that $\sim$ is reflexive and symmetric, so we are only going to prove its transitivity. Suppose $\varphi \sim \varphi^{\prime}$ and $\varphi^{\prime} \sim \varphi^{\prime \prime}$. We have, thus:

$$
(\forall i \in\{1, \cdots, n\})\left(\pi_{i} \circ \varphi-\pi_{i} \circ \varphi^{\prime} \in J\right)
$$

and

$$
(\forall i \in\{1, \cdots, n\})\left(\pi_{i} \circ \varphi^{\prime}-\pi_{i} \circ \varphi^{\prime \prime} \in J\right)
$$

Since $J$ is closed under sums, it follows that:

$$
(\forall i \in\{1, \cdots, n\})\left(\pi_{i} \circ \varphi-\pi_{i} \circ \varphi^{\prime \prime}=\left(\pi_{i} \circ \varphi-\pi_{i} \circ \varphi^{\prime}\right)+\left(\pi_{i} \circ \varphi^{\prime}-\pi_{i} \circ \varphi^{\prime \prime}\right) \in J\right) .
$$

Hence, $\sim$ is an equivalence relation.

The equivalence class of an element $\varphi \in \mathcal{F}$ will be denoted by $[\varphi]$.

Remark 1.4.8. Observe that for the map $\mu$ constructed in the Proposition 1.4.7 is such that given $\varphi, \varphi^{\prime} \in \mathcal{F}$ such that we have:

$$
\varphi \sim \varphi^{\prime} \Longleftrightarrow \mu(\varphi)=\mu\left(\varphi^{\prime}\right)
$$

Indeed, if $\varphi \sim \varphi^{\prime}$, the for every $i \in\{1, \cdots, n\}, \pi_{i} \circ \varphi-\pi_{i} \circ \varphi^{\prime} \in J$ so $q_{J}\left(\pi_{i} \circ \varphi-\pi_{i} \circ \varphi^{\prime}\right)=J$, that is:

$$
(\forall i \in\{1, \cdots, n\})\left(q_{J}\left(\pi_{i} \circ \varphi\right)=q_{J}\left(\pi_{i} \circ \varphi^{\prime}\right)\right) .
$$

Thus $q_{J} \circ \varphi_{*}=q_{J} \circ \varphi^{\prime}{ }_{*}$, and $\mu(\varphi)=\Phi=\mu\left(\varphi^{\prime}\right)$.

On the other hand, if $\varphi, \varphi^{\prime} \in \mathcal{F}$ are such that $\mu(\varphi)=\Phi=\mu\left(\varphi^{\prime}\right)$, then since $q_{J} \circ \varphi_{*}=\Phi \circ q_{I}=q_{J} \circ \varphi^{\prime}{ }_{*}$. Thus, for all $i \in\{1, \cdots, n\} q_{J}\left(\varphi_{*}\left(\pi_{i}\right)\right)=q_{J}\left(\varphi^{\prime}{ }_{*}\left(\pi_{i}\right)\right)$, so $\pi_{i} \circ \varphi-\pi_{i} \circ \varphi^{\prime} \in J$.

As a direct consequence of Propositions 1.4.6 and 1.4.7, we obtain the following:

Theorem 1.4.9. Let $\frac{\mathcal{C}^{\infty}\left(\mathbb{R}^{n}\right)}{I}$ and $\frac{\mathcal{C}^{\infty}\left(\mathbb{R}^{m}\right)}{J}$ be two finitely generated $\mathcal{C}^{\infty}$-rings and let $\mathcal{F}=\left\{\varphi: \mathbb{R}^{m} \rightarrow\right.$ $\left.\mathbb{R}^{n} \mid I \subseteq \varphi_{*}^{-}[J]\right\}$. There is a 1-1 correspondence (bijection):

$$
\begin{gathered}
\widetilde{\mu}: \frac{\mathcal{F}}{\sim} \rightarrow \operatorname{Hom}_{\mathcal{C} \infty} \operatorname{Rng}_{\mathrm{fp}}\left(\frac{\mathcal{C}^{\infty}\left(\mathbb{R}^{n}\right)}{I}, \frac{\mathcal{C}^{\infty}\left(\mathbb{R}^{m}\right)}{J}\right) \\
{[\varphi] \mapsto \widetilde{\mu}([\varphi])=\mu(\varphi)}
\end{gathered}
$$

Now we are going to describe coequalizers in the category of the finitely generated $\mathcal{C}^{\infty}$-rings, starting with the coequalizer between two free $\mathcal{C}^{\infty}$-rings on a finite number of variables. 
Proposition 1.4.10. Let $\mathcal{C}^{\infty}\left(\mathbb{R}^{n}\right)$ and $\mathcal{C}^{\infty}\left(\mathbb{R}^{m}\right)$ be two free $\mathcal{C}^{\infty}$-rings and $\Phi, \Psi: \mathcal{C}^{\infty}\left(\mathbb{R}^{n}\right) \rightarrow \mathcal{C}^{\infty}\left(\mathbb{R}^{m}\right)$ be two $\mathcal{C}^{\infty}$-homomorphism between them. Let $\varphi, \psi: \mathbb{R}^{m} \rightarrow \mathbb{R}^{n}$ be two smooth functions such that $\mu(\varphi)=\Phi$ and $\mu(\psi)=\Psi$, as described in Proposition 1.4.6. The quotient map:

$$
q: \mathcal{C}^{\infty}\left(\mathbb{R}^{m}\right) \rightarrow \frac{\mathcal{C}^{\infty}\left(\mathbb{R}^{m}\right)}{\left\langle\pi_{1} \circ \varphi-\pi_{1} \circ \psi, \cdots, \pi_{n} \circ \varphi-\pi_{n} \circ \psi\right\rangle}
$$

is the coequalizer of $\Phi$ and $\Psi$.

Proof. First we note that for every $i \in\{1, \cdots, n\}, q \circ \varphi_{*}\left(\pi_{i}\right)=\pi_{i} \circ \varphi+\left\langle\pi_{1} \circ \varphi-\pi_{1} \circ \psi, \cdots, \pi_{n} \circ \varphi-\pi_{n} \circ \psi\right\rangle$ and $q \circ \psi_{*}\left(\pi_{i}\right)=\pi_{i} \circ \psi+\left\langle\pi_{1} \circ \varphi-\pi_{1} \circ \psi, \cdots, \pi_{n} \circ \varphi-\pi_{n} \circ \psi\right\rangle$, so:

$$
q \circ \varphi\left(\pi_{i}\right)-q \circ \psi\left(\pi_{i}\right) \in\left\langle\pi_{1} \circ \varphi-\pi_{1} \circ \psi, \cdots, \pi_{n} \circ \varphi-\pi_{n} \circ \psi\right\rangle
$$

hence

$$
(\forall i \in\{1, \cdots, n\})\left(q \circ \varphi_{*}\left(\pi_{i}\right)=q \circ \psi_{*}\left(\pi_{i}\right)\right) .
$$

Since $\mathcal{C}^{\infty}\left(\mathbb{R}^{n}\right)$ is the free $\mathcal{C}^{\infty}$-ring on $n$ generators, given the $n$ following elements:

$$
q \circ \varphi_{*}\left(\pi_{1}\right), \cdots, q \circ \varphi_{*}\left(\pi_{n}\right) \in \frac{\mathcal{C}^{\infty}\left(\mathbb{R}^{m}\right)}{\left\langle\pi_{1} \circ \varphi-\pi_{1} \circ \psi, \cdots, \pi_{n} \circ \varphi-\pi_{n} \circ \psi\right\rangle}
$$

there is a unique $\mathcal{C}^{\infty}$-homomorphism $\rho: \mathcal{C}^{\infty}\left(\mathbb{R}^{n}\right) \rightarrow \frac{\mathcal{C}^{\infty}\left(\mathbb{R}^{m}\right)}{\left\langle\pi_{1} \circ \varphi-\pi_{1} \circ \psi, \cdots, \pi_{n} \circ \varphi-\pi_{n} \circ \psi\right\rangle}$ such that:

$$
(\forall i \in\{1, \cdots, n\})\left(\rho\left(\pi_{i}\right)=q \circ \varphi_{*}\left(\pi_{i}\right)=q \circ \psi_{*}\left(\pi_{i}\right)\right) .
$$

Since both $q \circ \Phi, q \circ \Psi: C^{\mathbb{R}^{n}} \rightarrow \frac{\mathcal{C}^{\infty}\left(\mathbb{R}^{m}\right)}{\left\langle\pi_{1} \circ \varphi-\pi_{1} \circ \psi, \cdots, \pi_{n} \circ \varphi-\pi_{n} \circ \psi\right\rangle}$ are such that for every $i \in$ $\{1, \cdots, n\}$,

$$
\begin{aligned}
& q \circ \Phi\left(\pi_{i}\right)=\pi_{i} \circ \varphi+\left\langle\pi_{1} \circ \varphi-\pi_{1} \circ \psi, \cdots, \pi_{n} \circ \varphi-\pi_{n} \circ \psi\right\rangle=\rho\left(\pi_{i}\right) \\
& q \circ \Psi\left(\pi_{i}\right)=\pi_{i} \circ \psi+\left\langle\pi_{1} \circ \psi-\pi_{1} \circ \psi, \cdots, \pi_{n} \circ \psi-\pi_{n} \circ \psi\right\rangle=\rho\left(\pi_{i}\right)
\end{aligned}
$$

it follows that:

$$
q \circ \Phi=q \circ \Psi
$$

so $q$ coequalizes $\Phi$ and $\Psi$.

Now we proceed to show that if $h: \mathcal{C}^{\infty}\left(\mathbb{R}^{m}\right) \rightarrow A$ is any $\mathcal{C}^{\infty}$-homomorphism such that $h \circ \Phi=h \circ \Psi$, then there is a unique $\mathcal{C}^{\infty}$-homomorphism $\alpha: \frac{\mathcal{C}^{\infty}\left(\mathbb{R}^{m}\right)}{\left\langle\pi_{1} \circ \varphi-\pi_{1} \circ \psi, \cdots, \pi_{n} \circ \varphi-\pi_{n} \circ \psi\right\rangle} \rightarrow A$ such that $h \circ \alpha=q$.

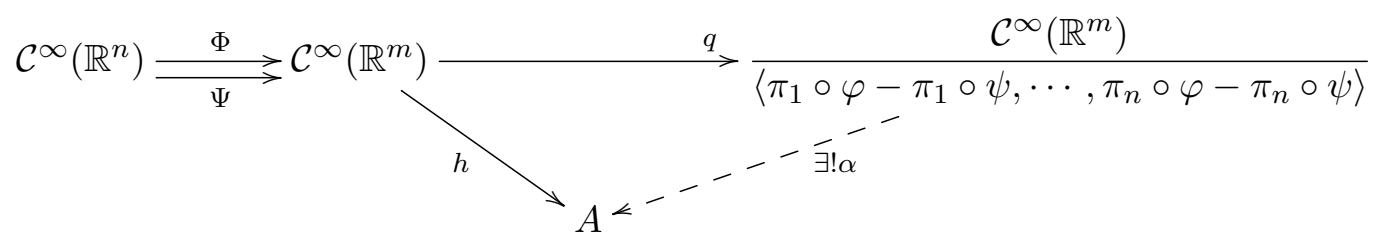

Since $h \circ \Phi=h \circ \Psi$, for every $i \in\{1, \cdots, n\}$ we have $h\left(\pi_{i} \circ \varphi-\pi_{i} \circ \psi\right)=h \circ \Phi\left(\pi_{i}\right)-h \circ \Psi\left(\pi_{i}\right)=0_{A}$, so:

$$
\left\langle\pi_{1} \circ \varphi-\pi_{1} \circ \psi, \cdots, \pi_{n} \circ \varphi-\pi_{n} \circ \psi\right\rangle \subseteq \operatorname{ker}(h) .
$$

By the Theorem of $\mathcal{C}^{\infty}$-Homomorphism, there is a unique $\mathcal{C}^{\infty}$-homomorphism 


$$
\bar{h}: \frac{\mathcal{C}^{\infty}\left(\mathbb{R}^{m}\right)}{\left\langle\pi_{1} \circ \varphi-\pi_{1} \circ \psi, \cdots, \pi_{n} \circ \varphi-\pi_{n} \circ \psi\right\rangle} \rightarrow A
$$

such that the following diagram commutes:

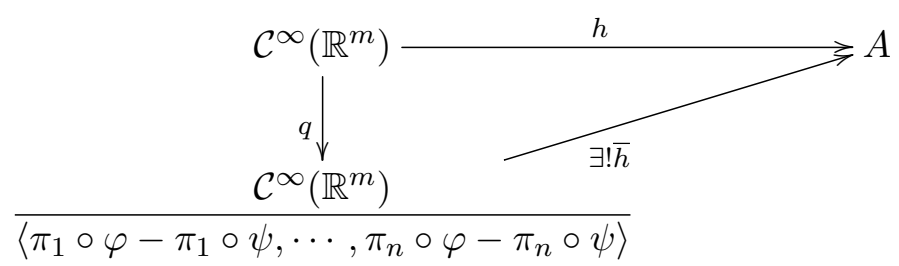

Hence, $\frac{\mathcal{C}^{\infty}\left(\mathbb{R}^{m}\right)}{\left\langle\pi_{1} \circ \varphi-\pi_{1} \circ \psi, \cdots, \pi_{n} \circ \varphi-\pi_{n} \circ \psi\right\rangle}$ is the coequalizer of $\Phi$ and $\Psi$.

By the Theorem of the Isomorphism for $\mathcal{C}^{\infty}$-rings, we obtain the following:

Lemma 1.4.11. Let $\mathcal{C}^{\infty}\left(\mathbb{R}^{n}\right)$ be the free $\mathcal{C}^{\infty}$-ring on $n$ generators, and $I$ and $J$ be two ideals of $\mathcal{C}^{\infty}\left(\mathbb{R}^{n}\right)$. There is an isomorphism:

$$
\widetilde{\rho}: \frac{\mathcal{C}^{\infty}\left(\mathbb{R}^{n}\right) / J}{(I+J) / J} \stackrel{\cong}{\longrightarrow} \frac{\mathcal{C}^{\infty}\left(\mathbb{R}^{n}\right)}{\langle I, J\rangle}
$$

where $\langle I, J\rangle$ stands for $I+J=\langle\{a+b \mid a \in I \& b \in J\}\rangle$, the ideal generated by the sum of the ideals $I$ and $J$.

Proposition 1.4.12. Let $\mathcal{C}^{\infty}\left(\mathbb{R}^{m}\right)$ be the free $\mathcal{C}^{\infty}$-ring on $m$ generators, $I, J \subseteq \mathcal{C}^{\infty}\left(\mathbb{R}^{m}\right)$ be any two ideals, $q_{I}, q_{J}, q_{I+J}$ be the canonical projections and $\widetilde{\rho}$ be the $\mathcal{C}^{\infty}$-isomorphism described in the Proposition 1.4.11. Then the $\mathcal{C}^{\infty}$-homomorphism:

$$
\begin{gathered}
\widetilde{q}: \frac{\mathcal{C}^{\infty}\left(\mathbb{R}^{m}\right)}{I} \rightarrow \frac{\mathcal{C}^{\infty}\left(\mathbb{R}^{m}\right)}{\langle I, J\rangle} \\
f+I \mapsto f+\langle I, J\rangle
\end{gathered}
$$

is the unique $\mathcal{C}^{\infty}$-homomorphism such that following diagram:

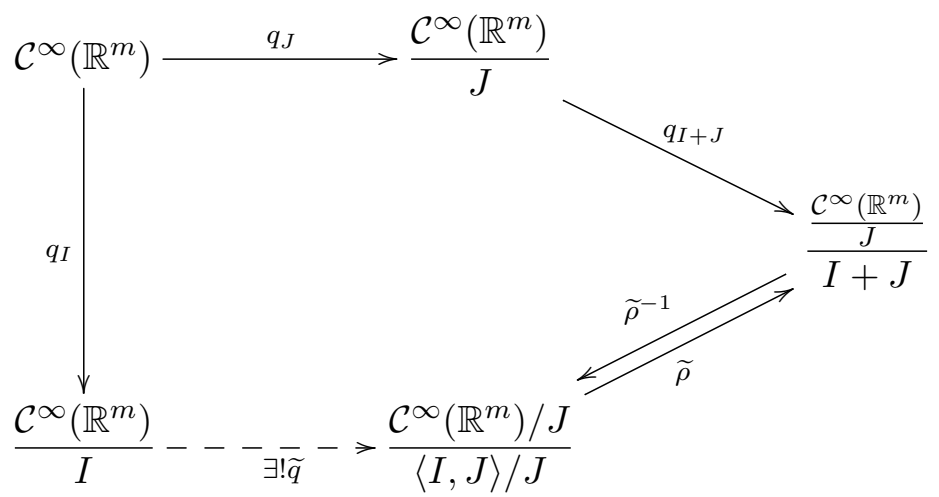

commutes.

Proof. By the Theorem of Homomorphism it suffices to show that $I \subseteq \operatorname{ker}\left(\widetilde{\rho}^{-1} \circ q_{I+J} \circ q_{J}\right)$.

Given $f \in I$, we have:

$$
\widetilde{\rho}^{-1} \circ q_{I+J} \circ q_{J}(f)=\widetilde{\rho}^{-1} \circ q_{I+J}(f+J)=\widetilde{\rho}^{-1}((f+J)+(I+J)) \stackrel{(*)}{=} \widetilde{\rho}^{-1}(0+I+J)=\langle I, J\rangle
$$


where $(*)$ is due to the fact that $f \in I \Rightarrow f+J \in(I+J)$.

Hence, there is a unique $\mathcal{C}^{\infty}$-homomorphism:

$$
\widetilde{q}: \frac{\mathcal{C}^{\infty}\left(\mathbb{R}^{m}\right)}{I} \rightarrow \frac{\mathcal{C}^{\infty}\left(\mathbb{R}^{m}\right)}{\langle I, J\rangle}
$$

such that:

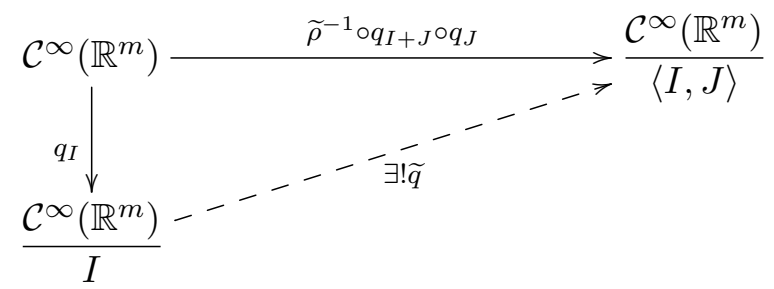

and $\widetilde{q}(f+I)=\widetilde{\rho}^{-1} \circ q_{I+J} \circ q_{J}(f)=f+(I+J)$.

Theorem 1.4.13. Let $\Phi, \Psi: \frac{\mathcal{C}^{\infty}\left(\mathbb{R}^{n}\right)}{I} \rightarrow \frac{\mathcal{C}^{\infty}\left(\mathbb{R}^{m}\right)}{J}$ be two $\mathcal{C}^{\infty}$-homomorphisms, and let $\varphi, \psi: \mathbb{R}^{m} \rightarrow \mathbb{R}^{n}$ be two $\mathcal{C}^{\infty}$-functions as in Proposition 1.4.6, that is, such that $I \subseteq \varphi_{*}^{-}[J], I \subseteq \psi_{*}^{-}[J]$ and that the following diagrams commute:
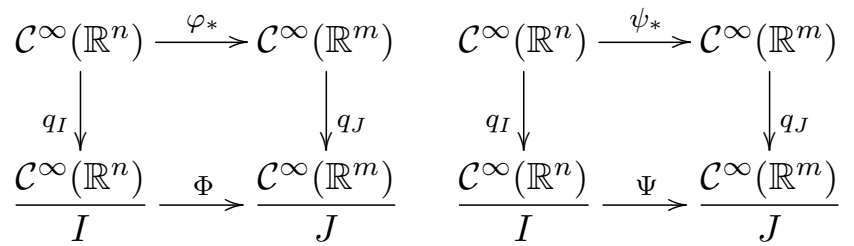

The coequalizer of $\Phi$ and $\Psi$ is given by the quotient map:

$$
\frac{\mathcal{C}^{\infty}\left(\mathbb{R}^{m}\right)}{J} \rightarrow \frac{\mathcal{C}^{\infty}\left(\mathbb{R}^{m}\right)}{J+\left\langle\left\{\pi_{1} \circ \varphi-\pi_{1} \circ \psi, \cdots, \pi_{n} \circ \varphi-\pi_{n} \circ \psi\right\}\right\rangle}
$$

Proof. By Proposition 1.4.10 we know that the following diagram is a coequalizer:

$$
\mathcal{C}^{\infty}\left(\mathbb{R}^{n}\right) \underset{\Psi}{\stackrel{\Phi}{\longrightarrow}} \mathcal{C}^{\infty}\left(\mathbb{R}^{m}\right) \stackrel{q}{\longrightarrow} \frac{\mathcal{C}^{\infty}\left(\mathbb{R}^{m}\right)}{\left\langle\pi_{1} \circ \varphi-\pi_{1} \circ \psi, \cdots, \pi_{n} \circ \varphi-\pi_{n} \circ \psi\right\rangle}
$$

By Proposition 1.4.11, we have:

$\frac{\mathcal{C}^{\infty}\left(\mathbb{R}^{n}\right) / J}{\left\langle\left\{\pi_{1} \circ \varphi-\pi_{1} \circ \psi+J, \cdots, \pi_{n} \circ \varphi-\pi_{n}+J \circ \psi\right\}\right\rangle / J} \cong \widetilde{\rho} \frac{\mathcal{C}^{\infty}\left(\mathbb{R}^{m}\right)}{\left\langle J,\left\langle\left\{\pi_{1} \circ \varphi-\pi_{1} \circ \psi, \cdots, \pi_{n} \circ \varphi-\pi_{n} \circ \psi\right\}\right\rangle\right\rangle}$

Taking $\left\langle\pi_{1} \circ \varphi-\pi_{1} \circ \psi, \cdots, \pi_{n} \circ \varphi-\pi_{n} \circ \psi\right\rangle$ in the place of $J$ and $J$ in the place of $I$ in the Proposition $\mathbf{1 . 4 . 1 2}$, it follows that there is a unique:

$$
\widetilde{q}: \frac{\mathcal{C}^{\infty}\left(\mathbb{R}^{m}\right)}{J} \rightarrow \frac{\mathcal{C}^{\infty}\left(\mathbb{R}^{m}\right)}{\langle I, J\rangle}
$$

such that the following diagram commutes: 


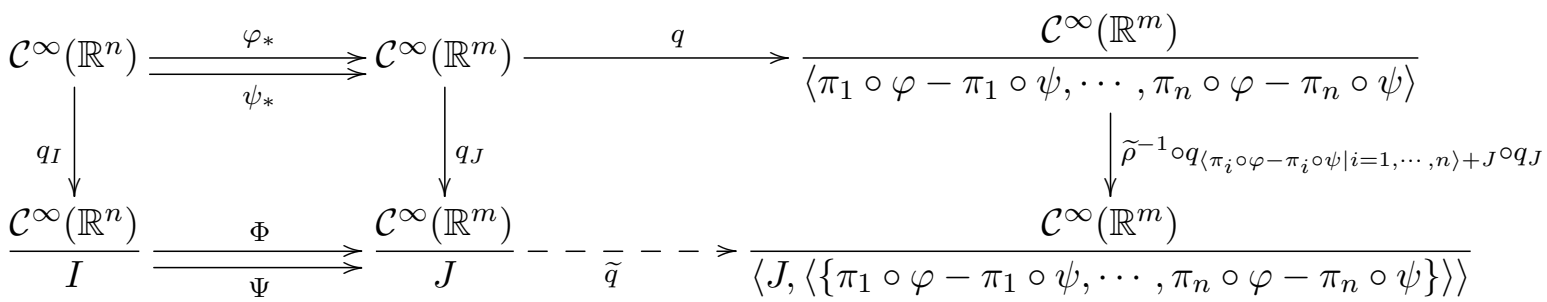

Fact: In the diagram above, the fact that $q$ is a coequalizer implies that $\widetilde{q}$ is a coequalizer.

Given any $f+I \in \frac{\mathcal{C}^{\infty}\left(\mathbb{R}^{n}\right)}{I}$, we have, on the one hand:

$$
\widetilde{q} \circ \Phi(f+I)=\widetilde{q} \circ\left(\Phi \circ q_{I}\right)(f)=\widetilde{q} \circ\left(q_{J} \circ \varphi_{*}\right)(f)
$$

and

$$
\widetilde{q} \circ \Psi(f+I)=\widetilde{q} \circ\left(\Psi \circ q_{I}\right)(f)=\widetilde{q} \circ\left(q_{J} \circ \psi_{*}\right)(f)
$$

On the other hand, since the right square commutes, $\widetilde{q} \circ q_{J}=\widetilde{\rho}^{-1} \circ q_{\left\langle\pi_{i} \circ \varphi-\pi_{i} \circ \psi \mid i=1, \cdots, n\right\rangle+J} \circ q_{J} \circ q$, so:

$$
\left(\widetilde{q} \circ q_{J}\right) \circ \varphi_{*}(f)=\widetilde{\rho}^{-1} \circ q_{\left\langle\pi_{i} \circ \varphi-\pi_{i} \circ \psi \mid i=1, \cdots, n\right\rangle+J} \circ q_{J} \circ\left(q \circ \varphi_{*}\right)(f)
$$

and since $q$ coequalizes $\varphi_{*}$ and $\psi_{*}$, we have:

$$
\widetilde{\rho}^{-1} \circ q_{\left\langle\pi_{i} \circ \varphi-\pi_{i} \circ \psi \mid i=1, \cdots, n\right\rangle+J} \circ q_{J} \circ\left(q \circ \varphi_{*}\right)(f)=\widetilde{\rho}^{-1} \circ q_{\left\langle\pi_{i} \circ \varphi-\pi_{i} \circ \psi \mid i=1, \cdots, n\right\rangle+J} \circ q_{J} \circ\left(q \circ \psi_{*}\right)(f)
$$

Also

$$
\widetilde{\rho}^{-1} \circ q_{\left\langle\pi_{i} \circ \varphi-\pi_{i} \circ \psi \mid i=1, \cdots, n\right\rangle+J} \circ q_{J} \circ\left(q \circ \psi_{*}\right)(f)=\left(\widetilde{q} \circ q_{J}\right)\left(\psi_{*}\right)(f)=\widetilde{q} \circ\left(\Phi \circ q_{I}\right)(f)=\widetilde{q} \circ \Psi(f+I)
$$

thus

$$
\widetilde{q} \circ \Phi=\widetilde{q} \circ \Psi
$$

Now, let $\widetilde{q}^{\prime}: \frac{\mathcal{C}^{\infty}\left(\mathbb{R}^{m}\right)}{J} \rightarrow Q^{\prime}$ be a $\mathcal{C}^{\infty}$-homomorphism which coequalizes $\Phi$ and $\Psi$, that is $\widetilde{q}^{\prime} \circ \Phi=\widetilde{q}^{\prime} \circ \Psi$. We have:

$$
\widetilde{q}^{\prime} \circ \Phi=\widetilde{q}^{\prime} \circ \Psi \Rightarrow \widetilde{q}^{\prime} \circ \Phi \circ q_{I}=\widetilde{q}^{\prime} \circ \Psi \circ q_{I},
$$

and since the left squares of the diagram commutes, that is, $\Phi \circ q_{I}=q_{J} \circ \varphi_{*}$ and $\Psi \circ q_{I}=q_{J} \circ \psi_{*}$, we have:

$$
\tilde{q}^{\prime} \circ \Phi \circ q_{I}=\widetilde{q}^{\prime} \circ q_{J} \circ \varphi_{*}
$$

and

$$
\widetilde{q}^{\prime} \circ \Psi \circ q_{I}=\widetilde{q}^{\prime} \circ q_{J} \circ \psi_{*}
$$

We have, thus:

$$
\left(\widetilde{q}^{\prime} \circ q_{J}\right) \circ \varphi_{*}=\widetilde{q}^{\prime} \circ \Phi \circ q_{I}=\widetilde{q}^{\prime} \circ \Psi \circ q_{I}=\left(\widetilde{q}^{\prime} \circ q_{J}\right) \circ \psi_{*}
$$


so $\left(\widetilde{q}^{\prime} \circ q_{J}\right)$ coequalizes $\varphi_{*}$ and $\psi_{*}$.

By the universal property of the coequalizer $q$, there is a unique

$$
\widetilde{\alpha}: \frac{\mathcal{C}^{\infty}\left(\mathbb{R}^{m}\right)}{\left\langle\pi_{1} \circ \varphi-\pi_{1} \circ \psi, \cdots, \pi_{n} \circ \varphi-\pi_{n} \circ \psi\right\rangle} \rightarrow Q^{\prime}
$$

such that $\widetilde{\alpha} \circ q=\widetilde{q}^{\prime} \circ q_{J}$ :

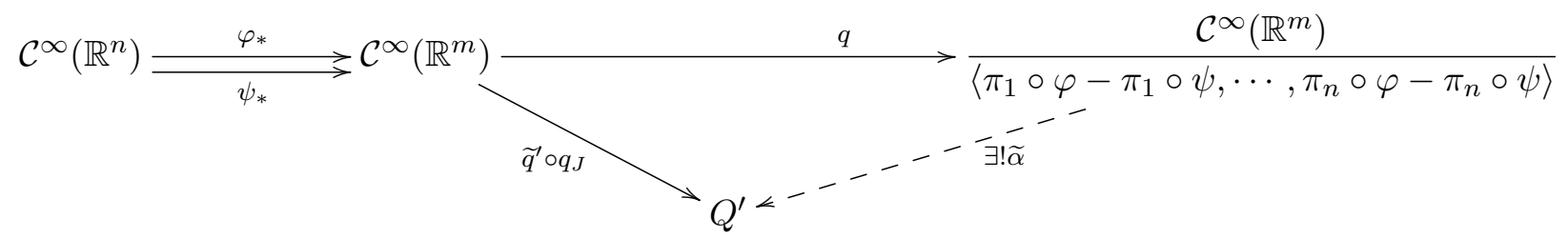

Now, $\left\langle J,\left\langle\left\{\pi_{1} \circ \varphi-\pi_{1} \circ \psi, \cdots, \pi_{n} \circ \varphi-\pi_{n} \circ \psi\right\}\right\rangle\right\rangle \subseteq \operatorname{ker}(\widetilde{\alpha})$, since given $f \in J, \widetilde{\alpha}(q(f))=\widetilde{q}^{\prime} \circ q_{J}(f)=$ $\widetilde{q}^{\prime}(0+J)=0_{Q^{\prime}}$. By the Theorem of Homomorphism there is a unique $\mathcal{C}^{\infty}$-homomorphism:

$$
\alpha^{\prime}: \frac{\mathcal{C}^{\infty}\left(\mathbb{R}^{m}\right)}{\left\langle J,\left\langle\left\{\pi_{1} \circ \varphi-\pi_{1} \circ \psi, \cdots, \pi_{n} \circ \varphi-\pi_{n} \circ \psi\right\}\right\rangle\right\rangle} \rightarrow Q^{\prime}
$$

such that $\alpha^{\prime} \circ \widetilde{\rho}^{-1} \circ q_{\left\langle\left\{\pi_{i} \circ \varphi-\pi_{i} \circ \psi \mid i=1, \cdots, n\right\}\right\rangle+J}=\widetilde{\alpha}$ :

$$
\begin{aligned}
& \frac{\mathcal{C}^{\infty}\left(\mathbb{R}^{m}\right)}{\left\langle\left\{\pi_{1} \circ \varphi-\pi_{1} \circ \psi, \cdots, \pi_{n} \circ \varphi-\pi_{n} \circ \psi\right\}\right\rangle} \frac{\widetilde{\alpha}}{{ }^{\prime} q_{\left\langle\left\{\pi_{i} \circ \varphi-\pi_{i} \circ \psi \mid i=1, \cdots, n\right\}\right\rangle+J} \downarrow} \ldots Q^{\prime} \\
& \frac{\mathcal{C}^{\infty}\left(\mathbb{R}^{m}\right)}{\left\langle J,\left\langle\left\{\pi_{1} \circ \varphi-\pi_{1} \circ \psi, \cdots, \pi_{n} \circ \varphi-\pi_{n} \circ \psi\right\}\right\rangle\right\rangle}
\end{aligned}
$$

Finally, we claim that:

$$
\alpha^{\prime} \circ \widetilde{q}=\widetilde{q}^{\prime}
$$

Indeed, $\widetilde{q}$ is the unique $\mathcal{C}^{\infty}$-homomorphism such that $\widetilde{q} \circ q_{J}=\left(\rho^{-1} \circ q_{\left\langle\pi_{i} \circ \varphi-\pi_{i} \circ \psi \mid i=1, \cdots, n\right\rangle+J} \circ q_{J}\right) \circ q$, $\alpha^{\prime}$ is the unique $\mathcal{C}^{\infty}$-homomorphism such that $\alpha^{\prime} \circ\left(\rho^{-1} \circ q_{\left\langle\pi_{i} \circ \varphi-\pi_{i} \circ \psi \mid i=1, \cdots, n\right\rangle+J} \circ q_{J}\right)=\widetilde{\alpha}$ and $\widetilde{\alpha}$ is the unique $\mathcal{C}^{\infty}$-homomorphism such that $\widetilde{\alpha} \circ q=\widetilde{q}^{\prime} \circ q_{J}$. thus:

$$
\begin{gathered}
\alpha^{\prime} \circ\left(\rho^{-1} \circ q_{\left\langle\pi_{i} \circ \varphi-\pi_{i} \circ \psi \mid i=1, \cdots, n\right\rangle+J} \circ q_{J}\right) \circ q=\widetilde{q}^{\prime} \circ q_{J} \\
\alpha^{\prime} \circ \widetilde{q} \circ q_{J}=\widetilde{q}^{\prime} \circ q_{J}
\end{gathered}
$$

Since $q_{J}$ is an epimorphism, it follows that:

$$
\alpha^{\prime} \circ \widetilde{q}=\widetilde{q}^{\prime}
$$

and the result is proved.

We can summarize the results above by saying that for any two $\mathcal{C}^{\infty}$-homomorphisms between finitely generated $\mathcal{C}^{\infty}$-rings, $\Phi, \Psi: \frac{\mathcal{C}^{\infty}\left(\mathbb{R}^{n}\right)}{I} \rightarrow \frac{\mathcal{C}^{\infty}\left(\mathbb{R}^{m}\right)}{J}$, if $\varphi, \psi: \mathbb{R}^{m} \rightarrow \mathbb{R}^{n}$ are such that $\Phi=\mu(\varphi)$ and $\Psi=\mu(\psi)$, the following diagram (with the arrows described in the results above) is a coequalizer: 


$$
\frac{\mathcal{C}^{\infty}\left(\mathbb{R}^{n}\right)}{I} \underset{\Psi}{\stackrel{\Phi}{\longrightarrow}} \frac{\mathcal{C}^{\infty}\left(\mathbb{R}^{m}\right)}{J} \stackrel{\widetilde{q}}{\longrightarrow} \frac{\mathcal{C}^{\infty}\left(\mathbb{R}^{m}\right)}{\left\langle J,\left\langle\pi_{1} \circ \varphi-\pi_{1} \circ \psi, \cdots, \pi_{n} \circ \varphi-\pi_{n} \circ \psi\right\rangle\right\rangle}
$$

Remark 1.4.14. Let $m, n \in \mathbb{N}$ and let $I \subseteq \mathcal{C}^{\infty}\left(\mathbb{R}^{n}\right)$ and $J \subseteq \mathcal{C}^{\infty}\left(\mathbb{R}^{m}\right)$ be ideals such that there are sets $X$ and $Y$ such that:

$$
I=\langle X\rangle
$$

and

$$
J=\langle Y\rangle
$$

Then we have:

$$
\frac{\mathcal{C}^{\infty}\left(\mathbb{R}^{n} \times \mathbb{R}^{m}\right)}{\left\langle\left\{f \circ \pi_{1} \mid f \in I\right\} \cup\left\{g \circ \pi_{2} \mid g \in J\right\}\right\rangle}=\frac{\mathcal{C}^{\infty}\left(\mathbb{R}^{n} \times \mathbb{R}^{m}\right)}{\left\langle\left\{f \circ \pi_{1} \mid f \in X\right\} \cup\left\{g \circ \pi_{2} \mid g \in Y\right\}\right\rangle}
$$

Remark 1.4.15. Note that the coequalizer of $\Phi, \Psi: \frac{\mathcal{C}^{\infty}\left(\mathbb{R}^{n}\right)}{I} \rightarrow \frac{\mathcal{C}^{\infty}\left(\mathbb{R}^{m}\right)}{J}$ does not depend on any particular choice of the representing $\varphi, \psi: \mathbb{R}^{m} \rightarrow \mathbb{R}^{n}$ of $\Phi$ and $\Psi$ chosen.

Indeed, given $\varphi^{\prime}, \psi^{\prime}: \mathbb{R}^{m} \rightarrow \mathbb{R}^{n}$ and $\varphi, \psi: \mathbb{R}^{m} \rightarrow \mathbb{R}^{n}$ such that $\mu(\varphi)=\Phi=\mu\left(\varphi^{\prime}\right)$ and $\mu(\psi)=\Psi=$ $\mu\left(\psi^{\prime}\right)$ with $\varphi \sim \varphi^{\prime}$ and $\psi \sim \psi^{\prime}$, for every $i \in\{1, \cdots, n\}$ :

$$
\pi_{i} \circ \varphi-\pi_{i} \circ \varphi^{\prime} \in J
$$

and

$$
\pi_{i} \circ \psi-\pi_{i} \circ \psi^{\prime} \in J
$$

so

$$
\left\langle\pi_{1} \circ \varphi-\pi_{1} \circ \psi+J, \cdots, \pi_{n} \circ \varphi-\pi_{n} \circ \psi+J\right\rangle=\left\langle\pi_{1} \circ \varphi^{\prime}-\pi_{1} \circ \psi^{\prime}+J, \cdots, \pi_{n} \circ \varphi^{\prime}-\pi_{n} \circ \psi^{\prime}+J\right\rangle
$$

and

$$
(\forall i \in\{1, \cdots, n\})\left(\pi_{i} \circ \varphi-\pi_{i} \circ \psi+J=\pi_{i} \circ \varphi^{\prime}-\pi_{i} \circ \psi^{\prime}+J\right)
$$

thus

$$
\frac{\frac{\mathcal{C}^{\infty}\left(\mathbb{R}^{m}\right)}{J}}{\left\langle\pi_{1} \circ \varphi-\pi_{1} \circ \psi+J, \cdots, \pi_{n} \circ \varphi-\pi_{n} \circ \psi+J\right\rangle}=\frac{\frac{\mathcal{C}^{\infty}\left(\mathbb{R}^{m}\right)}{J}}{\left\langle\pi_{1} \circ \varphi^{\prime}-\pi_{1} \circ \psi^{\prime}+J, \cdots, \pi_{n} \circ \varphi^{\prime}-\pi_{n} \circ \psi^{\prime}+J\right\rangle}
$$

and:

$$
\frac{\mathcal{C}^{\infty}\left(\mathbb{R}^{n}\right)}{\left\langle J,\left\langle\left\{\pi_{1} \circ \varphi-\pi_{1} \circ \psi, \cdots, \pi_{n} \circ \varphi-\pi_{n} \circ \psi\right\}\right\rangle\right\rangle}=\frac{\mathcal{C}^{\infty}\left(\mathbb{R}^{n}\right)}{\left\langle J,\left\langle\left\{\pi_{1} \circ \varphi^{\prime}-\pi_{1} \circ \psi^{\prime}, \cdots, \pi_{n} \circ \varphi^{\prime}-\pi_{n} \circ \psi^{\prime}\right\}\right\rangle\right\rangle}
$$

We shall use the following notation:

$$
\frac{\mathcal{C}^{\infty}\left(\mathbb{R}^{n}\right)}{\left\langle J,\left\langle\left\{\pi_{1} \circ \varphi-\pi_{1} \circ \psi, \cdots, \pi_{n} \circ \varphi-\pi_{n} \circ \psi\right\}\right\rangle\right\rangle} \doteq \frac{\mathcal{C}^{\infty}\left(\mathbb{R}^{m}\right)}{\left\langle J, \pi_{1} \circ \varphi-\pi_{1} \circ \psi, \cdots, \pi_{n} \circ \varphi-\pi_{n} \circ \psi\right\rangle}
$$

Theorem 1.4.16. The categories of finitely generated and finitely presented $\mathcal{C}^{\infty}$-rings, $\mathcal{C}^{\infty} \mathbf{R n g}_{\mathrm{fp}}$, are closed under initial object, binary coproducts and binary coequalizers. 
Proof. The initial object of these two categories is:

$$
\mathbb{R} \cong \mathcal{C}^{\infty}\left(\mathbb{R}^{0}\right) \cong \frac{\mathcal{C}^{\infty}\left(\mathbb{R}^{0}\right)}{\langle 0\rangle}
$$

thus it is a finitely presented $\mathcal{C}^{\infty}$-ring.

Let $\frac{\mathcal{C}^{\infty}\left(\mathbb{R}^{n}\right)}{\left\langle f_{1}, \cdots, f_{k}\right\rangle}$ and $\frac{\mathcal{C}^{\infty}\left(\mathbb{R}^{m}\right)}{\left\langle g_{1}, \cdots, g_{\ell}\right\rangle}$ be two finitely presented $\mathcal{C}^{\infty}$-rings.

By construction and by the Remark 1.4.14, their coproduct is given by:

$$
\frac{\mathcal{C}^{\infty}\left(\mathbb{R}^{n}\right)}{\left\langle f_{1}, \cdots, f_{k}\right\rangle} \otimes_{\infty} \frac{\mathcal{C}^{\infty}\left(\mathbb{R}^{m}\right)}{\left\langle g_{1}, \cdots, g_{\ell}\right\rangle} \cong \frac{\mathcal{C}^{\infty}\left(\mathbb{R}^{n+m}\right)}{\left\langle f_{1} \circ \pi_{1}, \cdots, f_{k} \circ \pi_{1}, g_{1} \circ \pi_{2}, \cdots, g_{\ell} \circ \pi_{2}\right\rangle}
$$

where

$$
\begin{aligned}
& \pi_{1}: \quad \mathbb{R}^{n+m} \quad \rightarrow \quad \mathbb{R}^{n} \\
& \left(x_{1}, \cdots, x_{n}, y_{1}, \cdots, y_{m}\right) \mapsto\left(x_{1}, \cdots, x_{n}\right)
\end{aligned}
$$

and

$$
\begin{aligned}
& \pi_{2}: \quad \mathbb{R}^{n+m} \quad \rightarrow \quad \mathbb{R}^{m} \\
& \left(x_{1}, \cdots, x_{n}, y_{1}, \cdots, y_{m}\right) \mapsto\left(y_{1}, \cdots, y_{m}\right)
\end{aligned}
$$

are the canonical projections. Thus:

$$
\frac{\mathcal{C}^{\infty}\left(\mathbb{R}^{n}\right)}{\left\langle f_{1}, \cdots, f_{k}\right\rangle} \otimes_{\infty} \frac{\mathcal{C}^{\infty}\left(\mathbb{R}^{m}\right)}{\left\langle g_{1}, \cdots, g_{\ell}\right\rangle} \cong \frac{\mathcal{C}^{\infty}\left(\mathbb{R}^{n+m}\right)}{\left\langle f_{1} \circ \pi_{1}, \cdots, f_{k} \circ \pi_{1}, g_{1} \circ \pi_{2}, \cdots, g_{\ell} \circ \pi_{2}\right\rangle}
$$

which is finitely presented.

Let

$$
\Phi, \Psi: \frac{\mathcal{C}^{\infty}\left(\mathbb{R}^{n}\right)}{\left\langle f_{1}, \cdots, f_{k}\right\rangle} \rightarrow \frac{\mathcal{C}^{\infty}\left(\mathbb{R}^{m}\right)}{\left\langle g_{1}, \cdots, g_{\ell}\right\rangle}
$$

be two $\mathcal{C}^{\infty}$-homomorphism between the finitely presented $\mathcal{C}^{\infty}$-rings $\frac{\mathcal{C}^{\infty}\left(\mathbb{R}^{n}\right)}{\left\langle f_{1}, \cdots, f_{k}\right\rangle}$ and $\frac{\mathcal{C}^{\infty}\left(\mathbb{R}^{m}\right)}{\left\langle g_{1}, \cdots, g_{\ell}\right\rangle}$ and let $\varphi, \psi: \mathbb{R}^{m} \rightarrow \mathbb{R}^{n}$ be $\mathcal{C}^{\infty}$-functions such that $\mu(\varphi)=\Phi$ and $\mu(\psi)=\Psi$ (which exist due to Proposition 1.4.5). By Theorem 1.4.13 and Remark 1.4.14, the coequalizer of $\Phi$ and $\Psi$ is given by:

$$
\frac{\mathcal{C}^{\infty}\left(\mathbb{R}^{n}\right)}{\left\langle f_{1}, \cdots, f_{k}\right\rangle} \stackrel{\Phi}{\underset{\Psi}{\longrightarrow}} \frac{\mathcal{C}^{\infty}\left(\mathbb{R}^{m}\right)}{\left\langle g_{1}, \cdots, g_{\ell}\right\rangle} \longrightarrow \frac{\mathcal{C}^{\infty}\left(\mathbb{R}^{m}\right)}{\left\langle g_{1}, \cdots, g_{\ell}, \pi_{1} \circ \varphi-\pi_{1} \circ \psi, \cdots, \pi_{n} \circ \varphi-\pi_{n} \circ \psi\right\rangle}
$$

which is a finitely presented $\mathcal{C}^{\infty}$-ring.

The proof that the category of the finitely generated $\mathcal{C}^{\infty}$-rings, $\mathcal{C}^{\infty} \mathbf{R n g}_{\mathrm{fg}}$ is closed under initial object, binary coproducts and binary coequalizers is analogous - and even simpler.

An important consequence of Theorem 1.4.16 , together with the fact that the final object $\mathcal{C}^{\infty}\left(\mathbb{R}^{0}\right)$ is a finitely presented $\mathcal{C}^{\infty}$-ring, is the following:

Corollary 1.4.17. The categories of finitely presented $\mathcal{C}^{\infty}$-rings an finitely generated $\mathcal{C}^{\infty}$-rings, $\mathcal{C}^{\infty} \mathbf{R n g}_{\mathrm{fp}}$ and $\mathcal{C}^{\infty} \mathbf{R n g}_{\mathrm{fg}}$, are finitely co-complete, that is, they have all the finite colimits.

Proof. Since finite colimits can be obtained by a combination of initial object, binary coproducts and binary coequalizers, the result follows. 


\subsection{The Free $\mathcal{C}^{\infty}$-Ring Associated with a Ring}

We have already seen that the forgetful functor $U^{\prime}: \mathcal{C}^{\infty} \mathbf{R n g} \rightarrow$ Set has a left adjoint, $L$, and it is a well known fact that the forgetful functor $U^{\prime \prime}:$ CRing $\rightarrow$ Set has a left adjoint, so both $\mathcal{C}^{\infty}$ Rng and CRing are concrete categories. Considering the forgetful functor $\widetilde{U}: \mathcal{C}^{\infty} \mathbf{R n g} \rightarrow \mathbf{C R i n g}$, we have the following diagram:

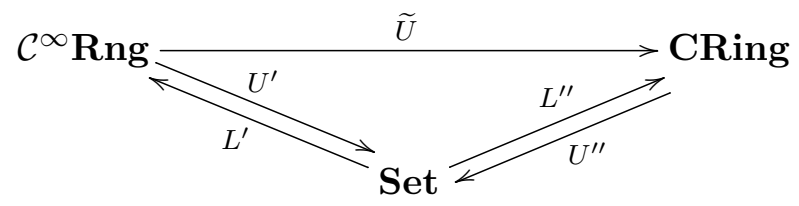

We would like to obtain a left-adjoint to the functor $\widetilde{U}$, which makes the following diagram commutative:

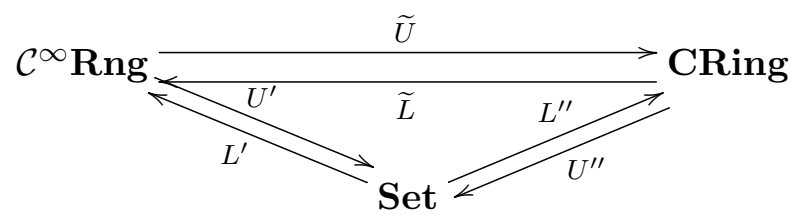

In this section we describe a construction of such a left adjoint.

Theorem 1.5.1. The forgetful functor:

$$
\widetilde{U}: \mathcal{C}^{\infty} \mathbf{R n g} \rightarrow \mathbf{C R n g}
$$

has a left adjoint.

Proof. First we note that both $\mathbf{C R n g}$ and $\mathcal{C}^{\infty} \mathbf{R n g}$ are concrete categories and the forgetful functors:

$$
U^{\prime}: \mathcal{C}^{\infty} \mathbf{R n g} \rightarrow \text { Set }
$$

and

$$
U^{\prime \prime}: \text { CRng } \rightarrow \text { Set }
$$

have left adjoints, $L^{\prime}:$ Set $\rightarrow \mathcal{C}^{\infty} \mathbf{R n g}$ (where, for a given set $E$, we have $L^{\prime}(E)=\mathcal{C}^{\infty}\left(\mathbb{R}^{E}\right)$ ) and $L^{\prime \prime}$ : Set $\rightarrow$ CRng (where, for a given set $E$, we have $L^{\prime \prime}(E)=\mathbb{Z}[E]$ ) so we have, for each $\mathcal{C}^{\infty}$-ring $B$, the following natural bijection:

$$
\mu_{E, B}: \operatorname{Set}\left(E, U^{\prime}(B)\right) \stackrel{\cong}{\rightarrow} \mathcal{C}^{\infty} \mathbf{R n g}\left(\mathcal{C}^{\infty}\left(\mathbb{R}^{E}\right), B\right)
$$

defined as follows:

Given a function $f: E \rightarrow U^{\prime}(B)$, by the universal property of $\imath_{E}^{\prime}: E \rightarrow U^{\prime}\left(\mathcal{C}^{\infty}\left(\mathbb{R}^{E}\right)\right)$, there is a unique $\mathcal{C}^{\infty}$-homomorphism $f^{\prime}: \mathcal{C}^{\infty}\left(\mathbb{R}^{E}\right) \rightarrow B$, such that the following diagram commutes:

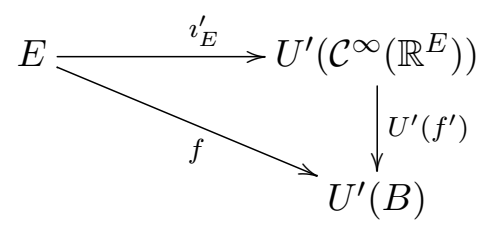

We define $\mu_{E, B}(f):=f^{\prime}$, and it is easy to see that it is natural and that the map which takes any $\mathcal{C}^{\infty}$-homomorphism $g: \mathcal{C}^{\infty}\left(\mathbb{R}^{E}\right) \rightarrow B$ to the function $U^{\prime}(g) \circ \imath_{E}^{\prime}: E \rightarrow U^{\prime}(B)$ is its inverse, i.e., 
$\mu_{E, B}^{-1}(g)=U^{\prime}(g) \circ \imath_{E}^{\prime}$.

We also have, for any commutative unital ring $R$, a natural bijection:

$$
\nu_{E, R}: \operatorname{Set}\left(E, U^{\prime \prime}(R)\right) \stackrel{\cong}{\rightrightarrows} \mathbf{C R n g}(\mathbb{Z}[E], R),
$$

which is defined in a similar way, as follows: given a function $f: E \rightarrow U^{\prime \prime}(R)$, by the universal property of $\imath_{E}^{\prime \prime}: E \rightarrow U^{\prime \prime}(\mathbb{Z}[E])$, there is a unique homomorphism of rings $f^{\prime \prime}: \mathbb{Z}[E] \rightarrow R$ such that the following diagram commutes:

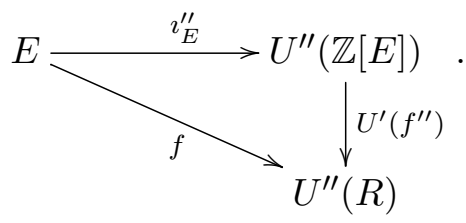

We define $\nu_{E, R}(f)=f^{\prime \prime}$, and it is easy to see that it is natural and that the map which takes any commutative unital rings homomorphism $g: \mathbb{Z}[E] \rightarrow R$ to the function $U^{\prime \prime}(g) \circ \imath_{E}^{\prime \prime}: E \rightarrow U^{\prime \prime}(R)$ is its inverse, i.e., $\nu_{E, R}^{-1}(g)=U^{\prime \prime}(g) \circ \imath_{E}^{\prime \prime}$.

Note that the following diagram commutes:

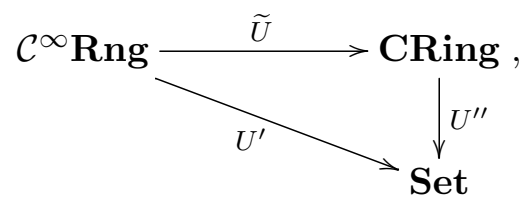

that is, $U^{\prime}=U^{\prime \prime} \circ \widetilde{U}$, and we have, for any set $E$ and any $\mathcal{C}^{\infty}$-ring $B$ :

$$
\operatorname{Set}\left(E, U^{\prime}(B)\right)=\operatorname{Set}\left(E, U^{\prime \prime}(\widetilde{U}(B))\right)
$$

and we get the following:

$$
\mathcal{C}^{\infty} \operatorname{Rng}\left(\mathcal{C}^{\infty}\left(\mathbb{R}^{E}\right), B\right) \stackrel{\mu_{E, B}^{-1}}{\rightarrow} \operatorname{Set}\left(E, U^{\prime}(B)\right)=\operatorname{Set}\left(E, U^{\prime \prime}(\widetilde{U}(B))\right) \stackrel{\nu_{E, \widetilde{U}(B)}}{\rightarrow} \mathbf{C R n g}(\mathbb{Z}[E], \widetilde{U}(B))
$$

so, composing these natural bijections yields the natural bijection:

$$
\varphi_{\mathbb{Z}[E], B}: \mathcal{C}^{\infty} \mathbf{R n g}\left(\mathcal{C}^{\infty}\left(\mathbb{R}^{E}\right), B\right) \stackrel{\nu_{E, \widetilde{U}(B)} \mu_{E, B}^{-1}}{\longrightarrow} \mathbf{C R n g}(\mathbb{Z}[E], \widetilde{U}(B)) .
$$

In the case that $\widetilde{U}$ has a left adjoint, $\widetilde{L}$, we must have $L^{\prime \prime} \cong \widetilde{L} \circ L^{\prime}($ cf. Corollary 1, p. 85 of $[49])$. This suggests us that, in order to compose the left adjoint we must define a function $\widetilde{L}_{0}$ on the free objects of CRng as follows:

$$
\widetilde{L}_{0}(\mathbb{Z}[E]):=\mathcal{C}^{\infty}\left(\mathbb{R}^{E}\right)
$$

In order to define the left adjoint $\widetilde{L}$, we are going to use Theorem 9, p. 116 of $[1]$, that is, we are going to show that $\widetilde{U}: \mathcal{C}^{\infty} \mathbf{R n g} \rightarrow \mathbf{C R i n g s}$ is a functor with the property that to every commutative unital ring, $R$ there corresponds a free $\mathcal{C}^{\infty}$-ring, call it $\left(\widetilde{L}_{0}(R), \gamma_{R}: R \rightarrow \widetilde{U}\left(\widetilde{L}_{0}(R)\right)\right)$. Given any commutative unital 
rings homomorphism $f: R \rightarrow R^{\prime}$, we define $\widetilde{L}_{1}(f): \widetilde{L}_{0}(R) \rightarrow \widetilde{L}_{0}\left(R^{\prime}\right)$ through the universal property of $\gamma_{R}$ : given the map $\gamma_{R^{\prime}} \circ f: R \rightarrow \widetilde{U}\left(\widetilde{L}_{0}\left(R^{\prime \prime}\right)\right)$, there is a unique $\mathcal{C}^{\infty}$-homomorphism $\widetilde{L}_{1}(f): \widetilde{L}_{0}(R) \rightarrow \widetilde{L}_{0}\left(R^{\prime}\right)$ such that the following diagram commutes:

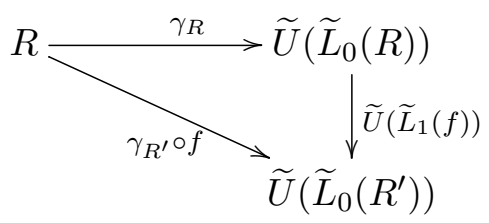

We begin with the definition of the free $\mathcal{C}^{\infty}$-rings corresponding to the free commutative unital rings. So, we start by commutative rings of the form $\mathbb{Z}[E]$ for some set $E$.

Given any set $E$ and given the map $\imath_{E}^{\prime}: E \rightarrow U^{\prime \prime}\left(\widetilde{U}\left(\mathcal{C}^{\infty}\left(\mathbb{R}^{E}\right)\right)\right)$, by the universal property of $\imath_{E}^{\prime \prime}: E \rightarrow$ $U^{\prime \prime}(\mathbb{Z}[E])$, there is a unique commutative unital rings homomorphism:

$$
\gamma_{E}: \mathbb{Z}[E] \rightarrow \widetilde{U}\left(\mathcal{C}^{\infty}\left(\mathbb{R}^{E}\right)\right)
$$

such that the following triangle commutes:

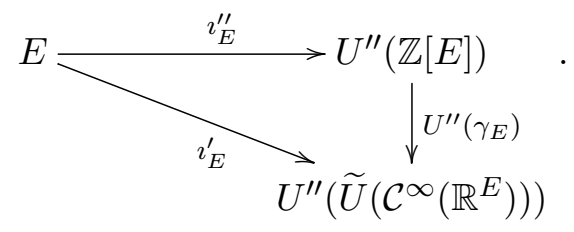

Note that $\gamma_{E}$ has the required universal property. In fact, for any $\mathcal{C}^{\infty}$-ring $A$, one has the following map:

$$
\begin{array}{cccc}
\widehat{\gamma}_{\mathbb{Z}[E], A}: \quad \mathcal{C}^{\infty} \operatorname{Rng}\left(\mathcal{C}^{\infty}\left(\mathbb{R}^{E}\right), A\right) & \rightarrow & \operatorname{CRing}(\mathbb{Z}[E], \widetilde{U}(A)) \\
f & \mapsto & \widetilde{U}(f) \circ \gamma_{E}
\end{array}
$$

which is a natural bijection since the following diagram commutes:

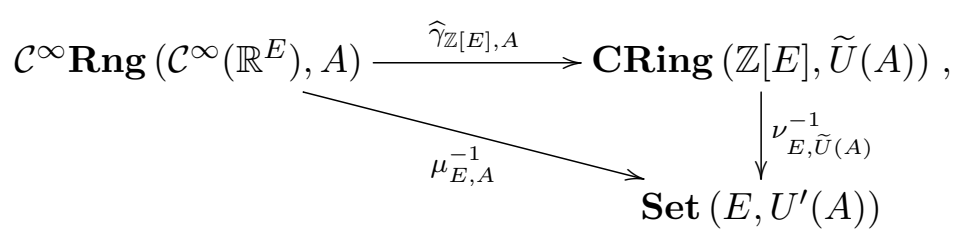

In fact, for every $f \in \mathcal{C}^{\infty} \mathbf{R n g}\left(\mathcal{C}^{\infty}\left(\mathbb{R}^{E}\right), A\right)$ we have:

$$
\begin{aligned}
& \left(\nu_{E, \widetilde{U}(A)}^{-1} \circ \widehat{\gamma}_{\mathbb{Z}[E], A}\right)(f)=\nu_{E, \widetilde{U}(A)}^{-1}\left(\widehat{\gamma}_{\mathbb{Z}[E], A}(f)\right)=\nu_{E, \widetilde{U}(A)}^{-1}\left(\widetilde{U}(f) \circ \gamma_{E}\right)= \\
& =U^{\prime \prime}\left(\widetilde{U}(f) \circ \gamma_{E}\right) \circ \imath_{E}^{\prime \prime}=\left(U^{\prime \prime}(\widetilde{U}(f)) \circ U^{\prime \prime}\left(\gamma_{E}\right)\right) \circ \imath_{E^{\prime \prime}}=U^{\prime}(f) \circ\left(U^{\prime \prime}\left(\gamma_{E}\right) \circ \imath_{E}^{\prime \prime}\right)= \\
& =U^{\prime}(f) \circ \imath_{E}^{\prime}=\mu_{E, A}^{-1}(f)
\end{aligned}
$$

and $\widehat{\gamma}_{\mathbb{Z}[E], A}$ is a natural bijection.

Now we are going to extend this definition to any commutative unital ring. 
Given any commutative unital ring of the form $\frac{\mathbb{Z}[E]}{I}$, we define $\widehat{I}=\left\langle\gamma_{E}[I]\right\rangle$, the ideal of the $\mathcal{C}^{\infty}$-ring $\mathcal{C}^{\infty}\left(\mathbb{R}^{E}\right)$ generated by $\gamma_{E}[I]$, and we define $\gamma_{E, I}$ as the unique commutative unital ring homomorphism such that the following diagram commutes:

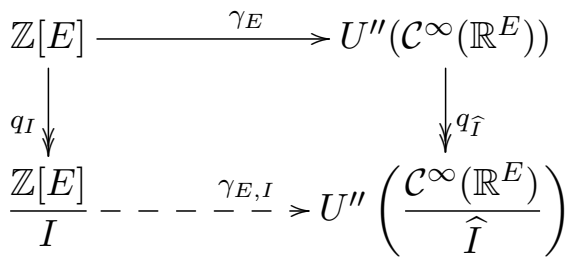

where $q_{I}$ and $q_{\widehat{I}}$ are the canonical quotient homomorphisms.

Thus we define:

$$
\widetilde{L}_{0}\left(\frac{\mathbb{Z}[E]}{I}\right):=\frac{\mathcal{C}^{\infty}\left(\mathbb{R}^{E}\right)}{\widehat{I}}
$$

Now we are going to prove that the map:

$$
\gamma_{E, I}: \frac{\mathbb{Z}[E]}{I} \rightarrow \widetilde{U}\left(\frac{\mathcal{C}^{\infty}\left(\mathbb{R}^{E}\right)}{\widehat{I}}\right)
$$

has the universal property required, by showing that $\widehat{\gamma}_{E, I}$ is a composition of bijections.

Consider the sets:

$$
\mathcal{A}=\left\{\mathcal{C}^{\infty}\left(\mathbb{R}^{E}\right) \stackrel{\varphi}{\longrightarrow} B \mid \varphi[\widehat{I}]=\{0\}\right\} \subseteq \mathcal{C}^{\infty} \mathbf{R n g}\left(\mathcal{C}^{\infty}\left(\mathbb{R}^{E}\right), B\right)
$$

and:

$$
\mathcal{B}=\{\mathbb{Z}[E] \stackrel{\psi}{\longrightarrow} \widetilde{U}(B) \mid \psi[I]=\{0\}\} \subseteq \mathbf{C R i n g}(\mathbb{Z}[E], \widetilde{U}(B)),
$$

and take:

$$
\widehat{\gamma}_{E} \uparrow_{\mathcal{A}}: \mathcal{A} \rightarrow \mathcal{B}
$$

Since $\widehat{\gamma}_{E}\left\lceil_{\mathcal{A}}[\mathcal{A}]=\mathcal{B}\right.$, we have a bijection between $\mathcal{A}$ and $\mathcal{B}$.

By the First Isomorphism Theorem, however, for every map $\mathcal{C}^{\infty}\left(\mathbb{R}^{E}\right) \stackrel{\varphi}{\longrightarrow} B$ such that $\widehat{I} \subseteq \operatorname{ker} \varphi$, i.e., for every element of $\mathcal{A}$ there is a unique element of $\mathcal{C}^{\infty} \mathbf{R n g}\left(\frac{\mathcal{C}^{\infty}\left(\mathbb{R}^{E}\right)}{\widehat{I}}, B\right)$, say $\bar{\varphi}: \frac{\mathcal{C}^{\infty}\left(\mathbb{R}^{E}\right)}{\widehat{I}} \rightarrow B$ such that the following diagram:

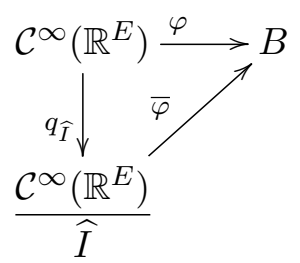

commutes, so there is a bijection between these classes. Let $\alpha: \mathcal{A} \rightarrow \mathcal{C}^{\infty} \mathbf{R n g}\left(\frac{\mathcal{C}^{\infty}\left(\mathbb{R}^{E}\right)}{\widehat{I}}, B\right)$ be such a bijection. 
By the Fundamental Homomorphism Theorem there is a natural bijection between $\mathcal{B}$ and CRng $\left(\frac{\mathbb{Z}[E]}{I}, \widetilde{U}(B)\right)$ such that for any $\psi \in \mathcal{B}$ there is a unique $\bar{\psi}$ such that the following diagram:

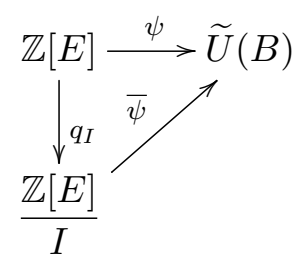

commutes. Let $\beta: \mathcal{B} \rightarrow \mathbf{C R n g}\left(\frac{\mathbb{Z}[E]}{I}, \widetilde{U}(B)\right)$ be such a bijection.

The map $\widehat{\gamma}_{E, I}:=\beta \circ\left(\widetilde{\gamma}_{E} \uparrow_{\mathcal{A}}\right) \circ \alpha^{-1}$ is a natural bijection, since it is a composition of natural bijections:

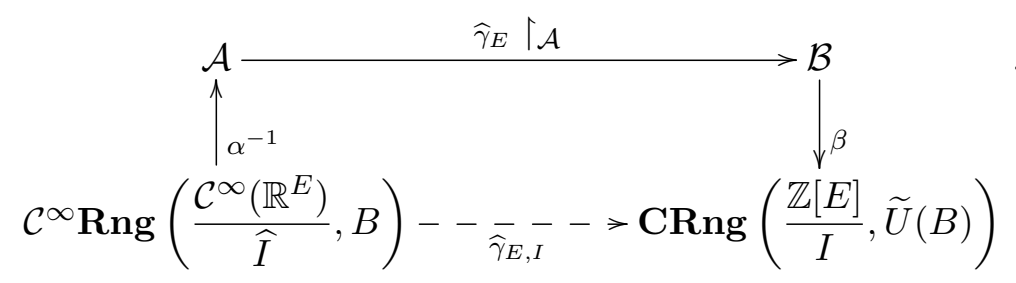

Now, given any commutative unital ring $R$, we have the counity of the adjunction $L^{\prime \prime} \dashv U^{\prime \prime}$ at $R$ :

$$
\varepsilon_{R}^{\prime \prime}: L^{\prime \prime}\left(U^{\prime \prime}(R)\right) \rightarrow R
$$

so we have the isomorphism:

$$
\overline{\varepsilon_{R}^{\prime \prime}}: \frac{\mathbb{Z}\left[U^{\prime \prime}(R)\right]}{\operatorname{ker} \varepsilon_{R}^{\prime \prime}} \rightarrow R
$$

which provides us the canonical presentation of $R$ by relations and generators:

$$
R \cong \frac{\mathbb{Z}\left[U^{\prime \prime}(R)\right]}{\operatorname{ker} \varepsilon_{R}^{\prime \prime}}
$$

so we define:

$$
\widetilde{L}_{0}(R)=\widetilde{L}_{0}\left(\frac{\mathbb{Z}\left[U^{\prime \prime}(R)\right]}{\operatorname{ker} \varepsilon_{R}^{\prime \prime}}\right)=\frac{\mathcal{C}^{\infty}\left(\mathbb{R}^{U^{\prime \prime}(R)}\right)}{\widehat{\operatorname{ker} \varepsilon_{R}^{\prime \prime}}}
$$

together with the universal map:

$$
\gamma_{R}=\gamma_{U^{\prime}(R), \operatorname{ker} \varepsilon_{R}^{\prime \prime}} \circ{\overline{\varepsilon_{R}^{\prime \prime}}}_{R}^{-1}: R \rightarrow \widetilde{U}\left(\frac{\mathcal{C}^{\infty}\left(\mathbb{R}^{U^{\prime \prime}(R)}\right)}{\widehat{\operatorname{ker} \varepsilon_{R}^{\prime \prime}}}\right)
$$

Applying the Theorem 9 (p. 116 of [1]), it follows that there is a unique way (up to natural isomorphism) to define a left-adjoint, $\widetilde{L}$ to $\widetilde{U}$.

Remark 1.5.2. Since $\widetilde{L}$, described above, is left-adjoint, it preserves all colimits, and in particular we have: 


$$
\widetilde{L}\left(\frac{\mathbb{Z}[E]}{I}\right) \cong \frac{\widetilde{L}(\mathbb{Z}[E])}{\left\langle\widetilde{\gamma_{E}}[I]\right\rangle}
$$

Note that $\widetilde{L}$ takes finitely generated (presented) commutative unital rings to finitely generated (presented) $\mathcal{C}^{\infty}$-rings.

In fact, let $B=\frac{\mathbb{Z}\left[x_{1}, \cdots, x_{n}\right]}{\left\langle p_{1}, \cdots, p_{k}\right\rangle}$, with $k, n \in \mathbb{N}$, be a finitely presented commutative unital ring, so it can be expressed by the coequalizer:

$$
\mathbb{Z}\left[x_{1}, \cdots, x_{k}\right] \underset{P}{\stackrel{0}{\longrightarrow}} \mathbb{Z}\left[x_{1}, \cdots, x_{n}\right] \longrightarrow B
$$

where for every $i \in\{1, \cdots, k\}, P\left(x_{i}\right)=p_{i}$ and $0\left(x_{i}\right)=0$.

Since $\widetilde{L}$ preserves free objects and colimits, it follows that $\widetilde{L}(B)$ is given by the coequalizer:

$$
\mathcal{C}^{\infty}\left(\mathbb{R}^{k}\right) \underset{0}{\stackrel{\widetilde{P}}{\longrightarrow}} \mathcal{C}^{\infty}\left(\mathbb{R}^{n}\right) \longrightarrow \widetilde{L}(B) \cong \frac{\mathcal{C}^{\infty}\left(\mathbb{R}^{n}\right)}{\left\langle\widetilde{\gamma_{E}}\left(p_{1}\right), \cdots, \widetilde{\gamma_{E}}\left(p_{k}\right)\right\rangle}
$$

Remark 1.5.3. Since $\mathbb{R} \cong \mathcal{C}^{\infty}(\{*\})$ is the initial object in $\mathcal{C}^{\infty} \mathbf{R n g}$, we can consider $\mathcal{C}^{\infty}$-rings as $\mathbb{R}$-algebras. In fact, we have a forgetful functor:

$$
\mathcal{U}: \mathcal{C}^{\infty} \text { Rng } \rightarrow \mathbb{R}-\text { Alg. }
$$

The functor $\mathcal{U}$ also has a left adjoint, whose construction is basically the same as the given in the previous theorem.

\subsection{Appendix on Free $\mathcal{C}^{\infty}-$ Rings}

Let $E$ be any set. We are going to describe the free $\mathcal{C}^{\infty}$-ring determined by $E$.

For any set $E$ (finite or infinite), we set:

$$
\mathbb{R}^{E}:=\{f: E \rightarrow \mathbb{R} \mid f \text { is a function }\}
$$

and

$$
\operatorname{Func}\left(\mathbb{R}^{E}, \mathbb{R}\right)=\left\{g: \mathbb{R}^{E} \rightarrow \mathbb{R} \mid g \text { is a function }\right\}
$$

Given any two finite subsets of $E, E_{i}, E_{j} \subseteq E$, whenever $E_{i} \subseteq E_{j}$ we have the restriction map:

$$
\begin{array}{rlc}
\mu_{i, j}: \mathbb{R}^{E_{j}} & \rightarrow & \mathbb{R}^{E_{i}} \\
f & \mapsto & f \circ \imath_{i, j}: E_{i} \rightarrow \mathbb{R}
\end{array}
$$

where

$$
\begin{aligned}
\imath_{i, j}: E_{i} & \hookrightarrow E_{j} \\
x & \mapsto x
\end{aligned}
$$


is the inclusion map of $E_{i}$ into $E_{j}$, and

$$
\begin{aligned}
\iota_{i}: E_{i} & \hookrightarrow E \\
x & \mapsto x
\end{aligned}
$$

is the inclusion map of $E_{i}$ into $E$.

We also have the injective pullback (defined by "composition") associated with $\mu_{i, j}$, namely:

$$
\begin{array}{rlll}
\widehat{\mu}_{i, j}: & \operatorname{Func}\left(\mathbb{R}^{E_{i}}, \mathbb{R}\right) & \mapsto & \operatorname{Func}\left(\mathbb{R}^{E_{j}}, \mathbb{R}\right) \\
\left(\mathbb{R}^{E_{i}} \stackrel{g}{\longrightarrow} \mathbb{R}\right) & \mapsto \quad\left(\mathbb{R}^{\left.E_{j} \stackrel{g \circ \mu_{i, j}}{\longrightarrow} \mathbb{R}\right)}\right.
\end{array}
$$

Analogously that for each finite subset $E_{i}$ of $E$ we have the injective map $\iota_{E_{i}, E}:$ Func $\left(\mathbb{R}^{E_{i}}, \mathbb{R}\right) \rightarrow$ Func $\left(\mathbb{R}^{E}, \mathbb{R}\right)$ given by

$$
\begin{array}{rlllll}
\iota_{E_{i}, E}: \quad & \operatorname{Func}\left(\mathbb{R}^{E_{i}}, \mathbb{R}\right) & \rightarrow & & \operatorname{Func}\left(\mathbb{R}^{E}, \mathbb{R}\right) \\
\left(\mathbb{R}^{E_{i}} \stackrel{f}{\longrightarrow} \mathbb{R}\right) & \mapsto & \tilde{f}: \quad \mathbb{R}^{E} & \rightarrow & \mathbb{R} \\
& & & g & \mapsto f\left(g \circ \iota_{i}: \mathbb{R}^{E_{i}} \rightarrow \mathbb{R}\right)
\end{array}
$$

Given $f \in \operatorname{Func}\left(\mathbb{R}^{E_{i}}, \mathbb{R}\right)$, on the one hand we have:

$$
\iota_{E_{j}, E} \circ \widehat{\mu_{i, j}}(f)=\iota_{E_{j}, E}\left(f \circ \mu_{i, j}\right)=\widetilde{f \circ \mu_{i, j}}
$$

where

$$
\begin{aligned}
\widetilde{f \circ \mu_{i, j}}: \mathbb{R}^{E} & \rightarrow \\
g & \mapsto f \circ \mu_{i, j}\left(g \circ \iota_{j}\right)=f \circ\left(g \circ \iota_{j} \circ \imath_{i, j}\right)=f\left(g \circ \iota_{i}\right)
\end{aligned}
$$

and on the other hand,

$$
\begin{aligned}
\tilde{f}: \mathbb{R}^{E} & \rightarrow \mathbb{R} \\
g & \mapsto f\left(g \circ \iota_{i}\right)
\end{aligned}
$$

so $\left(\forall f \in \operatorname{Func}\left(\mathbb{R}^{E_{i}}, \mathbb{R}\right)\right)\left(\iota_{E_{j}, E} \circ \widehat{\mu_{i, j}}(f)=\iota_{E_{i}, E}(f)\right)$.

Thus, for every $E_{i}, E_{j}$ such that $E_{i} \subseteq E_{j}$, the following diagram commutes:

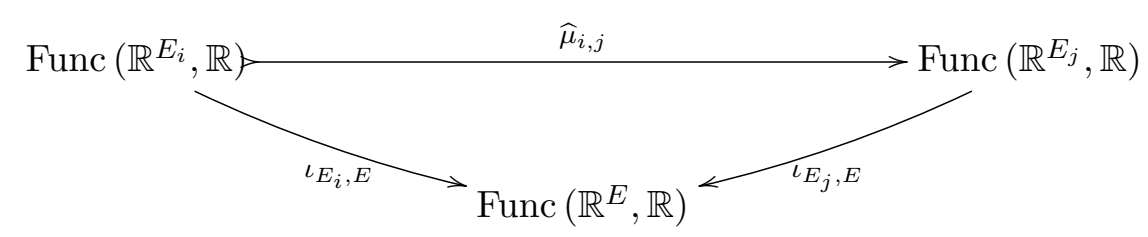

that is,

$$
\iota_{E_{j}, E} \circ \widehat{\mu}_{i, j}=\iota_{E_{i}, E}
$$

Consider the following commutative diagram: 


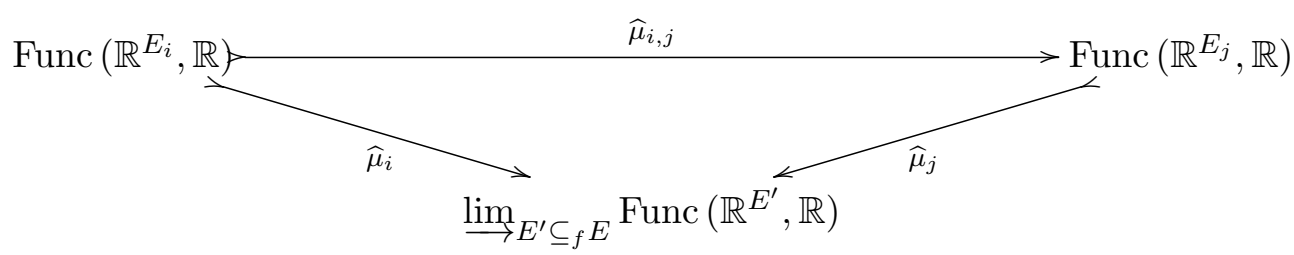

which is the colimit diagram of the directed system $\left(\right.$ Func $\left.\left(\mathbb{R}^{E_{i}}, \mathbb{R}\right), \widehat{\mu}_{i, j}\right)$.

Recall that, concretely we have:

$$
\underset{E^{\prime} \subseteq_{f} E}{\lim _{P}} \operatorname{Func}\left(\mathbb{R}^{E^{\prime}}, \mathbb{R}\right)=\frac{\bigcup_{E_{i} \subseteq_{f} E} \operatorname{Func}\left(\mathbb{R}^{E_{i}}, \mathbb{R}\right) \times\left\{E_{i}\right\}}{\sim}
$$

where

$$
\left(f_{i}, E_{i}\right) \sim\left(f_{j}, E_{j}\right) \Longleftrightarrow\left(\exists E_{k} \subseteq f E\right)\left(E_{i} \subseteq E_{k}\right)\left(E_{j} \subseteq E_{k}\right)\left(\widehat{\mu}_{i, k}\left(f_{i}\right)=\widehat{\mu}_{j, k}\left(f_{j}\right)\right)
$$

Given the cone (1.7), the universal property of the colimit yields a unique function

$$
u: \underset{E^{\prime} \subseteq_{f} E}{\lim _{\longrightarrow}} \operatorname{Func}\left(\mathbb{R}^{E^{\prime}}, \mathbb{R}\right) \rightarrow \operatorname{Func}\left(\mathbb{R}^{E}, \mathbb{R}\right)
$$

such that the following diagram commutes:

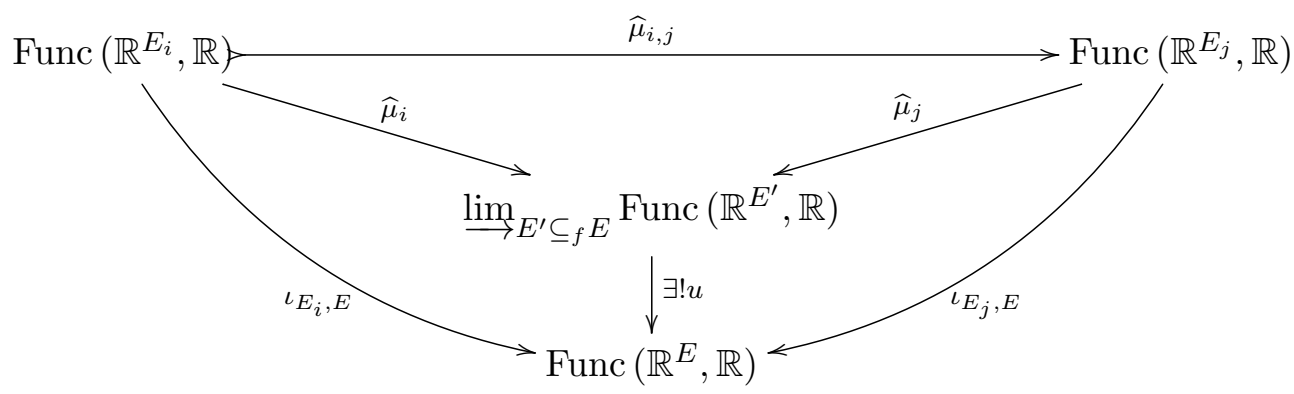

Now we claim that $u$ is an injective function.

Given any $\left[\left(f_{i}, E_{i}\right)\right],\left[\left(f_{j}, E_{j}\right)\right] \in \underline{\lim }_{E^{\prime} \subseteq_{f} E}$ Func $\left(\mathbb{R}^{E^{\prime}}, \mathbb{R}\right)$ such that:

$$
u\left(\left[\left(f_{i}, E_{i}\right)\right]\right)=u\left(\left[\left(f_{j}, E_{j}\right)\right]\right),
$$

and since $\widehat{\mu}_{i}\left(f_{i}\right)=\left[\left(f_{i}, E_{i}\right)\right]$ and $\widehat{\mu}_{j}\left(f_{j}\right)=\left[\left(f_{j}, E_{j}\right)\right]$, this is equivalent to:

$$
\iota_{E_{i}, E}\left(f_{i}\right)=\iota_{E_{j}, E}\left(f_{j}\right)
$$

so:

$$
\left(\forall g \in \mathbb{R}^{E}\right)\left(\widetilde{f}_{i}(g)=\widetilde{f}_{j}(g)\right)
$$

i.e.,

$$
\left(\forall g \in \mathbb{R}^{E}\right)\left(f_{i}\left(g \circ \iota_{i}\right)=f_{j}\left(g \circ \iota_{j}\right)\right)
$$


Now, given any finite $E_{i}$ and $E_{j}$, we can take $E_{k} \subseteq E$ such that $E_{i} \subseteq E_{k}$ and $E_{j} \subseteq E_{k}$, namely $E_{k}=E_{i} \cup E_{j}$. We are going to show that $\widehat{\mu}_{i, k}\left(f_{i}\right)=\widehat{\mu}_{j, k}\left(f_{j}\right)$.

Given any $g: E_{i} \cup E_{j} \rightarrow \mathbb{R}$ consider:

$$
\begin{aligned}
& \check{g}: \quad E \rightarrow \begin{array}{c}
\mathbb{R} \\
x
\end{array} \\
& \mapsto\left\{\begin{array}{l}
g(x), \text { if } x \in E_{k} \\
0 \text { otherwise }
\end{array}\right.
\end{aligned}
$$

and note that $\check{g} \circ \iota_{i}=g \circ \imath_{i k}$ and $\check{g} \circ \iota_{j}=g \circ \imath_{j k}$.

Thus we have:

$$
\widehat{\mu}_{i, k}\left(f_{i}\right)(g)=f_{i}\left(g \circ \imath_{i k}\right)=f_{i}\left(\check{g} \circ \iota_{i}\right) \stackrel{1.8}{=} f_{j}\left(\check{g} \circ \iota_{j}\right)=f_{j}\left(g \circ \imath_{j k}\right)=\widehat{\mu}_{j, k}\left(f_{j}\right)(g)
$$

Since $g$ is arbitrary, we have:

$$
\widehat{\mu}_{i, k}\left(f_{i}\right)=\widehat{\mu}_{j, k}\left(f_{j}\right)
$$

and

$$
\left[\left(f_{i}, E_{i}\right)\right]=\left[\left(f_{j}, E_{j}\right)\right]
$$

so $u$ is injective.

Therefore we can identify $\underline{\lim }_{E^{\prime} \subseteq_{f}}$ Func $\left(\mathbb{R}^{E^{\prime}}, \mathbb{R}\right)$ with a subset of Func $\left(\mathbb{R}^{E}, \mathbb{R}\right)$, namely:

$$
u\left[\underset{E^{\prime} \subseteq_{f} E}{\underset{\lim }{\longrightarrow}} \operatorname{Func}\left(\mathbb{R}^{E^{\prime}}, \mathbb{R}\right)\right] .
$$

In this sense, we say that $\underline{\lim }_{E^{\prime} \subseteq_{f} E}$ Func $\left(\mathbb{R}^{E^{\prime}}, \mathbb{R}\right)$ consists of all functions $f: \mathbb{R}^{E} \rightarrow \mathbb{R}$ for which there are some finite $E^{\prime} \subseteq E$ and some $f^{\prime}: \mathbb{R}^{E^{\prime}} \rightarrow \mathbb{R}$ with $u\left(\left[\left(f^{\prime}, E^{\prime}\right)\right]\right)=f$. As an "abus de langage", one says that $\lim _{E^{\prime} \subseteq_{f} E}$ Func $\left(\mathbb{R}^{E^{\prime}}, \mathbb{R}\right)$ is the set of all functions $f: \mathbb{R}^{E^{\prime}} \rightarrow \mathbb{R}$ for some $E^{\prime} \subseteq_{f} E$.

Now we proceed to describe the free $\mathcal{C}^{\infty}$-ring determined by an arbitrary set $E$.

Given two finite subsets of $E, E_{i}, E_{j} \subseteq E$, we set $\widehat{\mu}_{i, j}\left\lceil_{\mathcal{C}^{\infty}\left(\mathbb{R}^{\left.E_{i}\right)}\right.}: \mathcal{C}^{\infty}\left(\mathbb{R}^{E_{i}}\right) \longmapsto \mathcal{C} \infty\left(\mathbb{R}^{E_{j}}\right)\right.$

$$
\begin{array}{rlllll}
\widehat{\mu}_{i, j}\left\lceil_{\mathcal{C}^{\infty}\left(\mathbb{R}^{\left.E_{i}\right)}:\right.}: \mathcal{C}^{\infty}\left(\mathbb{R}^{E_{i}}\right)\right. & \rightarrow & & \mathcal{C}^{\infty}\left(\mathbb{R}^{E_{j}}\right) \\
\left(\mathbb{R}^{E_{i}} \stackrel{\widehat{g}}{\rightarrow} \mathbb{R}\right) & \mapsto & \widehat{g} \circ \mu_{i, j}: & \mathbb{R}^{E_{j}} & \rightarrow & \mathbb{R} \\
& & f & \mapsto & & \mapsto\left(f \circ \imath_{i j}: \mathbb{R}^{E_{i}} \rightarrow \mathbb{R}\right)
\end{array}
$$

to be the restriction of the map $\widehat{\mu}_{i, j}$ to $\mathcal{C}^{\infty}\left(\mathbb{R}^{E_{i}}\right)$, so the following rectangle commutes

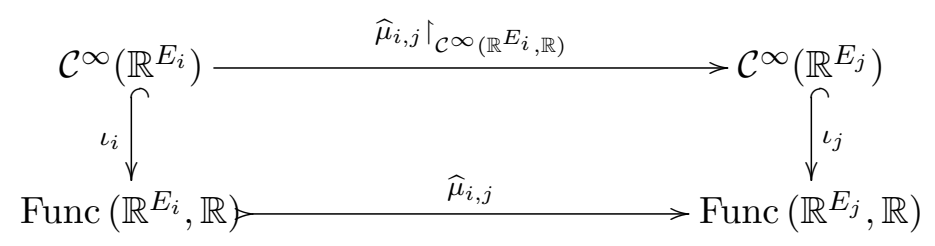


and consider the following colimit diagram in Set:

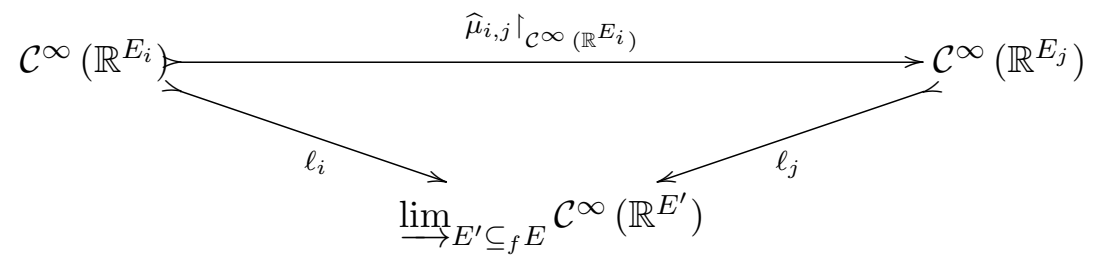

Recall that:

$$
\underset{\lim ^{\prime} \subseteq_{f} E}{\mathcal{C}^{\infty}}\left(\mathbb{R}^{E^{\prime}}\right)=\frac{\bigcup_{E_{i} \subseteq_{f} E} \mathcal{C}^{\infty}\left(\mathbb{R}^{E_{i}}\right) \times\left\{E_{i}\right\}}{\sim}
$$

where

$$
\left(f_{i}, E_{i}\right) \sim\left(f_{j}, E_{j}\right) \Longleftrightarrow\left(\exists E_{k} \subseteq f E\right)\left(E_{i} \subseteq E_{k}\right)\left(E_{j} \subseteq E_{k}\right)\left(\widehat{\mu}_{i, k} \Upsilon_{\mathcal{C}^{\infty}\left(\mathbb{R}^{\left.E_{i}\right)}\right.}\left(f_{i}\right)=\widehat{\mu}_{j, k} \Upsilon_{\mathcal{C}^{\infty}\left(\mathbb{R}^{E_{j}}\right)}\left(f_{j}\right)\right)
$$

so:

$$
\begin{array}{rlll}
\ell_{i}: & \mathcal{C}^{\infty}\left(\mathbb{R}^{E_{i}}\right) & \rightarrow & \lim _{E^{\prime} \subseteq_{f} E} \mathcal{C}^{\infty}\left(\mathbb{R}^{E^{\prime}}\right) \\
\left(\mathbb{R}^{E_{i}} \stackrel{f_{i}}{\rightarrow} \mathbb{R}\right) & \mapsto & {\left[\left(f_{i}, E_{i}\right)\right]}
\end{array}
$$

We now claim that $\widehat{\mu}_{i, j} \Upsilon_{\mathcal{C}^{\infty}\left(\mathbb{R}^{\left.E_{i}\right)}\right.}:\left(\mathcal{C}^{\infty}\left(\mathbb{R}^{E_{i}}\right), \Phi_{E_{i}}\right) \rightarrow\left(\mathcal{C}^{\infty}\left(\mathbb{R}^{E_{j}}\right), \Phi_{E_{j}}\right)$ is a $\mathcal{C}^{\infty}$-homomorphism.

Given any $f \in \mathcal{C}^{\infty}\left(\mathbb{R}^{n}, \mathbb{R}\right)$, we are going to show that the following diagram commutes:

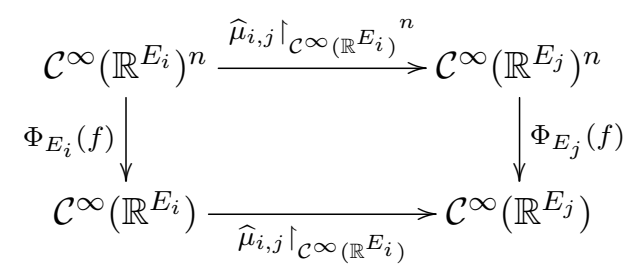

Let $\left(\widehat{g}_{1}, \cdots, \widehat{g}_{n}\right) \in \mathcal{C}^{\infty}\left(\mathbb{R}^{E_{i}}\right)^{n}$. On the one hand we have:

$$
\begin{gathered}
\Phi_{E_{j}}(f) \circ \widehat{\mu}_{i, j}\left\lceil_{\mathcal{C}^{\infty}\left(\mathbb{R}^{\left.E_{i}\right)}\right.}^{n}\left(\widehat{g}_{1}, \cdots, \widehat{g}_{n}\right)=\Phi_{E_{j}}(f)\left(\widehat{\mu}_{i, j}\left\lceil_{\mathcal{C}^{\infty}\left(\mathbb{R}^{\left.E_{i}\right)}\right.}\left(\widehat{g}_{1}\right), \cdots, \widehat{\mu}_{i, j}\left\lceil_{\mathcal{C}^{\infty}\left(\mathbb{R}^{\left.E_{i}\right)}\right.}\left(\widehat{g}_{n}\right)\right)\right)=\right.\right. \\
=\Phi_{E_{j}}(f)\left(\widehat{g}_{1} \circ \mu_{i j}, \cdots, \widehat{g}_{n} \circ \mu_{i, j}\right)
\end{gathered}
$$

and on the other hand we have:

$$
\begin{gathered}
\widehat{\mu}_{i, j}\left\lceil_{\mathcal{C} \infty\left(\mathbb{R}^{E_{i}}\right)} \circ \Phi_{E_{i}}(f)\left(\widehat{g}_{1}, \cdots, \widehat{g}_{n}\right)=\widehat{\mu}_{i, j}\left\lceil_{\mathcal{C}^{\infty}\left(\mathbb{R}^{\left.E_{i}\right)}\right.}\left(f \circ\left(\widehat{g_{1}, \cdots}, g_{n}\right)\right)=\right.\right. \\
=\left(f \circ\left(\widehat{g_{1}, \cdots}, g_{n}\right)\right) \circ \mu_{i, j}
\end{gathered}
$$

Given any $h \in \mathbb{R}^{E_{j}}$, we have, on the one side:

$$
\begin{gathered}
\Phi_{E_{j}}(f)\left(\widehat{g}_{1} \circ \mu_{i j}, \cdots, \widehat{g}_{n} \circ \mu_{i, j}\right)(h)=\Phi_{E_{j}}(f)\left(\widehat{g}_{1}\left(h \circ \imath_{i j}\right), \cdots, \widehat{g}_{n}\left(h \circ \imath_{i, j}\right)\right)= \\
=\Phi_{E_{j}}(f)\left(\widehat{g}_{1}, \cdots, \widehat{g}_{n}\right)\left(h \circ \imath_{i j}\right)=f \circ\left(\widehat{g_{1}, \cdots}, g_{n}\right)\left(h \circ \imath_{i j}\right)
\end{gathered}
$$


and on the other hand:

$$
\begin{gathered}
{\left[\widehat{\mu}_{i j}\left\lceil_{\mathcal{C}^{\infty}\left(\mathbb{R}^{\left.E_{i}\right)}\right.} \circ \Phi_{E_{i}}(f)\left(\widehat{g}_{1}, \cdots, \widehat{g}_{n}\right)\right](h)=\left[\widehat{\mu}_{i j}\left\lceil_{\mathcal{C}^{\infty}\left(\mathbb{R}^{\left.E_{i}\right)}\right.} \circ\left(f \circ\left(\widehat{g_{1}, \cdots}, g_{n}\right)\right)\right](h)=\right.\right.} \\
=\left[f \circ\left(\widehat{g_{1}, \cdots}, g_{n}\right) \circ \mu_{i j}\right](h)=f \circ\left(\widehat{g_{1}, \cdots}, g_{n}\right)\left(h \circ \imath_{i j}\right)
\end{gathered}
$$

so $\widehat{\mu}_{i j}\left\lceil_{\mathcal{C}^{\infty}\left(\mathbb{R}^{E_{i}}\right)}\right.$ is a $\mathcal{C}^{\infty}$-homomorphism.

We have, thus, the directed system $\left(\left(\mathcal{C}^{\infty}\left(\mathbb{R}^{E_{i}}\right), \Phi_{E_{i}}\right), \widehat{\mu}_{i j} \uparrow_{\mathcal{C}^{\infty}\left(\mathbb{R}^{\left.E_{i}\right)}\right.}\right)$ of $\mathcal{C}^{\infty}$-rings, and the following commutative diagrams (for any $E_{i}, E_{j} \subseteq_{f} E$ ) in $\mathcal{C}^{\infty} \mathbf{R n g}$ :

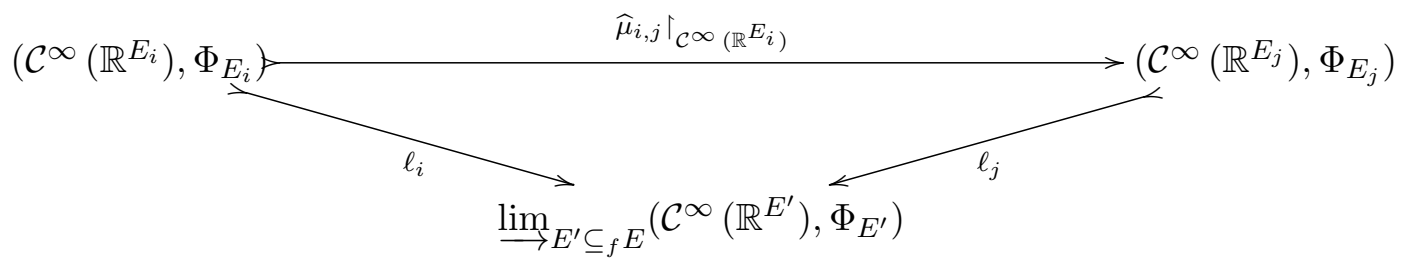

Note that each $\ell_{i}:\left(\mathcal{C}^{\infty}\left(\mathbb{R}^{E_{i}}\right), \Phi_{E_{i}}\right) \rightarrow \underline{\lim }_{E^{\prime} \subseteq_{f} E}\left(\mathcal{C}^{\infty}\left(\mathbb{R}^{E^{\prime}}\right), \Phi_{E^{\prime}}\right)$ is a $\mathcal{C}^{\infty}$-homomorphism.

Let us examine this colimit more closely. We denote:

$$
\mathcal{C}^{\infty}\left(\mathbb{R}^{E}\right)=\underset{E^{\prime} \underline{\lim }_{f}}{\lim } \mathcal{C}^{\infty}\left(\mathbb{R}^{E^{\prime}}\right)
$$

and describe the $\mathcal{C}^{\infty}$-structure on $\mathcal{C}^{\infty}\left(\mathbb{R}^{E}\right)$,

$$
\begin{aligned}
& \Phi_{E}: \bigcup_{n \in \mathbb{N}} \mathcal{C}^{\infty}\left(\mathbb{R}^{n}, \mathbb{R}\right) \rightarrow \bigcup_{n \in \mathbb{N}} \operatorname{Func}\left(\mathcal{C}^{\infty}\left(\mathbb{R}^{E}\right)^{n}, \mathcal{C}^{\infty}\left(\mathbb{R}^{E}\right)\right) \\
& \left(\mathbb{R}^{n} \stackrel{f}{\rightarrow} \mathbb{R}\right) \quad \mapsto \quad \Phi_{E}(f): \mathcal{C}^{\infty}\left(\mathbb{R}^{E}\right)^{n} \rightarrow \mathcal{C}^{\infty}\left(\mathbb{R}^{E}\right)
\end{aligned}
$$

where:

$$
\begin{array}{ccc}
\Phi_{E}(f): & \mathcal{C}^{\infty}\left(\mathbb{R}^{E}\right)^{n} & \mathcal{C}^{\infty}\left(\mathbb{R}^{E}\right) \\
\left(\left[\left(\widehat{f}_{1}, E_{1}\right)\right], \cdots,\left[\left(\widehat{f}_{n}, E_{n}\right)\right]\right) & \mapsto & {\left[\left(f \circ\left(\widehat{\mu}_{1 k}\left\lceil_{\mathcal{C}^{\infty}\left(\mathbb{R}^{\left.E_{1}\right)}\right.}\left(\widehat{f}_{1}\right), \cdots, \widehat{\mu}_{n k} \uparrow_{\mathcal{C}^{\infty}\left(\mathbb{R}^{E_{n}}\right)}\left(\widehat{f}_{n}\right)\right), E_{k}\right)\right]\right.}
\end{array}
$$

and $E_{k}=\cup_{i=1}^{n} E_{i}$. We have thus:

$$
f \circ\left(\widehat { \mu } _ { 1 k } \left\lceil_{\mathcal{C}^{\infty}\left(\mathbb{R}^{\left.E_{1}\right)}\right.}\left(\widehat{f}_{1}\right), \cdots, \widehat{\mu}_{n k}\left\lceil_{\mathcal{C}^{\infty}\left(\mathbb{R}^{\left.E_{n}\right)}\right.}\left(\widehat{f}_{n}\right)\right): \mathbb{R}^{E_{k}} \rightarrow \mathbb{R}\right.\right.
$$

which belongs to $\mathcal{C}^{\infty}\left(\mathbb{R}^{E_{k}}\right)$, since $\widehat{\mu}_{1 k} \Upsilon_{\mathcal{C}^{\infty}\left(\mathbb{R}^{\left.E_{1}\right)}\right.}\left(\widehat{f}_{1}\right), \cdots, \widehat{\mu}_{n k} \Upsilon_{\mathcal{C}^{\infty}\left(\mathbb{R}^{E_{n}}\right)}\left(\widehat{f}_{n}\right) \in \mathcal{C}^{\infty}\left(\mathbb{R}^{E_{k}}\right)$ and $f \in$ $\mathcal{C}^{\infty}\left(\mathbb{R}^{n}\right)$.

Thus we have the $\mathcal{C}^{\infty}$-ring:

$$
\left(\mathcal{C}^{\infty}\left(\mathbb{R}^{E}\right), \Phi_{E}\right)
$$

Let $E$ be any set, and write 


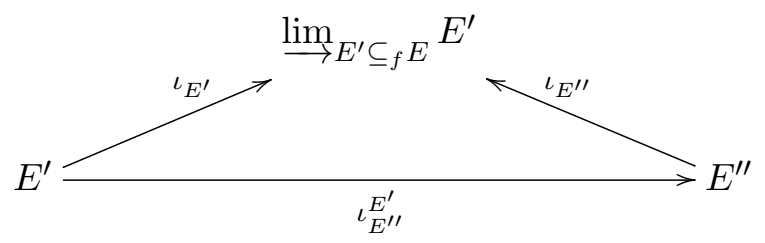

For every finite subset $E^{\prime} \subseteq_{f} E$, we have the free $\mathcal{C}^{\infty}$-ring:

$$
\jmath_{E^{\prime}}: E^{\prime} \rightarrow U\left(\mathcal{C}^{\infty}\left(\mathbb{R}^{E^{\prime}}\right), \Phi_{E^{\prime}}\right)
$$

so we can form the commutative cone:

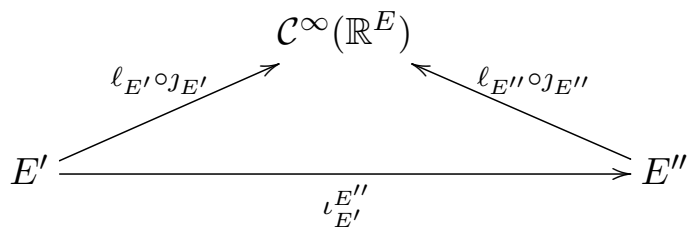

The universal property of $\lim _{E^{\prime} \subseteq_{f} E} E^{\prime}$ yields a unique function $\jmath_{E}: \varliminf_{\lim ^{\prime} \subseteq_{f} E} E^{\prime} \rightarrow \mathcal{C}^{\infty}\left(\mathbb{R}^{E}\right)$ such that the following prism commutes in Set:

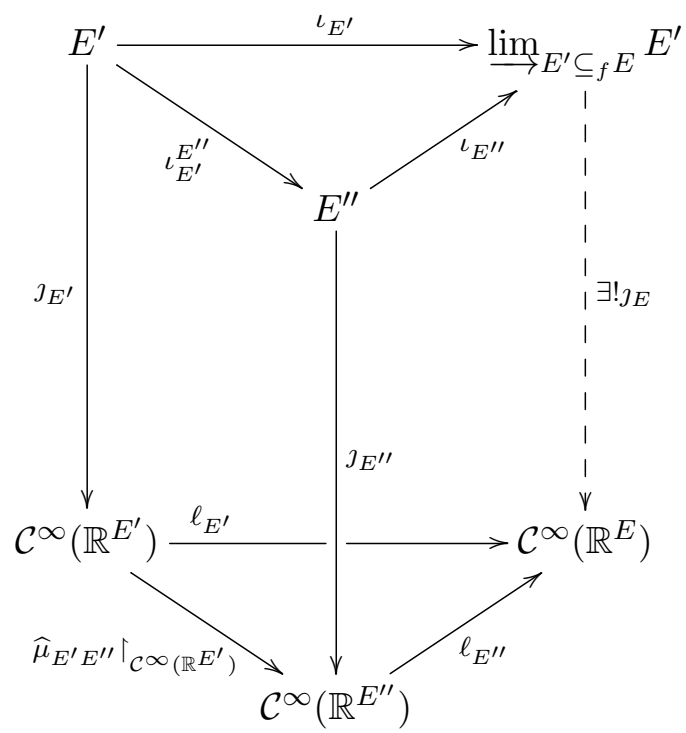

In Set we have:

$$
\underset{E^{\prime} \subseteq_{f} E}{\lim _{E^{\prime} \subseteq_{f} E}} E^{\prime} \cong \bigcup^{\prime} E^{\prime}=E
$$

so we have the function $\jmath_{E}: E \rightarrow \mathcal{C}^{\infty}\left(\mathbb{R}^{E}\right)$.

Remark 1.6.1. Given a set $E$, for every finite subset $E^{\prime} \subseteq_{f} E$, with $\sharp E^{\prime}=n$, there is a bijection $\omega_{n}:\{1, \cdots, n\} \rightarrow E^{\prime}$ and a corresponding $\mathcal{C}^{\infty}$-isomorphism:

$$
\widehat{\omega_{n}}:\left(\mathcal{C}^{\infty}\left(\mathbb{R}^{E^{\prime}}\right), \Phi_{E^{\prime}}\right) \rightarrow\left(\mathcal{C}^{\infty}\left(\mathbb{R}^{n}\right), \Omega\right)
$$

Also, given $E^{\prime \prime} \subseteq_{f} E$ and a bijection $\omega_{m}:\{1, \cdots, m\} \rightarrow E^{\prime \prime}$, with $E^{\prime} \subseteq E^{\prime \prime}$ (so with $n \leq m$ ) we have the composite: 


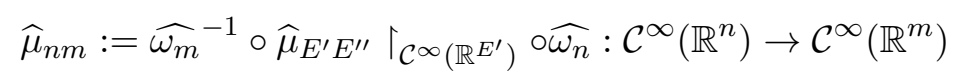

so the following rectangle commutes:

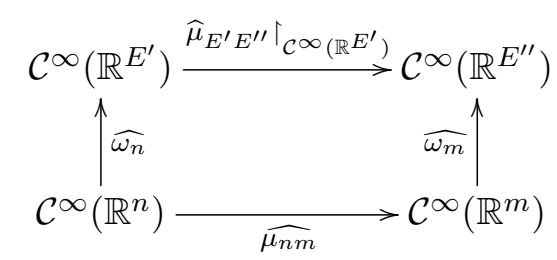

Considering $E_{i} \subseteq E_{j}$ and the inclusion maps $\iota_{i}: \mathcal{C}^{\infty}\left(\mathbb{R}^{E_{i}}\right) \hookrightarrow \operatorname{Func}\left(\mathbb{R}^{E_{i}}\right)$, we have the following commutative diagram:

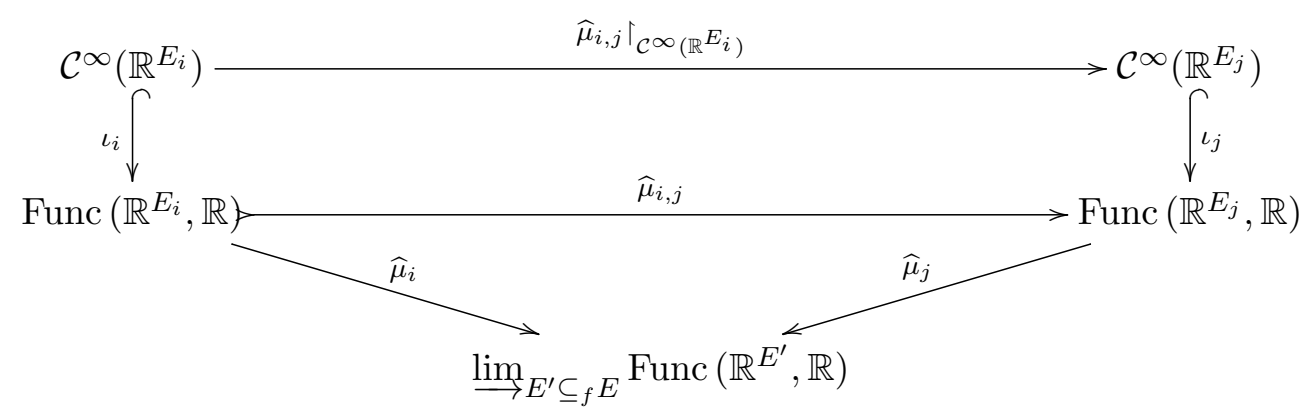

By the universal property of $\lim _{E_{i} \subseteq_{f} E} \mathcal{C}^{\infty}\left(\mathbb{R}^{E_{i}}, \mathbb{R}\right)$, in the category Set, given the cone:

$$
\left(\widehat{\mu}_{i} \circ \iota_{i}: \mathcal{C}^{\infty}\left(\mathbb{R}^{E_{i}}, \mathbb{R}\right) \rightarrow \underset{E^{\prime} \underline{\lim }_{E}}{\operatorname{lin}} \operatorname{Func}\left(\mathbb{R}^{E^{\prime}}, \mathbb{R}\right)\right),
$$

there is a unique function $v: \underline{\lim }_{E^{\prime} \subseteq_{f} E} \mathcal{C}^{\infty}\left(\mathbb{R}^{E^{\prime}}, \mathbb{R}\right) \rightarrow \underline{\lim }_{E^{\prime} \subseteq_{f} E}$ Func $\left(\mathbb{R}^{E^{\prime}}, \mathbb{R}\right)$ such that the following prism commutes:

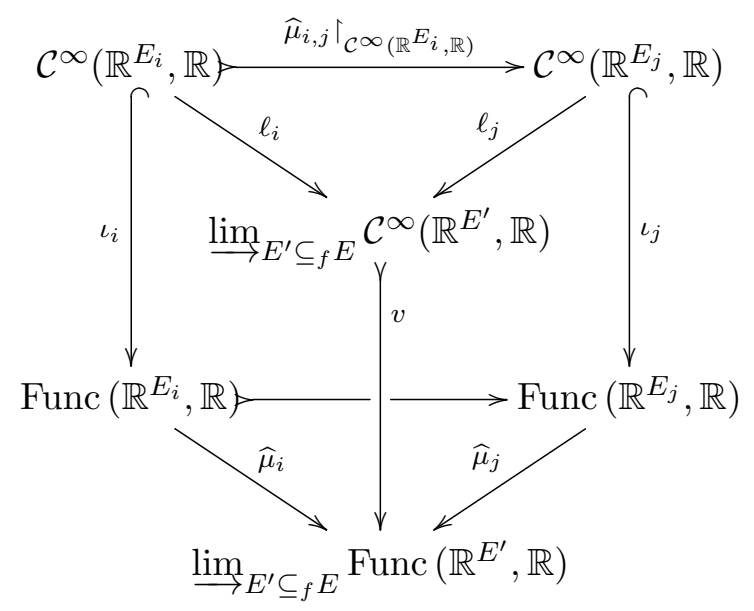

where $\ell_{i}: \mathcal{C}^{\infty}\left(\mathbb{R}^{E_{i}}, \mathbb{R}\right) \rightarrow \lim _{E^{\prime} \subseteq E} \mathcal{C}^{\infty}\left(\mathbb{R}^{E^{\prime}}, \mathbb{R}\right)$ are the canonic colimit arrows.

We claim that $v=\iota: \varliminf_{\lim _{E^{\prime}} \subseteq_{f}} \mathcal{C}^{\infty}\left(\mathbb{R}^{E^{\prime}}, \mathbb{R}\right) \hookrightarrow \underline{\lim }_{E^{\prime} \subseteq_{f} E}$ Func $\left(\mathbb{R}^{E^{\prime}}, \mathbb{R}\right)$ (the inclusion).

Given $f \in \lim _{E^{\prime} \subseteq_{f} E} \mathcal{C}^{\infty}\left(\mathbb{R}^{E^{\prime}}, \mathbb{R}\right)$, there is some $E_{k} \subseteq_{f} E$ and some $f_{k} \in \mathcal{C}^{\infty}\left(\mathbb{R}^{E_{k}}, \mathbb{R}\right)$ such that:

$$
f=\left[\left(f_{k}, E_{k}\right)\right]=\ell_{k}\left(f_{k}\right)
$$


We have:

$$
v(f)=v\left(\ell_{k}\left(f_{k}\right)\right)=\widehat{\mu}_{k} \circ \iota_{k}(f)=\widehat{\mu}_{k}(f)=\left[\left(f_{k}, E_{k}\right)\right]=f
$$

So we have $\lim _{E^{\prime} \subseteq_{f} E} \mathcal{C}^{\infty}\left(\mathbb{R}^{E^{\prime}}, \mathbb{R}\right) \subseteq \lim _{E^{\prime} \subseteq_{f} E} \operatorname{Func}\left(\mathbb{R}^{E^{\prime}}, \mathbb{R}\right) \longmapsto \operatorname{Func}\left(\mathbb{R}^{E}, \mathbb{R}\right)$.

Hence, we can regard $\lim _{E^{\prime} \subseteq_{f} E} \mathcal{C}^{\infty}\left(\mathbb{R}^{E^{\prime}}, \mathbb{R}\right)$ as (an actual) subset of $\lim _{E^{\prime} \subseteq_{f} E}$ Func $\left(\mathbb{R}^{E^{\prime}}, \mathbb{R}\right)$, and consider, as in 65, $\left(\mathcal{C}^{\infty}\left(\mathbb{R}^{E}\right), \Phi_{E}\right)$ as the ring of functions $\mathbb{R}^{E} \rightarrow \mathbb{R}$ which smoothly depend on finitely many variables only. 


\section{Chapter 2}

\section{Topics on Smooth Commutative Algebra}

In this chapter we study $\mathcal{C}^{\infty}$-rings mainly from the point of view of "Commutative Algebra", looking for results similar to those one finds in the latter. We present the analog concepts of radical ideal $\left(\mathcal{C}^{\infty}\right.$-radical ideal), rings of fractions ( $\mathcal{C}^{\infty}$-rings of fractions), local rings (local $\mathcal{C}^{\infty}$-rings), reduced rings $\left(\mathcal{C}^{\infty}\right.$-reduced $\mathcal{C}^{\infty}$-rings) and some other concepts, such as a von Neumann regular $\mathcal{C}^{\infty}$-ring.

We are going to see many similarities between Smooth Commutative Algebra and Ordinary Commutative Algebra, and also some of their differences. For example, the notion of " $\mathcal{C}^{\infty}$-radical ideal", found in [66], which is appropriate to the theory of $\mathcal{C}^{\infty}$-rings, differs from the one given in ordinary Commutative Algebra - which makes use only of powers of elements. We present an example of an ideal of a $\mathcal{C}^{\infty}$-ring which is radical in the ordinary sense (since it is prime) but that is not $\mathcal{C}^{\infty}$-radical (see Remark 2.2.32.

The difference between the notions of "radical" and " $\mathcal{C}^{\infty}$-radical" ideals brings us a whole new study of some important concepts, such as "smooth saturation", $\mathcal{C}^{\infty}$-reduced $\mathcal{C}^{\infty}$-rings, "smooth Zariski spectrum" an so on. We are going to see, for example, that the smooth version of the Zariski spectrum presents some crucial differences when compared to the ordinary Zariski spectrum.

We begin this chapter by giving a description of the fundamental concept of "smooth ring of fractions", presenting the axioms given in [66]. Also in this chapter, we are going to state and prove a stronger version of the Theorem 1.4 of [66], characterizing every $\mathcal{C}^{\infty}$-ring of fractions.

From this chapter on, we are going to write " $A$ " instead of $(A, \Phi)$, partly to simplify the notation and partly because the $\mathcal{C}^{\infty}$-structure will not play an important role as it did in the previous chapter.

\subsection{Smooth Ring of Fractions}

We now turn to the discussion of how to obtain the ring of fractions of a $\mathcal{C}^{\infty}$-ring $A$ with respect to some of its subsets, $S \subseteq U(A, \Phi)$. Recall that for a commutative ring $\left(R,+_{R}, \cdot_{R},-{ }_{R}, 1_{R}\right)$ and a multiplicative subset $S \subseteq R$, the ring of fractions is obtained by defining an equivalence relation on $R \times S$, 三, given by:

$$
\left(\left(r_{1}, s_{1}\right) \sim\left(r_{2}, s_{2}\right)\right) \Longleftrightarrow(\exists u \in S)\left(u \cdot\left(r_{1} \cdot s_{2}-r_{2} \cdot s_{1}\right)=0\right) .
$$

We denote

$$
S^{-1} R \doteq \frac{R \times S}{\sim}=\left\{[(r, s)] \doteq \frac{r}{s} \mid(r, s) \in R \times S\right\}
$$

and we put a ring structure on it by defining the addition and the multiplication of these fractions in 
the same way as in Elementary Algebra, that is:

$$
\begin{aligned}
& +: \quad S^{-1} R \times S^{-1} R \quad \rightarrow \quad S^{-1} R \\
& \left(\left[\left(r_{1}, s_{1}\right)\right],\left[\left(r_{2}, s_{2}\right)\right]\right) \mapsto\left[\left(r_{1} \cdot R s_{2}+{ }_{R} r_{2} \cdot R s_{1}, s_{1} \cdot R s_{2}\right)\right] \\
& \text {. : } \quad S^{-1} R \times S^{-1} R \quad \rightarrow \quad S^{-1} R \\
& \left(\left[\left(r_{1}, s_{1}\right)\right],\left[\left(r_{2}, s_{2}\right)\right]\right) \mapsto\left[\left(r_{1} \cdot R r_{2}, s_{1} \cdot R s_{2}\right)\right] \\
& \text { - : } \quad S^{-1} R \quad \rightarrow \quad S^{-1} R \\
& {\left[\left(r_{1}, s_{1}\right)\right] \mapsto\left[\left(-{ }_{R} r_{1}, s_{1}\right)\right]}
\end{aligned}
$$

Together with the element $\left[\left(1_{R}, 1_{R}\right)\right],\left(S^{-1} R,+_{R}, \cdot_{R},\left[\left(1_{R}, 1_{R}\right)\right]\right)$ is a commutative unital ring. We also have a canonical ring homomorphism that "embeds" $R$ into $S^{-1} R$ in such a way that the image of every element of $S$ is invertible, namely:

$$
\begin{aligned}
\eta_{S}: \quad R & \rightarrow S^{-1} R \\
r & \mapsto[(r, 1)]
\end{aligned}
$$

We can prove that $S^{-1} R$ has the following universal property (cf. Proposition 3.1 of [3]):

Proposition 2.1.1. Given a ring homomorphism $g: R \rightarrow B$ such that $(\forall s \in S)\left(g(s) \in B^{\times}\right)$, there is a unique ring homomorphism $\widetilde{g}: S^{-1} R \rightarrow B$ such that the following triangle commutes:

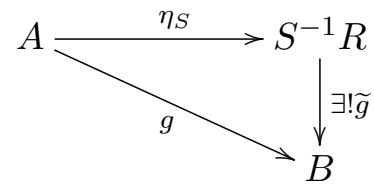

In order to extend the notion of the ring of fractions to the category $\mathcal{C}^{\infty} \mathbf{R n g}$ we make use of the universal property described in Proposition 2.1.1, as we can see in the following:

Definition 2.1.2. Let $A$ be $a \mathcal{C}^{\infty}$-ring and $S \subseteq A$ one of its subsets. The $\mathcal{C}^{\infty}$-ring of fractions of $A$ with respect to $S$ is a $\mathcal{C}^{\infty}$-ring together with a $\mathcal{C}^{\infty}$-homomorphism $\eta_{S}: A \rightarrow A\left\{S^{-1}\right\}$ with the following properties:

(1) $(\forall s \in S)\left(\eta_{S}(s) \in\left(A\left\{S^{-1}\right\}\right)^{\times}\right)$

(2) If $\varphi: A \rightarrow B$ is any $\mathcal{C}^{\infty}$-homomorphism such that for every $s \in S$ we have $\varphi(s) \in B^{\times}$, then there is a unique $\mathcal{C}^{\infty}$-homomorphism $\widetilde{\varphi}: A\left\{S^{-1}\right\} \rightarrow B$ such that the following triangle commutes:

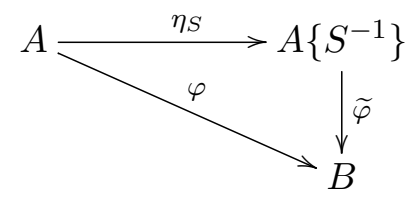

By this universal property, the $\mathcal{C}^{\infty}$-ring of fractions is unique, up to (unique) isomorphisms.

Now we prove the existence of such a $\mathcal{C}^{\infty}$-ring of fractions by constructing it. Recall that we can only make use of the constructions available within the category $\mathcal{C}^{\infty} \mathbf{R n g}$, such as the free $\mathcal{C}^{\infty}$-ring on a set of generators, their coproduct, their quotients and others described in Chapter 1.

Recall that given any set $S$, the $\mathcal{C}^{\infty}$-ring of "smooth polynomials" in the set $S$ of variables over a $\mathcal{C}^{\infty}$-ring $A$ is obtained as follows: 
We consider $\mathcal{C}^{\infty}\left(\mathbb{R}^{S}\right)$, the free $\mathcal{C}^{\infty}$-ring on the set $S$ of generators, together with its canonical map, $\jmath_{S}: S \rightarrow \mathcal{C}^{\infty}\left(\mathbb{R}^{S}\right)$. If we denote by:

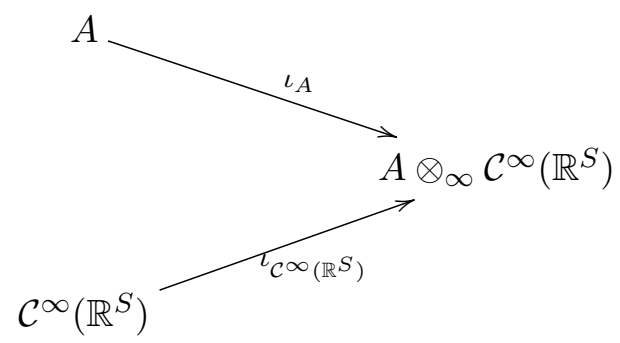

the coproduct of $A$ and $\mathcal{C}^{\infty}\left(\mathbb{R}^{S}\right)$, we define:

$$
x_{s}:=\iota_{\mathcal{C}^{\infty}\left(\mathbb{R}^{S}\right)}\left(\jmath_{S}(s)\right) .
$$

By definition, we have:

$$
A\left\{x_{s} \mid s \in S\right\}:=A \otimes_{\infty} \mathcal{C}^{\infty}\left(\mathbb{R}^{S}\right)
$$

so we can consider the quotient:

$$
\frac{A\left\{x_{s} \mid s \in S\right\}}{\left\langle\left\{x_{s} \cdot \iota_{A}(s)-1 \mid s \in S\right\}\right\rangle},
$$

where $\left\langle\left\{x_{s} \cdot \iota_{A}(s)-1 \mid s \in S\right\}\right\rangle$ is the ideal of $A$ generated by $\left\{x_{s} \cdot \iota_{A}(s)-1 \mid s \in S\right\}$.

In these settings we can formulate the following:

Theorem 2.1.3. Let $A$ be a $\mathcal{C}^{\infty}$-ring and $S \subseteq A$ be any of its subsets. The $\mathcal{C}^{\infty}$-ring of fractions of $A$ with respect to $S$ is given concretely by the $\mathcal{C}^{\infty}$-ring:

$$
A\left\{S^{-1}\right\}:=\frac{A\left\{x_{s} \mid s \in S\right\}}{\left\langle\left\{x_{s} \cdot \iota_{A}(s)-1 \mid s \in S\right\}\right\rangle}
$$

together with the $\mathcal{C}^{\infty}$-homomorphism:

$$
\eta_{S}:=q \circ \iota_{A}: A \rightarrow A\left\{S^{-1}\right\}
$$

where $q: A\left\{x_{s} \mid s \in S\right\} \rightarrow \frac{A\left\{x_{s} \mid s \in S\right\}}{\left\langle\left\{x_{s} \cdot \iota_{A}(s)-1 \mid s \in S\right\}\right\rangle}$ is the canonical quotient map and $\iota_{A}: A \rightarrow A\left\{x_{s} \mid s \in\right.$ $S\}=A \otimes_{\infty} \mathcal{C}^{\infty}\left(\mathbb{R}^{S}\right)$ is the canonical coproduct homomorphism corresponding to $A$.

Proof. We are going to show that $q \circ \iota_{A}: A \rightarrow \frac{A\left\{x_{s} \mid s \in S\right\}}{\left\langle\left\{x_{s} \cdot s-1\right\}\right\rangle}$ satisfies the universal property described in the Definition 2.1.2,

First we prove that for every $s \in S,\left(q \circ \imath_{A}\right)(s) \in\left(\frac{A\left\{x_{s} \mid s \in S\right\}}{\left\langle\left\{x_{s} \cdot s-1 \mid s \in S\right\}\right\rangle}\right)^{\times}$.

Given $s \in S$, we have that $x_{s}+\left\langle\left\{x_{s} \cdot \iota_{A}(s)-1 \mid s \in S\right\}\right\rangle$ is the multiplicative inverse of $\left(q \circ \imath_{A}\right)(s)=$ $q\left(\iota_{A}(s)\right)=\iota_{A}(s)+\left\langle\left\{x_{s} \cdot \iota_{A}(s)-1 \mid s \in S\right\}\right\rangle$, since $x_{s} \cdot \iota_{A}(s)-1 \in\left\langle\left\{x_{s} \cdot \iota_{A}(s)-1 \mid s \in S\right\}\right\rangle$. Thus $\eta_{S}(s)=\left(q \circ \imath_{A}\right)(s) \in\left(\frac{A\left\{x_{s} \mid s \in S\right\}}{\left\langle\left\{x_{s} \cdot \iota_{A}(s)-1 \mid s \in S\right\}\right\rangle}\right)^{\times}$. 
Now we need to check if the $\mathcal{C}^{\infty}$-homomorphism $q \circ \imath_{A}: A \rightarrow \frac{A\left\{x_{s} \mid s \in S\right\}}{\left\langle\left\{x_{s} \cdot s-1 \mid s \in S\right\}\right\rangle}$ satisfies the universal property described in the Definition 2.1.2

Let $B$ be a $\mathcal{C}^{\infty}$-ring and $g: A \rightarrow B$ be a $\mathcal{C}^{\infty}$-rings homomorphism such that for every $s \in S$, $g(s) \in B^{\times}$.

By the universal property of the free $\mathcal{C}^{\infty}$-ring with the set $S$ of generators, given the restriction of $g: A \rightarrow B$ to the function $g\left\lceil_{S}: S \rightarrow B\right.$ and considering the map:

$$
\begin{aligned}
& g_{S}^{-1}: S \rightarrow B \\
& s \mapsto g(s)^{-1}
\end{aligned}
$$

there is a unique $\mathcal{C}^{\infty}$-rings homomorphism $\widetilde{g_{S}^{-1}}: \mathcal{C}^{\infty}\left(\mathbb{R}^{S}\right) \rightarrow B$ such that the following diagram commutes:

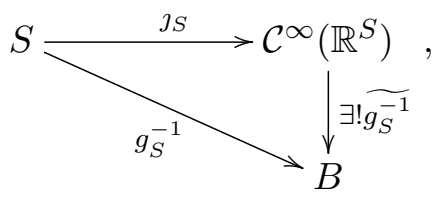

that is, $\widetilde{g_{S}^{-1}} \circ \jmath_{S}=g_{S}^{-1}$, or equivalently $(\forall s \in S)\left(g_{S}^{-1}\left(\jmath_{S}(s)\right)=g(s)^{-1}\right)$.

By the universal property of the coproduct, given the diagram:

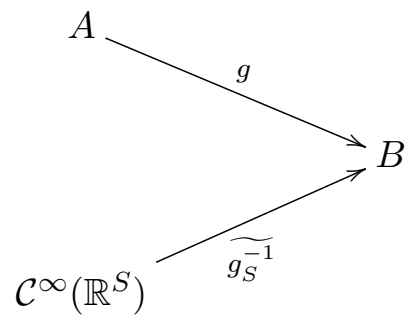

there is a unique $\mathcal{C}^{\infty}$-rings homomorphism, $\widetilde{g}: A \otimes_{\infty} \mathcal{C}^{\infty}\left(\mathbb{R}^{S}\right) \rightarrow B$ such that the following diagram commutes:

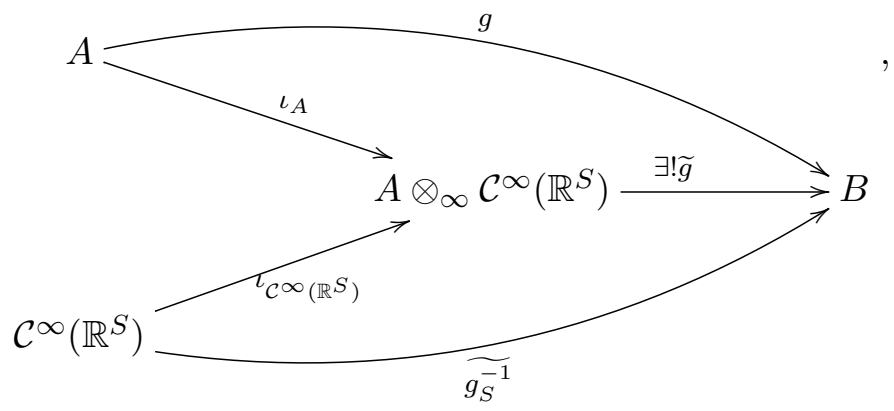

that is,

$$
\widetilde{g} \circ \iota_{\mathcal{C} \infty\left(\mathbb{R}^{S}\right)}=\widetilde{g} \uparrow_{S}
$$

and

$$
\widetilde{g} \circ \iota_{A}=g
$$

Note that such a $\widetilde{g}$ satisfies: 


$$
(\forall s \in S)\left(\widetilde{g}\left(x_{s}\right)=g(s)^{-1}\right),
$$

so for every $s \in S, \widetilde{g}\left(x_{s} \cdot \iota_{A}(s)-1\right)=\widetilde{g}\left(x_{s}\right) \cdot \widetilde{g}\left(\iota_{A}(s)\right)-\widetilde{g}(1)=g(s)^{-1} \cdot g(s)-1_{B}=0$ and $\left\langle\left\{x_{s} \cdot \iota_{A}(s)-1 \mid s \in S\right\}\right\rangle \subseteq \operatorname{ker} \widetilde{g}$.

Let $q: A \otimes_{\infty} \mathcal{C}^{\infty}\left(\mathbb{R}^{S}\right) \rightarrow \frac{A \otimes_{\infty} \mathcal{C}^{\infty}\left(\mathbb{R}^{S}\right)}{\left\langle\left\{x_{s} \cdot \iota_{A}(s)-1 \mid s \in S\right\}\right\rangle}$ be the canonical quotient map. Since $\left\langle\left\{x_{s} \cdot \iota_{A}(s)-\right.\right.$ $1 \mid s \in S\}\rangle \subseteq \operatorname{ker} \widetilde{g}$, by the Theorem of the Homomorphism, given the map $\widetilde{g}: A \otimes_{\infty} \mathcal{C}^{\infty}\left(\mathbb{R}^{S}\right) \rightarrow B$, there is a unique $\bar{g}: \frac{A \otimes_{\infty} \mathcal{C}^{\infty}\left(\mathbb{R}^{S}\right)}{\left\langle\left\{x_{s} \cdot \iota_{A}(s)-1 \mid s \in S\right\}\right\rangle} \rightarrow B$ such that the following diagram commutes:

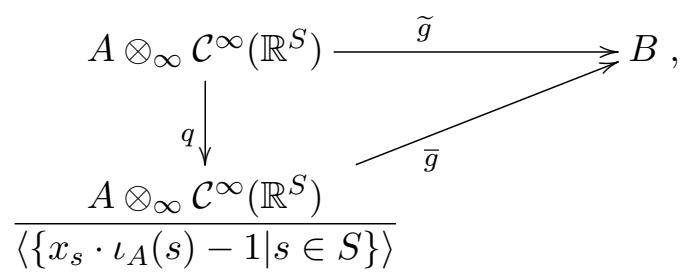

that is, such that:

$$
\bar{g} \circ q=\widetilde{g} .
$$

Note that since $\bar{g} \circ\left(q \circ \iota_{A}\right)=(\bar{g} \circ q) \circ \iota_{A} \stackrel{2.2]}{=} \widetilde{g} \circ \iota_{A} \stackrel{2.1]}{=} g$, the following diagram commutes:

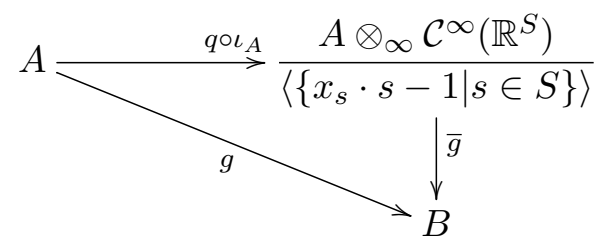

Moreover, the very construction of $\bar{g}$ tells us that it is the unique $\mathcal{C}^{\infty}$-homomorphism that makes the above diagram commute.

Thus, the pair

$$
\left(\frac{A\left\{x_{s} \mid s \in S\right\}}{\left\langle\left\{x_{s} \cdot \iota_{A}(s)-1 \mid s \in S\right\}\right\rangle}, q \circ \iota_{A}: A \rightarrow \frac{A \otimes_{\infty} \mathcal{C}^{\infty}\left(\mathbb{R}^{S}\right)}{\left\langle\left\{x_{s} \cdot \iota_{A}(s)-1 \mid s \in S\right\}\right\rangle}\right)
$$

is the $\mathcal{C}^{\infty}$-ring of fractions of $A$ with respect to $S$, up to isomorphism.

Remark 2.1.4. As we shall see later on, in Proposition 2.1.15, the universal map $\eta_{S}: A \rightarrow A\left\{S^{-1}\right\}$ is an epimorphism in $\mathcal{C}^{\infty} \mathbf{R n g}$, even though it is not always a surjective map.

\subsubsection{Smooth Saturation}

Within the theory of Commutative Algebra we have the following concept, namely the concept of "saturation" of a set:

Definition 2.1.5. Let $A$ be any commutative ring with unity and $S \subseteq A$ any of its subsets. Denoting by $\langle S\rangle$ the multiplicative submonoid of A generated by $S$, we define the saturation of $S$ as follows:

$$
S^{\mathrm{sat}}=\{a \in A \mid(\exists d \in A)(a \cdot d \in\langle S\rangle)\} .
$$

In other words, the saturation of a set $S$ is the set of all divisors of elements in $\langle S\rangle$. 
One easily sees that the saturation of a subset $S$ of a commutative ring $A$ is equal to the pre-image of the invertible elements of $A\left[S^{-1}\right]$ by the canonical map $\eta_{S}: A \rightarrow A\left\{S^{-1}\right\}$, i.e., $S^{\text {sat }}=\eta_{S}^{-}\left[\left(A\left[S^{-1}\right]\right)^{\times}\right]$.

We are going to use this characterization in order to define the smooth saturation of a subset $S$ of a $\mathcal{C}^{\infty}$-ring $A$, that we are going to denote by $S^{\infty-\text { sat }}$. First we need the following:

Proposition 2.1.6. Let $(A, \Phi)$ be a $\mathcal{C}^{\infty}$-ring and $S \subseteq A$ be any subset. If both $(F, \sigma)$ and $\left(F^{\prime}, \sigma^{\prime}\right)$ satisfy the Definition 2.1.2, then $\sigma^{-1}\left[F^{\times}\right]=\sigma^{\prime-1}\left[F^{\prime \times}\right]$.

Proof. Since both $(F, \sigma)$ and $\left(F^{\prime}, \sigma^{\prime}\right)$ have the universal property described in the Definition 2.1.2, there is a unique $\mathcal{C}^{\infty}$-isomorphism $\varphi: F \rightarrow F^{\prime}$, so the following triangle commutes:

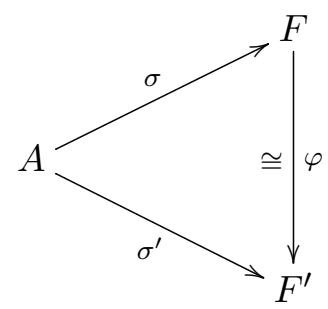

Because $\varphi: F \rightarrow F^{\prime}$ is a $\mathcal{C}^{\infty}$-isomorphism, $\varphi^{-1}\left[F^{\prime \times}\right]=F^{\times}$, so:

$$
\sigma^{\prime \dashv}\left[F^{\prime \times}\right]=\sigma^{\dashv}\left[\varphi^{\dashv}\left[F^{\prime \times}\right]\right]=\sigma^{\dashv}\left[F^{\times}\right] .
$$

Now we give the following:

Definition 2.1.7. Let $A$ be a $\mathcal{C}^{\infty}$-ring, $S \subseteq A$ and $(F, \sigma)$ be a ring of fractions of $A$ with respect to $S$. The smooth saturation of $S$ in $A$ is:

$$
S^{\infty-\text { sat }}:=\left\{a \in A \mid \sigma(a) \in F^{\times}\right\} .
$$

In virtue of the Proposition 2.1.6 the set $S^{\infty-\text { sat }}$ does not depend on any particular choice of the representation of the ring of fractions, rather it depends only on $A$ and $S$.

Remark 2.1.8. Since for every $s \in S, \eta_{S}(s) \in\left(A\left\{S^{-1}\right\}\right)^{\times}$, from now on we are going to use the more suggestive notation:

$$
(\forall s \in S)\left(\frac{1}{\eta_{S}(s)} \doteq \eta_{S}(s)^{-1}\right)
$$

and for any $a \in A$ and $s \in S$ we are going to denote:

$$
\frac{\eta_{S}(a)}{\eta_{S}(s)} \doteq \eta_{S}(a) \cdot \eta_{S}(s)^{-1}
$$

Definition 2.1.9. Let $A$ be $a \mathcal{C}^{\infty}$-ring and let $S \subseteq A^{\times}$be any subset. The smooth saturation of $S$ is $S^{\text {sat- }-\infty}=\eta_{S}{ }^{-1}\left[A\left\{S^{-1}\right\}^{\times}\right]$, where $\eta_{S}: A \rightarrow A\left\{S^{-1}\right\}$ is the canonical map of the ring of fractions of $A$ with respect to $S$.

Remark 2.1.10. Let $A$ be $a \mathcal{C}^{\infty}$-ring, $S \subseteq A$ and consider the forgetful functor:

$$
\begin{aligned}
& \mathcal{U}: \mathcal{C}^{\infty} \text { Rng } \rightarrow \quad \mathbb{R}-\mathbf{A l g} \\
& A \quad \mapsto \quad \mathcal{U}(A) \\
& A \stackrel{f}{\rightarrow} B \quad \mapsto \mathcal{U}(A) \stackrel{\mathcal{U}(f)}{\rightarrow} \mathcal{U}(B)
\end{aligned}
$$

We have always:

$$
S^{\text {sat }} \subseteq S^{\infty-\text { sat }}
$$


Theorem 2.1.11. Let $A$ be a $\mathcal{C}^{\infty}$-ring, $S \subseteq A$ and consider the forgetful functor:

$$
\begin{array}{rlc}
\mathcal{U}: \mathcal{C}^{\infty} \text { Rng } & \rightarrow & \mathbb{R}-\text { Alg } \\
A & \mapsto & \mathcal{U}(A) \\
A \stackrel{f}{\rightarrow} B & \mapsto & \mathcal{U}(A) \stackrel{\mathcal{U}(f)}{\rightarrow} \mathcal{U}(B)
\end{array}
$$

Since $\eta_{S}^{\infty}: A \rightarrow A\left\{S^{-1}\right\}$ is such that $\eta_{S}^{\infty}[S] \subseteq\left(A\left\{S^{-1}\right\}\right)^{\times}$, then $\left(\eta_{S}^{\infty}\right)[S] \subseteq\left(\mathcal{U}\left(A\left\{S^{1}\right\}\right)\right)^{\times}$, so by the universal property of the ring of fractions:

$$
\eta_{S}: \mathcal{U}(A) \rightarrow \mathcal{U}(A)\left[S^{-1}\right]
$$

there is a unique $\mathbb{R}$-algebras homomorphism, Can : $\mathcal{U}(A)\left[S^{-1}\right] \rightarrow \mathcal{U}\left(A\left\{S^{-1}\right\}\right)$ such that the following diagram commutes:

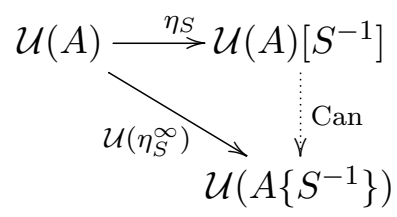

The following assertions are equivalent:

(1) $S^{\mathrm{sat}}=S^{\infty-\mathrm{sat}}$;

(2) Can is an isomorphism of $\mathbb{R}$-algebra.

Proof. Ad (2) $\rightarrow$ (1). Since the above diagram commutes, we have:

$$
S^{\infty-\mathrm{sat}}=\eta_{S}^{\infty \dashv}\left[\left(A\left\{S^{-1}\right\}\right)^{\times}\right]=\eta_{S}^{\dashv}\left[\operatorname{Can}^{-1}\left[\left(A\left\{S^{-1}\right\}\right)^{\times}\right]\right] \stackrel{(2)}{=} \eta_{S}^{\dashv}\left[\left(A\left[S^{-1}\right]\right)^{\times}\right]=S^{\text {sat }}
$$

Ad $(1) \rightarrow(2)$ : We already know that Can $: \mathcal{U}(A)\left[S^{-1}\right] \rightarrow \mathcal{U}\left(A\left\{S^{-1}\right\}\right)$ is an $\mathbb{R}$-algebras morphism. Note that since $S^{\text {sat }}=S^{\infty-\text { sat }}$, the morphism:

$$
\mathcal{U}\left(\eta_{S}^{\infty}\right): \mathcal{U}(A) \rightarrow \mathcal{U}\left(A\left\{S^{-1}\right\}\right)
$$

is such that:

(i) $\left(\forall \varphi^{\prime} \in \mathcal{U}(A)\left[S^{-1}\right]\right)(\exists a \in U(A))\left(\exists b \in S^{\mathrm{sat}}\right)\left(\mathcal{U}\left(\eta_{S}^{\infty}\right)(b) \cdot \varphi^{\prime}=\mathcal{U}\left(\eta_{S}^{\infty}\right)(a)\right)$;

(ii) $(\forall a \in U(A))\left(\mathcal{U}\left(\eta_{S}^{\infty}\right)(a)=0 \rightarrow\left(\exists \lambda^{\prime} \in S^{\text {sat }}\right)\left(\lambda^{\prime} \cdot a=0\right)\right)$;

(iii) $S \subseteq S^{\text {sat }}$.

which are precisely the hypotheses of Theorem 2.1.23, so $\mathcal{U}\left(\eta_{S}^{\infty}\right): \mathcal{U}(A) \rightarrow \mathcal{U}\left(A\left\{S^{-1}\right\}\right)$ is isomorphic to the localization. This fact implies that since Can is the only ring homomorphism which makes the diagram commute, Can must be the unique isomorphism between $\mathcal{U}(A)\left[S^{-1}\right]$ and $\mathcal{U}\left(A\left\{S^{-1}\right\}\right)$

In what follows we give some properties relating the inclusion relation among the subsets of a $\mathcal{C}^{\infty}$-ring and their smooth saturations.

Proposition 2.1.12. Let $A$ be $a \mathcal{C}^{\infty}$-ring and $T, S \subseteq A$ be any of its subsets. Then:

(i) $A^{\times} \subseteq S^{\infty-\text { sat }}$

(ii) $S \subseteq S^{\infty-\text { sat }}$

(iii) $S \subseteq T$ implies $S^{\infty-\text { sat }} \subseteq T^{\infty-\text { sat }}$ 
(iv) $S^{\infty-\text { sat }}=\langle S\rangle^{\infty-\text { sat }}$, where $\langle S\rangle$ is the submonoid generated by $S$.

Proof. - Ad (i): Since $\eta_{S}: A \rightarrow A\left\{S^{-1}\right\}$ is a $\mathcal{C}^{\infty}$-ring homomorphism, it preserves invertible elements, so $\eta_{S}\left[A^{\times}\right] \subseteq\left(A\left\{S^{-1}\right\}\right)^{\times}$, hence $A^{\times} \subseteq \eta_{S}^{-1}\left[\left(A\left\{S^{-1}\right\}\right)^{\times}\right]$.

- Ad (ii): It is immediate that $S \subseteq S^{\infty-\text { sat }}$, since by the very definition of $\eta_{S},(\forall a \in S)\left(\eta_{S}(a) \in\right.$ $\left.\left(A\left\{S^{-1}\right\}\right)^{\times}\right)$.

- Ad (iii): Since $S \subseteq T, \eta_{T}[S] \subseteq \eta_{T}[T] \subseteq\left(A\left\{T^{-1}\right\}\right)^{\times}$so, by the universal property of $\eta_{S}: A \rightarrow$ $A\left\{S^{-1}\right\}$ there exists a unique $\mu_{S T}: A\left\{S^{-1}\right\} \rightarrow A\left\{T^{-1}\right\}$ such that the following diagram commutes:

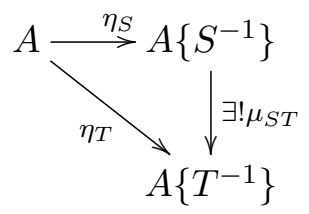

Hence, $T^{\infty-\text { sat }}=\eta_{T}^{-1}\left[\left(A\left\{T^{-1}\right\}\right)^{\times}\right]=\left(\mu_{S T} \circ \eta_{S}\right)^{-1}\left[\left(A\left\{T^{-1}\right\}\right)^{\times}\right]=\eta_{S}^{-1}\left[\mu_{S T}^{-1}\left[\left(A\left\{T^{-1}\right\}\right)^{\times}\right]\right]$. Now, since:

$$
\begin{gathered}
\mu_{S T}^{-}\left[\left(A\left\{T^{-1}\right\}\right)^{\times}\right] \supseteq A\left\{S^{-1}\right\}^{\times}, \\
T^{\infty-\text { sat }}=\eta_{S}^{-1}\left[\mu_{S T}^{-1}\left[A\left\{T^{-1}\right\}\right] \supseteq \eta_{S}^{-1}\left[\left(A\left\{S^{-1}\right\}\right)^{\times}\right]=S^{\infty-\text { sat }} .\right.
\end{gathered}
$$

- Ad (iv): Since $S \subseteq\langle S\rangle$, by the preceding item, $S^{\infty-\text { sat }} \subseteq\langle S\rangle^{\infty \text {-sat }}$. We need to show that $\langle S\rangle^{\infty-\text { sat }} \subseteq S^{\infty-\text { sat }}$.

We already know that, since $S \subseteq\langle S\rangle$, there exists a unique $\mathcal{C}^{\infty}$-morphism $\mu_{S\langle S\rangle}$ such that the following diagram commutes:

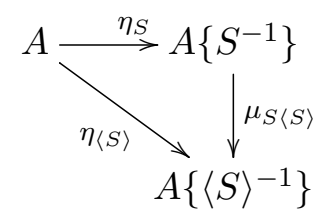

Given $x \in\langle S\rangle$, there exists some finite subset $S^{\prime} \subseteq S$ such that $x=\prod S^{\prime}$. Now, $\eta_{S}(x)=\prod \eta_{S}\left[S^{\prime}\right]$. Since $S^{\prime}$ is a finite subset of $S$ we have $\eta_{S}\left[S^{\prime}\right] \subseteq\left(A\left\{S^{-1}\right\}\right)^{\times}$. By the universal property of $\eta_{\langle S\rangle}$ : $A \rightarrow A\left\{\langle S\rangle^{-1}\right\}$, there exists a unique $\nu_{\langle S\rangle S}: A\left\{\langle S\rangle^{-1}\right\} \rightarrow A\left\{S^{-1}\right\}$ such that the following diagram commutes:

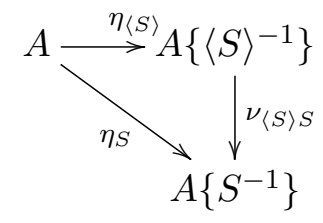

Now,

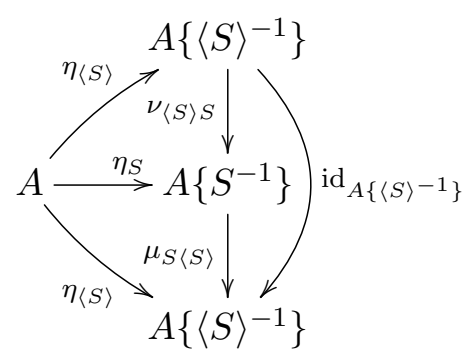


It follows that $\mu_{S\langle S\rangle} \circ \nu_{\langle S\rangle S}=\mathrm{id}_{A\left\{\langle A\rangle^{-1}\right\}}$. Also, we have the following diagram:

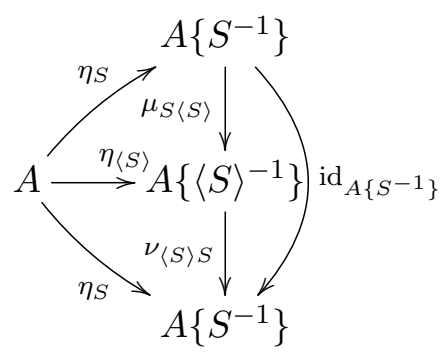

so $\nu_{\langle S\rangle S} \circ \mu_{S\langle S\rangle}=\operatorname{id}_{A\left\{S^{-1}\right\}}$, and $\mu_{S\langle S\rangle}: A\left\{S^{-1}\right\} \rightarrow A\left\{\langle S\rangle^{-1}\right\}$ and $\nu_{\langle S\rangle S}: A\left\{\langle S\rangle^{-1}\right\} \rightarrow A\left\{S^{-1}\right\}$ are inverse isomorphisms to each other. We conclude, from Proposition $\mathbf{2 . 1 . 2}$, that:

$$
S^{\infty-\mathrm{sat}}=\langle S\rangle^{\infty-\mathrm{sat}}
$$

Some necessary and sufficient conditions for the $\mathcal{C}^{\infty}$-homomorphism $\eta_{S}: A \rightarrow A\left\{S^{-1}\right\}$ be a $\mathcal{C}^{\infty}$-isomorphism is given below:

Proposition 2.1.13. Let $A$ be a $\mathcal{C}^{\infty}$-ring and $S \subseteq A$ any of its subsets. The following assertions are equivalent:

(i) $\eta_{S}: A \rightarrow A\left\{S^{-1}\right\}$ is an isomorphism;

(ii) $S^{\infty-\text { sat }} \subseteq A^{\times}$;

(iii) $S^{\infty-\text { sat }}=A^{\times}$

Proof. Ad (i) $\rightarrow$ (iii): Since $\eta_{S}$ is an isomorphism, both $\eta_{S}^{-1}$ and $\eta_{S}$ preserve the invertible elements, so $A^{\times}=\eta_{S}^{-1}\left[\left(A\left\{S^{-1}\right\}\right)^{\times}\right]=\eta_{S}^{-}\left[\left(A\left\{S^{-1}\right\}\right)^{\times}\right]=S^{\text {sat }}$.

Ad (ii) $\leftrightarrow$ (iii): Since we always have $A^{\times} \subseteq S^{\infty \text {-sat }}$, by (ii) we conclude that $A^{\times}=S^{\infty \text {-sat }}$.

Ad (iii) $\rightarrow\left(\right.$ i): Suppose that $S^{\infty-\text { sat }}=A^{\times}$. We need to show $\left.\eta_{A^{\times}}: A \rightarrow A\left\{\left(A^{\times}\right)^{-1}\right)\right\}$ is an isomorphism.

First we note that $\operatorname{id}_{A}: A \rightarrow A$ has the universal property of the ring of fractions of $A$ with respect to $A^{\times}$. Indeed, given any $\psi: A \rightarrow B$ such that $\psi\left[A^{\times}\right] \subseteq B^{\times}$(i.e., any $\psi$ which is a $\mathcal{C}^{\infty}$-homomorphism), there exists a unique $\mathcal{C}^{\infty}$-homomorphism from $A$ to $B$, namely $\psi: A \rightarrow B$, such that the following diagram commutes:

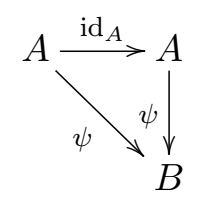

It follows that $\left(\operatorname{id}_{A}: A \rightarrow A\right) \cong\left(\eta_{A^{\times}}: A \rightarrow A\left\{\left(A^{\times}\right)^{-1}\right\}\right)$, since both satisfy the same universal property. Thus, $\eta_{A^{\times}}$is the composition of a $\mathcal{C}^{\infty}$-isomorphism with $\mathrm{id}_{A}$, hence it is a $\mathcal{C}^{\infty}$-isomorphism.

Moreover, by Proposition 2.1.2, we conclude that $A^{\times}=\eta_{A^{\times}}\left[\left(A\left\{\left(A^{\times}\right)^{-1}\right\}\right)^{\times}\right]=\left(A^{\times}\right)^{\infty-\text { sat }}$.

Next we prove that the smooth saturation of the smooth saturation of a set is again the smooth saturation of this set. 
Proposition 2.1.14. Let $A$ be $a \mathcal{C}^{\infty}$-ring and $S \subseteq A$ be any of its subsets. Then

$$
\left(S^{\infty-\mathrm{sat}}\right)^{\infty-\mathrm{sat}}=S^{\infty-\mathrm{sat}}
$$

Proof. Since $S \subseteq S^{\infty-\text { sat }}$, there exists a unique morphism $\mu_{S S^{\infty-\text { sat }}}: A\left\{S^{-1}\right\} \rightarrow A\left\{\left(S^{\infty-\text { sat }}\right)^{-1}\right\}$ such that the following diagram commutes:

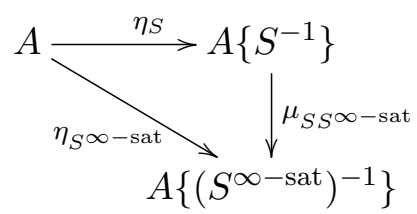

Now, $\eta_{S}\left[S^{\infty-\text { sat }}\right] \subseteq\left(A\left\{S^{-1}\right\}\right)^{\times}$by the very definition of $S^{\infty-\text { sat }}$, so, by the universal property of $\eta_{S^{\infty-\text { sat }}}: A \rightarrow A\left\{\left(S^{\infty-\mathrm{sat}}\right)^{-1}\right\}$, there exist a unique $\nu: A\left\{\left(S^{\infty-\mathrm{sat}}\right)^{-1}\right\} \rightarrow A\left\{S^{-1}\right\}$ such that the following diagram commutes:

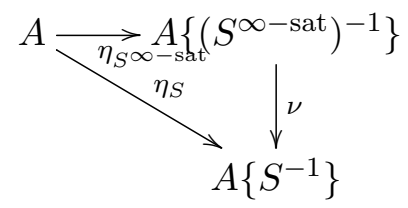

We have, then, the following commuting diagrams:
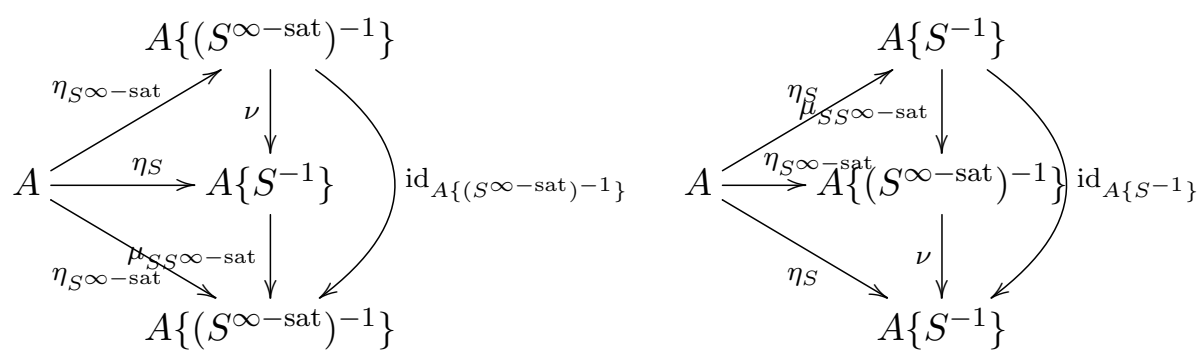

So $\left(\mu_{S S^{\infty-\text { sat }}}\right)^{-1}=\nu$, and $\mu_{S S^{\infty-\text { sat }}}$ is an isomorphism. Hence $A\left\{S^{-1}\right\} \cong A\left\{\left(S^{\infty-\text { sat }}\right)^{-1}\right\}$, so by Proposition 2.1.2,

$$
\left(S^{\infty-\mathrm{sat}}\right)^{\infty-\mathrm{sat}}=S^{\infty-\mathrm{sat}}
$$

Proposition 2.1.15. Let $A$ be a $\mathcal{C}^{\infty}$-ring, $S \subseteq A$ be any of its subsets, and $\eta_{S}: A \rightarrow A\left\{S^{-1}\right\}$ be the canonical morphism of the ring of fractions. If $g, h: A\left\{S^{-1}\right\} \rightarrow B$ are two morphisms such that $g \circ \eta_{S}=h \circ \eta_{S}$ then $g=h$. In other words, $\eta_{S}: A \rightarrow A\left\{S^{-1}\right\}$ is an epimorphism.

Proof. Note that since $g \circ \eta_{S}$ is such that $\left(g \circ \eta_{S}\right)[S] \subseteq B^{\times}$, there exists a unique morphism $\tilde{t}: A\left\{S^{-1}\right\} \rightarrow B$ such that $\tilde{t} \circ \eta_{S}=g \circ \eta_{S}$. By hypothesis we have $h \circ \eta_{S}=g \circ \eta_{S}$, so $g$ has the property which determines $\widetilde{t}$. Hence $g=\widetilde{t}=h$

Proposition 2.1.16. Let $A$ be a $\mathcal{C}^{\infty}$-ring and $S, T \subseteq A$ two of its subsets. The following assertions are equivalent:

(i) $S^{\infty-\text { sat }} \subseteq T^{\infty-\text { sat }}$ 
(ii) There is a unique morphism $\mu: A\left\{S^{-1}\right\} \rightarrow A\left\{T^{-1}\right\}$ such that the following diagram commutes:

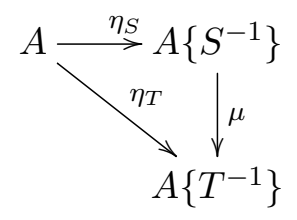

Proof. Ad (i) $\rightarrow$ (ii): Since $S^{\infty-\text { sat }} \subseteq T^{\infty-\text { sat }}$, by the universal property of $\eta_{S^{\infty-\text { sat }}}: A \rightarrow A\left\{\left(S^{\infty-\text { sat }}\right)^{-1}\right\}$

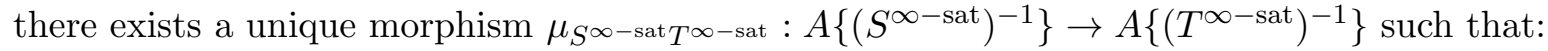

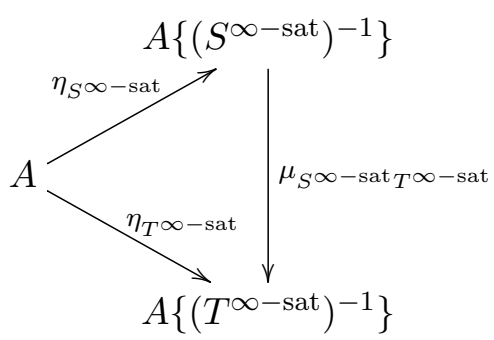

commutes.

Since $S \subseteq S^{\infty-\text { sat }}$ and $T \subseteq T^{\infty-\text { sat }}$, there are unique isomorphisms $\mu_{S S^{\infty-\text { sat }}}: A\left\{S^{-1}\right\} \rightarrow A\left\{S^{\infty-\text { sat }}{ }^{-1}\right\}$, $\mu_{T T^{\infty-\mathrm{sat}}}: A\left\{T^{-1}\right\} \rightarrow A\left\{T^{\infty-\mathrm{sat}^{-1}}\right\}$ such that the following diagram commutes:

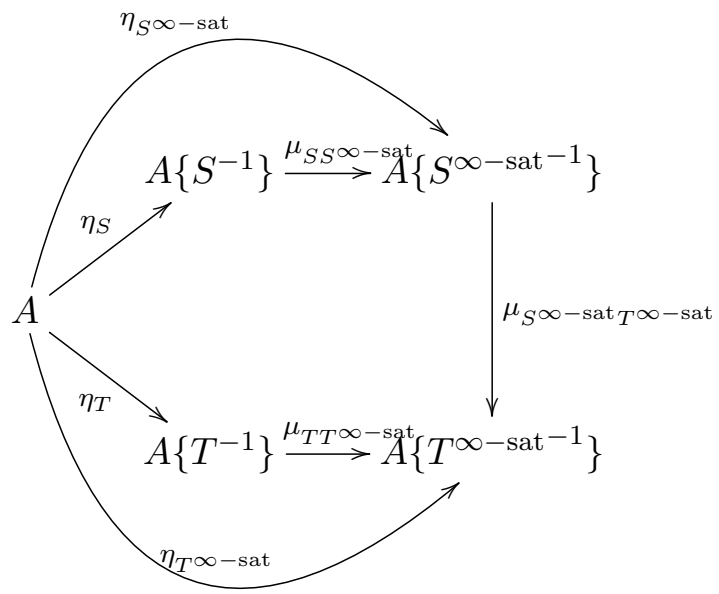

Since the upper and the lower triangles commute, we have:

$$
\mu_{S^{\infty-\mathrm{sat}} T^{\infty-\mathrm{sat}}} \circ \eta_{S^{\infty-\mathrm{sat}}}=\eta_{T^{\infty-\mathrm{sat}}} .
$$

We define $\mu:=\left(\mu_{T T^{\infty-\mathrm{sat}}}\right)^{-1} \circ \mu_{S^{\infty-\mathrm{sat}} T^{\infty-\mathrm{sat}}} \circ \mu_{S S^{\infty-\mathrm{sat}}}$. We claim that $\eta_{T}=\mu \circ \eta_{S}$.

On the one hand we have $\mu_{T T^{\infty-s a t}} \circ \eta_{T}=\eta_{T^{\infty-s a t}}$, and by the very definition of $\mu$ :

$$
\left(\mu_{T T^{\infty-\text { sat }}} \circ \mu\right) \circ \eta_{S}=\left(\mu_{S^{\infty-\mathrm{sat}} T^{\infty-\mathrm{sat}}} \circ \mu_{S S^{\infty-\mathrm{sat}}}\right) \circ \eta_{S}
$$

We also have:

$$
\mu_{S^{\infty-\mathrm{sat}} T^{\infty-\mathrm{sat}}} \circ\left(\mu_{S S^{\infty-\mathrm{sat}}} \circ \eta_{S}\right)=\mu_{T T^{\infty-\mathrm{sat}}} \circ \eta_{T}=\eta_{T^{\infty-\mathrm{sat}}}
$$

hence:

$$
\mu_{T T^{\infty-\mathrm{sat}}} \circ \eta_{T}=\eta_{T^{\infty-\mathrm{sat}}}=\mu_{T T^{\infty-\mathrm{sat}}} \circ\left(\mu \circ \eta_{S}\right)
$$


and since $\mu_{T T \infty-\text { sat }}$ is an isomorphism, it follows that:

$$
\eta_{T}=\mu \circ \eta_{S}
$$

In order to show that $\mu$ is unique, let $\nu: A\left\{S^{-1}\right\} \rightarrow A\left\{T^{-1}\right\}$ be a homomorphism such that the following diagram commutes:

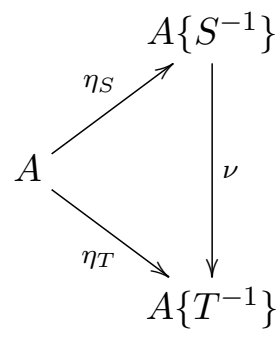

By composing both sides of the equation:

$$
\nu \circ \eta_{S}=\eta_{T}
$$

with $\mu_{T T^{\infty}-\text { sat }}$ we get:

$$
\mu_{T T^{\infty-\mathrm{sat}}} \circ \nu \circ \eta_{S}=\mu_{T T^{\infty-\mathrm{sat}}} \circ \eta_{T} .
$$

We know that $\mu_{T T^{\infty-\mathrm{sat}}} \circ \eta_{T}=\eta_{T^{\infty-\mathrm{sat}}}$, so

$$
\eta_{T^{\infty-\mathrm{sat}}}=\mu_{S^{\infty-\mathrm{sat}} T^{\infty-\mathrm{sat}}} \circ \eta_{S^{\mathrm{sat}}}=\mu_{S^{\infty-\mathrm{sat}} T^{\mathrm{sat}}} \circ \mu_{S S^{\infty-\mathrm{sat}}} \circ \eta_{S},
$$

so

$$
\mu_{T T^{\infty-\mathrm{sat}}} \circ \nu \circ \eta_{S}=\mu_{S^{\infty-\mathrm{sat}} T^{\infty-\mathrm{sat}}} \circ \mu_{S S^{\infty-\mathrm{sat}}} \circ \eta_{S}
$$

By Proposition 2.1.15, we conclude:

$$
\mu_{T T^{\infty-\mathrm{sat}}} \circ \nu=\mu_{S^{\infty-\mathrm{sat}} T^{\infty-\mathrm{sat}}} \circ \mu_{S S^{\infty-\mathrm{sat}}} .
$$

Composing both sides of the above equation with $\left(\mu_{T T^{\infty-s a t}}\right)^{-1}$ by the left we get:

$$
\nu=\left(\mu_{T T^{\infty-\mathrm{sat}}}\right)^{-1} \circ \mu_{S^{\infty-\mathrm{sat}} T^{\mathrm{sat}}} \circ \mu_{S S^{\infty-\mathrm{sat}}}=\mu
$$

Corollary 2.1.17. The following assertions are equivalent:

(i) $S^{\infty-\mathrm{sat}}=T^{\infty-\mathrm{sat}}$

(ii) There is an isomorphism $\mu: A\left\{S^{-1}\right\} \rightarrow A\left\{T^{-1}\right\}$ such that the following diagram commutes:

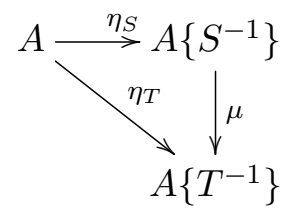

(iii) There is a unique isomorphism $\mu: A\left\{S^{-1}\right\} \rightarrow A\left\{T^{-1}\right\}$ such that the following diagram commutes:

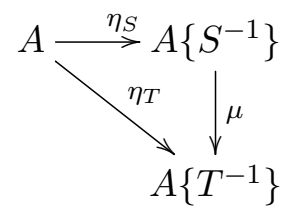


Proof. Ad (i) $\rightarrow$ (iii): Since $S^{\infty-\text { sat }}=T^{\infty-\text { sat }}$, we have both $S^{\infty-\text { sat }} \subseteq T^{\infty-\text { sat }}$, so by Proposition 2.1.16 there is a unique isomorphism $\nu: A\left\{S^{-1}\right\} \rightarrow A\left\{T^{-1}\right\}$ such that the following diagram commutes

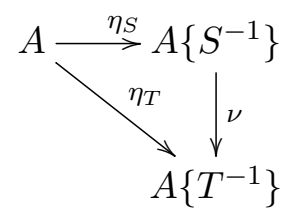

and $T^{\infty-\text { sat }} \subseteq S^{\infty-\text { sat }}$ so by Proposition 2.1.16 there is a unique homomorphism $\psi: A\left\{S^{-1}\right\} \rightarrow$ $A\left\{T^{-1}\right\}$ such that the following diagram commutes

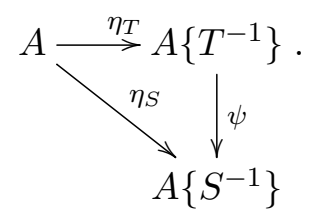

We have the following commutative diagrams:
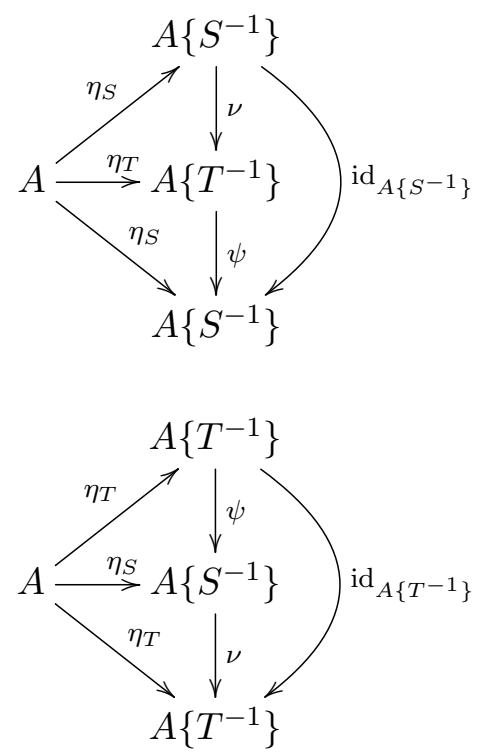

It follows that since $\eta_{T}$ and $\eta_{S}$ are epimorphisms and we have:

$$
\begin{aligned}
& \nu \circ \psi \circ \eta_{T}=\operatorname{id}_{A\left\{T^{-1}\right\}} \circ \eta_{T} \Rightarrow \nu \circ \psi=\operatorname{id}_{A\left\{T^{-1}\right\}} \\
& \psi \circ \nu \circ \eta_{S}=\operatorname{id}_{A\left\{S^{-1}\right\}} \circ \eta_{S} \Rightarrow \psi \circ \nu=\operatorname{id}_{A\left\{S^{-1}\right\}}
\end{aligned}
$$

so $\nu$ and $\psi$ are inverse isomorphisms to each other. We can take, then $\mu=\nu$.

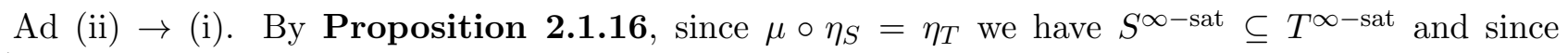
$\mu^{-1} \circ \eta_{T}=\eta_{S}$ we have $T^{\infty-\text { sat }} \subseteq S^{\infty-\text { sat }}$. Hence $S^{\infty-\text { sat }}=T^{\infty-\text { sat }}$.

Proposition 2.1.18. Let $A$ be a $\mathcal{C}^{\infty}$-ring and $S, T$ two of its subsets such that $S \subseteq T$. The following assertions are equivalent:

(i) $\mu_{S T}: A\left\{S^{-1}\right\} \rightarrow A\left\{T^{-1}\right\}$ is an isomorphism;

(ii) $S \subseteq T \subseteq S^{\infty-\text { sat }}$; 
(iii) $T^{\infty-\mathrm{sat}}=S^{\infty-\mathrm{sat}}$.

Proof. Ad (ii) $\rightarrow$ (iii): Since $S \subseteq T$ we have $S^{\infty-\text { sat }} \subseteq T^{\infty-\text { sat }}$, and since $T \subseteq S^{\infty-\text { sat }}$ we have $T^{\infty-\text { sat }} \subseteq$ $\left(S^{\infty-\text { sat }}\right)^{\infty-\text { sat }}=S^{\infty-\text { sat }}$. Hence:

$$
S^{\infty-\text { sat }} \subseteq T^{\infty-\text { sat }} \subseteq S^{\infty-\text { sat }}
$$

and

$$
S^{\infty-\mathrm{sat}}=T^{\infty-\mathrm{sat}}
$$

Ad (iii) $\rightarrow$ (ii): We always have $T \subseteq T^{\text {sat }}$, and since $T^{\text {sat }}=S^{\text {sat }}$, it follows that $T \subseteq S^{\text {sat }}$.

(i) $\leftrightarrow$ (iii) was established in Corollary 2.1.17.

Proposition 2.1.19. Let $A$ be a $\mathcal{C}^{\infty}$-ring and $S \subseteq A$. Whenever $\left\{S_{i}\right\}_{i \in I}$ is a directed system such that:

$$
S=\bigcup_{i \in I} S_{i}
$$

we have:

$$
S^{\infty-\mathrm{sat}}=\left(\bigcup_{i \in I} S_{i}\right)^{\infty-\mathrm{sat}}=\bigcup_{i \in I} S_{i}^{\infty-\mathrm{sat}}
$$

Proof. It is clear that:

$$
\bigcup_{i \in I} S_{i}^{\infty-\mathrm{sat}} \subseteq S^{\infty-\mathrm{sat}}
$$

In order to prove the other inclusion, we shall use the fact that $A\left\{S^{-1}\right\}$ is isomorphic to the vertex of the following directed colimit:

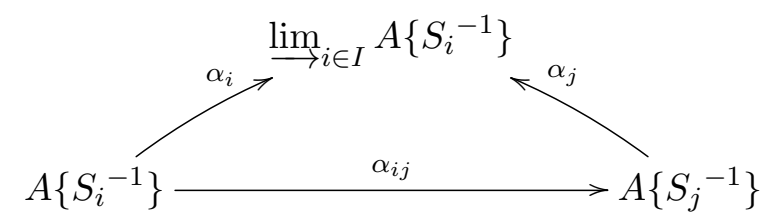

Note that $\eta_{S}: A \rightarrow A\left\{S^{-1}\right\}$ is such that for any $i \in I, \eta_{S}\left[S_{i}\right] \subseteq \eta_{S}[S] \subseteq\left(A\left\{S^{-1}\right\}\right)^{\times}$, so by the universal property of $\eta_{S_{i}}: A \rightarrow A\left\{\left(S_{i}\right)^{-1}\right\}$, there is a unique $\mathcal{C}^{\infty}$-rings homomorphism $\varphi_{i}: A\left\{\left(S_{i}\right)^{-1}\right\} \rightarrow A\left\{S^{-1}\right\}$ such that the following triangle commutes:

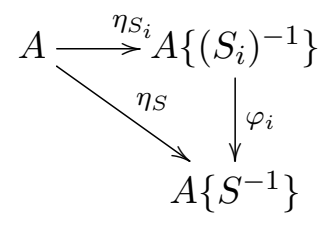

so

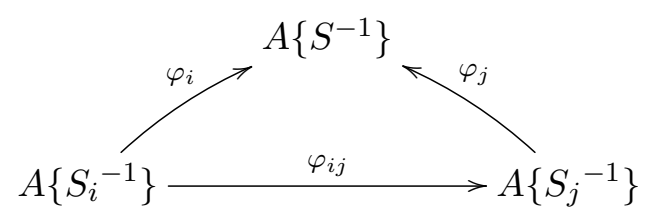

commutes for every $i, j \in I$ such that $i \leq j$, since $\eta_{S_{i}}: A \rightarrow A\left\{S_{i}^{-1}\right\}$ is an epimorphism. 
Thus, by the universal property of the colimit, there is a unique $\mathcal{C}^{\infty}$-homomorphism:

$$
\varphi: \underset{i \in I}{\lim _{i \in I}} A\left\{S_{i}^{-1}\right\} \rightarrow A\left\{S^{-1}\right\}
$$

such that:

$$
(\forall i \in I)\left(\varphi \circ \alpha_{i}=\varphi_{i}\right) .
$$

On the other hand, given $s \in S=\bigcup_{i \in I} S_{i}$ there is $i \in I$ such that $s \in S_{i}$, so $\eta_{S_{i}}(s) \in A\left\{S_{i}^{-1}\right\}^{\times}$. Taking $\left.\widetilde{\eta}:=\alpha_{i} \circ \eta_{S_{i}}: A \rightarrow{\underset{\lim }{\longrightarrow} i \in I}_{A} A S_{i}^{-1}\right\}$, we have:

$$
\widetilde{\eta}(s) \in\left(\lim _{i \in I} A\left\{S_{i}^{-1}\right\}\right)^{\times} .
$$

By the universal property of $\eta_{S}: A \rightarrow A\left\{S^{-1}\right\}$, there is a unique $\mathcal{C}^{\infty}$-homomorphism $\psi: A\left\{S^{-1}\right\} \rightarrow$ $\lim _{i \in I} A\left\{S_{i}^{-1}\right\}$ such that:

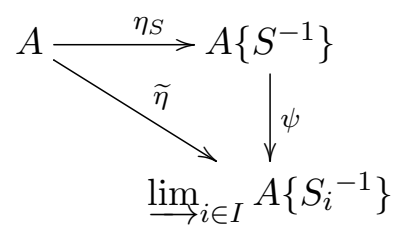
that:

It is easy to see, by the universal properties involved, that $\varphi$ and $\psi$ are inverse $\mathcal{C}^{\infty}$-isomorphism and

$$
\widetilde{\eta}^{-1}\left[\left(\lim _{i \in I} A\left\{S_{i}^{-1}\right\}\right)^{\times}\right]=\bigcup_{i \in I} S_{i}^{\infty-\mathrm{sat}} .
$$

Thus $S=\bigcup_{i \in I} S_{i}^{\infty-\text { sat }}$, as we claimed.

Proposition 2.1.20. Let $A$ be a $\mathcal{C}^{\infty}$-ring. The universal solution to freely adjoining a variable $x$ to $A$ in the category $\mathcal{C}^{\infty} \mathbf{R n g}$ is isomorphic to the smooth coproduct of $A$ and the free object in one generator of this category:

$$
A\{x\} \cong A \otimes_{\infty} \mathcal{C}^{\infty}(\mathbb{R})
$$

Proof. Let's first consider the inclusions $\jmath_{2}:\{x\} \rightarrow A\{x\}$ and $\eta:\{x\} \rightarrow \mathcal{C}^{\infty}(\mathbb{R})$, this latter given by the definition of free object in one generator. We are going to use the universal property of this free object together with the universal property of the smooth coproduct to show that $A \otimes_{\infty} \mathcal{C}^{\infty}(\mathbb{R})$ has the desired property.

In order to show that $A\{x\} \cong A \otimes_{\infty} \mathcal{C}^{\infty}(\mathbb{R})$ we use the universal property of the coproduct and of the localization, one at a time.

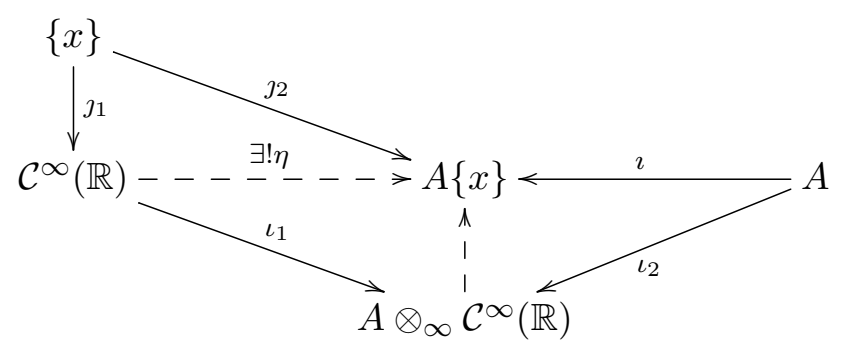


Since $\mathcal{C}^{\infty}(\mathbb{R})$ is the free object in one generator, $x$, given the inclusion $\jmath_{2}:\{x\} \rightarrow A\{x\}$ there is a unique $\mathcal{C}^{\infty}$-ring homomorphism $\eta: \mathcal{C}^{\infty}(\mathbb{R}) \rightarrow A\{x\}$ such that the upper left triangle of the above diagram commutes, i.e., such that $\eta \circ \jmath_{1}=\jmath_{2}$. We also have the inclusion map $\imath: A \rightarrow A\{x\}$ in the very definition of $A\{x\}$.

Now, by the universal property of the coproduct $\mathcal{C}^{\infty}(\mathbb{R}) \rightarrow A \otimes_{\infty} \mathcal{C}^{\infty}(\mathbb{R}) \leftarrow A$, given the $\mathcal{C}^{\infty}$-rings homomorphisms $\eta: \mathcal{C}^{\infty}(\mathbb{R}) \rightarrow A\{x\}$ and $\imath: A \rightarrow A\{x\}$ there exists a unique $\mathcal{C}^{\infty}$-homomorphism $\varphi: A \otimes_{\infty} \mathcal{C}^{\infty}(\mathbb{R})$ such that the following diagram commutes:

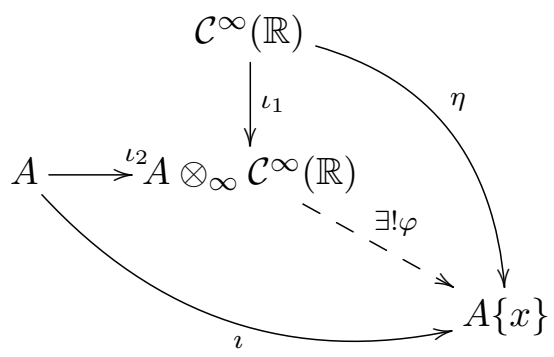

Conversely, since $\jmath_{2}:\{x\} \rightarrow A\{x\}$ is the solution to the universal problem of freely adjoining one element $t$ to $A$ and $\mathcal{C}^{\infty}(\mathbb{R})$ is the free object on one generator, there exists a unique $\ell: \mathcal{C}^{\infty}(\mathbb{R}) \rightarrow A\{t\}$ such that:

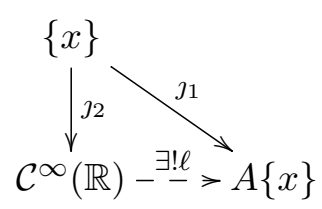

commutes. We will now consider the following diagram:

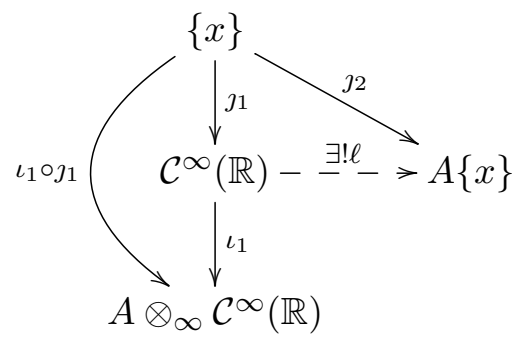

Now, given the $\mathcal{C}^{\infty}$-ring homomorphism $\iota_{1} \circ \jmath_{1}:\{x\} \rightarrow A \otimes_{\infty} \mathcal{C}^{\infty}(\mathbb{R})$, by the universal property of $\jmath_{2}:\{x\} \rightarrow A\{x\}$, there exists a unique $\mathcal{C}^{\infty}$-ring homomorphism $\varrho: A\{x\} \rightarrow A \otimes_{\infty} \mathcal{C}^{\infty}(\mathbb{R})$ such that the diagram below commutes:

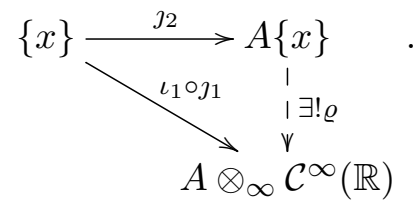

We have, then, the following diagram: 


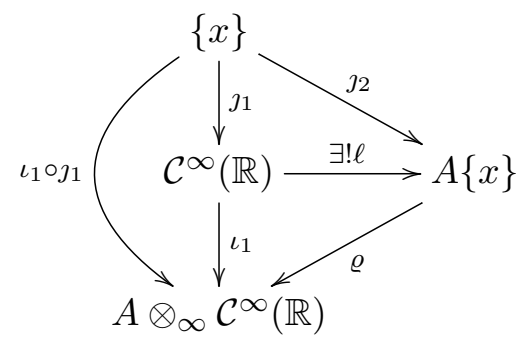

It can be proved that $\varrho \circ \varphi=\operatorname{id}_{A \otimes_{\infty} \mathcal{C} \infty(\mathbb{R})}$ and $\varphi \circ \varrho=\operatorname{id}_{A\{x\}}$, hence:

$$
A\{x\} \cong A \otimes_{\infty} \mathcal{C}^{\infty}(\mathbb{R}) .
$$

Our next goal is to give a characterization of ring of fractions in $\mathcal{C}^{\infty} \mathbf{R n g}$ using a similar axiomatization one has in Commutative Algebra. In order to motivate it, we first present some characterizations of rings of fractions in CRing.

Theorem 2.1.21. Let $A$ be a commutative ring with unity, $S \subseteq A$ a multiplicative subset, and $\eta: A \rightarrow$ $A\left[S^{-1}\right]$ its localization. Then:

(i) $\left(\forall \beta \in A\left[S^{-1}\right]\right)(\exists c \in S)(\exists d \in A)(\beta \cdot \eta(c)=\eta(d))$;

(ii) $(\forall \beta \in A)(\eta(\beta)=0 \rightarrow(\exists c \in S)(c \cdot \beta=0)$

Proof. Ad (i): Given any $\beta \in A\left[S^{-1}\right]$ we can write $\beta=\frac{a}{s}$ for some $a \in A$ and $s \in S$. Take $c=s$ and $d=a$, so we have:

$$
\beta \cdot \eta(c)=\frac{a}{s} \cdot \eta(c)=\frac{a}{s} \cdot \frac{s}{1}=\frac{a}{1}=\eta(a),
$$

so it suffices to take $d=a$.

Ad (ii): Given any $\beta \in A$ such that $\eta(b)=0$ we have, by the very construction of $A\left[S^{-1}\right]$, that $(\exists u \in S)(u(\beta \cdot 1-0 \cdot 1)=0)$, so it is enough to take $c=u$ in order to get $c \cdot \beta=u \cdot \beta=0$.

Conversely we have:

Proposition 2.1.22. Let $A$ be a commutative ring with unity and $S \subseteq A$. If $\varphi: A \rightarrow B$ is a ring homomorphism such that $\varphi[A] \subseteq B$ and:

(i) $(\forall \beta \in B)(\exists c \in S)(\exists d \in A)(\beta \cdot \varphi(c)=\varphi(d))$

(ii) $(\forall \beta \in A)(\varphi(\beta)=0 \rightarrow(\exists c \in S)(c \cdot \beta=0))$

then $B \cong A\left[S^{-1}\right]$.

Proof. Since $\varphi: A \rightarrow B$ is such that $\varphi[A] \subseteq B$, by the universal property of the localization there is a unique $\psi: A\left[S^{-1}\right] \rightarrow B$ such that the diagram below commutes:

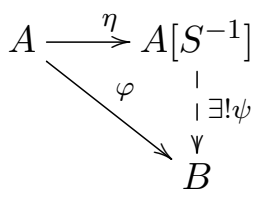


Now we claim that $\psi$ is a surjection. Given any $z \in B$, since $\varphi: A \rightarrow B$ satisfies $(i)$, there are elements $c \in S$ and $d \in A$ such that $z \cdot \varphi(c)=\varphi(d)$, that is to say $z=\varphi(d) \cdot(\varphi(c))^{-1}$. Taking $w=\eta(d) \cdot(\eta(c))^{-1} \in A\left[S^{-1}\right]$ we get $\psi(w)=\psi\left(\eta(d) \cdot(\eta(c))^{-1}\right)=\psi(\eta(d)) \cdot \psi(\eta(c))^{-1}=\varphi(d) \cdot(\varphi(c))^{-1}=z$.

We claim, also, that $\psi$ is an injective ring homomorphism. Let $w \in A\left[S^{-1}\right]$ be an element such that $\psi(w)=0$. Since $w \in A\left[S^{-1}\right]$ there are $c \in S$ and $d \in A$ such that $w=\eta(d) \cdot(\eta(c))^{-1}$. The condition $\psi(w)=0$ means that $\psi\left(\eta(d) \cdot(\eta(c))^{-1}\right)=\psi(\eta(d)) \cdot \psi((\eta(c)))^{-1}=0$, so $\psi(\eta(d))=0$, and since $\psi \circ \eta=\varphi$, it follows that $\varphi(d)=0$. By the property (ii), there exists $c^{\prime} \in S$ such that $c^{\prime} \cdot d=0$, so $\eta\left(c^{\prime}\right) \cdot \eta(d)=\eta\left(c^{\prime} \cdot d\right)=0$, and since $\eta\left(c^{\prime}\right) \in A\left[S^{-1}\right]^{\times}$, it follows that $\eta(d)=0$. Thus $w=\eta(d) \cdot\left(\eta\left(c^{\prime}\right)\right)^{-1}=0 \cdot\left(\eta\left(c^{\prime}\right)\right)^{-1}=0$.

It follows that $\psi$ is an isomorphism between $B$ and $A\left[S^{-1}\right]$.

The two preceding theorems gives us the following:

Theorem 2.1.23. Let $A$ be a commutative ring with unity and $S \subseteq A$. Then $\varphi: A \rightarrow B$ is isomorphic to the localization $\eta: A \rightarrow A\left[S^{-1}\right]$ if and only if:

(i) $(\forall b \in B)(\exists c \in S)(\exists d \in A)(b \cdot \varphi(c)=\varphi(d))$

(ii) $(\forall b \in A)(\varphi(b)=0 \rightarrow(\exists c \in S)(c \cdot b=0))$

hold.

We have, thus, obtained a characterization of the localization of a commutative ring. For $\mathcal{C}^{\infty}$-rings we have the analogous result, that generalizes Theorem 1.4 of [66]:

Theorem 2.1.24. Let $A$ be a $\mathcal{C}^{\infty}$-ring $\Sigma \subset A$ a set. Then $\varphi: A \rightarrow B$ is isomorphic to the smooth localization $\eta: A \rightarrow A\left\{\Sigma^{-1}\right\}$ if and only if:

(i) $(\forall b \in B)\left(\exists c \in \Sigma^{\infty-\mathrm{sat}}\right)(\exists d \in A)(b \cdot \varphi(c)=\varphi(d))$

(ii) $(\forall b \in A)\left(\varphi(b)=0 \rightarrow\left(\exists c \in \Sigma^{\infty-\mathrm{sat}}\right)(c \cdot b=0)\right)$

hold.

We postpone the proof of this theorem, giving it right after Remark 2.1.33.

Theorem 2.1.25. Let $A, \widetilde{A}$ be $\mathcal{C}^{\infty}$-rings and let $\eta: A \rightarrow \widetilde{A}$ be a $\mathcal{C}^{\infty}$-rings homomorphism such that:

(i) $(\forall d \in \widetilde{A})(\exists b \in A)(\exists c \in A)\left(\eta(c) \in \widetilde{A}^{\times} \&(d \cdot \eta(c)=\eta(b))\right)$;

(ii) $(\forall b \in A)\left(\left(\eta(b)=0_{\widetilde{A}}\right) \rightarrow(\exists c \in A)\left(\left(\eta(c) \in \widetilde{A}^{\times}\right) \wedge\left(b \cdot c=0_{A}\right)\right)\right)$

Then $\eta: A \rightarrow \widetilde{A}$ is isomorphic to $\operatorname{Can}_{S_{\eta}}: A \rightarrow A\left\{S_{\eta}{ }^{-1}\right\}$, where $S_{\eta}=\eta^{-1}\left[\widetilde{A}^{\times}\right]$.

Proof. First we show that $\eta: A \rightarrow \widetilde{A}$ has the universal property which characterize $\operatorname{Can}_{S_{\eta}}$.

Let $f: A \rightarrow B$ be a $\mathcal{C}^{\infty}$-rings homomorphism such that $f\left[S_{\eta}\right] \subseteq B^{\times}$. We are going to show there is a unique $\mathcal{C}^{\infty}$-rings homomorphism $\widetilde{f}: \widetilde{A} \rightarrow B$ such that the following diagram commutes:

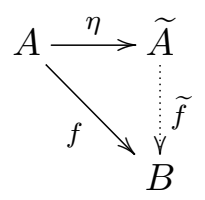


i.e., such that $\tilde{f} \circ \eta=f$.

First we notice that:

$$
\eta\left[S_{\eta}\right]=\eta\left[\eta^{-1}\left[\widetilde{A}^{\times}\right]\right] \subseteq \widetilde{A}^{\times}
$$

Candidate and Uniqueness of $\widetilde{f}$ : Let $\widetilde{f}_{1}, \widetilde{f}_{2}: \widetilde{A} \rightarrow B$ be two $\mathcal{C}^{\infty}$-rings homomorphisms such that the following diagram commutes:

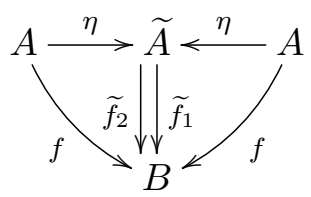

that is to say, such that $\widetilde{f}_{1} \circ \eta=f=\widetilde{f}_{2} \circ \eta$.

Given any $d \in \widetilde{A}$, by the hypothesis (i) there exist $b, c \in A, \eta(c) \in \widetilde{A}^{\times}$such that $d=\frac{\eta(b)}{\eta(c)}$, so:

$$
\begin{aligned}
& \tilde{f}_{1}(d)=\widetilde{f}_{1}\left(\eta(b) \cdot \eta(c)^{-1}\right)=\widetilde{f}_{1}(\eta(b)) \cdot \widetilde{f}_{1}(\eta(c))^{-1}= \\
&=\left(\widetilde{f}_{1} \circ \eta\right)(b) \cdot\left(\left(\widetilde{f}_{1} \circ \eta\right)(c)\right)^{-1}=f(b) \cdot f(c)^{-1}=\left(\widetilde{f}_{2} \circ \eta\right)(b) \cdot\left(\left(\widetilde{f}_{2} \circ \eta\right)(c)\right)^{-1}= \\
& \quad=\widetilde{f}_{2}(\eta(b)) \cdot \widetilde{f}_{2}\left(\eta(c)^{-1}\right)=\widetilde{f}_{2}\left(\eta(b) \cdot \eta(c)^{-1}\right)=\widetilde{f}_{2}(d)
\end{aligned}
$$

so we conclude that $(\forall d \in \widetilde{A})\left(\widetilde{f}_{1}(d)=\widetilde{f}_{2}(d)\right)$ and $\widetilde{f}\left(\frac{\eta(b)}{\eta(c)}\right)=f(b) \cdot f(c)^{-1}$. Thus $\widetilde{f}_{1}=\widetilde{f}_{2}$.

Existence of $\widetilde{f}$ : We know that for every $d \in \widetilde{A}$ there exist $b, c \in A, \eta(c) \in \widetilde{A}^{\times}$, such that $d=\eta(b) \cdot \eta(c)^{-1}$. Define the following relation: $\widetilde{f}=\left\{\left(d, f(b) \cdot f(c)^{-1}\right) \mid d \in \widetilde{A}\right\} \subseteq \widetilde{A} \times B$. We claim that $\tilde{f}$ is a function.

Claim: $\widetilde{f}$ is a univocal relation.

Given $d \in \widetilde{A}$, let $b, c, b^{\prime}, c^{\prime} \in A, \eta(c), \eta\left(c^{\prime}\right) \in \widetilde{A}^{\times}$be such that $\eta(b) \cdot \eta(c)^{-1}=d=\eta\left(b^{\prime}\right) \cdot \eta\left(c^{\prime}\right)^{-1}$, so $\eta(b) \cdot \eta\left(c^{\prime}\right)=\eta\left(b^{\prime}\right) \cdot \eta(c)$. Then we have $\eta\left(b \cdot c^{\prime}\right)=\eta(b) \cdot \eta\left(c^{\prime}\right)=\eta\left(b^{\prime}\right) \cdot \eta(c)$, so $\eta\left(b \cdot c^{\prime}-b^{\prime} \cdot c\right)=0$. By (ii), since $\eta\left(b \cdot c^{\prime}-b^{\prime} \cdot c\right)=0$, there is some $a \in A$ such that $\eta(c) \in \widetilde{A}^{\times}$, i.e., $a \in \eta^{-1}\left[\widetilde{A}^{\times}\right]=S_{\eta}$, and $a \cdot\left(b \cdot c^{\prime}-b^{\prime} \cdot c\right)=0$, i.e., $b \cdot c^{\prime} \cdot a=b^{\prime} \cdot c \cdot a$. We now have:

$$
f(b) \cdot f\left(c^{\prime}\right) \cdot f(a)=f\left(b \cdot c^{\prime} \cdot a\right)=f\left(b^{\prime} \cdot c \cdot a\right)=f\left(b^{\prime}\right) \cdot f(c) \cdot f(a) .
$$

Now since $f\left[S_{\eta}\right] \subseteq B^{\times}$and $a \in S_{\eta}$, from the preceding equations we obtain, by cancelling $f(a)$ :

$$
f(b) \cdot f\left(c^{\prime}\right)=f\left(b^{\prime}\right) \cdot f(c)
$$

and since $\eta(c), \eta\left(c^{\prime}\right) \in \widetilde{A}^{\times}, c, c^{\prime} \in \eta^{\dashv}\left[\widetilde{A}^{\times}\right]=S_{\eta}$ so $f(c), f\left(c^{\prime}\right) \in B^{\times}$, so:

$$
f(b) \cdot f(c)^{-1}=f\left(b^{\prime}\right) \cdot f\left(c^{\prime}\right)^{-1} .
$$

We conclude that $(\forall d \in \widetilde{A})\left(\left(\left(d, f(b) \cdot f(c)^{-1}\right) \in \widetilde{f}\right) \wedge\left(\left(d, f\left(b^{\prime}\right) \cdot f\left(c^{\prime}\right)^{-1}\right) \in \widetilde{f}\right) \rightarrow f(b) \cdot f(c)^{-1}=\right.$ $\left.f\left(b^{\prime}\right) \cdot f\left(c^{\prime}\right)^{-1}\right)$.

Claim: $\tilde{f}$ is a total relation. 
This follows immediately from item (i).

We are going to denote $\left(d, f(b) \cdot f(c)^{-1}\right) \in \widetilde{f}$ simply by $\widetilde{f}(d)=f(b) \cdot f(c)^{-1}$, as usually we do for functions.

Therefore, there exists exactly one function $\tilde{f}: \widetilde{A} \rightarrow B$ such that $\tilde{f} \circ \eta=f$.

Now we show that $\widetilde{A} \cong A\left\{S_{\eta}^{-1}\right\}$. We begin by noticing that $\operatorname{Can}_{S_{\eta}}\left[S_{\eta}\right] \subseteq A\left\{S_{\eta}^{-1}\right\}^{\times}$by the very

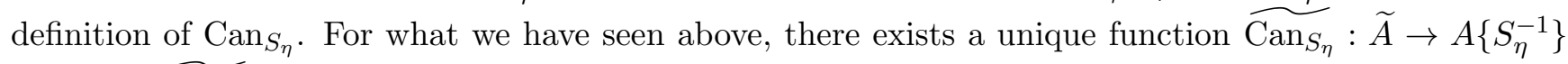
such that $\widetilde{\operatorname{Can}_{S_{\eta}}} \circ \eta=\operatorname{Can}_{S_{\eta}}$. Now, from the universal property of $\operatorname{Can}_{S_{\eta}}$ there exists a unique $\mathcal{C}^{\infty}$-rings homomorphism:

$$
\widehat{\eta}: A\left\{S_{\eta}^{-1}\right\} \rightarrow \widetilde{A}
$$

such that $\widehat{\eta} \circ \operatorname{Can}_{S_{\eta}}=\eta$.

Claim: $\widehat{\eta}$ is a bijection whose inverse is $\widetilde{\operatorname{Can}_{S_{\eta}}}$, and that will prove that $\widetilde{\operatorname{Can}_{S_{\eta}}}$ is a $\mathcal{C}^{\infty}$-rings isomorphism.

Now, $\left(\widehat{\eta} \circ \widetilde{\operatorname{Can}_{S_{\eta}}}\right) \circ \eta=\widehat{\eta} \circ\left(\widetilde{\operatorname{Can}_{S_{\eta}}} \circ \eta\right)=\widehat{\eta} \circ \operatorname{Can}_{S_{\eta}}=\eta=\mathrm{id}_{\widetilde{A}} \circ \eta$, so:

$$
\left(\widehat{\eta} \circ \widetilde{\operatorname{Can}_{S_{\eta}}}\right) \circ \eta=\operatorname{id}_{\widetilde{A}} \circ \eta \text {. }
$$

We have seen, however, that there is exactly one function $\widetilde{\varphi}$ such that $\widetilde{\varphi} \circ \eta=\eta$, so it follows that $\operatorname{id}_{\widetilde{A}}=\widehat{\eta} \circ \widetilde{\operatorname{Can}_{S_{\eta}}}$.

On the other hand,

$$
\widetilde{\operatorname{Can}_{S_{\eta}}} \circ \widehat{\eta} \circ \operatorname{Can}_{S_{\eta}}=\widetilde{\operatorname{Can}_{S_{\eta}}} \circ \eta=\operatorname{Can}_{S_{\eta}}=\mathrm{id}_{A\left\{S_{\eta}^{-1}\right\}} \circ \operatorname{Can}_{S_{\eta}} .
$$

Once again, by the universal property of $\mathrm{Can}_{S_{\eta}}$ we have:

$$
\operatorname{id}_{A\left\{S_{\eta}^{-1}\right\}}=\widetilde{\operatorname{Can}_{S_{\eta}}} \circ \widehat{\eta} \text {. }
$$

Hence $\widetilde{\operatorname{Can}_{S_{\eta}}}$ is the $\mathcal{C}^{\infty}$-rings isomorphism between $\eta$ and $\operatorname{Can}_{S_{\eta}}$, that is, it is a $\mathcal{C}^{\infty}$-rings isomorphism such that the following diagram commutes:

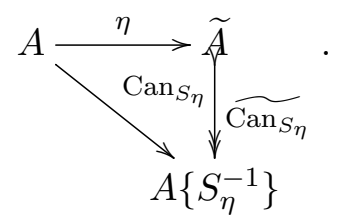

In order to smoothly localize larger subsets of $\mathcal{C}^{\infty}\left(\mathbb{R}^{n}\right)$ for some $n \in \mathbb{N}$, say $\Sigma$, which is a set that contains possibly a non-countable amount of elements, we can proceed as follows. First notice that we can obtain the $\mathcal{C}^{\infty}$-ring of fractions of $\mathcal{C}^{\infty}\left(\mathbb{R}^{n}\right)$ with respect to the singleton $\Sigma=\left\{f: \mathbb{R}^{n} \rightarrow \mathbb{R}\right\}$, provided that $f \not \equiv 0$. Whenever $\Sigma=\left\{f_{1}, \cdots, f_{k}\right\}$ for some $k \in \mathbb{N}$, inverting $\Sigma$ is equivalent to inverting $\prod \Sigma=f_{1} \cdot f_{2} \cdots f_{k-1} \cdot f_{k}$. In the case that $\Sigma$ is infinite, first we decompose it as the union of its finite subsets: 


$$
\Sigma=\bigcup_{\Sigma^{\prime} \subseteq_{\text {fin }} \Sigma} \Sigma^{\prime}
$$

Note that $\mathcal{S}=\left\{\Sigma^{\prime} \subseteq \Sigma \mid \Sigma^{\prime}\right.$ is finite $\}$ is partially ordered by the inclusion relation. Also, whenever $\Sigma^{\prime} \subseteq \Sigma^{\prime \prime}$, since $\eta_{\Sigma^{\prime \prime}}\left[\Sigma^{\prime}\right] \subseteq \eta_{\Sigma^{\prime \prime}}\left[\Sigma^{\prime \prime}\right] \subseteq\left(A\left\{\Sigma^{\prime \prime}-1\right\}\right)^{\times}$, by the universal property of $\eta_{\Sigma^{\prime \prime}}: A \rightarrow A\left\{\Sigma^{\prime \prime}-1\right\}$, there is a unique $\mathcal{C}^{\infty}$-homomorphism $\alpha_{\Sigma^{\prime} \Sigma^{\prime \prime}}: A\left\{\Sigma^{\prime-1}\right\} \rightarrow A\left\{\Sigma^{\prime \prime-1}\right\}$ such that the following diagram commutes:

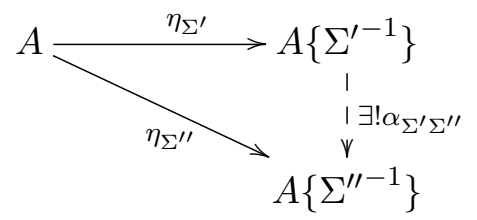

It is simple to prove, using the "uniqueness part" of the $\mathcal{C}^{\infty}$-homomorphism obtained via universal property, that for any finite $\Sigma^{\prime}$ we have $\alpha_{\Sigma^{\prime} \Sigma^{\prime}}=\mathrm{id}_{A\left\{\Sigma^{\prime-1}\right\}}$, and given any finite $\Sigma^{\prime}, \Sigma^{\prime \prime}$ and $\Sigma^{\prime \prime \prime}$ such that $\Sigma^{\prime} \subseteq \Sigma^{\prime \prime} \subseteq \Sigma^{\prime \prime \prime}, \alpha_{\Sigma^{\prime \prime} \Sigma^{\prime \prime \prime}} \circ \alpha_{\Sigma^{\prime} \Sigma^{\prime \prime}}=\alpha_{\Sigma^{\prime} \Sigma^{\prime \prime \prime}}$, so we have an inductive system:

$$
\left\{\alpha_{\Sigma^{\prime} \Sigma^{\prime \prime}}: A\left\{\Sigma^{\prime-1}\right\} \rightarrow A\left\{\Sigma^{\prime \prime-1}\right\} \mid\left(\Sigma^{\prime}, \Sigma^{\prime \prime} \in \mathcal{S}\right) \&\left(\Sigma^{\prime} \subseteq \Sigma^{\prime \prime}\right)\right\}
$$

We take, thus:

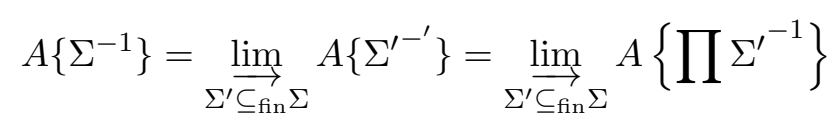

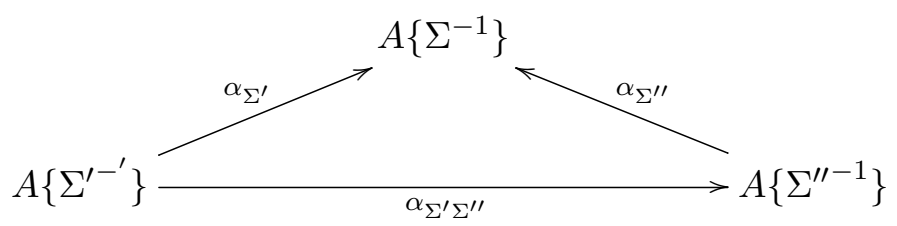

Hence, given any $\mathcal{C}^{\infty}$-ring $A$ and any $S \subseteq A$, we can construct:

$$
\eta_{\Sigma}=\alpha_{\Sigma^{\prime}} \circ \eta_{\Sigma^{\prime}}: A \rightarrow A\left\{\Sigma^{-1}\right\}
$$

It is easy to prove that $\eta_{\Sigma}$ has the universal property which characterizes the $\mathcal{C}^{\infty}$-ring of fractions of $A$ with respect to $\Sigma$.

Remark 2.1.26. In the case that $A=\mathcal{C}^{\infty}\left(\mathbb{R}^{n}\right)$ and $\Sigma=\left\{f: \mathbb{R}^{n} \rightarrow \mathbb{R}\right\}$, we have $\Sigma^{\infty-\text { sat }}=\{g \in$ $\left.\mathcal{C}^{\infty}\left(\mathbb{R}^{n}\right) \mid U_{f} \subseteq U_{g}\right\}=\left\{g \in \mathcal{C}^{\infty}\left(\mathbb{R}^{n}\right) \mid Z(g) \subseteq Z(f)\right\}$.

Let us now consider the following result, credited to Ortega and Muñoz by I. Moerdijk and G. Reyes in 66]:

Theorem 2.1.27. (5.0.6) Let $U \subseteq \mathbb{R}^{n}$ be open, and $g \in \mathcal{C}^{\infty}(U)$. Then there are $h, k \in \mathcal{C}^{\infty}\left(\mathbb{R}^{n}\right)$ with $U_{k}=U$ and $g \cdot k \uparrow_{U} \equiv h \uparrow_{U}$. where $U_{k}=\mathbb{R}^{n} \backslash Z(k)$ and $Z(k)=\left\{x \in \mathbb{R}^{n} \mid k(x)=0\right\}$.

Theorem 2.1.28. Let $A$ be $a \mathcal{C}^{\infty}$-ring and $S \subseteq A$. An element $\lambda=\frac{\eta_{S}(c)}{\eta_{S}(b)}$ (with $c \in A$ and $b \in S^{\infty \text {-sat }}$ ) is invertible in $A\left\{S^{-1}\right\}$ if, and only if, there are elements $d \in S^{\infty-\text { sat }}$ and $c^{\prime} \in A$ such that $d c^{\prime} c \in S^{\infty-\text { sat }}$, that is,

$$
\frac{\eta_{S}(c)}{\eta_{S}(b)} \in\left(A\left\{S^{-1}\right\}\right)^{\times} \Longleftrightarrow\left(\exists d \in S^{\infty-\mathrm{sat}}\right)\left(\exists c^{\prime} \in A\right)\left(d \cdot c^{\prime} \cdot c \in S^{\infty-\mathrm{sat}}\right) .
$$


Proof. Suppose $\frac{\eta_{S}(c)}{\eta_{S}(b)} \in\left(A\left\{S^{-1}\right\}\right)^{\times}$, so there are $c^{\prime} \in A$ and $b^{\prime} \in S^{\infty-\text { sat }}$ such that:

$$
\begin{gathered}
\frac{\eta_{S}(c)}{\eta_{S}(b)} \cdot \frac{\eta_{S}\left(c^{\prime}\right)}{\eta_{S}\left(b^{\prime}\right)}=1_{A\left\{S^{-1}\right\}}=\eta_{S}\left(1_{A}\right) . \\
\eta_{S}\left(c \cdot c^{\prime}\right)=\eta_{S}\left(b \cdot b^{\prime}\right) \\
\eta_{S}\left(c \cdot c^{\prime}-b \cdot b^{\prime}\right)=0
\end{gathered}
$$

By Theorem 2.1.24, there is some $d \in S^{\infty-\text { sat }}$ such that:

$$
\begin{gathered}
d \cdot\left(c \cdot c^{\prime}-b \cdot b^{\prime}\right)=0 \\
d \cdot c \cdot c^{\prime}=d \cdot b \cdot b^{\prime} \in S^{\infty-\mathrm{sat}}
\end{gathered}
$$

where $d \cdot b \cdot b^{\prime} \in S^{\infty-\text { sat }}$ because it is a product of elements of $S^{\infty-\text { sat }}$, which is a submonoid of $A$.

Conversely, suppose that $\frac{\eta_{S}(c)}{\eta_{S}(b)} \in A\left\{S^{-1}\right\}$ with $b \in S^{\infty-\text { sat }}$ is an element for which there are elements $d \in S^{\infty-\text { sat }}$ and $c^{\prime} \in A$ such that $d \cdot c \cdot c^{\prime} \in S^{\infty-\text { sat }}$. We have $\eta_{S}\left(d \cdot c^{\prime} \cdot c\right) \in\left(A\left\{S^{-1}\right\}\right)^{\times}$and $b \in S^{\infty-\text { sat }}$, so $\eta_{S}(b) \in\left(A\left\{S^{-1}\right\}\right)^{\times}$, hence

$$
\frac{\eta_{S}\left(d \cdot c^{\prime} \cdot c\right)}{\eta_{S}(b)} \in\left(A\left\{S^{-1}\right\}\right)^{\times}
$$

Since

$$
\frac{\eta_{S}(c)}{\eta_{S}(b)} \cdot \eta_{S}\left(d \cdot c^{\prime}\right)=\frac{\eta_{S}\left(d \cdot c^{\prime} \cdot c\right)}{\eta_{S}(b)} \in\left(A\left\{S^{-1}\right\}\right)^{\times}
$$

it follows that $\frac{\eta_{S}(c)}{\eta_{S}(b)} \in\left(A\left\{S^{-1}\right\}\right)^{\times}$, for if $\alpha \cdot \beta$ is invertible, then both $\alpha$ and $\beta$ are invertible.

Now we have the following:

Proposition 2.1.29. Let $U \subseteq \mathbb{R}^{n}$ be any open subset and define $S_{U}=\left\{g \in \mathcal{C}^{\infty}\left(\mathbb{R}^{n}\right) \mid U \subseteq U_{g}\right\} \subseteq \mathcal{C}^{\infty}\left(\mathbb{R}^{n}\right)$. The $\mathcal{C}^{\infty}$-ring of fractions of $\mathcal{C}^{\infty}\left(\mathbb{R}^{n}\right)$ with respect to the set $S_{U}$ :

$$
\eta_{S_{U}}: \mathcal{C}^{\infty}\left(\mathbb{R}^{n}\right) \rightarrow \mathcal{C}^{\infty}\left(\mathbb{R}^{n}\right)\left\{S_{U}^{-1}\right\}
$$

is isomorphic to the restriction map:

$$
\begin{aligned}
\rho: \mathcal{C}^{\infty}\left(\mathbb{R}^{n}\right) & \rightarrow \mathcal{C}^{\infty}(U) \\
h & \mapsto h \uparrow_{U}
\end{aligned}
$$

Proof. We are going to show that $\rho \cong \eta_{S_{U}}$ using Theorem 2.1.25 with $A=\mathcal{C}^{\infty}\left(\mathbb{R}^{n}\right), \widetilde{A}=\mathcal{C}^{\infty}(U)$ and $\eta=\rho$.

Note that $\rho\left[S_{U}\right] \subseteq \mathcal{C}^{\infty}(U)^{\times}$.

Let's verify the first item, (i):

By the Theorem 5.0.6 given any $g \in \mathcal{C}^{\infty}(U)$, there are $h, k \in \mathcal{C}^{\infty}\left(\mathbb{R}^{n}\right)$ with $U_{k}=U$, such that $g \cdot k \uparrow_{U}=h \uparrow_{U}$. Since $U=U_{k}, \rho(k) \in \mathcal{C}^{\infty}(U)^{\times}$, and we have, thus:

$$
\left(\rho(k) \in \mathcal{C}^{\infty}(U)^{\times}\right) \&(g \cdot \rho(k)=\rho(h)) .
$$


For item (ii), suppose that $g \in \mathcal{C}^{\infty}\left(\mathbb{R}^{n}\right)$ is such that $g \uparrow_{U}=0$, so $U \subseteq Z(g)$. We know, by a well-known theorem proved by Whitney (see Theorem $\mathbf{5 . 0 . 5}$ ), that given the open subset $U$ of $\mathbb{R}^{n}$, there is some $h \in \mathcal{C}^{\infty}\left(\mathbb{R}^{n}\right)$ such that $U=U_{h}$, so $Z(h \cdot g)=Z(h) \cup Z(g)=U \cup\left(\mathbb{R}^{n} \backslash U\right)=\mathbb{R}^{n}$. We have both:

$$
\rho(g)=0 \in \mathcal{C}^{\infty}(U)
$$

and

$$
g \cdot h=0 \in \mathcal{C}^{\infty}\left(\mathbb{R}^{n}\right),
$$

so item (ii) is also fulfilled. By Theorem 2.1.25, the result follows.

Remark 2.1.30. Let $A$ be $a \mathcal{C}^{\infty}$-ring and $a \in A$. In general, the $\mathcal{C}^{\infty}$-ring of fractions of $A$ with respect to a is not a local $\mathcal{C}^{\infty}$-ring. Let us consider the case on which $A=\mathcal{C}^{\infty}\left(\mathbb{R}^{n}\right)$ and $a=f: \mathbb{R}^{n} \rightarrow \mathbb{R}$ is such that $\neg(f \equiv 0)$. By the Theorem 5.0.6. $A\left\{a^{-1}\right\} \cong \mathcal{C}^{\infty}\left(\mathbb{R}^{n}\right)\left\{f^{-1}\right\}$, and $\mathcal{C}^{\infty}\left(\mathbb{R}^{n}\right)\left\{f^{-1}\right\} \cong \mathcal{C}^{\infty}\left(U_{f}\right)$, where $U_{f}=\operatorname{Coz}(f)=\mathbb{R}^{n} \backslash Z(f)$. For every $x \in U_{f}$, we have a maximal ideal:

$$
\mathfrak{m}_{x}=\left\{g \in \mathcal{C}^{\infty}\left(U_{f}\right) \mid g(x)=0\right\},
$$

hence a continuum of maximal ideals.

Proposition 2.1.31. Let $A$ be $a \mathcal{C}^{\infty}$-ring, $a, b, c \in A$ be three elements of its underlying subset and:

$$
\eta_{b, A}^{\infty}: A \rightarrow A\left\{b^{-1}\right\}
$$

the $\mathcal{C}^{\infty}$-ring of fractions of $A$ with respect to $b$ and

$$
\eta_{b c}^{\infty}: A \rightarrow A\left\{(b \cdot c)^{-1}\right\}
$$

the $\mathcal{C}^{\infty}$-ring of fractions of $A$ with respect to $b \cdot c$.

There is an isomorphism between $\left(A\left\{b^{-1}\right\}\right)\left\{\eta_{b, A}^{\infty}(c)^{-1}\right\}$ and $A\left\{(b \cdot c)^{-1}\right\}$.

Proof. Consider the following diagram:

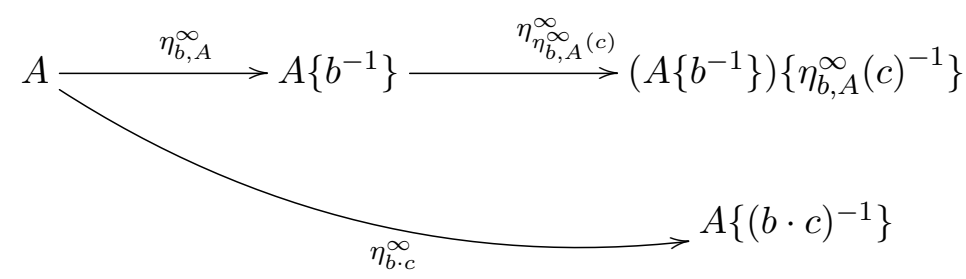

Note that $\eta_{b \cdot c}^{\infty}(b) \in\left(A\left\{(b \cdot c)^{-1}\right\}\right)^{\times}$, for since $\eta_{b \cdot c}^{\infty}(b \cdot c) \in\left(A\left\{(b \cdot c)^{-1}\right\}\right)^{\times}$, there is some $\psi \in A\left\{(b \cdot c)^{-1}\right\}$ such that:

$$
\eta_{b \cdot c}^{\infty}(b \cdot c) \cdot \psi=1
$$

so

$$
\eta_{b \cdot c}^{\infty}(b) \cdot\left[\eta_{b \cdot c}^{\infty}(c) \cdot \psi\right]=1
$$

and $\eta_{b \cdot c}^{\infty}(c) \cdot \psi$ is an inverse of $\eta_{b \cdot c}^{\infty}(b)$ in $A\left\{(b \cdot c)^{-1}\right\}$.

By the universal property of $\eta_{b, A}^{\infty}: A \rightarrow A\left\{b^{-1}\right\}$, there is a unique morphism $\zeta: A\left\{b^{-1}\right\} \rightarrow A\left\{(b \cdot c)^{-1}\right\}$ such that the following triangle commutes:

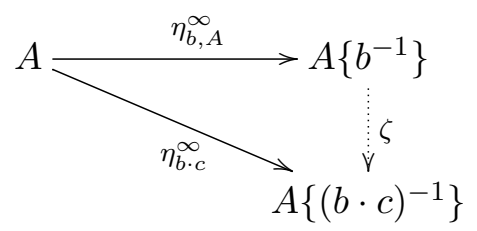


Now we can use a similar argument to conclude that $\eta_{b \cdot c}^{\infty}(c) \in\left(A\left\{(b \cdot c)^{-1}\right\}\right)^{\times}$. Since the above triangle commutes, it follows that:

$$
\zeta\left(\eta_{b, A}^{\infty}(c)\right)=\eta_{b \cdot c}^{\infty}(c) \in\left(A\left\{(b \cdot c)^{-1}\right\}\right)^{\times} .
$$

By the universal property of $\eta_{\eta_{b, A}^{\infty}(c)}^{\infty}: A\left\{b^{-1}\right\} \rightarrow\left(A\left\{b^{-1}\right\}\right)\left\{\eta_{b, A}(c)^{-1}\right\}$, there is a unique morphism $\theta:\left(A\left\{b^{-1}\right\}\right)\left\{\eta_{b, A}^{\infty}(c)^{-1}\right\} \rightarrow A\left\{(b \cdot c)^{-1}\right\}$ such that the following diagram commutes:

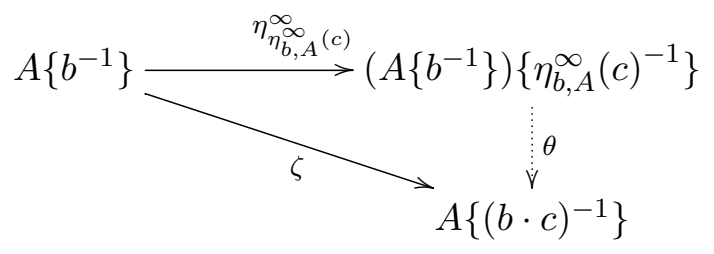

hence $\theta$ is the unique $\mathcal{C}^{\infty}$-homomorphism such that the following triangle commutes:

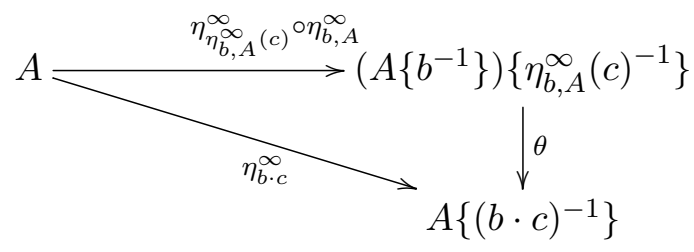

Now we are going to prove that $\eta_{\eta_{b, A}^{\infty}(c)}^{\infty} \circ \eta_{b, A}^{\infty}(b \cdot c) \in\left[\left(A\left\{b^{-1}\right\}\right)\left\{\eta_{b, A}^{\infty}(c)^{-1}\right\}\right]^{\times}$.

We have $\eta_{b, A}^{\infty}(b) \in\left(A\left\{b^{-1}\right\}\right)^{\times}$, hence $\eta_{\eta_{b, A}^{\infty}}\left(\eta_{b, A}^{\infty}(b)\right) \in\left[\left(A\left\{b^{-1}\right\}\right)\left\{\eta_{b, A}^{\infty}(c)^{-1}\right\}\right]^{\times}$, and by the very definition of $\eta_{\eta_{b, A}^{\infty}(c)}^{\infty}: A\left\{b^{-1}\right\} \rightarrow\left(A\left\{b^{-1}\right\}\right)\left\{\eta_{b, A}^{\infty}(c)^{-1}\right\}, \eta_{\eta_{b, A}^{\infty}}\left(\eta_{b, A}^{\infty}(c)\right) \in\left[\left(A\left\{b^{-1}\right\}\right)\left\{\eta_{b, A}^{\infty}(c)^{-1}\right\}\right]^{\times}$. Since each factor is invertible, it follows that $\eta_{\eta_{b, A}^{\infty}(c)}^{\infty} \circ \eta_{b, A}^{\infty}(b) \cdot \eta_{\eta_{b, A}^{\infty}(c)}^{\infty} \circ \eta_{b, A}^{\infty}(c)=\eta_{\eta_{b, A}^{\infty}(c)}^{\infty} \circ \eta_{b, A}^{\infty}(b \cdot c)$ is invertible.

By the universal property of $\eta_{b \cdot c}^{\infty}: A \rightarrow A\left\{(b \cdot c)^{-1}\right\}$, there is a unique morphism $\xi: A\left\{(b \cdot c)^{-1}\right\} \rightarrow$ $\left(A\left\{b^{-1}\right\}\right)\left\{\eta_{b, A}^{\infty}(c)^{-1}\right\}$ such that the following triangle commutes:

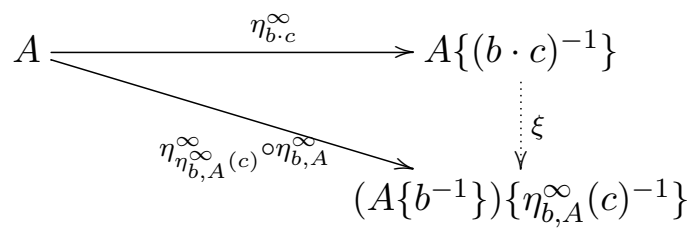

By the uniqueness of the $\mathcal{C}^{\infty}$-homomorphisms $\zeta$ and $\xi$, we conclude $\zeta \circ \xi=\operatorname{id}_{A\left\{(b \cdot c)^{-1}\right\}}$ and $\xi \circ \zeta=$ $\operatorname{id}_{\left(A\left\{b^{-1}\right\}\right)\left\{\eta_{b, A}(c)^{-1}\right\}}$, so the result follows.

In the same line of the previous proposition, we can prove the following:

Proposition 2.1.32. Let $A$ be $a \mathcal{C}^{\infty}$-ring and let $a \in A$ and $\beta \in A\left\{a^{-1}\right\}$. Since $\beta=\eta_{a}^{A}(b) / \eta_{a}^{A}(c)$ for some $b \in A$ and $c \in\{a\}^{\infty-s a t}$, then there is a unique $\mathcal{C}^{\infty}$-isomorphism of A-algebras:

$$
\theta_{a b}:\left(A\left\{a^{-1}\right\}\right)\left\{\beta^{-1}\right\} \stackrel{\cong}{\longrightarrow} A\left\{(a \cdot b)^{-1}\right\}
$$

I.e., $\theta_{a b}:\left(A\left\{a^{-1}\right\}\right)\left\{\beta^{-1}\right\} \rightarrow A\left\{(a \cdot b)^{-1}\right\}$ is a $\mathcal{C}^{\infty}$-rings isomorphism such that the following diagram commutes: 


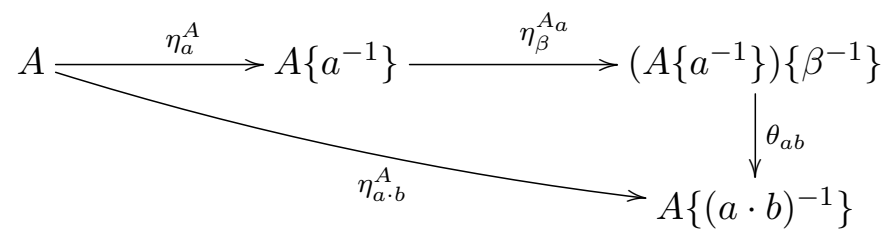

that is, $\left(\eta_{a \cdot b}^{A}: A \rightarrow A\left\{(a \cdot b)^{-1}\right\}\right) \cong\left(\eta_{\beta}^{A_{a}} \circ \eta_{a}^{A}: A \rightarrow\left(A\left\{a^{-1}\right\}\right)\left\{\beta^{-1}\right\}\right)$ in $A \downarrow \mathcal{C}^{\infty} \mathbf{R n g}_{\mathrm{fp}}$. Hence:

$$
\left\langle\left\{\eta_{a \cdot b}: A \rightarrow A\left\{(a \cdot b)^{-1}\right\} \mid a, b \in A\right\}\right\rangle=\left\langle\left\{\eta_{\beta} \circ \eta_{a}: A \rightarrow\left(A\left\{a^{-1}\right\}\right)\left\{\beta^{-1}\right\} \mid a, b \in A\right\}\right\rangle .
$$

Remark 2.1.33. In the context of Proposition 2.1.29, note that if $f \in \mathcal{C}^{\infty}\left(\mathbb{R}^{n}\right)$ is such that $U=U_{f}$, we have $S_{U_{f}}=\{f\}^{\infty-\text { sat }}$.

Now we are ready to prove Theorem $\mathbf{2 . 1 . 2 4}$.

Proof of Theorem 2.1.24: Let $A$ be a $\mathcal{C}^{\infty}$-ring. We are going to prove the result first for free $\mathcal{C}^{\infty}$-rings, then for quotients and finally for colimits.

The case where $A=\mathcal{C}^{\infty}\left(\mathbb{R}^{n}\right)$ was proved in Proposition 2.1.29.

Suppose, now, that $A=\frac{\mathcal{C}^{\infty}\left(\mathbb{R}^{n}\right)}{I}$ for some ideal $I$. By Corollary 2.1.45 it follows that:

$$
\frac{\mathcal{C}^{\infty}\left(\mathbb{R}^{n}\right)}{I}\left\{f+I^{-1}\right\} \cong \frac{\mathcal{C}^{\infty}\left(\mathbb{R}^{n}\right)\left\{f^{-1}\right\}}{\left\langle\eta_{f}[I]\right\rangle}
$$

so items (i) and (ii) of Theorem $\mathbf{2 . 1 . 2 5}$ hold for the quotient.

Up to this point, we have proved the result for a finitely generated $\mathcal{C}^{\infty}$-ring, $A$, and $\Sigma=\{f\}$. Given any finite $S$, say $S=\left\{f_{1}, \cdots, f_{\ell}\right\}$, since $A\left\{S^{-1}\right\}=A\left\{\prod_{i=1}^{\ell} f_{i}^{-1}\right\}$, the result follows too.

Let $A$ be a finitely generated $\mathcal{C}^{\infty}$-ring and $S \subseteq A$ be any set. Write:

$$
S=\bigcup_{S^{\prime} \subseteq \text { fin } S} S^{\prime}
$$

Since $A\left\{S^{-1}\right\} \cong \lim _{S^{\prime} \subseteq S} A\left\{S^{\prime-1}\right\}$, the items (i) and (ii) hold for $A\left\{S^{-1}\right\}$, for a finitely generated $\mathcal{C}^{\infty}$-ring $A$ and any set $S$.

Finally, given any $\mathcal{C}^{\infty}$-ring $B$ and any set $S \subseteq B$, write $B$ as a directed colimit of its finitely generated $\mathcal{C}^{\infty}$-subrings

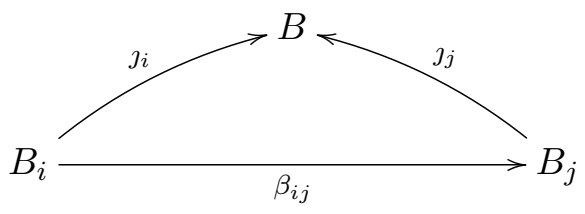

so $B \cong \lim _{B_{i} \subseteq_{\text {f.g. }} B} B_{i}$ and define $S_{i}=\jmath_{i}^{\dashv}[S]$. Since items (i) and (ii) hold for every $B_{i}\left\{S_{i}^{-1}\right\}$, the same is true for 


$$
B\left\{S^{-1}\right\} \cong \underset{B_{i} \underline{\lim }_{\mathrm{fin}} B}{ } B_{i}\left\{S_{i}^{-1}\right\}
$$

and the result follows.

\subsubsection{A Category of Pairs}

Let us now consider the category whose objects are pairs $(A, S)$, where $A$ is any $\mathcal{C}^{\infty}$-ring and $S \subseteq A$ is any of its subsets, and whose morphisms between $(A, S)$ and $(B, T)$ are precisely the $\mathcal{C}^{\infty}$-morphisms $f: A \rightarrow B$ such that $f[S] \subseteq T$. In order to distinguish the latter morphism of the category of pairs from the morphisms of $\mathcal{C}^{\infty} \mathbf{R n g}$, we shall denote it by $f_{S T}:(A, S) \rightarrow(B, T)$. We denote this category by $\mathcal{C}_{2}^{\infty}$.

In this section we are going to define a functor $F: \mathcal{C}_{2}^{\infty} \rightarrow \mathcal{C}^{\infty} \mathbf{R n g}$ with some "nice" properties.

Given $(A, S) \in \operatorname{Obj}\left(\mathcal{C}_{2}^{\infty}\right)$, let:

$$
F(A, S)=A\left\{S^{-1}\right\}
$$

Also, given $(A, S),(B, T) \in \operatorname{Obj}\left(\mathcal{C}_{2}^{\infty}\right)$ and $(A, S) \stackrel{f_{S T}}{\rightarrow}(B, T) \in \operatorname{Mor}\left(\mathcal{C}_{2}^{\infty}\right)$, since $f_{S T}[S] \subseteq T$ and $\eta_{T}[T] \subseteq B\left\{T^{-1}\right\}^{\times}$, we have $\left(\eta_{T} \circ f_{S T}\right)[S] \subseteq B\left\{T^{-1}\right\}^{\times}$, so by the universal property of $\eta_{S}: A \rightarrow A\left\{S^{-1}\right\}$ there is a unique $\mathcal{C}^{\infty}$-homomorphism:

$$
\widetilde{f_{S T}}: A\left\{S^{-1}\right\} \rightarrow B\left\{T^{-1}\right\}
$$

such that the following diagram commutes:

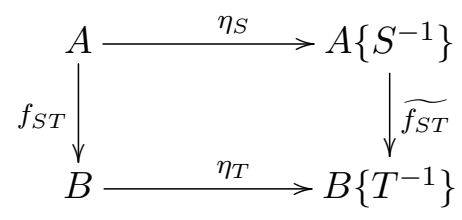

Let:

$$
F\left((A, S) \stackrel{f_{S T}}{\rightarrow}(B, T)\right):=A\left\{S^{-1}\right\} \stackrel{\widetilde{f_{S T}}}{\rightarrow} B\left\{T^{-1}\right\} .
$$

Theorem 2.1.34. The map:

$$
\begin{array}{cccc}
F: & \mathcal{C}_{2}^{\infty} & \rightarrow & \mathcal{C}^{\infty} \text { Rng } \\
(A, S) & \mapsto & A\left\{S^{-1}\right\} \\
(A, S) \stackrel{f_{S T}}{\longrightarrow}(B, T) & \mapsto & A\left\{S^{-1}\right\} \stackrel{\widetilde{f_{S T}}}{\longrightarrow} B\left\{T^{-1}\right\}
\end{array}
$$

is a functor.

Proof. Given any object $(A, S) \in \operatorname{Obj}\left(\mathcal{C}_{2}^{\infty}\right)$ and its identity map $\operatorname{id}_{(A, S)}:(A, S) \rightarrow(A, S), F\left(\operatorname{id}_{(A, S)}\right)$ is the unique $\mathcal{C}^{\infty}$-homomorphism such that the following rectangle commutes:

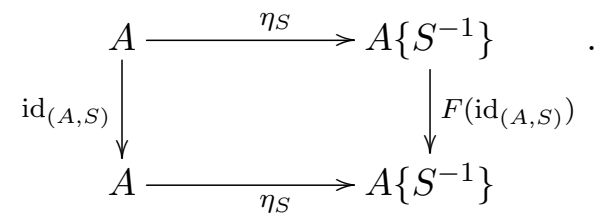


Since:

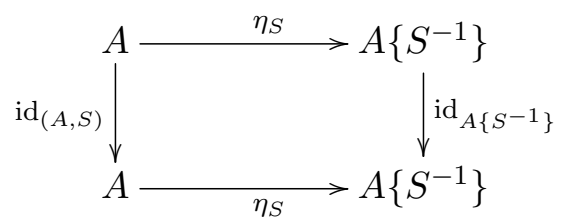

commutes, it follows that $F\left(\operatorname{id}_{(A, S)}\right)=\operatorname{id}_{A\left\{S^{-1}\right\}}$.

Let $(A, S),(B, T),(C, R) \in \operatorname{Obj}\left(\mathcal{C}_{2}^{\infty}\right)$ and $(A, S) \stackrel{f_{S T}}{\rightarrow}(B, T),(B, T) \stackrel{g_{T R}}{\rightarrow}(C, R) \in \operatorname{Mor}\left(\mathcal{C}_{2}^{\infty}\right)$. By definition, $F\left(f_{S T}\right): A\left\{S^{-1}\right\} \rightarrow B\left\{T^{-1}\right\}$ is the unique $\mathcal{C}^{\infty}$-homomorphism such that:

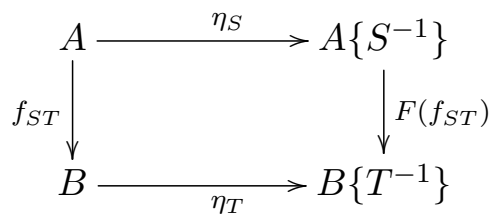

commutes, and $F\left(g_{T R}\right): B\left\{T^{-1}\right\} \rightarrow C\left\{R^{-1}\right\}$ is the unique $\mathcal{C}^{\infty}$-homomorphism such that:

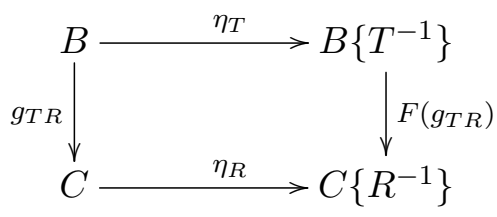

commutes. It follows that the following diagram commutes:

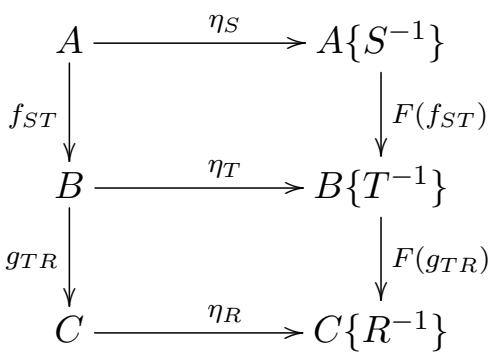

so

$$
\left[F\left(g_{T R}\right) \circ F\left(f_{S T}\right)\right] \circ \eta_{S}=\eta_{R} \circ\left(g_{T R} \circ f_{S T}\right)
$$

Also by definition, $F\left(g_{T R} \circ f_{S T}\right): A\left\{S^{-1}\right\} \rightarrow C\left\{R^{-1}\right\}$ is the unique $\mathcal{C}^{\infty}$-homomorphism such that:

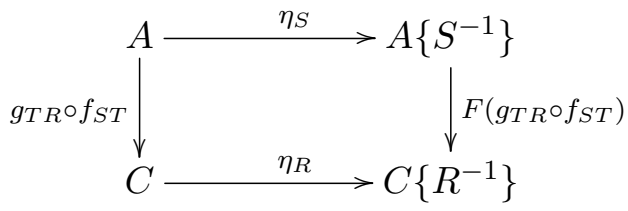

commutes, that is, such that

$$
F\left(g_{T R} \circ f_{S T}\right) \circ \eta_{S}=\eta_{R} \circ\left(g_{T R} \circ f_{S T}\right)
$$

By the uniqueness of the $\mathcal{C}^{\infty}$-homomorphism that satisfies $(2.3)$ and $(2.4)$, it follows that:

$$
F\left(g_{T R} \circ f_{S T}\right)=F\left(g_{T R}\right) \circ F\left(f_{S T}\right) .
$$

Hence $F$ is a functor. 
Proposition 2.1.35. Let $A$ and $B$ be two $\mathcal{C}^{\infty}$-rings and $S \subseteq A$ and $f: A \rightarrow B$ a $\mathcal{C}^{\infty}$-homomorphism. By the universal property of $\eta_{S}: A \rightarrow A\left\{S^{-1}\right\}$ we have a unique $\mathcal{C}^{\infty}$-homomorphism $f_{S}: A\left\{S^{-1}\right\} \rightarrow$ $B\left\{f[S]^{-1}\right\}$ such that the following square commutes:

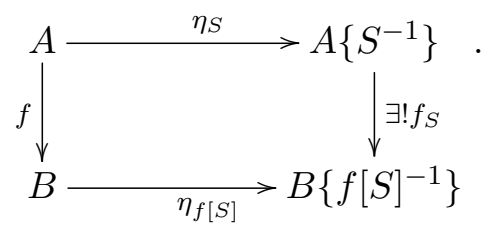

The diagram:

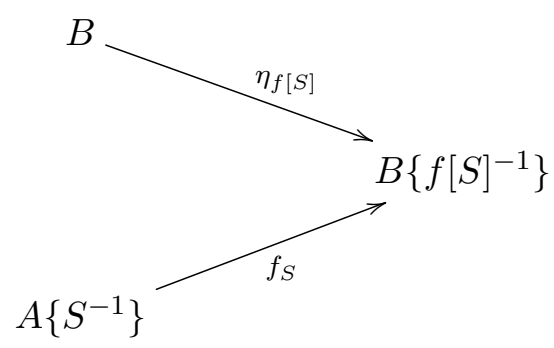

is a pushout of the diagram:

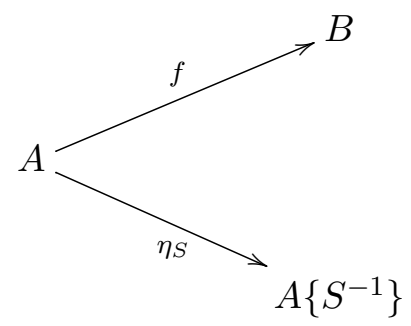

Proof. Let $C$ be any $\mathcal{C}^{\infty}$-ring and $g: B \rightarrow C$ and $h: A\left\{S^{-1}\right\} \rightarrow C$ be two $\mathcal{C}^{\infty}$-homomorphisms such that $g \circ f=h \circ \eta_{S}$, i.e., such that the following diagram commutes:

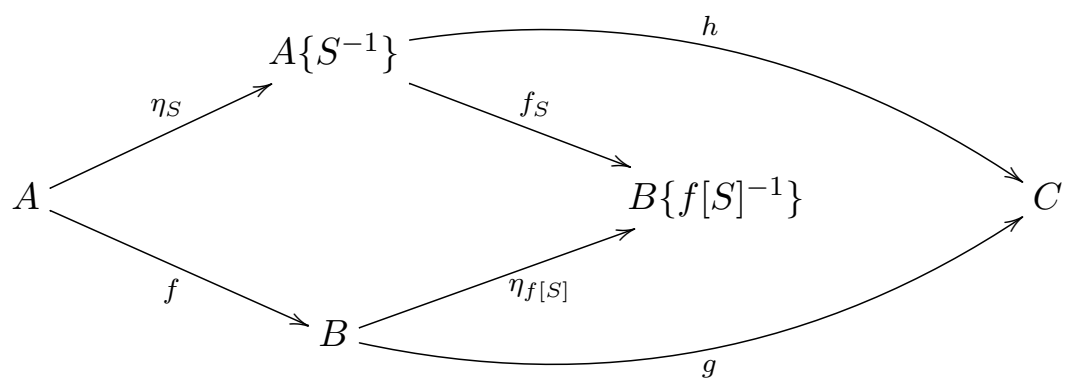

We claim that there is a unique $\mathcal{C}^{\infty}$-homomorphism $u: B\left\{f[S]^{-1}\right\} \rightarrow C$ such that the following diagram commutes:

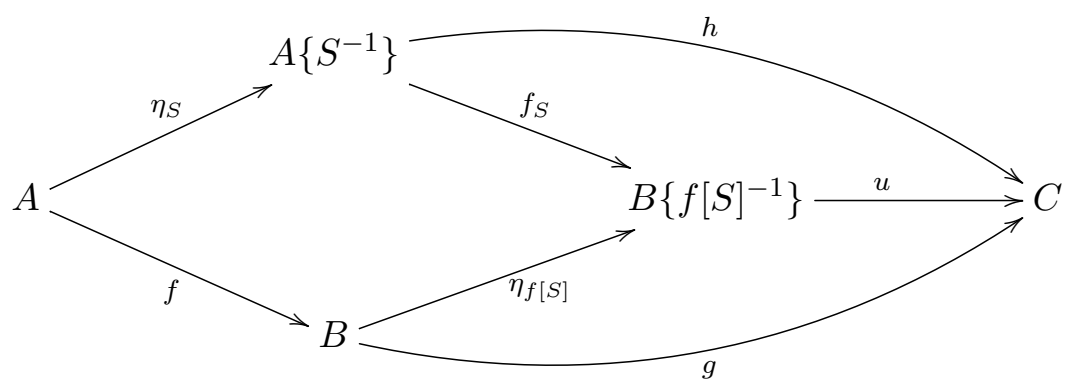

Given any $s \in S$, we have $f_{S}(s) \in B^{\times}$, so $h\left(\eta_{S}(s)\right) \in C^{\times}$. Since the former diagram commutes, $g(f(s))=h\left(\eta_{S}(s)\right) \in C^{\times}$and by the universal property of $\eta_{f[S]}: B \rightarrow B\left\{f[S]^{-1}\right\}$ there is a unique 
$\mathcal{C}^{\infty}$-homomorphism $u: B\left\{f[S]^{-1}\right\} \rightarrow C$ such that the following diagram commutes:

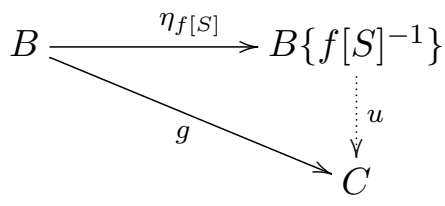

Now we need only to prove that the following triangle commutes:

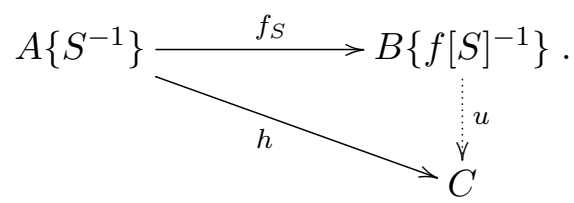

Note that, by the universal property of $\eta_{S}: A \rightarrow A\left\{S^{-1}\right\}$ there must exist a unique $\mathcal{C}^{\infty}$-homomorphism $\xi: A\left\{S^{-1}\right\} \rightarrow C$ such that:

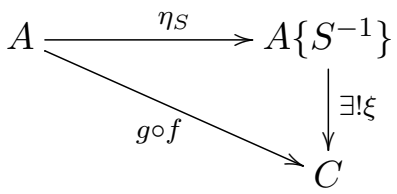

commutes, and since both $h: A\left\{S^{-1}\right\} \rightarrow C$ and $u \circ f_{S}: A\left\{S^{-1}\right\} \rightarrow C$ have this property, it follows that $h=u \circ f_{S}$.

Hence we have a pushout diagram.

Corollary 2.1.36. The following rectangle is a pushout:

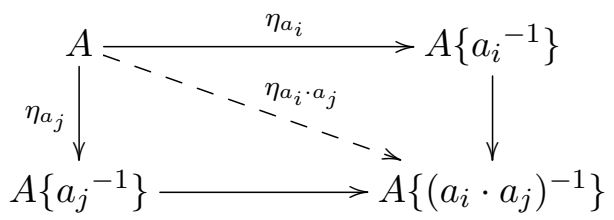

Proof. By the previous lemma, the following diagram is a pushout:

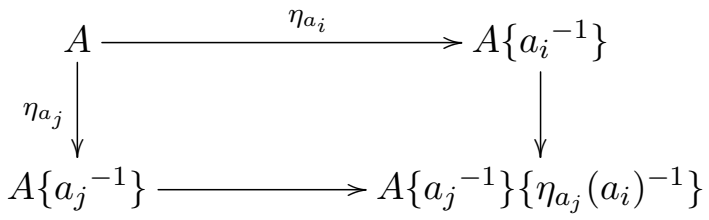

By Proposition 2.1.31, $A\left\{a_{j}^{-1}\right\}\left\{\eta_{a_{j}}\left(a_{i}\right)^{-1}\right\} \cong A\left\{\left(a_{i} \cdot a_{j}\right)^{-1}\right\}$, and the result follows.

Proposition 2.1.37. Let $(A, S)$ and $(B, T)$ be any two pairs in $\mathcal{C}_{2}^{\infty}$ and let $\jmath_{S T}:(A, S) \rightarrow(B, T)$ be a $\mathcal{C}^{\infty}$-monomorphism such that $\jmath_{S T}\left[S^{\infty-\mathrm{sat}}\right]=T^{\infty-\text { sat }}$. Under these circumstances, $\widetilde{J_{S T}}: A\left\{S^{-1}\right\} \rightarrow$ $B\left\{T^{-1}\right\}$ is also a $\mathcal{C}^{\infty}$-monomorphism.

Proof. Let $\theta \in A\left\{S^{-1}\right\}$ be such that $\widetilde{J_{S T}}(\theta)=0$. Since $\theta \in A\left\{S^{-1}\right\}$, there are $c \in A$ and $d \in S^{\infty-\text { sat }}$ such that $\theta=\frac{\eta_{S}(c)}{\eta_{S}(d)}$, so:

$$
\widetilde{\jmath_{S T}}\left(\eta_{S}(c) \cdot \eta_{S}(d)^{-1}\right)=\widetilde{\jmath_{S T}}\left(\eta_{S}(c)\right) \cdot \widetilde{\jmath_{S T}}\left(\eta_{S}(d)\right)^{-1}=\eta_{T}\left(\jmath_{S T}(c)\right) \cdot \eta_{T}\left(\jmath_{S T}(d)\right)^{-1}
$$


Thus

$$
\widetilde{\jmath_{S T}}\left(\eta_{S}(c) \cdot \eta_{S}(d)^{-1}\right)=0 \Rightarrow \eta_{T}\left(\jmath_{S T}(c)\right)=0
$$

Since $\eta_{T}\left(\jmath_{S T}(c)\right)=0$, there is some $u \in T^{\infty-\text { sat }}$ such that $u \cdot \jmath_{S T}(c)=0$.

By hypothesis, $\jmath_{S T}\left[S^{\infty-\mathrm{sat}}\right]=T^{\infty-\mathrm{sat}}$, so given $u \in T^{\infty-\mathrm{sat}}$, there is some $v \in S^{\infty-\text { sat }}$ such that $u=\jmath_{S T}(v)$. Thus,

$$
\jmath_{S T}(v \cdot c)=\jmath_{S T}(v) \cdot \jmath_{S T}(c)=u \cdot \jmath_{S T}(c)=0 .
$$

Since, by hypothesis, $\jmath_{S T}$ is a monomorphism, it follows that $v \cdot c=0$, so $\eta_{S}(v \cdot c)=\eta_{S}(v) \cdot \eta_{S}(c)=0$ with $v \in S^{\infty-\text { sat }}$. We have, thus, $\eta_{S}(c)=0$ and, therefore:

$$
\theta=\frac{\eta_{S}(c)}{\eta_{S}(d)}=0
$$

and $\widetilde{J_{S T}}$ is a $\mathcal{C}^{\infty}$-monomorphism.

Theorem 2.1.38. Let $\left(A_{1}, S_{1}\right)$ and $\left(A_{2}, S_{2}\right)$ be two pairs in $\operatorname{Obj}\left(\mathcal{C}_{2}^{\infty}\right), \jmath_{1}: S_{1} \hookrightarrow A_{1}$ and $\jmath_{2}: S_{2} \hookrightarrow A_{2}$ be the inclusion maps and let:

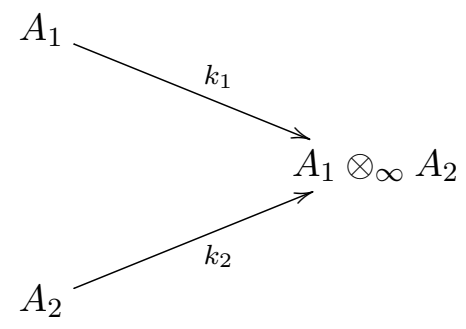

be the coproduct of $A_{1}$ and $A_{2}$ in $\mathcal{C}^{\infty} \mathbf{R n g}$. We have:

$$
\left(A_{1} \otimes_{\infty} A_{2}\right)\left\{\left(k_{1}\left[S_{1}\right] \cup k_{2}\left[S_{2}\right]\right)^{-1}\right\} \cong A_{1}\left\{S_{1}^{-1}\right\} \otimes_{\infty} A_{2}\left\{S_{2}^{-1}\right\},
$$

that is,

$$
F\left(A_{1}, S_{1}\right) \otimes_{\infty} F\left(A_{2}, S_{2}\right) \cong F\left(A_{1} \otimes_{\infty} A_{2}, k_{1}\left[S_{1}\right] \cup k_{2}\left[S_{2}\right]\right)
$$

Proof. Let:

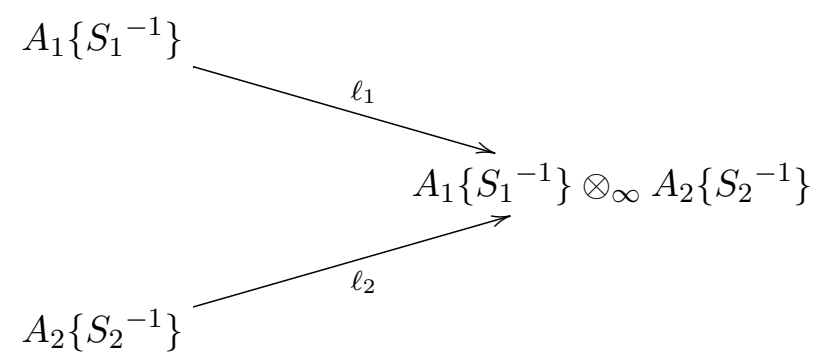

be the coproduct of $A_{1}\left\{S_{1}^{-1}\right\}$ and $A_{2}\left\{S_{2}^{-1}\right\}$.

Let $\eta_{k_{1}\left[S_{1}\right] \cup k_{2}\left[S_{2}\right]}: A_{1} \otimes_{\infty} A_{2} \rightarrow\left(A_{1} \otimes_{\infty} A_{2}\right)\left\{\left(k_{1}\left[S_{1}\right] \cup k_{2}\left[S_{2}\right]\right)^{-1}\right\}$ be the canonical map of the ring of fractions of $A_{1} \otimes_{\infty} A_{2}$ with respect to $k_{1}\left[S_{1}\right] \cup k_{2}\left[S_{2}\right]$. Since for every $s_{1} \in S_{1}$ we have $k_{1}\left(s_{1}\right) \in k_{1}\left[S_{1}\right] \subseteq$ $k_{1}\left[S_{1}\right] \cup k_{2}\left[S_{2}\right],\left(\eta_{k_{1}\left[S_{1}\right] \cup k_{2}\left[S_{2}\right]} \circ k_{1}\right)\left(s_{1}\right)=\eta_{k_{1}\left[S_{1}\right] \cup k_{2}\left[S_{2}\right]}\left(k_{1}\left(s_{1}\right)\right) \in\left(\left(A_{1} \otimes_{\infty} A_{2}\right)\left\{k_{1}\left[S_{1}\right] \cup k_{2}\left[S_{2}\right]^{-1}\right\}\right)^{\times}$, so the universal property of $\eta_{S_{1}}: A_{1} \rightarrow A_{1}\left\{S_{1}^{-1}\right\}$ there is a unique $\varphi_{1}: A_{1}\left\{S_{1}^{-1}\right\} \rightarrow\left(A_{1} \otimes_{\infty} A_{2}\right)\left\{k_{1}\left[S_{1}\right] \cup\right.$ 
$\left.k_{2}\left[S_{2}\right]^{-1}\right\}$ such that the following triangle commutes:

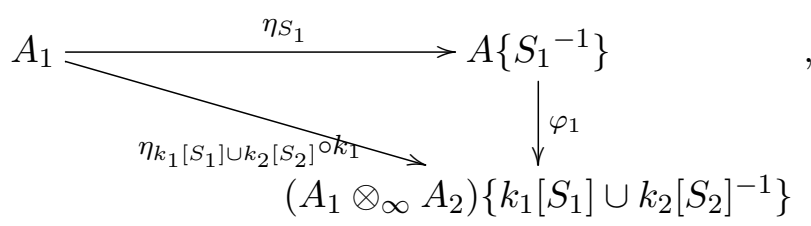

that is, $\varphi_{1} \circ \eta_{S_{1}}=\eta_{k_{1}\left[S_{1}\right] \cup k_{2}\left[S_{2}\right]}$.

The same argument yields a unique $\varphi_{2}: A_{2}\left\{S_{2}{ }^{-1}\right\} \rightarrow A_{1} \otimes_{\infty} A_{2}\left\{k_{1}\left[S_{1}\right] \cup k_{2}\left[S_{2}\right]^{-1}\right\}$ such that the following triangle commutes:

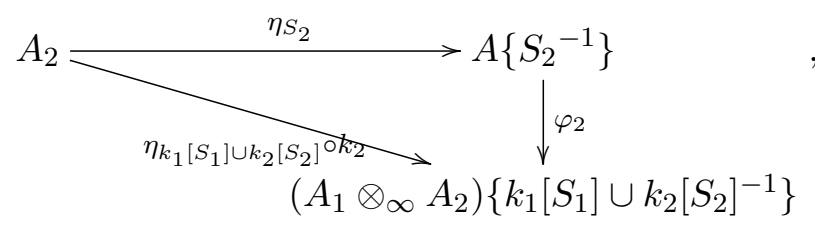

that is, $\varphi_{2} \circ \eta_{S_{2}}=\eta_{k_{1}\left[S_{1}\right] \cup k_{2}\left[S_{2}\right]}$.

By the universal property of the coproduct of $A_{1}\left\{S_{1}{ }^{-1}\right\} \otimes_{\infty} A_{2}\left\{S_{2}{ }^{-1}\right\}$, given the diagram:

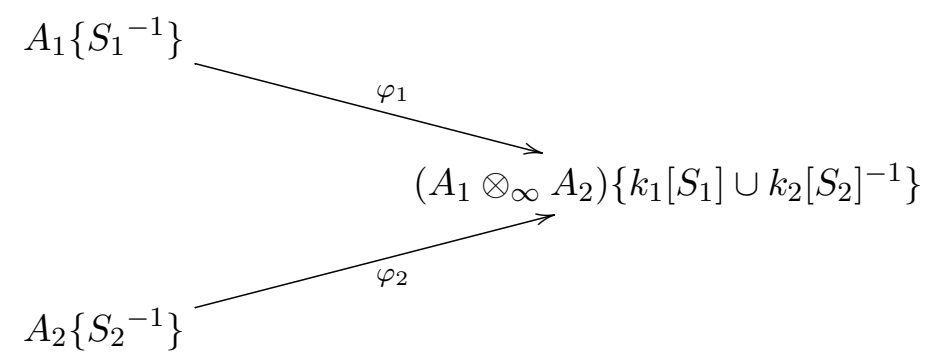

there is a unique $\mathcal{C}^{\infty}$-homomorphism $\widetilde{\varphi}: A_{1}\left\{S_{1}^{-1}\right\} \otimes_{\infty} A_{2}\left\{S_{2}^{-1}\right\} \rightarrow A_{1} \otimes_{\infty} A_{2}\left\{k_{1}\left[S_{1}\right] \cup k_{2}\left[S_{2}\right]^{-1}\right\}$ such that the following diagram commutes:

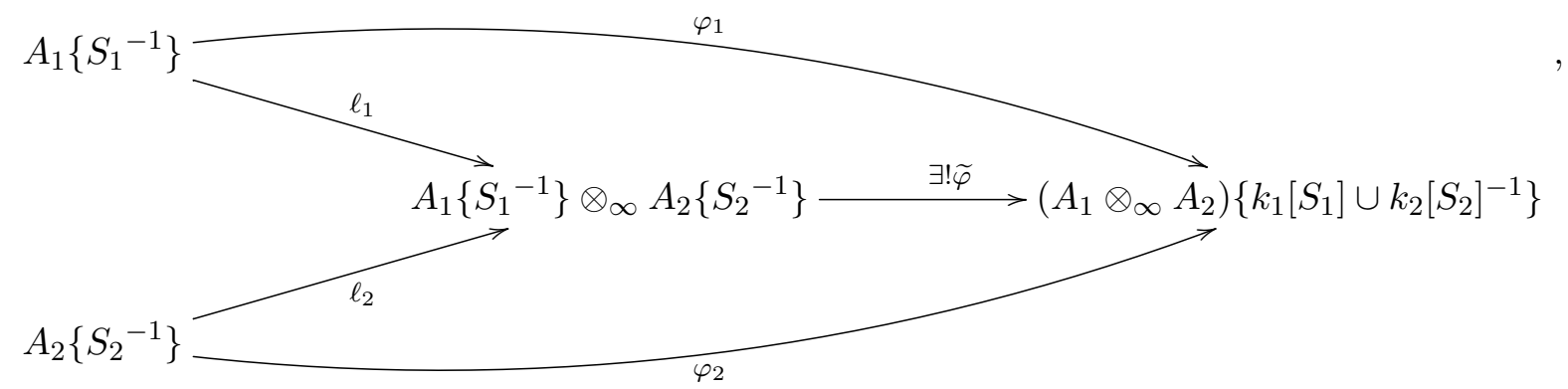

that is, such that $\widetilde{\varphi} \circ \ell_{i}=\varphi_{i}$ for $i \in\{1,2\}$.

Given the diagram:

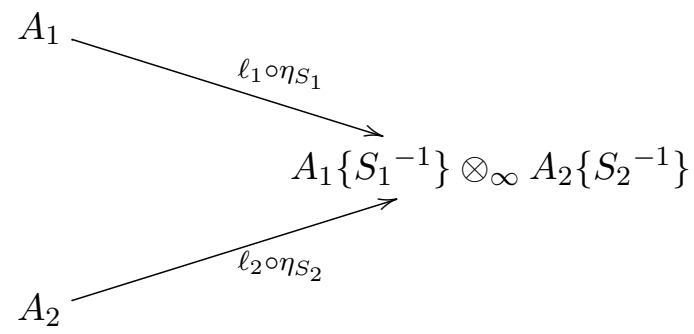


the universal property of the coproduct $A_{1} \otimes_{\infty} A_{2}$, there is a unique $\mathcal{C}^{\infty}$-homomorphism $\widetilde{\psi}: A_{1} \otimes_{\infty}$ $A_{2} \rightarrow A_{1}\left\{S_{1}^{-1}\right\} \otimes_{\infty} A\left\{S_{2}^{-1}\right\}$ such that the following diagram commutes:

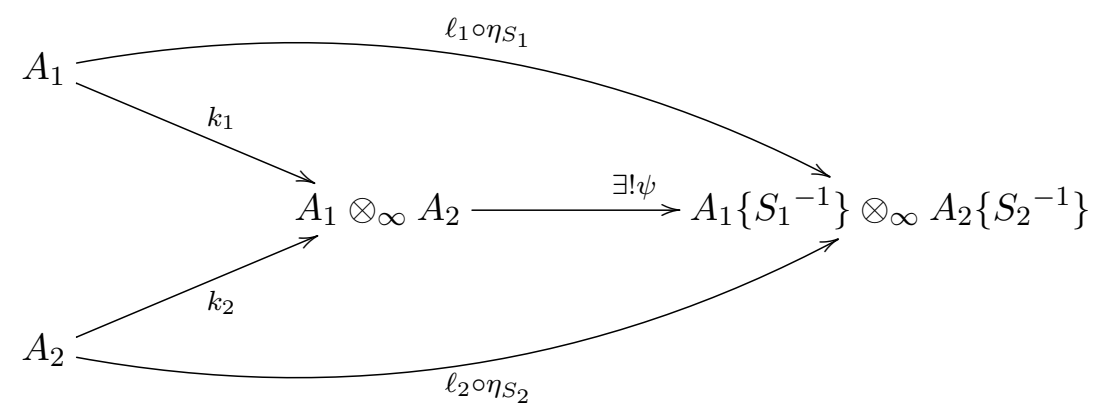

that is, such that $\psi \circ k_{1}=\ell_{1} \circ \eta_{S_{1}}$ and $\psi \circ k_{2}=\ell_{2} \circ \eta_{S_{2}}$.

Given any $y \in k_{1}\left[S_{1}\right] \cup k_{2}\left[S_{2}\right]$, then $y \in k_{1}\left[S_{1}\right]$ or $y \in k_{2}\left[S_{2}\right]$. Suppose, without loss of generality, $y \in k_{1}\left[S_{1}\right]$, so there is some $s_{1} \in S_{1}$ such that $y=k_{1}\left(s_{1}\right)$. We have:

$$
\widetilde{\psi}(y)=\psi\left(k_{1}\left(s_{1}\right)\right)=\ell_{1} \circ \eta_{S_{1}}\left(s_{1}\right)=\ell_{1}\left(\eta_{S_{1}}\left(s_{1}\right)\right) \in\left(A_{1}\left\{S_{1}^{-1}\right\} \otimes_{\infty} A_{2}\left\{S_{2}^{-1}\right\}\right)^{\times},
$$

and the universal property of $\eta_{k_{1}\left[S_{1}\right] \cup k_{2}\left[S_{2}\right]}: A_{1} \otimes_{\infty} A_{2} \rightarrow\left(A_{1} \otimes_{\infty} A_{2}\right)\left\{k_{1}\left[S_{1}\right] \cup k_{2}\left[S_{2}\right]^{-1}\right\}$, there is a unique $\widetilde{\psi}:\left(A_{1} \otimes_{\infty} A_{2}\right)\left\{k_{1}\left[S_{1}\right] \cup k_{2}\left[S_{2}\right]^{-1}\right\} \rightarrow A_{1}\left\{S_{1}{ }^{-1}\right\} \otimes_{\infty} A_{2}\left\{S_{2}^{-1}\right\}$ such that the following triangle commutes:

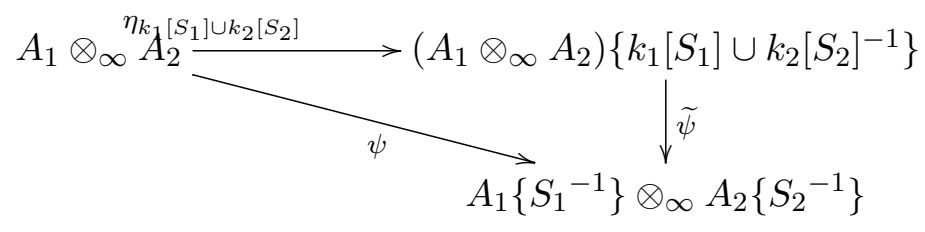

Claim:

$$
\widetilde{\varphi} \circ \widetilde{\psi}=\operatorname{id}_{\left(A_{1} \otimes_{\infty} A_{2}\right)\left\{\left(k_{1}\left[S_{1}\right] \cup k_{2}\left[S_{2}\right]\right)^{-1}\right\}}
$$

In fact, we are going to prove that

$$
(\widetilde{\varphi} \circ \widetilde{\psi}) \circ \eta_{k_{1}\left[S_{1}\right] \cup k_{2}\left[S_{2}\right]} \circ k_{i}=\eta_{k_{1}\left[S_{1}\right] \cup k_{2}\left[S_{2}\right]} \circ k_{i}, i=1,2 .
$$

and since $\operatorname{id}_{\left(A_{1} \otimes_{\infty} A_{2}\right)\left\{k_{1}\left[S_{1}\right] \cup k_{2}\left[S_{2}\right]^{-1}\right\}}$ is the unique $\mathcal{C}^{\infty}$-homomorphism such that:

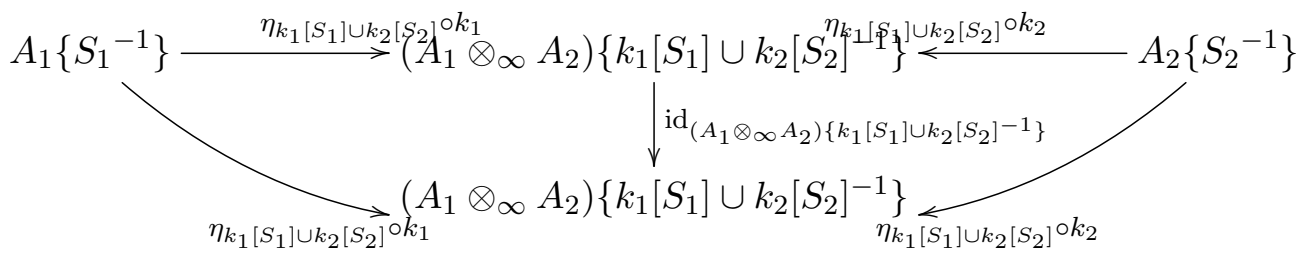

commutes, it will follow that $\widetilde{\varphi} \circ \widetilde{\psi}=\operatorname{id}_{\left(A_{1} \otimes_{\infty} A_{2}\right)\left\{k_{1}\left[S_{1}\right] \cup k_{2}\left[S_{2}\right]^{-1}\right\}}$.

By definition,

$$
\widetilde{\psi} \circ \eta_{k_{1}\left[S_{1}\right] \cup k_{2}\left[S_{2}\right]}=\psi
$$

so composing both sides with $k_{i}$ yields:

$$
\widetilde{\psi} \circ \eta_{k_{1}\left[S_{1}\right] \cup k_{2}\left[S_{2}\right]} \circ k_{i}=\psi \circ k_{i} .
$$


Since $\psi$ is such that $\psi \circ k_{i}=\ell_{i} \circ \eta_{S_{i}}$, we get:

$$
\widetilde{\psi} \circ \eta_{k_{1}\left[S_{1}\right] \cup k_{2}\left[S_{2}\right]} \circ k_{i}=\ell_{i} \circ \eta_{S_{i}} .
$$

Composing both sides of the above equation with $\widetilde{\varphi}$ we get:

$$
\widetilde{\varphi} \circ\left(\widetilde{\psi} \circ \eta_{k_{1}\left[S_{1}\right] \cup k_{2}\left[S_{2}\right]} \circ k_{i}\right)=\widetilde{\varphi} \circ\left(\ell_{i} \circ \eta_{S_{i}}\right)=\varphi_{i} \circ \eta_{S_{i}},
$$

and since $\eta_{k_{1}\left[S_{1}\right] \cup k_{2}\left[S_{2}\right]} \circ k_{i}=\varphi_{i} \circ \eta_{S_{i}}$, we have:

$$
(\widetilde{\varphi} \circ \widetilde{\psi}) \circ \eta_{k_{1}\left[S_{1}\right] \cup k_{2}\left[S_{2}\right]} \circ k_{i}=\eta_{k_{1}\left[S_{1}\right] \cup k_{2}\left[S_{2}\right]} \circ k_{i} .
$$

By the universal property of the coproduct, it follows that $\widetilde{\varphi} \circ \widetilde{\psi} \circ \eta_{k_{1}\left[S_{1}\right] \cup k_{2}\left[S_{2}\right]}=\eta_{k_{1}\left[S_{1}\right] \cup k_{2}\left[S_{2}\right]}$, and since $\eta_{k_{1}\left[S_{1}\right] \cup k_{2}\left[S_{2}\right]}: A_{1} \otimes_{\infty} A_{2} \rightarrow\left(A_{1} \otimes_{\infty} A_{2}\right)\left\{\left(k_{1}\left[S_{1}\right] \cup k_{2}\left[S_{2}\right]\right)^{-1}\right\}$ is an epimorphism, we have:

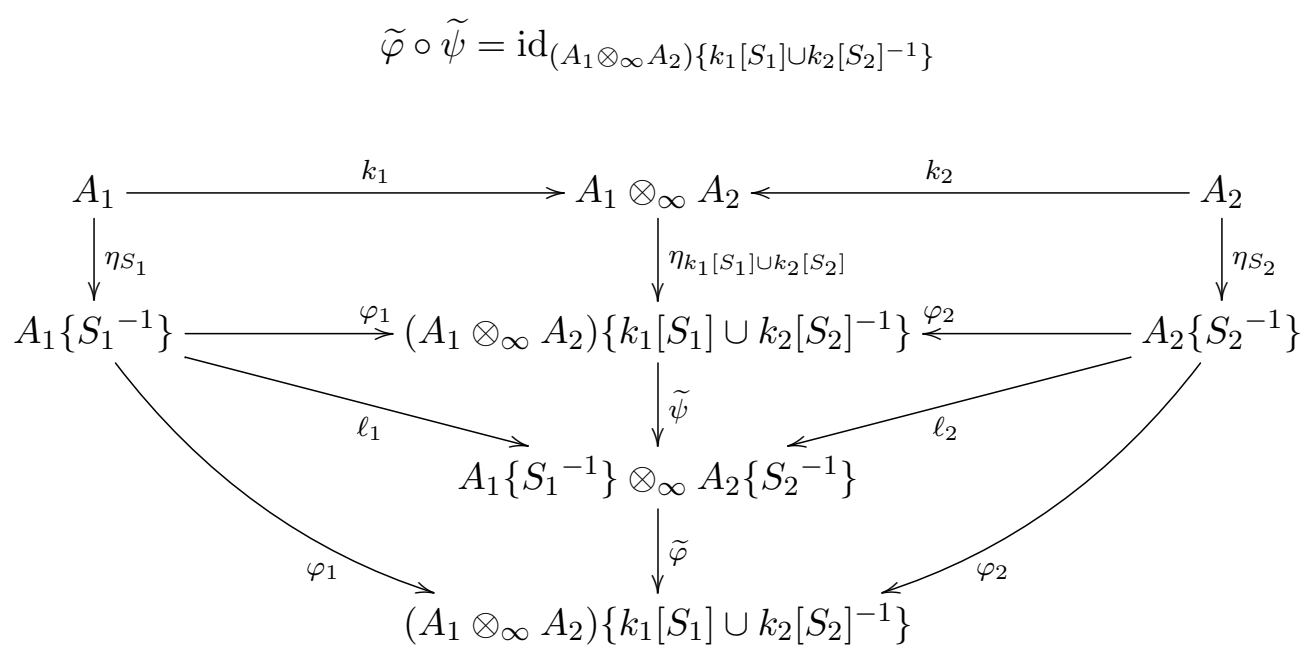

Claim: $\widetilde{\psi} \circ \widetilde{\varphi}=\operatorname{id}_{A_{1}\left\{S_{1}-1\right\} \otimes_{\infty} A_{2}\left\{S_{2}{ }^{-1}\right\}}$.

Note that, by the universal property of the coproduct:

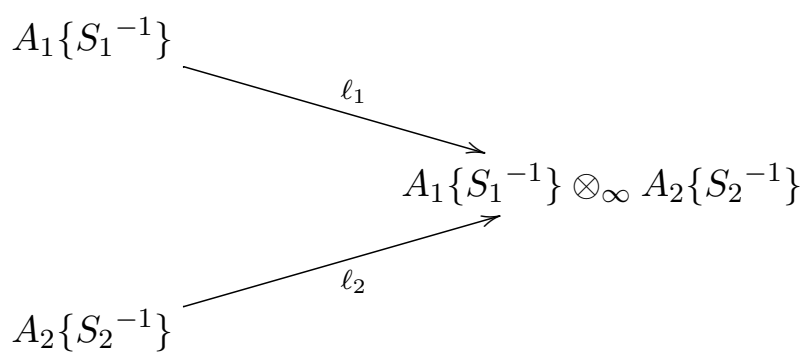

given the morphisms $\ell_{i}: A_{i}\left\{S_{i}^{-1}\right\} \rightarrow A_{1}\left\{S_{1}^{-1}\right\} \otimes_{\infty} A_{2}\left\{S_{2}^{-1}\right\}$ for $i=1,2$, there is a unique $\mathcal{C}^{\infty}$-homomorphism, namely:

$$
\operatorname{id}_{A_{1}\left\{S_{1}^{-1}\right\} \otimes_{\infty} A_{2}\left\{S_{2}^{-1}\right\}}: A_{1}\left\{S_{1}^{-1}\right\} \otimes_{\infty} A_{2}\left\{S_{2}^{-1}\right\} \rightarrow A_{1}\left\{S_{1}^{-1}\right\} \otimes_{\infty} A_{2}\left\{S_{2}^{-1}\right\}
$$

such that the following diagram commutes:

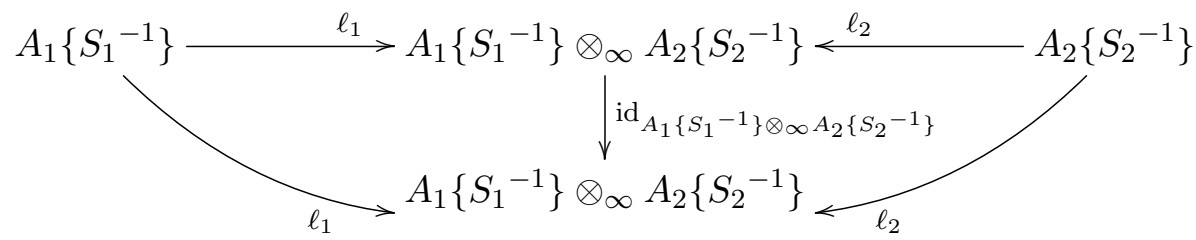


We are going to show that for $i=1,2$, we have:

$$
(\widetilde{\psi} \circ \widetilde{\varphi}) \circ \ell_{i}=\ell_{i} .
$$

We have:

$$
\widetilde{\varphi} \circ \ell_{i}=\varphi_{i}
$$

and composing both sides of the above equation with $\widetilde{\psi}$ yields:

$$
\widetilde{\psi} \circ \widetilde{\varphi} \circ \ell_{i}=\widetilde{\psi} \circ \varphi_{i} .
$$

Composing both sides of the above equation with $\eta_{S_{i}}$ yields

$$
(\widetilde{\psi} \circ \widetilde{\varphi}) \circ \ell_{i} \circ \eta_{S_{i}}=\tilde{\psi} \circ \varphi_{i} \circ \eta_{S_{i}} .
$$

Since $\varphi_{i} \circ \eta_{S_{i}}=\eta_{k_{1}\left[S_{1}\right] \cup k_{2}\left[S_{2}\right]} \circ k_{i}$, we have:

$$
(\widetilde{\psi} \circ \widetilde{\varphi}) \circ \ell_{i} \circ \eta_{S_{i}}=\widetilde{\psi} \circ \eta_{k_{1}\left[S_{1}\right] \cup k_{2}\left[S_{2}\right]} \circ k_{i}
$$

and since $\widetilde{\psi} \circ \eta_{k_{1}\left[S_{1}\right] \cup k_{2}\left[S_{2}\right]}=\psi$, it follows that

$$
(\widetilde{\psi} \circ \widetilde{\varphi}) \circ \ell_{i} \circ \eta_{S_{i}}=\psi \circ k_{i}
$$

But $\psi \circ k_{i}=\ell_{i} \circ \eta_{S_{i}}$ for $i=1,2$, so:

$$
(\widetilde{\psi} \circ \widetilde{\varphi}) \circ \ell_{i} \circ \eta_{S_{i}}=\ell_{i} \circ \eta_{S_{i}}
$$

and since $\eta_{S_{i}}$ is an epimorphism for $i=1,2$, we have:

$$
(\widetilde{\psi} \circ \widetilde{\varphi}) \circ \ell_{i}=\ell_{i}
$$

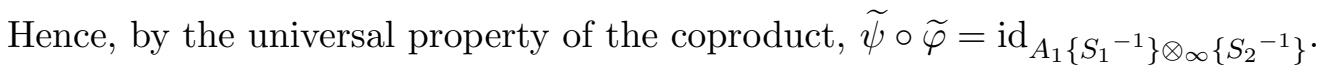

Remark 2.1.39. With an analogous proof, we can obtain a more general result: given any set of pairs, $\left\{\left(A_{i}, S_{i}\right) \mid i \in I\right\}$, we have:

$$
\bigotimes_{\infty} A_{i}\left\{S_{i}^{-1}\right\} \cong\left(\bigotimes_{\infty} A_{i}\right)\left\{\left(\bigcup_{i \in I} k_{i}\left[S_{i}\right]\right)^{-1}\right\}
$$

where $k_{i}: A_{i} \rightarrow \otimes_{\infty} A_{i}$, for each $i$, is the canonical $\mathcal{C}^{\infty}$-homomorphism into the coproduct.

Theorem 2.1.40. Let $B$ be the directed colimit of a system $\left\{A_{\ell} \stackrel{t_{\ell_{j}}}{\rightarrow} A_{j} \mid \ell, j \in I\right\}$ of $\mathcal{C}^{\infty}$-rings, that is,

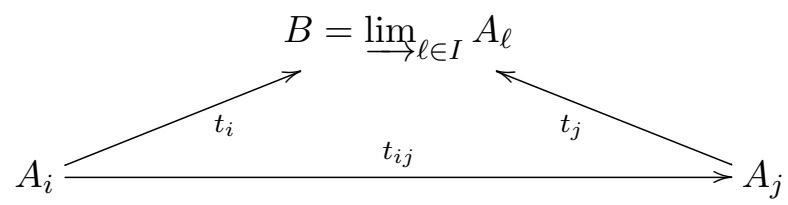

is a limit co-cone. Given any $u \in B$, there are $j \in I$ and $u_{j} \in A_{j}$ such that $t_{j}\left(u_{j}\right)=u$.

Under those circumstances, we have:

$$
\underset{k \geq j}{\lim _{k}} A_{k}\left\{u_{k}^{-1}\right\} \cong B\left\{u^{-1}\right\}
$$

where $u_{k}=t_{j k}\left(u_{j}\right)$. 
Proof. Let $\ell \geq k \geq j$. Note that for every $m \geq j$ we have $u=t_{j}\left(u_{j}\right)=t_{m} \circ t_{j m}\left(u_{j}\right):=t_{m}\left(u_{m}\right)$.

Since $\eta_{u_{\ell}} \circ t_{k \ell}\left(u_{k}\right) \in A_{\ell}\left\{u_{\ell}^{-1}\right\}^{\times}$, there is a unique $\mathcal{C}^{\infty}$-homomorphism $s_{k \ell}: A_{k}\left\{u_{k}^{-1}\right\} \rightarrow A_{\ell}\left\{u_{\ell}^{-1}\right\}$ such that:

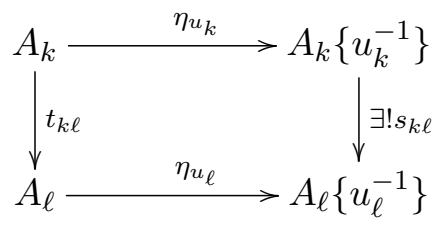

commutes.

Note also that for every $k \geq j$ we have:

$$
\eta_{u} \circ t_{k}\left(u_{k}\right)=\eta_{u}\left(t_{k}\left(u_{k}\right)\right)=\eta_{u}(u) \in B\left\{u^{-1}\right\}^{\times},
$$

so by the universal property of $\eta_{u_{k}}: A_{k} \rightarrow A_{k}\left\{u_{k}^{-1}\right\}$, there is a unique $s_{k}: A_{k}\left\{u_{k}^{-1}\right\} \rightarrow B\left\{u^{-1}\right\}$ such that the following diagram commutes:

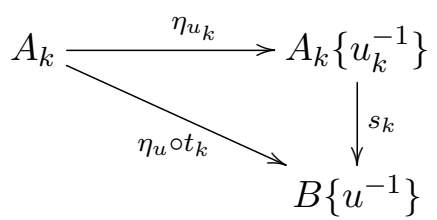

We claim that for every $\ell \geq k \geq j$ the following triangle commutes:

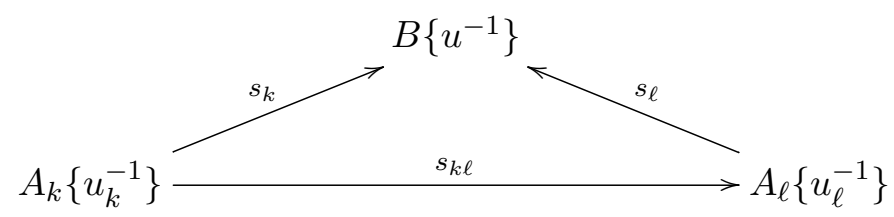

We are going to prove this by dividing our argumentation in two claims.

Claim 1: The following triangle commutes:

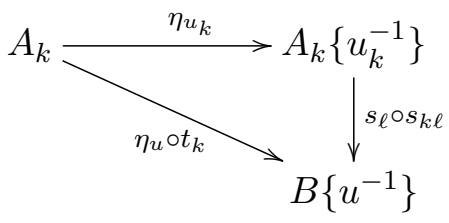

We have, for every $\ell \geq k \geq j$ :

$$
\begin{aligned}
\left(s_{\ell} \circ s_{k \ell}\right) \circ \eta_{u_{k}}=s_{\ell} \circ\left(s_{k \ell} \circ \eta_{u_{k}}\right) & =s_{\ell} \circ\left(\eta_{u_{\ell}} \circ t_{k \ell}\right)= \\
& =\left(s_{\ell} \circ \eta_{u_{\ell}}\right) \circ t_{k \ell}=\left(\eta_{u} \circ t_{\ell}\right) \circ t_{k \ell}=\eta_{u} \circ\left(t_{\ell} \circ t_{k \ell}\right)=\eta_{u} \circ t_{k}=s_{k} \circ \eta_{u_{k}}
\end{aligned}
$$

SO

$$
\left(s_{\ell} \circ s_{k \ell}\right) \circ \eta_{u_{k}}=s_{k} \circ \eta_{u_{k}}
$$

Since $\eta_{u_{k}}$ is an epimorphism, it follows that: 


$$
s_{\ell} \circ s_{k \ell}=s_{k}
$$

thus

$$
(\forall k, \ell \geq j)\left(s_{\ell} \circ s_{k \ell}=s_{k}\right)
$$

Claim 2: Given any other co-cone $\left\{v_{k}: A_{k}\left\{u_{k}^{-1}\right\} \rightarrow C \mid k \geq j\right\}$ such that for every $\ell \geq k \geq j$ :

$$
v_{\ell} \circ s_{k \ell}=v_{k}
$$

there is a unique $\mathcal{C}^{\infty}$-homomorphism:

$$
\widetilde{v}: B\left\{u^{-1}\right\} \rightarrow C
$$

such that for every $\ell \geq j$

$$
v_{\ell}=\widetilde{v} \circ s_{\ell}
$$

In other words, there is a unique $\widetilde{v}: B\left\{u^{-1}\right\} \rightarrow C$ such that the following diagram commutes:

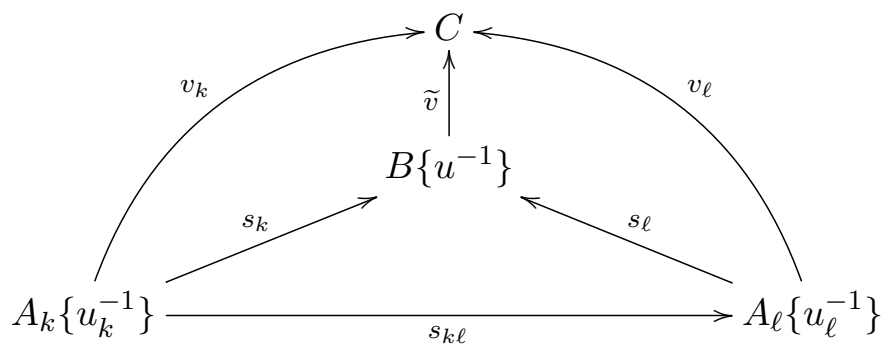

Considering the diagram:

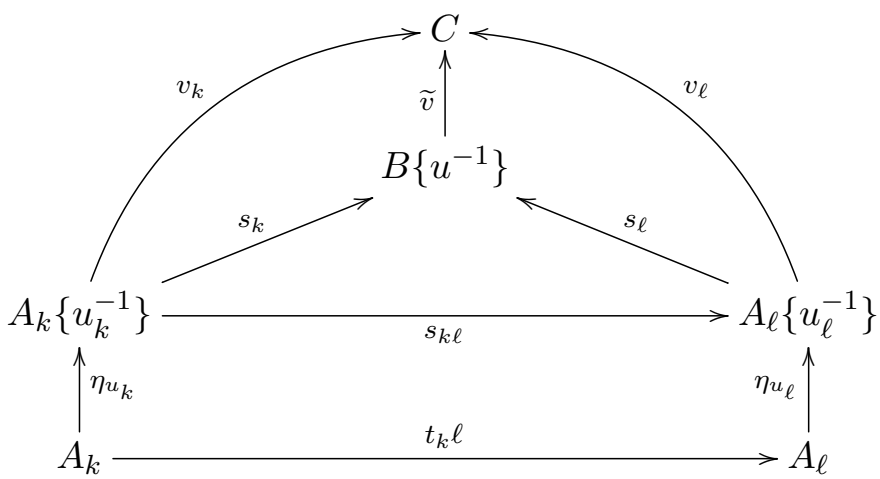

and noting that for every $\ell \geq k \geq j,\left(v_{\ell} \circ \eta_{u_{\ell}}\right) \circ t_{k \ell}=\left(v_{k} \circ \eta_{u_{k}}\right)$, by the universal property of the colimit $B$, there is a unique $\mathcal{C}^{\infty}$-homomorphism $\psi: B \rightarrow C$ such that for every $k \geq j, \psi \circ t_{k}=\left(v_{k} \circ \eta_{u_{k}}\right)$

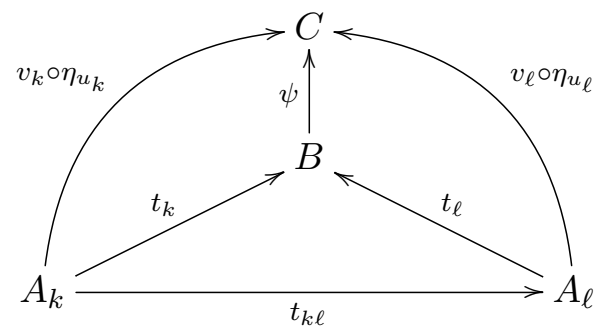

Note that $\psi(u)=\psi\left(t_{k}\left(u_{k}\right)\right)=\left(v_{k} \circ \eta_{u_{k}}\right)\left(u_{k}\right) \in C^{\times}$, for every $k \geq j$, and by the universal property of $\eta_{u}: B \rightarrow B\left\{u^{-1}\right\}$, there is a unique $\widetilde{v}: B\left\{u^{-1}\right\} \rightarrow C$ such that $\widetilde{v} \circ \eta_{u}=\psi$. 


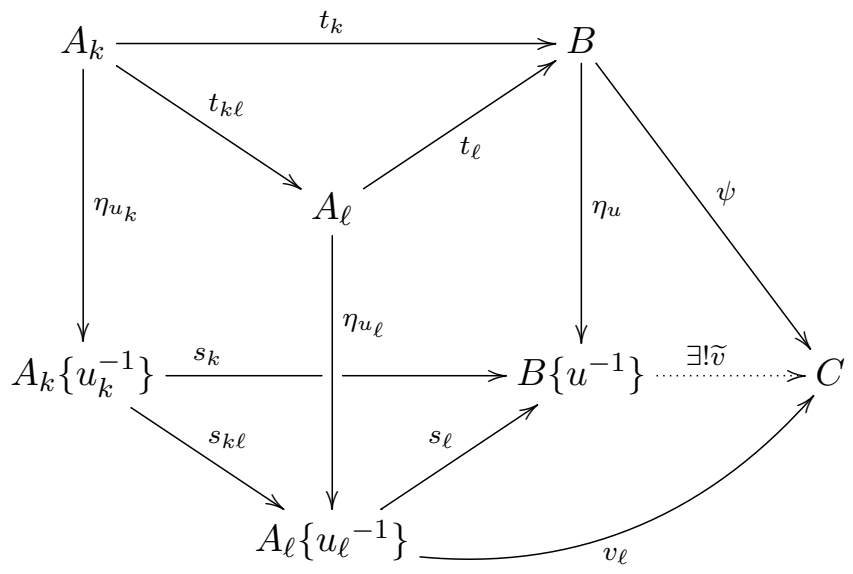

Claim: $(\forall \ell \geq j)\left(\widetilde{v} \circ s_{\ell}=v_{\ell}\right)$.

$$
\widetilde{v} \circ \eta_{u}=\psi \Rightarrow\left(\widetilde{v} \circ \eta_{u}\right) \circ t_{\ell}=\psi \circ t_{\ell} \Longleftrightarrow \widetilde{v} \circ\left(\eta_{u} \circ t_{\ell}\right)=v_{k} \circ \eta_{u_{\ell}} \Longleftrightarrow \widetilde{v} \circ\left(s_{\ell} \circ \eta_{u_{\ell}}\right)=v_{\ell} \circ \eta_{u_{\ell}}
$$

hence:

$$
\left(\widetilde{v} \circ s_{\ell}\right) \circ \eta_{u_{\ell}}=v_{\ell} \circ \eta_{u_{\ell}}
$$

Given any $\alpha \in A_{\ell}\left\{u_{\ell}^{-1}\right\}$, there are $x_{\ell} \in A_{\ell}$ and $y_{\ell} \in\left\{u_{\ell}\right\}^{\infty-\text { sat }}$ such that $\alpha=\frac{\eta_{u_{\ell}}\left(x_{\ell}\right)}{\eta_{u_{\ell}}\left(y_{\ell}\right)}$.

We have:

$$
\begin{aligned}
\left(\widetilde{v} \circ s_{\ell}\right)(\alpha)=( & \left(\widetilde{v} \circ s_{\ell}\right)\left(\frac{\eta_{u_{\ell}}\left(x_{\ell}\right)}{\eta_{u_{\ell}}\left(y_{\ell}\right)}\right)= \\
=\left(\widetilde{v} \circ s_{\ell}\right)\left(\eta_{u_{\ell}}\left(x_{\ell}\right) \cdot \eta_{u_{\ell}}\left(y_{\ell}\right)^{-1}\right)=\left(\widetilde{v} \circ s_{\ell} \circ \eta_{u_{\ell}}\right)\left(x_{\ell}\right) \cdot\left(\widetilde{v} \circ s_{\ell} \circ \eta_{u_{\ell}}\right)(y \ell)^{-1} & = \\
& =\frac{\left(\widetilde{v} \circ s_{\ell} \circ \eta_{u_{\ell}}\right)\left(x_{\ell}\right)}{\left(\widetilde{v} \circ s_{\ell} \circ \eta_{u_{\ell}}\right)\left(y_{\ell}\right)}
\end{aligned}
$$

and by the equation 2.5 ,

$$
\frac{\left(\widetilde{v} \circ s_{\ell} \circ \eta_{u_{\ell}}\right)\left(x_{\ell}\right)}{\left(\widetilde{v} \circ s_{\ell} \circ \eta_{u_{\ell}}\right)\left(y_{\ell}\right)}=\frac{\left(v_{\ell} \circ \eta_{u_{\ell}}\right)\left(x_{\ell}\right)}{\left(v_{\ell} \circ \eta_{u_{\ell}}\right)\left(y_{\ell}\right)}=v_{\ell}\left(\frac{\eta_{u_{\ell}}\left(x_{\ell}\right)}{\eta_{u_{\ell}}\left(y_{\ell}\right)}\right)=v_{\ell}(\alpha)
$$

Hence, for every $\alpha \in A_{\ell}\left\{u_{\ell}^{-1}\right\},\left(\widetilde{v} \circ s_{\ell}\right)(\alpha)=v_{\ell}(\alpha)$.

It follows, then, that if $B=\lim _{i \in I} A_{i}$, then for any $u \in B, B\left\{u^{-1}\right\}=\lim _{i \in I} A_{i}\left\{u_{i}^{-1}\right\}$.

Theorem 2.1.41. Let $(I, \leq)$ be a directed poset and let $(A, S)$ be the directed colimit of a system $\left\{\left(A_{\ell}, S_{\ell}\right) \stackrel{t_{\ell j}}{\rightarrow}\left(A_{j}, S_{j}\right) \mid \ell, j \in I\right\}$ of $\mathcal{C}_{2}^{\infty}$, that is,

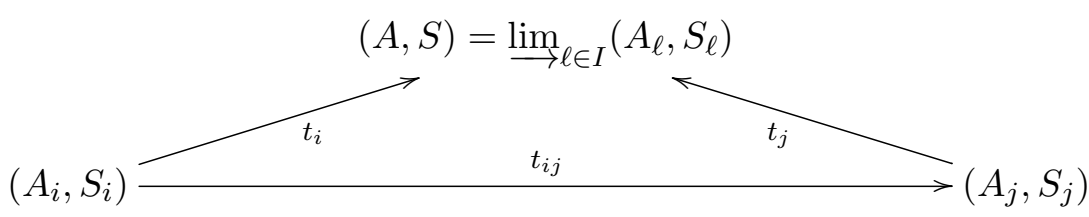


We have:

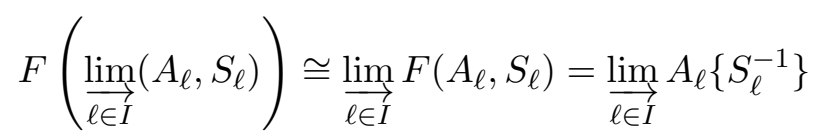

$$
\begin{aligned}
& \underset{i \in I}{\lim _{i \in I}} A_{i}\left\{S_{i}^{-1}\right\} \cong\left(\lim _{i \in I} A_{i}\right)\left\{\left(\lim _{i \in I} S_{i}\right)^{-1}\right\}
\end{aligned}
$$

Proof. (Sketch)

Denote by $\alpha_{i}: A_{i} \rightarrow \underline{\lim }_{i \in I} A_{i}$ and $\sigma_{i}: A_{i}\left\{S_{i}^{-1}\right\} \rightarrow \underline{\lim }_{i \in I} A_{i}\left\{S_{i}^{-1}\right\}$ the canonical colimit arrows for each $i \in I$.

We make use of the universal property of the colimit of $\mathcal{C}^{\infty}$-rings of fractions in order to show that there is a unique $\mathcal{C}^{\infty}$-homomorphism:

$$
\widetilde{\varphi}: \lim _{i \in I} A_{i}\left\{S_{i}^{-1}\right\} \rightarrow\left(\lim _{i \in I} A_{i}\right)\left\{\left(\lim _{i \in I} S_{i}\right)^{-1}\right\}
$$

such that the following diagram commutes:

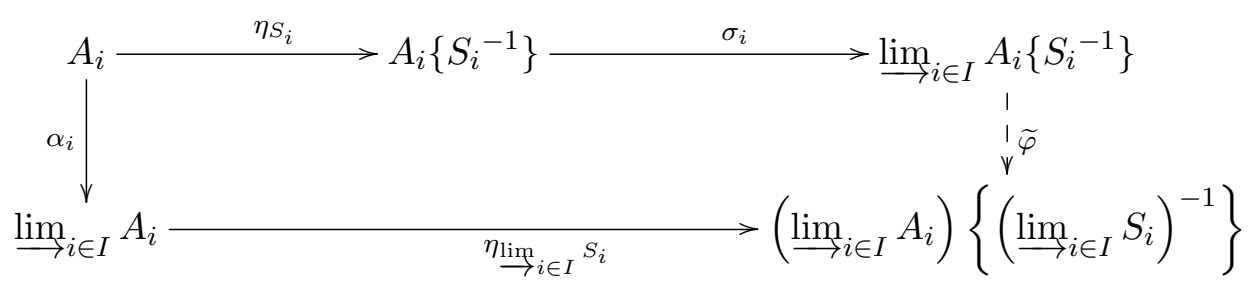

and that there is a unique $\mathcal{C}^{\infty}$-homomorphism:

$$
\widetilde{\psi}:\left(\lim _{i \in I} A_{i}\right)\left\{\left(\underset{i \in I}{\lim _{i \in I}} S_{i}\right)^{-1}\right\} \rightarrow \underset{i \in I}{\lim } A_{i}\left\{S_{i}^{-1}\right\}
$$

such that the following diagram commutes:

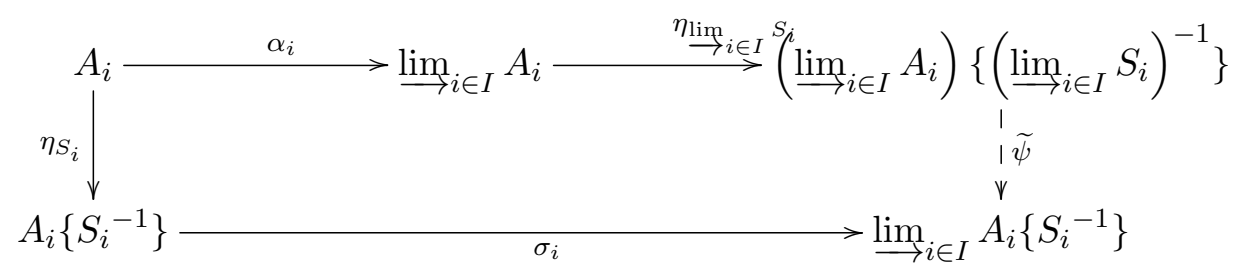

It follows, also by a "uniqueness argument", that $\widetilde{\varphi}$ and $\widetilde{\psi}$ are inverse $\mathcal{C}^{\infty}$-isomorphisms.

As a consequence of the above proposition we have:

Corollary 2.1.42. In the same context of Theorem 2.1.41, we have:

$$
\left(\underset{i \in I}{\lim _{i \in I}} S_{i}\right)^{\infty-\text { sat }}=\underset{i \in I}{\lim _{i \in I}} S_{i}^{\infty-\text { sat }} \subseteq \underset{i \in I}{\lim _{i \in I}} A_{i}
$$


Next we present some theorems about quotients of $\mathcal{C}^{\infty}$-rings by their ideals and some relationships between quotients and $\mathcal{C}^{\infty}$-rings of fractions. We are going to prove that, in the category of $\mathcal{C}^{\infty}$-rings, taking quotients and taking rings of fractions are constructions which commute in a sense that will become clear in the following considerations.

Let $A$ be a $\mathcal{C}^{\infty}$-ring, $I$ be any of its ideals, $S \subseteq A$ be any subset of $A$ and consider the canonical map of the ring of fractions of $A$ with respect to $S$ :

$$
\eta_{S}: A \rightarrow A\left\{S^{-1}\right\}
$$

and consider $\widetilde{I_{S}}=\left\langle\eta_{S}[I]\right\rangle$.

Since $\left(q_{\widetilde{I_{S}}} \circ \eta_{S}\right)[I] \subseteq \widetilde{I}_{S}$, then by the Theorem of Homomorphism there exists a unique $\eta_{S I}: \frac{A}{I} \rightarrow$ $\frac{A\left\{S^{-1}\right\}}{\widetilde{I_{S}}}$ such that:

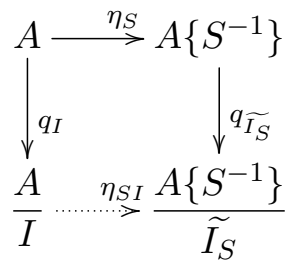

commutes.

Proposition 2.1.43. The morphism $\eta_{S I}: \frac{A}{I} \rightarrow \frac{A\left\{S^{-1}\right\}}{\widetilde{I_{S}}}$ has the same universal property which identifies the ring of quotients of $q_{I}[S]=S+I$.

Proof. Consider the following diagram:

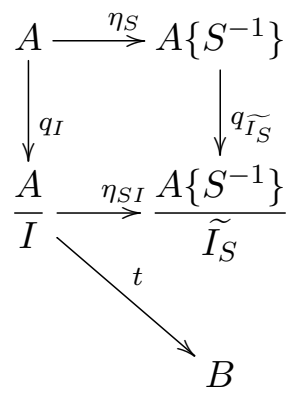

where $t: \frac{A}{I} \rightarrow B$ is any $\mathcal{C}^{\infty}$-homomorphism such that $t\left[\left(\frac{A}{I}\right)^{\times}\right] \subseteq B^{\times}$. Ipso facto, $\left(t \circ q_{I}\right)[S]=$ $t\left[q_{I}[S]\right] \subseteq B^{\times}$, so by the universal property of $\eta_{S}: A \rightarrow A\left\{S^{-1}\right\}$, there exists a unique $\mathcal{C}^{\infty}$-homomorphism $\varphi: A\left\{S^{-1}\right\} \rightarrow B$ such that the following diagram commutes:

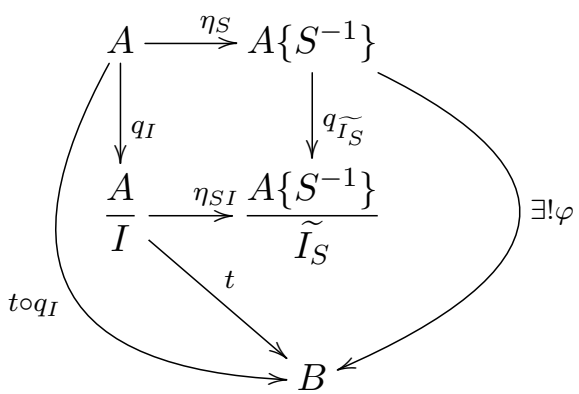


Claim: $\varphi\left[\widetilde{I}_{S}\right]=\{0\}$.

With regards to this, we note that $\left(\varphi \circ \eta_{S}\right)[I]=\left(t \circ q_{I}\right)[I]=t[\{0\}]=\{0\}$, so by linearity, $\varphi\left[\left\langle\eta_{S}[I]\right\rangle\right]=$ $\varphi\left[\widetilde{I_{S}}\right]=\{0\}$.

For this reason, and applying the Theorem of Homomorphism we get as a consequence the existence and uniqueness of a $\mathcal{C}^{\infty}$-homomorphism $\bar{\varphi}: \frac{A\left\{S^{-1}\right\}}{\widetilde{I}_{S}} \rightarrow B$ such that the following diagram commutes:

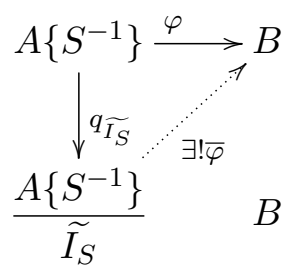

Claim: $\bar{\varphi} \circ \eta_{S I}=t$, that is, the following diagram commutes:

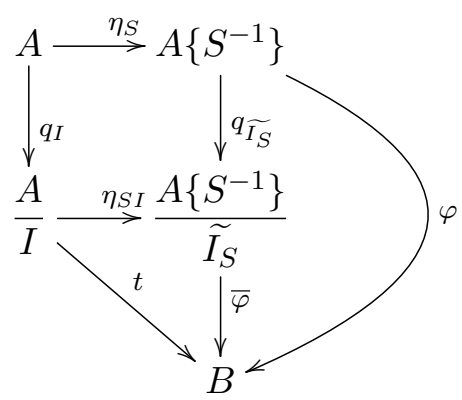

It suffices to show that $\bar{\varphi} \circ \eta_{S I} \circ q_{I}=t \circ q_{I}$, for since $q_{I}$ is an epimorphism we conclude $\bar{\varphi} \circ \eta_{S I}=t$.

Indeed, $t \circ q_{I}=\varphi \circ \eta_{S}=\left(\bar{\varphi} \circ q_{\widetilde{I_{S}}}\right) \circ \eta_{S}=\bar{\varphi} \circ \eta_{S I} \circ q_{I} \Rightarrow t=\bar{\varphi} \circ \eta_{S I}$.

Now we claim that if $\psi: \frac{A\left\{S^{-1}\right\}}{\widetilde{I_{S}}} \rightarrow B$ is a $\mathcal{C}^{\infty}$-morphism such that:

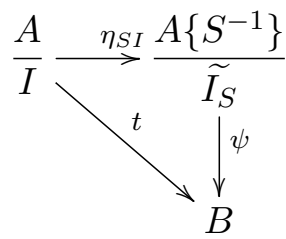

commutes, then $\psi=\varphi$.

Verily,

$$
\begin{aligned}
& \psi \circ \eta_{S I} \circ q_{I}=t \circ q_{I}=\varphi \circ \eta_{S}=\bar{\varphi} \circ q_{\widetilde{I_{S}}} \circ \eta_{S}=\bar{\varphi} \circ \eta_{S I} \circ q_{I} \\
& \psi \circ q_{\widetilde{I_{S}}} \circ \eta_{S}=\psi \circ \eta_{S I} \circ q_{I}=t \circ q_{I}=\varphi \circ \eta_{S}=\bar{\varphi} \circ q_{\widetilde{I_{S}}} \circ \eta_{S}
\end{aligned}
$$

hence

$$
\left(\psi \circ q_{\widetilde{I_{S}}}\right) \circ \eta_{S}=\left(\bar{\varphi} \circ q_{\widetilde{I_{S}}}\right) \circ \eta_{S}
$$

and since $\eta_{S}: A \rightarrow A\left\{S^{-1}\right\}$ is a $\mathcal{C}^{\infty}$-epimorphism, we get:

$$
\psi \circ q_{\widetilde{I_{S}}}=\bar{\varphi} \circ q_{\widetilde{I_{S}}},
$$


and because $q_{\widetilde{I_{S}}}$ is also an epimorphism, we get:

$$
\psi=\bar{\varphi}
$$

We have just proved that $\eta_{S I}: \frac{A}{I} \rightarrow \frac{A\left\{S^{-1}\right\}}{\widetilde{I}_{S}}$ has the same universal property as:

$$
\eta_{S+I}: \frac{A}{I} \rightarrow\left(\frac{A}{I}\right)\left\{(S+I)^{-1}\right\}
$$

Proposition 2.1.44. Let $A$ be a $\mathcal{C}^{\infty}$-ring, $I$ any of its ideals and $S \subseteq A$ any of its subsets. Since $\left(\eta_{S+I} \circ q_{I}\right)\left[A^{\times}\right] \subseteq\left(\frac{A}{I}\left\{(S+I)^{-1}\right\}\right)^{\times}$, there is a unique $\widehat{q}_{I S}$ such that the following diagram commutes:

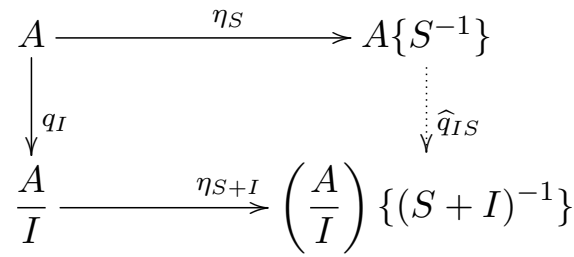

The $\mathcal{C}^{\infty}$-homomorphism $\mu^{-1}: \frac{A\left\{S^{-1}\right\}}{\widetilde{I_{S}}} \rightarrow \frac{A}{I}\left\{(S+I)^{-1}\right\}$ is an isomorphism.

Proof. As a consequence of the uniqueness of the ring of fractions map (up to isomorphism), it follows that there is an isomorphism $\mu:\left(\frac{A}{I}\right)\left\{(S+I)^{-1}\right\} \rightarrow \frac{A\left\{S^{-1}\right\}}{\widetilde{I}_{S}}$ such that the following diagrams commute:

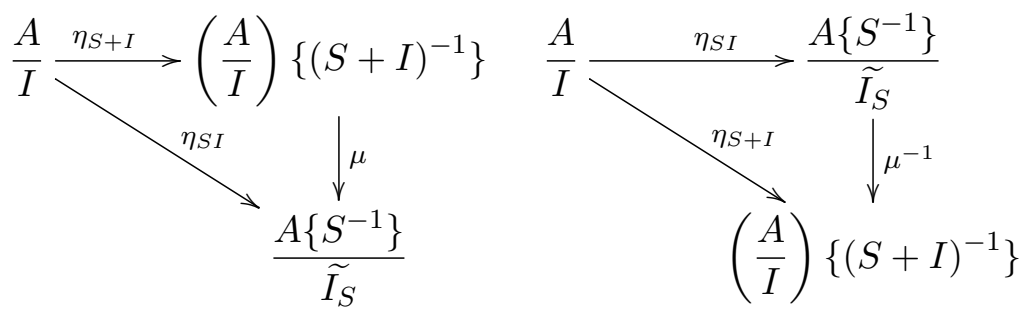

We claim that the upper right triangles of the following diagrams commute:
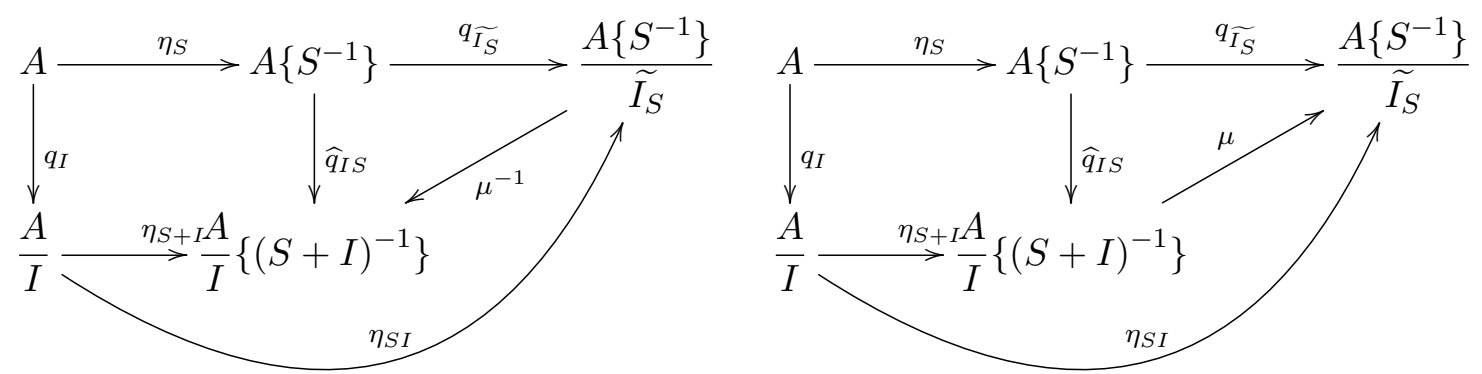

Indeed, since the left square commutes,

$$
q_{\widetilde{I}_{S}} \circ \eta_{S}=\eta_{S I} \circ q_{I}=\mu \circ \eta_{S+I} \circ q_{I}=\mu \circ \widehat{q}_{I S} \circ \eta_{S},
$$

and since $\eta_{S}$ is an epimorphism,

$$
q_{\widetilde{I}_{S}}=\mu \circ \widehat{q}_{I S}
$$

Since $\mu^{-1}$ is an isomorphism and $q_{\widetilde{I_{S}}}$ is an epimorphism, it follows that $\widehat{q}_{I S}=\mu^{-1} \circ q_{\widetilde{q_{S}}}$ is an epimorphism. As a result of this, $\widetilde{I}_{S}=\operatorname{ker}\left(\widehat{q}_{I S}\right)$, and due to the uniqueness of que quotient morphism, it follows that $\mu^{-1}$ is the quotient morphism, so the result is proved. 
Corollary 2.1.45. Let $A$ be a $\mathcal{C}^{\infty}$-ring, $I$ any of its ideals and $S \subseteq A$ any of its subsets. There are unique isomorphisms $\mu: \frac{A}{I}\left\{(S+I)^{-1}\right\} \rightarrow \frac{A\left\{S^{-1}\right\}}{\left\langle\eta_{S}[I]\right\rangle}$ such that the following pentagons commute:

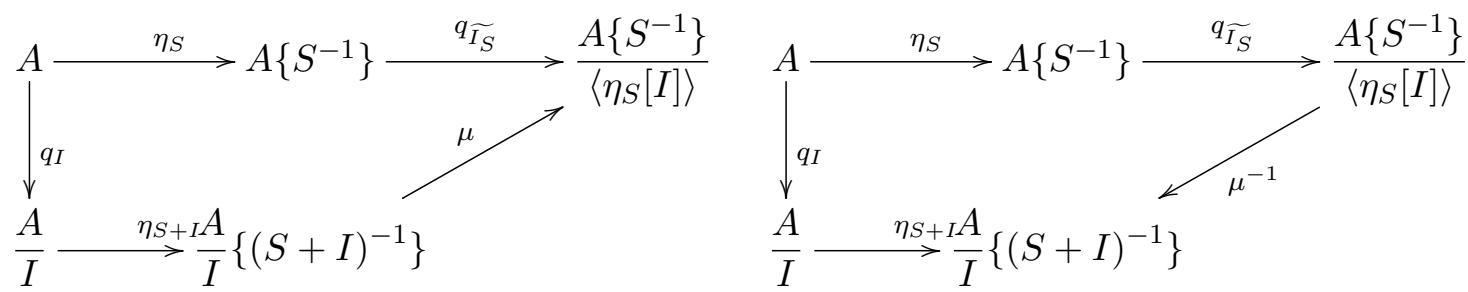

Now we analyze the concept of a "C ${ }^{\infty}$-radical ideal" in the theory of $\mathcal{C}^{\infty}$-rings, which plays a similar role to the one played by radical ideals in Commutative Algebra. This concept is presented by I. Moerdijk and G. Reyes in [66 in 1986 and explored in more details in [67].

Contrary to the concepts of $\mathcal{C}^{\infty}$-fields, $\mathcal{C}^{\infty}$-domains and local $\mathcal{C}^{\infty}$-rings, the concept of a $\mathcal{C}^{\infty}$-radical of an ideal can not be brought from Commutative Algebra via the forgetful functor. Recall that the radical of an ideal $I$ of a commutative unital ring $R$ is given by:

$$
\sqrt{I}=\left\{x \in R \mid(\exists n \in \mathbb{N})\left(x^{n} \in I\right)\right\}
$$

and this concept can be characterized by:

$$
\sqrt{I}=\bigcap\{\mathfrak{p} \in \operatorname{Spec}(R) \mid I \subseteq \mathfrak{p}\}=\left\{x \in R \mid\left(\frac{R}{I}\right)\left[(x+I)^{-1}\right] \cong 0\right\}
$$

We use the latter equality in order to motivate our definition.

Definition 2.1.46. Let $A$ be a $\mathcal{C}^{\infty}$-ring and let $I \subseteq A$ be a proper ideal. The $\mathcal{C}^{\infty}$-radical of I is given by:

$$
\sqrt[\infty]{I}:=\left\{a \in A \mid\left(\frac{A}{I}\right)\left\{(a+I)^{-1}\right\} \cong 0\right\}
$$

Proposition 2.1.47. Let $A$ be a $\mathcal{C}^{\infty}$-ring and let $I \subseteq A$ be any ideal. We have the following equalities:

$$
\sqrt[\infty]{I}=\left\{a \in A \mid(\exists b \in I) \wedge\left(\eta_{a}(b) \in\left(A\left\{a^{-1}\right\}\right)^{\times}\right)\right\}=\left\{a \in A \mid I \cap\{a\}^{\infty-\text { sat }} \neq \varnothing\right\}
$$

where $\eta_{a}: A \rightarrow A\left\{a^{-1}\right\}$ is the morphism of fractions with respect to $\{a\}$.

Proof. First we show that:

$$
\left\{a \in A \mid(\exists b \in I)\left(b \in A\left\{a^{-1}\right\}^{\times}\right)\right\} \subseteq \sqrt[\infty]{I} .
$$

Let $a \in A$ be such that $(\exists b \in I)\left(b \in A\left\{a^{-1}\right\}^{\times}\right)$. We have the following diagram:

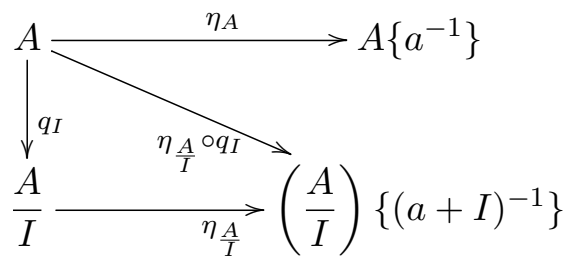


Note that the composite $\eta_{\frac{A}{I}} \circ q_{I}: A \rightarrow\left(\frac{A}{I}\right)\left\{(a+I)^{-1}\right\}$ maps $a$ to an invertible element of $\left(\frac{A}{I}\right)\{(a+$ $\left.I)^{-1}\right\}$, for

$$
\left(\eta_{\frac{A}{I}} \circ q_{I}\right)(a)=\eta_{\frac{A}{I}}(a+I) \in\left(\frac{A}{I}\left\{(a+I)^{-1}\right\}\right)^{\times} .
$$

By the universal property of $\eta_{a}: A \rightarrow A\left\{a^{-1}\right\}$ there is a unique morphism $q_{a}: A\left\{a^{-1}\right\} \rightarrow\left(\frac{A}{I}\right)\{(a+$ $\left.I)^{-1}\right\}$ such that the following rectangle commutes:

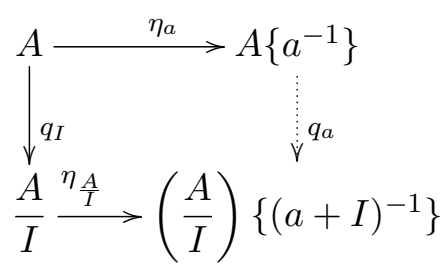

that is to say,

$$
q_{a} \circ \eta_{a}=\eta_{\frac{A}{I}} \circ q_{I}
$$

Since there is some $b \in I$ such that $\eta_{a}(b) \in\left(A\left\{a^{-1}\right\}\right)^{\times}$, we have:

$$
0_{\frac{A}{I}}=\eta_{\frac{A}{I}} \circ q_{I}(b)=q_{a} \circ \eta_{a}(b)=q_{a}\left(\eta_{a}(b)\right) \in\left(\frac{A}{I}\left\{(a+I)^{-1}\right\}\right)^{\times} .
$$

It follows that $\frac{A}{I}\left\{(a+I)^{-1}\right\} \cong 0$, hence $a \in \sqrt[\infty]{I}$.

Conversely, we are going to show that:

$$
\sqrt[\infty]{I} \subseteq\left\{a \in A \mid(\exists b \in I)\left(\eta_{a}(b) \in A\left\{a^{-1}\right\}^{\times}\right)\right\} .
$$

Given $a \in \sqrt[\infty]{I}$, we have by definition

$$
\frac{A}{I}\left\{(a+I)^{-1}\right\} \cong 0
$$

so $1_{\frac{A}{I}}=0_{\frac{A}{I}}$. Thus, the element $1_{A} \in A$ is such that $q_{a}\left(\eta_{a}\left(1_{A}\right)\right)=1_{\frac{A}{I}}=0_{\frac{A}{I}}=\eta_{\frac{A}{I}} \circ q_{I}\left(1_{A}\right)$.

Since by Corollary 2.1.45, taking quotients and taking rings of fractions commute, we have:

$$
0=\left(\frac{A}{I}\right)\left\{(a+I)^{-1}\right\} \cong \frac{A\left\{a^{-1}\right\}}{\widehat{I}_{a}},
$$

where $\widehat{I}_{a}=\operatorname{ker} q_{a}=\left\langle\eta_{a}[I]\right\rangle=\left\{\frac{\eta_{a}(i)}{\eta_{a}(c)} \mid(i \in I) \&\left(c \in\{a\}^{\infty-\text { sat }}\right)\right\}$ (see Theorem 2.2.7, the ideal generated by $\eta_{a}[I]$. Thus

$$
\frac{A\left\{a^{-1}\right\}}{\operatorname{ker} q_{a}}=\frac{A\left\{a^{-1}\right\}}{\left\langle\eta_{a}[I]\right\rangle} \cong 0,
$$

so $1 \in \widehat{I}_{a}$, i.e., there are $i \in I$ and $c \in\{a\}^{\infty-\text { sat }}$ such that:

$$
1=\frac{\eta_{a}(i)}{\eta_{a}(c)}
$$



Thus,

It follows that $\eta_{a}(i)=\eta_{a}(c)$, so $\eta_{a}(i-c)=0$ and there is some $d \in\{a\}^{\infty-\text { sat }}$ such that $d \cdot(i-c)=0$.

$$
\underbrace{d \cdot i}_{\in I}=\underbrace{d \cdot c}_{\in\{a\}^{\infty}-\mathrm{sat}}
$$

and $I \cap\{a\}^{\infty-\text { sat }} \neq \varnothing$.

Theorem 2.1.48. Let $A$ and $B$ be two $\mathcal{C}^{\infty}$-rings, $S \subseteq A, p \rightarrow B$ a surjective map and $\eta_{S}: A \rightarrow A\left\{S^{-1}\right\}$ and $\eta_{p[S]}: B \rightarrow B\left\{p[S]^{-1}\right\}$ be the canonical $\mathcal{C}^{\infty}$-ring of fractions. There is a unique surjective map $q: A\left\{S^{-1}\right\} \rightarrow B\left\{p[S]^{-1}\right\}$ such that the following square commutes:

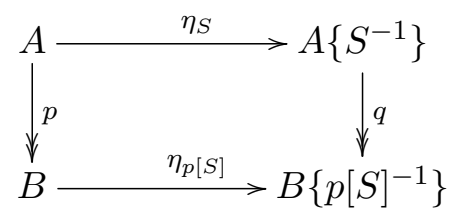

Proof. Since for all $s \in S, \eta_{p[S]}(p(s)) \in\left(B\left\{p[S]^{-1}\right\}\right)^{\times}$, there must exist a unique $\mathcal{C}^{\infty}$-homomorphism $q: A\left\{S^{-1}\right\} \rightarrow B\left\{p[S]^{-1}\right\}$ such that the following diagram commutes:

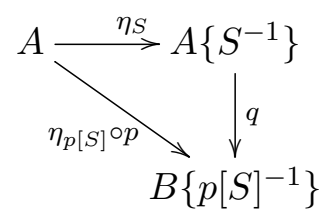

Now we need only to prove the map $q$ is a surjective map.

Since $p: A \rightarrow B$ is surjective, by the Theorem of the Isomorphism there exists a unique $\mathcal{C}^{\infty}$-isomorphism $\Phi: \frac{A}{\operatorname{ker} p} \rightarrow B$ such that the following diagram commutes:

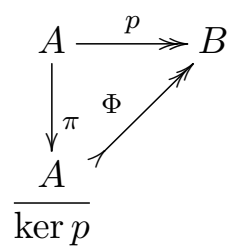

Note that $p[S]=(\Phi \circ \pi)[S]=\Phi[S+\operatorname{ker} p]$, so $\Phi^{-1}[p[S]]=\left(\Phi^{-1} \circ \Phi\right)[S+\operatorname{ker} p]=S+\operatorname{ker} p$, and $\eta_{S+\operatorname{ker} p}\left[\Phi^{-1}[p[S]]\right]=\eta_{S+\operatorname{ker} p}[S+\operatorname{ker} p] \subseteq\left(\left(\frac{A}{\operatorname{ker} p}\right)\left\{(S+\operatorname{ker} p)^{-1}\right\}\right)^{\times}$, so there exists a unique $\mathcal{C}^{\infty}$-homomorphism:

$$
\Psi: B\left\{p[S]^{-1}\right\} \rightarrow \frac{A}{\operatorname{ker} p}\left\{(S+\operatorname{ker} p)^{-1}\right\}
$$

such that:

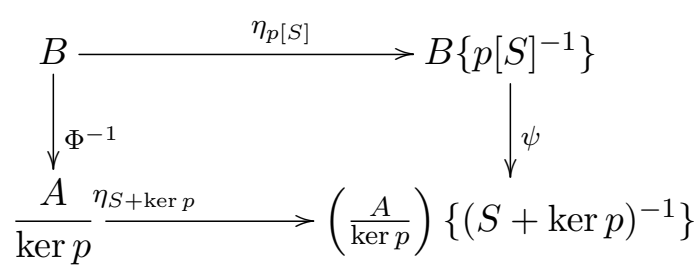

commutes.

Conversely, since $\Phi[S+\operatorname{ker} p]=p[S]$ and $\eta_{p[S]}[\Phi[S+\operatorname{ker} p]]=\eta_{p[S]}[p[S]] \subseteq\left(B\left\{p[S]^{-1}\right\}\right)^{\times}$, there is a unique $\mathcal{C}^{\infty}$-homomorphism: 


$$
\Xi:\left(\frac{A}{\operatorname{ker} p}\right)\left\{(S+\operatorname{ker} p)^{-1}\right\} \rightarrow B\left\{p[S]^{-1}\right\}
$$

such that:

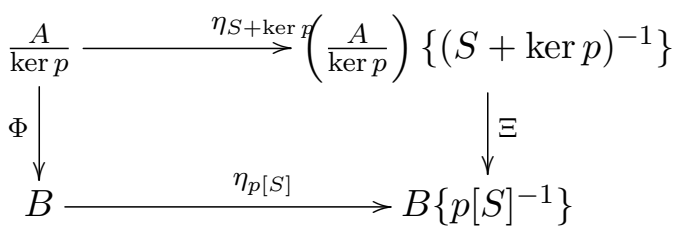

commutes.

It is clear that $\Xi=\Psi^{-1}$, so $B\left\{p[S]^{-1}\right\} \cong\left(\frac{A}{\operatorname{ker} p}\right)\left\{(S+\operatorname{ker} p)^{-1}\right\}$.

We have the following commutative diagram:

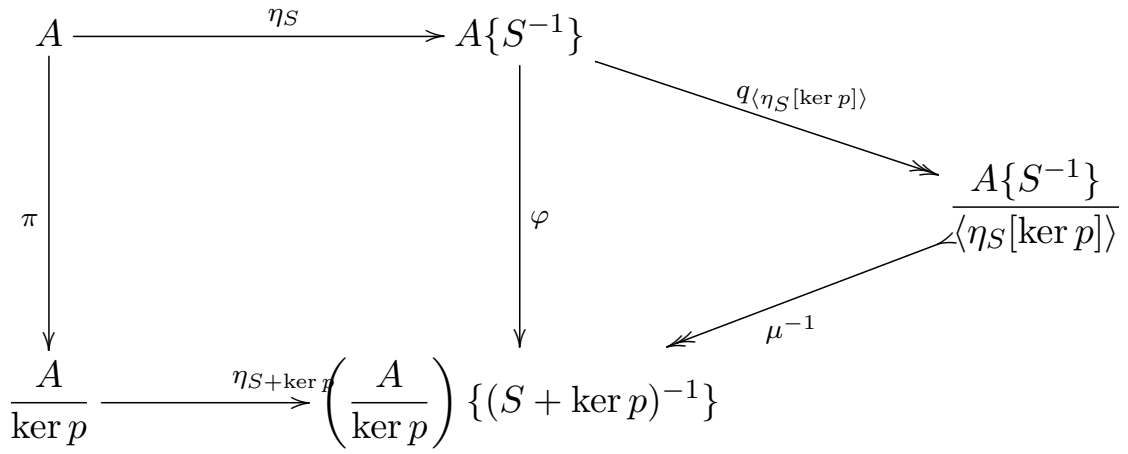

so $\varphi=\mu^{-1} \circ q_{\left\langle\eta_{S}[\operatorname{ker} p]\right\rangle}$, hence it is a composition of surjective $\mathcal{C}^{\infty}$-homomorphisms.

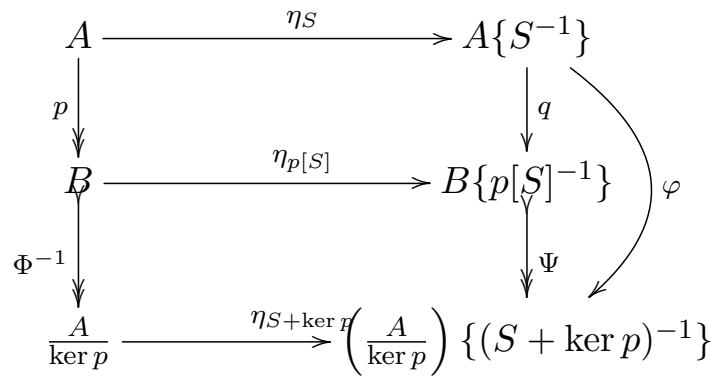

Since $\Psi$ is bijective, $\varphi$ is surjective and $\varphi=\Psi \circ q$ (by the uniqueness of $\varphi$ ), it follows that $q$ is surjective.

\subsection{Distinguished Classes of $\mathcal{C}^{\infty}$-rings}

In this section we present some distinguished classes of $\mathcal{C}^{\infty}$-rings, such as $\mathcal{C}^{\infty}$-fields, $\mathcal{C}^{\infty}$-domains, $\mathcal{C}^{\infty}$-local rings, reduced $\mathcal{C}^{\infty}$-rings and von Neumann regular $\mathcal{C}^{\infty}$-rings.

In [66], we find definitions of $\mathcal{C}^{\infty}$-fields, $\mathcal{C}^{\infty}$-domains and $\mathcal{C}^{\infty}$-local rings. We describe these concepts in the following: 
Definition 2.2.1. Let $\mathbb{R}$ - Alg be the category of $\mathbb{R}$-algebras, and consider the forgetful functor $U$ : $\mathcal{C}^{\infty} \mathbf{R n g} \rightarrow \mathbb{R}-\mathbf{A l g}$. We say that a $\mathcal{C}^{\infty}$-ring $A$ is:

- a $\mathcal{C}^{\infty}$-field whenever $U(A) \in \operatorname{Obj}(\mathbb{R}-\mathbf{A l g})$ is a field;

- a $\mathcal{C}^{\infty}$-domain whenever $U(A) \in \operatorname{Obj}(\mathbb{R}-\mathbf{A l g})$ is a domain;

- a local $\mathcal{C}^{\infty}$-ring whenever $U(A) \in \operatorname{Obj}(\mathbb{R}-\mathbf{A l g})$ is a local ring.

Thus a $\mathcal{C}^{\infty}$-field, a $\mathcal{C}^{\infty}$-domain and a local $\mathcal{C}^{\infty}$-ring are $\mathcal{C}^{\infty}$-rings such that their underlying $\mathbb{R}$-algebras are fields, domains and local rings in the ordinary sense, respectively.

The next lemma gives us a general method to obtain finitely generated local $\mathcal{C}^{\infty}$-rings using prime filters on the set of the closed parts of $\mathbb{R}^{n}$.

Lemma 2.2.2. Let $\Phi \subseteq \mathcal{P}\left(\mathbb{R}^{n}\right)$ be a prime filter. Then

$$
\underset{U \in \Phi}{\lim _{U \in \Phi}} \mathcal{C}^{\infty}(U)
$$

is a local $\mathcal{C}^{\infty}$-ring.

Proof. First we give an explicit description of $\lim _{U \in \Phi} \mathcal{C}^{\infty}(U)$ as:

$$
\underset{U \in \Phi}{\lim _{U \in \Phi}} \mathcal{C}^{\infty}(U)=\frac{\bigsqcup_{U \in \Phi} \mathcal{C}^{\infty}(U)}{\sim},
$$

where $\sim$ is the equivalence relation on $\bigsqcup_{U \in \Phi} \mathcal{C}^{\infty}(U)$ given by:

$$
(\alpha, U) \sim(\beta, V) \Longleftrightarrow(\exists W \in \Phi)\left(\rho_{W}^{U}(\alpha)=\alpha\left\lceil_{W}=\beta\left\lceil_{W}=\rho_{W}^{V}(\beta)\right) .\right.\right.
$$

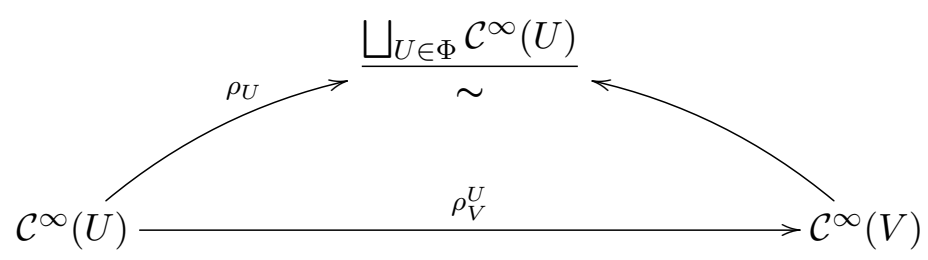

The sum and the product are defined in a rather obvious fashion:

$$
\begin{aligned}
& +:\left(\frac{\bigsqcup_{U \in \Phi} \mathcal{C}^{\infty}(U)}{\sim}\right) \times\left(\frac{\bigsqcup_{U \in \Phi} \mathcal{C}^{\infty}(U)}{\sim}\right) \rightarrow \quad \frac{\bigsqcup_{U \in \Phi} \mathcal{C}^{\infty}(U)}{\sim} \\
& ([(\alpha, U)],[(\beta, V)]) \quad \mapsto\left[\left((\alpha+\beta) \uparrow_{U \cap V}, U \cap V\right)\right]
\end{aligned}
$$

and:

$$
\begin{aligned}
\cdot:\left(\frac{\bigsqcup_{U \in \Phi} \mathcal{C}^{\infty}(U)}{\sim}\right) \times\left(\frac{\bigsqcup_{U \in \Phi} \mathcal{C}^{\infty}(U)}{\sim}\right) & \rightarrow \quad \frac{\bigsqcup_{U \in \Phi} \mathcal{C}^{\infty}(U)}{\sim} \\
([(\alpha, U)],[(\beta, V)]) & \mapsto \quad\left[\left((\alpha \cdot \beta) \uparrow_{U \cap V}, U \cap V\right)\right]
\end{aligned}
$$

Notice that since $\Phi$ is a proper filter, there is no danger of getting $U \cap V=\varnothing$, since $\varnothing \notin \Phi$, and for every $U, V \in \Phi$ we have $U \cap V \in \Phi$.

Let $a=(\alpha, U)$ and $b=(\beta, V) \in \bigsqcup_{U \in \Phi} \mathcal{C}^{\infty}(U)$ be such that $[a]+[b]=[1]$, i.e., $(\exists W \in \Phi)\left((\alpha+\beta) \uparrow_{W}=\right.$ $\left.1 \uparrow_{W}\right)$. 
Let $V_{\alpha}=\{x \in U \mid \alpha(x) \neq 0\}$ and $V_{\beta}=\{x \in U \mid \beta(x) \neq 0\}$.

Claim: $V_{\alpha} \cup V_{\beta}=W$.

If $V_{\alpha} \cup V_{\beta} \subsetneq W$, then there exists some $x_{0} \in U$ such that $\alpha\left(x_{0}\right)=0$ and $\beta\left(x_{0}\right)=0$, and we could not have $\alpha\left(x_{0}\right)+\beta\left(x_{0}\right)=1$, hence we have the equality.

Now we have $V_{\alpha} \cup V_{\beta}=W \in \Phi$, and since $\Phi$ is a prime filter, either $V_{\alpha} \in \Phi$ or $V_{\beta} \in \Phi$. In the former case, $\tilde{a}=\left(\alpha, V_{\alpha}\right) \in\left(\bigsqcup_{U \in \Phi} \mathcal{C}^{\infty}(U)\right)^{\times}$, and since $\left(\alpha, V_{\alpha}\right) \sim(\alpha, U),[a]=[\tilde{a}] \in\left(\frac{\bigsqcup_{U \in \Phi} \mathcal{C}^{\infty}(U)}{\sim}\right)^{\times}$, and in the latter case we have, analogously, $[b] \in\left(\frac{\bigsqcup_{U \in \Phi} \mathcal{C}^{\infty}(U)}{\sim}\right)^{\times}$.

Proposition 2.2.3. Let $A$ be a $\mathcal{C}^{\infty}$-ring and $\mathfrak{p} \in \operatorname{Spec}^{\infty}(A)$, that is to say that $\mathfrak{p}$ is a $\mathcal{C}^{\infty}$-radical ideal. Then $A_{\mathfrak{p}}:=A\left\{A \backslash \mathfrak{p}^{-1}\right\}$ is a local $\mathcal{C}^{\infty}$-ring whose unique maximal ideal is given by $\mathfrak{m}_{\mathfrak{p}}=$ $\left\{\frac{\eta_{A \backslash \mathfrak{p}}(x)}{\eta_{A \backslash \mathfrak{p}}(y)} \mid(x \in \mathfrak{p}) \&(y \in A \backslash \mathfrak{p})\right\}$.

Proof. As for the fact of $A_{\mathfrak{p}}$ being a local $\mathcal{C}^{\infty}$-ring, cf. Lemma 1.1 of 62$]$.

Let $A=\mathcal{C}^{\infty}\left(\mathbb{R}^{n}\right)$. We know that

$$
\mathcal{C}^{\infty}\left(\mathbb{R}^{n}\right)_{\mathfrak{p}}=\underset{f \notin \mathfrak{p}}{\lim _{f \notin}} \mathcal{C}^{\infty}\left(U_{f}\right)
$$

where $U_{f}=\operatorname{Coz}(f)=\left\{x \in \mathbb{R}^{n} \mid f(x) \neq 0\right\}$, so it remains only to prove that:

$$
\Phi=\left\{U \subseteq \mathbb{R}^{n} \mid(\exists f \notin \mathfrak{p})\left(U=U_{f}\right)\right\}
$$

is a prime filter, and by Lemma $2 \mathbf{2 . 2 . 2}$ the will result follow.

We have $\mathbb{R}^{n}=U_{1} \in \Phi$ and for every $f, g \in \mathcal{C}^{\infty}\left(\mathbb{R}^{n}\right)$ we have $U_{f} \cap U_{g}=U_{f \cdot g} \in \Phi$. Moreover, if $U_{f} \subseteq V$, taking the smooth characteristic function of $V, \chi_{V}$ we have $Z\left(\chi_{V}\right) \subseteq Z(f)$, (note that $\widehat{\mathfrak{p}}=\{Z(h) \mid h \in \mathfrak{p}\}$ is a filter).

If $V \cup W \in \Phi$, say $V \cup W=U_{f}$ for some $f \notin \mathfrak{p}$, then writing $V=U_{\chi_{V}}$ and $W=U_{\chi_{W}}$, then $Z(f)=Z\left(\chi_{V}\right) \cap Z\left(\chi_{U}\right)=Z\left(\chi_{V}^{2}+\chi_{U}^{2}\right)$, so $\chi_{V}^{2}+\chi_{U}^{2} \notin \mathfrak{p}$, since $\mathfrak{p}$ is $\mathcal{C}^{\infty}$-radical, hence $\chi_{V}^{2} \notin \mathfrak{p}$ or $\chi_{W}{ }^{2} \notin \mathfrak{p}$, that is, $V \in \Phi$ or $W \in \Phi$, so $\Phi$ is prime.

Let us show that $\mathfrak{m}_{\mathfrak{p}}=A_{\mathfrak{p}} \backslash A_{\mathfrak{p}} \times$.

By the very definition of $A_{\mathfrak{p}}=A\left\{A \backslash \mathfrak{p}^{-1}\right\}$, any element of it is of the form $\frac{\eta_{A \backslash \mathfrak{p}}(x)}{\eta_{A \backslash \mathfrak{p}}(y)}$ for some $x \in A$ and $y \in A \backslash \mathfrak{p}^{\infty-\text { sat }}$. Note that if $x^{\prime}, y^{\prime} \in A$ are such that $\frac{\eta_{A \backslash \mathfrak{p}}\left(x^{\prime}\right)}{\eta_{A \backslash \mathfrak{p}}\left(y^{\prime}\right)}=\frac{\eta_{A \backslash \mathfrak{p}}(x)}{\eta_{A \backslash \mathfrak{p}}(y)}$, then $\left(\exists s \in A \backslash \mathfrak{p}^{\infty-\mathrm{sat}}=\right.$ $A \backslash \mathfrak{p})\left(s \cdot\left(x^{\prime} \cdot y-x \cdot y^{\prime}\right)=0\right)$, so $x^{\prime} \cdot \underbrace{s \cdot y}_{\in A \backslash \mathfrak{p}}=\underbrace{x \cdot s \cdot y^{\prime}}_{\in \mathfrak{p}}$ and $x^{\prime} \notin A \backslash \mathfrak{p}$, thus $x^{\prime} \in \mathfrak{p}$.

Let $\frac{\eta_{A \backslash \mathfrak{p}}(x)}{\eta_{A \backslash \mathfrak{p}}(y)} \in A_{\mathfrak{p}}$ be such that $x \in \mathfrak{p}$ and $y \in A \backslash \mathfrak{p}$. By Theorem 2.1.28, it follows that $\frac{\eta_{A \backslash \mathfrak{p}}(x)}{\eta_{A \backslash \mathfrak{p}}(y)} \in A_{\mathfrak{p}} \backslash A_{\mathfrak{p}}{ }^{\times}$occurs if, and only if, $(\forall d \in A \backslash \mathfrak{p})\left(\forall c^{\prime} \in A\right)\left(d \cdot c^{\prime} \cdot x \in \mathfrak{p}\right)$, which is the case since $x \in \mathfrak{p}$ and $\mathfrak{p}$ is an ideal. Hence: 


$$
\left\{\frac{\eta_{A \backslash \mathfrak{p}}(x)}{\eta_{A \backslash \mathfrak{p}}(y)} \mid(x \in \mathfrak{p}) \&(y \in A \backslash \mathfrak{p})\right\} \subseteq A_{\mathfrak{p}} \backslash A_{\mathfrak{p}} \times
$$

Conversely, suppose

$$
\frac{\eta_{A \backslash \mathfrak{p}}(x)}{\eta_{A \backslash \mathfrak{p}}(y)} \in A_{\mathfrak{p}} \backslash A_{\mathfrak{p}} \times
$$

so by Theorem 2.1.28, $(\forall d \in A \backslash \mathfrak{p})\left(\forall c^{\prime} \in A\right)\left(d \cdot c^{\prime} \cdot x \in \mathfrak{p}\right)$. In particular, taking $c^{\prime}=1$ yields $(\forall d \in A \backslash \mathfrak{p})(d \cdot x \in \mathfrak{p})$. Since $\mathfrak{p}$ is a prime ideal, then either $d \in \mathfrak{p}$ (which is not the case since $d \in A \backslash \mathfrak{p}$ ) or $x \in \mathfrak{p}$. Hence $x \in \mathfrak{p}$.

Proposition 2.2.4. Let $A$ be a $\mathcal{C}^{\infty}$-ring. The following assertions are equivalent.

i) $A$ is a $\mathcal{C}^{\infty}$-field;

ii) For every subset $S \subseteq A \backslash\{0\}$, the canonical map:

$$
\mathrm{Can}_{S}: A \rightarrow A\left\{S^{-1}\right\}
$$

is a $\mathcal{C}^{\infty}$-ring isomorphism;

iii) For any $a \in A \backslash\{0\}$, we have that:

$$
\mathrm{Can}_{a}: A \rightarrow A\left\{a^{-1}\right\}
$$

is a $\mathcal{C}^{\infty}$-isomorphism.

Proof. $i) \Rightarrow$ ii) : Since $S \subseteq A^{\times}$, it is easy to see that $\operatorname{Can}_{S}: A \rightarrow A\left\{S^{-1}\right\}$ is isomorphic to id $A: A \rightarrow A$, so $\operatorname{Can}_{S}: A \rightarrow A\left\{S^{-1}\right\}$ is a $\mathcal{C}^{\infty}$-isomorphism.

ii) $\Rightarrow$ iii) It is immediate, since $\{a\} \subseteq A^{\times}$.

iii) $\Rightarrow$ i) Suppose that for every $a \in A \backslash\{0\}, \operatorname{Can}_{a}: A \rightarrow A\left\{a^{-1}\right\}$ is a $\mathcal{C}^{\infty}$-isomorphism. We must show that $a$ has an inverse in $A$. Let $\theta \in A\left\{a^{-1}\right\}$ be the inverse of $\operatorname{Can}_{a}(a)$, i.e.,

$$
\theta \cdot \operatorname{Can}_{a}(a)=1_{U(A)}=\operatorname{Can}_{a}(a) \cdot \theta .
$$

Since $\operatorname{Can}_{a}$ is an isomorphism, there is a unique $b \in A$ such that $\operatorname{Can}_{a}(b)=\theta$. Now, $\operatorname{Can}_{a}(a) \cdot \theta=$ $\operatorname{Can}_{a}(a) \cdot \operatorname{Can}_{a}(b)=\operatorname{Can}_{a}(a \cdot b)=1_{U(A)\left\{a^{-1}\right\}}$, so $a \cdot b=1_{A}$, thus $b=a^{-1}$.

Definition 2.2.5. $A \mathcal{C}^{\infty}$-ring $A$ is $\mathcal{C}^{\infty}$-reduced if, and only if, $\sqrt[\infty]{(0)}=(0)$.

Proposition 2.2.6. Every $\mathcal{C}^{\infty}$-field, $A$, is a $\mathcal{C}^{\infty}$-reduced $\mathcal{C}^{\infty}$-ring.

Proof. Suppose $A$ is a $\mathcal{C}^{\infty}$-field. For every $a \in A$, we have $\frac{A}{(0)}\left\{(a+(0))^{-1}\right\} \cong A\left\{a^{-1}\right\}$, so $A\left\{a^{-1}\right\} \cong A$ occurs if, and only if, $a \neq 0$. Hence $\sqrt[\infty]{(0)}=(0)$

Theorem 2.2.7. Let $A$ be $a \mathcal{C}^{\infty}-$ ring, $S \subseteq A$, and $I \subset A$ any ideal. Then:

$$
\left\langle\eta_{S}[I]\right\rangle=\left\{\frac{\eta_{S}(b)}{\eta_{S}(d)} \mid b \in I \& d \in S^{\infty-\mathrm{sat}}\right\}
$$


Proof. Given $h \in\left\langle\eta_{S}[I]\right\rangle$, there are $n \in \mathbb{N}, b_{1}, \cdots, b_{n} \in I$ and $\alpha_{1}, \cdots, \alpha_{n} \in A\left\{S^{-1}\right\}$ such that:

$$
h=\sum_{i=1}^{n} \alpha_{i} \cdot \eta_{S}\left(b_{i}\right)
$$

For each $i \in\{1, \cdots, n\}$ there are $c_{i} \in A$ and $d_{i} \in S^{\infty-\text { sat }}$ such that:

$$
\alpha_{i} \cdot \eta_{S}\left(d_{i}\right)=\eta_{S}\left(c_{i}\right)
$$

so

$$
h=\sum_{i=1}^{n} \alpha_{i} \cdot \eta_{S}\left(b_{i}\right)=\sum_{i=1}^{n} \frac{1}{\eta_{S}\left(d_{i}\right)} \cdot \eta_{S}\left(c_{i}\right) \cdot \eta_{S}\left(b_{i}\right),
$$

and denoting $b_{i}^{\prime}:=c_{i} \cdot b_{i} \in I$, we get:

$$
h=\sum_{i=1}^{n} \frac{\eta_{S}\left(b_{i}^{\prime}\right)}{\eta_{S}\left(d_{i}\right)}
$$

For each $i=1, \cdots, n$, let

$$
b_{i}^{\prime \prime}=b_{i}^{\prime} \prod_{j \neq i} d_{j}
$$

so

$$
h=\frac{\eta_{S}\left(\prod_{j \neq 1} d_{j}\right) \eta_{S}\left(b_{1}^{\prime}\right)}{\eta_{S}\left(d_{1} \cdots d_{n}\right)}+\frac{\eta_{S}\left(\prod_{j \neq 2} d_{j}\right) \eta_{S}\left(b_{2}^{\prime}\right)}{\eta_{S}\left(d_{1} \cdots d_{n}\right)}+\cdots+\frac{\eta_{S}\left(\prod_{j \neq n} d_{j}\right) \eta_{S}\left(b_{n}^{\prime}\right)}{\eta_{S}\left(d_{1} \cdots d_{n}\right)}
$$

hence

$$
h \cdot \eta_{S}\left(d_{1} \cdots d_{n}\right)=\eta_{S}\left(\prod_{j \neq 1} d_{j}\right) \eta_{S}\left(b_{1}^{\prime}\right)+\eta_{S}\left(\prod_{j \neq 2} d_{j}\right) \eta_{S}\left(b_{2}^{\prime}\right)+\cdots+\eta_{S}\left(\prod_{j \neq n} d_{j}\right) \eta_{S}\left(b_{n}^{\prime}\right) .
$$

Let $b_{i}^{\prime \prime}:=\left(\prod_{j \neq i} d_{i}\right) \cdot \overbrace{b_{i}^{\prime}}^{\in I} \in I$, so we have $h \cdot \eta_{S}\left(d_{1} \cdots d_{n}\right)=\sum_{i=1}^{n} \eta_{S}\left(b_{i}^{\prime \prime}\right)=\eta_{S}\left(\sum_{i=1}^{n} b_{i}^{\prime \prime}\right)$

Since $\eta_{S}\left(d_{1} \cdots d_{n}\right) \in A\left\{S^{-1}\right\}^{\times}, d=d_{1} \cdots d_{n} \in S^{\infty-\text { sat }}$, so taking $b=\sum_{i=1}^{n} b_{i}^{\prime \prime}$, we have $b \in I$, since it is a sum of elements of $I$, we can write:

$$
h \cdot \eta_{S}(d)=\eta_{S}(b)
$$

and $h=\frac{\eta_{S}(b)}{\eta_{S}(d)}$, with $b \in I$ and $d \in S^{\infty-\text { sat }}$.

The other way round is immediate.

As a consequence of Theorem 2.2.7, we have:

Corollary 2.2.8. If $A$ is a reduced $\mathcal{C}^{\infty}$-ring, then we have:

$$
(\forall a \in A)\left(\left(0 \in\{a\}^{\infty-\mathrm{sat}}\right) \leftrightarrow(a=0)\right)
$$

Proposition 2.2.9. Let $I \subsetneq \mathcal{C}^{\infty}\left(\mathbb{R}^{n}\right)$ any ideal. Then:

$$
\widehat{I}=\left\{A \subseteq \mathbb{R}^{n} \mid A \text { is closed and }(\exists f \in I)(A=Z(f))\right\}
$$

is a filter on the set of all the closed subsets of $\mathbb{R}^{n}$. Moreover, $I$ is a proper ideal if, and only if, $\widehat{I}$ is a proper filter. 
Proof. First we note that: $\varnothing \notin \widehat{I}$ if, and only if, there is some $f \in I$ such that $Z(f)=\varnothing$, if, and only if, there is some invertible $f \in I$, which happens if, and only if, $1 \in I$ so $I$ is not be proper.

Since $0 \in I$, we have $\mathbb{R}^{n}=Z(0) \in \widehat{I}$.

Let $A, B \in \widehat{I}$ and let $f, g \in I$ be smooth functions such that $A=Z(f)$ and $B=Z(g)$, so $f^{2}+g^{2} \in I$. We claim that:

$$
Z(f) \cap Z(g)=Z\left(f^{2}+g^{2}\right) .
$$

Indeed, for every $x \in Z\left(f^{2}+g^{2}\right)$ we have $\left(f^{2}+g^{2}\right)(x)=f^{2}(x)+g^{2}(x)=(f(x))^{2}+(g(x))^{2}=0$, and since $f(x), g(x) \in \mathbb{R},(f(x))^{2}+(g(x))^{2}=0$ implies $f(x)=0=g(x)$, so $x \in Z(f) \cap Z(g)$. Conversely, given any $x \in Z(f) \cap Z(g), f(x)=0=g(x)$, so $f^{2}(x)+g^{2}(x)=0$, so $x \in Z(f) \cap Z(g)$.

Now, since $A \cap B=Z\left(f^{2}+g^{2}\right)$ and $f^{2}+g^{2} \in I$, it follows that $A \cap B \in \widehat{I}$.

Finally, if $A \in \widehat{I}$ then there is $f \in I$ such that $A=Z(f)$. If $B \subseteq \mathbb{R}^{n}$ is closed and and $A \subset B$, then there is a smooth characteristic function $\chi_{B}: \mathbb{R}^{n} \rightarrow \mathbb{R}$ such that $\left.Z\left(\chi_{B}\right)=B\right)$. Consider the following smooth function:

$$
g=f \cdot \chi_{B} \in I .
$$

We notice that $Z(g)=Z(f) \cup Z\left(\chi_{B}\right)=A \cup B=B$, so $B \in \widehat{I}$.

From the above considerations it follows that $\widehat{I}$ is a filter on the closed parts of $\mathbb{R}^{n}$.

Proposition 2.2.10. Let $\mathcal{F}$ be any filter on the set of all the closed subsets of $\mathbb{R}^{n}$. Then:

$$
\check{\mathcal{F}}=\left\{f \in \mathcal{C}^{\infty}\left(\mathbb{R}^{n}\right) \mid Z(f) \in \mathcal{F}\right\}
$$

is an ideal of $\mathcal{C}^{\infty}\left(\mathbb{R}^{n}\right)$. Moreover, $\mathcal{F}$ is a proper filter if, and only if, $\check{\mathcal{F}}$ is a proper ideal.

Proof. Note that $0 \in \check{\mathcal{F}}$, since $Z(0)=\mathbb{R}^{n} \in \mathcal{F}$.

We claim that for any $f \in \check{\mathcal{F}}$ and any $g \in \mathcal{C}^{\infty}\left(\mathbb{R}^{n}\right)$ we have $f \cdot g \in \check{\mathcal{F}}$.

Indeed, since $f \in \check{\mathcal{F}}, Z(f) \in \mathcal{F}$. Since $Z(f) \subseteq Z(f) \cup Z(g)$ and $Z(f) \in \mathcal{F}$, it follows that $f \cdot g \in \check{\mathcal{F}}$.

Now, given $f, g \in \check{\mathcal{F}}, Z(f), Z(g) \in \mathcal{F}$, and since $\mathcal{F}$ is a filter, $Z(f) \cap Z(g) \in \mathcal{F}$. Since $Z(f) \cap Z(g) \subseteq$ $Z(f+g)$, it follows that $f+g \in \check{\mathcal{F}}$.

Finally, $\varnothing \in \mathcal{F}$ occurs if, and only of there is some invertible $f \in \check{\mathcal{F}}$, thus $1 \in \check{\mathcal{F}}$.

Remark 2.2.11. Let $I \subseteq \mathcal{C}^{\infty}\left(\mathbb{R}^{n}\right)$ be any ideal. Then we have, by definition, $\widehat{I}=\left\{A \subseteq \mathbb{R}^{n} \mid(\exists h \in I)(A=\right.$ $Z(h))\}$, and therefore

$$
\check{\widehat{I}}=\left\{g \in \mathcal{C}^{\infty}\left(\mathbb{R}^{n}\right) \mid(\exists h \in I)(Z(g)=Z(h))\right\} .
$$

Let $\mathcal{F}$ be a proper filter on the set of all closed subsets of $\mathbb{R}^{n}$. Then:

$$
\widehat{\mathcal{F}}=\left\{A \subseteq \mathbb{R}^{n} \mid(\exists g \in \check{\mathcal{F}})(A=Z(g))\right\}=\left\{A \subseteq \mathbb{R}^{n} \mid(\exists g \in \check{\mathcal{F}})((Z(g) \in \mathcal{F}) \&(A=Z(g)))\right\}=\mathcal{F}
$$

since every closed subset $B \subseteq \mathbb{R}^{n}$ is a zero set of some smooth function. 
Proposition 2.2.12. Let $I \subseteq \mathcal{C}^{\infty}\left(\mathbb{R}^{n}\right)$ be an ideal. Then:

$$
\check{\widehat{I}}=\left\{g \in \mathcal{C}^{\infty}\left(\mathbb{R}^{n}\right) \mid(\exists h \in I)(Z(g)=Z(h))\right\}=\sqrt[\infty]{I}
$$

In particular, the $\mathcal{C}^{\infty}$-radical of an ideal I of the free $\mathcal{C}^{\infty}$-ring on finitely many generators, $\mathcal{C}^{\infty}\left(\mathbb{R}^{n}\right)$, is again an ideal.

Proof. Let $g \in \check{\widehat{I}}$, so there is some $h \in I$ such that $Z(g)=Z(h)$, so a fortiori, $Z(g) \supseteq Z(h)$, and $\left(h \uparrow_{U_{g}}\right)$ is invertible in $\mathcal{C}^{\infty}\left(U_{g}\right)$, where $U_{g}=\mathbb{R}^{n} \backslash Z(g)$ (see Proposition 2.1.29). Since there exists $h \in I$ such that $h \in\left(\mathcal{C}^{\infty}\left(\mathbb{R}^{n}\right)\{g\}^{-1}\right)^{\times}$it follows that $g \in \sqrt[\infty]{I}$.

Conversely, given $g \in \sqrt[\infty]{I}$, there must be some $h \in I$ such that $h \in\left(\mathcal{C}^{\infty}\left(\mathbb{R}^{n}\right)\left\{g^{-1}\right\}\right)^{\times}$. But such an $h$ must not be zero when restricted to $U_{g}$ (otherwise $h$ would not be invertible), so $U_{h} \supseteq U_{g}$, and therefore $Z(g) \supseteq Z(h)$. Since $\widehat{I}$ is a filter, $Z(h) \in \widehat{I}$ and $Z(g) \supseteq Z(h)$, it follows that $Z(g) \in \widehat{I}$. But if $Z(g) \in \widehat{I}$ then there is $h^{\prime} \in I$ such that $Z(g)=Z\left(h^{\prime}\right)$ so $g \in \widehat{I}$.

The following proposition is a consequence of Proposition $\mathbf{2 . 2 . 1 2}$

Corollary 2.2.13. Let $A=\mathcal{C}^{\infty}\left(\mathbb{R}^{n}\right)$ be a finitely generated free $\mathcal{C}^{\infty}$-ring. $I \subseteq \mathcal{C}^{\infty}\left(\mathbb{R}^{n}\right)$ is a $\mathcal{C}^{\infty}$-radical ideal, that is, $\sqrt[\infty]{I}=I$, if, and only if:

$$
\left(\forall g \in \mathcal{C}^{\infty}\left(\mathbb{R}^{n}\right)\right)((g \in I) \leftrightarrow(\exists f \in I)(Z(f)=Z(g))) .
$$

The following proposition is a consequence of a comment made by Moerdijk and Reyes in the page 330 of 65$]$ :

Corollary 2.2.14. Let $\mathcal{C}^{\infty}\left(\mathbb{R}^{n}\right)$ be the free $\mathcal{C}^{\infty}$-ring and let $I \subseteq \mathcal{C}^{\infty}\left(\mathbb{R}^{n}\right)$ be a finitely generated ideal, that is,

$$
I=\left\langle g_{1}, \cdots, g_{k}\right\rangle
$$

for some $g_{1}, \cdots, g_{k} \in \mathcal{C}^{\infty}\left(\mathbb{R}^{n}\right)$.

I is a $\mathcal{C}^{\infty}$-radical ideal if, and only if:

$$
\left(\forall x \in Z\left(g_{1}, \cdots, g_{k}\right)\right)(f(x)=0) \rightarrow(f \in I)
$$

Proof. Suppose $I=\sqrt[\infty]{I}$ and let $f \in \mathcal{C}^{\infty}\left(\mathbb{R}^{n}\right)$ be such that:

$$
\left(\forall x \in Z\left(g_{1}, \cdots, g_{k}\right)\right)(f(x)=0) .
$$

We have $g=g_{1}^{2}+\cdots+g_{k}^{2} \in I$ such that $Z(g) \subseteq Z(f)$, so $Z(f) \in \widehat{I}$ (since $\widehat{I}$ is a filter) and by Proposition 2.2.12, $f \in \check{\widehat{I}}=\sqrt[\infty]{I}=I$.

Now, suppose

$$
\left(\forall x \in Z\left(g_{1}, \cdots, g_{k}\right)\right)(f(x)=0) \rightarrow(f \in I)
$$

Given $h \in \sqrt[\infty]{I}=\check{\widehat{I}}$, we have $Z(h) \in \widehat{I}$, so there exists some $g \in I$ such that $Z(h)=Z(g)$, thus $\left(\forall x \in Z\left(g_{1}, \cdots, g_{k}\right)\right)(h(x)=0)$. By hypothesis, this means that $h \in I$, so $\sqrt[\infty]{I} \subseteq I$. Since $I \subseteq \sqrt[\infty]{I}$ always holds, it follows that $I$ is a $\mathcal{C}^{\infty}$-radical ideal. 
By Proposition 4.5 of [59], it follows that any finitely generated $\mathcal{C}^{\infty}$-radical ideal $I$ of a free $\mathcal{C}^{\infty}$-ring on $n$ generators, $\mathcal{C}^{\infty}\left(\mathbb{R}^{n}\right)$ we have:

$$
(\forall x \in Z(I))\left(f \uparrow_{x} \in I \uparrow_{x} \rightarrow f \in I\right),
$$

where $Z(I):=\bigcap_{f \in I} Z(f)$.

As a consequence, we have a rudimentary particular version of the weak Nullstellensatz:

Proposition 2.2.15. For any finitely generated $\mathcal{C}^{\infty}$-radical ideal I of $\mathcal{C}^{\infty}\left(\mathbb{R}^{n}\right)$, we have:

$$
1 \in I \Longleftrightarrow Z(I)=\varnothing
$$

Proof. Let $I=\left\langle g_{1}, \cdots, g_{k}\right\rangle$ be a $\mathcal{C}^{\infty}$-radical ideal of $\mathcal{C}^{\infty}\left(\mathbb{R}^{n}\right)$, so

$$
\left(\forall x \in Z\left(g_{1}, \cdots, g_{k}\right)\right)(f(x)=0) \rightarrow(f \in I)
$$

Suppose $Z\left(g_{1}, \cdots, g_{k}\right)=\varnothing$, so we have, for each $f \in \mathcal{C}^{\infty}\left(\mathbb{R}^{n}\right)$ :

$$
\varnothing=Z\left(g_{1}, \cdots, g_{k}\right) \subseteq Z(f)
$$

so

$$
\left(\forall x \in Z\left(g_{1} \cdots g_{k}\right)\right)(f(x)=0)
$$

Thus $\mathcal{C}^{\infty}\left(\mathbb{R}^{n}\right) \subseteq I$ and, in particular, $1 \in I$.

On the other hand, if $1 \in I$, since $1^{-1}[\{0\}]=\varnothing \in\left\{f^{-1}[\{0\}] \mid f \in I\right\}$, so $Z(I)=\bigcap\left\{f^{\dashv}[\{0\}] \mid f \in I\right\}=$ $\varnothing$.

Example 2.2.16. As a consequence of the fact pointed out by Moerdijk and Reyes in [65], any countably generated prime ideal of $\mathcal{C}^{\infty}\left(\mathbb{R}^{n}\right)$, for $n \in \mathbb{N}$, is a $\mathcal{C}^{\infty}$-radical ideal.

Let $\mathfrak{F}$ be the set of all the filters on the closed parts of $\mathbb{R}^{n}$ and $\mathfrak{I}$ be the set of all the ideals of $\mathcal{C}^{\infty}\left(\mathbb{R}^{n}\right)$. We have, so far, established that for every ideal $I \subseteq \mathcal{C}^{\infty}\left(\mathbb{R}^{n}\right)$ we have $\sqrt[\infty]{I}=\breve{\widehat{I}}$ and for every filter $\mathcal{F} \in \mathfrak{F}$ we have $\widehat{\tilde{\mathcal{F}}}$.

Consider the following diagram:

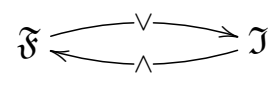

In what follows, we show that $\wedge \vdash \vee$ is a Galois connection.

Proposition 2.2.17. The adjunction $\wedge \vdash \vee$ is a covariant Galois connection between the posets $(\mathfrak{F}, \subseteq)$ and $(\mathfrak{I}, \subseteq)$, i.e.,

a) Given $\mathcal{F}_{1}, \mathcal{F}_{2} \in \mathfrak{F}$ such that $\mathcal{F}_{1} \subseteq \mathcal{F}_{2}$ then $\check{\mathcal{F}}_{1} \subseteq \check{\mathcal{F}}_{2}$;

b) Given $I_{1}, I_{2} \in \mathfrak{I}$ such that $I_{1} \subseteq I_{2}$ then $\widehat{I}_{1} \subseteq \widehat{I}_{2}$;

c) For every $\mathcal{F} \in \mathfrak{F}$ and every $I \in \mathfrak{I}$ we have:

$$
\widehat{I} \subseteq \mathcal{F} \Longleftrightarrow I \subseteq \check{\mathcal{F}} .
$$


Proof. Ad a): Given any $f \in \check{\mathcal{F}}_{1}$ we have $Z(f) \in \mathcal{F}_{1}$, and since $\mathcal{F}_{1} \subseteq \mathcal{F}_{2}, Z(f) \in \mathcal{F}_{2}$, so $f \in \check{\mathcal{F}}_{2}$.

Ad b) Given $A \in \widehat{I_{1}}$, there is $f_{1} \in I_{1}$ such that $A=Z\left(f_{1}\right)$. Since $f_{1} \in I_{1} \subseteq I_{2}, f_{1} \in I_{2}$, so $A=Z\left(f_{1}\right) \in \widehat{I_{2}}$.

Ad c): First we show that $\widehat{I} \subseteq \mathcal{F} \Rightarrow I \subseteq \check{\mathcal{F}}$.

Given any $f \in I, Z(f) \in \widehat{I}$, and since $\widehat{I} \subseteq \mathcal{F}, Z(f) \in \mathcal{F}$ so $f \in \check{\mathcal{F}}$, by definition.

Conversely we show that $I \subseteq \check{\mathcal{F}} \Rightarrow \widehat{I} \subseteq \mathcal{F}$. $\mathcal{F}$.

Given $A \in \widehat{I}$, there exists some $h \in I$ such that $A=Z(h)$, so $h \in \check{\mathcal{F}}$ and, by definition, $Z(h)=A \in$

Proposition 2.2.18. Let $A=\mathcal{C}^{\infty}\left(\mathbb{R}^{n}\right)$ for some $n \in \mathbb{N}$. The Galois connection $\wedge \vdash \vee$ establishes a bijective correspondence between:

(a) proper filters of $(\mathfrak{F}, \subseteq)$ and proper ideals of $(\mathfrak{I}, \subseteq)$;

(b) maximal filters of $(\mathfrak{F}, \subseteq)$ and maximal ideals of $(\mathfrak{I}, \subseteq)$;

(c) prime filters of $(\mathfrak{F}, \subseteq)$ and prime ideals of $(\mathfrak{I}, \subseteq)$.

(d) filters on the closed parts of $\mathbb{R}^{n}, \mathfrak{F}$, and the set of all $\mathcal{C}^{\infty}$-radical ideals of $\mathcal{C}^{\infty}\left(\mathbb{R}^{n}\right), \mathfrak{I}^{\infty}=\{I \subseteq$ $\left.\mathcal{C}^{\infty}\left(\mathbb{R}^{n}\right) \mid \sqrt[\infty]{I}=I\right\}$

Proof. Ad (a): By Proposition 2.2.9, $I$ is a proper ideal if, and only if, $\check{I}$ is a proper filter, and by Proposition 2.2.10 $F$ is a proper filter if, and only if, $\widehat{\mathcal{F}}$ is a proper ideal. In particular, whenever $I$ is a proper ideal, $\sqrt[\infty]{I}=\widehat{I}$ is a proper ideal.

Ad (b): If $I$ is a maximal ideal then it is proper, so $\check{I}$ is a proper filter. By item (a), $\widehat{\tilde{I}}$ is a proper ideal. Since $I \subseteq \widehat{\tilde{I}}$ and $I$ is a maximal ideal, we have $I=\widehat{\widetilde{I}}=\sqrt[\infty]{I}$. In particular, every maximal ideal of $\mathcal{C}^{\infty}\left(\mathbb{R}^{n}\right)$ is a $\mathcal{C}^{\infty}$-radical ideal.

On the other hand, for any filter $\mathcal{F}$ we have $\mathcal{F}=\breve{\hat{\mathcal{F}}}$. If $\check{I} \subseteq \mathcal{F}$ for some proper filter $\mathcal{F}$, then $I=\widehat{\tilde{I}} \subseteq \widehat{\mathcal{F}}$, and since $\widehat{\mathcal{F}}$ is a proper ideal, by (a), it follows that:

$$
I=\widehat{\mathcal{F}} \Rightarrow \check{I}=\check{\widehat{\mathcal{F}}}=\mathcal{F},
$$

so $\check{I}$ is a maximal filter.

Conversely, suppose $\mathcal{F}$ is a maximal filter. If $J$ is some proper ideal of $\mathcal{C}^{\infty}\left(\mathbb{R}^{n}\right)$ such that $\widehat{\mathcal{F}} \subseteq J$, then $\breve{\widehat{\mathcal{F}}} \subseteq \check{J}$, so $\mathcal{F} \subseteq \breve{J}$. Since $\check{J}$ is a proper filter, we have $\mathcal{F}=\breve{J}$, so $\widehat{\mathcal{F}}=\widehat{\breve{J}}$. Since $J \subseteq \widehat{\widetilde{J}}$, we have $\widehat{\mathcal{F}}=\widehat{\tilde{J}}=J$, and $\widehat{\mathcal{F}}$ is a maximal ideal.

Ad (c): Let $\mathcal{F}$ be a prime filter. If $f, g \in \mathcal{C}^{\infty}\left(\mathbb{R}^{n}\right)$ are such that $f \cdot g \in \check{\mathcal{F}}$, then $Z(f \cdot g)=Z(f) \cup Z(g) \in$ $\mathcal{F}$, so we have $Z(f) \in \mathcal{F}$ or $Z(g) \in \mathcal{F}$. Thus, $f \in \check{\mathcal{F}}$ or $g \in \check{\mathcal{F}}$, so $\check{\mathcal{F}}$ is a prime ideal.

Let $I$ be a prime ideal of $\mathcal{C}^{\infty}\left(\mathbb{R}^{n}\right)$, that is, if $f, g \in \mathcal{C}^{\infty}\left(\mathbb{R}^{n}\right)$ are such that $f \cdot g \in I$ then $f \in I$ or $g \in I$. We need to show that $\widehat{I}=\{Z(h) \mid h \in I\}$ is a prime filter. 
Let $F, G \subseteq \mathbb{R}^{n}$ be two closed sets such that $F \cup G \in \widehat{I}$. By definition of $\widehat{I}$, there is some $\varphi: \mathbb{R}^{n} \rightarrow \mathbb{R}$ such that $F \cup G=Z(\varphi)$. Consider:

$$
\begin{aligned}
\widetilde{\varphi}: \quad \mathbb{R}^{n} & \rightarrow \mathbb{R} \\
\vec{x} & \mapsto \varphi^{2}(\vec{x})
\end{aligned}
$$

and we have $F \cup G=Z(\widetilde{\varphi})$.

Since $F, G$ are closed subsets of $\mathbb{R}^{n}$ there are $f, g \in \mathcal{C}^{\infty}\left(\mathbb{R}^{n}\right)$ such that $F=Z(f)$ and $G=Z(g)$. Considering:

$$
\begin{aligned}
\tilde{f}: \mathbb{R}^{n} & \rightarrow \mathbb{R} \\
\vec{x} & \mapsto f^{2}(\vec{x})
\end{aligned}
$$

and

$$
\begin{aligned}
\widetilde{g}: \quad \mathbb{R}^{n} & \rightarrow \mathbb{R} \\
\vec{x} & \mapsto g^{2}(\vec{x})
\end{aligned}
$$

we have $\widetilde{\varphi}, \widetilde{f}$ and $\widetilde{g}$ non-negative functions, and both $F=Z(\widetilde{f})$ and $G=Z(\widetilde{g})$.

Let

$$
\begin{aligned}
& \psi: \mathbb{R}^{n} \rightarrow \quad \mathbb{R} \\
& \vec{x} \mapsto \widetilde{f}(\vec{x})-\widetilde{g}(\vec{x})
\end{aligned}
$$

and define $H=\left\{\vec{x} \in \mathbb{R}^{n} \mid \psi(\vec{x}) \leq 0\right\}$ and $K=\left\{\vec{x} \in \mathbb{R}^{n} \mid \psi(\vec{x}) \geq 0\right\}$, so $\mathbb{R}^{n}=H \cup K$

Claim: $H \cap Z(\widetilde{\varphi})=F$.

Given $\vec{x} \in F$, we have $\widetilde{f}(\vec{x})=0$, hence $\psi(\vec{x})=0-\widetilde{g}(\vec{x}) \leq 0$ and $x \in H$. Also, since $Z(\widetilde{f}) \subseteq Z(\widetilde{\varphi})$, $\widetilde{\varphi}(\vec{x})=0$.

On the other hand, given $\vec{x} \in H \cap Z(\widetilde{\varphi})$, since $\vec{x} \in Z(\widetilde{\varphi})=Z(\widetilde{f}) \cup Z(\widetilde{g})$, then either $\vec{x} \in Z(\widetilde{f})$ or $\vec{x} \in Z(\widetilde{g})$. If $\vec{x} \in Z(\widetilde{g})$ then $\widetilde{g}(\vec{x})=0$ and $\psi(\vec{x})=\widetilde{f}(\vec{x})-0 \leq 0$, thus $\psi(\vec{x})=\widetilde{f}(\vec{x})=0$. Hence $\vec{x} \in Z(\widetilde{f})=F$.

Analogously we prove that $K \cap Z(\widetilde{\varphi})=G$.

Let $h, k \in \mathcal{C}^{\infty}\left(\mathbb{R}^{n}\right)$ be non-negative functions such that $K=Z(k)$ and $H=Z(h)$. Note that $Z(\widetilde{\varphi}+\widetilde{h})=$ $F(\widetilde{\varphi}(x)+\widetilde{h}(x)=0 \Longleftrightarrow \widetilde{\varphi}(x)=\widetilde{h}(x)=0)$ and $Z(\widetilde{\varphi}+\widetilde{k})=G$. For every $\vec{x} \in \mathbb{R}^{n}=Z(h \cdot k)=H \cup K$. Since $\widetilde{\varphi} \in I$ and $I$ is an ideal, $[(\widetilde{h}+\widetilde{k}) \cdot \widetilde{\varphi}] \in I$, so:

$$
[(\widetilde{\varphi}+\widetilde{h}) \cdot(\widetilde{\varphi}+\widetilde{k})]=\widetilde{\varphi}^{2}+[(\widetilde{h}+\widetilde{k}) \cdot \widetilde{\varphi}] \in I
$$
filter.

Since $I$ is a prime ideal, either $(\widetilde{\varphi}+\widetilde{h}) \in I$ or $(\widetilde{\varphi}+\widetilde{k}) \in I$, i.e., $F \in \widehat{I}$ or $G \in \widehat{I}$, hence $\widehat{I}$ is a prime

Ad (d): Consider the following maps:

$$
\begin{aligned}
\varphi: \mathfrak{F} & \rightarrow \mathfrak{I}^{\infty} \\
\mathcal{F} & \mapsto \check{\mathcal{F}}
\end{aligned}
$$

and

$$
\begin{aligned}
\psi: \mathfrak{I}^{\infty} & \rightarrow \mathfrak{F} \\
I & \mapsto \widehat{I}
\end{aligned}
$$


We have already seen that whenever $I$ is an ideal, we have $\sqrt[\infty]{I}=\breve{\widehat{I}}$. We must verify that $\varphi$ takes filters to $\mathcal{C}^{\infty}$-radical ideals, i.e., $\mathcal{F} \in \mathfrak{F} \rightarrow \check{\mathcal{F}} \in \mathfrak{I}^{\infty}$. Now, for every $\mathcal{F} \in \mathfrak{F}$ we have $\stackrel{\widehat{\mathcal{F}}}{\mathcal{F}}$, so $\sqrt[\infty]{\check{\mathcal{F}}}=\stackrel{\vee}{\widetilde{\mathcal{F}}}=\check{\mathcal{F}}$. Thus, $\psi \circ \varphi(\mathcal{F})=\mathcal{F}$.

On the other hand, if $J \in \mathfrak{I}^{\infty}$ then $\varphi \circ \psi(J)=\check{\widehat{J}}=\sqrt[\infty]{J}=J=\operatorname{id}_{\mathfrak{I}^{\infty}}(J)$. It follows that $\varphi$ and $\psi$ are inverse bijections.

Corollary 2.2.19. Let $A=\mathcal{C}^{\infty}\left(\mathbb{R}^{n}\right)$ and $\mathfrak{p}$ be a prime ideal of $A$. Under those circumstances $\sqrt[\infty]{\mathfrak{p}}$ is a prime ideal.

Proof. By the Corollary 2.2.18, since $\mathfrak{p}$ is a prime ideal, the filter associated with $\mathfrak{p}, \widehat{\mathfrak{p}}$ is a prime filter. Again by Corollary 2.2.18, it follows that $\breve{\mathfrak{p}}=\sqrt[\infty]{\mathfrak{p}}$ is a prime ideal.

Corollary 2.2.20. The composite $\vee \circ \wedge: \mathfrak{I} \rightarrow \mathfrak{I}$ is a closure operator, thus we have the following three properties:

(a) $I \subseteq \check{\widehat{I}}$;

(b) $I_{1} \subseteq I_{2} \Rightarrow \check{\widehat{I}_{1}} \subseteq \check{\widehat{I_{2}}}$;

(c) If $J=\breve{\widehat{I}}$ then $\breve{\widehat{J}}=J$.

Theorem 2.2.21. Let $I, I_{1}, I_{2} \subseteq \mathcal{C}^{\infty}\left(\mathbb{R}^{n}\right)$ be ideals. Then:

(a) $\sqrt[\infty]{I}$ is an ideal of $\mathcal{C}^{\infty}\left(\mathbb{R}^{n}\right)$ and $I \subseteq \sqrt[\infty]{I}$;

(b) $I_{1} \subseteq I_{2} \Rightarrow \sqrt[\infty]{I_{1}} \subseteq \sqrt[\infty]{I_{2}}$

(c) $\sqrt[\infty]{\sqrt[\infty]{I}}=\sqrt[\infty]{I}$

Proof. Ad (a): From Proposition 2.2.12 we have $\sqrt[\infty]{I}=\breve{\hat{I}}$, and from Proposition 2.2.10 it follows that $\breve{\hat{I}}$ is an ideal. Moreover, since $\vee \circ \wedge$ is a closure operator, by item a) of Corollary 2.2.20 we have $I \subseteq \check{\widehat{I}}=\sqrt[\infty]{I}$.

Ad (b): From item (b) of Corollary 2.2.20, $I_{1} \subseteq I_{2} \Rightarrow \check{I_{1}} \subseteq \check{\widetilde{I_{2}}}$, so $\sqrt[\infty]{I_{1}} \subseteq \sqrt[\infty]{I_{2}}$.

Ad (c): From item (c) of Corollary $\mathbf{2 . 2 . 2 0}$ it follows immediately by the idempotence of $\vee \circ \wedge$ that $\sqrt[\infty]{\sqrt{I}}=\sqrt[\infty]{I}$

Theorem 2.2.22. Let $A$ be a $\mathcal{C}^{\infty}$-ring. We have:

$$
\bigcap \operatorname{Spec}^{\infty}(A)=\sqrt[\infty]{(0)}
$$

Proof. In order to prove the inclusion $\sqrt[\infty]{(0)} \subseteq \bigcap \operatorname{Spec}^{\infty}(A)$, we observe that for every $\mathfrak{p} \in \operatorname{Spec}^{\infty}(A)$ we have $(0) \subseteq \mathfrak{p}$. By item b) of Theorem $2.2 .21, \sqrt[\infty]{(0)} \subseteq \sqrt[\infty]{\mathfrak{p}}=\mathfrak{p}$

We prove the other inclusion by contradiction. $A b$ absurdo, suppose $\bigcap \operatorname{Spec}^{\infty}(A) \nsubseteq \sqrt[\infty]{(0)}$. This implies that there exists some $x \in \mathfrak{p}$ such that $x \notin \sqrt[\infty]{(0)}$ and, therefore, $D^{\infty}(x) \nsubseteq D^{\infty}((0))=\varnothing$, so $D^{\infty}(x) \neq \varnothing$. Taking into account that $D^{\infty}(x)=\left\{\mathfrak{p} \in \operatorname{Spec}^{\infty}(A) \mid x \notin \mathfrak{p}\right\}$, we have $\left(D^{\infty}(x) \neq \varnothing\right) \Longleftrightarrow$ $\left(\left(\exists \mathfrak{p} \in \operatorname{Spec}^{\infty}(A)\right)(x \notin \mathfrak{p})\right)$ and we achieve the absurdity (we have simultaneously $\left(\forall \mathfrak{p} \in \operatorname{Spec}^{\infty}(A)\right)(x \in \mathfrak{p})$ and $\left.\left(\exists \mathfrak{p} \in \operatorname{Spec}^{\infty}(A)\right)(x \notin \mathfrak{p})\right)$. 
The following definition will be helpful to prove that whenever $I$ is an ideal of any $\mathcal{C}^{\infty}-\operatorname{ring} A$, then $\sqrt[\infty]{I}$ is an ideal too.

Definition 2.2.23. Let $A$ be a $\mathcal{C}^{\infty}$-ring. We say that $A$ is admissible if for every ideal $I \subseteq A, \sqrt[\infty]{I}$ is an ideal in $A$.

In what follows we sketch a proof that every $\mathcal{C}^{\infty}$-ring $A$ is admissible.

Lemma 2.2.24. Let $A$ and $A^{\prime}$ be $\mathcal{C}^{\infty}$-rings such that $A \cong A^{\prime}$. Then if $A$ is an admissible $\mathcal{C}^{\infty}$-ring, $A^{\prime}$ is admissible too.

Lemma 2.2.25. The free $\mathcal{C}^{\infty}$-ring in $n$ generators, $\mathcal{C}^{\infty}\left(\mathbb{R}^{n}\right)$, is an admissible $\mathcal{C}^{\infty}$-ring.

Proof. It follows immediately from Proposition 2.2.12.

Lemma 2.2.26. Let $A$ be an admissible $\mathcal{C}^{\infty}$-ring and $J \subset A$ be an ideal. Then $\frac{A}{J}$ is an admissible $\mathcal{C}^{\infty}$-ring.

Lemma 2.2.27. Let $\left\{A_{i} \stackrel{h_{i j}}{\rightarrow} A_{j}\right\}$ be a filtered diagram of admissible $\mathcal{C}^{\infty}$-rings. Then $\stackrel{\lim }{\longrightarrow} A_{i}$ is an admissible $\mathcal{C}^{\infty}$-ring.

Theorem 2.2.28. Every $\mathcal{C}^{\infty}$-ring is admissible.

Proof. Let $A$ be any $\mathcal{C}^{\infty}$-ring. We know that every $\mathcal{C}^{\infty}$-ring is a filtered colimit of finitely presented $\mathcal{C}^{\infty}$-rings. Since every finitely presentable $\mathcal{C}^{\infty}$-ring is admissible, the result follows from Lemma 2.2.27.

Let $B$ be any finitely presentable $\mathcal{C}^{\infty}$-ring. We know that there exist some $n \in \mathbb{N}$ and some ideal $J \subset \mathcal{C}^{\infty}\left(\mathbb{R}^{n}\right)$ such that $A \cong \frac{\mathcal{C}^{\infty}\left(\mathbb{R}^{n}\right)}{J}$. From Lemma 2.2.24 if we prove that $\frac{\mathcal{C}^{\infty}\left(\mathbb{R}^{n}\right)}{J}$ is admissible, then it follows that $A$ is admissible.

From Lemma 4.3.6 we have that $\mathcal{C}^{\infty}\left(\mathbb{R}^{n}\right)$ is admissible, and from Lemma 2.2.26 it follows that $\frac{\mathcal{C}^{\infty}\left(\mathbb{R}^{n}\right)}{J}$ is admissible.

Now we present some properties of taking the $\mathcal{C}^{\infty}$-radical of an ideal.

Proposition 2.2.29. Let $A$ be a $\mathcal{C}^{\infty}-$ ring, $I, J \subseteq A$ any of its ideals. Then:

(i) $I \subseteq J \Rightarrow \sqrt[\infty]{I} \subseteq \sqrt[\infty]{J}$

(ii) $I \subseteq \sqrt[\infty]{I}$

Proof. Ad (i): Given $a \in \sqrt[\infty]{I}$, there is $b \in I$ such that $\eta_{a}(b) \in\left(A\left\{a^{-1}\right\}\right)^{\times}$. Since $I \subseteq J$, the same $b$ is a witness of the fact that $a \in \sqrt[\infty]{J}$, for $b \in J$ and $\eta_{a}(b) \in\left(A\left\{a^{-1}\right\}\right)$.

Ad (ii): We are going to show that $A \backslash \sqrt[\infty]{I} \subseteq A \backslash I$.

Given $a \in A \backslash \sqrt[\infty]{I}$, we have that $(\forall b \in I)\left(\eta_{a}(b) \notin\left(A\left\{a^{-1}\right\}\right)^{\times}\right)$, so $\eta_{a}[I] \cap\left(A\left\{a^{-1}\right\}\right)^{\times}=\varnothing$. Since $\eta_{a}(a) \in\left(A\left\{a^{-1}\right\}\right)^{\times}$, it follows that $\eta_{a}(a) \notin \eta_{a}[I]$, so $a \notin I$. 
Proposition 2.2.30. Let $B$ be a $\mathcal{C}^{\infty}$-ring and $J \subseteq B$ any of its ideals. We have the following equality:

$$
\sqrt[\infty]{\left\{0_{\frac{B}{J}}\right\}}=\frac{\sqrt[\infty]{J}}{J}
$$

Proof.

$$
a \in \sqrt[\infty]{J} \Longleftrightarrow(\exists b \in J)\left(\eta(b) \in\left(B\left\{a^{-1}\right\}\right)^{\times}\right),
$$

hence

$$
\begin{aligned}
a+J=\bar{a} \in \sqrt[\infty]{\left\{0_{\frac{B}{J}}\right\}} \Longleftrightarrow\left(\exists \bar{b} \in\left\{0_{\frac{B}{J}}\right\}\right)\left(\overline{\eta_{a}}(\bar{b}) \in\left(\frac{B}{J}\left\{(a+J)^{-1}\right\}\right)^{\times}\right) & \Longleftrightarrow \frac{B}{J}\left\{(a+J)^{-1}\right\} \cong\{0\} \Longleftrightarrow \\
& \Longleftrightarrow a \in \sqrt[\infty]{J} \Longleftrightarrow a+J \in \frac{\sqrt[\infty]{J}}{J}
\end{aligned}
$$

Now, since $J \subseteq \sqrt[\infty]{J}$, if $a^{\prime}+J=a+J$, then $a \in \sqrt[\infty]{J} \Longleftrightarrow a^{\prime} \in \sqrt[\infty]{J}$.

Corollary 2.2.31. Let $A$ be a $\mathcal{C}^{\infty}$-ring. We have:

(a) An ideal $J \subseteq A$ is a $\mathcal{C}^{\infty}$-radical ideal if, and only if, $\frac{A}{J}$ is a $\mathcal{C}^{\infty}$-reduced $\mathcal{C}^{\infty}$-ring

(b) A proper prime ideal $\mathfrak{p} \subseteq A$ is $\mathcal{C}^{\infty}$-radical if, and only if, $\frac{A}{\mathfrak{p}}$ is a $\mathcal{C}^{\infty}$-reduced $\mathcal{C}^{\infty}$-domain.

Remark 2.2.32. Not every prime proper ideal of a $\mathcal{C}^{\infty}$-ring $A$ is a $\mathcal{C}^{\infty}$-radical ideal. Consider the ideal of functions which are flat at $0, \mathfrak{m}_{0}^{\infty} \subseteq \mathcal{C}^{\infty}(\mathbb{R})$, which is a maximal - and thus a prime - ideal of $\mathcal{C}^{\infty}(\mathbb{R})$. We claim that this ideal is not a $\mathcal{C}^{\infty}$-radical ideal (cf. Example 1.2 of [67]).

Consider $\operatorname{id}_{\mathbb{R}}: \mathbb{R} \rightarrow \mathbb{R}$ and $\mathcal{C}^{\infty}(\mathbb{R})\left\{\operatorname{id}_{\mathbb{R}}^{-1}\right\} \cong{ }_{\varphi} \mathcal{C}^{\infty}(\mathbb{R} \backslash\{0\})$. Consider:

$$
\begin{aligned}
& f: \mathbb{R} \backslash\{0\} \rightarrow \quad \mathbb{R} \quad g: \mathbb{R} \backslash\{0\} \rightarrow \quad \rightarrow \quad \mathbb{R} \\
& x \mapsto\left\{\begin{array} { l l } 
{ 0 , } & { \text { if } x < 0 } \\
{ 1 , } & { \text { if } x > 0 . }
\end{array} \quad x \quad \mapsto \left\{\begin{array}{ll}
1, & \text { if } x<0 \\
0, & \text { if } x>0 .
\end{array}\right.\right.
\end{aligned}
$$

We have $f+g=1 \in \mathcal{C}^{\infty}(\mathbb{R} \backslash\{0\})$, thus $\varphi(f+g)=\varphi(f)+\varphi(g)=\varphi(1)=1 \in \mathcal{C}^{\infty}(\mathbb{R})_{\mathfrak{m}_{0}^{\infty}}$. But neither $\varphi(f)$ nor $\varphi(g)$ can be inverted in $\mathcal{C}^{\infty}(\mathbb{R})_{\mathfrak{m}_{0}^{\infty}}$. For, if $\varphi(f)$ were invertible, then $f$ would be invertible and there would be an $h \notin \mathfrak{m}_{0}^{\infty}$ with $U_{f} \supset U_{h}$, i.e., $h \uparrow_{]-\infty, 0[} \cong 0$, contradicting $h \notin \mathfrak{m}_{0}^{\infty}$; Hence $\mathcal{C}^{\infty}(\mathbb{R})_{\mathfrak{m}_{0}^{\infty}}$ is not a local $\mathcal{C}^{\infty}$-ring.

Proposition 2.2.33. Let $A^{\prime}, B^{\prime}$ be two $\mathcal{C}^{\infty}$-rings and $\jmath: A^{\prime} \rightarrow B^{\prime}$ be a monomorphism. If $B^{\prime}$ is $\mathcal{C}^{\infty}$-reduced, then $A^{\prime}$ is also $\mathcal{C}^{\infty}$-reduced.

Proof. Since $B^{\prime}$ is reduced, $\left\{0_{B^{\prime}}\right\}=\sqrt[\infty]{\left\{0_{B^{\prime}}\right\}}$.

Suppose, ab absurdo, that $\left(\exists a^{\prime} \in \sqrt[\infty]{\left\{0_{A^{\prime}}\right\}}\right)\left(a^{\prime} \neq 0_{A^{\prime}}\right)$. Consider the following commutative diagram:

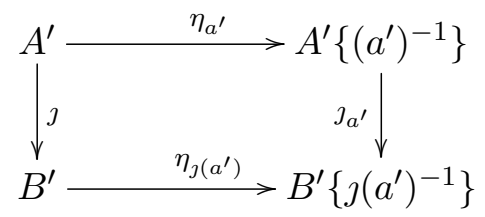

where $\jmath_{a^{\prime}}$ is the $\mathcal{C}^{\infty}$-homomorphism which is induced by $\eta_{\jmath\left(a^{\prime}\right)} \circ \jmath: A^{\prime} \rightarrow B^{\prime}\left\{\jmath\left(a^{\prime}\right)^{-1}\right\}$. 
Since $a^{\prime} \neq 0_{A^{\prime}}$ and $\jmath: A^{\prime} \rightarrow B^{\prime}$ is injective, it follows that $\jmath\left(a^{\prime}\right) \neq 0_{B^{\prime}}$. Since $B^{\prime}$ is reduced, $B^{\prime}\left\{\jmath\left(a^{\prime}\right)^{-1}\right\} \not\{0\}$. On the other hand, since $a^{\prime} \in \sqrt[\infty]{\left\{0_{A^{\prime}}\right\}}, A^{\prime}\left\{a^{\prime-1}\right\} \cong\{0\}$. Thus, $\jmath_{a}: A^{\prime}\left\{a^{\prime-1}\right\} \rightarrow$ $B^{\prime}\left\{\jmath\left(a^{\prime}\right)^{-1}\right\}$ is a $\mathcal{C}^{\infty}$-rings morphism such that $\jmath_{a}\left(1_{A^{\prime}\left\{a^{\prime-1}\right\}}\right)=1_{B^{\prime}\left\{\jmath\left(a^{\prime}\right)^{-1}\right\}}$ from the trivial $\mathcal{C}^{\infty}$-ring $A^{\prime}\left\{a^{\prime-1}\right\} \cong\{0\}$ into a non-trivial $\mathcal{C}^{\infty}$-ring $B^{\prime}\left\{\jmath\left(a^{\prime}\right)^{-1}\right\}$, which is absurd.

Hence, $\sqrt[\infty]{\left\{0_{A^{\prime}}\right\}}=\left\{0_{A^{\prime}}\right\}$ and $A^{\prime}$ is reduced.

As a consequence of Proposition 2.2.33 and of Proposition 2.1.47, we have the following:

Corollary 2.2.34. Every $\mathcal{C}^{\infty}$-subring of a $\mathcal{C}^{\infty}$-field is a $\mathcal{C}^{\infty}$-reduced $\mathcal{C}^{\infty}$-domain

Next we show that the directed limit of reduced $\mathcal{C}^{\infty}$-rings is also reduced.

Proposition 2.2.35. Let $(I, \leq)$ be a directed set and suppose that $\left\{A_{i}\right\}_{i \in I}$ is a directed family of $\mathcal{C}^{\infty}$-reduced $\mathcal{C}^{\infty}$-rings. Then

$$
B=\underset{i \in I}{\lim _{i \in I}} A_{i}
$$

is a $\mathcal{C}^{\infty}$-reduced $\mathcal{C}^{\infty}$-ring.

Proof. Let $u \in \sqrt[\infty]{\left\{0_{B}\right\}}$, so $B\left\{u^{-1}\right\} \cong 0$, and let $j \in I$ and $u_{j} \in A_{j}$ be such that $u=t_{j}\left(u_{j}\right)$. By Theorem 2.1.40,

$$
B\left\{u^{-1}\right\} \cong \varliminf_{i \geq j} A_{i}\left\{u_{i}^{-1}\right\} .
$$

so there is some $\ell \geq j$ such that $A_{\ell}\left\{u_{\ell}^{-1}\right\} \cong 0$. Indeed, $1=0$ in $\lim _{i \geq j} A_{i}\left\{u_{i}{ }^{-1}\right\}$.

Since $A_{\ell}$ is $\mathcal{C}^{\infty}$-reduced, it follows that $u_{\ell}=0$. Hence, $u=t_{\ell}\left(u_{\ell}\right)=t_{\ell}(0)=0$, and $B$ is $\mathcal{C}^{\infty}$-reduced.

Theorem 2.2.36. Let $A$ and $B$ be two $\mathcal{C}^{\infty}$-rings, $J \subseteq B$ a $\mathcal{C}^{\infty}$-radical ideal in $B$ and $f: A \rightarrow B$ any $\mathcal{C}^{\infty}$-homomorphism. Then $f^{-1}[J]$ is a $\mathcal{C}^{\infty}$-radical ideal in $A$.

Proof. Since $\sqrt[\infty]{J}=J$ and $J$ is an ideal, it follows that $f^{-1}[\sqrt[\infty]{J}]=f^{-}[J]$ is also an ideal.

We always have:

$$
f^{\dashv}[J] \subseteq \sqrt[\infty]{f^{\dashv}[J]}
$$

so $f^{-1}[\sqrt[\infty]{J}]=f^{-1}[J] \subseteq \sqrt[\infty]{f^{-}[J]}$. It remains to show the other inclusion, namely:

$$
\sqrt[\infty]{f^{\dashv}[J]} \subseteq f^{\dashv}[\sqrt[\infty]{J}] .
$$

Now, given the morphism $f: A \rightarrow B$, we compose it with the projection map:

$$
\begin{aligned}
q_{J}: \quad B & \rightarrow \frac{B}{J} \\
b & \mapsto b+J
\end{aligned},
$$

in order to get the following commutative triangle:

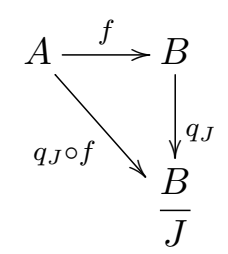


By the Theorem of Homomorphism, there exists a unique morphism $f_{J}: \frac{A}{f^{\dashv}[J]} \rightarrow \frac{B}{J}$ such that the following triangle commutes:

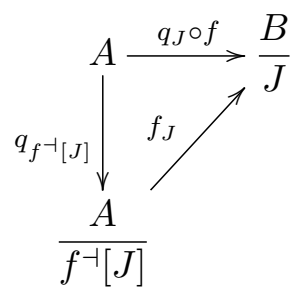

We note that $f_{J}: \frac{A}{f^{-1}[J]} \rightarrow \frac{B}{J}$ is injective, since:

$f_{J}^{\dashv}\left[0_{\frac{B}{J}}\right]=\left\{a+f^{-}[J] \mid f_{J}\left(a+f^{-}[J]\right)=J\right\}=\left\{a+f^{\dashv}[J] \mid f(a)+J=J\right\}=\left\{a+f^{\dashv}[J] \mid f(a) \in J\right\}=\left\{0_{\frac{A}{f^{\dashv}[J]}}\right\}$

Since $J$ is a $\mathcal{C}^{\infty}$-radical ideal of $B$, it follows by Corollary 2.2 .34 that $\frac{\sqrt[\infty]{J}}{J} \cong\left\{0_{\frac{B}{J}}\right\}$, so $\frac{B}{J}$ is $\mathcal{C}^{\infty}$-reduced. By Proposition 2.2.33 since $f_{J}$ is a monomorphism, we conclude that $\frac{A}{f^{\dashv}[J]}$ is also reduced, so:

$$
\frac{\infty}{f^{-1}[J]}=\infty \sqrt{\left\{0 \frac{A}{f^{-}[J]}\right\}}=\left\{0 \frac{A}{f^{-1}[J]}\right\}
$$

Hence:

$$
\sqrt[\infty]{f^{-}[J]}=f^{\dashv}[J]
$$

We register that, in general, holds:

Proposition 2.2.37. Let $A, B$ be $\mathcal{C}^{\infty}$-rings, $f: A \rightarrow B$ a $\mathcal{C}^{\infty}$-homomorphism and $J \subseteq B$ any ideal. Then:

$$
\sqrt[\infty]{f^{-1}[J]} \subseteq f^{-1}[\sqrt[\infty]{J}]
$$

Proof. Given the $\mathcal{C}^{\infty}$-homomorphism $f: A \rightarrow B$, there exists a unique morphism $f_{J}: \frac{A}{f^{-}[J]} \rightarrow \frac{B}{J}$ such that the following diagram commutes:

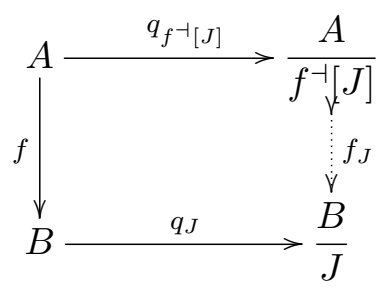

Consider the rings of fractions:

$$
\begin{aligned}
& \eta_{a+f^{\dashv}[J]}: \frac{A}{f^{\dashv}[J]} \rightarrow\left(\frac{A}{f^{\dashv}[J]}\right)\left\{a+f^{\dashv}[J]\right\} \\
& \eta_{f(a)+J}:\left(\frac{B}{J}\right) \rightarrow\left(\frac{B}{J}\right)\left\{(f(a)+J)^{-1}\right\}
\end{aligned}
$$

and note that the $\mathcal{C}^{\infty}$-homomorphism:

$$
\eta_{f(a)+J} \circ f_{J}: \frac{A}{f^{-}[J]} \rightarrow\left(\frac{B}{J}\right)\left\{(f(a)+J)^{-1}\right\}
$$


is such that $\left(\eta_{f(a)+J}\left(f_{J}\left(a+f^{-}[J]\right)\right)\right)=f(a)+J \in\left[\left(\frac{B}{J}\right)\left\{(f(a)+J)^{-1}\right\}\right]^{\times}$. By the universal property of the ring of fractions $\eta_{a+f^{\dashv}[J]}$, there exists a unique morphism $f_{(J, a)}$ such that the following triangle commutes:

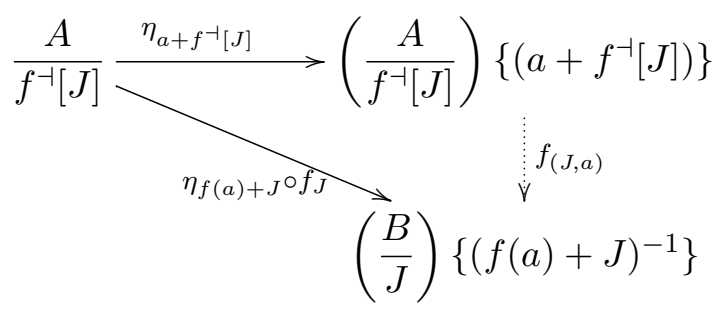

so we have the following commutative rectangle:

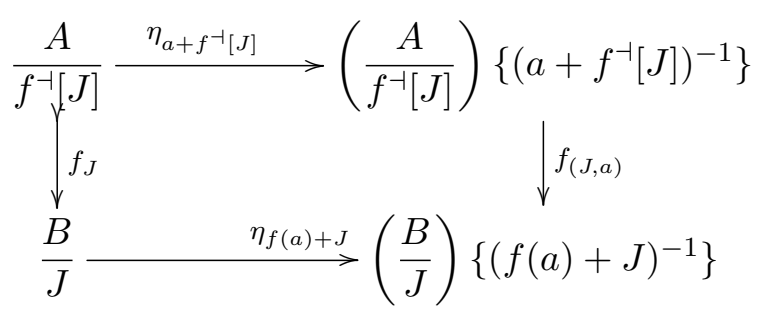

Hence, given $a \in \sqrt[\infty]{f^{-[}[J]}$, we have $\left(\frac{A}{f^{-}[J]}\right)\left\{\left(a+f^{-}[J]\right)^{-1}\right\} \cong\{0\}$, and since the rectangle commutes,

$$
\left(\eta_{f(a)+J} \circ f_{J}\right)(a+\sqrt[\infty]{J})=0 .
$$

However, we know that $\left(\eta_{f(a)+J} \circ f_{J}\right)\left(a+f^{-1}[J]\right) \in\left(\frac{B}{J}\right)\left\{(f(a)+J)^{-1}\right\}^{\times}$, so equation 2.6 tells us that $0 \in\left(\frac{B}{J}\right)\left\{(f(a)+J)^{-1}\right\}^{\times}$, hence:

$$
\left(\frac{B}{J}\right)\left\{(f(a)+J)^{-1}\right\} \cong\{0\}
$$

so $f(a) \in \sqrt[\infty]{J}$.

Remark 2.2.38. With exactly the same method used in the proof of Theorem 2.2.28, one proves that whenever $\mathfrak{p} \subseteq A$ is a prime ideal, $\sqrt[\infty]{\mathfrak{p}}$ is also a prime ideal.

At this point it is natural to look for a $\mathcal{C}^{\infty}$-analog of the Zariski spectrum of a commutative unital ring. With this motivation, we give the following:

Definition 2.2.39. For a $\mathcal{C}^{\infty}$-ring $A$, we define:

$$
\operatorname{Spec}^{\infty}(A)=\left\{\mathfrak{p} \in \operatorname{Spec}(A) \mid \mathfrak{p} \text { is } \mathcal{C}^{\infty}-\text { radical }\right\}
$$

equipped with the smooth Zariski topology defined by its basic open sets:

$$
D^{\infty}(a)=\left\{\mathfrak{p} \in \operatorname{Spec}^{\infty}(A) \mid a \notin \mathfrak{p}\right\}
$$

A more detailed study of the smooth Zariski spectrum will be given in the next chapter.

Now we prove some properties of $\mathcal{C}^{\infty}$-radical ideals of a $\mathcal{C}^{\infty}$-ring $A$

Proposition 2.2.40. The following results hold:

(a) Suppose that $(\forall \alpha \in \Lambda)\left(I_{\alpha} \in \mathfrak{I}_{A}^{\infty}\right)$. Then $\bigcap_{\alpha \in \Lambda} I_{\alpha} \in \mathfrak{I}_{A}^{\infty}$, that is, if $(\forall \alpha \in \Lambda)\left(I_{\alpha} \in \mathfrak{I}_{A}^{\infty}\right)$, then:

$$
\sqrt[\infty]{\bigcap_{\alpha \in \Lambda} I_{\alpha}}=\bigcap_{\alpha \in \Lambda} I_{\alpha}=\bigcap_{\alpha \in \Lambda} \sqrt[\infty]{I_{\alpha}}
$$


(b) If $I \subseteq A$ is any ideal, then $\sqrt[\infty]{I}=\bigcap\left\{\mathfrak{p} \in \operatorname{Spec}^{\infty}(A) ; I \subseteq \mathfrak{p}\right\}$

(c) Let $\left\{I_{\alpha} \mid \alpha \in \Sigma\right\}$ an upward directed family of elements of $\mathfrak{I}_{A}^{\infty}$. Then $\bigcup_{\alpha \in \Sigma} I_{\alpha} \in \mathfrak{I}_{A}^{\infty}$.

Proof. (a) Let $u \in \sqrt[\infty]{\bigcap_{\alpha \in \Lambda} I_{\alpha}}$. There exists $b \in \bigcap_{\alpha \in \Lambda} I_{\alpha}$ such that $\operatorname{Can}_{u}(b) \in\left(A\left\{u^{-1}\right\}\right)^{\times}$.

Since $b \in \bigcap_{\alpha \in \Lambda} I_{\alpha}$, then $(\forall \alpha \in \Lambda)\left(\exists b_{\alpha} \in I_{\alpha}\right)\left(b_{\alpha=b}\right)\left(\operatorname{Can}_{u}(b) \in\left(A\left\{u^{-1}\right\}\right)^{\times}\right)$, so $u \in \sqrt[\infty]{I_{\alpha}}=I_{\alpha}$, and therefore $u \in \bigcap_{\alpha \in \Lambda} I_{\alpha}$. This proves that $\sqrt[\infty]{\bigcap_{\alpha \in \Lambda} I_{\alpha}} \subseteq \bigcap_{\alpha \in \Lambda} I_{\alpha}$, so:

$$
\sqrt[\infty]{\bigcap_{\alpha \in \Lambda} I_{\alpha}}=\bigcap_{\alpha \in \Lambda} I_{\alpha}
$$

and

$$
\bigcap_{\alpha \in \Lambda} I_{\alpha} \in \mathfrak{I}_{A}^{\infty}
$$

(b) As we have proved in item e), the arbitrary intersection of $\mathcal{C}^{\infty}$-radical ideals is again $\mathcal{C}^{\infty}$-radical, so given any ideal $I$ there is a unique prime maximal $\mathcal{C}^{\infty}$-radical ideal $\mathfrak{q}$ such that $I \subseteq \mathfrak{q}$, so $I \subseteq \bigcap\left\{\mathfrak{p} \in \operatorname{Spec}^{\infty}(A) \mid I \subseteq \mathfrak{p}\right\}$

(c) Given any $u \in \sqrt[\infty]{\bigcup_{\alpha \in \Sigma} I_{\alpha}}$, then there is some $b \in \bigcup_{\alpha \in \Sigma} I_{\alpha}$ such that $\operatorname{Can}_{u}(b) \in\left(A\left\{u^{-1}\right\}\right)^{\times}$. Since $\Sigma$ is upward directed, there is $\alpha_{0} \in \Sigma$ such that $b \in I_{\alpha_{0}}$, so $\operatorname{Can}_{u}(b) \in\left(A\left\{u^{-1}\right\}\right)^{\times}$, therefore $u \in \sqrt[\infty]{I_{\alpha_{0}}}=I_{\alpha_{0}} \subseteq \bigcup_{\alpha \in \Sigma} I_{\alpha}$. Thus,

$$
\sqrt[\infty]{\bigcup_{\alpha \in \Sigma} I_{\alpha}}=\bigcup_{\alpha \in \Sigma} I_{\alpha}
$$

Remark 2.2.41. Due to the previous proposition, we have that $\mathfrak{I}_{A}^{\infty}$ is a complete Heyting algebra.

Lemma 2.2.42. Let $A$ be $a \mathcal{C}^{\infty}$-ring and $S \subset A$ any multiplicative subset. Consider the following posets:

$$
\left(\operatorname{Spec}^{\infty}\left(A\left\{S^{-1}\right\}\right), \subseteq\right)
$$

and

$$
\left(\left\{\mathfrak{p} \in \operatorname{Spec}^{\infty}(A) \mid \mathfrak{p} \cap S=\varnothing\right\}, \subseteq\right)
$$

The following poset maps:

$$
\begin{array}{ccc}
\operatorname{Can}_{S}^{*}:\left(\operatorname{Spec}^{\infty}\left(A\left\{S^{-1}\right\}\right), \subseteq\right) & \rightarrow & \left(\left\{\mathfrak{p} \in \operatorname{Spec}^{\infty}(A) \mid \mathfrak{p} \cap S=\varnothing\right\}, \subseteq\right) \\
Q & \mapsto & \operatorname{Can}_{S}^{-1}[Q]
\end{array}
$$

and

$$
\begin{array}{ccc}
\operatorname{Can}_{S_{*}}: \quad\left(\left\{\mathfrak{p} \in \operatorname{Spec}^{\infty}(A) \mid \mathfrak{p} \cap S=\varnothing\right\}, \subseteq\right) & \rightarrow & \left(\operatorname{Spec}^{\infty}\left(A\left\{S^{-1}\right\}\right), \subseteq\right) \\
P & \mapsto & \left\langle\operatorname{Can}_{S}[P]\right\rangle
\end{array}
$$

are poset isomorphisms, each one inverse of the other.

Proof. See p. 286 of 66 .

Proposition 2.2.43. Let $A$ be a $\mathcal{C}^{\infty}$-ring. For every $\mathfrak{p} \in \operatorname{Spec}^{\infty}(A)$ let $\hat{\mathfrak{p}}$ denote the maximal ideal of $A_{\{\mathfrak{p}\}}=A\left\{A \backslash \mathfrak{p}^{-1}\right\}$ and consider:

$$
\mathrm{Can}_{\mathfrak{p}}: A \rightarrow A_{\{\mathfrak{p}\}} .
$$

We have the following equalities:

$$
\operatorname{Can}_{\mathfrak{p}}^{-1}[\hat{\mathfrak{p}}]=\mathfrak{p}
$$

and

$$
\left(\operatorname{Can}_{\mathfrak{p}}[\mathfrak{p}]\right)=\hat{\mathfrak{p}}
$$


Proof. Let us take $S=A \backslash \mathfrak{p}$. Since $\hat{\mathfrak{p}}$ is a maximal ideal, it is the largest element of $\operatorname{Spec}^{\infty}\left(A\left\{(A \backslash \mathfrak{p})^{-1}\right\}\right)$. Hence $\operatorname{Can}_{\mathfrak{p}}^{-1}[\hat{\mathfrak{p}}]$ is the largest element of $\left(\left\{\mathfrak{p}^{\prime} \in \operatorname{Spec}^{\infty}(A) \mid \mathfrak{p}^{\prime} \cap(A \backslash \mathfrak{p})=\varnothing\right\}\right)$. Thus, by Lemma 2.2.42 $\mathrm{Can}_{\mathfrak{p}}^{-1}[\hat{\mathfrak{p}}]=\mathfrak{p}$.

Proposition 2.2.44. If $D$ is a reduced $\mathcal{C}^{\infty}$-domain, then $D\left\{a^{-1}\right\} \cong\{0\}$ implies $a=0$.

Proof. If $D\left\{a^{-1}\right\} \cong\{0\}$ then $\left(\frac{D}{(0)}\right)\left\{a+(0)^{-1}\right\} \cong\{0\}$, so $a \in \sqrt[\infty]{(0)}=(0)$ (for $D$ is reduced), and $a=0$.

Proposition 2.2.45. Any free $\mathcal{C}^{\infty}$-ring is a reduced $\mathcal{C}^{\infty}-$ ring.

Proof. First we prove the result for a free $\mathcal{C}^{\infty}$-ring on finitely many generators.

Suppose, by contraposition, that $f$ is not constant equal to zero. Then there is some $f \in \mathcal{C}^{\infty}\left(\mathbb{R}^{k}\right)$ such that $\left(\exists y_{0} \in \mathbb{R}^{k}\right)\left(f\left(y_{0}\right) \neq 0\right)$ and without loss of generality, suppose $f\left(y_{0}\right)>0$. By the Theorem of Sign Permanence, there is some open subset $V \subseteq \mathbb{R}^{k}$ such that $y_{0} \in V$ and $f \uparrow_{V}>0$. It is clear that $V \subseteq U_{f}=\operatorname{Coz}(f)$, so $f$ is a non-zero element of $\mathcal{C}^{\infty}\left(U_{f}\right) \cong \mathcal{C}^{\infty}\left(\mathbb{R}^{n}\right)\left\{f^{-1}\right\}$, hence $\mathcal{C}^{\infty}\left(\mathbb{R}^{n}\right)\left\{f^{-1}\right\} \supsetneqq 0$.

Thus, if $f \in \mathcal{C}^{\infty}\left(\mathbb{R}^{k}\right)$ is such that $\mathcal{C}^{\infty}\left(\mathbb{R}^{k}\right)\left\{f^{-1}\right\} \cong 0$ then $f=0$, so $\sqrt[\infty]{(0)}=\left\{f \in \mathcal{C}^{\infty}\left(\mathbb{R}^{k}\right) \mid \mathcal{C}^{\infty}\left(\mathbb{R}^{k}\right)\left\{f^{-1}\right\} \cong\right.$ $0\}=(0)$.

Now consider the free $\mathcal{C}^{\infty}$-rings on the set $E$ of generators. We know that:

$$
\mathcal{C}^{\infty}\left(\mathbb{R}^{E}\right)=\underset{E^{\prime} \underline{\mathrm{C}_{\text {fin }}} E}{\lim } \mathcal{C}^{\infty}\left(\mathbb{R}^{E^{\prime}}\right),
$$

and since the directed colimit of $\mathcal{C}^{\infty}$-reduced $\mathcal{C}^{\infty}$-rings is again a $\mathcal{C}^{\infty}$-reduced $\mathcal{C}^{\infty}$-ring (by Proposition 2.2.35 it follows that $\mathcal{C}^{\infty}\left(\mathbb{R}^{E}\right)$ is a $\mathcal{C}^{\infty}$-reduced $\mathcal{C}^{\infty}$-ring.

Proposition 2.2.46. Let $\left\{A_{i}\right\}_{i \in I}$ be a directed family of $\mathcal{C}^{\infty}$-rings, so we have the diagram:

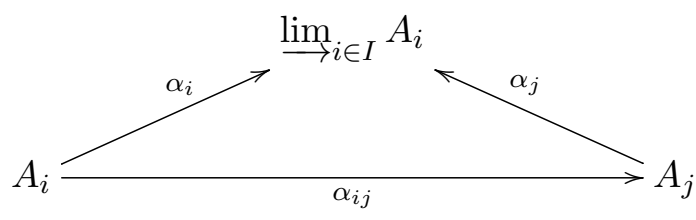

and let $\left(\mathfrak{p}_{i}\right)_{i \in I}$ be a compatible family of prime $\mathcal{C}^{\infty}$-radical ideals, that is:

$$
\left(\mathfrak{p}_{i}\right)_{i \in I} \in \varliminf_{i \in I}^{\lim _{i \in I}} \operatorname{Spec}^{\infty}\left(A_{i}\right) .
$$

Under those circumstances,

$$
\underset{i \in I}{\lim _{i \in I}} \mathfrak{p}_{i}=\bigcup_{i \in I} \alpha_{i}\left[\mathfrak{p}_{i}\right]
$$

is a $\mathcal{C}^{\infty}$-radical prime ideal of $\underline{\lim }_{i \in I} A_{i}$.

Proof. It suffices to prove that

$$
\frac{\lim _{i \in I} A_{i}}{\lim _{i \in I} \mathfrak{p}_{i}}
$$

is a $\mathcal{C}^{\infty}$-reduced domain.

It is a fact that colimits commute with quotients, so: 


$$
\underset{\lim _{i \in I} A_{i}}{\mathfrak{l i m}_{i}} \cong \lim _{i \in I} \frac{A_{i}}{\mathfrak{p}_{i}}
$$

Now, since every $\mathfrak{p}_{i}$ is a $\mathcal{C}^{\infty}$-radical prime ideal of $A_{i}$, we have, for every $i \in I$, that $\frac{A_{i}}{\mathfrak{p}_{i}}$ is a $\mathcal{C}^{\infty}$-reduced $\mathcal{C}^{\infty}$-domain.

The colimit of $\mathcal{C}^{\infty}$-reduced $\mathcal{C}^{\infty}$-domains is again a $\mathcal{C}^{\infty}$-reduced $\mathcal{C}^{\infty}$-domain, so $\frac{\lim _{i \in I} A_{i}}{\varliminf_{i \in I} \mathfrak{p}_{i}}$ is a domain and:

$$
\underset{i \in I}{\lim _{i \in I}} \mathfrak{p}_{i}
$$

is a $\mathcal{C}^{\infty}$-radical prime ideal of $\lim _{i \in I} A_{i}$.

Theorem 2.2.47 (Separation Theorems). Let $A$ be a $\mathcal{C}^{\infty}$-ring, $S \subseteq A$ be a subset of $A$ and $I$ be an ideal of $A$. Denote by $\langle S\rangle$ the multiplicative submonoid of $A$ generated by $S$. We have:

(a) If I is a $\mathcal{C}^{\infty}$-radical ideal, then:

$$
I \cap\langle S\rangle=\varnothing \Longleftrightarrow I \cap S^{\infty-\text { sat }}=\varnothing
$$

(b) If $S \subseteq A$ is a $\mathcal{C}^{\infty}$-saturated subset, then:

$$
I \cap S=\varnothing \Longleftrightarrow \sqrt[\infty]{I} \cap S=\varnothing
$$

(c) If $\mathfrak{p} \in \operatorname{Spec}^{\infty}(A)$ then $A \backslash \mathfrak{p}=(A \backslash \mathfrak{p})^{\infty-\text { sat }}$

(d) If $S \subseteq A$ is a $\mathcal{C}^{\infty}$-saturated subset, then:

$$
I \cap S=\varnothing \Longleftrightarrow\left(\exists \mathfrak{p} \in \operatorname{Spec}^{\infty}(A)\right)((I \subseteq \mathfrak{p}) \&(\mathfrak{p} \cap S=\varnothing)) .
$$

(e) $\sqrt[\infty]{I}=\bigcap\left\{\mathfrak{p} \in \operatorname{Spec}^{\infty}(A) \mid I \subseteq \mathfrak{p}\right\}$

Proof. Ad (a): Since $\langle S\rangle \subseteq S^{\infty-\text { sat }}$, it is clear that $(i i) \rightarrow(i)$.

We are going to show that $(i) \rightarrow(i i)$ via contraposition.

Suppose there exists some $b \in I \cap S^{\infty-\text { sat }}$, so $\eta_{S}(b) \in\left(B\left\{S^{-1}\right\}\right)^{\times}$.

We have:

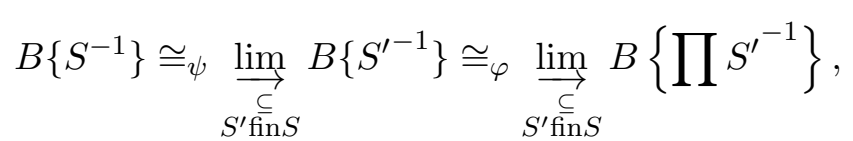

so $(\varphi \circ \psi)\left(\eta_{S}(b)\right) \in \lim _{S^{\prime} \text { fin } S} B\left\{\prod S^{\prime-1}\right\}$ implies that there is some finite $S^{\prime \prime} \subseteq S$ such that $\eta_{S^{\prime \prime}}(b) \in$ $B\left\{S^{\prime \prime-1}\right\} \cong B\left\{\prod S^{\prime \prime-1}\right\}$. Let $a=\prod S^{\prime \prime}$, so $a \in\langle S\rangle$. We have that $\eta_{a}(b) \in B\left\{S^{\prime \prime-1}\right\}$ implies $\eta_{a}(b) \in$ $\left(B\left\{a^{-1}\right\}\right)^{\times}$and by hypothesis, $b \in I$ so $a \in \sqrt[\infty]{I}=I$. Hence $a \in I \cap\langle S\rangle \neq \varnothing$, and the result is proved.

Ad (b): Given $b \in \sqrt[\infty]{I} \cap S$, there must be some $x \in I$ such that $\eta_{b}(x) \in A\left\{b^{-1}\right\}^{\times}$, so $x \in\{b\}^{\infty-\text { sat }} \subseteq$ $S^{\infty-\text { sat }}=S$. Thus $x \in I \cap S$ and $I \cap S=\varnothing$. The other way round is immediate since $I \subseteq \sqrt[\infty]{I}$.

Ad (c): Since $A \backslash \mathfrak{p}=\langle A \backslash \mathfrak{p}\rangle$, by item (a) we have: 


$$
\mathfrak{p} \cap(A \backslash \mathfrak{p}) \Longleftrightarrow \mathfrak{p} \cap(A \backslash \mathfrak{p})^{\infty-\text { sat }}=\varnothing
$$

so $(A \backslash \mathfrak{p})^{\infty-\text { sat }} \subseteq A \backslash \mathfrak{p}$. The other inclusion always holds, so $A \backslash \mathfrak{p}=(A \backslash \mathfrak{p})^{\infty-\text { sat }}$.

Ad (d): Consider the set:

$$
\Gamma_{S}:=\{J \in \Im(A) \mid(I \subseteq J) \&(S \cap J=\varnothing)\}
$$

ordered by inclusion. It is straightforward to check that $\left(\Gamma_{S}, \subseteq\right)$ satisfies the hypotheses of Zorn's Lemma. Let $M$ be a maximal member of $\Gamma_{S}$. We are going to show that $M \in \operatorname{Spec}^{\infty}(A)$.

Claim: $M$ is a proper prime ideal of $A$.

In fact, $M$ is proper since $1 \in S$ and $S \cap M=\varnothing$.

The proof that $M$ is prime is made by contradiction.

If $a, a^{\prime} \notin M$, then by maximality there are $\alpha, \alpha^{\prime} \in A$ and $m, m^{\prime} \in M$ such that $m+\alpha \cdot a \in S$ and $m^{\prime}+\alpha^{\prime} \cdot a^{\prime} \in S$. Since $\mathcal{C}^{\infty}$-saturated sets are submonoids, it follows that $(m+\alpha \cdot a) \cdot\left(m^{\prime}+\alpha^{\prime} \cdot a^{\prime}\right) \in S$. Thus,

$$
\underbrace{\left(m \cdot m^{\prime}+m \cdot \alpha^{\prime} \cdot a^{\prime}+m^{\prime} \cdot \alpha \cdot a\right)}_{\in M}+\underbrace{\left(\alpha \cdot \alpha^{\prime}\right) \cdot\left(a \cdot a^{\prime}\right)}_{\in S} \in S
$$

If $a, a^{\prime} \in M$, we get $M \cap S \neq \varnothing$, a contradiction. Thus, $a, a^{\prime} \notin M$, and $M$ is a prime ideal.

Claim: $M=\sqrt[\infty]{M}$

Since $M \cap S=\varnothing$ and $S$ is $\mathcal{C}^{\infty}$-saturated, by item (b) it follows that $\sqrt[\infty]{M} \cap S=\varnothing$, so $\sqrt[\infty]{M} \in \Gamma_{S}$. Since $M \subseteq \sqrt[\infty]{M}, \sqrt[\infty]{M} \in \Gamma_{S}$ and $M$ is a maximal element of $\Gamma_{S}$, it follows that $M=\sqrt[\infty]{M}$.

Hence, $M \in \operatorname{Spec}^{\infty}(A)$.

Ad (e): Clearly,

$$
\sqrt[\infty]{I} \subseteq \bigcap\left\{\mathfrak{p} \in \operatorname{Spec}^{\infty}(A) \mid I \subseteq \mathfrak{p}\right\}
$$

so we need only to prove the reverse inclusion.

Let $a \notin \sqrt[\infty]{I}$, so $S_{a}=\left\{1, a, a^{2}, \cdots\right\} \cap \sqrt[\infty]{I}=\varnothing$. Since $\frac{A}{I}\left\{(a+I)^{-1}\right\} \cong \frac{A}{I}\left\{\left(a^{k}+I\right)^{-1}\right\}$ for any $k \in \mathbb{N}$ such that $k \geq 1$ and since $\sqrt[\infty]{I}$ is a $\mathcal{C}^{\infty}$-radical ideal, by item (a), we have $\left(S_{a}\right)^{\infty-\text { sat }} \cap \sqrt[\infty]{I}=\varnothing$, and by item (d), there is some $\mathfrak{p} \in \operatorname{Spec}^{\infty}(A)$ such that $\mathfrak{p} \supseteq \sqrt[\infty]{I} \supseteq I$ such that $a \notin \mathfrak{p}$.

Proposition 2.2.48. Let $A$ be $a C^{\infty}$-ring and let $\left\{a_{i}: i \in I\right\} \subseteq A$. Denote $\mathcal{I}:=\left\langle\left\{a_{i}: i \in I\right\}\right\rangle$. Then the following are equivalent:

(a) $\operatorname{Spec}^{\infty}(A)=\bigcup_{i \in I} D^{\infty}\left(a_{i}\right)$

(b) $A=\mathcal{I}$ 
Proof. (b) $\Rightarrow$ (a): Since there exists $\left\{i_{1}, \cdots, i_{n}\right\} \subseteq I$ such that $1_{A}=\sum_{j=1}^{n} \lambda_{j} . a_{i_{j}}$ for some $\lambda_{1}, \cdots, \lambda_{n} \in A$, then there is no $\mathfrak{p} \in \operatorname{Spec}^{\infty}(A)$ such that $\left\{a_{i_{1}}, \cdots, a_{i_{n}}\right\} \subseteq \mathfrak{p}$, i.e. $\operatorname{Spec}^{\infty}(A) \subseteq \bigcup_{i \in I} D^{\infty}\left(a_{i}\right)$.

(a) $\Rightarrow$ (b): Suppose that $A \neq \mathcal{I}$. Then

$$
A^{\times} \cap \mathcal{I}=\emptyset .
$$

Since $A^{\times} \subseteq A$ is a $\infty$-saturated subset of $A\left(A^{\times}=\eta_{1}^{-1}\left[\left(A\left\{1^{-1}\right\}\right)^{\times}\right]\right)$, then by the Separation Theorem 2.2.47, (b),

$$
A^{\times} \cap \sqrt[\infty]{\mathcal{I}}=\emptyset .
$$

By the Separation Theorem 2.2.47. (d), there is $\mathfrak{p} \in \operatorname{Spec}^{\infty}(A)$ such that

$$
\sqrt[\infty]{\mathcal{I}} \subseteq \mathfrak{p}
$$

thus $\mathfrak{p} \in \operatorname{Spec}^{\infty}(A) \backslash \bigcup_{i \in I} D^{\infty}\left(a_{i}\right)$.

Proposition 2.2.49. Let $A$ be a $\mathcal{C}^{\infty}$-ring. The following assertions are equivalent:

(1) $A$ is a $\mathcal{C}^{\infty}$-reduced $\mathcal{C}^{\infty}$-domain;

(2) There is some $\mathcal{C}^{\infty}$-field $\mathbb{K}$ and an injective $\mathcal{C}^{\infty}$-rings homomorphism

$$
\jmath: A \longmapsto \mathbb{K}
$$

(3) $A\left\{(A \backslash\{0\})^{-1}\right\}$ is a $\mathcal{C}^{\infty}$-field, $\eta_{A \backslash\{0\}}^{\infty}: A \rightarrow A\left\{(A \backslash\{0\})^{-1}\right\}$ is an injective $\mathcal{C}^{\infty}$-rings homomorphism with the following universal property: for every $\mathcal{C}^{\infty}$-field $\mathbb{K}$ and every injective $\mathcal{C}^{\infty}$-homomorphism $\jmath: A \rightarrow \mathbb{K}$ there is a unique $\mathcal{C}^{\infty}$-fields homomorphism:

$$
\bar{\jmath}: A\left\{(A \backslash\{0\})^{-1}\right\} \longmapsto \mathbb{K}
$$

such that the following diagram commutes:

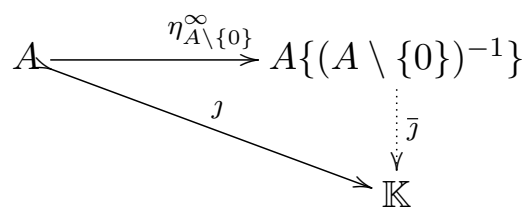

Proof. Ad $(3) \rightarrow(2):$ Suppose $A\left\{(A \backslash\{0\})^{-1}\right\}$ is a $\mathcal{C}^{\infty}$-field with the claimed property.

We always have the following inclusions:

$$
A \backslash\{0\} \subseteq(A \backslash\{0\})^{\text {sat }} \subseteq(A \backslash\{0\})^{\infty-\text { sat }}
$$

so we must prove that:

$$
x \notin A \backslash\{0\} \rightarrow x \notin(A \backslash\{0\})^{\infty-\text { sat }} .
$$

Now, $x \notin A \backslash\{0\} \Longleftrightarrow x=0$ and $\eta_{A \backslash\{0\}}^{\infty}(x)=0$ in a field implies $\eta_{A \backslash\{0\}}^{\infty}(x) \notin(A \backslash\{0\})^{\times}$, since fields are non-trivial rings (0 is not an invertible element). Hence $(A \backslash\{0\})^{\infty-\text { sat }} \subseteq A \backslash\{0\}$, so

$$
(A \backslash\{0\})^{\text {sat }}=(A \backslash\{0\})^{\infty-\text { sat }} .
$$

Now we prove $\eta_{A \backslash\{0\}}^{\infty}: A \rightarrow A\left\{(A \backslash\{0\})^{-1}\right\}$ is injective. Let $x \in U(A)$ be such that $\eta_{A \backslash\{0\}}^{\infty}(x)=0$. Then $x \notin(A \backslash\{0\})^{\infty-\text { sat }}=A \backslash\{0\}$, i.e., $x=0$, so the result follows. 
Ad $(2) \rightarrow(1)$ : Since $\jmath: A \rightarrow \mathbb{K}$ is an injective $\mathcal{C}^{\infty}$-rings homomorphism, we have $\left\{0_{A}\right\}=\jmath^{\dashv}\left[\left\{0_{\mathbb{K}}\right\}\right]$. Since $\mathbb{K}$ is a $\mathcal{C}^{\infty}$-field, it is, in particular, a $\mathcal{C}^{\infty}$-reduced $\mathcal{C}^{\infty}$-ring, so $\sqrt[\infty]{\left(0_{\mathbb{K}}\right)}=\left(0_{\mathbb{K}}\right)$. We have:

$$
\left\{0_{A}\right\}=\jmath^{-1}\left[\left\{0_{\mathbb{K}}\right\}\right]=\jmath^{-1}\left[\left(0_{\mathbb{K}}\right)\right]=\jmath^{-1}\left[\sqrt[\infty]{\left(0_{\mathbb{K}}\right)}\right]
$$

Since by Theorem 2.2.36 the pre-image of a $\mathcal{C}^{\infty}$-radical ideal is again a $\mathcal{C}^{\infty}$-radical ideal, we have $\jmath^{-1}\left[\sqrt[\infty]{\left(0_{\mathbb{K}}\right)}\right]=\sqrt[\infty]{\jmath^{-1}\left[\left(0_{\mathbb{K}}\right)\right]}$, and since $\jmath$ is injective, $\jmath^{-1}\left[\left(0_{\mathbb{K}}\right)\right]=\left(0_{\mathbb{K}}\right)$. Hence:

$$
\left\{0_{A}\right\}=\jmath^{\dashv}\left[\left\{0_{\mathbb{K}}\right\}\right]=\jmath^{\dashv}\left[\left(0_{\mathbb{K}}\right)\right]=\jmath^{\dashv}\left[\sqrt[\infty]{\left(0_{\mathbb{K}}\right)}\right]=\sqrt[\infty]{\jmath^{\dashv}\left[\left(0_{\mathbb{K}}\right)\right]}=\sqrt[\infty]{\left(0_{A}\right)},
$$

so $A$ is indeed a $\mathcal{C}^{\infty}$-reduced $\mathcal{C}^{\infty}$-ring.

Ad $(1) \rightarrow(3)$ : Suppose $A$ is a $\mathcal{C}^{\infty}$-reduced $\mathcal{C}^{\infty}$-ring.

We claim that:

$$
\eta_{A \backslash\{0\}}^{\infty}: A \rightarrow A\left\{A \backslash\{0\}^{-1}\right\}
$$

is injective.

Let $a \in A$ be such that $\eta_{A \backslash\{0\}}^{\infty}(a)=0$. By the item (ii), Theorem 2.1.24, there exists some $\lambda \in A \backslash\{0\}^{\infty-\text { sat }}$ with $\lambda \cdot a=0$ in $A$. By Proposition 2.1.19. we can write:

$$
A \backslash\{0\}=\bigcup_{\substack{\subseteq \\ S^{\prime} \text { fin } A \backslash\{0\}}} S^{\prime}
$$

so given this $\lambda$ there is some finite $S^{\prime} \subseteq A \backslash\{0\}$ such that $\lambda \in S^{\prime \infty-\text { sat }}$ and $\lambda \cdot a=0$. We have, then, $\eta_{S^{\prime}}^{\infty}(a)=0$.

Since $S^{\prime}$ is a finite set, $S^{\prime \infty-\text { sat }}=\left\langle\left\{\prod S^{\prime}\right\}\right\rangle^{\infty-\text { sat }}$. Denote $\prod S^{\prime}$ by $s^{\prime}$, and we have:

$$
\eta_{s^{\prime}}(a)=0 \rightarrow\left(A\left\{s^{-1}\right\}\right)\{\underbrace{\eta_{s^{\prime}}(a)^{-1}}_{=0}\} \cong\{0\}
$$

for 0 invertible in a $\mathcal{C}^{\infty}$-reduced $\mathcal{C}^{\infty}$-ring implies the ring is trivial. By the Proposition 2.1.31,

$$
A\left\{s^{\prime} \cdot a^{-1}\right\} \cong\left(A\left\{s^{-1}\right\}\right)\left\{\eta_{s^{\prime}}(a)^{-1}\right\} \cong\{0\} \rightarrow s^{\prime} \cdot a \in \sqrt[\infty]{(0)} \subseteq A .
$$

Since $A$ is a $\mathcal{C}^{\infty}$-reduced $\mathcal{C}^{\infty}$-ring, it follows that $s^{\prime} \cdot a=0$. Since $S^{\prime} \subseteq A \backslash\{0\}, s^{\prime}=\prod S^{\prime} \neq 0$, and since $A$ is a $\mathcal{C}^{\infty}$-domain, $\left(s^{\prime} \cdot a=0\right) \wedge\left(s^{\prime} \neq 0\right) \rightarrow a=0$, so $\eta_{A \backslash\{0\}}^{\infty}$ is an injective $\mathcal{C}^{\infty}$-homomorphism.

Now we prove $\eta_{A \backslash\{0\}}^{\infty}$ has the desired universal property.

Let $\mathbb{K}$ be a $\mathcal{C}^{\infty}$-field and $\jmath: A \rightarrow \mathbb{K}$ be an injective $\mathcal{C}^{\infty}$-morphism. Under those circumstances,

$$
\jmath[A \backslash\{0\}] \subseteq \mathbb{K} \backslash\{0\}=\mathbb{K}^{\times},
$$

so by the universal property of $\eta_{A \backslash\{0\}}^{\infty}$ of inverting $A \backslash\{0\}$, there is a unique $\mathcal{C}^{\infty}$-homomorphism $\bar{\jmath}$ : $A\left\{A \backslash\{0\}^{-1}\right\} \rightarrow \mathbb{K}$ such that:

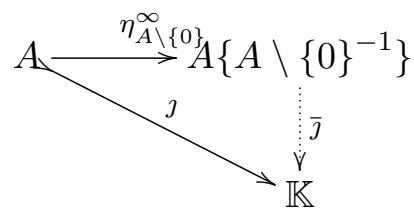


commutes.

Finally we show that $A\left\{A \backslash\{0\}^{-1}\right\}$ is a $\mathcal{C}^{\infty}$-field. We are going to show that any non-zero element of $A\left\{A \backslash\{0\}^{-1}\right\}$ is invertible.

By item (i) of Theorem 2.1.24 for every $\varphi \in A\left\{A \backslash\{0\}^{-1}\right\}$ there are $a \in A$ and $b \in A \backslash\{0\}^{\infty-\text { sat }}=$ $A \backslash\{0\}$, the last equality occurring because $\eta_{A \backslash\{0\}}^{\infty}$ is injective and $A \neq\{0\}$. Hence $b \in A \backslash\{0\}$.

Since $\varphi \neq 0, \eta_{A \backslash\{0\}}^{\infty}(a) \neq 0$, and since $\eta_{A \backslash\{0\}}^{\infty}$ is injective, this implies $a \neq 0$. We define:

$$
\psi:=\frac{\eta_{A \backslash\{0\}}^{\infty}(b)}{\eta_{A \backslash\{0\}}^{\infty}(a)},
$$

and we note that:

$$
\varphi \cdot \psi=1
$$

so $\varphi$ is invertible.

Lemma 2.2.50. Let $A$ be a $\mathcal{C}^{\infty}$-ring and $\mathfrak{p}$ be any of its prime ideals. There is a unique $\mathcal{C}^{\infty}$-homomorphism:

$$
\alpha_{\mathfrak{p}}: A\left\{A \backslash \mathfrak{p}^{-1}\right\} \rightarrow\left(\frac{A}{\mathfrak{p}}\right)\left\{\left(\frac{A}{\mathfrak{p}}\right) \backslash\{0+\mathfrak{p}\}^{-1}\right\}
$$

such that the following rectangle commutes:

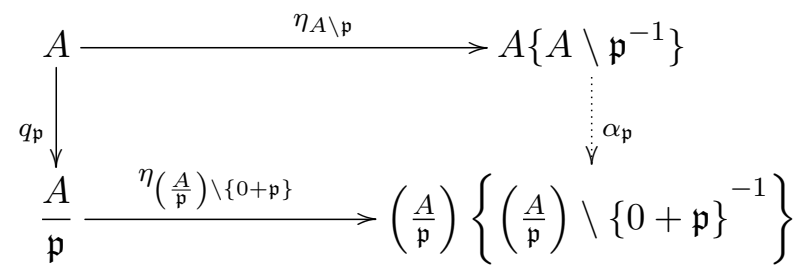

Proof. We are going to show that $\eta_{\left(\frac{A}{\mathfrak{p}}\right) \backslash\{0+\mathfrak{p}\}}\left[q_{\mathfrak{p}}[A \backslash \mathfrak{p}]\right] \subseteq\left[\left(\frac{A}{\mathfrak{p}}\right)\left\{\left(\frac{A}{\mathfrak{p}}\right) \backslash\{0+\mathfrak{p}\}^{-1}\right\}\right]^{\times}$.

Since $q_{\mathfrak{p}}[\mathfrak{p}]=\{0\}, q_{\mathfrak{p}}[A \backslash \mathfrak{p}]=\left(\frac{A}{\mathfrak{p}}\right) \backslash\{0+\mathfrak{p}\}$, and by the very property that defines $\eta_{\left(\frac{A}{\mathfrak{p}}\right) \backslash\{0+\mathfrak{p}\}}$, we have $\eta_{\left(\frac{A}{\mathfrak{p}}\right) \backslash\{0+\mathfrak{p}\}}\left[q_{\mathfrak{p}}[A \backslash \mathfrak{p}]\right] \subseteq\left[\left(\frac{A}{\mathfrak{p}}\right)\left\{\left(\frac{A}{\mathfrak{p}}\right) \backslash\{0+\mathfrak{p}\}^{-1}\right\}\right]^{\times}$. By the universal property of $\eta_{A \backslash \mathfrak{p}}: A \rightarrow A\left\{A \backslash \mathfrak{p}^{-1}\right\}$, there exists a unique $\mathcal{C}^{\infty}$-homomorphism $\alpha_{\mathfrak{p}}$ such that:

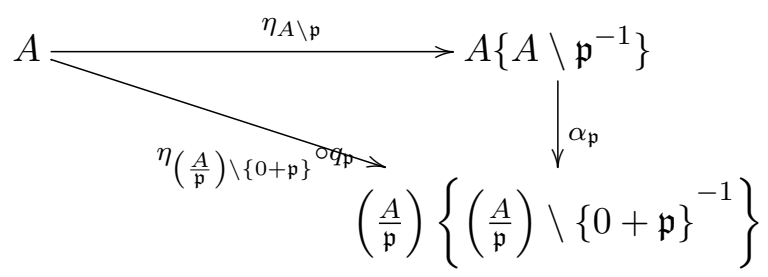

commutes, so the result follows.

Note that $\mathfrak{p} \in \operatorname{Spec}^{\infty}(A) \Longleftrightarrow\left(\frac{A}{\mathfrak{p}}\right)$ is a reduced $\mathcal{C}^{\infty}$-domain, so whenever $\mathfrak{p} \in \operatorname{Spec}^{\infty}(A)$ we have $A\left\{A \backslash \mathfrak{p}^{-1}\right\}$ a $\mathcal{C}^{\infty}$-local ring. Let $\mathfrak{m}_{\mathfrak{p}}$ denote its unique maximal ideal. 
We note that for $\mathfrak{p} \in \operatorname{Spec}^{\infty}(A), \mathfrak{p} \cap(A \backslash \mathfrak{p})=\varnothing$. Since $\mathfrak{p}$ is a proper prime ideal of $A$, it follows that $A \backslash \mathfrak{p}$ is a submonoid of $A$, so by item (a) of Theorem $\mathbf{2 . 2 . 4 7}$ it follows that:

$$
\mathfrak{p} \cap(A \backslash \mathfrak{p})^{\infty-\text { sat }}=\varnothing
$$

hence

$$
(A \backslash \mathfrak{p})^{\infty-\text { sat }} \subseteq A \backslash \mathfrak{p}
$$

so $A \backslash \mathfrak{p}$ is a $\mathcal{C}^{\infty}$-saturated set whenever $\mathfrak{p} \in \operatorname{Spec}^{\infty}(A)$.

We have the following:

Theorem 2.2.51. Let $A$ be a $\mathcal{C}^{\infty}$-ring and let $\mathfrak{p} \in \operatorname{Spec}^{\infty}(A)$. The ring $A\left\{A \backslash \mathfrak{p}^{-1}\right\}$ is a local $\mathcal{C}^{\infty}$-ring.

Proof. We are going to show that $\left(A\left\{A \backslash \mathfrak{p}^{-1}\right\}\right) \backslash\left(A\left\{A \backslash \mathfrak{p}^{-1}\right\}\right)^{\times}$is an ideal by showing that:

$$
\left(A\left\{A \backslash \mathfrak{p}^{-1}\right\}\right) \backslash\left(A\left\{A \backslash \mathfrak{p}^{-1}\right\}\right)^{\times}=\left\langle\eta_{A \backslash \mathfrak{p}}[\mathfrak{p}]\right\rangle=\left\{\eta_{A \backslash \mathfrak{p}}(a) \cdot \eta_{A \backslash \mathfrak{p}}(b) \mid a \in \mathfrak{p} \& b \in A \backslash \mathfrak{p}\right\}
$$

\section{By Theorem 2.1.28,}

$$
\lambda=\frac{\eta_{S}(c)}{\eta_{S}(b)} \in\left(A\left\{S^{-1}\right\}\right)^{\times} \Longleftrightarrow\left(\exists c^{\prime} \in A\right)\left(\exists d \in(A \backslash \mathfrak{p})^{\infty-\mathrm{sat}}\right)\left(d \cdot c^{\prime} \cdot c \in(A \backslash \mathfrak{p})\right)
$$

If $c \in \mathfrak{p}$, then $\lambda$ would not be an invertible element, since for every $d, c^{\prime} \in A$ we would have $d \cdot c^{\prime} \cdot c \in \mathfrak{p}$, hence $\left(\forall d \in(A \backslash \mathfrak{p})^{\infty-\text { sat }}\right)(\forall c \in A)\left(d \cdot c^{\prime} \cdot c \notin(A \backslash \mathfrak{p})\right)$. We conclude that $c \in(A \backslash \mathfrak{p})$, so taking $d=1 \in(A \backslash \mathfrak{p})$ (for $\mathfrak{p}$ is a proper prime ideal) and $c=1 \in A$ we have $1 \cdot 1 \cdot c^{\prime} \in(A \backslash \mathfrak{p})$, so there are, indeed, $d \in(A \backslash \mathfrak{p})^{\infty-\text { sat }}$ and $c \in A$ such that $d \cdot c^{\prime} \cdot c=1 \cdot 1 \cdot c \in(A \backslash \mathfrak{p})^{\infty-\text { sat }}$.

From these considerations, it follows that $\lambda=\frac{\eta_{S}(c)}{\eta_{S}(b)}$ is non-invertible if, and only if, $c \in \mathfrak{p}$, that is:

$$
\lambda=\underbrace{\eta_{S}(c)}_{\in \eta_{A \backslash \mathfrak{p}}[\mathfrak{p}]} \cdot \overbrace{\eta_{S}(b)^{-1}}^{\in A\left\{A \backslash \mathfrak{p}^{-1}\right\}} \in \eta_{A \backslash \mathfrak{p}}[\mathfrak{p}],
$$

so $A\left\{A \backslash \mathfrak{p}^{-1}\right\} \backslash\left(A\left\{A \backslash \mathfrak{p}^{-1}\right\}\right)^{\times}$is an ideal and $A\left\{A \backslash \mathfrak{p}^{-1}\right\}$ is a local $\mathcal{C}^{\infty}$-ring.

Theorem 2.2.52. Let $A$ be a $\mathcal{C}^{\infty}$-ring and $\mathfrak{p}$ one of its $\mathcal{C}^{\infty}$-radical prime ideals. As proven in the previous theorem, $A\left\{A \backslash \mathfrak{p}^{-1}\right\}$ is a local $\mathcal{C}^{\infty}$-ring, so we denote by $\mathfrak{m}_{\mathfrak{p}}$ its unique maximal ideal. There is a unique $\mathcal{C}^{\infty}$-homomorphism:

$$
\beta_{\mathfrak{p}}:\left(\frac{A}{\mathfrak{p}}\right) \rightarrow \frac{A\left\{A \backslash \mathfrak{p}^{-1}\right\}}{\mathfrak{m}_{\mathfrak{p}}}
$$

such that the following rectangle commutes:

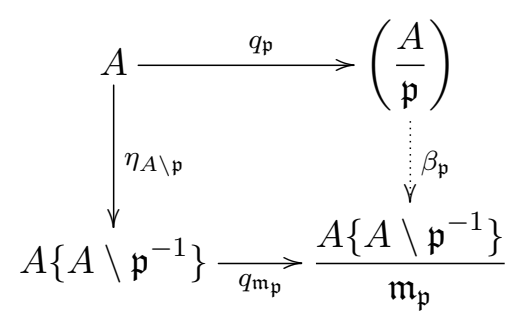


Proof. We are going to show that $\mathfrak{p} \subseteq \operatorname{ker}\left(q_{\mathfrak{m}_{\mathfrak{p}}} \circ \eta_{A \backslash \mathfrak{p}}\right)$, so the result will follow from the Homomorphism Theorem, i.e., there will be a unique $\beta_{\mathfrak{p}}$ such that the following triangle commutes:

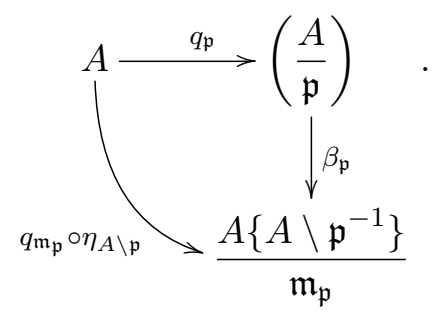

Since by the proof of Teorema 2.2.51 we have $\mathfrak{m}_{\mathfrak{p}}=\left\langle\eta_{A \backslash \mathfrak{p}}[\mathfrak{p}]\right\rangle=\left\{\eta_{A \backslash \mathfrak{p}}(a) \cdot \eta_{A \backslash \mathfrak{p}}(b) \mid a \in \mathfrak{p} \& b \in(A \backslash \mathfrak{p})\right\}$, it follows that:

$$
q_{\mathfrak{m}_{\mathfrak{p}}}\left[\eta_{A \backslash \mathfrak{p}}[\mathfrak{p}]\right]=0+\mathfrak{m}_{\mathfrak{p}}
$$

what proves the result.

Theorem 2.2.53. Let $A$ be $a \mathcal{C}^{\infty}$ _ring and $\mathfrak{p}$ be any of its prime ideals. There are unique isomorphisms:

$$
\varphi_{\mathfrak{p}}: \frac{A\left\{A \backslash \mathfrak{p}^{-1}\right\}}{\mathfrak{m}_{\mathfrak{p}}} \rightarrow\left(\frac{A}{\mathfrak{p}}\right)\left\{\left(\frac{A}{\mathfrak{p}}\right) \backslash\{0+\mathfrak{p}\}^{-1}\right\}
$$

and

$$
\psi_{\mathfrak{p}}:\left(\frac{A}{\mathfrak{p}}\right)\left\{\left(\frac{A}{\mathfrak{p}}\right) \backslash\{0+\mathfrak{p}\}^{-1}\right\} \rightarrow \frac{A\left\{A \backslash \mathfrak{p}^{-1}\right\}}{\mathfrak{m}_{\mathfrak{p}}}
$$

such that the following diagram commutes:

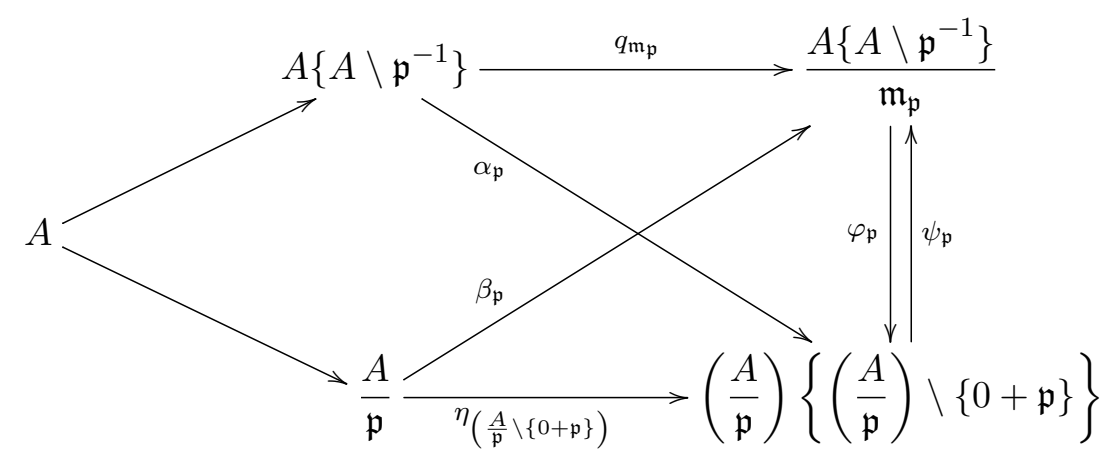

Proof. First we are going to show that there exists a unique $\psi_{\mathfrak{p}}$ such that:

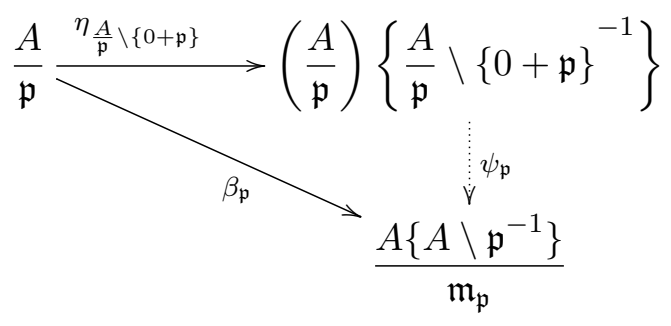

is a commutative diagram.

Note that $\beta_{\mathfrak{p}}\left[\frac{A}{\mathfrak{p}} \backslash\{0+\mathfrak{p}\}\right] \subseteq\left(\frac{A\left\{A \backslash \mathfrak{p}^{-1}\right\}}{\mathfrak{m}_{\mathfrak{p}}}\right)^{\times}$ 
By definition, $\beta_{\mathfrak{p}}$ is the only $\mathcal{C}^{\infty}$-homomorphism such that the following diagram commutes:

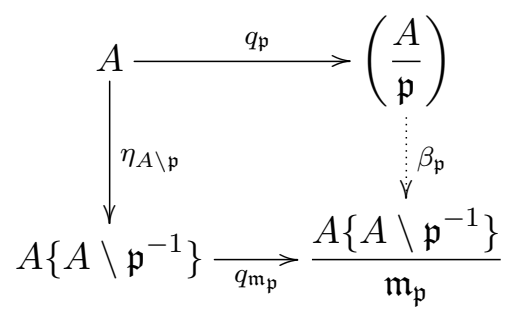

so

$$
\beta_{\mathfrak{p}}[\{a+\mathfrak{p} \mid a \notin \mathfrak{p}\}]=\left\{\eta_{\mathfrak{p}}(a)+\mathfrak{m}_{\mathfrak{p}} \mid a \in(A \backslash \mathfrak{p})\right\} \subseteq\left(\frac{A\left\{A \backslash \mathfrak{p}^{-1}\right\}}{\mathfrak{m}_{\mathfrak{p}}}\right)^{\times}
$$

and $\left(\frac{A\left\{A \backslash \mathfrak{p}^{-1}\right\}}{\mathfrak{m}_{\mathfrak{p}}}\right)^{\times}=\frac{A\left\{A \backslash \mathfrak{p}^{-1}\right\}}{\mathfrak{m}_{\mathfrak{p}}} \backslash\left\{0+\mathfrak{m}_{\mathfrak{p}}\right\}$, for $a \in(A \backslash \mathfrak{p}) \rightarrow \eta_{A \backslash \mathfrak{p}}(a) \notin \mathfrak{m}_{\mathfrak{p}}=\left\langle\eta_{A \backslash \mathfrak{p}}[\mathfrak{p}]\right\rangle$. By the universal property of $\eta_{\frac{A}{\mathfrak{p}} \backslash\{0+\mathfrak{p}\}}$, it follows that there is a unique $\psi_{\mathfrak{p}}$ with the desired property.

Now we claim that there is a unique $\varphi_{\mathfrak{p}}$ such that the following triangle commutes:

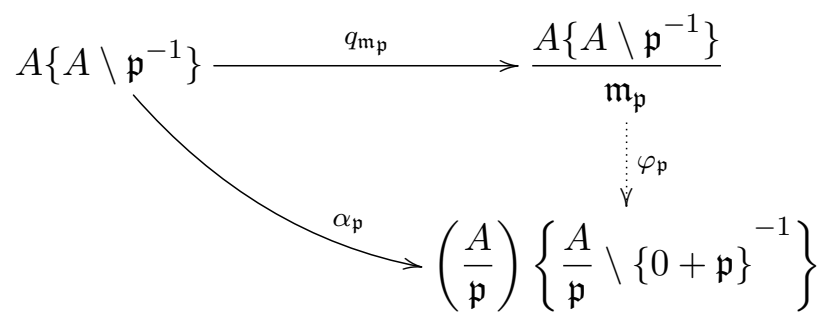

Note that $\alpha_{\mathfrak{p}}\left[\mathfrak{m}_{\mathfrak{p}}\right] \subseteq\left\{0+\mathfrak{m}_{\mathfrak{p}}\right\}$. Indeed, let $\frac{\eta_{A \backslash \mathfrak{p}}(a)}{\eta_{A \backslash \mathfrak{p}}(b)} \in \mathfrak{m}_{\mathfrak{p}}$, that is, an element such that $a \in \mathfrak{p}$ and $b \in A \backslash \mathfrak{p}$. By the very definition of $\alpha_{\mathfrak{p}}$ given in the Lemma 2.2.50.

$$
\alpha_{\mathfrak{p}}\left(\frac{\eta_{A \backslash \mathfrak{p}}(a)}{\eta_{A \backslash \mathfrak{p}}(b)}\right)=\frac{\eta_{\frac{A}{\mathfrak{p}} \backslash\{0+\mathfrak{p}\}} \overbrace{(a+\mathfrak{p})}^{\in \mathfrak{p}}}{\eta_{\eta_{\frac{A}{\mathfrak{p}}} \backslash\{0+\mathfrak{p}\}}(b)}=0+\mathfrak{m}_{\mathfrak{p}} .
$$

By the Homomorphism Theorem, there is a unique $\varphi_{\mathfrak{p}}$ such that the following diagram commutes:

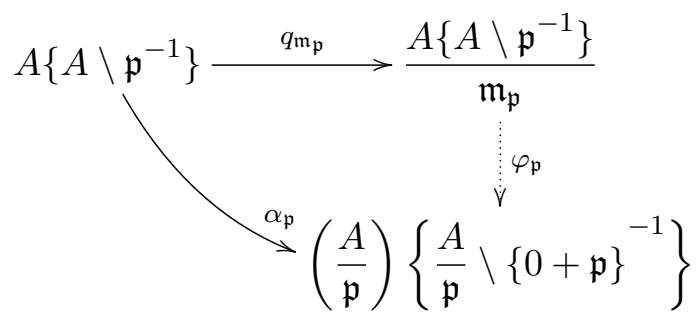

Finally, we are going to show that $\varphi_{\mathfrak{p}} \circ \psi_{\mathfrak{p}}=\operatorname{id}_{\frac{A\left\{A \backslash p^{-1}\right\}}{\mathfrak{m}_{\mathfrak{p}}}}$.

Consider the following diagram: 


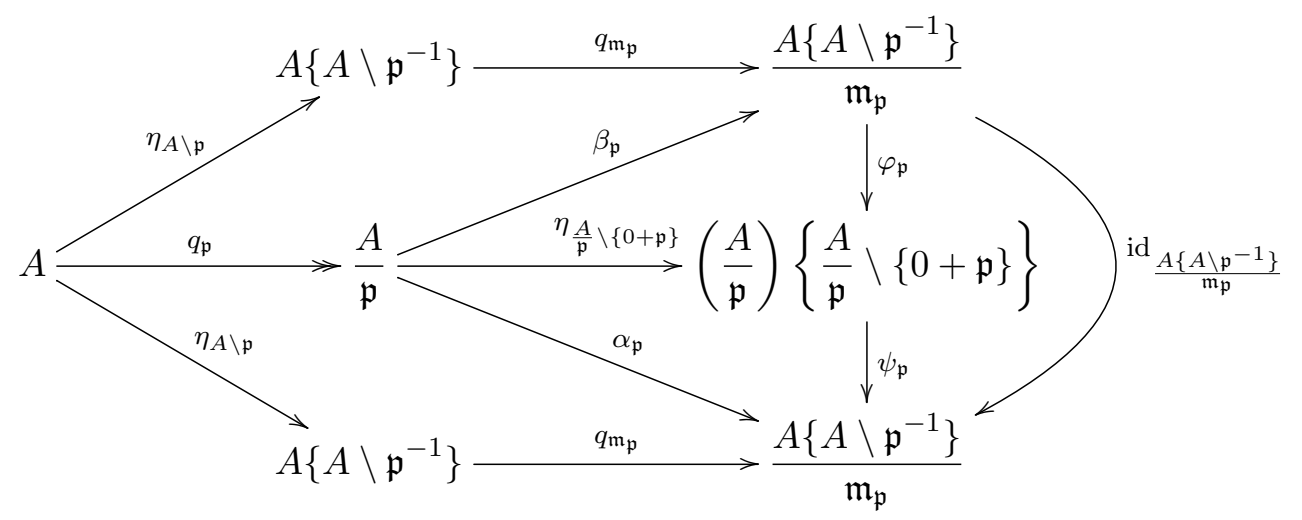

Note that the upper inner triangle in the above diagram commutes because $q_{\mathfrak{p}}$ is an epimorphism and

$$
\left(\varphi_{\mathfrak{p}} \circ \beta_{\mathfrak{p}}\right) \circ q_{\mathfrak{p}}=\eta_{\frac{A}{\mathfrak{p}} \backslash\{0+\mathfrak{p}\}}
$$

By the uniqueness granted by the Homomorphism Theorem, it follows that $\psi_{\mathfrak{p}} \circ \varphi_{\mathfrak{p}}=\mathrm{id}_{\frac{A\{A \backslash \mathfrak{p}-1\}}{\mathfrak{m} \mathfrak{p}}}$.

Similarly, by the uniqueness granted by the universal property of the ring of fractions $\eta_{\frac{A}{\mathfrak{p}} \backslash\{0+\mathfrak{p}\}}$, it follows that:

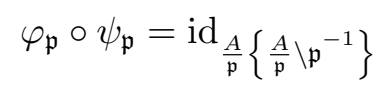

Hence,

$$
\frac{A\left\{A \backslash \mathfrak{p}^{-1}\right\}}{\mathfrak{m}_{\mathfrak{p}}} \cong\left(\frac{A}{\mathfrak{p}}\right)\left\{\frac{A}{\mathfrak{p}} \backslash\{0+\mathfrak{p}\}^{-1}\right\}
$$

We now present a result that relates the saturation of $S$ and the saturation of $q_{I}[S]$.

Theorem 2.2.54. Let $A$ be a $\mathcal{C}^{\infty}$-ring, $S \subseteq A$ and $I$ be an ideal of $A$. Then

$$
(\beta+I) \in(S+I)^{\infty-\mathrm{sat}} \Longleftrightarrow(\exists \gamma \in A)\left(\exists s \in S^{\infty-\mathrm{sat}}\right)\left(s \cdot\left(\beta \gamma-1_{A}\right) \in I\right)
$$

Proof. By definition, $(\beta+I) \in(S+I)^{\infty-\text { sat }}$ occurs if, and only if, $\eta_{S+I}(\beta+I) \in\left(\frac{A}{I}\right)\left\{S+I^{-1}\right\}^{\times}$.

By Corollary 2.1.45, we have the following $\mathcal{C}^{\infty}$-isomorphism:

$$
\mu^{-1}:\left(\frac{A}{I}\right) \cong \frac{A\left\{S^{-1}\right\}}{\left\langle\eta_{S}[I]\right\rangle}
$$

and since $\mu^{-1}\left(\eta_{S+I}(\beta+I)\right) \in \frac{A\left\{S^{-1}\right\}}{\left\langle\eta_{S}[I]\right\rangle}$, then:

$$
\mu^{-1}\left(\eta_{S+I}(\beta)\right)=\eta_{S}(\beta)+\left\langle\eta_{S}[I]\right\rangle \frac{A\left\{S^{-1}\right\}}{\left\langle\eta_{S}[I]\right\rangle} .
$$

Now, $\mu^{-1}\left(\eta_{S+I}(\beta+I)\right) \in\left(\frac{A\left\{S^{-1}\right\}}{\left\langle\eta_{S}[I]\right\rangle}\right)^{\times}$occurs if, and only if, there is some $\gamma \in A$ such that:

$$
\left(\mu^{-1}\left(\eta_{S+I}(\beta+I)\right)\right) \cdot\left(\eta_{S}(\gamma)+\left\langle\eta_{S}[I]\right\rangle\right)=1_{A}+\left\langle\eta_{S}[I]\right\rangle
$$




$$
\left(\eta_{S}(\beta)+\left\langle\eta_{S}[I]\right\rangle\right) \cdot\left(\eta_{S}(\gamma)+\left\langle\eta_{S}[I]\right\rangle\right)=1_{A}+\left\langle\eta_{S}[I]\right\rangle
$$

or, equivalently, if and only if,

$$
\eta_{S}\left(\beta \cdot \gamma-1_{A}\right) \in\left\langle\eta_{S}[I]\right\rangle
$$

By Corollary 2.2.7, $\left\langle\eta_{S}[I]\right\rangle=\left\{\eta_{S}(b) \cdot \eta_{S}(d) \mid(b \in I) \&\left(d \in S^{\infty-\text { sat }}\right)\right\}$, so

$$
\eta_{S}\left(\beta \cdot \gamma-1_{A}\right) \in\left\langle\eta_{S}[I]\right\rangle \Longleftrightarrow(\exists i \in I)\left(\exists d \in S^{\infty-\mathrm{sat}}\right)\left(\eta_{S}\left(\beta \cdot \gamma-1_{A}\right)=\frac{\eta_{S}(i)}{\eta_{S}(d)}\right),
$$

that is, if and only if:

$$
\eta_{S}\left(\beta \cdot \gamma-1_{A}\right) \cdot \eta_{S}(d)=\eta_{S}(i)
$$

or

$$
\eta_{S}\left(d \cdot\left(\beta \cdot \gamma-1_{A}\right)-i\right)=0
$$

By item (ii) of Theorem 1.4 of 63 ,

$$
\left(\eta_{S}\left(d \cdot\left(\beta \cdot \gamma-1_{A}\right)-i\right)=0\right) \Longleftrightarrow\left(\exists c \in S^{\infty-\mathrm{sat}}\right)\left(c \cdot\left[d \cdot\left(\beta \cdot \gamma-1_{A}\right)-i\right]=0\right) .
$$

and

$$
c \cdot\left[d \cdot\left(\beta \cdot \gamma-1_{A}\right)-i\right]=\underbrace{(c \cdot d)}_{\in S^{\infty-\text { sat }}}\left[\left(\beta \cdot \gamma-1_{A}\right)-i\right]=0
$$

so it is sufficient to take $s=c \cdot d \in S^{\infty-\text { sat }}$ in order to get:

$$
s \cdot(\beta \cdot \gamma-1) \in I
$$

Conversely, suppose that:

$$
(\exists \gamma \in A)\left(\exists s \in S^{\infty-\mathrm{sat}}\right)\left(s \cdot\left(\beta \cdot \gamma-1_{A}\right) \in I\right)
$$

Let $s \cdot\left(\beta \cdot \gamma-1_{A}\right)=i \in I$. We have:

$$
\eta_{S}\left(s \cdot\left(\beta \cdot \gamma-1_{A}\right)\right)=\eta_{S}(i) \in \eta_{S}[I]
$$

hence:

$$
\eta_{S}\left(\beta \cdot \gamma-1_{A}\right)=\frac{\eta_{S}(i)}{\eta_{S}(s)} \in\left\langle\eta_{S}[I]\right\rangle
$$

and

$$
\eta_{S}(\beta) \cdot \eta_{S}(\gamma)-\eta_{S}\left(1_{A}\right) \in\left\langle\eta_{S}[I]\right\rangle
$$

so

$$
\left(\eta_{S}(\beta)+\left\langle\eta_{S}[I]\right\rangle\right) \cdot\left(\eta_{S}(\gamma)+\left\langle\eta_{S}[I]\right\rangle\right)=\eta_{S}\left(1_{A}\right)+\left\langle\eta_{S}[I]\right\rangle,
$$

hence 


$$
\begin{gathered}
\eta_{S}(\beta)+\left\langle\eta_{S}[I]\right\rangle \in\left(\frac{A\left\{S^{-1}\right\}}{\left\langle\eta_{S}[I]\right\rangle}\right)^{\times} \\
\mu^{-1}\left(\eta_{S}(\beta)+\left\langle\eta_{S}[I]\right\rangle\right) \in \mu^{-1}\left[\left(\frac{A\left\{S^{-1}\right\}}{\left\langle\eta_{S}[I]\right\rangle}\right)^{\times}\right]=\left(\left(\frac{A}{I}\right)\left\{S+I^{-1}\right\}\right)^{\times}
\end{gathered}
$$

and

$$
\eta_{S+I}(\beta+I)=\mu^{-1}\left(\eta_{S}(\beta)+\left\langle\eta_{S}[I]\right\rangle\right) \in\left(\left(\frac{A}{I}\right)\left\{S+I^{-1}\right\}\right)^{\times}
$$

hence

$$
(\beta+I) \in(S+I)^{\infty-\mathrm{sat}} .
$$

Proposition 2.2.55. Let $A$ be $\mathcal{C}^{\infty}$-ring, $I$ be any of its ideals $I, q_{I}: A \rightarrow \frac{A}{I}$ be the standard projection map and $a \in A$ be any element. We have a relation between $\{a\}^{\infty-\text { sat }}$ and $\{a+I\}^{\infty \text {-sat }}$ given by the following implication:

$$
(\forall b \in A)\left(\left(b \in\{a\}^{\infty-\mathrm{sat}}\right) \rightarrow\left(q_{I}(b) \in\{a+I\}^{\infty-\mathrm{sat}}\right)\right)
$$

Proof. Consider the following diagram:

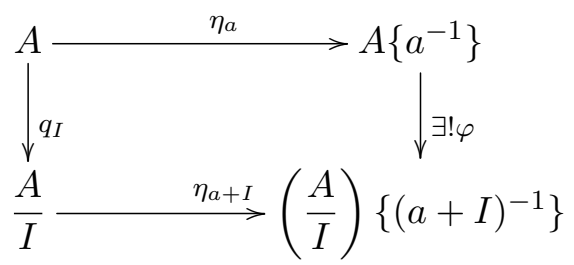

where $\varphi$ is given by the universal property of $A\left\{a^{-1}\right\}$, since $\eta_{a+I}\left(q_{I}(a)\right)=\eta_{a+I}(a+I) \in\left(\frac{A}{I}\left\{(a+I)^{-1}\right\}\right)^{\times}$.

Given $b \in\{a\}^{\infty \text {-sat }}$, so $\eta_{a}(b) \in\left(A\left\{a^{-1}\right\}\right)^{\times}$, we have $\varphi\left(\eta_{a}(b)\right) \in\left(\frac{A}{I}\left\{(a+I)^{-1}\right\}\right)^{\times}$(for, the homomorphic image of invertible elements are invertible). But since the diagram above commutes, it follows that $\eta_{a+I}(b+I)=\eta_{a+I}\left(q_{I}(b)\right)=\varphi\left(\eta_{a}(b)\right) \in\left(\frac{A}{I}\left\{(a+I)^{-1}\right\}\right)^{\times}$, so $q_{I}(b) \in\left(\frac{A}{I}\left\{(a+I)^{-1}\right\}\right)^{\times}$and $b+I \in\{a+I\}^{\infty-\mathrm{sat}}$.

Now suppose $(s+I) \in\{a+I\}^{\infty-\text { sat }}$, so by Theorem 2.2 .54 there are $\sigma \in\{a\}^{\infty-\text { sat }}$ and $\gamma \in A$ such that $\sigma \cdot(s \cdot \gamma-1) \in I$. By Theorem 2.1.28 in order to show that $s \in\{a\}^{\infty-\text { sat }}$ it suffices to show that these $\sigma \in\{a\}^{\infty-\text { sat }}$ and $\gamma \in A$ are such that $\sigma \cdot \gamma \cdot s \in\{a\}^{\infty-\text { sat }}$.

$$
(\sigma \cdot(s \cdot \gamma-1) \in I) \Longleftrightarrow(\sigma \cdot \gamma \cdot s-\sigma \in I) .
$$

Since $I \cap\{a\}^{\infty-\text { sat }}=\varnothing$ (for if it was not the case we would have $\left(\frac{A}{I}\right)\left\{(a+I)^{-1}\right\} \cong\{0\}$ ), it follows that $\sigma \cdot(\gamma \cdot s-1) \notin\{a\}^{\infty-\text { sat }}$. Since $\{a\}^{\infty-\text { sat }}$ is a multiplicative set,

$$
\left.\sigma \cdot(\gamma \cdot s-1) \notin\{a\}^{\infty-\mathrm{sat}} \rightarrow\left(\sigma \notin\{a\}^{\infty-\mathrm{sat}}\right) \vee\left(\gamma \cdot s-1 \notin\{a\}^{\infty-\mathrm{sat}}\right)\right)
$$


It is not the case that $\sigma \notin\{a\}^{\infty-\text { sat }}$, so we must have $\gamma \cdot s-1 \notin\{a\}^{\infty-\text { sat }}$, that is, $\eta_{a}(\gamma) \cdot \eta_{a}(s)-1 \notin$ $\left(A\left\{a^{-1}\right\}\right)^{\times}$.

Remark 2.2.56. The converse of the above implication is not always true, that is

$$
(\exists b \in A)\left(\left(q_{I}(b) \in\{a+I\}^{\infty-\mathrm{sat}}\right) \&\left(b \notin\{a\}^{\infty-\mathrm{sat}}\right)\right) .
$$

Consider $A=\mathcal{C}^{\infty}(\mathbb{R})$,

$$
\begin{aligned}
a: \mathbb{R} & \rightarrow \mathbb{R} \\
x & \mapsto \begin{cases}e^{-\frac{1}{1-x^{2}}}, & \text { if }|x|<1 \\
0, & \text { otherwise }\end{cases}
\end{aligned}
$$

and $I=\mathfrak{m}_{0}=\left\{g \in \mathcal{C}^{\infty}(\mathbb{R}) \mid g(0)=0\right\}$, which is a maximal ideal. Since $\mathfrak{m}_{0}$ is a maximal ideal, $\frac{\mathcal{C}^{\infty}(\mathbb{R})}{\mathfrak{m}_{0}}$ is a field, so every non-zero element of it must be invertible and $\frac{\mathcal{C}^{\infty}(\mathbb{R})}{\mathfrak{m}_{0}}\left\{\left(a+\mathfrak{m}_{0}\right)^{-1}\right\} \cong \frac{\mathcal{C}^{\infty}(\mathbb{R})}{\mathfrak{m}_{0}}$

Consider $h(x)=a(x)-a\left(\frac{1}{2}\right)$, which is a smooth function. Since $h\left(\frac{1}{2}\right)=0, h \notin \mathcal{C}^{\infty}(\mathbb{R})\left\{a^{-1}\right\}$, for $\frac{1}{2} \in \operatorname{Coz}(a)$. However, since $h(0)=a(0)-a\left(\frac{1}{2}\right)=e^{-1}-e^{\frac{4}{3}} \neq 0, h \notin \mathfrak{m}_{0}$, so $h+\mathfrak{m}_{0} \neq 0+\mathfrak{m}_{0} \in \frac{\mathcal{C}^{\infty}(\mathbb{R})}{\mathfrak{m}_{0}}$, hence it is invertible.

Remark 2.2.57. Let $A$ be $a \mathcal{C}^{\infty}$-ring, $a \in A$ and $b \in A$ be any two elements of $A$ such that $(a)=(b)$. Under those circumstances there is a unique $\mathcal{C}^{\infty}$-isomorphism $\sigma_{b}^{a}: A\left\{a^{-1}\right\} \rightarrow A\left\{b^{-1}\right\}$ such that the following diagram commutes:

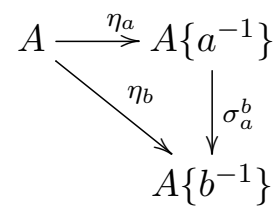

Proof. Suppose $a \neq 0$. Since $(a)=(b)$, in particular we have $a \in(b)$, so there is some $y \in A$ such that $a=y \cdot b$. Also, since $b \in(a)$, there is some $z \in A$ such that $b=z \cdot a$.

We have $a=y \cdot b=y \cdot(z \cdot a)=(y \cdot z) \cdot a$, so $A\left\{a^{-1}\right\}=A\left\{(y z a)^{-1}\right\}$, hence $\eta_{a}(y z a) \in\left(A\left\{a^{-1}\right\}\right)^{\times}$, i.e., $\eta_{a}(y z) \cdot \eta_{a}(a) \in\left(A\left\{a^{-1}\right\}\right)^{\times}$. Since $\eta_{a}(a) \in\left(A\left\{a^{-1}\right\}\right)^{\times}$, it follows that $\eta_{a}(y z)=\eta_{a}(y) \cdot \eta_{a}(z) \in\left(A\left\{a^{-1}\right\}\right)^{\times}$, so we have both $\eta_{a}(y) \in\left(A\left\{a^{-1}\right\}\right)^{\times}$and $\eta_{a}(z) \in\left(A\left\{a^{-1}\right\}\right)^{\times}$.

Under those circumstances, $\eta_{a}(b)=\eta_{a}(z) \cdot \eta_{a}(a) \in\left(A\left\{a^{-1}\right\}\right)^{\times}$. By the universal property of $\eta_{b}: A \rightarrow$ $A\left\{b^{-1}\right\}$, there must exist a unique $\sigma_{a}^{b}: A\left\{b^{-1}\right\} \rightarrow A\left\{a^{-1}\right\}$ such that the following diagram commutes:

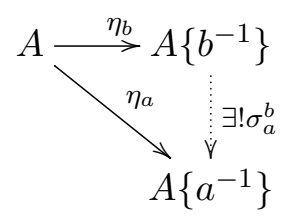

Analogously, we conclude that there is a unique $\mathcal{C}^{\infty}$-homomorphism $\sigma_{b}^{a}: A\left\{a^{-1}\right\} \rightarrow A\left\{b^{-1}\right\}$ such that:

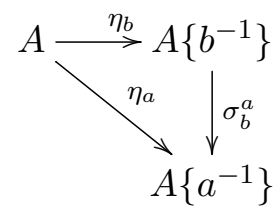


commutes.

By uniqueness, it follows that $\left(\sigma_{b}^{a}\right)^{-1}=\left(\sigma_{a}^{b}\right)$, and the result follows.

Proposition 2.2.58. If $A$ is a $\mathcal{C}^{\infty}$-reduced $\mathcal{C}^{\infty}$-domain, then:

(i) $A\left\{A \backslash\{0\}^{-1}\right\}$ is a $\mathcal{C}^{\infty}$-field;

(ii) $\operatorname{Can}_{A \backslash\{0\}}: A \rightarrow A\left\{A \backslash\{0\}^{-1}\right\}$ is a $\mathcal{C}^{\infty}$-monomorphism;

(iii) If $\mathbb{K}$ is a $\mathcal{C}^{\infty}$-field and $\jmath: A \rightarrow \mathbb{K}$ is a $\mathcal{C}^{\infty}$-monomorphism, then there is a unique $\mathcal{C}^{\infty}$-homomorphism $\tilde{\jmath}: A\left\{A \backslash\{0\}^{-1}\right\} \rightarrow \mathbb{K}$ such that the following diagram commutes:

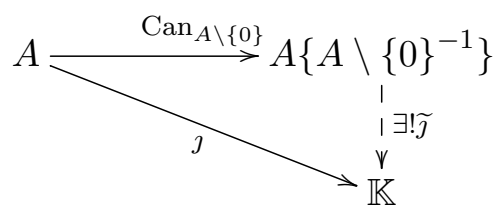

Thus, $\operatorname{Frac}(A):=A\left\{A \backslash\{0\}^{-1}\right\}$ is the $\mathcal{C}^{\infty}$-field of fractions of $A$,

Proof. Ad (i): By item (c) of Theorem 2.2.47, since $(0) \in \operatorname{Spec}^{\infty}(A)$ then $A \backslash\{0\}=(A \backslash\{0\})^{\infty-\text { sat }}$.

Given any $\alpha \in A\left\{A \backslash\{0\}^{-1}\right\}$, there are $x \in A$ and $y \in(A \backslash\{0\})^{\infty-\text { sat }}=A \backslash\{0\}$ such that:

$$
\alpha=\frac{\operatorname{Can}_{A \backslash\{0\}}(x)}{\operatorname{Can}_{A \backslash\{0\}}(y)} .
$$

If $\alpha \neq 0$, then $x \neq 0$ and $x \in A \backslash\{0\}$. Thus, $\beta=\frac{\operatorname{Can}_{A \backslash\{0\}}(y)}{\operatorname{Can}_{A \backslash p}(x)}$ is a multiplicative inverse of $\alpha$.

Since every non-zero element of $A\left\{A \backslash\{0\}^{-1}\right\}$ is invertible, it follows that $A\left\{A \backslash\{0\}^{-1}\right\}$ is a $\mathcal{C}^{\infty}$-field;

Ad (ii): If $a \neq 0$, then $a \in A \backslash\{0\}$ so $\operatorname{Can}_{A \backslash\{0\}}(a) \in\left(A\left\{A \backslash\{0\}^{-1}\right\}\right)^{\times}$. Since $A\left\{A \backslash\{0\}^{-1}\right\}$ is a $\mathcal{C}^{\infty}$-field, it is not the trivial $\mathcal{C}^{\infty}$-ring, thus $\operatorname{Can}_{A \backslash\{0\}}(a) \neq 0$. We conclude that if $a \in A \backslash\{0\}$ is such that $\operatorname{Can}_{A \backslash\{0\}}(a)=0$ implies $a=0$.

Ad (iii): Note that $\jmath[A \backslash\{0\}] \subseteq \mathbb{K}^{\times}$, and the result follows from the universal property of the $\mathcal{C}^{\infty}$-ring of fractions, $A\left\{A \backslash\{0\}^{-1}\right\}$.

Now we present a result that says that every $\mathcal{C}^{\infty}$-radical prime ideal is the kernel of a $\mathcal{C}^{\infty}$-homomorphism into a $\mathcal{C}^{\infty}$-field.

First we notice that whenever $\mathfrak{p}$ is a $\mathcal{C}^{\infty}$-radical prime ideal of $A$ the localization:

$$
\mathrm{Can}_{A \backslash \mathfrak{p}}: A \rightarrow A_{\{\mathfrak{p}\}},
$$

where $A_{\{\mathfrak{p}\}}=A\left\{(A \backslash \mathfrak{p})^{-1}\right\}$ is a local ring. Let $\hat{\mathfrak{p}}$ be the maximal ideal of $A_{\{\mathfrak{p}\}}$ and $q: A_{\{\mathfrak{p}\}} \rightarrow \frac{A_{\{\mathfrak{p}\}}}{\hat{\mathfrak{p}}}$ be the quotient map into the residual field. Notice that $q$ is a local homomorphism, since $q[\hat{\mathfrak{p}}]=(0)$.

Lemma 2.2.59. Let $S$ be a multiplicative subset of a $\mathcal{C}^{\infty}-$ ring $A$, and write $A\left\{S^{-1}\right\}=\varliminf_{a \in S} A\left\{a^{-1}\right\}$. There is an inclusion-preserving bijection between the set of $\mathcal{C}^{\infty}$-radical prime ideals in $A\left\{S^{-1}\right\}$ and $\mathcal{C}^{\infty}$-prime ideals in $A$ which are disjoint from $S$. 
Proof. A proof of this fact can be found on page 286 of [65].

We register another technical result:

Lemma 2.2.60. Let $A$ be $a \mathcal{C}^{\infty}-$ ring and $e \in A$ an idempotent element. There are unique isomorphisms:

$$
A\left\{e^{-1}\right\} \cong \frac{A}{(1-e)} \cong A \cdot e:=\{a \cdot e \mid a \in A\}
$$

Proof. Let

$$
\begin{aligned}
m_{e}: A & \rightarrow A \cdot e \\
a & \mapsto a \cdot e
\end{aligned}
$$

and

$$
\begin{aligned}
q \quad A & \rightarrow \frac{A}{(1-e)} \\
a & \mapsto a+(1-e)
\end{aligned}
$$

Since $(1-e) \cdot e=e-e^{2}=e-e=0$, given any $a=x(1-e) \in(1-e), m_{e}(a)=m_{e}(x(1-e))=$ $x(1-e) e=x \cdot 0=0$, so $(1-e) \subseteq \operatorname{ker} m_{e}$.

Noting that $(1-e) \subseteq \operatorname{ker} m_{e}$ and that $m_{e}$ is a surjective map, by the Theorem of the Isomorphism there is a unique isomorphism $\psi: \frac{A}{(1-e)} \rightarrow A \cdot e$ such that the following triangle commutes:

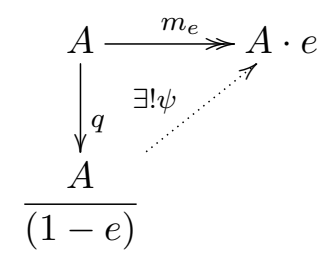

so $\frac{A}{(1-e)} \cong A \cdot e$.

Finally we show that $A\left\{e^{-1}\right\} \cong \frac{A}{(1-e)}$. First we note that since $\eta_{e}(e) \in\left(A\left\{e^{-1}\right\}\right)^{\times}$and $\left(\eta_{e}(e)\right)^{2}=$ $\eta_{e}\left(e^{2}\right)=\eta_{e}(e)$ (that is, $\eta_{e}(e)$ is an idempotent element of $\left.A\left\{e^{-1}\right\}\right)$, it follows that $\eta_{e}(e)=1$.

We have $\eta_{e}(1-e)=\eta_{e}(1)-\eta_{e}(e)=1-1=0$, so $(1-e) \subseteq$ ker $\eta_{a}$, so applying the Theorem of the Homomorphism we get a unique $\mathcal{C}^{\infty}$-homomorphism $\varphi: \frac{A}{(1-e)} \rightarrow A\left\{e^{-1}\right\}$ such that the following diagram commutes:

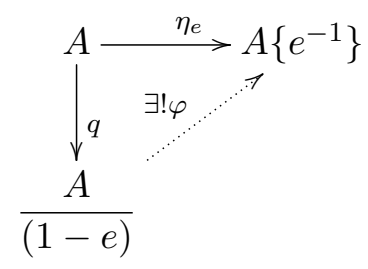

We have also:

$$
q(e)-q(1)=q(e-1) \in(e-1)
$$

hence

$$
q(e)=q(1) \text { in } \frac{A}{(1-e)}
$$


Since $(q(e))^{2}=q\left(e^{2}\right)=q(e)$, i.e., it is idempotent, it follows that $q(e)=1+(e-1) \in\left(\frac{A}{(1-e)}\right)^{\times}$. By the universal property of $\eta_{e}: A \rightarrow A\left\{e^{-1}\right\}$, there is a unique $\mathcal{C}^{\infty}$-homomorphism $\psi: A\left\{e^{-1}\right\} \rightarrow \frac{A}{(1-e)}$ such that the following diagram commutes:

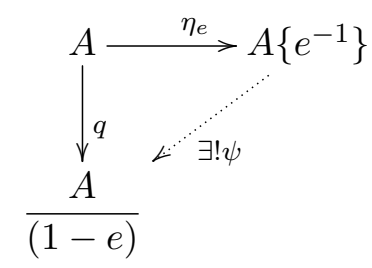

By the uniqueness of the arrows $\varphi$ and $\psi$, we conclude that $\varphi \circ \psi=\operatorname{id}_{A\left\{e^{-1}\right\}}$ and $\psi \circ \varphi=\operatorname{id}_{\frac{A}{(1-e)}}$, hence $A\left\{e^{-1}\right\} \cong \frac{A}{(1-e)}$.

We give a definition of a von Neumann regular $\mathcal{C}^{\infty}$-ring: it is a $\mathcal{C}^{\infty}$-ring $(A, \Phi)$ such that $\widetilde{U}(A, \Phi)$ is a von Neumann regular commutative unital ring (here $\widetilde{U}: \mathcal{C}^{\infty} \mathbf{R n g} \rightarrow \mathbf{C R i n g}$ ).

Definition 2.2.61. Let $A$ be a $\mathcal{C}^{\infty}$-ring. We say that $A$ is a von Neumann regular $\mathcal{C}^{\infty}$-ring if one (and thus all) of the following equivalen 1 , conditions is satisfied:

(i) $(\forall a \in A)(\exists x \in A)\left(a=a^{2} x\right)$;

(ii) Every principal ideal of $A$ is generated by an idempotent element, i.e.,

$$
(\forall a \in A)(\exists e \in A)(\exists y \in A)(\exists z \in A)\left(\left(e^{2}=e\right) \&(e y=a) \&(a z=e)\right)
$$

(iii) $(\forall a \in A)(\exists ! b \in A)\left(\left(a=a^{2} b\right) \&\left(b=b^{2} a\right)\right)$

Proposition 2.2.62. Let $A$ be a local von Neumann-regular $\mathcal{C}^{\infty}$-ring. Then $A$ is a $\mathcal{C}^{\infty}$-field.

Proof. Since $A$ is a local $\mathcal{C}^{\infty}$-ring there is a unique maximal ideal, $\mathfrak{m} \subseteq A$. Since $A$ is a von Neumannregular $\mathcal{C}^{\infty}$-ring, by Corollary $\mathbf{2 . 2 . 7 7}$.

$$
A_{\mathfrak{m}} \cong \frac{A}{\mathfrak{m}}
$$

and the latter is a $\mathcal{C}^{\infty}$-field.

Also, $A_{\mathfrak{m}}=A\left\{A \backslash \mathfrak{m}^{-1}\right\}=A\left\{A^{\times-1}\right\} \cong A$, and since $A_{\mathfrak{m}}$ is isomorphic to a $\mathcal{C}^{\infty}$-field, it follows that $A$ is a $\mathcal{C}^{\infty}$-field.

Definition 2.2.63. Let $\mathcal{C}^{\infty} \mathbf{v N R n g}$ be the category whose objects are von Neumann-regular $\mathcal{C}^{\infty}$-rings and whose morphisms are the $\mathcal{C}^{\infty}$-homomorphisms between them.

Remark 2.2.64. Since the inclusion functor $\iota: \mathcal{C}^{\infty} \mathbf{v N R n g} \hookrightarrow \mathcal{C}^{\infty} \mathbf{R n g}$ is closed under quotients, it follows that the congruences of von Neumann-regular $\mathcal{C}^{\infty}$-rings are also classified by their ring-theoretic ideals.

Remark 2.2.65. Let $A$ be a von Neumann-regular $\mathcal{C}^{\infty}$-ring and $e \in A$ be any idempotent element. Then $A \cdot e$ is a von Neumann-regular $\mathcal{C}^{\infty}$-ring. Indeed, we have $A \cdot e \cong \frac{A}{(1-e)}$ and the latter is an homomorphic image of a von Neumann-regular $\mathcal{C}^{\infty}$-ring, namely $\frac{A}{(1-e)}=q[A]$. Since $\frac{A}{(1-e)}$ is the

\footnotetext{
${ }^{1}$ See for instance 2 for a proof of these equivalences in the ring setting.
} 
homomorphic image of a von Neumann-regular $\mathcal{C}^{\infty}$-ring, $\frac{A}{(1-e)}$ is a von Neumann-regular $\mathcal{C}^{\infty}$-ring. Since $A \cdot e \cong \frac{A}{(1-e)}$, it follows that $A \cdot e$ is a von Neumann-regular $\mathcal{C}^{\infty}$-ring.

The following lemma tells us that, in $\mathcal{C}^{\infty} \mathbf{v N R n g}$, taking localizations and taking the ring of fractions with respect to a special element yield the same object.

Lemma 2.2.66. If $A$ is a von Neumann regular $\mathcal{C}^{\infty}$-ring, then given any $a \in A$ there is some idempotent element $e \in A$ such that $A\left\{a^{-1}\right\} \cong \frac{A}{(1-e)}$.

Proof. Let $a \in A$ be any element. Since $A$ is a von Neumann regular $\mathcal{C}^{\infty}$-ring, there exists some idempotent element $e \in A$ such that $a \in(e)$ and $e \in(a)$, so

$$
(a)=(e) \text {. }
$$

We claim that:

$$
\frac{A}{(1-e)} \cong A\left\{a^{-1}\right\}
$$

First notice that the quotient map $q: A \rightarrow \frac{A}{(1-e)}$ inverts $a$, i.e., $q(a) \in\left(\frac{A}{(1-e)}\right)^{\times}$. Indeed, since $(a)=(e)$, there must exist some $y \in A$ such that $a y=e$, so $1-a y+(1-e)$ is the multiplicative inverse of $q(a)=a+(1-e)$, hence $q(a) \in\left(\frac{A}{(1-e)}\right)^{\times}$.

Given $f: A \rightarrow B$ a $\mathcal{C}^{\infty}$-rings homomorphism such that $f(a) \in B^{\times}$, consider the following diagram:

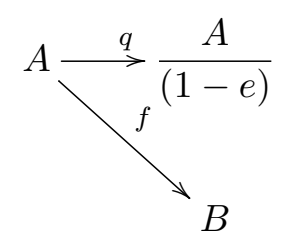

Claim: $1-e \in \operatorname{ker} f$.

Since $f(a) \in B^{\times}, f(a)=f(a e)=f(a) \cdot f(e) \in B^{\times}$, so there is some $b \in B$ such that $f(e) \cdot[f(a) \cdot b]=$ $[f(e) f(a)] \cdot b=1$. Hence $f(e)^{-1}=f(a) \cdot b$.

Note that $f(e) \in B^{\times}$and $f(e)=f\left(e^{2}\right)=f(e)^{2}$, that is, $f(e)$ is an invertible idempotent of $A$, hence $f(e)=1$ and $1-e \in \operatorname{ker} f$.

By the Theorem of the Homomorphism, since $(1-e) \subseteq$ ker $f$ there is a unique $\mathcal{C}^{\infty}$-homomorphism $\widetilde{f}: \frac{A}{(1-e)} \rightarrow B$ such that

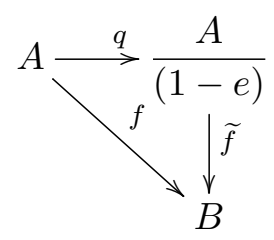

commutes.

Since $\frac{A}{(1-e)}$ has the universal property of the ring of fractions $A\left\{a^{-1}\right\}$, it follows that:

$$
\frac{A}{(1-e)} \cong A\left\{a^{-1}\right\}
$$


Lemma 2.2.67. Let $A$ be a von Neumann-regular $\mathcal{C}^{\infty}-$ ring, $S \subseteq A$ and let $\widetilde{U}: \mathcal{C}^{\infty} \mathbf{R n g} \rightarrow \mathbf{C R i n g}$ be the forgetful functor. Then:

$$
\widetilde{U}\left(A\left\{S^{-1}\right\}\right)=\widetilde{U}(A)\left[S^{-1}\right]
$$

Proof. We prove the result first in the case $S=\{a\}$ for some $a \in A$.

Since $A$ is a von Neumann-regular $\mathcal{C}^{\infty}$-ring, by Lemma 2.2.66 given $a \in A$ there is some idempotent element such that:

$$
A\left\{a^{-1}\right\} \cong A\left\{e^{-1}\right\} \cong \frac{A}{(1-e)}
$$

Now, $\frac{A}{(1-e)} \cong A\left[e^{-1}\right]$, and $A\left[e^{-1}\right] \cong \widetilde{U}(A)\left[e^{-1}\right]$, and since $\widetilde{U}(A)\left[e^{-1}\right] \cong \widetilde{U}(A)\left[a^{-1}\right]$, the result follows.

Whenever $S$ is finite, we have $A\left\{S^{-1}\right\}=A\left\{a^{-1}\right\}$, for $a=\prod S$, and we can use the proof we have just made.

For a general $S \subseteq A$, we write:

$$
S=\bigcup_{S^{\prime} \subseteq_{\text {fin }} S} S^{\prime}
$$

and use the fact that $\widetilde{U}: \mathcal{C}^{\infty} \mathbf{R n g} \rightarrow \mathbf{C R i n g}$ preserves directed colimits.

As a corollary, we have the following:

Proposition 2.2.68. $\mathcal{C}^{\infty} \mathbf{v N R n g} \subseteq \mathcal{C}^{\infty} \mathbf{R n g}$ is closed under localizations.

The following result is an adaptation of Proposition 1 of [2] for the $\mathcal{C}^{\infty}$-case.

Theorem 2.2.69. If $A$ is a von Neumann regular $\mathcal{C}^{\infty}$-ring then $A$ is a reduced $\mathcal{C}^{\infty}$-ring.

Proof. By the Lemma 2.2.66,

$$
\sqrt[\infty]{(0)}=\left\{a \in A \mid A\left\{a^{-1}\right\} \cong\{0\}\right\}=\left\{a \in A \mid \frac{A}{(1-e)} \cong\{0\}\right\}
$$

Now $\frac{A}{(1-e)} \cong\{0\}$ yields $1 \in(1-e)$, so there must exist some $z \in A$ such that $1=z \cdot(1-e)$, and $(1-e)$ is an invertible idempotent of $A$, so:

$$
\begin{gathered}
1-e=1 \\
e=0
\end{gathered}
$$

Therefore $a=0$, so $\sqrt[\infty]{(0)} \subseteq\{0\}$. The other inclusion is always true, so:

$$
\sqrt[\infty]{(0)} \cong\{0\}
$$

and $A$ is reduced. 
The following result shows us that whenever $A$ is a von Neumann regular $\mathcal{C}^{\infty}$-ring, the notions of $\mathcal{C}^{\infty}$-spectrum, Zariski spectrum, maximal spectrum and thus, the structure sheaf of its affine scheme coincide.

Theorem 2.2.70. Let $A$ be a von Neumann regular $\mathcal{C}^{\infty}$-ring. Then:

1) $\sqrt[\infty]{(0)}=\sqrt{(0)}=(0)$;

2) $\operatorname{Spec}^{\infty}(A)=\operatorname{Specm}(A)=\operatorname{Spec}(A)$, as topological spaces;

3) The structure sheaf of $A$ in the category $\mathcal{C}^{\infty} \mathbf{R n g}$ is equal to the structure sheaf of $U(A)$ in the category CRing.

Proof. Ad 1): By Theorem 2.2.69, since $A$ is a von Neumann regular $\mathcal{C}^{\infty}-\operatorname{ring}, \sqrt[\infty]{(0)} \cong(0)$. But $\widetilde{U}(A)$ is also a von Neumann regular ring, so given any $a \in A$ there exists some idempotent element $e \in A$ such that $(a)=(e)$, i.e.,

$$
a \in(e)
$$

and

$$
e \in(a)
$$

The former condition implies the existence of some $x \in A$ such that $a=e x$ so

$$
a e=e^{2} x=e x=a
$$

and latter condition implies the existence of some $y \in A$ such that $e=a y$, so

$$
a y=e=e^{2}=a^{2} y^{2} .
$$

Now, given $a \in \sqrt{(0)}$, there is some $n \in \mathbb{N}$ such that $a^{n}=0$, so

$$
0=a^{n}=(a e)^{n}=a^{n} e^{n}=a^{n} e .
$$

Since $e^{n}=a^{n} y^{n}=0$, then $e=0$, so $a=a \cdot 0=0$.

Hence, $\sqrt{(0)}=(0)=\sqrt[\infty]{(0)}$.

Ad 2):

Claim: In a von Neumann regular $\mathcal{C}^{\infty}$-ring every prime ideal is a maximal ideal.

Let $\mathfrak{p}$ be a prime ideal in $A$. Given $a+\mathfrak{p} \neq \mathfrak{p}$ in $\frac{A}{\mathfrak{p}}$, then $a+\mathfrak{p} \in\left(\frac{A}{\mathfrak{p}}\right)^{\times}$.

Since $A$ is a von Neumann regular ring, there exists some $b \in A$ such that $a b a=a$, so

$$
\begin{gathered}
a+\mathfrak{p}=a b a+\mathfrak{p} \\
a+\mathfrak{p}=(a b+\mathfrak{p}) \cdot(a+\mathfrak{p}) \\
a b+\mathfrak{p}=1+\mathfrak{p}
\end{gathered}
$$

so

$$
a b=1 .
$$

Hence, every non-zero element of $\frac{A}{\mathfrak{p}}$ is invertible, so $\frac{A}{\mathfrak{p}}$ is a field. Under those circumstances, it follows that $\mathfrak{p}$ is a maximal ideal, so $\operatorname{Spec}(A)=\operatorname{Specm}(A)$. 
We always have $\operatorname{Specm}(A) \subseteq \operatorname{Spec}^{\infty}(A)$ and $\operatorname{Spec}^{\infty}(A) \subseteq \operatorname{Spec}(A)$, so:

$$
\operatorname{Spec}(A) \subseteq \operatorname{Specm}(A) \subseteq \operatorname{Spec}^{\infty}(A) \subseteq \operatorname{Spec}(A)
$$

and

$$
\operatorname{Spec}^{\infty}(A)=\operatorname{Spec}(A) .
$$

We note, also, that both the topological spaces $\operatorname{Spec}(A)$ and $\operatorname{Spec}^{\infty}(A)$ have the same basic open sets:

$$
D^{\infty}(a)=\left\{\mathfrak{p} \in \operatorname{Spec}^{\infty}(A) \mid a \notin \mathfrak{p}\right\}=\{\mathfrak{p} \in \operatorname{Spec}(A) \mid a \notin \mathfrak{p}\}=D(a),
$$

hence

$$
\operatorname{Spec}^{\infty}(A)=\operatorname{Spec}(A)
$$

as topological spaces.

Ad 3). Immediate.

Proposition 2.2.71. Let $A$ be a $\mathcal{C}^{\infty}$-ring. Then the following are equivalent:

(i) A is von Neumann-regular, i.e.,

$$
(\forall a \in A)(\exists x \in A)\left(a=a^{2} x\right)
$$

(ii) Every principal ideal of $A$ is generated by an idempotent element, i.e.,

$$
(\forall a \in A)(\exists e \in A)(\exists y \in A)(\exists z \in A)\left(\left(e^{2}=e\right) \wedge(e y=a) \wedge(a z=e)\right)
$$

(iii) $(\forall a \in A)(\exists ! b \in A)\left(\left(a=a^{2} b\right) \wedge\left(b=b^{2} a\right)\right)$

Moreover, when $A$ is von Neumann-regular, then $A$ is reduced (i.e., $\sqrt[\infty]{(0)}=(0)$ ) and for each $a \in A$ the idempotent element e satisfying (ii) and the element b satisfying (iii) are uniquely determined.

Proof. The implication (iii) $\rightarrow$ (i) is obvious, so we omit the proof.

Ad (i) $\rightarrow$ (ii): Let $I=(a)$ be a principal ideal of $A$. By (i), there is $x \in A$ such that $a=a^{2} x$, so we define $e:=a x$, which is idempotent since $e^{2}=(a x)^{2}=a^{2} x^{2}=\left(a^{2} x\right) x=a x=e$. By definition, $e=a x \in(a)=I$, so $(e) \subseteq I$, and since $a=a^{2} x=(a x) a=e a$ we also have $a \in(e)$, so $I=(a) \subseteq(e)$. Hence, $I=(e)$.

Ad (ii) $\rightarrow$ (i): Let $a \in A$ be any element. By (ii) there are $e \in A, y \in A$ and $z \in A$ such that $e^{2}=e$, $a=e y$ and $e=a z$. Define $x:=z^{2} y$, and we have $a^{2} x=a^{2} z^{2} y=e^{2} y=e y=a$.

Ad (i) $\rightarrow$ (iii): Let $a \in A$ be any element. By (i), there is some $x \in A$ such that $a=a^{2} x$. There can be many $x \in A$ satisfying this role, but there is a "minimal" one: the element $a x$ is idempotent and we can project any chosen $x$ down with this idempotent, obtaining $b:=a x^{2}$. Then $a b a=a a b^{2} a=(a x)(a x) a=a x a=a$ and $b a b=\left(a x^{2}\right) a\left(a x^{2}\right)=(a x)^{3} x=(a x) x=b$.

Now suppose that $A$ is a von Neumann-regular $\mathcal{C}^{\infty}$-ring, and let $a \in A$ be such that $a \in \sqrt[\infty]{(0)}$. Then let $e$ be an idempotent such that $e y=a, a z=e$, for some $y, z \in A$. Then $a$ is such that $A\left\{a^{-1}\right\} \cong\{0\}$, and by Lemma 2.2.66 there is some idempotent $e \in A$ such that $A\left\{a^{-1}\right\} \cong \frac{A}{(1-e)}$. Now, $A\left\{a^{-1}\right\} \cong\{0\}$ occurs if and only if, $\frac{A}{(1-e)} \cong\{0\}$, i.e., if and only if, $(1-e)=A$. Since $(1-e)=A$, it follows that 
$1-e \in A^{\times}$, and since $e \cdot(1-e)=0$, it follows by cancellation that $e=0$, hence $a=e y=0 y=0$.

Let $e, e^{\prime} \in A$ be idempotents in an arbitrary ring satisfying $(e)=\left(e^{\prime}\right)$. Select $r, r^{\prime} \in A$ such that $e r^{\prime}=e^{\prime}$ and $e^{\prime} r=e$. Then $e^{\prime}=e r^{\prime}=e r^{\prime} e=e^{\prime} e=e^{\prime} r e^{\prime}=e^{\prime} r=e$. Thus, if an ideal is generated by an idempotent element, this element is uniquely determined.

Finally, let $A$ be a von Neumann-regular $\mathcal{C}^{\infty}$-ring. Select a member $a \in A$ and consider $b, b^{\prime} \in A$ such that $a^{2} b^{\prime}=a=a^{2} b, b=b^{2} a, b^{\prime}=b^{\prime 2} a$. Then $\left(b-b^{\prime}\right) a^{2}=\left(b-b^{\prime}\right) a^{2}=\left(b-b^{\prime}\right)\left(b a^{2}-b^{\prime} a^{2}\right)=\left(b-b^{\prime}\right)(a-a)=$ $\left(b-b^{\prime}\right) \cdot 0=0$ and $\left[\left(b-b^{\prime}\right) \cdot a\right]^{2} \in(0)$. Since $A$ is $\mathcal{C}^{\infty}$-reduced, $\left[\left(b-b^{\prime}\right) \cdot a\right]^{2} \in(0)=\sqrt[\infty]{(0)}$. By item (1) of Theorem 2.2.70, $\sqrt[\infty]{(0)}=\sqrt{(0)}$, so $\left[\left(b-b^{\prime}\right) \cdot a\right]^{2} \in \sqrt[\infty]{(0)}=\sqrt{(0)}$ and $\left(b-b^{\prime}\right) \cdot a=0$. Therefore $b-b^{\prime}=b^{2} a-b^{\prime 2} a=\left(b^{2}-b^{\prime 2}\right) a=\left(b+b^{\prime}\right)\left(b-b^{\prime}\right) a=\left(b+b^{\prime}\right) \cdot 0=0$.

Lemma 2.2.72. Let $A$ be a local $\mathcal{C}^{\infty}$-ring. The only idempotent elements of $A$ are 0 and 1 .

Proof. Since $A$ is a local $\mathcal{C}^{\infty}$-ring, we have:

$$
(\forall x \in A)(\forall y \in A)\left(\left(x+y \in A^{\times}\right) \rightarrow\left(x \in A^{\times}\right) \vee\left(y \in A^{\times}\right)\right)
$$

Let $e \in A$ be an idempotent. We have $\left(1=e+(1-e) \in A^{\times}\right) \rightarrow\left(\left(e \in A^{\times}\right) \vee\left(1-e \in A^{\times}\right)\right)$. If $e \in A^{\times}$ then $e=1$ (since 1 is the only invertible idempotent), and if $1-e \in A^{\times}$then, by the same reasons, $1-e=1$, hence $e=0$.

Proposition 2.2.73. Let $A$ be a von Neumann-regular $\mathcal{C}^{\infty}$-ring whose only idempotent elements are 0 and 1 . Then the following assertions are equivalent:

(i) $A$ is a $\mathcal{C}^{\infty}$-field;

(ii) $A$ is a $\mathcal{C}^{\infty}$-domain;

(iii) $A$ is a local $\mathcal{C}^{\infty}$-ring.

Proof. The implications (i) $\rightarrow$ (ii), (i) $\rightarrow$ (iii) are immediate, so we omit their proofs.

Ad (iii) $\rightarrow$ (i): Suppose $A$ is a local $\mathcal{C}^{\infty}$-ring. Since $A$ is a von Neumann-regular $\mathcal{C}^{\infty}$-ring, given any $x \in A \backslash\{0\}$ there exists some idempotent element $e \in A$ such that $(x)=(e)$. However, the only idempotent elements of $A$ are, by Lemma 2.2.72, 0 and 1 . We claim that $(x)=(1)$, otherwise we would have $(x)=(0)$, so $x=0$.

Now, $(x)=(1)$ implies $1 \in(x)$, so there is some $y \in A$ such that $1=x \cdot y=y \cdot x$, and $x$ is invertible. Thus $A$ is a $\mathcal{C}^{\infty}$-field.

Ad (ii) $\rightarrow$ (i):Suppose $A$ is a $\mathcal{C}^{\infty}$-domain. Given any $x \in A \backslash\{0\}$, we have:

$$
(\forall y \in A \backslash\{0\})(x \cdot y \neq 0),
$$

so $(x) \neq(0)$. Since $A$ is a von Neumann-regular $\mathcal{C}^{\infty}-\operatorname{ring},(x)$ is generated by some non-zero idempotent element, namely, 1 . Hence $(x)=(1)$ and $x \in A^{\times}$.

Proposition 2.2.74. The inclusion functor $\imath: \mathcal{C}^{\infty} \mathbf{v N R n g} \hookrightarrow \mathcal{C}^{\infty} \mathbf{R n g}$ creates filtered colimits, i.e., $\mathcal{C}^{\infty}$ vNRng $\subset \mathcal{C}^{\infty}$ Rng 
Proof. A filtered colimit of von Neumann-regular $\mathcal{C}^{\infty}$-rings, taken in $\mathcal{C}^{\infty} \mathbf{R n g}$ is a von Neumann-regular $\mathcal{C}^{\infty}$-ring again. Indeed, filtered colimits in $\mathcal{C}^{\infty} \mathbf{R n g}$ are formed by taking the colimit of the underlying sets and defining the $n$-ary functional symbol $f^{(n)}$ of an $n$-tuple $\left(a_{1}, \cdots, a_{n}\right)$ into a common $\mathcal{C}^{\infty}$-ring occurring in the diagram and taking the element $f^{(n)}\left(a_{1}, \cdots, a_{n}\right)$ there.

Let $(I, \leq)$ be a filtered poset and $\left\{A_{i}\right\}_{i \in I}$ be a filtered family of $\mathcal{C}^{\infty}$-ring. Given any element $\alpha \in$ $\lim _{i} A_{i}$, there is some $i \in I$ and $a_{i} \in A_{i}$ such that $\alpha=\left[\left(a_{i}, i\right)\right]$. Since $A_{i}$ is a von Neumann-regular $\mathcal{C}^{\infty}$-ring, there must exist some idempotent $e_{i} \in A_{i}$ such that $\left(a_{i}\right)=\left(e_{i}\right)$. It suffices to take $\eta=\left[\left(e_{i}, i\right)\right] \in \lim A_{i}$, which is an idempotent element of $\underset{\lim }{\longrightarrow} A_{i}$ such that $(\alpha)=\left(\left[\left(a_{i}, i\right)\right]\right)=\left(\left[\left(e_{i}, i\right)\right]\right)=(\eta)$. Hence $\underset{\longrightarrow}{\longrightarrow} \overleftarrow{A_{i}}$ is a von Neumann-regular $\mathcal{C}^{\infty}$-ring.

We have the following important result, which relates von Neumann-regular $\mathcal{C}^{\infty}$-rings to the topology of its smooth Zariski spectrum:

Theorem 2.2.75. Let $A$ be a $\mathcal{C}^{\infty}$-ring. The following assertions are equivalent:

(i) $A$ is a von Neumann-regular $\mathcal{C}^{\infty}$-ring;

(ii) $A$ is a $\mathcal{C}^{\infty}$-reduced $\mathcal{C}^{\infty}$-ring (i.e., $\sqrt[\infty]{(0)}=(0)$ ) and $\operatorname{Spec}^{\infty}(A)$ is a Boolean space, i.e., a compact, Hausdorff and totally disconnected space.

Proof. Ad $(i) \rightarrow($ ii $)$ : Suppose $A$ is a von Neumann regular $\mathcal{C}^{\infty}$-ring. We are going to show that $\sqrt[\infty]{(0)}=(0)$

Given $a \in \sqrt[\infty]{(0)}$, since $A$ is a von Neumann regular $\mathcal{C}^{\infty}$-ring, there are some $e \in A$ such that $e^{2}=e$, some $x \in A$ and some $y \in A$ such that $a=e x$ and $e=a y$. that:

Since $a \in \sqrt[\infty]{(0)}$ and $\sqrt[\infty]{(0)}$ is an ideal, we have $e=a y \in \sqrt[\infty]{(0)}$. From Theorem 2.2 .22 , we conclude

$$
\left(\forall \mathfrak{p} \in \operatorname{Spec}^{\infty}(A)\right)(e \in \mathfrak{p})
$$

Now, $e^{2}=e$ implies that $(1-e) \cdot e=0$, so either $e=0$ or $e=1$. The latter does not occur, since $\mathfrak{p}$ is a proper prime ideal, hence $e=0$ and $a=0$. Hence $\sqrt[\infty]{(0)} \subseteq(0)$ and $\sqrt[\infty]{(0)}=(0)$.

It remains to show that $\operatorname{Spec}^{\infty}(A)$ is a Boolean space. Since $\operatorname{Spec}^{\infty}(A)$ is a spectral space, we only need to show that:

$$
\mathcal{B}=\left\{D^{\infty}(a) \mid a \in A\right\}
$$

is a clopen basis for its topology.

Given any $a \in A$, since $A$ is a von Neumann regular $\mathcal{C}^{\infty}$-ring, there is some idempotent element $e \in A$ such that $(a)=(e)$, so $D^{\infty}(a)=D^{\infty}(e)$.

We claim that $\operatorname{Spec}^{\infty}(A) \backslash D^{\infty}(e)=D^{\infty}(1-e)$, hence $D^{\infty}(a)=D^{\infty}(e)$ is a clopen set.

From item (iii) of Lemma $\mathbf{1 . 2}$ of [67,

$$
D^{\infty}(e) \cap D^{\infty}(1-e)=D^{\infty}(e \cdot(1-e))=D^{\infty}(0)=\left\{\mathfrak{p} \in \operatorname{Spec}^{\infty}(A) \mid 0 \notin \mathfrak{p}\right\}=\varnothing .
$$

Also by item (iii) of Lemma 1.2 of 67,

$$
D^{\infty}(e) \cup D^{\infty}(1-e)=D^{\infty}(e+(1-e))=D^{\infty}(1)=\operatorname{Spec}^{\infty}(A)
$$


so it follows that $\operatorname{Spec}^{\infty}(A)$ is a Boolean space and $A$ is a $\mathcal{C}^{\infty}$-reduced $\mathcal{C}^{\infty}$-ring.

Ad $(i i) \rightarrow(i)$. Suppose that $A$ is a $\mathcal{C}^{\infty}$-reduced $\mathcal{C}^{\infty}$-ring and that $\operatorname{Spec}^{\infty}(A)$ is a Boolean space.

Since $\operatorname{Spec}^{\infty}(A)$ is a Boolean space, it is a Hausdorff space and for every $a \in A, D^{\infty}(a)$ is compact, hence it is closed. From that we conclude that for every $a \in A, D^{\infty}(a)$ is a clopen set.

We conclude that $\operatorname{Spec}^{\infty}(A) \backslash D^{\infty}(a)$ is a clopen subset of $\operatorname{Spec}^{\infty}(A)$.

We claim that for every clopen $C$ in $\operatorname{Spec}^{\infty}(A)$ there is some $b \in A$ such that $C=D^{\infty}(b)$.

Since $C$ is clopen in $\operatorname{Spec}^{\infty}(A)$, it is in particular an open set, and since $\left\{D^{\infty}(a) \mid a \in A\right\}$ is a basis for the topology of $\operatorname{Spec}^{\infty}(A)$, there is a family $\left\{b_{i}\right\}_{i \in I}$ of elements of $A$ such that:

$$
C=\bigcup_{i \in I} D^{\infty}\left(b_{i}\right)
$$

Since $C$ is compact, there is a finite subset $I^{\prime} \subseteq I$ such that:

$$
C=\bigcup_{i \in I^{\prime}} D^{\infty}\left(b_{i}\right)
$$

By item (iii) of Lemma 1.4 of [67],

$$
\bigcup_{i \in I^{\prime}} D^{\infty}\left(b_{i}\right)=D^{\infty}\left(\sum_{i \in I^{\prime}} b_{i}{ }^{2}\right) .
$$

Hence, given a clopen set $C$, there is an element $b=\sum_{i \in I^{\prime}} b_{i}{ }^{2}$ such that $C=D^{\infty}(b)$.

Applying the above result to the clopen $\operatorname{Spec}^{\infty}(A) \backslash D^{\infty}(a)$, we obtain an element $d \in A$ such that:

$$
\operatorname{Spec}^{\infty}(A) \backslash D^{\infty}(a)=D^{\infty}(d) .
$$

We have:

$$
\varnothing=D^{\infty}(a) \cap D^{\infty}(d)=D^{\infty}(a \cdot d)=\left\{\mathfrak{p} \in \operatorname{Spec}^{\infty}(A) \mid a \cdot d \notin \mathfrak{p}\right\}
$$

so

$$
\left(\forall \mathfrak{p} \in \operatorname{Spec}^{\infty}(A)\right)(a \cdot d \in \mathfrak{p})
$$

hence

$$
a \cdot d \in \bigcap \operatorname{Spec}^{\infty}(A)=\sqrt[\infty]{(0)}=(0)
$$

where the last equality is due to the fact that $A$ is supposed to be a $\mathcal{C}^{\infty}$-reduced $\mathcal{C}^{\infty}$-ring.

We have, then,

$$
a \cdot d=0 .
$$

We have, also:

$$
D^{\infty}\left(a^{2}+d^{2}\right)=D^{\infty}(a) \cup D^{\infty}(b)=\operatorname{Spec}^{\infty}(A)=D^{\infty}(1) .
$$

By item (i) of Lemma 1.4 of [67], $D^{\infty}(1) \subseteq D^{\infty}\left(a^{2}+d^{2}\right)$ implies $1 \in\left\{a^{2}+d^{2}\right\}^{\infty-\text { sat }}$, which happens if, and only if, $a^{2}+d^{2} \in \sqrt[\infty]{(1)}$. 
Now, $a^{2}+d^{2} \in \sqrt[\infty]{(1)}$ occurs if, and only if, $\left(\exists c \in\{1\}^{\infty-\text { sat }}\right)$ and $(\exists \lambda \in A)$ such that:

$$
\lambda \cdot c \cdot\left(a^{2}+d^{2}\right) \in\langle 1\rangle=\{1\}
$$

Let $y=\lambda \cdot c$, and we get:

$$
\begin{gathered}
y \cdot\left(a^{2}+d^{2}\right)=1 \\
y a^{2}+y d^{2}=1
\end{gathered}
$$

Since $a \cdot d=0$, we get:

$$
\begin{aligned}
a\left(a^{2} y\right)+a\left(b^{2} y\right) & =a \cdot 1=a \\
a^{2}(a \cdot y) & =a
\end{aligned}
$$

and considering the forgetful functor:

$$
\widetilde{U}: \mathcal{C}^{\infty} \text { Rng } \rightarrow \text { CRing }
$$

Let $a \in \sqrt[\infty]{(0)}$, i.e., $a \in A$ is such that $\left(\frac{A}{(0)}\right)\left\{(a+(0))^{-1}\right\} \cong A\left\{a^{-1}\right\} \cong 0$. Since $A\left\{a^{-1}\right\} \cong \frac{A\{x\}}{(a x-1)}$, $A\left\{a^{-1}\right\} \cong 0$ implies

$$
1_{\frac{A\{x\}}{(a x-1)}}=0_{\frac{A\{x\}}{(a x-1)}},
$$

so

$$
1_{A\{x\}} \in(a x-1),
$$

which occurs if and only if, there exists some $h \in A\{x\} \cong A \otimes_{\infty} \mathcal{C}^{\infty}(\mathbb{R})$ such that $1=h(x) \cdot[\Psi(\jmath(a))$. $\left.\Psi\left(\jmath^{\prime}\left(\operatorname{id}_{\mathbb{R}}\right)\right)-1\right]=h(x) \cdot(a x-1)$, where $\jmath: A \rightarrow A\{x\}$ and $\jmath^{\prime}: \mathcal{C}^{\infty}(\mathbb{R}) \rightarrow A\{x\}$ are the coproduct morphisms and $\Psi: A \otimes_{\infty} \mathcal{C}^{\infty}(\mathbb{R}) \rightarrow A\{x\}$ is the isomorphism between them, as we see in the following diagram:

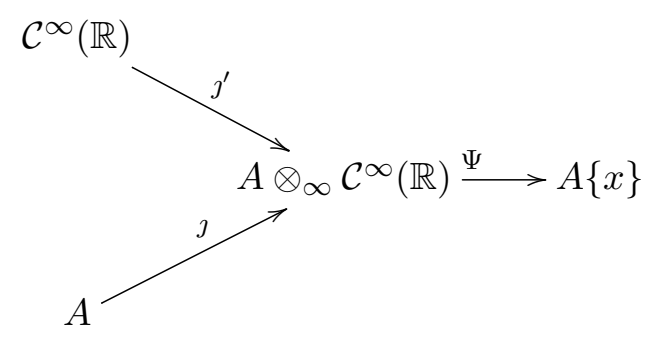

Under those circumstances we conclude that if $A$ is a reduced $\mathcal{C}^{\infty}$-ring, then there is $h \in A\{x\}$ such that $1=h(x) \cdot(a x-1)$, so $1 \in(a x-1)$ and

$$
\frac{1}{(a x-1)}^{(a\{x\}}=0 \frac{A\{x\}}{(a x-1)},
$$

or

$$
1_{A\left\{a^{-1}\right\}}=0_{A\left\{a^{-1}\right\}}
$$

so

$$
a \in \sqrt[\infty]{(0)}
$$

The following proposition will be useful to characterize the von Neumann-regular $\mathcal{C}^{\infty}$-rings by means of the ring of global sections of the structure sheaf of its affine scheme.

Proposition 2.2.76. If a $\mathcal{C}^{\infty}$-ring $A$ is a von-Neumann-regular $\mathcal{C}^{\infty}$-ring and $\mathfrak{p} \in \operatorname{Spec}^{\infty}(A)$, then $A\left\{A \backslash \mathfrak{p}^{-1}\right\}$ is a $\mathcal{C}^{\infty}$-field. 
Proof. We are going to show that the only maximal ideal of $A\left\{A \backslash \mathfrak{p}^{-1}\right\}, \mathfrak{m}_{\mathfrak{p}}$ is such that $\mathfrak{m}_{\mathfrak{p}} \cong\{0\}$.

Let $\eta_{\mathfrak{p}}: A \rightarrow A\left\{A \backslash \mathfrak{p}^{-1}\right\}$ be the localization morphism of $A$ with respect to $A \backslash \mathfrak{p}$. We have $\mathfrak{m}_{\mathfrak{p}}=\left\langle\eta_{\mathfrak{p}}[A \backslash \mathfrak{p}]\right\rangle=\left\{\frac{\eta_{\mathfrak{p}}(a)}{\eta_{\mathfrak{p}}(b)} \mid(a \in \mathfrak{p}) \wedge(b \in A \backslash \mathfrak{p})\right\}$. We must show that for every $a \in \mathfrak{p}, \eta_{\mathfrak{p}}(a)=0$, which is equivalent, by Theorem 1.4 of [67], to assert that for every $a \in \mathfrak{p}$ there is some $c \in(A \backslash \mathfrak{p})^{\infty-\text { sat }}=A \backslash \mathfrak{p}$ such that $c \cdot a=0$ in $A$.

Ab absurdo, suppose $\mathfrak{m}_{\mathfrak{p}} \neq\{0\}$, so there is $a \in \mathfrak{p}$ such that $\eta_{\mathfrak{p}}(a) \neq 0$, i.e., such that for every $c \in A \backslash \mathfrak{p}$, $c \cdot a \neq 0$. Since $A$ is a von Neumann-regular $\mathcal{C}^{\infty}$-ring, for this $a$ there is some idempotent $e \in \mathfrak{p}$ such that $(a)=(e)$.

Since $a \in(e)$, there is some $\lambda \in A$ such that $a=\lambda \cdot a$, hence:

$$
0 \neq \eta_{\mathfrak{p}}(a)=\eta_{\mathfrak{p}}(\lambda \cdot e)=\eta_{\mathfrak{p}}(\lambda) \cdot \eta_{\mathfrak{p}}(e)
$$

and $\eta_{\mathfrak{p}}(e) \neq 0$.

Since $\eta_{\mathfrak{p}}(e) \neq 0$,

$$
(\forall d \in A \backslash \mathfrak{p})(d \cdot e \neq 0) .
$$

Since $e$ is an idempotent element, $1-e \notin \mathfrak{p}$, for if $1-e \in \mathfrak{p}$ then $e+(1-e)=1 \in \mathfrak{p}$ and $\mathfrak{p}$ would not be a proper prime ideal.

We have also:

$$
e \cdot(1-e)=0
$$

The equation (2.8) contradicts (2.7), so $\mathfrak{m}_{\mathfrak{p}} \cong\{0\}$ and $A\left\{A \backslash \mathfrak{p}^{-1}\right\}$ is a $\mathcal{C}^{\infty}$-field.

Corollary 2.2.77. Let $A$ be a von Neumann-regular $\mathcal{C}^{\infty}-$ ring and $\mathfrak{p} \in \operatorname{Spec}^{\infty}(A)$. We have:

$$
\frac{A}{\mathfrak{p}} \cong A\left\{A \backslash \mathfrak{p}^{-1}\right\}
$$

Proof. Since $A$ is a von Neumann-regular $\mathcal{C}^{\infty}-\operatorname{ring}, \operatorname{Spec}^{\infty}(A)=\operatorname{Specm}^{\infty}(A)$ and for every $\mathfrak{p} \in \operatorname{Spec}^{\infty}(A)=$ $\operatorname{Specm}^{\infty}(A), \frac{A}{\mathfrak{p}}$ is a $\mathcal{C}^{\infty}$-field, so $\left(\frac{A}{\mathfrak{p}}\right)\left\{\left(\frac{A}{\mathfrak{p}} \backslash\{0+\mathfrak{p}\}\right)^{-1}\right\} \cong \frac{A}{\mathfrak{p}}$. Also, since $A\left\{A \backslash \mathfrak{p}^{-1}\right\}$ is a $\mathcal{C}^{\infty}$-field (by Proposition 2.2.76, the quotient map $q_{\mathfrak{m}_{\mathfrak{p}}}: A\left\{A \backslash \mathfrak{p}^{-1}\right\} \rightarrow \frac{A\left\{A \backslash \mathfrak{p}^{-1}\right\}}{\mathfrak{m}_{\mathfrak{p}}}$ is an isomorphism, so $A\left\{A \backslash \mathfrak{p}^{-1}\right\} \cong \frac{A\left\{A \backslash \mathfrak{p}^{-1}\right\}}{\mathfrak{m}_{\mathfrak{p}}}$.

$$
\begin{aligned}
& \text { By Theorem 2.2.53. }\left(\frac{A}{\mathfrak{p}}\right)\left\{\left(\frac{A}{\mathfrak{p}} \backslash\{0+\mathfrak{p}\}\right)^{-1}\right\} \cong \frac{A\left\{A \backslash \mathfrak{p}^{-1}\right\}}{\mathfrak{m}_{\mathfrak{p}}} \text {, hence: } \\
& A\left\{A \backslash \mathfrak{p}^{-1}\right\} \cong \frac{A\left\{A \backslash \mathfrak{p}^{-1}\right\}}{\mathfrak{m}_{\mathfrak{p}}} \cong\left(\frac{A}{\mathfrak{p}}\right)\left\{\left(\frac{A}{\mathfrak{p}} \backslash\{0+\mathfrak{p}\}\right)^{-1}\right\} \cong \frac{A}{\mathfrak{p}}
\end{aligned}
$$

Summarizing, we have the following result: 
Theorem 2.2.78. If $A$ is a von Neumann-regular $\mathcal{C}^{\infty}$-ring, then $\left(\operatorname{Spec}^{\infty}(A)\right.$, Zar $\left.{ }^{\infty}\right)$ (which we denote simply by $\left.\operatorname{Spcc}^{\infty}(A)\right)$ is a Boolean topological space, by Theorem 2.2.75. Moreover, by Proposition 2.2.76, for every $\mathfrak{p} \in \operatorname{Spec}^{\infty}(A)$,

$$
A_{\mathfrak{p}}=\underset{a \notin \mathfrak{p}}{\lim _{a \notin}} A\left\{a^{-1}\right\} \cong A\{A \backslash \mathfrak{p}\}
$$

is a $\mathcal{C}^{\infty}$-field.

The above theorem suggests us that von Neumann-regular $\mathcal{C}^{\infty}$-rings behave much like ordinary von Neumann-regular commutative unital rings. In the next chapter we are going to explore this result using sheaf theoretic machinery.

Proposition 2.2.79. The limit in $\mathcal{C}^{\infty} \mathbf{R n g}$ of a diagram of von Neumann-regular $\mathcal{C}^{\infty}$-rings is a von Neumann-regular $\mathcal{C}^{\infty}$-ring. In particular, $\mathcal{C}^{\infty} \mathbf{v N R n g}$ is a complete category and the inclusion functor $\imath: \mathcal{C}^{\infty} \mathbf{v N R n g} \hookrightarrow \mathcal{C}^{\infty} \mathbf{R n g}$ preserves all limits.

Proof. It is clear from the definition that the class $\mathcal{C}^{\infty} \mathbf{v N R n g}$ of von Neumann-regular $\mathcal{C}^{\infty}$-rings is closed under arbitrary products in the class $\mathcal{C}^{\infty} \mathbf{R n g}$ of all $\mathcal{C}^{\infty}$-rings. Thus it suffices to show that it is closed under equalizers. So let $A, B$ be von Neumann-regular $\mathcal{C}^{\infty}$-rings and $f, g: A \rightarrow B \mathcal{C}^{\infty}$-ring homomorphisms. Their equalizer in the category $\mathcal{C}^{\infty} \mathbf{R n g}$ is given by the set $E:=\{a \in A \mid f(a)=g(a)\}$, endowed with the restricted ring operations from $A$.

To see that $E$ is a von Neumann-regular $\mathcal{C}^{\infty}$-ring, we need to show that for $a \in E$, the unique element $b \in A$ satisfying $a^{2} b=a$ and $b^{2} a=b$ also belongs to $E$.

First we show that the idempotent element $a b$ belongs to $E$. Indeed, we have $f(a b)=f(a) f(b)=$ $g(a) f(b)=g\left(a^{2} b\right) f(b)=g(a) g(a b) f(b)=f(a) g(a b) f(b)=f(a b) g(a b)$. Exchanging $f$ and $g$ in this chain of equations, we also get $g(a b)=f(a b) g(a b)$. Altogether we obtain $g(a b)=f(a b)$, and hence $a b \in E$.

Now we use this, as well as the fact that we also have the equation $b=a b^{2}$, and conclude $f(b)=$ $f\left(a b^{2}\right)=f(b) f(a b)=f(b) g(a b)=f(b) g(a) g(b)=f(b) f(a) g(b)=f(a b) g(b)=g(a b) g(b)=g\left(a b^{2}\right)=$ $g(b)$.

Proposition 2.2.80. The category $\mathcal{C}^{\infty} \mathbf{v N R n g}$ is the smallest subcategory of $\mathcal{C}^{\infty} \mathbf{R n g}$ closed under limits containing all $\mathcal{C}^{\infty}$-fields.

Proof. Clearly all $\mathcal{C}^{\infty}$-fields are von Neumann-regular $\mathcal{C}^{\infty}$-rings, and by Proposition 2.2 .79 so are limits of $\mathcal{C}^{\infty}$-fields. Thus $\mathcal{C}^{\infty} \mathbf{v N R n g}$ contains all limits of $\mathcal{C}^{\infty}$-fields. On the other hand the ring of global sections of a sheaf can be expressed as a limit of a diagram of products and ultraproducts of the stalks (by Lemma 2.5 of $\left[99 \mid\right.$ ). All these occurring (ultra)products are von Neumann-regular $\mathcal{C}^{\infty}$-rings as well and hence so is their limit, by Proposition 2.2.79.

\subsection{Order Theory of $\mathcal{C}^{\infty}$-rings}

The class of $\mathcal{C}^{\infty}$-rings carries good notions of order theory for rings. As pointed out by Moerdijk and Reyes in [67], every $\mathcal{C}^{\infty}$-ring $A$ has a canonical pre-order. We describe it in the following:

Definition 2.3.1. Given a $\mathcal{C}^{\infty}-$ ring $(A, \Phi)$, we write:

$$
(\forall a \in A)(\forall b \in A)\left(a \prec b \Longleftrightarrow\left(\exists c \in A^{\times}\right)\left(b-a=c^{2}\right)\right)
$$

Note that if $A \neq\{0\}, \prec$ is an irreflexive relation, i.e., $(\forall a \in A)(\neg(a \prec a))$. 
According to [66], we have the following facts about the order $\prec$ defined above.

Fact 2.3.2. Let $A=\frac{\mathcal{C}^{\infty}\left(\mathbb{R}^{E}\right)}{I}$ for some set $E$. Then, given any $f+I, g+I \in A$, with respect to the relation $\prec$, given in Definition 2.3.1, we have:

$$
f \prec g \Longleftrightarrow(\exists \varphi \in I)((\forall x \in Z(\varphi))(f(x)<g(x)))
$$

so $\prec$ is compatible with the ring structure which underlies $A$, i.e.:

(i) $0 \prec f+I, g+I \Rightarrow 0 \prec(f+I) \cdot(g+I)$;

(ii) $0 \prec f+I, g+I \Rightarrow 0 \prec f+g+I$

Fact 2.3.3. Let $A=\frac{\mathcal{C}^{\infty}\left(\mathbb{R}^{E}\right)}{I}$ for some set $E$ be a $\mathcal{C}^{\infty}$-field, so $I=\sqrt[\infty]{I}$. The relation $\prec$, given in Definition 2.3.1, is such that:

$$
(\forall f+I \in A)(f+I \neq 0+I \rightarrow(f+I \prec 0) \vee(0 \prec f+I))
$$

We have the following:

Proposition 2.3.4. For any $\mathcal{C}^{\infty}$-ring $A$, we have:

$$
1+\sum A^{2} \subseteq A^{\times}
$$

where $\sum A^{2}=\left\{\sum_{i=1}^{n} a_{i}^{2} \mid n \in \mathbb{N}, a_{i} \in A\right\}$. In particular, every $\mathcal{C}^{\infty}$ ring $A$ is such that its underlying commutative unital ring, $\widetilde{U}(A)$ is a semi-real ring.

Proof. First suppose $A=\mathcal{C}^{\infty}\left(\mathbb{R}^{n}\right)$ for some $n \in \mathbb{N}$. Given any $k \in \mathbb{N}$ and any $k$-tuple, $f_{1}, \cdots, f_{k} \in$ $\mathcal{C}^{\infty}\left(\mathbb{R}^{n}\right)$, we have $Z\left(1+\sum_{i=1}^{k} f_{i}^{2}\right)=\varnothing$, so $1+\sum_{i=1}^{k} f_{i}^{2} \in \mathcal{C}^{\infty}\left(\mathbb{R}^{n}\right)^{\times}$.

Suppose, now, that $A$ is a finitely generated $\mathcal{C}^{\infty}$-ring, that is, that there are $n \in \mathbb{N}$ and an ideal $I \subseteq \mathcal{C}^{\infty}\left(\mathbb{R}^{n}\right)$ such that:

$$
A=\frac{\mathcal{C}^{\infty}\left(\mathbb{R}^{n}\right)}{I}
$$

We have, for every $k \in \mathbb{N}$ and for every $k$-tuple $f_{1}+I, \cdots, f_{k}+I \in \frac{\mathcal{C}^{\infty}\left(\mathbb{R}^{n}\right)}{I}$ :

$$
(1+I)+\sum_{i=1}^{k}\left(f_{i}+I\right)^{2}=\left(1+\sum_{i=1}^{k} f_{i}^{2}\right)+I
$$

Since $1+\sum_{i=1}^{k} f_{i}^{2} \in \mathcal{C}^{\infty}\left(\mathbb{R}^{n}\right)^{\times}$, it follows that $\left(1+\sum_{i=1}^{k} f_{i}^{2}\right)+I \in\left(\frac{\mathcal{C}^{\infty}\left(\mathbb{R}^{n}\right)}{I}\right)^{\times}$.

Finally, for an arbitrary $\mathcal{C}^{\infty}$-ring $A$, we can always write:

$$
A=\underset{i \in I}{\lim _{i \in I}} A_{i}
$$

for the directed family $\left\{A_{i} \mid i \in I\right\}$ consisting of its finitely generated $\mathcal{C}^{\infty}$-subrings. We are going to denote the canonical colimit arrow by $\alpha_{i}: A_{i} \rightarrow \underline{\lim }_{i \in I} A_{i}$, for every $i \in I$. 
For every $k \in \mathbb{N}$ and for every $k$-tuple, $\left[a_{i_{1}}\right], \cdots,\left[a_{i_{k}}\right] \in \lim _{i \in I} A_{i}$, there is some $\ell \geq i_{1}, \cdots, i_{k}$ such that for every $j \in\{1, \cdots, k\}\left[a_{i_{j}}\right]=\left[\alpha_{\ell}\left(\alpha_{i_{j} \ell}\left(a_{i_{j}}\right)\right)\right]$. Since $\overrightarrow{A_{\ell}}$ is finitely generated, it follows that $1+\sum_{j=1}^{k} \alpha_{i_{j} \ell}\left(a_{i_{j}}\right)^{2} \in A_{\ell}^{\times}$, so

$$
1+\sum_{j=1}^{k}\left[a_{i_{j}}\right]^{2}=\alpha_{\ell}\left(1+\sum_{j=1}^{k} \alpha_{i_{j} \ell}\left(a_{i_{j}}\right)^{2}\right) \in\left(\lim _{i \in I} A_{i}\right)^{\times} .
$$

Fact 2.3.5. Let $n \in \mathbb{N}$. We have:

$$
h+I \in\left(\frac{\mathcal{C}^{\infty}\left(\mathbb{R}^{n}\right)}{I}\right)^{\times} \Longleftrightarrow(\exists \varphi \in I)(\forall x \in Z(\varphi))(h(x) \neq 0)
$$

Recall that a totally ordered field $(F, \leq)$ is real closed if it satisfies:

(a) $(\forall x \in F)\left(0<x \rightarrow(\exists y \in F)\left(x=y^{2}\right)\right)$;

(b) every polynomial of odd degree has, at least, one root;

Remark 2.3.6. A $\mathcal{C}^{\infty}$-polynomial in one variable is an element of $\mathcal{C}^{\infty}\left(\mathbb{R}^{n}\right)\{t\}$. More generally, a $\mathcal{C}^{\infty}$-polynomial in set $S$ of variables is an element of $A\{S\}$.

As pointed out in Theorem $\mathbf{2 . 1 0}$ of [66], we have the following:

Fact 2.3.7. Every $\mathcal{C}^{\infty}$-field, $F$, together with its canonical preorder $\prec$ given in Definition 2.3.1, is such that $\widetilde{U}(F)$ is a real closed field.

As a consequence of the above fact, given any polynomial $p \in F[x]$ and any $f, g \in F$ such that $f \prec g$, if $p(f)<p(g)$ then there is some $h \in] f, g[$ such that $p(h)=0$.

We have the $\mathcal{C}^{\infty}$-analog of the notion of "real closedness":

Definition 2.3.8. Let $(F, \prec)$ be a $\mathcal{C}^{\infty}$-field. We say that $(F, \prec)$ is $\mathcal{C}^{\infty}$-real closed if, and only if:

$$
(\forall f \in F\{x\})\left((f(0) \cdot f(1)<0) \&\left(1 \in\left\langle\left\{f, f^{\prime}\right\}\right\rangle \subseteq F\{x\}\right) \rightarrow(\exists \alpha \in] 0,1[\subseteq F)(f(\alpha)=0)\right)
$$

Fact 2.3.9. As proved in Theorem 2.10' of [66], every $\mathcal{C}^{\infty}$-field is $\mathcal{C}^{\infty}$-real closed.

From the preceding proposition, we conclude that $\bar{f} \prec \bar{g}$ occurs if, and only if, there is a "witness" $\varphi \in I$ such that $(\forall x \in Z(\varphi))(f(x)<g(x))$.

Given any $\mathcal{C}^{\infty}$-ring $A$ and any $\mathfrak{p} \in \operatorname{Spec}^{\infty}(A)$, let:

$$
k_{\mathfrak{p}}:=\left(\frac{A}{\mathfrak{p}}\right)\left\{\frac{A}{\mathfrak{p}} \backslash\{0+\mathfrak{p}\}^{-1}\right\}
$$

that is, $k_{\mathfrak{p}}(A)$ is the $\mathcal{C}^{\infty}$-field obtained by taking the quotient $\frac{A}{\mathfrak{p}}$ :

$$
q_{\mathfrak{p}}: A \rightarrow \frac{A}{\mathfrak{p}}
$$

and then taking its $\mathcal{C}^{\infty}$-ring of fractions with respect to $\frac{A}{\mathfrak{p}} \backslash\{0+\mathfrak{p}\}$, 


$$
\eta_{\frac{A}{\mathfrak{p}} \backslash\{0+\mathfrak{p}\}}: \frac{A}{\mathfrak{p}} \rightarrow\left(\frac{A}{\mathfrak{p}}\right)\left\{\frac{A}{\mathfrak{p}} \backslash\{0+\mathfrak{p}\}^{-1}\right\} .
$$

The family of $\mathcal{C}^{\infty}$-fields $\left\{k_{\mathfrak{p}}(A) \mid \mathfrak{p} \in \operatorname{Spec}^{\infty}(A)\right\}$ has the following multi-universal property:

"Given any $\mathcal{C}^{\infty}$-homomorphism $f: A \rightarrow \mathbb{K}$, where $\mathbb{K}$ is a $\mathcal{C}^{\infty}$-field, there is a unique $\mathcal{C}^{\infty}$-radical prime ideal $\mathfrak{p}$ and a unique $\mathcal{C}^{\infty}$-homomorphism $\widetilde{f}: k_{\mathfrak{p}}(A) \rightarrow \mathbb{K}$ such that the following diagram commutes:

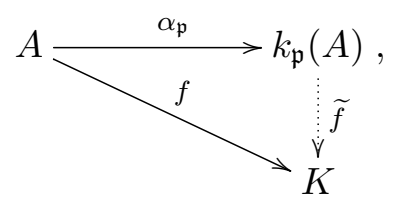

where $\alpha_{\mathfrak{p}}=\eta_{\frac{A}{\mathfrak{p}} \backslash\{0+\mathfrak{p}\}} \circ q_{\mathfrak{p}}: A \rightarrow k_{\mathfrak{p}}(A) . "$

Thus, given $f: A \rightarrow K$, take $\mathfrak{p}=\operatorname{ker}(f)$, so $\bar{f}$ is injective, $\frac{A}{\mathfrak{p}}$ is a $\mathcal{C}^{\infty}$-reduced $\mathcal{C}^{\infty}$-ring. By the universal property of the smooth fraction field $k_{\mathfrak{p}}(A)$, there is a unique arrow $\tilde{f}: k_{\mathfrak{p}}(A) \rightarrow \mathbb{K}$ such that the following diagram commutes:

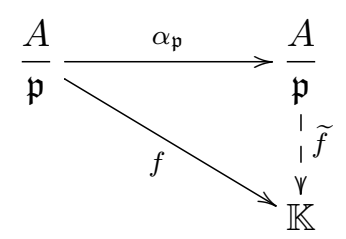

Definition 2.3.10. Let $\mathcal{F}$ be the (proper) class of all the $\mathcal{C}^{\infty}$-homomorphisms of $A$ to some $\mathcal{C}^{\infty}$-field. We define the following relation $\mathcal{R}:$ given $h_{1}: A \rightarrow F_{1}$ and $h_{2}: A \rightarrow F_{2}$, we say that $h_{1}$ is related with $h_{2}$ if, and only if, there is some $\mathcal{C}^{\infty}$-field $\widetilde{F}$ and some $\mathcal{C}^{\infty}$-fields homomorphisms $\mathcal{C}^{\infty} f_{1}: F_{1} \rightarrow \widetilde{F}$ and $f_{2}: F_{2} \rightarrow \widetilde{F}$ such that the following diagram commutes:

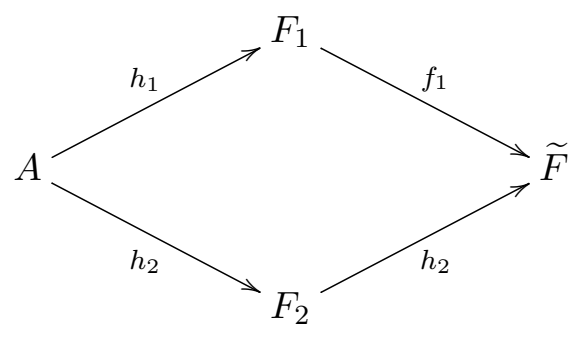

The relation $\mathcal{R}$ defined above is symmetric and reflexive.

Let $h_{1}: A \rightarrow F_{1}$ and $h_{2}: A \rightarrow F_{2}$ be two $\mathcal{C}^{\infty}$-homomorphisms of $A$ to the $\mathcal{C}^{\infty}$-fields $F_{1}, F_{2}$ such that $\left(h_{1}, h_{2}\right) \in \mathcal{R}$, and let $f_{1}: F_{1} \rightarrow \widetilde{F}$ and $f_{2}: F_{2} \rightarrow \widetilde{F}$ be two $\mathcal{C}^{\infty}$-homomorphisms to the $\mathcal{C}^{\infty}$-field $\widetilde{F}$ such that $f_{1} \circ h_{1}=f_{2} \circ h_{2}$, so:

$$
\left(f_{1} \circ h_{1}\right)^{-1}[\{0\}]=\left(f_{2} \circ h_{2}\right)^{-1}[\{0\}]
$$

Then

$$
\operatorname{ker}\left(h_{1}\right)=h_{1}^{\dashv}[\{0\}]=h_{1}^{\dashv}\left[f_{1}^{-1}[\{0\}]\right]=h_{2}^{\dashv}\left[f_{2}^{\dashv}[\{0\}]\right]=h_{2}^{\dashv}[\{0\}]=\operatorname{ker}\left(h_{2}\right) .
$$

The above considerations prove the following:

Proposition 2.3.11. If $h_{1}: A \rightarrow F_{1}$ and $h_{2}: A \rightarrow F_{2}$ be two $\mathcal{C}^{\infty}$-homomorphisms from the $\mathcal{C}^{\infty}$-ring $A$ to the $\mathcal{C}^{\infty}$-fields $F_{1}, F_{2}$ such that $\left(h_{1}, h_{2}\right) \in \mathcal{R}$, then $\operatorname{ker}\left(h_{1}\right)=\operatorname{ker}\left(h_{2}\right)$. 
The above proposition has the following immediate consequence:

Corollary 2.3.12. Keeping the same notations of the above result, let $\mathcal{R}^{t}$ be the transitive closure of $\mathcal{R}$. Then $\left(h_{1}, h_{2}\right) \in \mathcal{R}^{t}$ implies $\operatorname{ker}\left(h_{1}\right)=\operatorname{ker}\left(h_{2}\right)$. Thus, $\mathcal{R}^{t}$ is an equivalence relation on $\mathcal{F}$. We are going to denote the quotient set $\frac{\mathcal{F}}{\mathcal{R}^{t}}$ by $\widetilde{\mathcal{F}}$.

Let $F_{1}, F_{2}, \widetilde{F}$ be $\mathcal{C}^{\infty}$-fields and $f_{1}: F_{1} \rightarrow \widetilde{F}$ and $f_{2}: F_{2} \rightarrow \widetilde{F}$ be two following $\mathcal{C}^{\infty}$-fields homomorphisms. Since $\mathcal{C}^{\infty}$-fields homomorphisms must be injective maps, we have the following:

Proposition 2.3.13. The following relation

$$
\beta=\left\{([h: A \rightarrow F], \operatorname{ker}(h)) \mid F \text { is aC } \mathcal{C}^{\infty}-\text { field }\right\} \subseteq \widetilde{\mathcal{F}} \times \operatorname{Spec}^{\infty}(A)
$$

is a functional relation whose domain is $\widetilde{\mathcal{F}}$.

Proof. Suppose $\left[h: A \rightarrow F_{1}\right]=\left[g: A \rightarrow F_{2}\right]$, so there are maps $f_{1}: F_{1} \rightarrow \widetilde{F}$ and $f_{2}: F_{2} \rightarrow \widetilde{F}$ for some $\mathcal{C}^{\infty}$-field $\widetilde{F}$, such that the following diagram commutes:

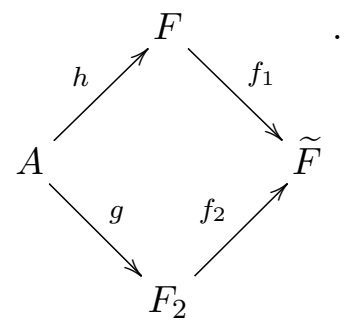

Now, if $\left(\left[h: A \rightarrow F_{1}\right], \operatorname{ker}(h)\right),\left(\left[g: A \rightarrow F_{2}\right], \operatorname{ker}(g)\right) \in \beta$ are such that $\left[h: A \rightarrow F_{1}\right]=\left[g: A \rightarrow F_{2}\right]$, then:

$$
f_{1} \circ h=f_{2} \circ g
$$

so

$$
\operatorname{ker}(h)=h^{\dashv}[\{0\}]=h^{\dashv-}\left[f_{1}^{\dashv}[\{0\}]\right]=\operatorname{ker}\left(f_{1} \circ h\right)=\operatorname{ker}\left(f_{2} \circ g\right)=g^{\dashv}\left[f_{2}^{\dashv}[\{0\}]\right]=g^{-1}[\{0\}]=\operatorname{ker}(g)
$$

Definition 2.3.14. Let $A$ be a $\mathcal{C}^{\infty}$-ring. $A \mathcal{C}^{\infty}$-ordering in $A$ is a subset $P \subseteq A$ such that:

(O1) $P+P \subseteq P$

(O2) $P \cdot P \subseteq P$;

(O3) $P \cup(-P)=A$

(O4) $P \cap(-P)=\mathfrak{p} \in \operatorname{Spec}^{\infty}(A)$

Definition 2.3.15. Let $A$ be a $\mathcal{C}^{\infty}$-ring. Given a $\mathcal{C}^{\infty}$-ordering $P$ in $A$, the $\mathcal{C}^{\infty}$-support of $A$ is given by:

$$
\operatorname{supp}^{\infty}(P):=P \cap(-P)
$$

Definition 2.3.16. Let $A$ be a $\mathcal{C}^{\infty}$-ring. The $\mathcal{C}^{\infty}$-real spectrum of $A$ is given by:

$$
\operatorname{Sper}^{\infty}(A)=\{P \subset A \mid P \text { is an ordering of the elements of } A\}
$$


together with the (spectral) topology generated by the sets:

$$
H^{\infty}(a)=\left\{P \in \operatorname{Sper}^{\infty}(A) \mid a \in P \backslash \operatorname{supp}^{\infty}(P)\right\}
$$

for every $a \in A$. The topology generated by these sets will be called "smooth Harrison topology", and will be denoted by $\mathrm{Har}^{\infty}$.

Remark 2.3.17. Given a $\mathcal{C}^{\infty}$-ring $A$, we have a function given by:

$$
\begin{array}{ccc}
\operatorname{supp}^{\infty}:\left(\operatorname{Sper}^{\infty}(A), \operatorname{Har}^{\infty}\right) & \rightarrow & \left(\operatorname{Spec}^{\infty}(A), \operatorname{Zar}^{\infty}\right) \\
P & \mapsto & P \cap(-P)
\end{array}
$$

which is spectral, and thus continuous, since given any $a \in A, \operatorname{supp}^{\infty-1}\left[D^{\infty}(a)\right]=H^{\infty}(a) \cup H^{\infty}(-a)$.

Contrary to what happens to a general commutative ring $R$, for which the mapping:

$$
\begin{aligned}
& \text { supp : } \operatorname{Sper}(R) \rightarrow \operatorname{Spec}(R) \\
& P \quad \mapsto P \cap(-P)
\end{aligned}
$$

is seldom surjective or injective, within the category of $\mathcal{C}^{\infty}$-rings supp ${ }^{\infty}$ is, as matter of fact, a bijection.

In order to prove this fact, we are going to need some preliminary results, given below.

Lemma 2.3.18. Let $A$ be a $\mathcal{C}^{\infty}$-ring and $\mathfrak{p}$ any $\mathcal{C}^{\infty}$-radical prime ideal, and let $\hat{\mathfrak{p}}$ be the maximal ideal of $A_{\{\mathfrak{p}\}}$. Then:

$$
\operatorname{Can}_{\mathfrak{p}}^{-1}[\hat{\mathfrak{p}}]=\mathfrak{p} .
$$

Proof. Let $a \in \mathfrak{p}$, then $\operatorname{Can}_{\mathfrak{p}}(a) \in \hat{\mathfrak{p}}$, and $\mathfrak{p} \subseteq \operatorname{Can}_{\mathfrak{p}}^{-1}[\hat{\mathfrak{p}}]$. Now, if $a \in A \backslash \mathfrak{p}$ then $\operatorname{Can}_{\mathfrak{p}}(a) \in \mathcal{U}\left(A\left\{(A \backslash \mathfrak{p})^{-1}\right\}\right)$. Since $A_{\{\mathfrak{p}\}}$ is a local ring, $A_{\{\mathfrak{p}\}}=\hat{\mathfrak{p}} \cup \mathcal{U}\left(A\left\{(A \backslash \mathfrak{p})^{-1}\right\}\right)$, so $\operatorname{Can}_{\mathfrak{p}}(a) \in A\left\{(A \backslash \mathfrak{p})^{-1}\right\} \backslash \hat{\mathfrak{p}}$, and therefore $\operatorname{Can}_{\mathfrak{p}}^{-1}[\hat{\mathfrak{p}}] \subseteq \mathfrak{p}$.

Theorem 2.3.19. Let $A$ be a $\mathcal{C}^{\infty}$-ring and $\mathcal{R}^{t}$ be the relation defined above. The function:

$$
\begin{array}{ccc}
\alpha^{\prime}: \operatorname{Sper}^{\infty}(A) & \rightarrow & \frac{\mathcal{F}}{\mathcal{R}^{t}} \\
P & \mapsto & {\left[\eta_{P \cap(-P)}\right]}
\end{array}
$$

is the inverse function of:

$$
\beta^{\prime}: \begin{array}{ccc}
\frac{\mathcal{F}}{\mathcal{R}^{t}} & \rightarrow & \operatorname{Sper}^{\infty}(A) \\
{[h: A \rightarrow K]} & \mapsto & h^{\dashv}\left[K^{2}\right]
\end{array}
$$

Proof. Note that:

$$
\left(\alpha^{\prime} \circ \beta^{\prime}\right)([h: A \rightarrow F])=\alpha^{\prime}\left(h^{\dashv}\left[F^{2}\right]\right)=\left[\eta_{\operatorname{supp}\left(h^{\dashv}\left[F^{2}\right]\right)}\right],
$$

where $\operatorname{supp}^{\infty}\left(h^{\dashv}\left[F^{2}\right]\right)=h^{\dashv}\left[F^{2}\right] \cap\left(-h^{\dashv}[F]^{2}\right)=h^{\dashv}[\{0\}]=\operatorname{ker}(h)$.

Thus we have:

$$
\left(\alpha^{\prime} \circ \beta^{\prime}\right)([h: A \rightarrow F])=\left[\eta_{\operatorname{ker}(h)}: A \rightarrow k_{\operatorname{ker}(h)}(A)\right] .
$$

We claim that $\beta^{\prime}$ is the left inverse function for $\alpha^{\prime}$, that is:

$$
\left(\forall P \in \operatorname{Sper}^{\infty}(A)\right)\left(\left(\beta^{\prime} \circ \alpha^{\prime}\right)(P)=P\right) .
$$


Thus, it will follow that $\alpha^{\prime}$ is injective and $\beta^{\prime}$ is surjective.

We have $\beta^{\prime}\left(\alpha^{\prime}(P)\right)=\eta_{\text {supp } \infty(P)}^{\dashv}\left[k_{\mathfrak{p}}(A)^{2}\right]$, so we need to show that:

$$
\eta_{\text {supp }(P)}^{\dashv}\left[k_{\mathfrak{p}}(A)^{2}\right]=P
$$

Let $\mathfrak{p}=\operatorname{supp}^{\infty}(P)$.

Ab absurdo, suppose

$$
\eta_{\mathfrak{p}}^{-1}\left[k_{\mathfrak{p}}(A)^{2}\right] \nsubseteq P
$$

There must exist some $x \in A$ such that $x \in \eta_{\mathfrak{p}}^{\dashv}\left[k_{\mathfrak{p}}(A)^{2}\right]$ and $x \notin P$. We have:

$$
\eta_{\mathfrak{p}}(x) \in\left(\frac{A\left\{A \backslash \mathfrak{p}^{-1}\right\}}{\widehat{\mathfrak{p}}}\right)^{2} \text { and } x \notin P .
$$

Now, since by Theorem 2.2 .53 (denoting $\mathfrak{m}_{\mathfrak{p}}$ by $\widehat{\mathfrak{p}}$ instead) the following diagram commutes:

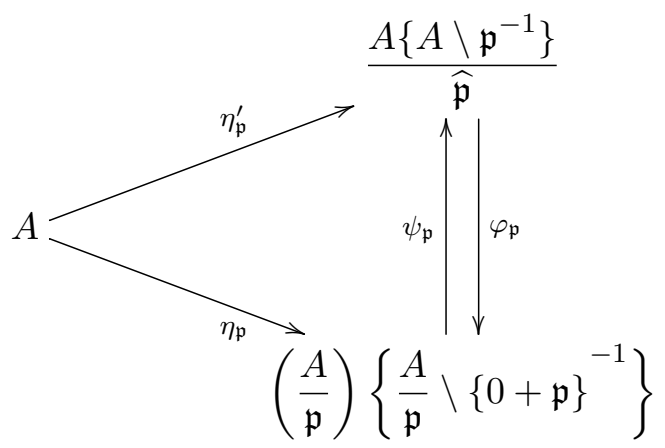

where $\eta_{\mathfrak{p}}=\eta_{\overline{\mathfrak{p}} \backslash\{0+\mathfrak{p}\}} \circ q_{\mathfrak{p}}$ and $\eta_{\mathfrak{p}}^{\prime}=q_{\widehat{p}} \circ \eta_{A \backslash \mathfrak{p}}$ and $\varphi_{\mathfrak{p}}$ and $\psi_{\mathfrak{p}}$ are the isomorphisms described in that theorem. Thus, we have:

$$
\eta_{\mathfrak{p}}(x) \in\left(k_{\mathfrak{p}}(A)\right)^{2} \Rightarrow \psi_{\mathfrak{p}}\left(\eta_{\mathfrak{p}}(x)\right)=\eta_{\mathfrak{p}}^{\prime}(x) \in\left(\frac{A\left\{A \backslash \mathfrak{p}^{-1}\right\}}{\widehat{\mathfrak{p}}}\right)^{2}
$$

and

$$
\eta_{\mathfrak{p}}(x) \in\left(k_{\mathfrak{p}}(A)\right)^{2} \Rightarrow\left(\exists(g+\widehat{\mathfrak{p}}) \in \frac{A\left\{A \backslash \mathfrak{p}^{-1}\right\}}{\widehat{\mathfrak{p}}}\right)\left(\eta_{\mathfrak{p}}^{\prime}(x)=g^{2}+\widehat{\mathfrak{p}}\right)
$$

Since $q_{\widehat{p}}$ is surjective, given this $g+\widehat{\mathfrak{p}} \in\left(\frac{A\left\{A \backslash \mathfrak{p}^{-1}\right\}}{\mathfrak{p}^{\prime}}\right)$, there is some $\theta \in A\left\{A \backslash \mathfrak{p}^{-1}\right\}$ such that $q_{\widehat{p}}(\theta)=g+\widehat{\mathfrak{p}}$.

By Theorem 1.4, item (i) of 66, given this $\theta \in A\left\{A \backslash \mathfrak{p}^{-1}\right\}$, there are $a \in A$ and $b \in A \backslash \mathfrak{p}^{\infty-\text { sat }}$, that is,

$$
\operatorname{Can}_{\mathfrak{p}}(b) \in\left(A\left\{A \backslash \mathfrak{p}^{-1}\right\}\right)^{\times}
$$

such that:

$$
\theta=\frac{\operatorname{Can}_{\mathfrak{p}}(a)}{\operatorname{Can}_{\mathfrak{p}}(b)}
$$


or equivalently, since $(A \backslash \mathfrak{p})^{\infty-\text { sat }}=A \backslash \mathfrak{p}$ :

$$
b \notin \mathfrak{p}
$$

Hence,

$$
\begin{gathered}
\eta_{\mathfrak{p}}^{\prime}(x)=g^{2}+\widehat{\mathfrak{p}}=q_{\widehat{\mathfrak{p}}}\left(\frac{\operatorname{Can}_{\mathfrak{p}}(a)}{\operatorname{Can}_{\mathfrak{p}}(b)}\right)^{2}=\left(\frac{\operatorname{Can}_{\mathfrak{p}}(a)}{\operatorname{Can}_{\mathfrak{p}}(b)}\right)^{2}+\widehat{\mathfrak{p}} \\
\eta_{\mathfrak{p}}^{\prime}(x) \cdot\left(\operatorname{Can}_{\mathfrak{p}}^{2}(b)+\widehat{\mathfrak{p}}\right)=\operatorname{Can}_{\mathfrak{p}}^{2}(a)+\widehat{\mathfrak{p}} \\
\operatorname{Can}_{\mathfrak{p}}\left(x \cdot b^{2}-a^{2}\right) \in \widehat{\mathfrak{p}} . \\
\left.\left(x \cdot b^{2}-a^{2}\right) \in \operatorname{Can}_{\mathfrak{p}}^{-1} \widehat{\mathfrak{p}}\right] .
\end{gathered}
$$

By Lemma 2.3.18, $\mathfrak{p}=\operatorname{Can}_{\mathfrak{p}}^{-1}[\widehat{\mathfrak{p}}]$, so

$$
x \cdot b^{2}-a^{2} \in \mathfrak{p} \subseteq P
$$

Let $y=x \cdot b^{2}+\left(-a^{2}\right) \in \mathfrak{p} \subseteq P$. Note that since $x \notin P, x \in(-P) \backslash \mathfrak{p}$ and

$$
x \cdot b^{2} \in(-P)
$$

Since $y \in P$,

$$
\begin{gathered}
x \cdot b^{2}=\underbrace{y}_{\in P}+\overbrace{a^{2}}^{\in P} \in P, \\
x \cdot b^{2} \in P
\end{gathered}
$$

By (2.10) and (2.11), it follows that $x \cdot b^{2} \in P \cap(-P)=\mathfrak{p}$. Since $\mathfrak{p}$ is prime, either $x \in \mathfrak{p}$ or $b^{2} \in \mathfrak{p}$. However, since $x \notin P$, a fortiori, $x \notin \mathfrak{p}$, so we must have $b^{2} \in \mathfrak{p}$. Once again, since $\mathfrak{p}$ is prime, it follows that $b \in \mathfrak{p}$ which contradicts $(2.9)$. Hence,

$$
\eta_{\text {supp }^{\infty}(P)}^{\prime}\left[\left(\frac{A\left\{A \backslash \operatorname{supp}^{\infty}(P)^{-1}\right\}}{\widehat{\mathfrak{p}}}\right)^{2}\right] \subseteq P
$$

Now we claim that:

$$
P \subseteq \eta_{\operatorname{supp}(P)}^{\prime} \dashv\left[\left(\frac{A\left\{A \backslash \operatorname{supp}^{\infty}(P)^{-1}\right\}}{\widehat{\mathfrak{p}}}\right)^{2}\right]
$$

Conversely, suppose, ab absurdo that

$$
P \nsubseteq \eta_{\text {supp }(P)}^{\prime}{ }^{\dashv}\left[\left(\frac{A\left\{A \backslash \operatorname{supp}^{\infty}(P)^{-1}\right\}}{\widehat{\mathfrak{p}}}\right)^{2}\right]
$$

so there must exist some $x \in P$ such that 


$$
\left(\forall(g+\widehat{\mathfrak{p}}) \in \frac{A\left\{A \backslash \mathfrak{p}^{-1}\right\}}{\widehat{\mathfrak{p}}}\right)\left(\eta_{\mathfrak{p}}^{\prime}(x) \neq g^{2}+\widehat{\mathfrak{p}}\right)
$$

Equivalently, there must exist some $(h+\widehat{\mathfrak{p}}) \in \frac{A\left\{A \backslash \mathfrak{p}^{-1}\right\}}{\tilde{\mathfrak{p}}}$ such that $\eta_{\mathfrak{p}}(x)=-h^{2}+\widehat{\mathfrak{p}}$.

Thus, since $q_{\mathfrak{p}}$ is surjective, given such an $h+\widehat{\mathfrak{p}} \in \frac{A\left\{A \backslash \mathfrak{p}^{-1}\right\}}{\widehat{\mathfrak{p}}}$ there must be some $\zeta \in A\left\{A \backslash \mathfrak{p}^{-1}\right\}$ such that $q_{\mathfrak{p}}(\zeta)=h+\widehat{\mathfrak{p}}$. By item (i) of Theorem 2.1.25, there are $c \in A$ and $d \in A$ with:

$$
\operatorname{Can}_{\mathfrak{p}}(d) \in A\left\{A \backslash \mathfrak{p}^{-1}\right\}^{\times},
$$

equivalently

$$
d \in(A \backslash \mathfrak{p})^{\infty-\text { sat }},
$$

and since $(A \backslash \mathfrak{p})^{\infty-\text { sat }}=A \backslash \mathfrak{p}$,

$$
d \notin \mathfrak{p}
$$

such that:

$$
\zeta=\frac{\operatorname{Can}_{\mathfrak{p}}(c)}{\operatorname{Can}_{\mathfrak{p}}(d)}
$$

Hence,

$$
\begin{gathered}
\eta_{\mathfrak{p}}(x)=-\frac{\operatorname{Can}_{\mathfrak{p}}^{2}(c)}{\operatorname{Can}_{\mathfrak{p}}^{2}(d)}+\widehat{\mathfrak{p}} \\
\operatorname{Can}_{\mathfrak{p}}^{2}(c)+\operatorname{Can}_{\mathfrak{p}}(x) \cdot \operatorname{Can}_{\mathfrak{p}}^{2}(d) \in \widehat{\mathfrak{p}} \\
\operatorname{Can}_{\mathfrak{p}}\left(c^{2}+x \cdot d^{2}\right) \in \widehat{\mathfrak{p}}
\end{gathered}
$$

so

$$
\begin{gathered}
\underbrace{c^{2}}_{\in P}+\overbrace{x \cdot d^{2}}^{\in P}=z=\operatorname{Can}_{\mathfrak{p}}^{-1}[\widehat{\mathfrak{p}}]=\mathfrak{p} \subseteq(-P) \\
x \cdot d^{2}=z-c^{2} \in(-P)
\end{gathered}
$$

Since $x \in P$, we also have $x \cdot d^{2} \in P$, hence $x \cdot d^{2} \in \mathfrak{p}$. Since $\mathfrak{p}$ is prime, either $x \in \mathfrak{p}$ or $d^{2} \in \mathfrak{p}$. Now, if $x \in \mathfrak{p}$ then $\operatorname{Can}_{\mathfrak{p}}(x)=0^{2}+\mathfrak{p}$, which contradicts our hypothesis (2.13). On the other hand, if $d^{2} \in \mathfrak{p}$, then $d \in \mathfrak{p}$, and this contradicts (2.14). Hence $x \notin \mathfrak{p}$ and $d^{2} \notin \mathfrak{p}$, so $x \cdot d^{2} \notin \mathfrak{p}$. Thus we achieved an absurdity: $\left(x \cdot d^{2} \in \mathfrak{p}\right) \&\left(x \cdot d^{2} \notin \mathfrak{p}\right)$. It follows that our premise 2.12$)$ must be false, so:

$$
P \subseteq \eta_{\operatorname{supp}(P)}^{\prime}{ }^{\dashv}\left[\left(\frac{A\left\{A \backslash \operatorname{supp}^{\infty}(P)^{-1}\right\}}{\widehat{\mathfrak{p}}}\right)^{2}\right] .
$$

Hence $P=\eta_{\operatorname{supp}(P)}^{\prime} \overbrace{}^{\dashv}\left[\left(\frac{A\left\{A \backslash \operatorname{supp}^{\infty}(P)^{-1}\right\}}{\widehat{\mathfrak{p}}}\right)^{2}\right]$.

Now we need only to show that $\alpha^{\prime} \circ \beta^{\prime}=\mathrm{id}_{\tilde{\mathcal{F}}}$.

Let $[h: A \rightarrow F] \in \widetilde{\mathcal{F}}$. We have: 


$$
\left(\alpha^{\prime} \circ \beta^{\prime}\right)([h: A \rightarrow F])=\alpha^{\prime}\left(h^{\dashv}\left[F^{2}\right]\right)=\left[\eta_{\operatorname{supp}^{\infty}\left(h^{\dashv}\left[F^{2}\right]\right)}: A \rightarrow k_{\operatorname{supp}^{\infty}\left(h^{\dashv}\left[F^{2}\right]\right)}(A)\right] .
$$

It suffices to show that $[h]=\left[\eta_{\operatorname{supp}^{\infty}\left(h^{\dashv}\left[F^{2}\right]\right)}\right]$. Note that $\operatorname{supp}^{\infty}\left(h^{-1}\left[F^{2}\right]\right)=h^{-1}\left[F^{2} \cap\left(-F^{2}\right)\right]=\operatorname{ker}(h)$.

By the universal property of the $\mathcal{C}^{\infty}$-field of fractions of $\left(\frac{A}{\operatorname{ker}(h)}\right), k_{\operatorname{ker}(h)}(A)$, since $h\left[A^{\times}\right] \subseteq F^{\times}$(for $\mathcal{C}^{\infty}$-homomorphisms preserve invertible elements), there is a unique $\mathcal{C}^{\infty}$-homomorphism $\widetilde{h}: k_{\operatorname{ker}(h)}(A) \rightarrow$ $F$ such that the following diagram commutes:

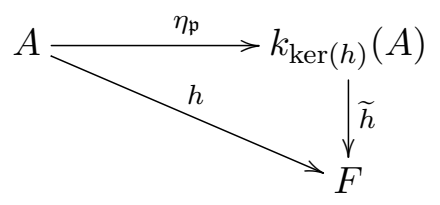

We have, then, the following commutative diagram:

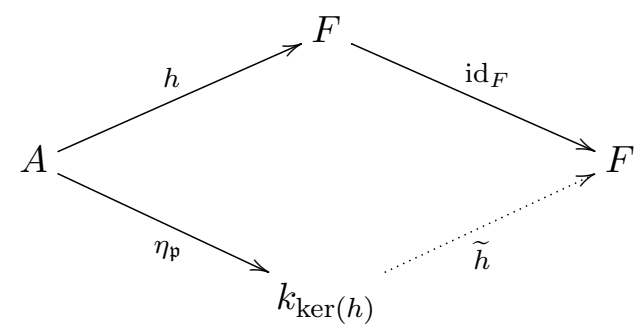

so $[h: A \rightarrow F]=\left[\eta_{\operatorname{ker}(h)}: A \rightarrow k_{\operatorname{ker}(h)}\right]$ and

$$
\left(\alpha^{\prime} \circ \beta^{\prime}\right)([h: A \rightarrow F])=[h: A \rightarrow F] .
$$

Hence it follows that $\alpha^{\prime}$ and $\beta^{\prime}$ are inverse bijections of each other.

Theorem 2.3.20. The map:

$$
\begin{array}{ccc}
\alpha: \operatorname{Spec}^{\infty}(A) & \rightarrow & \widetilde{\mathcal{F}} \\
\mathfrak{p} & \mapsto & {\left[\eta_{\mathfrak{p}}\right]=\left[q \circ \operatorname{Can}_{\mathfrak{p}}\right]}
\end{array}
$$

is a bijection whose inverse is given by:

$$
\beta: \begin{array}{ccc}
\widetilde{\mathcal{F}} & \rightarrow & \operatorname{Spec}^{\infty}(A) \\
{[h: A \rightarrow F]} & \mapsto & \operatorname{ker}(h)
\end{array}
$$

Proof. First we are going to show that $\alpha \circ \beta=\mathrm{id}_{\tilde{\mathcal{F}}}$, so $\beta$ is an injective map and $\alpha$ is its left inverse, hence it is surjective.

Let $[h: A \rightarrow F] \in \widetilde{F}$. We have:

$$
(\alpha \circ \beta)([h: A \rightarrow F])=\alpha(\operatorname{ker}(h))=\left[\eta_{\operatorname{ker}(h)}^{\prime}: A \rightarrow\left(\frac{A\left\{A \backslash \operatorname{ker}(h)^{-1}\right\}}{\widehat{\operatorname{ker}(h)}}\right)\right]
$$

It suffices to show that $[h: A \rightarrow F]=\left[\eta_{\operatorname{ker}(h)}^{\prime}: A \rightarrow\left(\frac{A\left\{A \backslash \operatorname{ker}(h)^{-1}\right\}}{\operatorname{ker}(h)}\right)\right]$. 
Since $\left(\frac{A\left\{A \backslash \operatorname{ker}(h)^{-1}\right\}}{\operatorname{ker}(h)}\right)$ is (up to $\mathcal{C}^{\infty}$-isomorphism) the $\mathcal{C}^{\infty}$-field of fractions of $\left(\frac{A}{\operatorname{ker}(h)}\right), k_{\operatorname{ker}(h)}(A)$, there is a unique $\mathcal{C}^{\infty}$-homomorphism $\widetilde{h}:\left(\frac{A\left\{A \backslash \operatorname{ker}(h)^{-1}\right\}}{\operatorname{ker}(h)}\right) \rightarrow F$ such that the following diagram commutes:

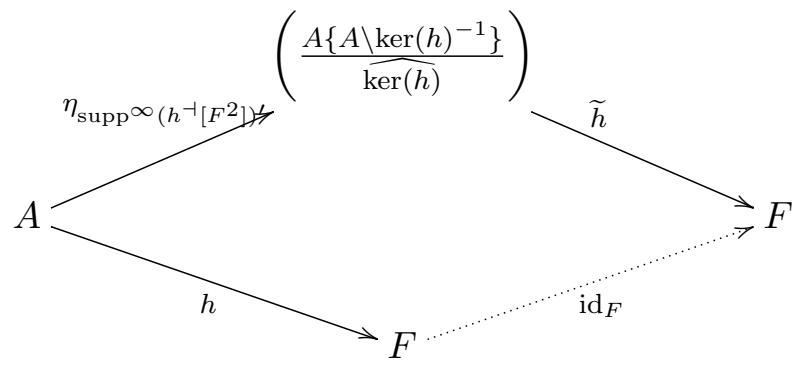

and the equality holds, i.e.,

$$
[h: A \rightarrow F]=\left[\eta_{\operatorname{ker}(h)}: A \rightarrow\left(\frac{A\left\{A \backslash \operatorname{ker}(h)^{-1}\right\}}{\widehat{\operatorname{ker}(h)}}\right)\right]
$$

It follows that $\alpha \circ \beta=\operatorname{id}_{\widetilde{F}}, \alpha$ is a surjective map and $\beta$ is an injective map.

On the other hand, given $\mathfrak{p} \in \operatorname{Spec}^{\infty}(A)$, we have:

$$
(\beta \circ \alpha)(P)=\beta\left(\left[\eta_{\mathfrak{p}}: A \rightarrow k_{\mathfrak{p}}\right]\right)=\operatorname{ker}\left(\eta_{\mathfrak{p}}\right)=\operatorname{Can}_{\mathfrak{p}}^{-1}[\widehat{\mathfrak{p}}]=\mathfrak{p}
$$

so:

$$
(\beta \circ \alpha)=\operatorname{id}_{\operatorname{Spec}^{\infty}(A)}
$$

as a Corollary of the theorems 2.3 .19 and 2.3 .20 , we have:

Lemma 2.3.21. Let $A$ be a $\mathcal{C}^{\infty}$-ring, and define:

$$
\begin{array}{cl}
\operatorname{supp}^{\infty}: \operatorname{Sper}^{\infty}(A) & \rightarrow \operatorname{Spec}^{\infty}(A) \\
P & \mapsto P \cap(-P)
\end{array}
$$

The following diagram commutes:

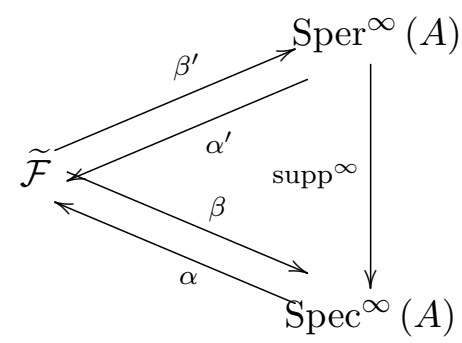

that is to say that:

$$
\alpha \circ \operatorname{supp}^{\infty}=\alpha^{\prime}
$$

and

$$
\operatorname{supp}^{\infty} \circ \beta^{\prime}=\beta
$$


Proof. Note that if we prove that $\alpha \circ \operatorname{supp}^{\infty}=\alpha^{\prime}$, then composing both sides with $\beta^{\prime}$ yields:

$$
\left(\alpha \circ \operatorname{supp}^{\infty}\right) \circ \beta^{\prime}=\alpha^{\prime} \circ \beta^{\prime}=\mathrm{id}_{\widetilde{\mathcal{F}}}
$$

so

$$
\alpha \circ\left(\operatorname{supp}^{\infty} \circ \beta^{\prime}\right)=\operatorname{id}_{\widetilde{\mathcal{F}}}
$$

and by the uniqueness of the inverse of $\alpha$, it follows that:

$$
\operatorname{supp}^{\infty} \circ \beta^{\prime}=\beta \text {. }
$$

Now we are going to prove that $\alpha \circ \operatorname{supp}^{\infty}=\alpha^{\prime}$.

Given $P \in \operatorname{Sper}^{\infty}(A)$ we have:

$$
\left(\alpha \circ \operatorname{supp}^{\infty}\right)(P)=\alpha\left(\operatorname{supp}^{\infty}(P)\right)=\left[\eta_{\text {supp }^{\infty}(P)}: A \rightarrow k_{\text {supp }^{\infty}(P)}(A)\right]=: \alpha^{\prime}(P),
$$

so the result holds.

As an important result of the theory of $\mathcal{C}^{\infty}$-rings which distinguishes it from the theory of the rings, we have the following:

Theorem 2.3.22. Let $A$ be a $\mathcal{C}^{\infty}$-ring. The following map:

$$
\begin{array}{cl}
\operatorname{supp}^{\infty}: \operatorname{Sper}^{\infty}(A) & \rightarrow \operatorname{Spec}^{\infty}(A) \\
P & \mapsto P \cap(-P)
\end{array}
$$

is a spectral bijection.

Proof. In Remark 2.3.17, we have already seen that $\operatorname{supp}^{\infty}$ is a spectral function, so we need only to show that is is a bijection.

Just note that $\operatorname{supp}^{\infty}=\beta \circ \alpha^{\prime}=\alpha \circ \beta^{\prime}$, and since $\operatorname{supp}^{\infty}$ is a composition of bijections, it is a bijection.

\subsection{Algebraic Concepts versus Smooth Algebraic Concepts}

In this section we comment some differences between some concepts and results of Commutative Algebra and Smooth Commutative Algebra. Some notions of Smooth Commutative Algebra, such as local $\mathcal{C}^{\infty}$-rings, $\mathcal{C}^{\infty}$-fields, $\mathcal{C}^{\infty}$-domains and von Neumann regular $\mathcal{C}^{\infty}$-rings are obtained via the adjunctions:

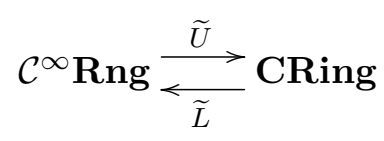

and

$$
\mathcal{C}^{\infty} \mathbf{R n g} \underset{\mathcal{L}}{\stackrel{\mathcal{U}}{\longrightarrow}} \mathbb{R}-\text { Alg }
$$

so many results about these concepts are very similar to those of Algebra. As an example, we can cite the fact that $\widetilde{L}$ "preserves" the concept of "object of fractions", as we see in the following: 
Remark 2.4.1. Let $A$ be a commutative unital ring and let $a \in A$. The functor $\widetilde{L}: \mathbf{C R i n g} \rightarrow \mathcal{C}^{\infty} \mathbf{R n g}$, which is left adjoint to $\widetilde{U}: \mathcal{C}^{\infty} \mathbf{R n g} \rightarrow \mathbf{C R i n g}$, preserves all colimits, so it takes the ring of fractions $A\left[a^{-1}\right]$ to the $\mathcal{C}^{\infty}$-ring of fractions, $B\left\{b^{-1}\right\}$, that is:

$$
\widetilde{L}\left(A\left[a^{-1}\right]\right) \cong \widetilde{L}\left(\frac{A \otimes \mathbb{Z}[x]}{\left\langle\left\{\iota_{A}(a) \cdot x-1\right\}\right\rangle}\right) \cong \frac{\widetilde{L}(A) \otimes_{\infty} \mathcal{C}^{\infty}(\mathbb{R})}{\left\langle\left\{\gamma_{A}(a) \cdot x-1\right\}\right\rangle}
$$

For a general $S \subseteq A$, we have:

$$
\widetilde{L}\left(A\left[S^{-1}\right]\right) \cong \widetilde{L}(A)\left\{\gamma_{A}[S]^{-1}\right\}
$$

The ring $A$ to be a domain does not entail that $\widetilde{L}(A)$ is a $\mathcal{C}^{\infty}$-domain. For example, despite $\mathbb{Z}[x]$ is a domain, $\widetilde{L}(\mathbb{Z}[x]) \cong C^{\infty}(\mathbb{R})$ is not a $\mathcal{C}^{\infty}$-domain - since its underlying ring is not a domain. However, some domains can be taken to $\mathcal{C}^{\infty}$-fields, e.g., $\widetilde{L}(\mathbb{Z}) \cong \mathbb{R}$ and $\widetilde{L}(\mathbb{Q}) \cong \mathbb{R}$.

Fields can be taken by $\widetilde{L}$ to the trivial $\mathcal{C}^{\infty}$-ring. Since every $\mathcal{C}^{\infty}$-ring $A$ is such that $1+\sum A^{2} \subseteq A^{\times}$, any field with non zero characteristic is mapped into the trivial $\mathcal{C}^{\infty}$-ring. More generally, every ring with non zero characteristic is taken to the trivial $\mathcal{C}^{\infty}$-ring.

The left adjoint $\widetilde{L}$ also maps free rings to free $\mathcal{C}^{\infty}$-rings, that is, given any set $E$ we have $\widetilde{L}(\mathbb{Z}[E]) \cong$ $\mathcal{C}^{\infty}\left(\mathbb{R}^{E}\right)$. Note that $\mathbb{Z}[E]$ is a reduced ring and that $\mathcal{C}^{\infty}\left(\mathbb{R}^{E}\right)$ is a $\mathcal{C}^{\infty}$-reduced $\mathcal{C}^{\infty}$-ring.

As we have already commented, every $\mathcal{C}^{\infty}$-ring can be regarded as an $\mathbb{R}$-algebra. However, the converse of this claim is not true: $\mathbb{C} \cong \frac{\mathbb{R}[x]}{\left\langle x^{2}+1\right\rangle}$ is an $\mathbb{R}$-algebra but it is not a $\mathcal{C}^{\infty}$-ring. In fact, every $\mathcal{C}^{\infty}$-ring is semi-real, so the sum of 1 with any sum of squares must be invertible. Thus, since we have $-1=x^{2}$ in $\frac{\mathbb{R}[x]}{\left\langle x^{2}+1\right\rangle}$, we would have $0+\left\langle x^{2}+1\right\rangle$ invertible in $\widetilde{L}\left(\frac{\mathbb{R}[x]}{\left\langle x^{2}+1\right\rangle}\right) \cong \widetilde{L}(\mathbb{C})$ - hence it must be the trivial $\mathcal{C}^{\infty}$-ring (cf. Proposition 2.3.4).

However, both theories have "ad hoc" notions of radical ideals, rings of fractions and polynomials. Contrary to what occurs in Commutative Algebra, in which every prime ideal is radical, in the smooth case we have seen that not every prime ideal of a $\mathcal{C}^{\infty}$-ring is $\mathcal{C}^{\infty}$-radical. Due to this fact, we have different notions in these theories, such as "saturation", "Zariski spectrum", "reducedness", "real spectrum" and others.

In both theories one has very similar results, such as the separation theorems. Perhaps the most "striking" difference between Commutative and Smooth Commutative Algebra is the closer relation that the latter holds with Real Algebraic Geometry than the former, in virtue of the spectral bijection between the $\mathcal{C}^{\infty}$-real spectrum and the smooth Zariski spectrum of a $\mathcal{C}^{\infty}$-ring, given in Theorem 2.3.22. Such a relationship will be explored later from a more logical ("model-theoretic") viewpoint. 


\section{Chapter 3}

\section{Sheaves of $\mathcal{C}^{\infty}$-Rings and Applications}

In this chapter we approach $\mathcal{C}^{\infty}$-rings from a Sheaf Theoretic viewpoint. We are going to study a $\mathcal{C}^{\infty}$-ring by means of its "affine $\mathcal{C}^{\infty}$-scheme". For this purpose, given an arbitrary $\mathcal{C}^{\infty}$-ring $A$, we construct a spectral topological space, namely its smooth Zariski spectrum, $\left(\operatorname{Spec}^{\infty}(A), Z^{\infty}{ }^{\infty}\right)$ and a sheaf of $\mathcal{C}^{\infty}$-rings, $\Sigma_{A}$ : Open $\left(\operatorname{Spec}^{\infty}(A), Z_{\text {Zar }}^{\infty}\right)^{\text {op }} \rightarrow \mathcal{C}^{\infty}$ Rng such that for every $a \in A, \Sigma_{A}\left(D^{\infty}(a)\right) \cong A\left\{a^{-1}\right\}$ and whose stalks are local $\mathcal{C}^{\infty}$-rings. In this chapter we give a detailed account of this topological space and we prove, for example, that it is indeed a spectral space (a fact that has been used in the last chapter without proof). A complete proof of this fact could not be found anywhere in the literature.

As a motivation we gave for the definition of the smooth version of Zariski spectrum, we have seen that given a $\mathcal{C}^{\infty}$-ring $A$ and a prime ideal, $\mathfrak{p} \subseteq A$, it is not always true that

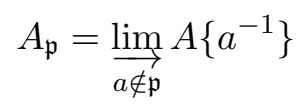

is a local $\mathcal{C}^{\infty}$-ring (cf. Example 1.2 of $[67 \mid)$. In fact, $A_{\mathfrak{p}}$ is a local $\mathcal{C}^{\infty}$-ring if, and only if, $\sqrt[\infty]{\mathfrak{p}}=\mathfrak{p}$ - so we have defined the smooth Zariski spectrum as consisting only of those $\mathcal{C}^{\infty}$-radical prime ideals.

Whenever $A$ is a von Neumann regular $\mathcal{C}^{\infty}$-ring, $\operatorname{Spec}^{\infty}(A)$ is a Boolean space and $A_{\mathfrak{p}}$ is a $\mathcal{C}^{\infty}$-field. It is a fact that a commutative unital ring is a von Neumann regular ring if, and only if, it is isomorphic to the ring of global sections of sheaves of rings over a Boolean space such that the stalks are fields (see Proposition 5.6 of [27]). A $\mathcal{C}^{\infty}$-ring $A$ is a von Neumann regular $\mathcal{C}^{\infty}$-ring if, and only if, $\widetilde{U}(A)$ is a von Neumann regular ring. In its turn, $\widetilde{U}(A)$ is a von Neumann regular ring if, and only if, it is isomorphic to the ring of global sections of sheaves of rings over the booleanization of $\operatorname{Spec}(\widetilde{U}(A))$ whose stalks are fields. As we have seen in Theorem 2.2 .70 , in the case in which $A$ is a von Neumann regular $\mathcal{C}^{\infty}$-ring, $\operatorname{Spec}(\widetilde{U}(A))=\operatorname{Spec}^{\infty}(A)$ and the structure sheaf coincides in both categories. As commented by R. S. Pierce in [116], sheaves over "enormously simplifies the theory, since local results can usually be blown up to global theorems".

As defined by D. Joyce in [30, a $\mathcal{C}^{\infty}$-ringed space is a pair $\left(X, \mathcal{O}_{X}\right)$, where $X$ is a topological space and $\mathcal{O}_{X}$ : Open $(X)^{\mathrm{op}} \rightarrow \mathcal{C}^{\infty} \mathbf{R n g}$ is a sheaf of $\mathcal{C}^{\infty}$-rings whose stalks are local $\mathcal{C}^{\infty}$-rings. As in Algebraic Geometry, we define a sheaf of (local) $\mathcal{C}^{\infty}$-rings on $\operatorname{Spec}^{\infty}(A)$, for any $\mathcal{C}^{\infty}$-ring, $A$, the "structure sheaf".

As an application of sheaf-theoretic ideas in $\mathcal{C}^{\infty}$-ring theory, we obtain some interesting results concerning the notion of von Neumann regular $\mathcal{C}^{\infty}$-ring -a concept introduced in the previous chapter, that has many equivalent descriptions-, together with a categorial and logical treatment for them. In particular, the subcategory of $\mathcal{C}^{\infty} \mathbf{R n g}$ consisting of all von Neumann regular $\mathcal{C}^{\infty}$-rings is characterized as the closure under small limits of the category of $\mathcal{C}^{\infty}$-fields, i.e., it is the smallest subcategory of $\mathcal{C}^{\infty} \mathbf{R n g}$ 
which contain all $\mathcal{C}^{\infty}$-fields and is closed under small limits. Moreover, it was proven, by two different methods (with a sheaf-theoretic one, following [2]), that the subcategory of von Neumann regular $\mathcal{C}^{\infty}$-rings is reflective in the category of all $\mathcal{C}^{\infty}$-rings: for every $\mathcal{C}^{\infty}$-ring $A$ there is a universal von Neumann regular $\mathcal{C}^{\infty}$-ring, vN $(A)$ (that we are going to call "von Neumann regular hull of $A$ ") and a $\mathcal{C}^{\infty}$-homomorphism $\nu_{A}: A \rightarrow \mathrm{vN}(A)$ which is initial among maps from $A$ to von Neumann regular $\mathcal{C}^{\infty}$-rings.

Some interesting results about the functorial relationship between von Neumann-regular $\mathcal{C}^{\infty}$-rings and Boolean spaces are proved: We apply von Neumann regular $\mathcal{C}^{\infty}$-ring to naturally represent Boolean Algebras in a strong sense: i.e., not only all Boolean algebras are isomorphic to the Boolean algebra of idempotents of a von Neumann regular $\mathcal{C}^{\infty}$-ring, as every homomorphism between such boolean algebras of idempotents is induced by a $C^{\infty}$-homomorphism.

\subsection{Some topological features of $\operatorname{Spec}^{\infty}(A)$}

In this section we make a detailed study of the smooth Zariski spectrum. Recall that, given any $\mathcal{C}^{\infty}$-ring $A$,

$$
\operatorname{Spec}^{\infty}(A):=\{\mathfrak{p} \in \operatorname{Spec}(\widetilde{U}(A)) \mid \sqrt[\infty]{\mathfrak{p}}=\mathfrak{p}\}
$$

We begin with the following:

Definition 3.1.1. Let $A$ be a $\mathcal{C}^{\infty}$-ring.

1. Given any $\mathcal{C}^{\infty}$-radical ideal $\mathfrak{a} \subseteq A$ (not necessarily prime ideal), we define:

$$
Z^{\infty}(\mathfrak{a}):=\left\{\mathfrak{p} \in \operatorname{Spec}^{\infty}(A) \mid \mathfrak{p} \supseteq \mathfrak{a}\right\}
$$

2. Given any element $a \in A$, we define:

$$
D^{\infty}(a):=\left\{\mathfrak{p} \in \operatorname{Spec}^{\infty}(A) \mid a \notin \mathfrak{p}\right\}
$$

Proposition 3.1.2. Let $A$ be $a \mathcal{C}^{\infty}$-ring and $\mathfrak{a} \subseteq A$ be any ideal. Then:

$$
Z^{\infty}(\mathfrak{a})=Z^{\infty}(\sqrt[\infty]{\mathfrak{a}})
$$

Proof. Given $\mathfrak{p} \in Z^{\infty}(\mathfrak{a}), \mathfrak{a} \subseteq \mathfrak{p}$. By the item b of the Theorem $2.2 .21, \sqrt[\infty]{\mathfrak{a}} \subseteq \sqrt[\infty]{\mathfrak{p}}=\mathfrak{p}$, so $\mathfrak{p} \in Z^{\infty}(\sqrt[\infty]{\mathfrak{a}})$

Conversely, given $\mathfrak{p} \in Z^{\infty}(\sqrt[\infty]{\mathfrak{a}})$, then $\mathfrak{p} \supseteq \sqrt[\infty]{\mathfrak{a}} \supseteq \mathfrak{a}$, so $\mathfrak{p} \in Z^{\infty}(\mathfrak{a})$.

Proposition 3.1.3. Let $A$ be $a \mathcal{C}^{\infty}$-ring, $a, b \in A$. The following conditions are equivalent:

(1) $D^{\infty}(a) \subseteq D^{\infty}(b)$;

(2) $Z^{\infty}(a) \supseteq Z^{\infty}(b)$;

(3) $a \in \sqrt[\infty]{(b)}$

(4) $\sqrt[\infty]{(a)} \subseteq \sqrt[\infty]{(b)}$ 
Proof. The equivalence (1) $\Longleftrightarrow(2)$ follows immediately from the definition of $Z^{\infty}$.

Ad $(2) \rightarrow(4)$

$$
\sqrt[\infty]{(a)}=\bigcap Z^{\infty}(a) \subseteq \bigcap Z^{\infty}(b)=\sqrt[\infty]{(b)}
$$

$\operatorname{Ad}(4) \Longleftrightarrow(3)$.

$$
\sqrt[\infty]{(a)} \subseteq \sqrt[\infty]{(b)} \Longleftrightarrow(a) \subseteq \sqrt[\infty]{(b)} \Longleftrightarrow a \in \sqrt[\infty]{(b)}
$$

Ad $(3) \rightarrow(2)$

Let $a \in \sqrt[\infty]{(a)}$. Given $\mathfrak{p} \in Z^{\infty}(b)$, since $\sqrt[\infty]{(b)}=\bigcap\left\{\mathfrak{p} \in \operatorname{Spec}^{\infty}(A) \mid(b) \subseteq \mathfrak{p}\right\}$, it follows that $\sqrt[\infty]{(b)} \subseteq \mathfrak{p}$. Since $(4) \Longleftrightarrow(3)$, we have that $\sqrt[\infty]{(a)} \subseteq \sqrt[\infty]{(b)} \subseteq \mathfrak{p}$ implies $(a) \subseteq \sqrt[\infty]{(a)} \subseteq \mathfrak{p}$. Hence $\mathfrak{p} \in Z^{\infty}(a)$.

Proposition 3.1.4. Given any ideals $\mathfrak{a}$ and $\mathfrak{b}$, we have:

$$
\sqrt[\infty]{\mathfrak{a}} \subseteq \sqrt[\infty]{\mathfrak{b}} \Longleftrightarrow Z^{\infty}(\mathfrak{b}) \subseteq Z^{\infty}(\mathfrak{a}) .
$$

Proof. In fact, if $\sqrt[\infty]{\mathfrak{a}} \subseteq \sqrt[\infty]{\mathfrak{b}}$, then for every $\mathfrak{p} \in \operatorname{Spec}^{\infty}(A)$, whenever $\mathfrak{p} \in Z^{\infty}(\sqrt[\infty]{b})$, i.e., whenever $\sqrt[\infty]{b} \subseteq \mathfrak{p}, \sqrt[\infty]{\mathfrak{a}} \subseteq \mathfrak{p}$, so $\mathfrak{p} \in Z^{\infty}(\sqrt[\infty]{\mathfrak{a}})$. Thus:

$$
Z^{\infty}(\mathfrak{b})=Z^{\infty}(\sqrt[\infty]{\mathfrak{b}}) \subseteq Z^{\infty}(\sqrt[\infty]{\mathfrak{a}})=Z^{\infty}(\mathfrak{a})
$$

Conversely, if $Z^{\infty}(\mathfrak{b}) \subseteq Z^{\infty}(\mathfrak{a})$, then:

$$
\sqrt[\infty]{\mathfrak{b}}=\bigcap_{\substack{\mathfrak{b} \subseteq \mathfrak{p} \\ \mathfrak{p} \in \operatorname{Spec}^{\infty}(A)}} \supseteq \bigcap_{\substack{\mathfrak{a} \subseteq \mathfrak{p} \\ \mathfrak{p} \in \operatorname{Spec}^{\infty}(A)}} \mathfrak{p}=\sqrt[\infty]{\mathfrak{a}} .
$$

The next theorem shows us that the sets of the form $Z^{\infty}(\mathfrak{b})$, for some $\mathcal{C}^{\infty}$-radical ideal $\mathfrak{b}$, are the closed subsets of a topology in $\operatorname{Spec}^{\infty}(A)$, that we shall refer to as the smooth Zariski topology.

Theorem 3.1.5. Let $A$ be a $\mathcal{C}^{\infty}$-ring. Then:

1. $Z^{\infty}((0))=\operatorname{Spec}^{\infty}(A)$ and $Z^{\infty}\left(\left(1_{A}\right)\right)=\varnothing$;

2. $Z^{\infty}(\mathfrak{a}) \cup Z^{\infty}(\mathfrak{b})=Z^{\infty}(\mathfrak{a} \cdot \mathfrak{b})=Z^{\infty}(\mathfrak{a} \cap \mathfrak{b})$;

3. $\bigcap_{i \in I} Z^{\infty}\left(\mathfrak{a}_{i}\right)=Z^{\infty}\left(\sum_{i \in I} \mathfrak{a}_{i}\right)$

where $\sum_{i \in I} \mathfrak{a}_{i}$ denotes the ideal generated by the family $\left\{\mathfrak{a}_{i}\right\}_{i \in I}$.

Proof. Ad $1 . Z^{\infty}((0))=\left\{\mathfrak{p} \in \operatorname{Spec}^{\infty}(A) \mid(0) \subseteq \mathfrak{p}\right\}=\operatorname{Spec}^{\infty}(A)$ and:

$$
Z^{\infty}\left(\left(1_{A}\right)\right)=\left\{\mathfrak{p} \in \operatorname{Spec}^{\infty}(A) \mid\left(1_{A}\right) \subseteq \mathfrak{p}\right\}=\left\{\mathfrak{p} \in \operatorname{Spec}^{\infty}(A) \mid 1_{A} \in \mathfrak{p}\right\}=\varnothing .
$$

Hence, by the Proposition 3.1.2 , we can write $\operatorname{Spec}^{\infty}(A)=Z^{\infty}(\sqrt[\infty]{(0)})$ and $\varnothing=Z^{\infty}\left(\sqrt[\infty]{\left(1_{A}\right)}\right)$. 
Ad 2. Let $\mathfrak{p} \in Z^{\infty}(\mathfrak{a} \cap \mathfrak{b})$, so $\mathfrak{a} \cap \mathfrak{b} \subseteq \mathfrak{p}$, and since $\mathfrak{a} \cdot \mathfrak{b} \subseteq \mathfrak{a} \cap \mathfrak{b}, \mathfrak{a} \cdot \mathfrak{b} \subseteq \mathfrak{a} \cap \mathfrak{b} \subseteq \mathfrak{p}$ and $\mathfrak{p} \in Z^{\infty}(\mathfrak{a} \cdot \mathfrak{b})$. Since $\mathfrak{p}$ is a prime ideal, $\mathfrak{a} \cdot \mathfrak{b} \subseteq \mathfrak{p} \Rightarrow \mathfrak{a} \subseteq \mathfrak{p}$ or $\mathfrak{b} \subseteq \mathfrak{p}$, hence $\mathfrak{p} \in Z^{\infty}(\mathfrak{a}) \cup Z^{\infty}(\mathfrak{b})$.

Now, if $\mathfrak{p} \in Z^{\infty}(\mathfrak{a}) \cup Z^{\infty}(\mathfrak{b})$, either $\mathfrak{p} \in Z^{\infty}(\mathfrak{a})$ or $\mathfrak{p} \in Z^{\infty}(\mathfrak{b})$, so $\mathfrak{a} \cap \mathfrak{b} \subseteq \mathfrak{p}$ and $\mathfrak{p} \in Z^{\infty}(\mathfrak{a} \cap \mathfrak{b})$.

Ad 3. We have:

$$
\begin{aligned}
\mathfrak{p} \in Z^{\infty}\left(\sum_{i \in I} \mathfrak{a}_{i}\right) \Longleftrightarrow \sum_{i \in I} \mathfrak{a}_{i} \subseteq \mathfrak{p} \Longleftrightarrow(\forall i \in I)\left(\mathfrak{a}_{i} \subseteq \mathfrak{p}\right) & \Longleftrightarrow \\
& \Longleftrightarrow(\forall i \in I)\left(\mathfrak{p} \in Z^{\infty}\left(\mathfrak{a}_{i}\right)\right) \Longleftrightarrow \mathfrak{p} \in \bigcap_{i \in I} Z^{\infty}\left(\mathfrak{a}_{i}\right)
\end{aligned}
$$

Because of the Proposition 3.1.2 , we have the $\mathcal{C}^{\infty}$-radical ideal $\sqrt[\infty]{\sum_{i \in I} \mathfrak{a}_{i}}$ such that $Z^{\infty}\left(\sum_{i \in I} \mathfrak{a}_{i}\right)=$ $Z^{\infty}\left(\sqrt[\infty]{\sum_{i \in I} \mathfrak{a}_{i}}\right)$.

In virtue of the Theorem 3.1.5 the sets of the form $\operatorname{Spec}^{\infty}(A) \backslash Z^{\infty}(\mathfrak{p})$ satisfy the axioms of open sets of a topology.

We have, thus:

Definition 3.1.6. Let $A$ be a $\mathcal{C}^{\infty}$-ring. The smooth Zariski topology on $\operatorname{Spec}^{\infty}(A)$ is:

$$
\operatorname{Zar}^{\infty}:=\left\{\operatorname{Spec}^{\infty}(A) \backslash Z^{\infty}(\mathfrak{p}) \mid \mathfrak{p} \in \mathfrak{I}_{A}^{\infty}\right\}
$$

Definition 3.1.7. Let $A$ be any $\mathcal{C}^{\infty}$-ring. The $\mathcal{C}^{\infty}$-spectrum of $A$ is the following topological space:

$$
\left(\operatorname{Spec}^{\infty}(A), \operatorname{Zar}^{\infty}\right) .
$$

Henceforth we are going to denote the topological space $\left(\operatorname{Spec}^{\infty}(A), Z_{a r}^{\infty}\right) \operatorname{simply}$ by $\operatorname{Spec}^{\infty}(A)$, omitting its topology.

Theorem 3.1.8. Let $A$ be a $\mathcal{C}^{\infty}$-ring. The family $\left\{D^{\infty}(a) \mid a \in A\right\}$ is a basis for the smooth Zariski topology, Zar ${ }^{\infty}$.

Proof. Note that given any $\mathcal{C}^{\infty}$-radical ideal, $\mathfrak{a}$ and a $\mathcal{C}^{\infty}$-radical prime ideal $\mathfrak{p}$, we have:

$$
\mathfrak{a} \not \mathfrak{p} \Longleftrightarrow(\exists a \in \mathfrak{a})(a \notin \mathfrak{p}),
$$

Hence, any open set of Zar ${ }^{\infty}$, say:

$$
\operatorname{Spec}^{\infty}(A) \backslash Z^{\infty}(\mathfrak{a})=\bigcup_{a \in \mathfrak{a}} D^{\infty}(a)
$$

Proposition 3.1.9. Let $A$ be a $\mathcal{C}^{\infty}$-ring and $\operatorname{Spec}^{\infty}(A)$ be its $\mathcal{C}^{\infty}$-spectrum. We have, for any point $\mathfrak{p} \in \operatorname{Spec}^{\infty}(A):$

$$
\text { Cl. }\{\mathfrak{p}\}=Z^{\infty}(\mathfrak{p}) .
$$

Proof. By definition, the closure of a set is the intersection of all the closed sets which includes it, so:

$$
\mathrm{Cl} .\{\mathfrak{p}\}=\bigcap_{\substack{\mathfrak{p} \in Z^{\infty}(\mathfrak{a}) \\ \mathfrak{a} \in \mathfrak{I}_{A}^{\infty}}} Z^{\infty}(\mathfrak{a})=Z^{\infty}(\mathfrak{p}) .
$$


Theorem 3.1.10. Let $A$ be a $\mathcal{C}^{\infty}$-ring. The topological space $\left(\operatorname{Spec}^{\infty}(A), Z^{\infty}{ }^{\infty}\right)$ is such that, given $\mathfrak{p}, \mathfrak{q} \in \operatorname{Spec}^{\infty}(A)$ with $\mathfrak{p} \neq \mathfrak{q}$, there is either $U \in \operatorname{Zar}^{\infty}$ such that $\mathfrak{p} \in U$ and $\mathfrak{q} \notin U$ or $V \in \mathrm{Zar}^{\infty}$ such that $\mathfrak{p} \notin V$ and $\mathfrak{q} \in V$. In other words, the topological space $\left(\operatorname{Spec}^{\infty}(A), \mathrm{Zar}^{\infty}\right)$ is a $T_{0}$ space.

Proof. Given $\mathfrak{p}, \mathfrak{q} \in \operatorname{Spec}^{\infty}(A)$ with $\mathfrak{p} \neq \mathfrak{q}$, then either $\mathfrak{p} \nsubseteq \mathfrak{q}$ or $\mathfrak{q} \nsubseteq \mathfrak{p}$.

If $\mathfrak{q} \not \nsubseteq \mathfrak{p}$, then there is some $b \in \mathfrak{q}$ such that $b \notin \mathfrak{p}$, so $\mathfrak{q} \notin D^{\infty}(b)$ and $\mathfrak{p} \in D^{\infty}(b)$. Thus it suffices to take $U=D^{\infty}(b)$.

Now, if $\mathfrak{p} \nsubseteq \mathfrak{q}$, then there is $a \in \mathfrak{p}$ such that $a \notin \mathfrak{q}$, so $\mathfrak{p} \notin D^{\infty}(a)$ and $\mathfrak{q} \in D^{\infty}(a)$. Thus, it suffices to take $V=D^{\infty}(a)$.

As a corollary of the Proposition 3.1.4, we can prove the following:

Proposition 3.1.11. Given any two $\mathcal{C}^{\infty}$-radical ideals of a $\mathcal{C}^{\infty}$-ring $A, I, J \in \mathfrak{I}_{A}^{\infty}$, we have:

$$
\sqrt[\infty]{I \cdot J}=\sqrt[\infty]{I \cap J}
$$

Proof. Since $I \cdot J \subseteq I \cap J$, we have $\sqrt[\infty]{I \cdot J} \subseteq \sqrt[\infty]{I \cap J}$.

Ab absurdo, suppose $\sqrt[\infty]{I \cap J} \nsubseteq \sqrt[\infty]{I \cdot J}$, so by Proposition 3.1.4, $Z^{\infty}(I \cdot J) \nsubseteq Z^{\infty}(I \cap J)$. This latter condition means that there is some $\mathfrak{p} \in \operatorname{Spec}^{\infty}(A)$ such that $I \cdot J \subseteq \mathfrak{p}$ and $I \cap J \nsubseteq \mathfrak{p}$, that is, there is $a \in I \cap J$ such that $a \notin \mathfrak{p}$. Thus, we have $a^{2} \in I \cdot J \subseteq \mathfrak{p}$, so $a^{2} \in \mathfrak{p}$ and $a \notin \mathfrak{p}$ - which contradicts the fact that the ideal $\mathfrak{p}$ is prime. Hence:

$$
\sqrt[\infty]{I \cap J} \subseteq \sqrt[\infty]{I \cdot J}
$$

Theorem 3.1.12 (Lemma 1.4 of $[65]$ ). Let $A$ be a $\mathcal{C}^{\infty}$-ring.

(i) $D^{\infty}(a) \subseteq D^{\infty}(b)$ if, and only if, $b \in A\left\{a^{-1}\right\}^{\times}$, if, and only if, $a \in \sqrt[\infty]{(b)}$;

(ii) Each basic open $D^{\infty}(a)$ of $\operatorname{Spec}^{\infty}(A)$ is compact; in fact $D^{\infty}(a) \subset \bigcup_{i \in I} D^{\infty}\left(a_{i}\right)$ if, and only if, there are finitely many indices $i_{1}, \cdots, i_{n} \in I$ such that $D^{\infty}(a) \subseteq D^{\infty}\left(a_{i_{1}}^{2}+\cdots+a_{i_{n}}^{2}\right)$.

(iii) The basic opens $D^{\infty}($ a) form a distributive lattice, with:

$$
D^{\infty}(a) \cap D^{\infty}(b)=D^{\infty}(a \cdot b) \text { and } D^{\infty}(a) \cup D^{\infty}(b)=D^{\infty}\left(a^{2}+b^{2}\right)
$$

Definition 3.1.13. Let $(X, \tau)$ be a topological space, $E \subseteq X$ and $x \in X$.

(a) $E$ is irreducible in $X$ if, and only if, for all closed sets, $F_{1}, F_{2}$, in $X$

$$
\left(E \subseteq F_{1} \cup F_{2}\right) \rightarrow\left(\left(E \subseteq F_{1}\right) \vee\left(E \subseteq F_{2}\right)\right)
$$

(b) $x$ is a generic point of $E$ if $x \in E$ and $E \subseteq \mathrm{Cl} .\{x\}$

Note that if $E \subseteq X$ is closed, since the finite intersection of closed sets is closed, $E$ is irreducible in $X$ if, and only if, for all closed sets $F_{1}$ and $F_{2}$ in $X$

$$
\left(E=F_{1} \cup F_{2}\right) \rightarrow\left(\left(E=F_{1}\right) \vee\left(E=F_{2}\right)\right) .
$$

Note also that if $E \subseteq X$ is closed, then a point $x \in E$ is generic if, and only if, $E=\mathrm{Cl}$. $\{x\}$. 
Definition 3.1.14. Let $(X, \tau)$ be a topological space. The space $(X, \tau)$ is a spectral space if, and only if:

(s1) $(X, \tau)$ is a compact $T_{0}$ topological space;

$(s 2) \stackrel{\circ}{\mathcal{K}}(X):=\{U \subseteq X \mid U$ is compact and open $\}$ constitute a basis of open subsets of $X$ that is closed under finite intersections;

(s3) Every irreducible closed subset E has a unique generic point.

Remark 3.1.15. The condition (s2) in the definition above can be replaced by the equivalent condition stated as follows: $(X, \tau)$ has some basis of compact open subsets of $X$ that is closed under finite intersections.

Theorem 3.1.16. Let $A$ be any $\mathcal{C}^{\infty}$-ring. The topological space $\operatorname{Spec}^{\infty}(A)$ is a spectral space.

Proof. By the Theorem 3.1.10 $\operatorname{Spec}^{\infty}(A)$ is a $T_{0}$ topological space.

By item (ii) of the Theorem 3.1.12, for any $a \in A, D^{\infty}(a)$ is compact, since given any open cover $\left\{D^{\infty}\left(a_{i}\right) \mid i \in I\right\}$ of $D^{\infty}(a)$ :

$$
D^{\infty}(a) \subseteq \bigcup_{i \in I} D^{\infty}\left(a_{i}\right)
$$

there are finitely many indices $i_{1}, \cdots, i_{n} \in I$ such that:

$$
D^{\infty}(a) \subseteq D^{\infty}\left(a_{i_{1}}^{2}+\cdots a_{i_{n}}^{2}\right) .
$$

Claim: $D^{\infty}\left(a_{i_{1}}^{2}+\cdots a_{i_{n}}^{2}\right) \subseteq \bigcup_{j=1}^{n} D^{\infty}\left(a_{i_{j}}\right)$.

Given $\mathfrak{p} \in D^{\infty}\left(a_{i_{1}}^{2}+\cdots a_{i_{n}}^{2}\right), a_{i_{1}}^{2}+\cdots a_{i_{n}}^{2} \notin \mathfrak{p}$, there must exist some $k \in\{1,2, \cdots, n\}$ such that $a_{i_{k}}^{2} \notin \mathfrak{p}$, and since $\mathfrak{p}$ is prime, $a_{i_{k}} \notin \mathfrak{p}$. Thus, $\mathfrak{p} \in D^{\infty}\left(a_{i_{k}}\right) \subseteq \bigcup_{j=1}^{n} D^{\infty}\left(a_{i_{j}}\right)$.

It follows that

$$
D^{\infty}(a) \subseteq \bigcup_{j=1}^{n} D^{\infty}\left(a_{i_{j}}\right) .
$$

It is also clear, by the item (iii) of Lemma 1.4 of [65], that $\mathcal{B}$ is closed under finite intersections, since given $D^{\infty}\left(a_{1}\right), D^{\infty}\left(a_{2}\right), \cdots, D^{\infty}\left(a_{n}\right) \in \mathcal{B}$,

$$
D^{\infty}\left(a_{1}\right) \cap \cdots \cap D^{\infty}\left(a_{n}\right)=D^{\infty}\left(a_{1} \cdot a_{2} \cdots a_{n}\right) \in \mathcal{B}
$$

Finally, we are going to show that each irreducible closed subset of $\operatorname{Spec}^{\infty}(A)$ has a unique generic point.

By the Definition 3.1.6 $E$ is closed in $\operatorname{Spec}^{\infty}(A)$ if, and only if, there is some $\mathcal{C}^{\infty}$-radical ideal, $P \in \mathfrak{I}^{\infty}(A)$, such that $E=Z^{\infty}(P)=\left\{\mathfrak{p} \in \operatorname{Spec}^{\infty}(A) \mid P \subseteq \mathfrak{p}\right\}$.

We claim that if $P$ is not a prime ideal, then $E$ is not irreducible, which is equivalent to assert that if $E$ is irreducible then $P$ is prime, via modus tollens.

Suppose $P$ is not prime, so there are ideals $I, J$ of $A$ such that $I \cdot J \subseteq P, I \nsubseteq P$ and $J \nsubseteq P$. 
We have:

$$
E=Z^{\infty}(P) \subseteq Z^{\infty}(I) \cup Z^{\infty}(J)
$$

In fact, if $K \in E=Z^{\infty}(P)$, since $I \cdot J \subseteq P, I \cdot J \subseteq K$. Because $K$ is prime, $I \cdot J \subseteq K$ implies either $I \subseteq K$ (so $\left.K \in Z^{\infty}(I)\right)$ or $J \subseteq K$ (so $\left.K \in Z^{\infty}(J)\right)$, hence $K \in Z^{\infty}(I) \cup Z^{\infty}(J)$.

By definition, both $Z^{\infty}(I)$ and $Z^{\infty}(J)$ are both closed.

Finally, we have both $Z^{\infty}(P) \subsetneq Z^{\infty}(I)$ and $Z^{\infty}(P) \subsetneq Z^{\infty}(J)$.

If $Z^{\infty}(I)=Z^{\infty}(P)$, then $\left\{\mathfrak{p} \in \operatorname{Spec}^{\infty}(A) \mid \mathfrak{p} \supseteq I\right\}=\left\{\mathfrak{p} \in \operatorname{Spec}^{\infty}(A) \mid \mathfrak{p} \supseteq P\right\}$ and:

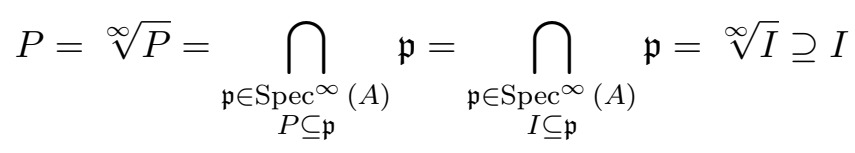

which contradicts our assumption that $I \nsubseteq P$. A similar argument holds for $Z^{\infty}(J)$.

Thus we have expressed the closed subset, $E \subseteq \operatorname{Spec}^{\infty}(A)$ as the union of two proper closed subsets, so $E$ is not irreducible.

Hence, if $E=Z^{\infty}(P)$ is an irreducible closed subset of $\operatorname{Spec}^{\infty}(A)$, then $P$ is a $\mathcal{C}^{\infty}$-radical prime ideal of $A$.

Thus,

$$
\text { Cl. }\{P\}:=\bigcap_{\mathfrak{p} \in \operatorname{Spec}^{\infty}(A)} Z^{\infty}(\mathfrak{p})=Z^{\infty}(P)=E,
$$

and $P$ is a generic point of $E$.

The uniqueness of the generic point is proved as follows.

Suppose, ab absurdo, that there is some $P^{\prime} \in \operatorname{Spec}^{\infty}(A)$ with $P \neq P^{\prime}$ and $\operatorname{Cl} .\{P\}=E=\operatorname{Cl} .\left\{P^{\prime}\right\}$. Since $P \neq P^{\prime}$, we can assume without loss of generality, $P \nsubseteq P^{\prime}$, so there is some $a \in P$ such that $a \notin P^{\prime}$, and $P^{\prime} \in D^{\infty}(a)$, so $P^{\prime} \notin Z^{\infty}((a))$ and $P \notin D^{\infty}(a)$, so $P \in Z^{\infty}((a))$.

Thus:

$$
E=\mathrm{Cl} .\left\{P^{\prime}\right\} \nsubseteq Z^{\infty}((a))
$$

and

$$
E=\mathrm{Cl} .\{P\} \subseteq Z^{\infty}((a))
$$

which is a contradiction. Hence such a $P^{\prime}$ does not exist and the generic point $P$ is unique.

Remark 3.1.17. Contrarily to Commutative Algebra, every compact open set $K$ of the spectral topology $\operatorname{Spec}^{\infty}(A)$ is of the form $D^{\infty}(a)$ for some $a \in A$, since $K=D^{\infty}\left(a_{1}\right) \cup \cdots \cup D^{\infty}\left(a_{n}\right)=D^{\infty}\left(a_{1}^{2}+\cdots+a_{n}^{2}\right)$, for some $a_{1}, \cdots, a_{n} \in A$.

Definition 3.1.18. Let $(X, \tau)$ and $(Y, \sigma)$ be two spectral spaces. A map $f: X \rightarrow Y$ is a spectral map if, and only if, for every compact open $K \subseteq Y, f^{\dashv}[K] \subseteq X$ is compact open. 
Proposition 3.1.19. Let $A, A^{\prime}$ be two $\mathcal{C}^{\infty}$-rings and let $f: A \rightarrow A^{\prime}$ be a $\mathcal{C}^{\infty}$-homomorphism. The function:

$$
\begin{aligned}
& h^{*}: \operatorname{Spec}^{\infty}\left(A^{\prime}\right) \rightarrow \operatorname{Spec}^{\infty}(A) \\
& \mathfrak{p} \quad \mapsto \quad h^{\dagger}[\mathfrak{p}]
\end{aligned}
$$

is a spectral map.

Proof. We are going to show that the inverse image of every basic compact-open subset of $\operatorname{Spec}^{\infty}(A)$ is a basic compact open subset of $\operatorname{Spec}^{\infty}\left(A^{\prime}\right)$.

Let $D^{\infty}(a)$, for some $a \in A$, be a basic open subset of $\operatorname{Spec}^{\infty}(A)$. We have:

$$
\begin{aligned}
h^{*-1}\left[D^{\infty}(a)\right]=\left\{\mathfrak{p}^{\prime} \in \operatorname{Spec}^{\infty}\left(A^{\prime}\right) \mid h^{*}\left(\mathfrak{p}^{\prime}\right) \in D^{\infty}(a)\right\}=\left\{\mathfrak{p}^{\prime} \in \operatorname{Spec}^{\infty}\left(A^{\prime}\right) \mid h^{\dashv}\left[\mathfrak{p}^{\prime}\right] \in D^{\infty}(a)\right\}= \\
\left\{\mathfrak{p}^{\prime} \in \operatorname{Spec}^{\infty}\left(A^{\prime}\right) \mid a \notin h^{-1}\left[\mathfrak{p}^{\prime}\right]\right\}=\left\{\mathfrak{p}^{\prime} \in \operatorname{Spec}^{\infty}\left(A^{\prime}\right) \mid h(a) \notin \mathfrak{p}^{\prime}\right\}=D^{\infty}(h(a))
\end{aligned}
$$

so $h^{*-1}\left[D^{\infty}(a)\right]$ is a basic compact open subset of $\operatorname{Spec}^{\infty}\left(A^{\prime}\right)$. It follows that $h^{*}$ is a spectral map. In particular, it follows that $h^{*}$ is a continuous function.

Thus, we are able to define a contravariant functor from the category of $\mathcal{C}^{\infty}$-rings to the category of topological spaces:

$$
\begin{aligned}
& \text { Spec }^{\infty}: \quad \mathcal{C}^{\infty} \text { Rng } \quad \rightarrow \quad \text { Top } \\
& (A \stackrel{h}{\longrightarrow} B) \mapsto\left(\operatorname{Spec}^{\infty}(B) \stackrel{h^{*}}{\longrightarrow} \operatorname{Spec}^{\infty}(A)\right)
\end{aligned}
$$

Proposition 3.1.20. Let $A, B$ be two reduced $\mathcal{C}^{\infty}$-rings. By the Proposition 1.3.19, the ideals of $A \times B$ are precisely those of the form $\mathfrak{a} \times \mathfrak{b}$, where $\mathfrak{a}$ is an ideal of $A$ and $\mathfrak{b}$ is an ideal of $B$. Moreover, $\left(\mathfrak{a} \times \mathfrak{b} \in \operatorname{Spec}^{\infty}(A \times B)\right) \Longleftrightarrow\left(\left(\mathfrak{a} \in \operatorname{Spec}^{\infty}(A)\right) \&(\mathfrak{b}=B) \vee\left((\mathfrak{a}=A) \&\left(\mathfrak{b} \in \operatorname{Spec}^{\infty}(B)\right)\right)\right)$

Proof. One can easily see that the map:

$$
\begin{aligned}
\varphi: A \times B & \rightarrow \frac{A}{\mathfrak{a}} \times \frac{B}{\mathfrak{b}} \\
(a, b) & \mapsto \quad(a+\mathfrak{a}, b+\mathfrak{b})
\end{aligned}
$$

is a $\mathcal{C}^{\infty}$-rings homomorphism such that $\operatorname{ker} \varphi=\mathfrak{a} \times \mathfrak{b}$. By the Theorem of the Isomorphism, there is a unique $\widetilde{\varphi}: \frac{A \times B}{\mathfrak{a} \times \mathfrak{b}} \rightarrow \frac{A}{\mathfrak{a}} \times \frac{B}{\mathfrak{b}}$ such that:

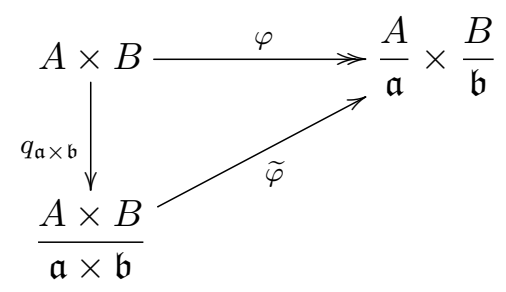

hence $\widetilde{\varphi}$ is a $\mathcal{C}^{\infty}$-isomorphism:

$$
\frac{A \times B}{\mathfrak{a} \times \mathfrak{b}} \stackrel{\widetilde{\varphi}}{\cong} \frac{A}{\mathfrak{a}} \times \frac{B}{\mathfrak{b}}
$$

It follows that $\mathfrak{a} \times \mathfrak{b} \in \operatorname{Spec}^{\infty}(A \times B)$ if, and only if, $\frac{A \times B}{\mathfrak{a} \times \mathfrak{b}}$ is a $\mathcal{C}^{\infty}$-domain. Since $\frac{A \times B}{\mathfrak{a} \times \mathfrak{b}} \cong \frac{\tilde{q}}{\mathfrak{a}} \times \frac{B}{\mathfrak{b}}$, it follows that $\frac{A \times B}{\mathfrak{a} \times \mathfrak{b}}$ is a $\mathcal{C}^{\infty}$-domain if, and only if, its isomorphic image, $\frac{A}{\mathfrak{a}} \times \frac{B}{\mathfrak{b}}$ is a $\mathcal{C}^{\infty}$-domain. 
Claim: $\frac{A}{\mathfrak{a}} \times \frac{B}{\mathfrak{b}}$ is a $\mathcal{C}^{\infty}$-domain if, and only if, one of these factors is 0 and the other is a $\mathcal{C}^{\infty}$-domain.

We prove that $\frac{A}{\mathfrak{a}} \times \frac{B}{\mathfrak{b}}$ is a $\mathcal{C}^{\infty}$-domain $\Rightarrow\left(\left(\frac{A}{\mathfrak{a}}\right.\right.$ is a $\mathcal{C}^{\infty}$-domain and $\left.\mathfrak{b}=B\right) \vee\left(\mathfrak{a}=A\right.$ and $\frac{B}{\mathfrak{b}}$ is a $\mathcal{C}^{\infty}$-domain)) via modus tollens.

Suppose we have both $\mathfrak{a} \neq A$ and $\mathfrak{b} \neq B$, so $\frac{A}{\mathfrak{a}} \neq\{0\}$ and $\frac{B}{\mathfrak{b}} \neq\{0\}$, and the $\mathcal{C}^{\infty}$-ring $\frac{A}{\mathfrak{a}} \times \frac{B}{\mathfrak{b}}$ has two non-trivial idempotent elements, namely $\left(1_{A}, 0_{B}\right)$ and $\left(0_{A}, 1_{B}\right)$, hence $\frac{A \times B}{\mathfrak{a} \times \mathfrak{b}} \cong \frac{A}{\mathfrak{a}} \times \frac{B}{\mathfrak{b}}$ is not a $\mathcal{C}^{\infty}$-domain.

Now we prove that $\left(\left(\frac{A}{\mathfrak{a}}\right.\right.$ is a $\mathcal{C}^{\infty}$-domain and $\left.\mathfrak{b}=B\right) \vee\left(\mathfrak{a}=A\right.$ and $\frac{B}{\mathfrak{b}}$ is a $\mathcal{C}^{\infty}$-domain $\left.)\right) \Rightarrow \frac{A}{\mathfrak{a}} \times \frac{B}{\mathfrak{b}}$ is a $\mathcal{C}^{\infty}$-domain.

Suppose that $\frac{A}{\mathfrak{a}}$ is a reduced $\mathcal{C}^{\infty}$-domain and $\mathfrak{b}=B$, then $\frac{A \times B}{\mathfrak{a} \times \mathfrak{b}} \cong \frac{A}{\mathfrak{a}} \times\{0\} \cong \frac{A}{\mathfrak{a}}$ is a reduced $\mathcal{C}^{\infty}$-domain. Now, if $\frac{B}{\mathfrak{b}}$ is a $\mathcal{C}^{\infty}$-domain and $\mathfrak{a}=A$, then $\frac{A \times B}{\mathfrak{a} \times \mathfrak{b}} \cong\{0\} \times \frac{B}{\mathfrak{b}} \cong \frac{B}{\mathfrak{b}}$ is again a $\mathcal{C}^{\infty}$-domain.

We have that $\frac{A \times B}{\mathfrak{a} \times \mathfrak{b}}$ is a $\mathcal{C}^{\infty}$-domain if, and only if, $\frac{A}{\mathfrak{a}} \times \frac{B}{\mathfrak{b}}$ is a $\mathcal{C}^{\infty}$-domain, and that $\frac{A}{\mathfrak{a}} \times \frac{B}{\mathfrak{b}}$ is a $\mathcal{C}^{\infty}$-domain if, and only if, one (and only one) of the following conditions holds:

(i) $\frac{A}{\mathfrak{a}} \cong\{0\}$ and $\frac{B}{\mathfrak{b}}$ is a $\mathcal{C}^{\infty}$-domain;

(ii) $\frac{A}{\mathfrak{a}}$ is a $\mathcal{C}^{\infty}$-domain and $\frac{B}{\mathfrak{b}} \cong\{0\}$;

If (i) is the case, then $\mathfrak{a}=A$ and $\mathfrak{b} \in \operatorname{Spec}^{\infty}(B)$, otherwise $\mathfrak{a} \in \operatorname{Spec}^{\infty}(A)$ and $\mathfrak{b}=B$. Since these two cases are the only possibilities, we have established that:

$$
\left(\mathfrak{a} \times \mathfrak{b} \in \operatorname{Spec}^{\infty}(A \times B)\right) \Longleftrightarrow\left(\left(\mathfrak{a} \in \operatorname{Spec}^{\infty}(A)\right) \&(\mathfrak{b}=B)\right) \vee\left((\mathfrak{a}=A) \&\left(\mathfrak{b} \in \operatorname{Spec}^{\infty}(B)\right)\right)
$$

Remark 3.1.21. The topology of $\operatorname{Spec}^{\infty}\left(A_{i}\right) \sqcup \operatorname{Spec}^{\infty}\left(A_{j}\right)$ is, by definition, the finest one such that:

$$
\begin{array}{ccc}
\imath_{k}: \operatorname{Spec}^{\infty}\left(A_{k}\right) & \rightarrow & \operatorname{Spec}^{\infty}\left(A_{i}\right) \sqcup \operatorname{Spec}^{\infty}\left(A_{j}\right) \\
\mathfrak{p}_{k} & \mapsto & \left(\mathfrak{p}_{k}, k\right)
\end{array}
$$

is continuous for $k=i, j$. Moreover:

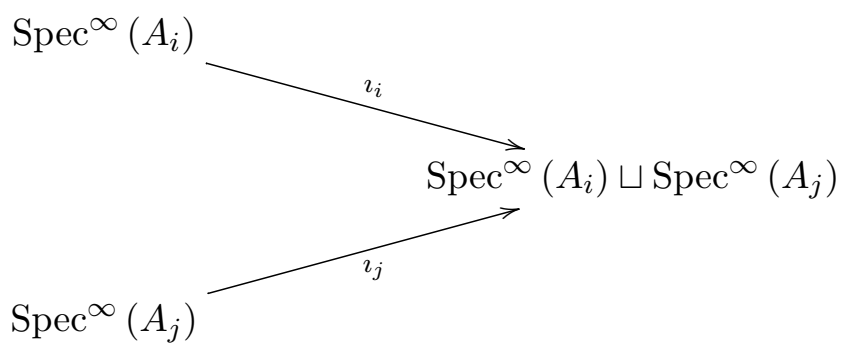

is the coproduct of $\operatorname{Spec}^{\infty}\left(A_{i}\right)$ and $\operatorname{Spec}^{\infty}\left(A_{j}\right)$ in the category Top. 
Proposition 3.1.22. Let $A_{i}, A_{j}$ be any two $\mathcal{C}^{\infty}$-rings.

The maps:

$$
\begin{array}{ccc}
\varphi_{i}: \operatorname{Spec}^{\infty}\left(A_{i}\right) & \rightarrow & \operatorname{Spec}^{\infty}\left(A_{i} \times A_{j}\right) \\
\mathfrak{p}_{i} & \mapsto & \mathfrak{p}_{i} \times A_{j}
\end{array}
$$

and

$$
\begin{array}{ccc}
\varphi_{j}: \operatorname{Spec}^{\infty}\left(A_{j}\right) & \rightarrow & \operatorname{Spec}^{\infty}\left(A_{i} \times A_{j}\right) \\
\mathfrak{p}_{j} & \mapsto & A_{i} \times \mathfrak{p}_{j}
\end{array}
$$

are both spectral maps (in particular, they are continuous).

Proof. Without loss of generality we prove that $\varphi_{i}$ is continuous.

Let $\left(a_{i}, a_{j}\right) \in A_{i} \times A_{j}$ and let $D^{\infty}\left(a_{i}, a_{j}\right):=\left\{\mathfrak{q} \in \operatorname{Spec}^{\infty}\left(A_{i} \times A_{j}\right) \mid\left(a_{i}, a_{j}\right) \notin \mathfrak{q}\right\}$ be any basic open subset of

$$
\operatorname{Spec}^{\infty}\left(A_{i} \times A_{j}\right)=\left\{\mathfrak{p}_{i} \times A_{j} \mid \mathfrak{p}_{i} \in \operatorname{Spec}^{\infty}\left(A_{i}\right)\right\} \dot{\cup}\left\{A_{i} \times \mathfrak{p}_{j} \mid \mathfrak{p}_{j} \in \operatorname{Spec}^{\infty}\left(A_{j}\right)\right\}
$$

We have

$$
D^{\infty}\left(a_{i}, a_{j}\right)=\left\{\mathfrak{p}_{i} \times A_{j} \mid \mathfrak{p}_{i} \in D^{\infty}\left(a_{i}\right)\right\} \dot{\cup}\left\{A_{i} \times \mathfrak{p}_{j} \mid \mathfrak{p}_{j} \in D^{\infty}\left(a_{j}\right)\right\}
$$

Thus,

$$
\varphi_{i}^{\dashv}\left[D^{\infty}\left(a_{i}, a_{j}\right)\right]=\left\{\mathfrak{p}_{i} \in \operatorname{Spec}^{\infty}\left(A_{i}\right) \mid\left(a_{i}, a_{j}\right) \notin \mathfrak{p}_{i} \times A_{j}\right\}
$$

Since $\left\{\mathfrak{p}_{i} \in \operatorname{Spec}^{\infty}\left(A_{i}\right) \mid\left(a_{i}, a_{j}\right) \notin \mathfrak{p}_{i} \times A_{j}\right\}=\left\{\mathfrak{p}_{i} \in \operatorname{Spec}^{\infty}\left(A_{i}\right) \mid a_{i} \notin \mathfrak{p}_{i}\right\}=D^{\infty}\left(a_{i}\right)$, it follows that:

$$
\varphi_{i}^{-1}\left[D^{\infty}\left(a_{i}, a_{j}\right)\right]=D^{\infty}\left(a_{i}\right),
$$

which is a basic open subset of $\operatorname{Spec}^{\infty}\left(A_{i}\right)$. Hence $\varphi_{i}$ is an spectral map, and in particular it is a continuous function. An analogous reasoning shows us that $\varphi_{j}$ is also a continuous map.

Remark 3.1.23. By the universal property of $\operatorname{Spec}^{\infty}\left(A_{i}\right) \sqcup \operatorname{Spec}^{\infty}\left(A_{j}\right)$, given the continuous maps $\varphi_{i}, \varphi_{j}$ of Proposition 3.1.22, there is a unique continuous function $\widetilde{\varphi}: \operatorname{Spec}^{\infty}\left(A_{i}\right) \sqcup \operatorname{Spec}^{\infty}\left(A_{j}\right) \rightarrow$ $\operatorname{Spec}^{\infty}\left(A_{i} \times A_{j}\right)$ such that:

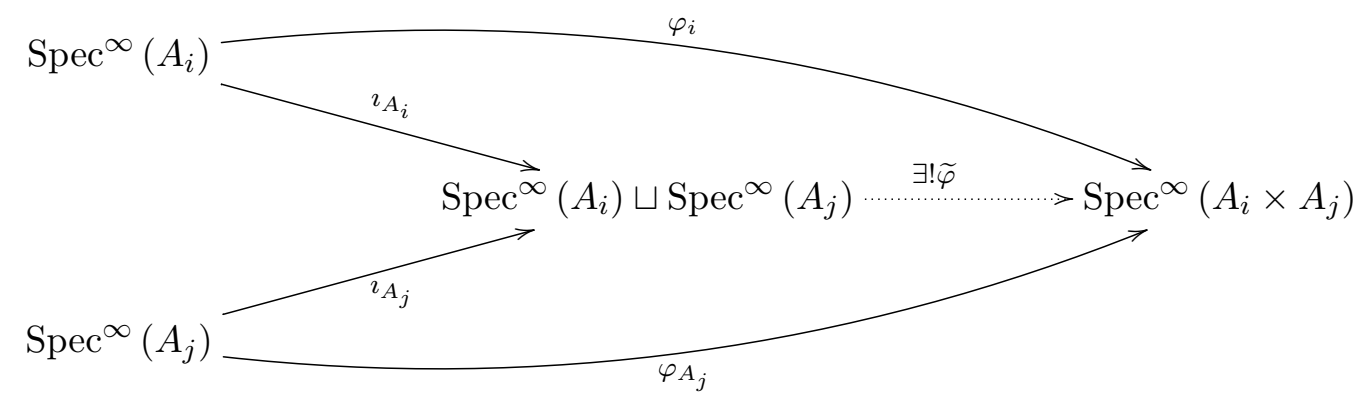

commutes, and since the function:

$$
\begin{aligned}
& \varphi: \operatorname{Spec}^{\infty}\left(A_{i}\right) \sqcup \operatorname{Spec}^{\infty}\left(A_{j}\right) \rightarrow \operatorname{Spec}^{\infty}\left(A_{i} \times A_{j}\right) \\
& \left(\mathfrak{p}_{i}, i\right) \quad \mapsto \quad \mathfrak{p}_{i} \times A_{j} \\
& \left(\mathfrak{p}_{j}, j\right) \quad \mapsto \quad A_{i} \times \mathfrak{p}_{j}
\end{aligned}
$$


is such that $\varphi \circ \imath_{A_{i}}=\varphi_{A_{i}}$ and $\varphi \circ \imath_{A_{j}}=\varphi_{A_{j}}$, it follows that $\varphi=\widetilde{\varphi}$, and $\varphi$ is a continuous map. by:

In virtue of the Propositions 1.3.19 and 3.1.20, it is clear that $\varphi$ is a bijection whose inverse is given

$$
\begin{array}{cccc}
\psi: \operatorname{Spec}^{\infty}\left(A_{i} \times A_{j}\right) & \rightarrow & \operatorname{Spec}^{\infty}\left(A_{i}\right) \sqcup \operatorname{Spec}^{\infty}\left(A_{j}\right) \\
\mathfrak{p}_{i} \times A_{j} & \mapsto & \left(\mathfrak{p}_{i}, i\right) \\
A_{i} \times \mathfrak{p}_{j} & \mapsto & \left(\mathfrak{p}_{j}, j\right)
\end{array}
$$

Theorem 3.1.24. Let $A_{i}, A_{j}$ be two $\mathcal{C}^{\infty}$-rings. The function:

$$
\begin{aligned}
& \varphi: \operatorname{Spec}^{\infty}\left(A_{i}\right) \sqcup \operatorname{Spec}^{\infty}\left(A_{j}\right) \rightarrow \operatorname{Spec}^{\infty}\left(A_{i} \times A_{j}\right) \\
& \left(\mathfrak{p}_{i}, i\right) \quad \mapsto \quad \mathfrak{p}_{i} \times A_{j} \\
& \left(\mathfrak{p}_{j}, j\right) \quad \mapsto \quad A_{i} \times \mathfrak{p}_{j}
\end{aligned}
$$

is an homeomorphism.

Proof. We saw above that $\varphi$ is a spectral/continuous bijection. To show that $\psi=\varphi^{-1}$ is a continuous map is equivalent to prove that $\varphi$ is an open map.

Let $D^{\infty}\left(a_{k}\right) \times\{k\}, k \in\{i, j\}$, be any basic open subset of $\operatorname{Spec}^{\infty}\left(A_{i}\right) \sqcup \operatorname{Spec}^{\infty}\left(A_{j}\right)$. We have, for $k=i$ (the case when $k=j$ is analogous):

$$
\varphi\left[D^{\infty}\left(a_{i}\right) \times\{i\}\right]=\left\{\mathfrak{q}_{i} \times A_{j} \mid\left(\mathfrak{q}_{i}, i\right) \in D^{\infty}\left(a_{i}\right) \times\{i\}\right\}=\left\{\mathfrak{q}_{i} \times A_{j} \mid a_{i} \notin \mathfrak{q}_{i}\right\}
$$

and we have:

$$
D^{\infty}\left(a_{i}, 0_{j}\right)=\left\{\mathfrak{q}_{i} \times A_{j} \mid a_{i} \notin \mathfrak{q}_{i}\right\} \dot{\cup}\left\{A_{i} \times \mathfrak{q}_{j} \mid 0_{j} \notin \mathfrak{q}_{j}\right\}=\left\{\mathfrak{q}_{i} \times A_{j} \mid a_{i} \notin \mathfrak{q}_{i}\right\}
$$

Thus $\varphi\left[D^{\infty}\left(a_{i}\right) \times\{i\}\right]=\left\{\mathfrak{q}_{i} \times A_{j} \mid a_{i} \notin \mathfrak{q}_{i}\right\}=D^{\infty}\left(a_{i}, 0_{j}\right)$, and $\varphi$ maps (basic) open sets to (basic) open sets. Since $\varphi$ is an open spectral/continuous bijection, it is an homeomorphism.

By induction, we obtain the following:

Theorem 3.1.25. Let $\left\{A_{i} \mid i \in\{1,2, \cdots, n\}\right\}$ be any finite family of $\mathcal{C}^{\infty}$-rings. We have:

$$
\operatorname{Spec}^{\infty}\left(\prod_{i=1}^{n} A_{i}\right) \approx \coprod_{i=1}^{n} \operatorname{Spec}^{\infty}\left(A_{i}\right)
$$

We also register the following consequence of the above theorem:

Theorem 3.1.26. Let $\mathbb{K}$ be any $\mathcal{C}^{\infty}$-field, let $I$ be a finite set and let Discr. $(I)=(I, \wp(I))$ be the corresponding discrete topological space. We have:

$$
\operatorname{Spec}^{\infty}\left(\mathbb{K}^{I}\right) \approx \operatorname{Discr} .(I)
$$

Proof. First note that $\operatorname{Spec}^{\infty}\left(\mathbb{K}^{I}\right)$ is a finite Boolean space.

Indeed, since $\mathbb{K}$ is a $\mathcal{C}^{\infty}$-field, $\mathbb{K}^{I}$ is a von Neumann regular $\mathcal{C}^{\infty}$-ring, so $\operatorname{Spec}^{\infty}\left(\mathbb{K}^{I}\right)=\operatorname{Specm}{ }^{\infty}\left(\mathbb{K}^{I}\right)$. Since $I$ is a finite set, we have:

$$
\operatorname{Spec}^{\infty}\left(\mathbb{K}^{I}\right)=\left\{\mathfrak{m}_{i}=\widehat{\left(\mathbb{K}^{I}\right)} \mid i \in I\right\} \cup\{0\} \cup\left\{\mathbb{K}^{I}\right\}
$$


Since $\mathbb{K}$ is a $\mathcal{C}^{\infty}$-field, the only $\mathcal{C}^{\infty}$-radical prime ideals of $\mathbb{K}$ are the maximal ones. Consider:

$$
\begin{aligned}
\varphi: \operatorname{Discr} .(I) & \rightarrow \\
i & \mapsto \mathfrak{m}_{i}=\widehat{\left.\mathbb{K}^{I}\right)_{i}}:=\mathbb{K} \times \cdots \times \mathbb{K} \times\{0\} \times \mathbb{K} \times \cdots \times \mathbb{K},
\end{aligned}
$$

which is obviously continuous since Discr. $(I)$ is a discrete space.

Theorem 3.1.27. Let $(I, \preceq)$ be a filtered partially ordered set and let $\left\{\left(A_{i}, \Phi_{i}\right) \mid i \in I\right\}$ be a direct filtered system of $\mathcal{C}^{\infty}$-rings, so we have the following colimit diagram:

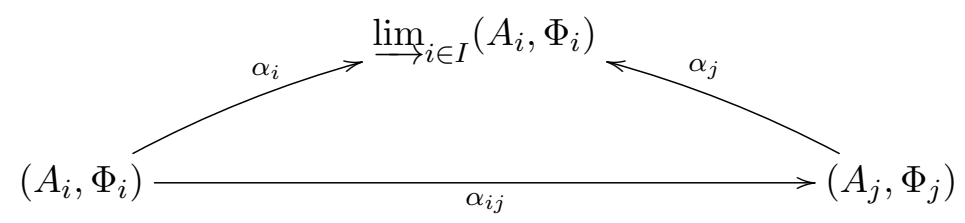

The following map is an homeomorphism

$$
\begin{aligned}
\kappa: \operatorname{Spec}^{\infty}\left({\underset{\lim }{\mathfrak{p}}}_{i \in I} A_{i}\right) & \underset{\rightarrow}{\rightarrow} \lim _{i \in I} \operatorname{Spec}^{\infty}\left(A_{i}\right) \\
& \mapsto\left(\alpha_{i}^{\dagger}[\mathfrak{p}]\right)_{i \in I}
\end{aligned}
$$

whose inverse is given by:

$$
\begin{aligned}
\kappa^{\prime}: \lim _{i \in I} \operatorname{Spec}^{\infty}\left(A_{i}\right) & \stackrel{\approx}{\rightarrow} \operatorname{Spec}^{\infty}\left(\mathfrak{\operatorname { l i m }}_{i \in I} A_{i \in I}\right) \\
& \mapsto \underline{\lim }_{i \in I} \mathfrak{p}_{i}=\bigcup_{i \in I} \alpha_{i}\left[\mathfrak{p}_{i}\right]
\end{aligned}
$$

Proof. Since for every $i \in I, \operatorname{Spec}^{\infty}\left(A_{i}\right)$ is a spectral space and the category of spectral spaces and spectral maps is closed under projective limits, it follows that:

$$
\varliminf_{i \in I}^{\lim } \operatorname{Spec}^{\infty}\left(A_{i}\right)
$$

is a spectral space, and for every $i \in I$,

$$
\begin{array}{rlc}
\alpha_{i}{ }^{*}: \lim _{i \in I} \operatorname{Spec}_{\left(\mathfrak{p}_{i}\right)_{i \in I}\left(A_{i}\right)} & \rightarrow & \operatorname{Spec}^{\infty}\left(A_{i}\right) \\
& \mapsto & \mathfrak{p}_{i}
\end{array}
$$

is a spectral map.

Also, since each

$$
\begin{aligned}
& \alpha_{i}: \quad\left(A_{i}, \Phi_{i}\right) \quad \rightarrow \quad \lim _{i \in I}\left(A_{i}, \Phi_{i}\right) \\
& a_{i} \quad \mapsto \quad \longrightarrow\left[\left(a_{i}, i\right)\right]
\end{aligned}
$$

is a $\mathcal{C}^{\infty}$-homomorphism, it follows that given any $\mathfrak{p} \in \operatorname{Spec}^{\infty}\left(\underline{\lim }_{i \in I}\left(A_{i}, \Phi_{i}\right)\right), \alpha_{i}^{*}(\mathfrak{p})=\alpha_{i}^{\dashv}[\mathfrak{p}] \in$ $\operatorname{Spec}^{\infty}\left(A_{i}\right)$.

We note, thus, that $\kappa$ is a spectral map, since each of its coordinates is spectral and the category of the spectral spaces is closed under projective limits.

Since the colimit diagram commutes, we have $\alpha_{i j}^{-1}\left[\mathfrak{p}_{j}\right]=\mathfrak{p}_{i}$, so:

$$
\mathfrak{p}_{i} \subseteq \alpha_{i j}^{-1}\left[\mathfrak{p}_{j}\right] \Longleftrightarrow \alpha_{i j}\left[\mathfrak{p}_{i}\right] \subseteq \mathfrak{p}_{j}
$$


thus we have the following commutative square:

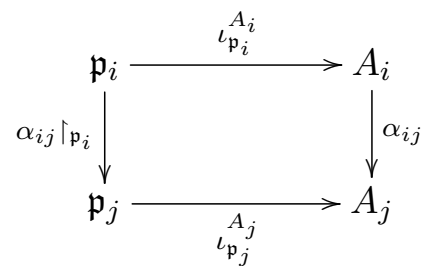

Given $\left(\mathfrak{p}_{i}\right)_{i \in I} \in \lim _{i \in I} \operatorname{Spec}^{\infty}\left(A_{i}\right)$, by Corollary 2.2.46 $\lim _{\rightarrow} \mathfrak{p}_{i}$ is a prime $\mathcal{C}^{\infty}$-radical ideal of $\lim _{i \in I} A_{i}$, so

$$
\begin{aligned}
& \kappa^{\prime}: \lim _{i \in I} \operatorname{Spec}^{\infty}\left(A_{i}\right) \rightarrow \operatorname{Spec}^{\infty}\left(\underline{\lim }_{i \in I} A_{i}\right) \\
& \left(\mathfrak{p}_{i}\right)_{i \in I} \quad \mapsto \quad \bigcup_{i \in I} \alpha_{i}\left[\mathfrak{p}_{i}\right]
\end{aligned}
$$

is indeed a function.

We have, thus:

$$
\kappa^{\prime}\left[\varliminf_{i \in I} \operatorname{Spec}^{\infty}\left(A_{i}, \Phi_{i}\right)\right] \subseteq \operatorname{Spec}^{\infty}\left(\lim _{i \in I}\left(A_{i}, \Phi_{i}\right)\right)
$$

so

$$
\kappa=\alpha \Gamma_{\operatorname{Spec}^{\infty}}\left(\lim _{\rightarrow \in I}\left(A_{i}, \Phi_{i}\right)\right)
$$

and

$$
\kappa^{\prime}=\alpha^{\prime}\left\lceil_{\lim _{i \in I}} \operatorname{Spec}^{\infty}\left(A_{i}, \Phi_{i}\right)\right.
$$

where $\alpha$ is given in Proposition 1.3.11 and $\alpha^{\prime}$ is given in its proof.

Since:

$$
\alpha\left[\operatorname{Spec}^{\infty}\left(\lim _{i \in I} A_{i}\right)\right]=\lim _{i \in I} \operatorname{Spec}^{\infty}\left(A_{i}\right)
$$

we have:

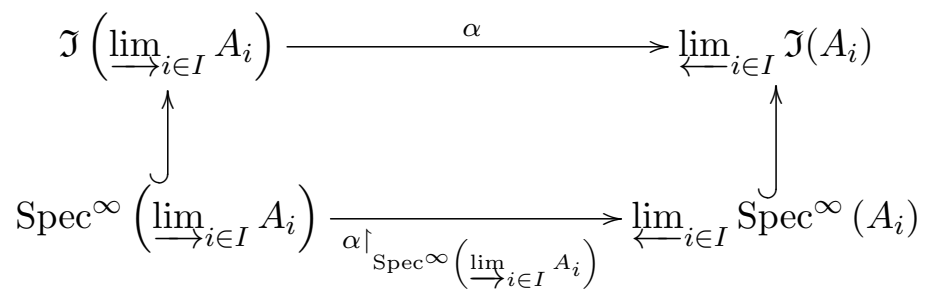

Since $\kappa$ and $\kappa^{\prime}$ are restrictions of inverse bijections, it follows that $\kappa$ and $\kappa^{\prime}$ are inverse bijections. 
Definition 3.1.28. Given a $\mathcal{C}^{\infty}$-ring $A,\left(\operatorname{Spec}^{\infty}(A), \mathrm{Zar}^{\infty}\right)$ is a spectral space (see Theorem 3.1.16). The constructible topology on $\operatorname{Spec}^{\infty}(A)$, denoted by $\operatorname{Spec}^{\infty-c o n s t}(A)$ is the smalest boolean topology in this set such that the identity function is continuous/spectral id: $\operatorname{Spec}^{\infty-c o n s t}(A) \rightarrow \operatorname{Spec}^{\infty}(A)$. It can be constructed by taking the sub-basis

$$
\left\{D^{\infty}(a) \cap Z^{\infty}(b) \mid a, b \in A\right\} .
$$

It is easy to see that, given any $\mathcal{C}^{\infty}$-ring $A, \operatorname{Spec}^{\infty-\text { const }}$ is indeed a Boolean space.

Remark 3.1.29. Contrary to what occurs in Commutative Algebra, the sub-basis of the constructible topology on $\operatorname{Spec}^{\infty}(A)$ given by $\left\{D^{\infty}(a) \cap Z^{\infty}(b) ; a, b \in A\right\}$ is closed under finite intersections (thus it is a basis), since

$$
D^{\infty}\left(a_{1}\right) \cap Z^{\infty}\left(b_{1}\right) \cap \cdots \cap D^{\infty}\left(a_{n}\right) \cap Z^{\infty}\left(b_{n}\right)=D^{\infty}\left(a_{1} . \cdots . a_{n}\right) \cap Z^{\infty}\left(b_{1}^{2}+\cdots+b_{n}^{2}\right) .
$$

\section{$3.2 \quad C^{\infty}$-rings and $C^{\infty}$-locally ringed spaces}

Like in Algebraic Geometry, there is a structural sheaf for each $C^{\infty}$-ring, such that the global section of this sheaf is canonically isomorphic to the original $C^{\infty}$-ring. The main reference for this section is [67].

Definition 3.2.1. $A \mathcal{C}^{\infty}$-ringed space is an ordered pair, $\left(X, \mathcal{O}_{X}\right)$, where $X$ is a topological space and:

$$
\mathcal{O}_{X}: \text { Open }(X)^{\text {op }} \rightarrow \mathcal{C}^{\infty} \mathbf{R n g}
$$

is a sheaf of $\mathcal{C}^{\infty}$-rings on $X$ such that all the stalks are local $\mathcal{C}^{\infty}$-rings, that is, for every $x \in X$, $\mathcal{O}_{X, x}$ is a local $\mathcal{C}^{\infty}$-ring.

Definition 3.2.2. Let $\left(X, \mathcal{O}_{X}\right)$ and $\left(Y, \mathcal{O}_{Y}\right)$ be two $\mathcal{C}^{\infty}$-ringed spaces. A morphism of $\mathcal{C}^{\infty}$-ringed spaces is a pair:

$$
\left(f, f^{\sharp}\right):\left(X, \mathcal{O}_{X}\right) \rightarrow\left(Y, \mathcal{O}_{Y}\right)
$$

where $f: X \rightarrow Y$ is a continuous map and $f^{\sharp}: f^{-}\left[\mathcal{O}_{Y}\right] \rightarrow \mathcal{O}_{X}$ is a morphism of sheaves of $\mathcal{C}^{\infty}$-rings on $X$ which induces a local $\mathcal{C}^{\infty}$-homomorphism of local $\mathcal{C}^{\infty}$-rings at each stalk,

$$
f_{x}^{\sharp}: f^{-}\left[\mathcal{O}_{Y, f(x)}\right] \rightarrow \mathcal{O}_{X, x}
$$

The category of all $\mathcal{C}^{\infty}$-locally ringed spaces, together with its morphisms, is going to be denoted by $\mathcal{C}^{\infty}$ RngSp.

Given a $\mathcal{C}^{\infty}$-ring $A$, we define a sheaf on $\operatorname{Spec}^{\infty}(A), \Sigma_{A}$ : Open $\left(\operatorname{Spec}^{\infty}(A)\right)^{\text {op }} \rightarrow \mathcal{C}^{\infty} \mathbf{R n g}$, such that for very $a \in A$,

$$
\Sigma_{A}\left(D^{\infty}(a)\right)=A\left\{a^{-1}\right\}
$$

In order to define the action of $\Sigma_{A}$ on the arrows between basic open subsets of $\operatorname{Spec}^{\infty}(A)$, we need some results.

Theorem 3.2.3. Let $A$ be $a \mathcal{C}^{\infty}$-ring and $a, b \in A$. The following conditions are equivalent: 
(1) $\eta_{a}(b) \in\left(A\left\{a^{-1}\right\}\right)^{\times}$;

(2) There is a unique $\mathcal{C}^{\infty}$-homomorphism $\sigma_{a, b}: A\left\{b^{-1}\right\} \rightarrow A\left\{a^{-1}\right\}$ such that the following triangle commutes:

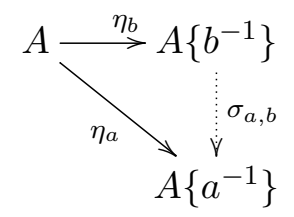

Proof. Ad (1) $\rightarrow(2)$. By the universal property of $\eta_{b}: A \rightarrow A\left\{b^{-1}\right\}$, since $\eta_{a}(b) \in\left(A\left\{a^{-1}\right\}\right)^{\times}$there is a unique $\mathcal{C}^{\infty}$-homomotphism $\sigma_{a, b}: A\left\{b^{-1}\right\} \rightarrow A\left\{a^{-1}\right\}$ such that the triangle commutes.

Ad $(2) \rightarrow(1)$. Suppose there is a unique $\mathcal{C}^{\infty}$-homomorphism $\sigma_{a, b}: A\left\{b^{-1}\right\} \rightarrow A\left\{a^{-1}\right\}$ such that $\sigma_{a, b} \circ \eta_{b}=\eta_{a}$. Then $\left(\sigma_{a, b} \circ \eta_{b}\right)(b)=\eta_{a}(b)$, i.e., $\sigma_{a, b}\left(\eta_{b}(b)\right)=\eta_{a}(b)$. Since $\eta_{b}(b) \in\left(A\left\{b^{-1}\right\}\right)^{\times}$, it follows that $\eta_{a}(b)=\sigma_{a, b}\left(\eta_{b}(b)\right) \in\left(A\left\{a^{-1}\right\}\right)^{\times}$.

Theorem 3.2.4. Let $A$ be a $\mathcal{C}^{\infty}$-ring. Suppose that the equivalent conditions in Theorem 3.1.3 hold. Then the equivalent conditions of Theorem 3.2.3 hold.

Proof. We are going to show that for any $a, b \in A, D^{\infty}(a)=D^{\infty}(b)$ implies $\eta_{a}(b) \in\left(A\left\{a^{-1}\right\}\right)^{\times}$.

We know that $\sqrt[\infty]{(b)}=\left\{x \in A \mid(\exists c \in(b))\left(\eta_{x}(c) \in\left(A\left\{a^{-1}\right\}\right)^{\times}\right)\right\}$, so $a \in \sqrt[\infty]{(b)} \Longleftrightarrow(\exists c \in(b))\left(\eta_{a}(c) \in\right.$ $\left.\left(A\left\{a^{-1}\right\}\right)^{\times}\right)$. Now, $c \in(b)$ occurs if, and only if, there is some $\lambda \in A$ such that $c=\lambda \cdot b$. Hence,

$$
\eta_{a}(c)=\eta_{a}(\lambda \cdot b)=\eta_{a}(\lambda) \cdot \eta_{a}(b)
$$

and since $\eta_{a}(c) \in\left(A\left\{a^{-1}\right\}\right)^{\times}$it follows that both $\eta_{a}(\lambda) \in\left(A\left\{a^{-1}\right\}\right)^{\times}$and $\eta_{a}(b) \in\left(A\left\{a^{-1}\right\}\right)^{\times}$.

The following two remarks will prove that $\left\{\sigma_{a, b}: A\left\{a^{-1}\right\} \rightarrow A\left\{b^{-1}\right\}\right\}_{a, b \in A}$ is a directed system of $\mathcal{C}^{\infty}$-homomorphisms.

Remark 3.2.5. Note that whenever we have $D^{\infty}(a)=D^{\infty}(b)$, by Theorem 3.2.4 we have a unique $\mathcal{C}^{\infty}$-isomorphism between $A\left\{a^{-1}\right\}$ and $A\left\{b^{-1}\right\}$, that follows from the uniqueness of the $\mathcal{C}^{\infty}$-homomorphisms $\sigma_{a, b}$ and $\sigma_{b, a}$ described in Theorem 3.2.3. In particular, when $a=b$ there exists a unique $\mathcal{C}^{\infty}$-homomorphism $\sigma_{a, a}: A\left\{a^{-1}\right\} \rightarrow A\left\{a^{-1}\right\}$ such that the following diagram commutes:

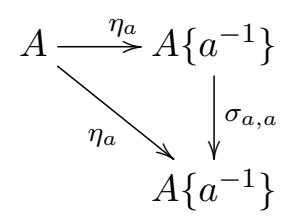

and since $\operatorname{id}_{A\left\{a^{-1}\right\}}$ also has this property, it follows that $\sigma_{a, a}=\operatorname{id}_{A\left\{a^{-1}\right\}}$.

Remark 3.2.6. Let $a, b, c \in A$ be such that $D^{\infty}(a) \subseteq D^{\infty}(b) \subseteq D^{\infty}(c)$. Then the following diagram commutes:

$$
A\left\{a^{-1}\right\} \underset{\sigma_{a, c}}{\stackrel{\sigma_{a, b}}{\longrightarrow} A\left\{b^{-1}\right\}}
$$


Proof. Since $D^{\infty}(a) \subseteq D^{\infty}(b) \subseteq D^{\infty}(c)$, we have that $D^{\infty}(a) \subseteq D^{\infty}(c)$, so by Theorem 3.2 .3 there exists a unique $\mathcal{C}^{\infty}$-homomorphism $\sigma_{a, c}: A\left\{a^{-1}\right\} \rightarrow A\left\{c^{-1}\right\}$ such that the following triangle commutes:

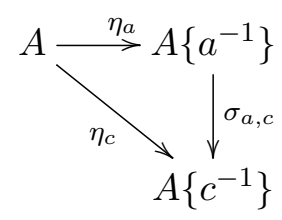

Since:

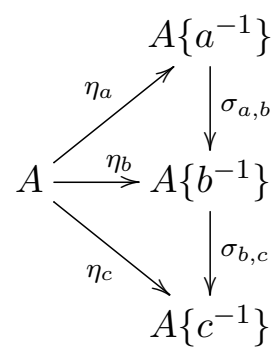

commutes, it follows that $\sigma_{b, c} \circ \sigma_{a, b}$ has the same property that defines $\sigma_{a, c}$, and by its uniqueness, $\sigma_{a, c}=\sigma_{b, c} \circ \sigma_{a, b}$

Now we build a pre-sheaf over the basic open subsets of $\operatorname{Spec}^{\infty}(A)$, i.e., the compact open subsets of $\operatorname{Spec}^{\infty}(A)$. Any basic open subset $U$ of $\operatorname{Spec}^{\infty}(A)$ is $U=D^{\infty}(a)$ for some $a \in A$. Given any basic open subset $U$ of $\operatorname{Spec}^{\infty}(A)$, define:

$$
\Sigma_{A}(U):=\lim _{U=D^{\infty}(a)} A\left\{a^{-1}\right\}
$$

that is, $\Sigma_{A}(U)$ is the colimit of the system $\left\{A\left\{a^{-1}\right\} \rightarrow^{\sigma_{a^{\prime}, a}} A\left\{a^{\prime-1}\right\} \mid a, a^{\prime} \in A\right.$ suchthat $U=D^{\infty}(a)=$ $\left.D^{\infty}\left(a^{\prime}\right)\right\}$, where $\sigma_{a^{\prime}, a}$ is the $\mathcal{C}^{\infty}$-isomorphism described by Remark $\mathbf{3 . 2 . 5}$

We note that given any basic open subset of $\operatorname{Spec}^{\infty}(A), U, \Sigma_{A}(U)$ has an $A$-algebra structure that is induced by the universal property of $\Sigma_{A}(U):=\lim _{\longleftarrow} D^{\infty}(a)=U$ A $A\left\{a^{-1}\right\}$. Indeed, given any $a, a^{\prime} \in A$ such that $D^{\infty}(a)=U=D^{\infty}\left(a^{\prime}\right)$, we have a unique $\mathcal{C}^{\infty}$-homomorphism $\nu_{A}: A \rightarrow \Sigma_{A}(U)$ such that the following diagram commutes:

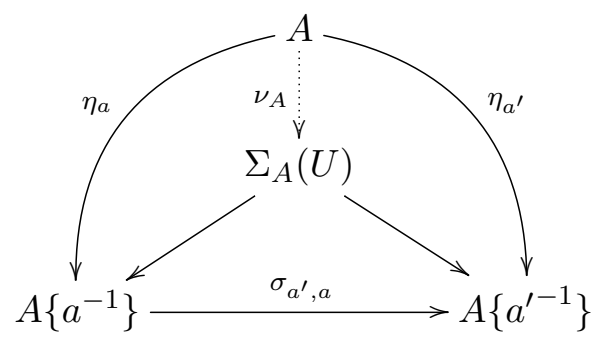

Let $U, V$ be two open subsets of $\operatorname{Spec}^{\infty}(A)$ such that $\imath_{U}^{V}: U \hookrightarrow V$ is the inclusion map, i.e., $U=$ $D^{\infty}(a) \subseteq D^{\infty}(c)=V$, and let $c, a \in A$ be two elements such that $V=D^{\infty}(c)$ and $U=D^{\infty}(a)$. Since $D^{\infty}(a) \subseteq D^{\infty}(c)$, by Theorem 3.2.4 there is a unique $\mathcal{C}^{\infty}$-homomorphism $\sigma_{c, a}: A\left\{a^{-1}\right\} \rightarrow A\left\{c^{-1}\right\}$, that we shall denote by $\rho_{U}^{V}:=\sigma_{c, a}$ such that the following triangle commutes:

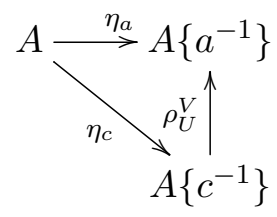

Let $p_{c}^{V}: \lim _{D^{\infty}\left(c^{\prime}\right)=V} A\left\{c^{\prime^{-1}}\right\} \rightarrow A\left\{c^{-1}\right\}$ and $p_{a}^{U}: \lim _{D^{\infty}\left(a^{\prime}\right)=U} A\left\{a^{\prime-1}\right\} \rightarrow A\left\{a^{-1}\right\}$ be the canonical colimit arrows, that are also $\mathcal{C}^{\infty}$-isomorphisms. We have the following diagram: 


$$
\begin{array}{ll}
\Sigma_{A}(V)=\lim _{D^{\infty}(c)=V} A\left\{c^{-1}\right\} \stackrel{p_{c}^{V}}{\longrightarrow} & A\left\{c^{-1}\right\} \\
\Sigma_{A}(U)=\lim _{U} \uparrow & \\
D^{\infty}(a)=U & A\left\{a^{-1}\right\} \stackrel{p_{a}^{U}}{\longrightarrow} A\left\{a^{-1}\right\}
\end{array}
$$

Since $\left\{\sigma_{c^{\prime}, a^{\prime}} \circ p_{a^{\prime}}^{U}: \Sigma_{A}(U) \rightarrow A\left\{c^{\prime-1}\right\} \mid c^{\prime} \in A\right.$ such that $\left.D^{\infty}\left(c^{\prime}\right)=V\right\}$ is a cone, by the universal property of $\Sigma_{A}(V)$ there exists a unique $\mathcal{C}^{\infty}$-homomorphism such that the following prism commutes for every $a, a^{\prime}, c, c^{\prime} \in A$ such that $U=D^{\infty}(a)=D^{\infty}\left(a^{\prime}\right)$ and $V=D^{\infty}(c)=D^{\infty}\left(c^{\prime}\right)$ :

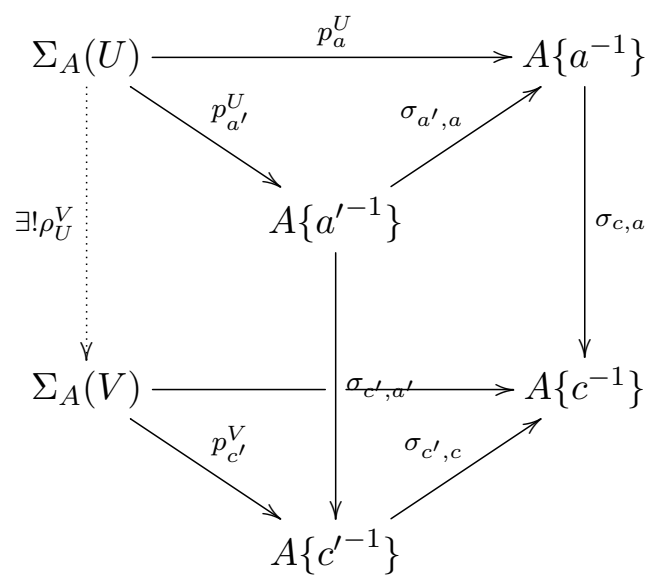

By the uniqueness of the arrow $\rho_{U}^{V}$, it follows that given basic open subsets $U, V, W$ of $\operatorname{Spec}^{\infty}(A)$ such that $U \subset V \subset W$,

$$
\Sigma_{A}\left(\imath_{V}^{W} \circ \imath_{U}^{V}\right)=\Sigma_{A}\left(\imath_{U}^{W}\right)=\Sigma_{A}\left(\imath_{V}^{W}\right) \circ \Sigma_{A}\left(\imath_{U}^{V}\right)
$$

and

$\Sigma_{A}\left(\imath_{U}^{U}\right)=\mathrm{id}_{\Sigma_{A}(U)}^{\Sigma_{A}(U)}$

Thus we have defined a functor:

$$
\begin{array}{clc}
\Sigma_{A}: \operatorname{Open}\left(\mathcal{B}\left(\operatorname{Spec}^{\infty}(A)\right)\right)^{\mathrm{op}} & \rightarrow & \mathcal{C}^{\infty} \mathbf{R n g} \\
U & \mapsto \Sigma_{A}(U)={\underset{\lim }{U}}_{U=D^{\infty}(a)} A\left\{a^{-1}\right\} \\
\imath_{U}^{V}: U \hookrightarrow V & \mapsto & \rho_{U}^{V}: \Sigma_{A}(U) \rightarrow \Sigma_{A}(V)
\end{array}
$$

hence a pre-sheaf of $\mathcal{C}^{\infty}$-rings on the basic open sets of $\operatorname{Spec}^{\infty}(A)$.

We extend this definition from the basic open sets to any open set $U$ of $\left.\operatorname{Spec}^{\infty}(A)\right)$ :

$$
\Sigma_{A}(U):=\lim _{D^{\infty} \overleftarrow{(a)} \subseteq U} \Sigma_{A}\left(D^{\infty}(a)\right) .
$$

Given $U, V \in$ Open $\left(\operatorname{Spec}^{\infty}(A)\right)$ such that $U \subseteq V$, we denote:

$$
\Sigma_{A}(V) \stackrel{\sigma_{U}^{V}}{\rightarrow} \Sigma_{A}(U)
$$

the $\mathcal{C}^{\infty}$-homomorphism induced by the inclusion $\iota_{U}^{V}: U \hookrightarrow V$, via the universal property of the limit.

In 67], we can find a proof of the following: 
Proposition 3.2.7 (Proposition 1.6 of 67]). Let $A$ be a $\mathcal{C}^{\infty}$-ring and let $\operatorname{Spec}^{\infty}(A)$ be its smooth Zariski spectrum. Using the notations of the above considerations,

$$
\Sigma_{A}: \text { Open }\left(\operatorname{Spec}^{\infty}(A)\right)^{\mathrm{op}} \rightarrow \mathcal{C}^{\infty} \mathbf{R n g}
$$

is a sheaf and all its stalks are local $\mathcal{C}^{\infty}$-rings. This means that, given any $\mathfrak{p} \in \operatorname{Spec}^{\infty}(A), A_{\mathfrak{p}} \cong$ $A\left\{A \backslash \mathfrak{p}^{-1}\right\}$ is a local $\mathcal{C}^{\infty}$-ring.

Summarizing the previous results, the $\mathcal{C}^{\infty}$-Zariski spectrum of a $\mathcal{C}^{\infty}$-ring $A$ can be regarded as a $\mathcal{C}^{\infty}$-ringed space, $\left(\operatorname{Spec}^{\infty}(A), \Sigma_{A}\right)$. In [67], we find the following:

Theorem 3.2.8 (Theorem 1.7 of $[67])$. Let $A$ be any $\mathcal{C}^{\infty}$-ring.

$$
\begin{aligned}
\text { Spec }^{\infty}: \mathcal{C}^{\infty} \text { Rng } & \rightarrow \quad \mathcal{C}^{\infty} \text { RngdSp } \\
A & \mapsto \quad\left(\operatorname{Spec}^{\infty}(A), \Sigma_{A}\right)
\end{aligned}
$$

is a contravariant functor from the category of $\mathcal{C}^{\infty}$-rings and the category of the $\mathcal{C}^{\infty}$-ringed spaces, which is adjoint to the global sections functor, i.e., for every $\mathcal{C}^{\infty}$-ring $A$ and for every $\mathcal{C}^{\infty}$-ringed space $\left(X, \mathcal{O}_{X}\right)$ there is a natural bijection:

$$
\frac{A \rightarrow \Gamma\left(X, \mathcal{O}_{X}\right)}{\left(X, \mathcal{O}_{X}\right) \rightarrow\left(\operatorname{Spec}^{\infty}(A), \Sigma_{A}\right)}
$$

Moreover, $\Gamma\left(\operatorname{Spec}^{\infty}(A), \Sigma_{A}\right) \cong A$, so $\operatorname{Spec}^{\infty}$ is a full and faithful functor.

\subsection{Sheaves and von Neumann Regular $\mathcal{C}^{\infty}$-Rings}

In this section we present applications of sheaf-theoretic notions to von Neumann Regular $\mathcal{C}^{\infty}$-Rings.

\subsubsection{The von Neumann Regular Hull of a $\mathcal{C}^{\infty}-$ Ring}

In this subsection we follow [2].

As defined, the affine scheme of a $\mathcal{C}^{\infty}$-ring $A$ is the $\mathcal{C}^{\infty}$-locally ringed space $\left(\operatorname{Spec}^{\infty}(A), \Sigma_{A}\right)$, where given any basic open set $D^{\infty}(a)$, for some $a \in A$, one defines $\Sigma_{A}\left(D^{\infty}(a)\right):=A\left\{a^{-1}\right\}$. The stalks of this sheaf are local $\mathcal{C}^{\infty}$-rings. In particular we have, for any $\mathcal{C}^{\infty}$-ring $A$ :

$$
\Gamma\left(\operatorname{Spec}^{\infty}(A), \Sigma_{A}\right) \cong A
$$

as stated in Theorem $\mathbf{3 . 2 . 8}$. This fact suggests us that if we get a sheaf of $\mathcal{C}^{\infty}$-rings over the booleanization of the spectral space $\operatorname{Spec}^{\infty}(A)$, that is, over $\operatorname{Spec}^{\infty}(A)$ together with the constructible topology, whose stalk at each prime $\mathcal{C}^{\infty}$-radical ideal $\mathfrak{p}$ is the residue field $k_{\mathfrak{p}}(A)$ of the local $\mathcal{C}^{\infty}$-ring $A_{\mathfrak{p}}$, then the ring of global sections of this sheaf should be the "closest" von Neumann regular $\mathcal{C}^{\infty}$-ring to $A$.

In the following, we are to see that if a $\mathcal{C}^{\infty}$-ring $A$ has a von Neumann regular hull, that is, a pair $\left(\nu_{A}, \mathrm{vN}(A)\right)$ where $\nu_{A}: A \rightarrow \mathrm{vN}(A)$ is such that for any von Neumann regular $\mathcal{C}^{\infty}$-ring $B$ and every $\mathcal{C}^{\infty}$-homomorphism $f: A \rightarrow B$ there is a unique $\mathcal{C}^{\infty}$-homomorphism $\tilde{f}: \operatorname{vN}(A) \rightarrow B$ such that the following diagram commutes: 


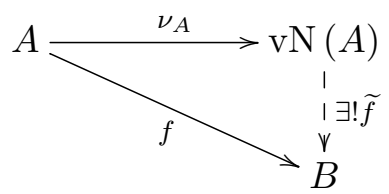

then the spectral sheaf of $\mathrm{vN}(A)$ must be such that the topological space $\operatorname{Spec}^{\infty}(\mathrm{vN}(A))$ is homeomorphic to the booleanization of $\operatorname{Spec}^{\infty}(A)$ and the stalks $\mathrm{vN}(A)_{\mathfrak{p}}$, for $\mathfrak{p} \in \operatorname{Spec}^{\infty}(\mathrm{vN}(A)$ ), are isomorphic to the residue fields of the local $\mathcal{C}^{\infty}$-ring $A_{\mathfrak{p}}$, for $\mathfrak{p} \in \operatorname{Spec}^{\infty}(A)$.

In fact, every $\mathcal{C}^{\infty}$-ring has a von Neumann-regular hull.

Given any $\mathcal{C}^{\infty}$-ring $A$, there exists a von Neumann regular $\mathcal{C}^{\infty}$-ring and a $\mathcal{C}^{\infty}$-homomorphism $\nu: A \rightarrow V$ such that for every $\mathcal{C}^{\infty}$-ring $B$ and any $\mathcal{C}^{\infty}$-homomorphism $g: A \rightarrow B$ there is a unique $\mathcal{C}^{\infty}$-homomorphism $f: V \rightarrow B$ such that the following diagram commutes:

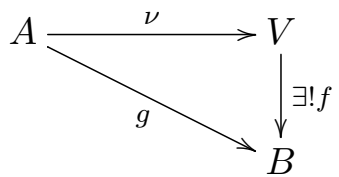

It will follow that the inclusion functor $i: \mathcal{C}^{\infty} \mathbf{v N R n g} \hookrightarrow \mathcal{C}^{\infty} \mathbf{R n g}$ has a left-adjoint $\nu$, that is, $\mathcal{C}^{\infty} \mathbf{v N R n g}$ is a reflexive subcategory of $\mathcal{C}^{\infty} \mathbf{R n g}$ (cf. p. 91 of $[49]$ ).

Given the $\mathcal{C}^{\infty}$-ring $(A, \Phi)$, we first take the coproduct of $A$ with the free $\mathcal{C}^{\infty}$-ring generated by $A$, $\eta_{A}: A \rightarrow(L(A), \Omega):$

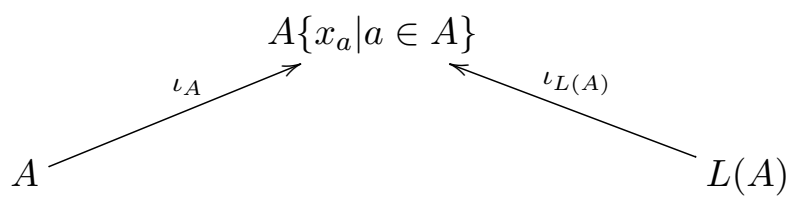

where:

$$
\begin{array}{ccc}
\iota_{A}: \quad A & \rightarrow & A\left\{x_{a} \mid a \in A\right\} \\
a & \mapsto & \iota_{A}(a)
\end{array}
$$

and:

$$
\begin{aligned}
\iota_{L(A)}: \quad L(A) & \rightarrow A\left\{x_{a} \mid a \in A\right\} \\
\widetilde{a} & \mapsto \iota_{L(A)}(\widetilde{a})
\end{aligned}
$$

and then we divide it by the ideal generated by the set:

$\left\{\left(\iota_{L(A)} \circ \eta_{A}\right)(a) \cdot \iota_{A}(a) \cdot\left(\iota_{L(A)} \circ \eta_{A}\right)(a)-\left(\iota_{L(A)} \circ \eta_{A}\right)(a), \iota_{A}(a) \cdot\left(\iota_{L(A)} \circ \eta_{A}\right)(a) \cdot \iota_{A}(a)-\iota_{A}(a) \mid a \in A\right\}$

so we have:

$\frac{A\left\{x_{a} \mid a \in A\right\}}{\left\langle\left\{\left(\iota_{L(A)} \circ \eta_{A}\right)(a) \cdot \iota_{A}(a) \cdot\left(\iota_{L(A)} \circ \eta_{A}\right)(a)-\left(\iota_{L}(A) \circ \eta_{A}\right)(a), \iota_{A}(a) \cdot\left(\iota_{L(A)} \circ \eta_{A}\right)(a) \cdot \iota_{A}(a)-\iota_{A}(a) \mid a \in A\right\}\right\rangle}$ 
In order to simplify our exposition, within this context we are going to write $x_{a}$ to denote $\left(\iota_{L(A)} \circ\right.$ $\left.\eta_{A}\right)(a) \in A\left\{x_{a} \mid a \in A\right\}$ for any $a \in A$, and $a$ to denote $\iota_{A}(a) \in A\left\{x_{a} \mid a \in A\right\}$. Thus we write:

$$
\frac{A\left\{x_{a} \mid a \in A\right\}}{\left\langle\left\{x_{a} \cdot a \cdot x_{a}-x_{a}, a \cdot x_{a} \cdot a-a \mid a \in A\right\}\right\rangle}
$$

instead of (3.1).

Given a von Neumann regular $\mathcal{C}^{\infty}$-ring $V$ and a $\mathcal{C}^{\infty}$-homomorphism $f: A \rightarrow V$, define the function:

$$
\begin{aligned}
F: A & \rightarrow V \\
a & \mapsto f(a)^{*}
\end{aligned}
$$

where $f(a)^{*}$ denotes the quasi-inverse of $f(a)$ in $V$.

From the universal property of the free $\mathcal{C}^{\infty}$-ring generated by $A, L(A)$, there is a unique $\mathcal{C}^{\infty}$-homomorphism $\widetilde{F}: L(A) \rightarrow V$ such that the following diagram commutes:

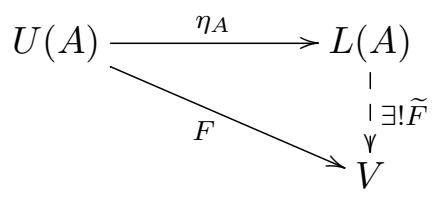

By the universal property of the $\mathcal{C}^{\infty}$-coproduct, given the $\mathcal{C}^{\infty}$-homomorphisms $f: A \rightarrow V$ and $\widetilde{F}$ : $L(A) \rightarrow V$ (the latter is constructed from $f$ ), there is a unique $\mathcal{C}^{\infty}$-homomorphism $\widehat{f}: A\left\{x_{a} \mid a \in A\right\} \rightarrow V$ such that:

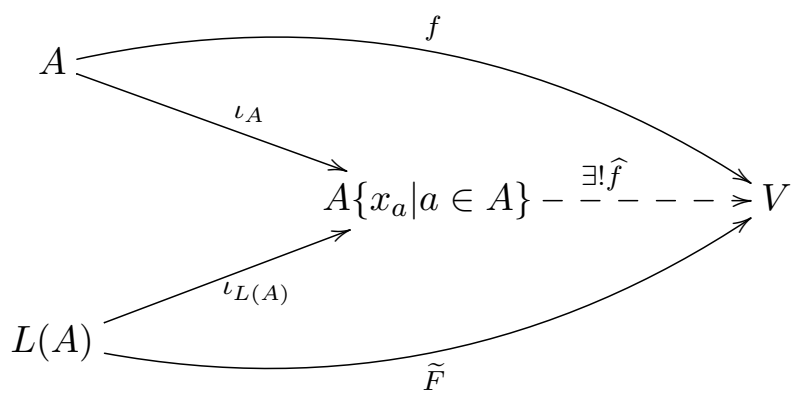

commutes.

Note that $\left\{x_{a} \cdot a \cdot x_{a}-x_{a}, a \cdot x_{a} \cdot a-a \mid a \in A\right\} \subseteq \operatorname{ker}(\widehat{f})$, for given any $\iota_{A}(a)=a,\left(\iota_{L(A)} \circ \eta_{A}\right)(a)=$ $x_{a} \in A\left\{x_{a} \mid a \in A\right\}$ :

$$
\widehat{f}\left(x_{a} \cdot a \cdot x_{a}-x_{a}\right)=\widehat{f}\left(x_{a}\right) \cdot \widehat{f}(a) \cdot \widehat{f}\left(x_{a}\right)-\widehat{f}\left(x_{a}\right)=f(a)^{*} \cdot f(a) \cdot f(a)^{*}-f(a)^{*}=0
$$

and

$$
\widehat{f}\left(a \cdot x_{a} \cdot a-a\right)=\widehat{f}(a) \cdot \widehat{f}\left(x_{a}\right) \cdot \widehat{f}(a)-\widehat{f}(a)=f(a) \cdot f(a)^{*} \cdot f(a)-f(a)=0 .
$$

By the Theorem of Homomorphism, there is a unique $\mathcal{C}^{\infty}$-homomorphism:

$$
\bar{f}: \frac{A\left\{x_{a} \mid a \in A\right\}}{\left\langle\left\{x_{a} \cdot a \cdot x_{a}-x_{a}, a \cdot x_{a} \cdot a-a \mid a \in A\right\}\right\rangle} \rightarrow V
$$


such that the following diagram commutes:

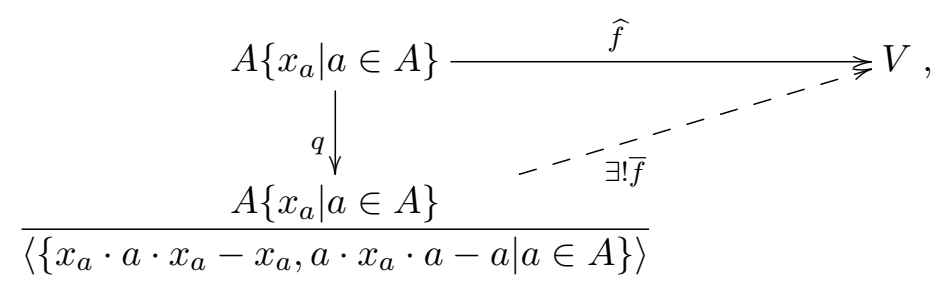

where $q: A\left\{x_{a} \mid a \in A\right\} \rightarrow \frac{A\left\{x_{a} \mid a \in A\right\}}{\left\langle\left\{x_{a} \cdot a \cdot x_{a}-x_{a}, a \cdot x_{a} \cdot a-a \mid a \in A\right\}\right\rangle}$ is the canonical quotient map.

Thus we obtain a $\mathcal{C}^{\infty}$-ring $\frac{A\left\{x_{a} \mid a \in A\right\}}{\left\langle\left\{x_{a} \cdot a \cdot x_{a}-x_{a}, a \cdot x_{a} \cdot a-a \mid a \in A\right\}\right\rangle}$ together with a $\mathcal{C}^{\infty}$-homomorphism $\nu_{0,1}=q \circ \iota_{A}: A \rightarrow \frac{A\left\{x_{a} \mid a \in A\right\}}{\left\langle\left\{x_{a} \cdot a \cdot x_{a}-x_{a}, a \cdot x_{a} \cdot a-a \mid a \in A\right\}\right\rangle}$ that have the following universal property: given any von Neumann regular $\mathcal{C}^{\infty}$-ring $V$ and any $\mathcal{C}^{\infty}$-homomorphism $f: A \rightarrow V$, there is a unique $\mathcal{C}^{\infty}$-homomorphism $\bar{f}: \frac{A\left\{x_{a} \mid a \in A\right\}}{\left\langle\left\{x_{a} \cdot a \cdot x_{a}-x_{a}, a \cdot x_{a} \cdot a-a \mid a \in A\right\}\right\rangle} \rightarrow V$ such that the following diagram commutes:

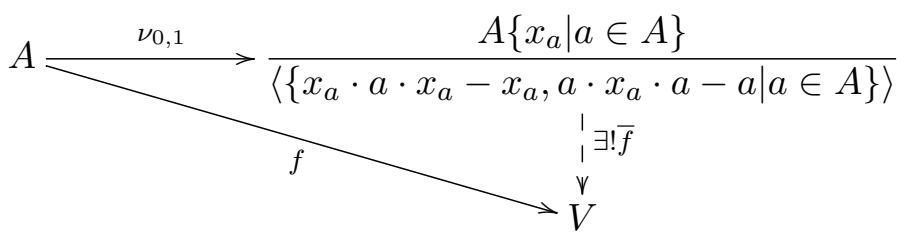

We have the following:

Lemma 3.3.1. Given a $\mathcal{C}^{\infty}-$ ring $B$, let:

$$
B^{+}:=\frac{B\left\{x_{b} \mid b \in B\right\}}{\left\langle\left\{x_{b} \cdot b \cdot x_{b}-x_{b}, b \cdot x_{b} \cdot b-b \mid b \in B\right\}\right\rangle}
$$

and $\nu_{0,1}: B \rightarrow B^{+}$be the following $\mathcal{C}^{\infty}$-homomorphism:

$$
\begin{aligned}
& \nu_{0,1}: \quad B \quad \rightarrow \quad B^{+} \\
& b \mapsto q\left(\iota_{B}(b)\right)
\end{aligned}
$$

where $\iota_{B}: B \rightarrow B\left\{x_{b} \mid b \in B\right\}$ is the inclusion map in the coproduct of $B$ and $L(B)$, and $q: B\left\{x_{b} \mid b \in\right.$ $B\} \rightarrow \frac{B\left\{x_{b} \mid b \in B\right\}}{\left\langle\left\{x_{b} \cdot b \cdot x_{b}-x_{b}, b \cdot x_{b} \cdot b-b \mid b \in B\right\}\right\rangle}$ is the canonical quotient map.

Given any von Neumann regular $\mathcal{C}^{\infty}$-ring $V$ and any $\mathcal{C}^{\infty}$-homomorphism $f: B \rightarrow V$, there is a unique $\mathcal{C}^{\infty}$-homomorphism $\bar{f}: B^{+} \rightarrow V$ such that the following diagram commutes:

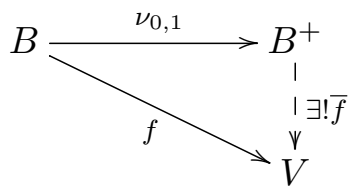

Proof. The existence of such a $\mathcal{C}^{\infty}$-homomorphism $\bar{f}$ has already been established in the construction, so we need only to prove its uniqueness. 
Let $f^{\prime}: \frac{B\left\{x_{b} \mid b \in B\right\}}{\left\langle\left\{x_{b} \cdot b \cdot x_{b}-x_{b}, b \cdot x_{b} \cdot b-b \mid b \in B\right\}\right\rangle} \rightarrow V$ be a $\mathcal{C}^{\infty}$-homomorphism such that $\overline{f^{\prime}} \circ \nu_{0,1}=0$, that is, such that for every $b \in A, \overline{f^{\prime}} \circ\left(q \circ \iota_{B}\right)(b)=f(b)$. This implies $\left(\left(\overline{f^{\prime}} \circ q \circ \iota_{B}\right)(b)\right)^{*}=f(b)^{*}$.

Also, since $\overline{f^{\prime}}$ is a $\mathcal{C}^{\infty}$-homomorphism, it follows that:

$$
\overline{f^{\prime}}\left(q\left(\iota_{B}(b)\right) \cdot q\left(\iota_{L(B)}(b)\right) \cdot q\left(\iota_{B}(b)\right)-q\left(\iota_{B}(b)\right)\right)=\overline{f^{\prime}}(0)=0
$$

so

$$
\overline{f^{\prime}}\left(q\left(\iota_{B}(b)\right)\right) \cdot \overline{f^{\prime}}\left(q\left(\iota_{L(B)}(b)\right)\right) \cdot \overline{f^{\prime}}\left(q\left(\iota_{B}(b)\right)\right)-\overline{f^{\prime}}\left(q\left(\iota_{B}(b)\right)\right)=0
$$

and

$$
f(b) \cdot\left[\overline{f^{\prime}}\left(q\left(\iota_{B}(b)\right)\right)\right] \cdot f(b)-f(b)=0 .
$$

Analogously we prove that:

$$
\left[\overline{f^{\prime}}\left(q\left(\iota_{B}(b)\right)\right)\right] \cdot f(b) \cdot\left[\overline{f^{\prime}}\left(q\left(\iota_{B}(b)\right)\right)\right]-\left[\overline{f^{\prime}}\left(q\left(\iota_{B}(b)\right)\right)\right]=0,
$$

so

$$
(\forall b \in B)\left(\overline{f^{\prime}}\left(q\left(\iota_{B}(b)\right)\right)=f(b)^{*}\right)
$$

and the following diagram commutes:

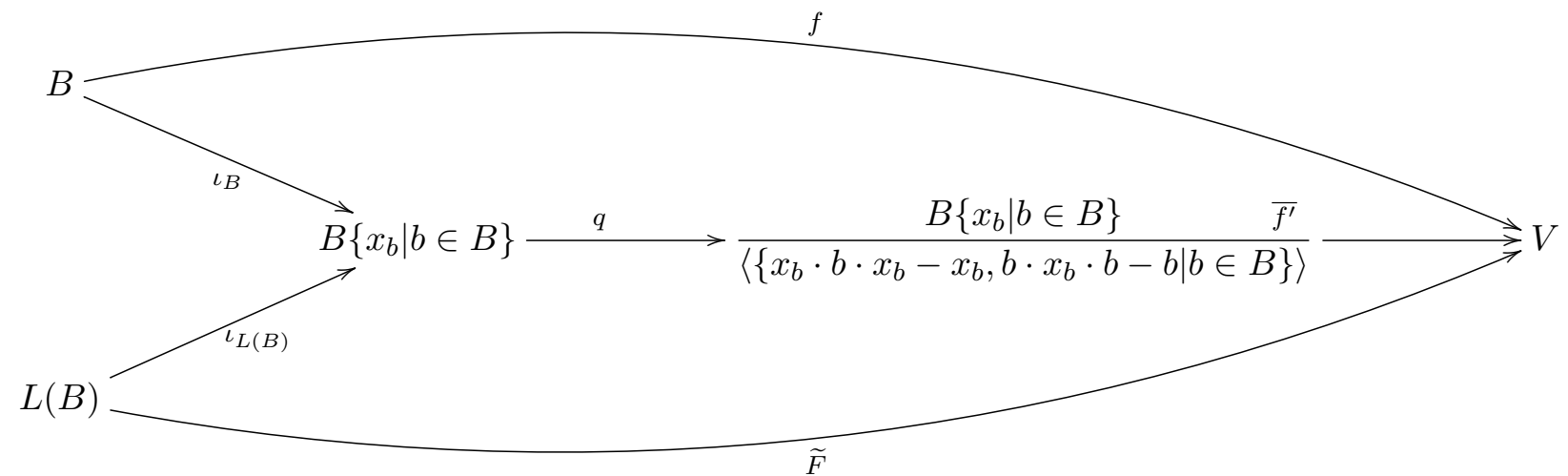

where for every $x_{b} \in L(B), \widetilde{F}\left(x_{b}\right)=f(b)^{*}$.

Now, $\bar{f}$ is the unique $\mathcal{C}^{\infty}$-homomorphism such that $\bar{f} \circ q=\widehat{f}$, and $\widehat{f}$ is the unique $\mathcal{C}^{\infty}$-homomorphism such that both $\widehat{f} \circ \iota_{B}=f$ and $\widehat{f} \circ \iota_{L(B)}=\widetilde{F}$. Thus, since $\overline{f^{\prime}} \circ q \circ \iota_{B}=f$ and $\overline{f^{\prime}} \circ q \circ \iota_{L(B)}=\widetilde{F}$, it follows that $\bar{f}=\overline{f^{\prime}}$.

Let $A$ be any $\mathcal{C}^{\infty}$-ring, so we define:

$$
\left\{\begin{array}{l}
A_{0}:=A \\
A_{n+1}:=A_{n}^{+}
\end{array}\right.
$$

and for every $n \in \mathbb{N}$ we define $\nu_{n, n+1}: A_{n} \rightarrow A_{n}^{+}=A_{n+1}$ as: 


$$
\begin{aligned}
\nu_{n, n+1}: \quad A_{n} & \rightarrow \quad \frac{A_{n}\left\{x_{a} \mid a \in A_{n}\right\}}{\left\langle\left\{a \cdot x_{a} \cdot a-a, x_{a} \cdot a \cdot x_{a}-x_{a} \mid a \in A_{n}\right\}\right\rangle} \\
a & \mapsto \quad \iota_{A_{n}}(a)+\left\langle\left\{a \cdot x_{a} \cdot a-a, x_{a} \cdot a \cdot x_{a}-x_{a} \mid a \in A_{n}\right\}\right\rangle
\end{aligned}
$$

For every $n, m \in \mathbb{N}$ such that $n<m$, we define:

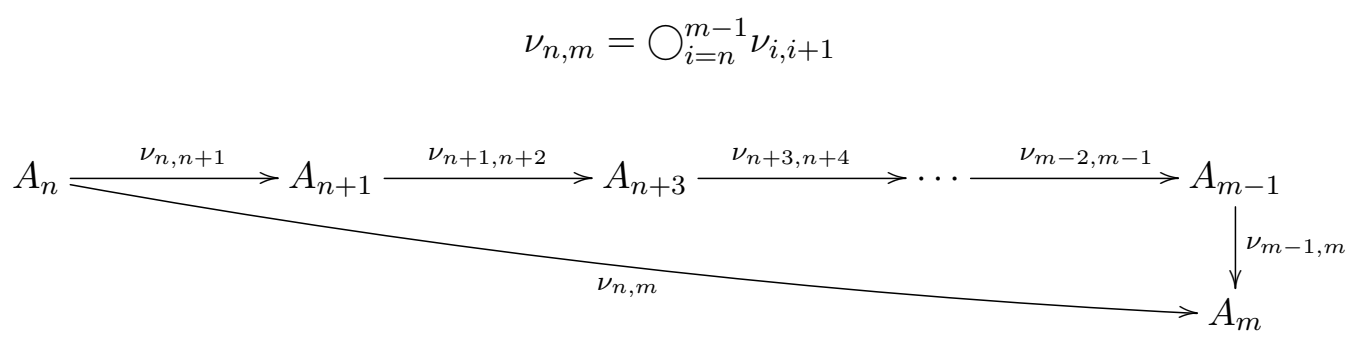

so we have an inductive system, $\left(\left\{A_{n}\right\}_{n \in \mathbb{N}},\left\{A_{n} \stackrel{\nu_{n, m}}{\rightarrow} A_{m} \mid n \leq m\right\}_{n, m \in \mathbb{N}}\right)$.

Let $A_{\omega}:=\lim _{n \in \mathbb{N}} A_{n}$ be the colimit of the above inductive system, so the following diagram commutes for every $n, m \in \mathbb{N}$ such that $n<m$ :

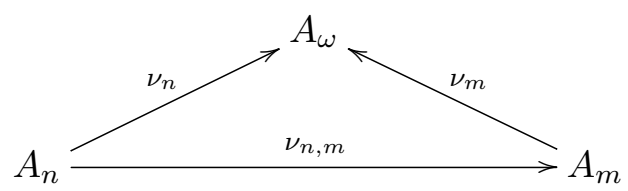

We claim that $A_{\omega}$ is a von Neumann regular $\mathcal{C}^{\infty}$-ring.

Given $[(a, n)] \in A_{\omega}$, there is some $a \in A_{n}$ such that $\nu_{n}(a)=[(a, n)]$. By construction, $\nu_{n, n+1}(a) \in A_{n+1}$ has a unique quasi-inverse, $\nu_{n, n+1}(a)^{*} \in A_{n+1}$, that is:

$$
\nu_{n, n+1}(a) \cdot \nu_{n, n+1}(a)^{*} \cdot \nu_{n, n+1}(a)=\nu_{n, n+1}(a)
$$

and

$$
\nu_{n, n+1}(a)^{*} \cdot \nu_{n, n+1}(a) \cdot \nu_{n, n+1}(a)^{*}=\nu_{n, n+1}(a)^{*} .
$$

Take $\nu_{n+1}\left(\nu_{n, n+1}(a)^{*}\right)=\left[\left(\nu_{n, n+1}(a)^{*}, n+1\right)\right]$. We have $[(a, n)]^{*}=\left[\left(\nu_{n, n+1}(a)^{*}, n+1\right)\right]$.

In fact, $\nu_{n}(a)=[(a, n)]=\left[\left(\nu_{n, n+1}(a), n+1\right)\right]=\nu_{n} \circ \nu_{n, n+1}(a)$, so:

$$
\begin{gathered}
{\left[\left(\nu_{n, n+1}(a)^{*}, n+1\right)\right] \cdot[(a, n)] \cdot\left[\left(\nu_{n, n+1}(a)^{*}, n+1\right)\right]=\left[\left(\nu_{n, n+1}(a)^{*}, n+1\right)\right] \cdot\left[\left(\nu_{n, n+1}(a), n+1\right)\right] \cdot\left[\left(\nu_{n, n+1}(a)^{*}, n+1\right)\right]=} \\
=\left[\left(\nu_{n, n+1}(a) \cdot \nu_{n, n+1}(a)^{*} \cdot \nu_{n, n+1}(a), n+1\right)\right]=\left[\left(\nu_{n, n+1}(a)^{*}, n+1\right)\right]
\end{gathered}
$$

and

$$
\begin{gathered}
{[(a, n)] \cdot\left[\left(\nu_{n, n+1}(a)^{*}, n+1\right)\right] \cdot[(a, n)]=\left[\left(\nu_{n, n+1}(a), n+1\right)\right] \cdot\left[\left(\nu_{n, n+1}(a)^{*}, n+1\right)\right] \cdot\left[\left(\nu_{n, n+1}(a), n+1\right)\right]=} \\
=\left[\left(\nu_{n, n+1}(a) \cdot \nu_{n, n+1}(a)^{*} \cdot \nu_{n, n+1}(a), n+1\right)\right]=\left[\left(\nu_{n, n+1}(a), n+1\right)\right]=[(a, n)]
\end{gathered}
$$

We register the following: 
Definition 3.3.2. Let $A$ be a $\mathcal{C}^{\infty}$-ring. The $\mathcal{C}^{\infty}$-von Neumann-regular hull of $A$, is a von Neumannregular $\mathcal{C}^{\infty}$-ring, denoted by $\mathrm{vN}(A)$, together with a $\mathcal{C}^{\infty}$-homomorphism $\nu_{A}: A \rightarrow \mathrm{vN}(A)$ with the following universal property: for every von Neumann-regular $\mathcal{C}^{\infty}$-ring, $B$ and any $\mathcal{C}^{\infty}$-homomorphism $\mu: A \rightarrow B$, there is a unique $\mathcal{C}^{\infty}$-homomorphism $\varphi: \mathrm{vN}(A) \rightarrow B$ such that the following triangle commutes:

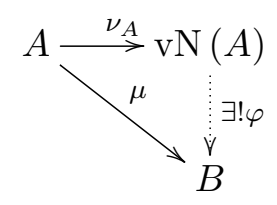

Theorem 3.3.3. Let $A$ be any $\mathcal{C}^{\infty}$-ring. The pair $\left(A_{\omega}, \nu_{0}: A \rightarrow A_{\omega}\right)$ (where $\nu_{0}$ is the colimit arrow) is the $\mathcal{C}^{\infty}$-von Neumann regular hull of $A$, that is, for every von Neumann-regular $\mathcal{C}^{\infty}$-ring $V$ and for every $\mathcal{C}^{\infty}$-homomorphism $f: A \rightarrow V$ there is a unique $\mathcal{C}^{\infty}$-homomorphism $\tilde{f}: A_{\omega} \rightarrow V$ such that the following diagram commutes:

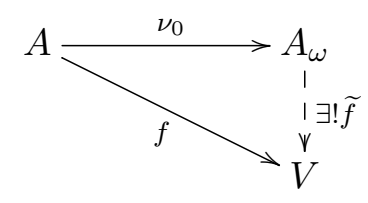

Proof. Given $f: A \rightarrow V$, by Lemma 3.3.1 there is a unique $f_{1}: A_{1} \rightarrow V$ such that $f_{1} \circ \nu_{0,1}=f$, that is, such that the diagram:

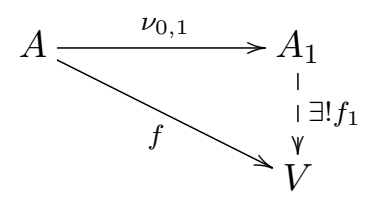

commutes. By induction, one proves that for this fixed $f: A \rightarrow V$, for every $n \in \mathbb{N}$ there is a unique $f_{n}: A_{n} \rightarrow V$ such that

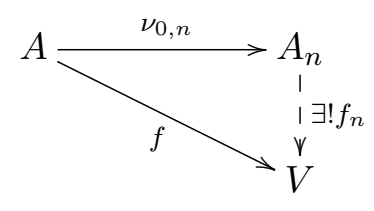

commutes, that is, such that $f_{n} \circ \nu_{0, n}=f$, so the for every $n, m \in \mathbb{N}$ with $n<m$, the following diagram commutes:

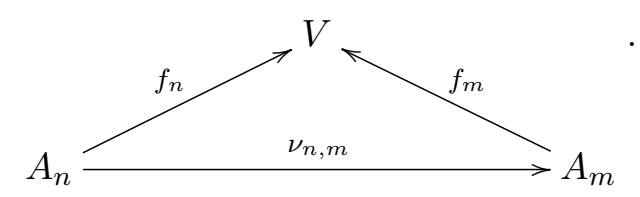

By the universal property of the colimit $A_{\omega}$, there is a unique $\mathcal{C}^{\infty}$-homomorphism:

$$
\widehat{f}: A_{\omega} \rightarrow V
$$

such that the following diagram commutes: 


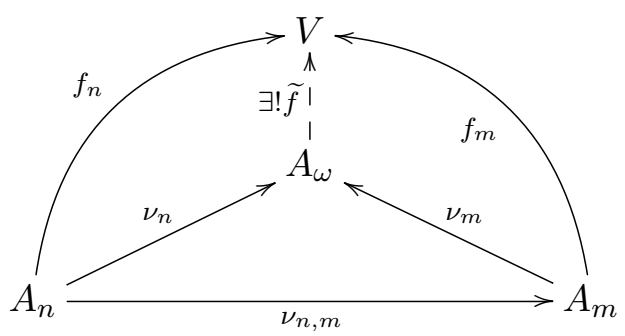

for every $n, m \in \mathbb{N}$ such that $n<m$. In particular, for $n=0$, we have the following commutative diagram:

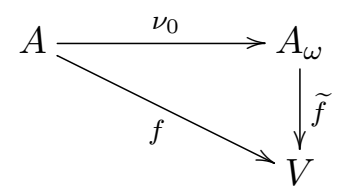

Remark 3.3.4. Whenever there is some $n \in \mathbb{N}$ such that $A_{n}$ is a von Neumann regular $\mathcal{C}^{\infty}$-ring, we have $\operatorname{vN}(A)=A_{n}$ and $\nu=\nu_{0, n}: A \rightarrow A_{n}$.

Theorem 3.3.5. The forgetful functor:

$$
U: \mathcal{C}^{\infty} \text { vNRng } \rightarrow \text { Set }
$$

has a left-adjoint, $L:$ Set $\rightarrow \mathcal{C}^{\infty} \mathbf{v N R n g}$.

Proof. By Proposition 2.2.80, the inclusion functor:

$$
\iota: \mathcal{C}^{\infty} \mathbf{v N R n g} \rightarrow \mathcal{C}^{\infty} \mathbf{R n g}
$$

has a left adjoint, $\nu: \mathcal{C}^{\infty} \mathbf{R n g} \rightarrow \mathcal{C}^{\infty}$ vNRng.

By Proposition 1.3.6, the forgetful functor $U^{\prime}: \mathcal{C}^{\infty} \mathbf{R n g} \rightarrow$ Set has a left adjoint, $L^{\prime}:$ Set $\rightarrow$ $\mathcal{C}^{\infty}$ Rng.

Since $U$ is the composition of the forgetful functor $U^{\prime}: \mathcal{C}^{\infty} \mathbf{v N R} \mathbf{n g} \rightarrow \mathcal{C}^{\infty} \mathbf{R n g}$ with the inclusion $\iota: \mathcal{C}^{\infty} \mathbf{R n g} \rightarrow$ Set, that is, $U=U^{\prime} \circ \iota$, it suffices to define $L:=\nu \circ L^{\prime}:$ Set $\rightarrow \mathcal{C}^{\infty}$ vNRng, so $L \dashv U$.

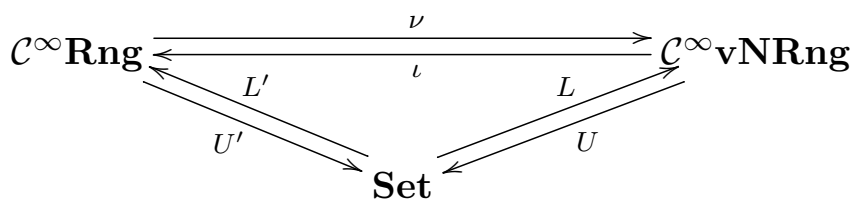

Remark 3.3.6. Let $A$ and $B$ be two $\mathcal{C}^{\infty}$-rings, $f: A \rightarrow B$ a $\mathcal{C}^{\infty}$-homomorphism, $\mathfrak{p} \in \operatorname{Spec}^{\infty}(A)$ and $\mathfrak{q} \in \operatorname{Spec}^{\infty}(B)$.

We write $\pi_{\mathfrak{p}}^{A}: A \rightarrow \frac{A}{\mathfrak{p}}$ for the quotient homomorphism and $\alpha_{\mathfrak{p}}^{A}: A \rightarrow k_{\mathfrak{p}}(A)$ for the composition $A \rightarrow \frac{A}{\mathfrak{p}} \longmapsto k_{\mathfrak{p}}(A)$, where $k_{\mathfrak{p}}(A)$ is the residue field of the local ring $A\left\{A \backslash \mathfrak{p}^{-1}\right\}$. 
Consider the quotient maps $\pi_{f^{*}(\mathfrak{q})}: A \rightarrow \frac{A}{f^{*}(\mathfrak{q})}$ and $\pi_{\mathfrak{q}}: B \rightarrow \frac{B}{\mathfrak{q}}$. Since $\operatorname{ker}\left(\pi_{\mathfrak{q}} \circ f\right)=f^{-}\left[\pi_{\mathfrak{q}}^{-}[\{0+\mathfrak{q}\}]\right]=$ $f^{\dashv}[\mathfrak{q}]=f^{*}(\mathfrak{q})$, by the Theorem of the Isomorphism there exists a unique $\overline{f_{\mathfrak{q}}}: \frac{A}{f^{*}(\mathfrak{q})} \rightarrow \frac{B}{\mathfrak{q}}$ such that the following diagram commutes:

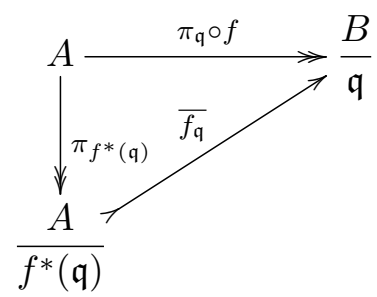

Moreover, $\overline{f_{\mathfrak{q}}}$ is a monomorphism, since ker $\overline{f_{\mathfrak{q}}}=\left\{0+f^{*}(\mathfrak{q})\right\}$. We call $\overline{f_{\mathfrak{q}}}$ "the quotient monomorphism between the associated $\mathcal{C}^{\infty}$-domains".

Consider the localization maps $\eta_{f^{*}(\mathfrak{q})}: A \rightarrow A\left\{A \backslash f^{*}(\mathfrak{q})^{-1}\right\}$ and $\eta_{\mathfrak{q}}: B \rightarrow B\left\{B \backslash \mathfrak{q}^{-1}\right\}$. Since $\eta_{\mathfrak{q}}\left[f\left[A \backslash f^{\star}(\mathfrak{q})\right]\right] \subseteq \eta_{\mathfrak{q}}[B \backslash \mathfrak{q}] \subseteq\left(B\{B \backslash \mathfrak{q}\}^{-1}\right)^{\times}$, by the universal property of the map $\eta_{f^{*}(\mathfrak{q})}: A \rightarrow A\left\{f^{*}(\mathfrak{q})^{-1}\right\}$ there is a unique $\check{f}_{\mathfrak{q}}: A\left\{A \backslash f^{\star}(\mathfrak{q})^{-1}\right\} \rightarrow B\left\{B \backslash \mathfrak{q}^{-1}\right\}$ such that the following square commutes:

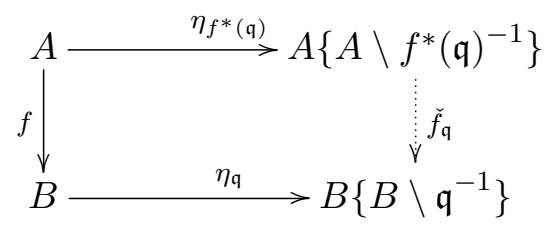

We call $\check{f}_{\mathfrak{q}}$ "the canonical local $\mathcal{C}^{\infty}$-homomorphism between the associated local $\mathcal{C}^{\infty}$-rings".

Let $k_{\mathfrak{q}}(B)=\operatorname{Res}\left(B\left\{B \backslash \mathfrak{q}^{-1}\right\}\right)$ and $k_{f^{*}(\mathfrak{q})}(A)=\operatorname{Res}\left(A\left\{A \backslash f^{*}(\mathfrak{q})^{-1}\right\}\right)$ and consider the canonical $\mathcal{C}^{\infty}$-homomorphisms $B\left\{B \backslash \mathfrak{q}^{-1}\right\} \rightarrow k_{\mathfrak{q}}(B)$ and $A\left\{A \backslash f^{*}(\mathfrak{q})^{-1}\right\} \rightarrow k_{f^{*}(\mathfrak{q})}(A)$. We have already seen that both $A\left\{A \backslash f^{*}(\mathfrak{q})^{-1}\right\}$ and $B\left\{B \backslash \mathfrak{q}^{-1}\right\}$ are local $\mathcal{C}^{\infty}$-rings, so let $\mathfrak{m}_{f^{*}(\mathfrak{q})}$ and $\mathfrak{m}_{\mathfrak{q}}$ be the (unique) maximal ideals of $A\left\{A \backslash f^{*}(\mathfrak{q})^{-1}\right\}$ and $B\left\{B \backslash \mathfrak{q}^{-1}\right\}$, respectively. We have:

$$
k_{\mathfrak{q}}(B)=\operatorname{Res}\left(B\left\{B \backslash \mathfrak{q}^{-1}\right\}\right)=\frac{B\left\{B \backslash \mathfrak{q}^{-1}\right\}}{\mathfrak{m}_{\mathfrak{q}}}
$$

and

$$
k_{f^{*}(\mathfrak{q})}(A)=\operatorname{Res}\left(A\left\{A \backslash f^{*}(\mathfrak{q})^{-1}\right\}\right)=\frac{A\left\{A \backslash f^{*}(\mathfrak{q})^{-1}\right\}}{\mathfrak{m}_{f^{*}(\mathfrak{q})}}
$$

By the Theorem of the Homomorphism, since $\check{f}_{\mathfrak{q}}{ }^{-1}\left[\mathfrak{m}_{\mathfrak{q}}\right]=\mathfrak{m}_{f^{*}(\mathfrak{q})}$ (for $\mathfrak{m}_{f^{*}(\mathfrak{q})}$ is a local $\mathcal{C}^{\infty}$-rings homomorphism), there is a unique $\mathcal{C}^{\infty}$-homomorphism $\widehat{f}_{\mathfrak{q}}: k_{f^{*}(\mathfrak{q})}(A) \rightarrow k_{\mathfrak{q}}(B)$ such that the following diagram commutes:

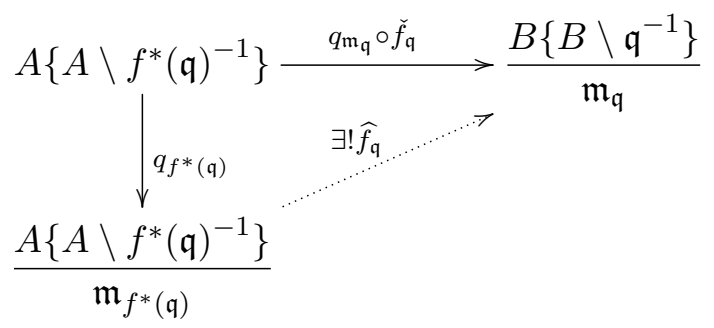

Thus, given a $\mathcal{C}^{\infty}$-homomorphism $f: A \rightarrow B$ we have a "canonical monomorphism" between the associated fields, that will be denoted by:

$$
\widehat{f}_{\mathfrak{q}}: k_{f^{*}(\mathfrak{q})}(A) \rightarrow k_{\mathfrak{q}}(B) .
$$


We will write:

$$
\widehat{f}: \prod_{\mathfrak{p} \in \operatorname{Spec}^{\infty}(A)} k_{\mathfrak{p}}(A) \rightarrow \prod_{\mathfrak{q} \in \operatorname{Spec}^{\infty}(B)} k_{\mathfrak{q}}(B)
$$

for the unique $\mathcal{C}^{\infty}$-homomorphism such that for every $\mathfrak{q} \in \operatorname{Spec}^{\infty}(B)$ the following diagram commutes:

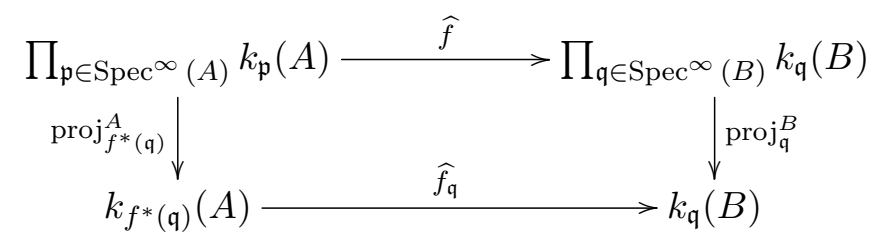

Theorem 3.3.7. Let $A$ be a $\mathcal{C}^{\infty}$-ring and $\left(\nu_{A}, \mathrm{vN}(A)\right)$ be its von Neumann-regular hull. Then:

(i) $\left(\nu_{A}\right)^{*}: \operatorname{Spec}^{\infty}(\mathrm{vN}(A)) \rightarrow \operatorname{Spec}^{\infty}(A)$ is a surjective spectral function;

(ii) For any $\mathfrak{q} \in \operatorname{Spec}^{\infty}(\mathrm{vN}(A)), \widehat{\nu_{A}}: k_{\left(\nu_{A}\right)^{*}(\mathfrak{q})}(A) \rightarrow k_{\mathfrak{q}}(\mathrm{vN}(A))$ is a $C^{\infty}$-ring isomorphims.

(iii) $\left(\nu_{A}\right)^{*}: \operatorname{Spec}^{\infty}(\mathrm{vN}(A)) \rightarrow \operatorname{Spec}^{\infty-c o n s t}(A)$ is a homeomorphism, where $\operatorname{Spec}^{\infty-c o n s t}(A)$ is the boolean topological space on the set of prime $\mathcal{C}^{\infty}$-radical ideals of $A$ with the constructible topology.

(iv) $\operatorname{ker}\left(\nu_{A}\right)=\sqrt[\infty]{(0)}=\bigcap \operatorname{Spec}^{\infty}(A)$. Thus $\nu_{A}$ is injective if and only if, $A$ is $\mathcal{C}^{\infty}$-reduced.

Proof. Ad (i): Take $\mathfrak{p} \in \operatorname{Spec}^{\infty}(A)$ and consider the canonical $\mathcal{C}^{\infty}$-homomorphism $\alpha_{\mathfrak{p}}: A \rightarrow k_{\mathfrak{p}}(A)$, where $k_{\mathfrak{p}}(A)$ is a von Neumann-regular $\mathcal{C}^{\infty}$-ring, for it is a $\mathcal{C}^{\infty}$-field. By the universal property of $\nu_{A}: A \rightarrow \mathrm{vN}(A)$ we have a unique "extension" $\widetilde{\alpha}_{\mathfrak{p}}^{A}: \mathrm{vN}(A) \rightarrow k_{\mathfrak{p}}(A)$ such that:

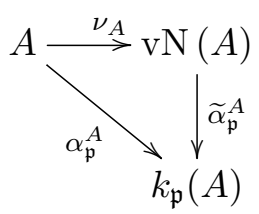

commutes, i.e., $\widetilde{\alpha_{\mathfrak{p}}^{A}} \circ \nu_{A}=\alpha_{\mathfrak{p}}^{A}$. Now take $\left.\gamma_{A}(\mathfrak{p}):={\widetilde{\alpha_{\mathfrak{p}}^{A}}}^{*}\right)[\{0\}] \in \operatorname{Spec}^{\infty}(\mathrm{vN}(A))$. In this way we have $\left(\nu_{A}\right)^{*}\left(\gamma_{A}(\mathfrak{p})\right)=\left(\nu_{A}\right)^{*}\left(\left(\widetilde{\alpha_{\mathfrak{p}}}\right)^{*}\right)(\{0\})=\left(\widetilde{\alpha_{\mathfrak{p}}} \circ \nu_{A}\right)^{*}(\{0\})=\mathfrak{p}$, thus $\left(\nu_{A}\right)^{*} \circ \gamma_{A}=\operatorname{id}_{\operatorname{Spec}^{\infty}(A)}$ is surjective.

Ad (ii): Let $\mathfrak{q} \in \operatorname{Spec}^{\infty}(\mathrm{vN}(A))$, by definition $\widehat{\nu_{A}}$ we have:

$$
\alpha_{\mathfrak{q}}^{\mathrm{vN}(A)} \circ \nu_{A}=\left(\widehat{\nu_{A}}\right)_{\mathfrak{q}} \circ \alpha_{\nu^{*}(\mathfrak{q})}^{A}
$$

so we have the $\mathcal{C}^{\infty}$-field monomorphism:

$$
\widehat{\eta_{A}}: k_{\nu_{A}^{*}(\mathfrak{q})}(A) \rightarrow k_{\mathfrak{q}}(\mathrm{vN}(A)) .
$$

Let us prove that it is surjective: consider the extension of $\alpha_{\nu_{A}^{*}(\mathfrak{q})}^{A}$ to $\mathrm{vN}(A): \widetilde{\alpha_{\nu_{A}^{*}(\mathfrak{q})}^{\mathrm{vN}(A)}}: \mathrm{vN}(A) \rightarrow$ $k_{\nu_{A}^{*}(\mathfrak{q})}(A)$, then $\widehat{\nu_{A_{\mathfrak{q}}}} \circ \widetilde{\alpha}_{\nu_{A}^{*}(\mathfrak{q})}^{A} \circ \nu_{A}=\widehat{\nu_{A_{\mathfrak{q}}}} \circ \alpha_{\nu_{A}^{*}(\mathfrak{q})}^{A}=\alpha_{\mathfrak{q}}^{\mathrm{vN}(A)} \circ \nu_{A}$, thus $\widehat{A_{\mathfrak{q}}} \circ \widetilde{\alpha}_{\nu_{A}^{*}(\mathfrak{q})}^{A}=\alpha_{\mathfrak{q}}^{\mathrm{vN}(A)}$, by the universal property of $\nu_{A}$. But $\mathfrak{q} \in \operatorname{Spec}^{\infty}(\mathrm{vN}(A))$ is a maximal ideal, so $\frac{\mathrm{vN}(A)}{\mathfrak{q}} \cong k_{\mathfrak{q}}(\mathrm{vN}(A))$ and $\alpha_{\mathfrak{q}}^{\mathrm{vN}(A)}$ : $\mathrm{vN}(A) \rightarrow \frac{\mathrm{vN}(A)}{\mathfrak{q}} \rightarrow k_{\mathfrak{q}}(\mathrm{vN}(A))$ is surjective, therefore $\widehat{\nu_{A_{\mathfrak{q}}}}$ is surjective too, since $\widehat{\nu_{A_{\mathfrak{q}}}} \circ \widetilde{\alpha}_{\nu_{A}^{\star}(\mathfrak{q})}^{A}=\alpha_{\mathfrak{q}}^{\mathrm{vN}(A)}$.

Ad (iii): Since $\left(\nu_{A}\right)^{\star}$ is a map between Boolean spaces and it is a spectral map, in order to prove that $\left(\nu_{A}\right)^{\star}: \operatorname{Spec}^{\infty}(\mathrm{vN}(A)) \rightarrow \operatorname{Spec}^{\infty-\text { const }}(A)$ is a homeomorphism, it is necessary and sufficient to 
prove that the spectral map $\left(\nu_{A}\right)^{\star}: \operatorname{Spec}^{\infty}(\mathrm{vN}(A)) \rightarrow \operatorname{Spec}^{\infty}(A)$ is a bijection from the Boolean space $\operatorname{Spec}^{\infty}(\mathrm{vN}(A))$ to the spectral space $\operatorname{Spec}^{\infty}(A)$. Keeping the notation in the proof of item (i), we will show that $\gamma_{A}$ is the inverse map of $\nu_{A}^{\star}$. By the proof of (i), it is enough to prove that $\gamma \circ\left(\nu_{A}\right)^{\star}=\operatorname{id}_{\operatorname{Spec}^{\infty}(\operatorname{vN}(A))}$. Let $\mathfrak{q} \in \operatorname{Spec}^{\infty}(\operatorname{vN}(A))$, then $\gamma_{A}\left(\nu_{A}^{\star}(\mathfrak{q})\right)=\operatorname{ker}\left(\widetilde{\alpha}_{\nu_{A}^{\star}(\mathfrak{q})}\right)=\operatorname{ker}\left(\alpha_{\mathfrak{q}}^{\mathrm{vN}(A)}\right)=\mathfrak{q}$, since $\widetilde{\nu_{A_{\mathfrak{q}}}} \circ \widetilde{\alpha}_{\nu_{A}^{\star}(\mathfrak{q})}^{A}=\alpha_{\mathfrak{q}}^{\mathrm{vN}(A)}$ and $\widehat{\nu_{A_{\mathfrak{q}}}}$ is injective.

Ad (iv): We will see that the result follows from the fact that $\operatorname{Spec}^{\infty}(\mathrm{vN}(A))$ is homeomorphic to the booleanization of $\operatorname{Spec}^{\infty}(A)$. Take any $\mathcal{C}^{\infty}$-ring $B$ and consider the "diagonal" $\mathcal{C}^{\infty}$-homomorphism:

$$
\delta_{B}:=\left(\alpha_{\mathfrak{p}}^{B}\right)_{\mathfrak{p} \in \operatorname{Spec}^{\infty}(B)}: B \rightarrow \prod_{\mathfrak{p} \in \operatorname{Spec}^{\infty}(B)} k_{\mathfrak{p}}(B) .
$$

From the equality of morphisms

$$
\begin{aligned}
\left(\alpha_{\mathfrak{p}}^{B}\right)_{\mathfrak{p} \in \operatorname{Spec}^{\infty}(B)}: B \rightarrow \prod_{\mathfrak{p} \in \operatorname{Spec}^{\infty}(B)} k_{\mathfrak{p}}(B)= & \\
& =B \stackrel{\left(\pi_{\mathfrak{p}}^{B}\right)_{\mathfrak{p} \in \operatorname{Spec} \infty}(B)}{\longrightarrow} \prod_{\mathfrak{p} \in \operatorname{Spec}^{\infty}(B)} \frac{B}{\mathfrak{p}} \longmapsto \prod_{\mathfrak{p} \in \operatorname{Spec}^{\infty}(B)} k_{\mathfrak{p}}(B)
\end{aligned}
$$

we have $\operatorname{ker}\left(\delta_{B}\right)=\bigcap \operatorname{Spec}^{\infty}(B)=\sqrt[\infty]{(0)}$.

In particular, for $B=\mathrm{vN}(A)$, we have $\operatorname{ker}\left(\delta_{\mathrm{vN}(A)}\right)=\sqrt[\infty]{(0)}=(0)$ and therefore obtain that $\delta_{\mathrm{vN}(A)}: \mathrm{vN}(A) \rightarrow \prod_{\mathfrak{q} \in \operatorname{Spec}^{\infty}(\mathrm{vN}(A))} k_{\mathfrak{q}}(\mathrm{vN}(A))$ is injective.

As $\nu_{A}^{\star}: \operatorname{Spec}^{\infty}(\mathrm{vN}(A)) \rightarrow \operatorname{Spec}^{\infty}(A)$ is bijective we get that the arrow $\widehat{\nu_{A}}: \prod_{\mathfrak{p} \in \operatorname{Spec}^{\infty}(A)} k_{\mathfrak{p}}(A) \rightarrow$ $\prod_{\mathfrak{q} \in \operatorname{Spec}^{\infty}(\mathrm{vN}(A))} k_{\mathfrak{q}}(\mathrm{vN}(A))$ is isomorphic to the $\mathcal{C}^{\infty}$-homomorphism

$$
\left(\widehat{\nu_{A_{\mathfrak{q}}}}\right)_{\mathfrak{q} \in \operatorname{Spec}^{\infty}(\mathrm{vN}(A)):} \prod_{\mathfrak{q} \in \operatorname{Spec}^{\infty}(\mathrm{vN}(A))} k_{\nu_{A}^{\star}(\mathfrak{q})}(A) \rightarrow \prod_{\mathfrak{q} \in \operatorname{Spec}^{\infty}(\mathrm{vN}(A))} k_{\mathfrak{q}}(\mathrm{vN}(A))
$$

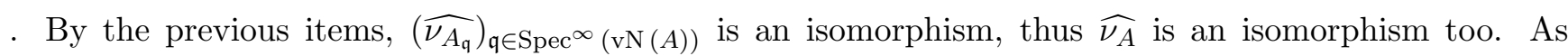
$\widehat{\nu_{A}} \circ \delta_{A}=\delta_{\mathrm{vN}(A)} \circ \nu_{A}$ and $\widehat{\nu_{A}}, \delta_{\mathrm{vN}(A)}$ are injective, we have $\operatorname{ker}\left(\nu_{A}\right)=\operatorname{ker}\left(\nu_{A} \circ \delta_{\mathrm{vN}(A)}\right)=\operatorname{ker}\left(\widehat{\nu_{A}} \circ \delta_{A}\right)=$ $\operatorname{ker}\left(\delta_{A}\right)=\sqrt[\infty]{(0)}$

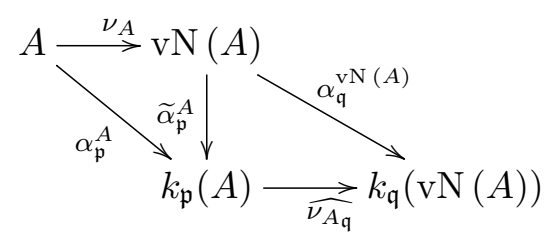

where $\mathfrak{p}:=\nu_{A}^{\star}(\mathfrak{q})$.

Theorem 3.3.8. Suppose that we have a functor $R: \mathcal{C}^{\infty} \mathbf{R n g} \rightarrow \mathcal{C}^{\infty} \mathbf{v N R n g}$ and a natural transforma-

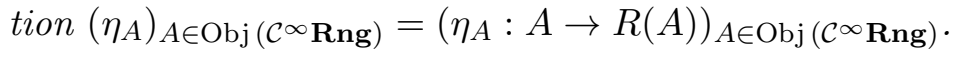

(i) Suppose that the following condition is satisfied:

"(E) For each von Neumann-regular $\mathcal{C}^{\infty}$-ring $V$ the arrow $\eta_{V}: V \rightarrow R(V)$ is a section (i.e., it has a left inverse)."

Then every $\mathcal{C}^{\infty}$-homomorphism $f: A \rightarrow V$ to a von Neumann-regular $\mathcal{C}^{\infty}$-ring factors through $\eta_{A}$. 
(ii) Suppose the following conditions are satisfied:

"(U) $\eta_{A}{ }^{*}: \operatorname{Spec}^{\infty}(R(A)) \rightarrow \operatorname{Spec}^{\infty}(A)$ is a bijection, i.e., $\operatorname{Spec}^{\infty}(R(A))$ is homeomorphic to the booleanization of $\operatorname{Spec}^{\infty}(A) . "$

"(U') The stalk of the spectral sheaf of $R(A)$ at a prime ideal $\mathfrak{p}$ "in $A$ " is isomorphic to $k_{\mathfrak{p}}(A)$, more precisely $\widehat{\eta_{A_{\mathfrak{q}}}}: k_{\eta_{A^{*}(\mathfrak{q})}}(A) \rightarrow k_{\mathfrak{q}}(R(A))$ is an isomorphism, $\mathfrak{q} \in \operatorname{Spec}^{\infty}(R(A))$."

Then a $\mathcal{C}^{\infty}$-homomorphism $f: A \rightarrow V$ to a von Neumann-regular $\mathcal{C}^{\infty}$-ring $V$ admits at most one factorization through $\eta_{A}$.

Thus, if all of $(\boldsymbol{E}),(\boldsymbol{U})$ and $\left(\boldsymbol{U}^{\prime}\right)$ are satisfied, then the map $\eta_{A}$ is initial among maps to von Neumann-regular $\mathcal{C}^{\infty}$-rings, i.e., a $\mathcal{C}^{\infty}$-homomorphism $f: A \rightarrow V$ to a von Neumann-regular $\mathcal{C}^{\infty}$-ring factors uniquely through $\eta_{A}$.

Proof. Ad (i): Let $V$ be a von Neumann-regular $\mathcal{C}^{\infty}$-ring and $f: A \rightarrow V$ a $\mathcal{C}^{\infty}$-homomorphism. As $\left(\eta_{A}\right)_{A \in \mathrm{Obj}\left(\mathcal{C}^{\infty} \mathrm{Rng}\right)}$ is a natural tranformation, we have $R(f) \circ \eta_{A}=\eta_{V} \circ f$. By hypothesis (E) there is a $\mathcal{C}^{\infty}$-homomorphism $r_{V}: R(V) \rightarrow V$ such that $r_{V} \circ \eta_{V}=\mathrm{id}_{V}$. Now define $F: R(A) \rightarrow V$ as the composition $F:=r_{V} \circ R(f)$, which makes the following diagram commute:

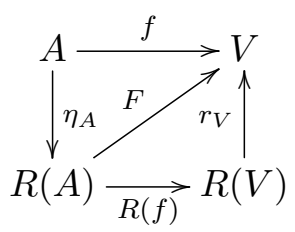

Indeed,

$$
F \circ \eta_{A}:=\left(r_{V} \circ R(f)\right) \circ \eta_{A}=r_{V} \circ\left(R(f) \circ \eta_{A}\right)=r_{V} \circ\left(\eta_{V} \circ f\right)=\left(r_{V} \circ \eta_{V}\right) \circ f=\mathrm{id}_{V} \circ f=f,
$$

as we claimed.

Ad (ii): Let $V$ be a von Neumann-regular $\mathcal{C}^{\infty}$-ring and let $F_{0}, F_{1}: R(A) \rightarrow V$ be two factorizations of $f$ through $\eta_{A}$, i.e., $\mathcal{C}^{\infty}$-homomorphisms such that $F_{0} \circ \eta_{A}=f=F_{1} \circ \eta_{A}$, or such that the following diagram commutes:

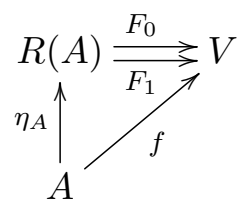

Then we get $\widehat{F_{0}} \circ \widehat{\eta_{A}}=\widehat{f}=\widehat{F_{1}} \circ \widehat{\eta_{A}}$ (to see that just compose these $\mathcal{C}^{\infty}$-homomorphisms with $\operatorname{proj}_{\mathfrak{s}}^{V}, \mathfrak{s} \in \operatorname{Spec}^{\infty}(V)$ and note that both $\widehat{F_{0}} \circ \widehat{\eta_{A}}$ and $\widehat{F_{1}} \circ \widehat{\eta_{A}}$ have the property which characterizes $\left.\widehat{f}\right)$.

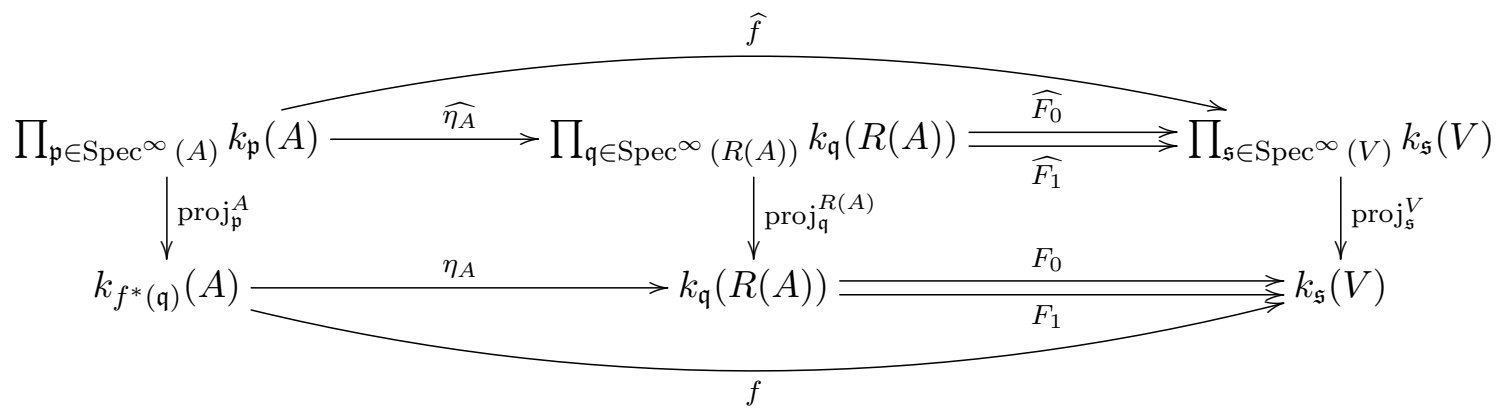


From the hypotheses (U) and (U') we obtain that the arrow:

$$
\widehat{\eta_{A}}: \prod_{\mathfrak{p} \in \operatorname{Spec}^{\infty}(A)} k_{\mathfrak{p}}(A) \rightarrow \prod_{\mathfrak{q} \in \operatorname{Spec}^{\infty}(R(A))} k_{\mathfrak{q}}(R(A))
$$

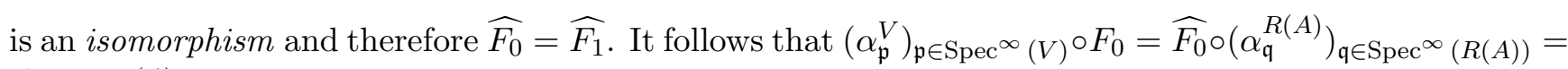
$\widehat{F_{1}} \circ\left(\alpha_{\mathfrak{q}}^{R(A)}\right)_{\mathfrak{q} \in \operatorname{Spec}^{\infty}(R(A))}=\left(\alpha_{\mathfrak{p}}^{V}\right)_{\mathfrak{p} \in \operatorname{Spec}^{\infty}(V)} \circ F_{1}$.

As $V$ is $\infty$-reduced, we get $\operatorname{ker}\left(\alpha_{\mathfrak{p}}^{V}\right)_{\mathfrak{p} \in \operatorname{Spec}^{\infty}(V)}=\sqrt[\infty]{(0)}=(0)$, so $\left(\alpha_{\mathfrak{p}}^{V}\right)_{\mathfrak{p} \in \operatorname{Spec}^{\infty}(V)}$ is injective and, by the commutativity of the following diagram, we can conclude that $F_{0}=F_{1}$, proving the uniqueness of the extensions.

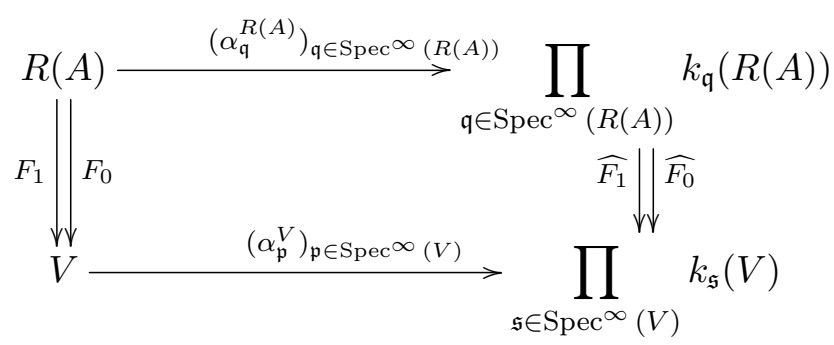

\subsubsection{Sheaves for the von Neumann regular hull of $\mathcal{C}^{\infty}$-ring}

In this subsection, we present another construction of the vN-hull of a $C^{\infty}$-ring by a sheaf-theoretic method. In fact, this alternative construction is useful to obtain a preservation result of the vN-hull functor (Proposition 3.3.15) and should be useful to proceed with other calculations.

The major part of the work here (that we will do in details) is: for each $\mathcal{C}^{\infty}$-ring, $A$, we are going to build a presheaf $P_{A}$ on the basis of the topology of $\left(\operatorname{Spec}^{\infty-\text { const }}(A)\right.$, Zar $\left.{ }^{\infty}\right)$ such that its stalks at each $\mathfrak{p} \in \operatorname{Spec}^{\infty}(A)$ are the $\mathcal{C}^{\infty}$-fields $k_{\mathfrak{p}}(A)$.

Consider $\operatorname{Spec}^{\infty}(A)$ together with the constructible topology, that we shall denote by $\operatorname{Spec}^{\infty \text {-const }}(A)$. We are going to construct a sheaf over this space, starting by the basic open sets of the constructible topology.

Given any basic open set $V$ of $\operatorname{Spec}^{\infty-\text { const }}(A)$, there are $a, b \in A$ such that $V=D^{\infty}(a) \cap Z^{\infty}(b)$. Given $\mathfrak{p} \in V=D^{\infty}(a) \cap Z^{\infty}(b), b \in \mathfrak{p}$ and $a \notin \mathfrak{p}$, so

$$
\sqrt[\infty]{(b)}=\bigcap\left\{\mathfrak{p}^{\prime} \in \operatorname{Spec}^{\infty}(A) \mid b \in \mathfrak{p}^{\prime}\right\} \subseteq \mathfrak{p}=\sqrt[\infty]{\mathfrak{p}} .
$$

Let $q_{b, \mathfrak{p}}: \frac{A}{\sqrt[\infty]{(b)}} \rightarrow \frac{A}{\mathfrak{p}}$ be the only $\mathcal{C}^{\infty}$-homomorphism such that:

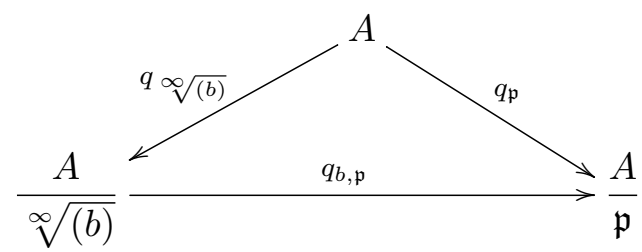

commutes. Consider the following diagram: 


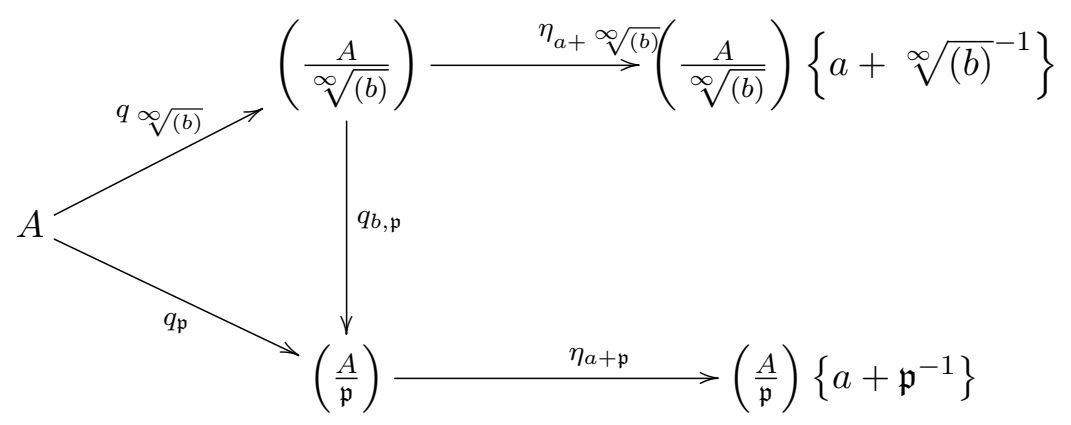

Note that $\left(\eta_{a+\mathfrak{p}} \circ q_{b, \mathfrak{p}}\right)(a+\sqrt[\infty]{(b)})=\eta_{a+\mathfrak{p}}\left(q_{b, \mathfrak{p}}(a+\sqrt[\infty]{(b)})\right)=\eta_{a+\mathfrak{p}}(a+\mathfrak{p}) \in\left(\frac{A}{\mathfrak{p}}\left\{a+\mathfrak{p}^{-1}\right\}\right)^{\times}$, and due to the universal property of $\eta_{a+\sqrt[\infty]{(b)}}:\left(\frac{A}{\sqrt[\infty]{(b)}}\right) \rightarrow\left(\frac{A}{\sqrt[\infty]{(b)}}\right)\{a+\sqrt[\infty]{(b)}-1\}$ there is a unique $\mathcal{C}^{\infty}$-homomorphism $\eta_{b, \mathfrak{p}}:\left(\frac{A}{\sqrt[\infty]{(b)}}\right)\left\{a+\sqrt[\infty]{(b)}^{-1}\right\} \rightarrow\left(\frac{A}{\mathfrak{p}}\right)\left\{a+\mathfrak{p}^{-1}\right\}$ such that the following diagram commutes:

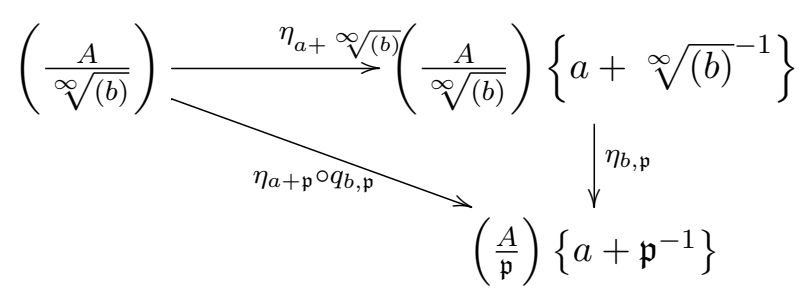

We now have the following commutative diagram:

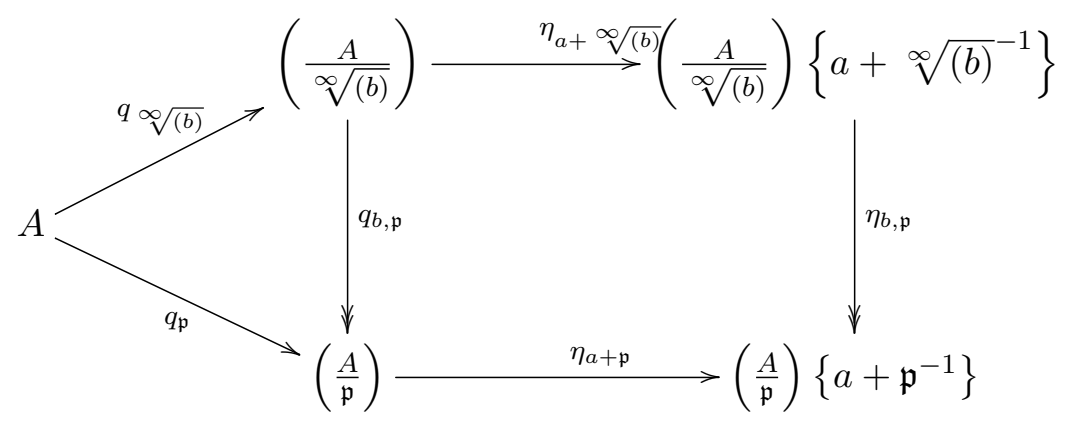

where $\eta_{b, \mathfrak{p}}$ is surjective due to the following:

Since $b \in \mathfrak{p}, \quad \sqrt[\infty]{(b)} \subseteq \mathfrak{p}$ so $q_{b, \mathfrak{p}}:\left(\frac{A}{\sqrt[\infty]{(b)}}\right) \rightarrow\left(\frac{A}{\mathfrak{p}}\right)$ is surjective and $\left\langle\eta_{a}[\sqrt[\infty]{(b)}]\right\rangle \subseteq\left\langle\eta_{a}[\mathfrak{p}]\right\rangle$. Also, by Corollary 2.1.45, rings of fractions commute with quotients, so

$$
\left\{\begin{array}{l}
\left(\frac{A}{\sqrt[\infty]{(b)}}\right)\left\{a+\sqrt[\infty]{(b)}-1, \stackrel{\mu_{a, b}}{\cong}\left(\frac{A\left\{a^{-1}\right\}}{\left\langle\eta_{a}[\sqrt[\infty]{(b)]\rangle})\right.}\right.\right. \\
\left(\frac{A}{\mathfrak{p}}\right)\left\{a+\mathfrak{p}^{-1}\right\} \stackrel{\mu_{a, \mathfrak{p}}}{\cong}\left(\frac{A\left\{a^{-1}\right\}}{\eta_{a}[\mathfrak{p}]}\right)
\end{array}\right.
$$

and we have the following commutative diagram: 


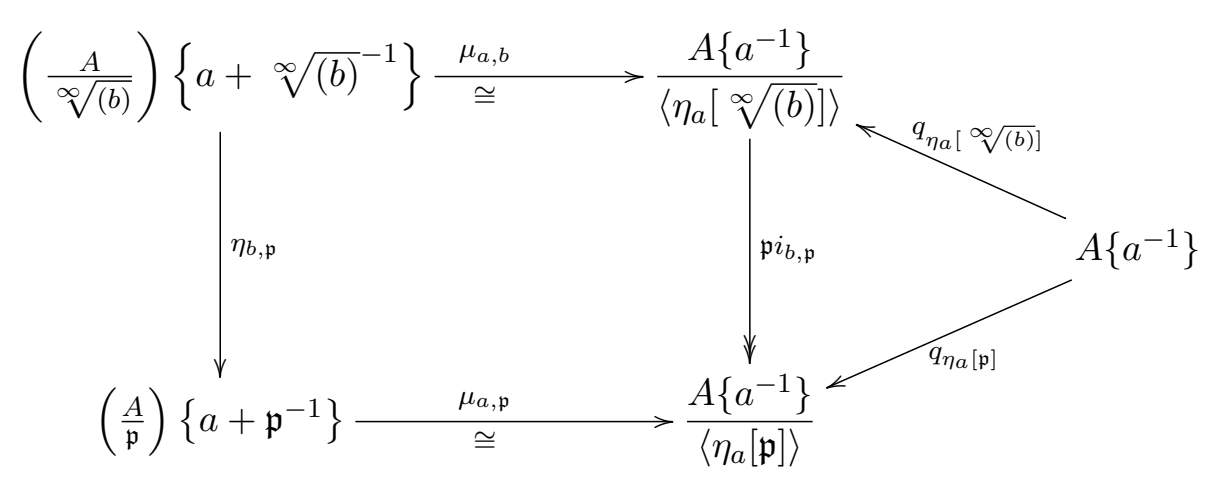

where $\mu_{a, b}$ and $\mu_{a, \mathfrak{p}}$ are the isomorphisms described in Corollary 2.1.45. We have:

$$
\eta_{b, \mathfrak{p}}=\left(\mu_{a, \mathfrak{p}}\right)^{-1} \circ \mathfrak{p} i_{b, \mathfrak{p}} \circ \mu_{a, b},
$$

so $\eta_{b, \mathfrak{p}}$ is a composition of surjective maps, hence it is a surjective map.

Considering the map:

$$
\eta_{\frac{A}{\mathfrak{p}} \backslash\{0+\mathfrak{p}\}}: \frac{A}{\mathfrak{p}}\left\{a+\mathfrak{p}^{-1}\right\} \rightarrow \frac{A}{\mathfrak{p}}\left\{\frac{A}{\mathfrak{p}} \backslash\{0+\mathfrak{p}\}^{-1}\right\}=k_{\mathfrak{p}}(A)
$$

we define $f_{\mathfrak{p}}^{a, b}:=\eta_{\frac{A}{\mathfrak{p}} \backslash\{0+\mathfrak{p}\}} \circ \eta_{b, \mathfrak{p}}: \frac{A}{\sqrt[\infty]{(b)}}\{a+\sqrt[\infty]{(b)}-1\} \rightarrow k_{\mathfrak{p}}(A)$, i.e., such that the following diagram commutes:

$$
\begin{aligned}
& \frac{A}{\sqrt[\infty]{(b)}}\left\{a+\sqrt[\infty]{(b)}^{-1}\right\} \\
& \frac{A}{\mathfrak{p}}\left\{a+\mathfrak{p}^{-1}\right\} \stackrel{\eta_{b, \mathfrak{p}}}{\eta_{\mathfrak{A}} \backslash\{0+\mathfrak{p}\}} \longrightarrow f_{\mathfrak{p}}^{a, b}(A)
\end{aligned}
$$

Let $t_{a, b}:=\eta_{a+\infty} \sqrt[\infty]{(b)} \circ q_{\infty}: A \rightarrow \frac{A}{\sqrt[\infty]{(b)}}\{a+\sqrt[\infty]{(b)}\}$ and define:

$$
\begin{aligned}
& t_{\mathfrak{p}}:= \eta_{\frac{A}{\mathfrak{p}} \backslash\{0+\mathfrak{p}\}} \circ \eta_{\frac{A}{\mathfrak{p}}}^{a+\mathfrak{p}} \circ q_{\mathfrak{p}}: A \rightarrow k_{\mathfrak{p}}(A), \\
& \frac{A}{\mathfrak{p}} \stackrel{\eta_{a+\mathfrak{p}}}{\longrightarrow} \frac{A}{\mathfrak{p}}\left\{a+\mathfrak{p}^{-1}\right\} \\
&\left.\right|_{q_{\mathfrak{p}}} \\
& A \longrightarrow k_{\mathfrak{p}}(A)
\end{aligned}
$$

where $\eta_{a+\mathfrak{p}}: \frac{A}{\mathfrak{p}} \rightarrow \frac{A}{\mathfrak{p}}\left\{a+\mathfrak{p}^{-1}\right\}$ is the ring of fractions of $\frac{A}{\mathfrak{p}}$ with respect to $a+\mathfrak{p}$.

We claim that $f_{\mathfrak{p}}^{a, b}:\left(\frac{A}{\sqrt[\infty]{(b)}}\right) \rightarrow\left(\frac{A}{\mathfrak{p}}\right)\left\{\frac{A}{\mathfrak{p}} \backslash\{0+\mathfrak{p}\}^{-1}\right\}$ is the unique $\mathcal{C}^{\infty}$-homomorphism such that the triangle:

$$
\begin{aligned}
A \longrightarrow & \frac{t_{a, b_{A}}}{\sqrt[\infty]{(b)}}\{a+\sqrt[\infty]{(b)}-1 \\
t_{\mathfrak{p}} & f_{\mathfrak{p}}^{a, b}
\end{aligned}
$$


commutes.

Let $g: \frac{A}{\sqrt[\infty]{(b)}} \rightarrow\left(\frac{A}{\mathfrak{p}}\right)\left\{\frac{A}{\mathfrak{p}} \backslash\{0+\mathfrak{p}\}^{-1}\right\}$ be a $\mathcal{C}^{\infty}$-homomorphism such that the following triangle commutes:

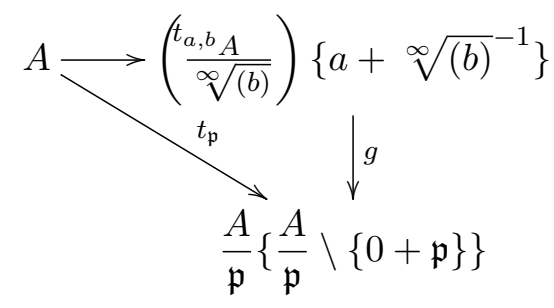

that is, such that $g \circ t_{a, b}=t_{\mathfrak{p}}$. We have:

$$
g \circ \eta_{\frac{A}{\sqrt[\infty]{(b)}}} \circ q_{b}=t_{\mathfrak{p}}=\eta_{\frac{A}{\mathfrak{p}} \backslash\{0+\mathfrak{p}\}} \circ q_{\mathfrak{p}}^{b} \circ q_{b} .
$$

Since $q_{b}$ is an epimorphism, it follows that:

$$
g \circ \eta_{\frac{A}{\infty \sqrt{(b)}}}=\eta_{\frac{A}{\mathfrak{p}} \backslash\{0+\mathfrak{p}\}} \circ q_{b, \mathfrak{p}}
$$

and

$$
\eta_{\frac{A}{\mathfrak{p}} \backslash\{0+\mathfrak{p}\}} \circ q_{b, \mathfrak{p}}=\eta_{a+\mathfrak{p}, \frac{A}{\mathfrak{p}} \backslash\{0+\mathfrak{p}\}} \circ \eta_{b, \mathfrak{p}} \circ \eta_{\frac{A}{\sqrt[\infty]{(b)}}},
$$

where:

$$
\eta_{a+\mathfrak{p}, \frac{A}{\mathfrak{p}} \backslash\{0+\mathfrak{p}\}}: \frac{A}{\mathfrak{p}}\left\{a+\mathfrak{p}^{-1}\right\} \rightarrow k_{\mathfrak{p}}(A .)
$$

By the universal property of $\eta_{\frac{A}{\infty} \sqrt[\infty]{(b)}}$, it follows that $g=\eta_{a+\mathfrak{p}, \frac{A}{\mathfrak{p}} \backslash\{0+\mathfrak{p}\}}$, hence $g=f_{\mathfrak{p}}^{a, b}$ and $f_{\mathfrak{p}}^{a, b}$ is unique.

We have now the following commutative diagram:

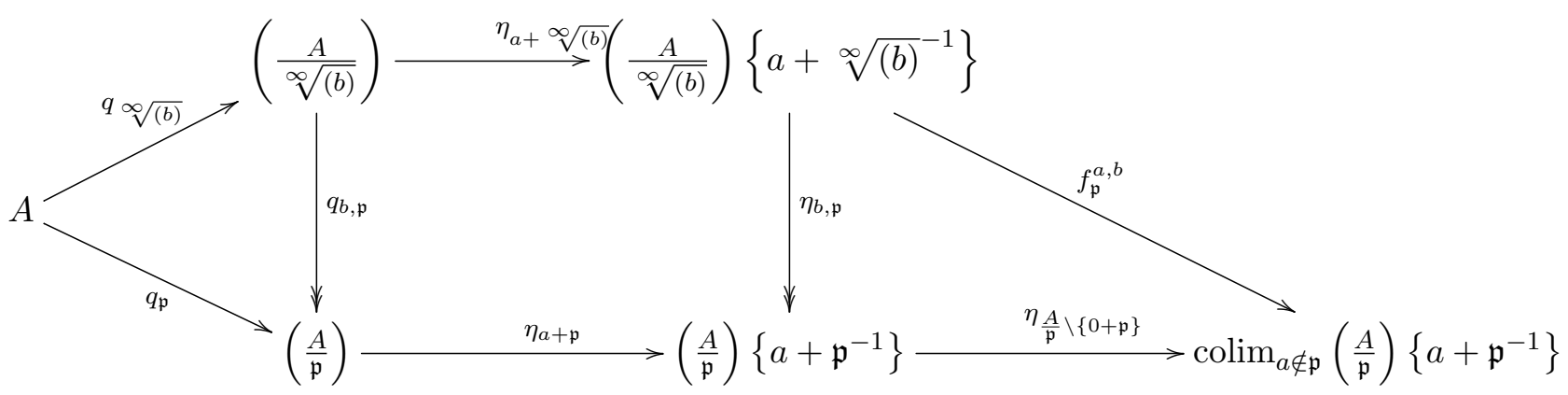

Remark 3.3.9. Since $\sqrt[\infty]{(b)} \subseteq \mathfrak{p}$, it follows that $q_{b, \mathfrak{p}}$ is surjective and since $\bigcup \operatorname{im} \jmath_{a}=\operatorname{colim}_{a \notin \mathfrak{p}} \frac{A}{\mathfrak{p}}\left\{a+\mathfrak{p}^{-1}\right\}$ (because it is a colimit and the right triangle of the above diagram commutes), it will follow that $\bigcup \operatorname{im} f_{\mathfrak{p}}^{a, b}=$ $\operatorname{colim}_{a \notin \mathfrak{p}} \frac{A}{\mathfrak{p}}\left\{a+\mathfrak{p}^{-1}\right\}$. Hence

$$
\left\{f_{\mathfrak{p}}^{a, b}:\left(\frac{A}{\sqrt[\infty]{(b)}}\right)\left\{a+\sqrt[\infty]{(b)}^{-1}\right\} \rightarrow \operatorname{colim}_{a \notin \mathfrak{p}} \frac{A}{\mathfrak{p}}\left\{a+\mathfrak{p}^{-1}\right\} \mid \mathfrak{p} \in D^{\infty}(a) \cap Z^{\infty}(b)\right\}
$$

is collectively surjective; 
Let $a, a^{\prime}, b, b^{\prime} \in A$ be such that $D^{\infty}(a) \cap Z^{\infty}(b) \subseteq D^{\infty}\left(a^{\prime}\right) \cap Z^{\infty}\left(b^{\prime}\right)$. Then

$$
D^{\infty}(a) \cap Z^{\infty}(b) \subseteq Z^{\infty}\left(b^{\prime}\right)
$$

and

$$
D^{\infty}(a) \cap Z^{\infty}(b) \subseteq D^{\infty}\left(a^{\prime}\right)
$$

We claim that $(3.2)$ implies that $\sqrt[\infty]{\left(b^{\prime}\right)} \subseteq \operatorname{ker}\left(t_{a, b}\right)$.

It suffices to show that:

$$
\bigcap D^{\infty}(a) \cap Z^{\infty}(b) \subseteq \operatorname{ker}\left(t_{a, b}\right)
$$

for

$$
\sqrt[\infty]{\left(b^{\prime}\right)}=\bigcap Z^{\infty}\left(b^{\prime}\right) \subseteq \bigcap D^{\infty}(a) \cap Z^{\infty}(b) .
$$

We have:

$$
\begin{gathered}
\bigcap D^{\infty}(a) \cap Z^{\infty}(b)=\left\{x \in A \mid\left(\forall \mathfrak{p} \in \operatorname{Spec}^{\infty}(A)\right)(a \notin \mathfrak{p}) \wedge(b \in \mathfrak{p}) \rightarrow(x \in \mathfrak{p})\right\} \\
t_{a, b}(x)=\eta_{\frac{A}{\sqrt[a]{(b)}}}^{a+\infty}\left(q_{b}(x)\right)=0 \Longleftrightarrow\left(\exists \theta \in\{a+\sqrt[\infty]{(b)}\}^{\infty-\operatorname{sat}}\right)\left(\theta \cdot q_{b}(x)=0 \text { in } \frac{A}{\sqrt[\infty]{(b)}}\right)
\end{gathered}
$$

Claim: $x \in \bigcap D^{\infty}(a) \cap Z^{\infty}(b) \rightarrow a \cdot x \in \sqrt[\infty]{(b)}$.

$A b$ absurdo, suppose $b \in \mathfrak{p}$ and $a \cdot x \notin \sqrt[\infty]{(b)}$. Since $\sqrt[\infty]{(b)}=\bigcap Z^{\infty}(b)$, there must exist some $\mathfrak{p} \in Z^{\infty}(b)$ such that $a \cdot x \in \mathfrak{p}$. Since $\mathfrak{p}$ is a prime ideal, it follows that $a \notin \mathfrak{p}$ and $x \notin \mathfrak{p}$.

Thus we have a contradiction between $\left(x \in \bigcap D^{\infty}(a) \cap Z^{\infty}(b)\right)$ and $\left(\exists \mathfrak{p} \in \operatorname{Spec}^{\infty}(A)\right)((a \notin \mathfrak{p}) \wedge(b \in$ $\mathfrak{p}) \wedge(x \notin \mathfrak{p}))$.

Now,

$$
\begin{aligned}
& t_{a, b}(x)=\eta_{\frac{A}{\infty} \sqrt{(b)}}\left(q_{b}(x)\right) \cdot \underbrace{\eta_{\frac{A}{(b)}}^{a}}_{\frac{A}{\eta_{(b)}}\left(q_{b}(a)\right)}\left(q_{b}(a \cdot x)\right)=\eta_{\frac{A}{\infty} \sqrt[\infty]{(b)}}(0)=0 \\
& \in\left(\frac{A}{\sqrt[\infty]{(b)}}\{a+\sqrt[\infty]{(b)}-1\}\right)^{\times}
\end{aligned}
$$

so $x \in \bigcap D^{\infty}(a) \cap Z^{\infty}(b)$ implies $x \in \operatorname{ker}\left(t_{a, b}\right)$, i.e.,

$$
\bigcap D^{\infty}(a) \cap Z^{\infty}(b) \subseteq \operatorname{ker}\left(t_{a, b}\right)
$$

hence

$$
\begin{gathered}
\sqrt[\infty]{\left(b^{\prime}\right) \subseteq \bigcap} D^{\infty}(a) \cap Z^{\infty}(b) \subseteq \operatorname{ker}\left(t_{a, b}\right) \\
\sqrt[\infty]{\left(b^{\prime}\right)} \subseteq \operatorname{ker}\left(t_{a, b}\right)
\end{gathered}
$$

By the Theorem of the $\mathcal{C}^{\infty}$-Homomorphism, there is a unique $\mathcal{C}^{\infty}$-homomorphism $r_{a, b}^{b^{\prime}}: \frac{A}{\sqrt[\infty]{\left(b^{\prime}\right)}} \rightarrow$ $\frac{A}{\sqrt[\infty]{(b)}}\{a+\sqrt[\infty]{(b)}-1\}$ such that $r_{a, b}^{b^{\prime}} \circ q_{\sqrt[\infty]{\left(b^{\prime}\right)}}=t_{a, b}$, that is, such that the following diagram commutes: 


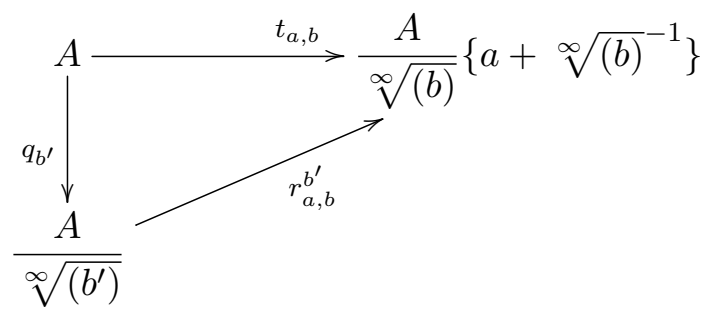

Now we claim that there is a unique $\mathcal{C}^{\infty}$-homomorphism $t_{a, b}^{a^{\prime}, b^{\prime}}: \frac{A}{\sqrt[\infty]{\left(b^{\prime}\right)}}\left\{a^{\prime}+\sqrt[\infty]{\left(b^{\prime}\right)}-1\right\} \rightarrow \frac{A}{\sqrt[\infty]{(b)}}\{a+\sqrt[\infty]{(b)}-1\}$ such that the following diagram commutes:

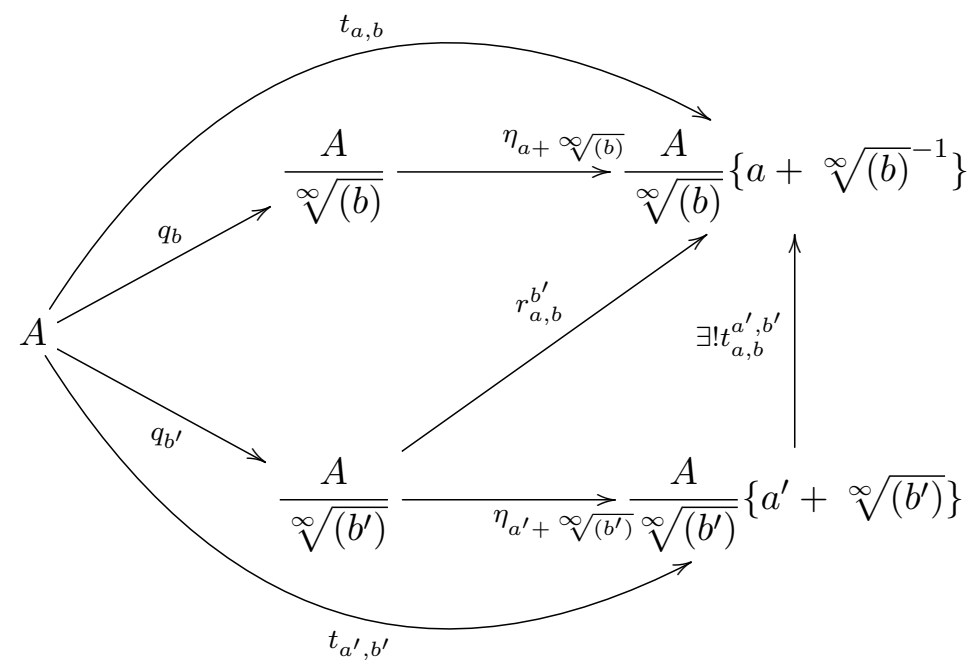

We claim that (3.3) implies that $r_{a, b}^{b^{\prime}}\left(a^{\prime}+\sqrt[\infty]{\left(b^{\prime}\right)}\right) \in\left(\frac{A}{\sqrt[\infty]{(b)}}\{a+\sqrt[\infty]{(b)}\}\right)^{\times}$. This is equivalent to assert that $t_{a, b}\left(a^{\prime}\right) \in\left(\frac{A}{\sqrt[\infty]{(b)}}\{a+\sqrt[\infty]{(b)}\}\right)^{\times}$or that $q_{b}\left(a^{\prime}\right) \in\{a+\sqrt[\infty]{(b)}\}^{\infty-\text { sat }}$.

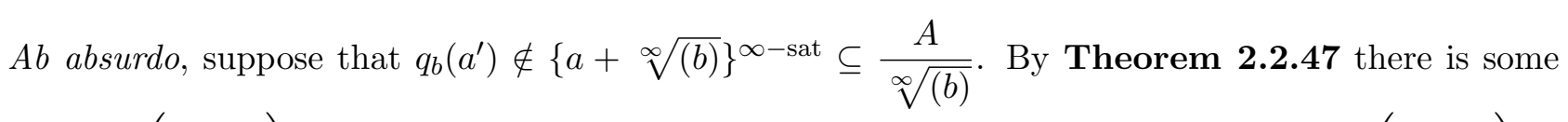
$\mathfrak{P} \in \operatorname{Spcc}^{\infty}\left(\frac{A}{\sqrt[\infty]{(b)}}\right)(\sqrt[\infty]{(b)} \subseteq \mathfrak{P})$ such that $q_{b}\left(a^{\prime}\right) \in \mathfrak{P}$ and $a+\sqrt[\infty]{(b)} \notin \mathfrak{P}$. Since $\operatorname{Spec}^{\infty}\left(\frac{A}{\sqrt[\infty]{(b)}}\right) \cong$ $\operatorname{Spec}_{\sqrt[\infty]{(b)}}^{\infty}(A)=\left\{\mathfrak{s} \in \operatorname{Spec}^{\infty}(A) \mid \sqrt[\infty]{(b)} \subseteq \mathfrak{s}\right\}$, there is a unique $\mathfrak{p} \in \operatorname{Spec}_{\sqrt[\infty]{(b)}}^{\infty}(A)$ such that $\mathfrak{P}=\mathfrak{p}+\sqrt[\infty]{(b)}$ and $\mathfrak{p} \in Z^{\infty}(b)$ (because $b \in \sqrt[\infty]{(b)} \subseteq \mathfrak{P}=\mathfrak{p}+\sqrt[\infty]{(b)}$ ).

Since $q_{b}\left(a^{\prime}\right) \in \mathfrak{P}=\mathfrak{p}+\sqrt[\infty]{(b)}$ and $a+\sqrt[\infty]{(b)} \notin \mathfrak{P}=\mathfrak{p}+\sqrt[\infty]{(b)}$, it follows that $a^{\prime} \in \mathfrak{p}\left(\mathfrak{p} \in Z^{\infty}\left(a^{\prime}\right)\right)$ and $a \notin \mathfrak{p}\left(\mathfrak{p} \in D^{\infty}(a)\right)$. Since $b \in \mathfrak{p}\left(\mathfrak{p} \in Z^{\infty}(b)\right)$, it follows that:

$$
\mathfrak{p} \in D^{\infty}(a) \cap Z^{\infty}(b) \cap Z^{\infty}\left(a^{\prime}\right)
$$

hence

$$
\begin{gathered}
D^{\infty}(a) \cap Z^{\infty}(b) \cap Z^{\infty}\left(a^{\prime}\right) \neq \varnothing \\
D^{\infty}(a) \cap Z^{\infty}(b) \nsubseteq D^{\infty}\left(a^{\prime}\right),
\end{gathered}
$$

which contradicts 3.3 . 
Hence:

$$
q_{b}\left(a^{\prime}\right) \in\{a+\sqrt[\infty]{(b)}\}^{\infty-\text { sat }}
$$

and by the universal property of $\eta_{a^{\prime}+\sqrt[\infty]{\left(b^{\prime}\right)}}: \frac{A}{\sqrt[\infty]{\left(b^{\prime}\right)}} \rightarrow \frac{A}{\sqrt[\infty]{\left(b^{\prime}\right)}}\left\{a^{\prime}+\sqrt[\infty]{\left(b^{\prime}\right)}-1\right\}$, there exists a unique $\mathcal{C}^{\infty}$-homomorphism $t_{a, b}^{a^{\prime}, b^{\prime}}: \frac{A}{\sqrt[\infty]{\left(b^{\prime}\right)}}\left\{a^{\prime}+\sqrt[\infty]{\left(b^{\prime}\right)}-1\right\} \rightarrow \frac{A}{\sqrt[\infty]{(b)}}\{a+\sqrt[\infty]{(b)}-1\}$ such that the following triangle commutes:

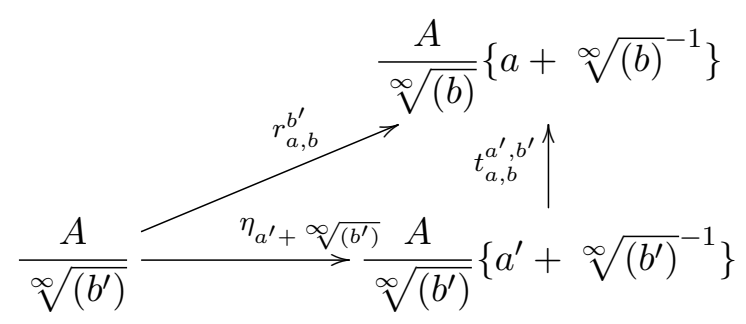

Now we claim that the following diagram is a colimit:

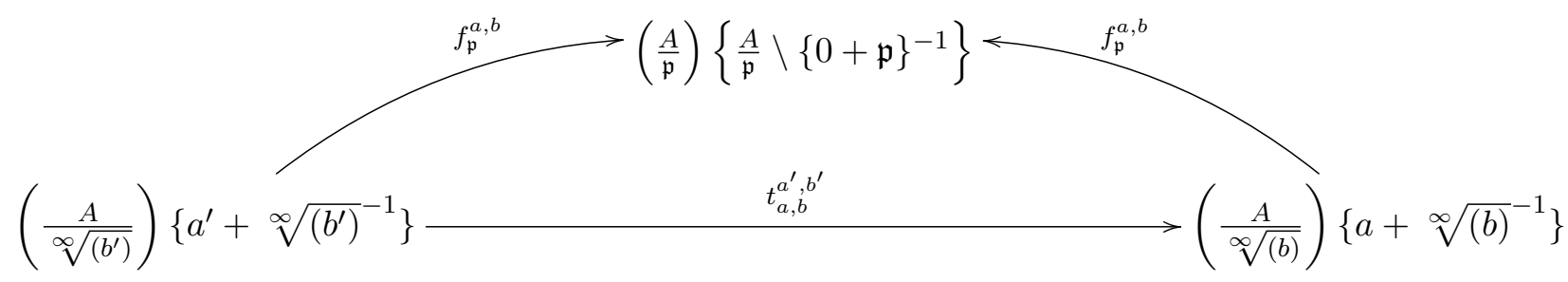

In order to show that, three conditions must hold:

1) For every $a, b, a^{\prime}, b^{\prime}$ such that $\mathfrak{p} \in D^{\infty}(a) \cap Z^{\infty}(b)$ and $\mathfrak{p} \in D^{\infty}\left(a^{\prime}\right) \cap Z^{\infty}\left(b^{\prime}\right)$ the above diagram commutes;

2) The family $\left\{f_{\mathfrak{p}}^{a, b}:\left(\frac{A}{\sqrt[\infty]{(b)}}\right)\left\{a+\sqrt[\infty]{(b)}^{-1}\right\} \rightarrow\left(\frac{A}{\mathfrak{p}}\right)\left\{\frac{A}{\mathfrak{p}} \backslash\{0+\mathfrak{p}\}^{-1}\right\}\right\}$ is collectively surjective;

3) Given $a_{1}, b_{1}, a_{2}, b_{2} \in A$ such that $D^{\infty}\left(a_{2}\right) \cap Z^{\infty}\left(b_{2}\right) \subseteq D^{\infty}\left(a_{1}\right) \cap Z^{\infty}\left(b_{1}\right), \theta_{1} \in\left(\frac{A}{\sqrt[\infty]{\left(b_{1}\right)}}\right)\left\{a_{1}+{\sqrt[\infty]{\left(b_{1}\right)}}^{-1}\right\}$ and $\theta_{2} \in\left(\frac{A}{\sqrt[\infty]{\left(b_{2}\right)}}\right)\left\{a_{2}+\sqrt[\infty]{\left(b_{2}\right)}-1\right\}$ such that $t_{\mathfrak{p}}^{a_{1}, b_{1}}\left(\theta_{1}\right)=t_{\mathfrak{p}}^{a_{2}, b_{2}}\left(\theta_{2}\right)$, there are $a, b \in A$ such that $\mathfrak{p} \in D^{\infty}(a) \cap Z^{\infty}(b) \subseteq D^{\infty}\left(a_{2}\right) \cap Z^{\infty}\left(b_{2}\right)$ and $t_{a, b}^{a_{1}, b_{1}}\left(\theta_{1}\right)=t_{a, b}^{a_{2}, b_{2}}\left(\theta_{2}\right)$, where $t_{a, b}^{a_{1}, b_{1}}$ and $t_{a, b}^{a_{2}, b_{2}}$ are given in the following commutative diagram:

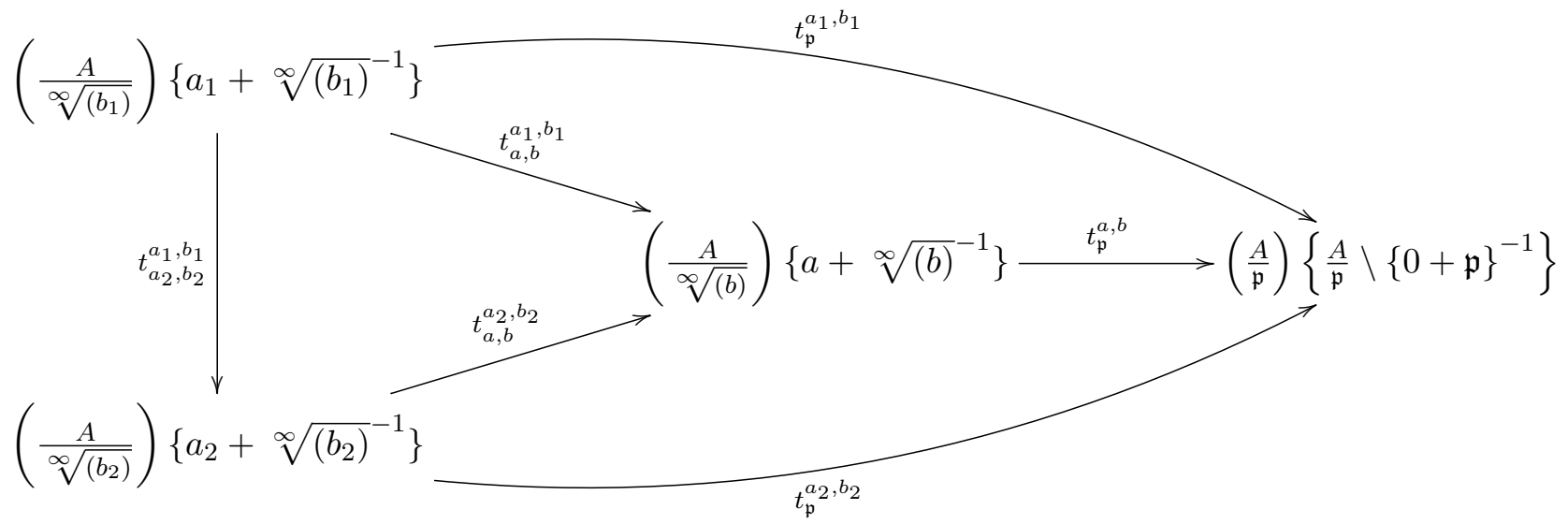


By Remark 3.3.9, the condition 2) is already satisfied.

Note that for each basic open subset $U$ of $\operatorname{Spec}^{\infty-\text { const }}(A), F_{A}(U)$ has the structure of an $A$-algebra. The universal property of $F_{A}(U)=\lim _{U} \underset{\substack{(a, b) \in A \times A \\ D^{\infty}(a) \cap Z^{\infty}(b)}}{ } \frac{A}{\sqrt[\infty]{(b)}}\{a+\sqrt[\infty]{(b)}-1\}$ induces a unique $\mathcal{C}^{\infty}$-homomorphism $u: A \rightarrow F_{A}(U)$ such that the following diagram commutes:

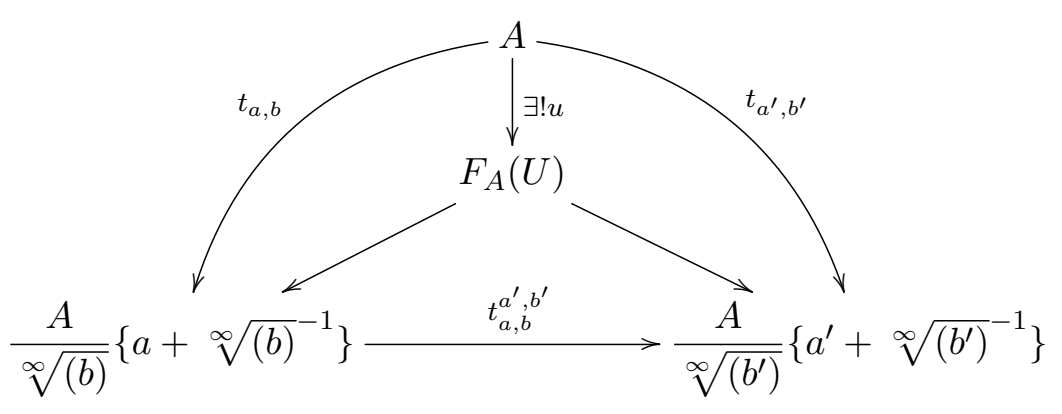

for every $a, a^{\prime}, b, b^{\prime} \in A$ such that $D^{\infty}(a) \cap Z^{\infty}(b)=U=D^{\infty}\left(a^{\prime}\right) \cap Z^{\infty}\left(b^{\prime}\right)$.

Now let $U$ and $V$ be any two basic open sets of $\operatorname{Spec}^{\infty-\text { const }}(A)$ such that $U \subseteq V$, and let $\iota_{U}^{V}: U \hookrightarrow V$ be the inclusion map. Since $U$ and $V$ are basic open subsets of the constructible topology, there are $a, b, c, d \in A$ such that $U=D^{\infty}(a) \cap Z^{\infty}(b)$ and $V=D^{\infty}(c) \cap Z^{\infty}(d)$. Let $a^{\prime}, b^{\prime}, c^{\prime}, d^{\prime} \in A$ be such that $U=D^{\infty}(a) \cap Z^{\infty}(b)=D^{\infty}\left(a^{\prime}\right) \cap Z^{\infty}\left(b^{\prime}\right)$ and $V=D^{\infty}(c) \cap Z^{\infty}(d)=D^{\infty}\left(c^{\prime}\right) \cap Z^{\infty}\left(d^{\prime}\right)$, so:

$$
t_{a^{\prime}, b^{\prime}}^{a, b}: \frac{A}{\sqrt[\infty]{(b)}}\{a+\sqrt[\infty]{(b)}-1\} \rightarrow \frac{A}{\sqrt[\infty]{\left(b^{\prime}\right)}}\left\{a^{\prime}+\sqrt[\infty]{\left(b^{\prime}\right)}-1\right\}
$$

and

$$
t_{c^{\prime}, d^{\prime}}^{c, d}: \frac{A}{\sqrt[\infty]{(d)}}\{c+\sqrt[\infty]{(d)}-1\} \rightarrow \frac{A}{\sqrt[\infty]{\left(d^{\prime}\right)}}\left\{c^{\prime}+\sqrt[\infty]{\left(d^{\prime}\right)}-1\right\}
$$

are $\mathcal{C}^{\infty}$-isomorphisms. Consider the following commutative diagram:

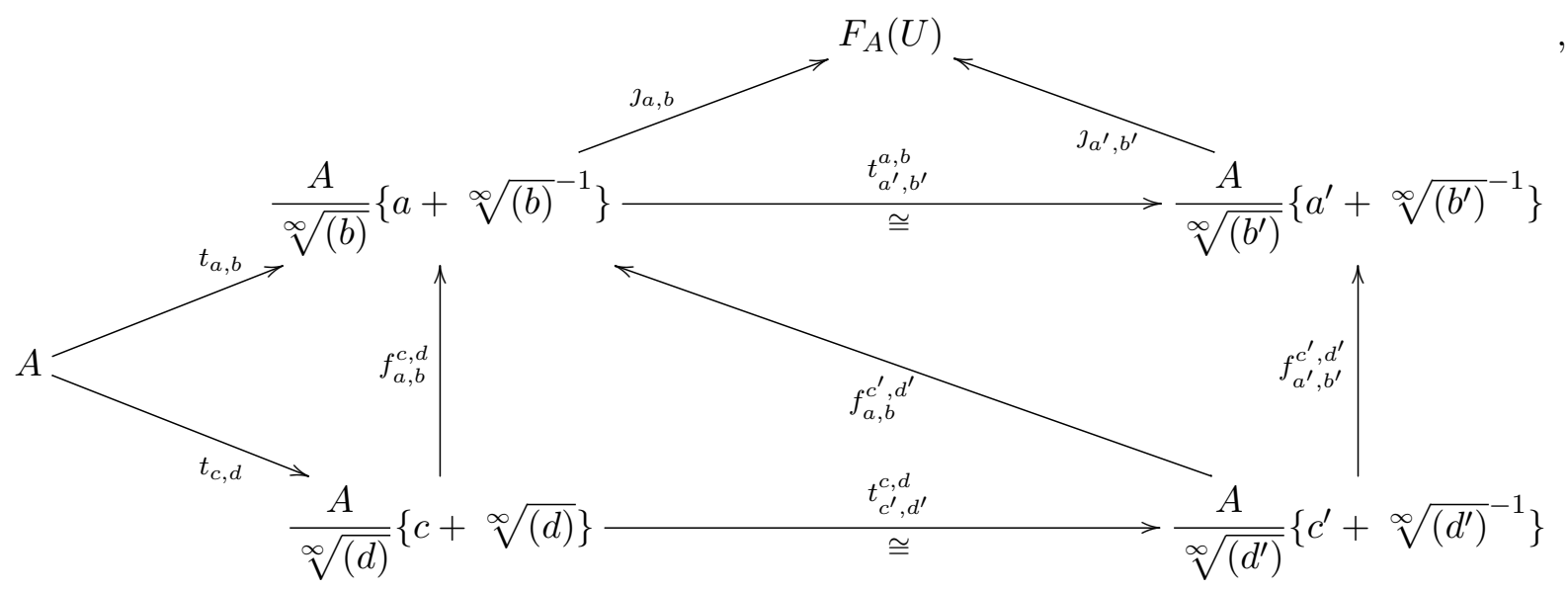

where $f_{a, b}^{c, d}$ is the unique $\mathcal{C}^{\infty}$-homomorphism such that $f_{a, b}^{c, d} \circ t_{c, d}=t_{a, b}, f_{a^{\prime}, b^{\prime}}^{c^{\prime}, d^{\prime}}$ is the unique $\mathcal{C}^{\infty}$-homomorphism such that $f_{a^{\prime}, b^{\prime}}^{c^{\prime}, d^{\prime}} \circ t_{c^{\prime}, d^{\prime}}=t_{a^{\prime}, b^{\prime}}$ and $f_{a, b}^{c^{\prime}, d^{\prime}}$ is the unique $\mathcal{C}^{\infty}$-homomorphism such that $f_{a, b}^{c^{\prime}, d^{\prime}} \circ t_{c^{\prime}, d^{\prime}}^{c, d}=f_{a, b}^{c, d}$

We have, then, the following cone: 


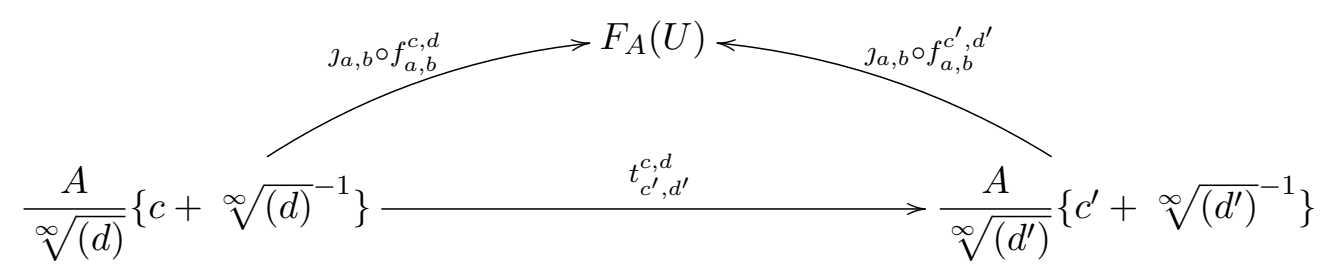

that induces, by the universal property of $F_{A}(V)$ as a colimit, a unique $\mathcal{C}^{\infty}$-homomorphism $f_{V}^{U}$ : $F_{A}(V) \rightarrow F_{A}(U)$ such that for every $a, b, c, d, c^{\prime}, d^{\prime} \in A$ the following diagram commutes:

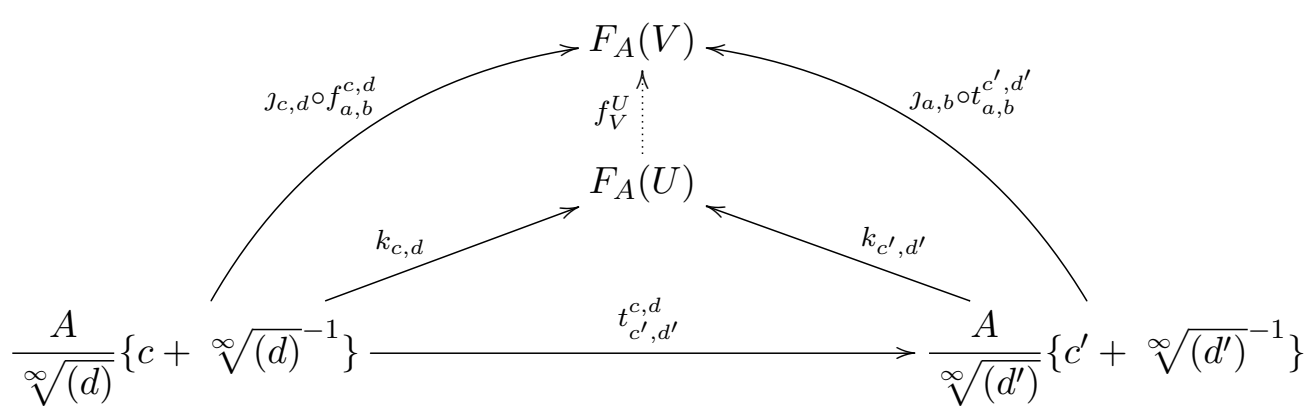

where $k_{c, d}$ and $k_{c^{\prime}, d^{\prime}}$ are the canonical colimit $\mathcal{C}^{\infty}$-homomorphisms.

Let $f_{c, d}^{U}:=J_{a, b}^{U} \circ f_{a, b}^{c, d}, f_{c, d}^{U}:=J_{a^{\prime}, b^{\prime}}^{U} \circ f_{a^{\prime}, b^{\prime}}^{c^{\prime}, d^{\prime}}, f_{e, f}^{V}:=J_{c, d}^{V} \circ j_{c, d}^{e, f}$ and $f_{e^{\prime}, f^{\prime}}^{V}:=J_{c^{\prime}, d^{\prime}}^{V} \circ j_{c^{\prime}, d^{\prime}}^{e^{\prime}, f^{\prime}}$.

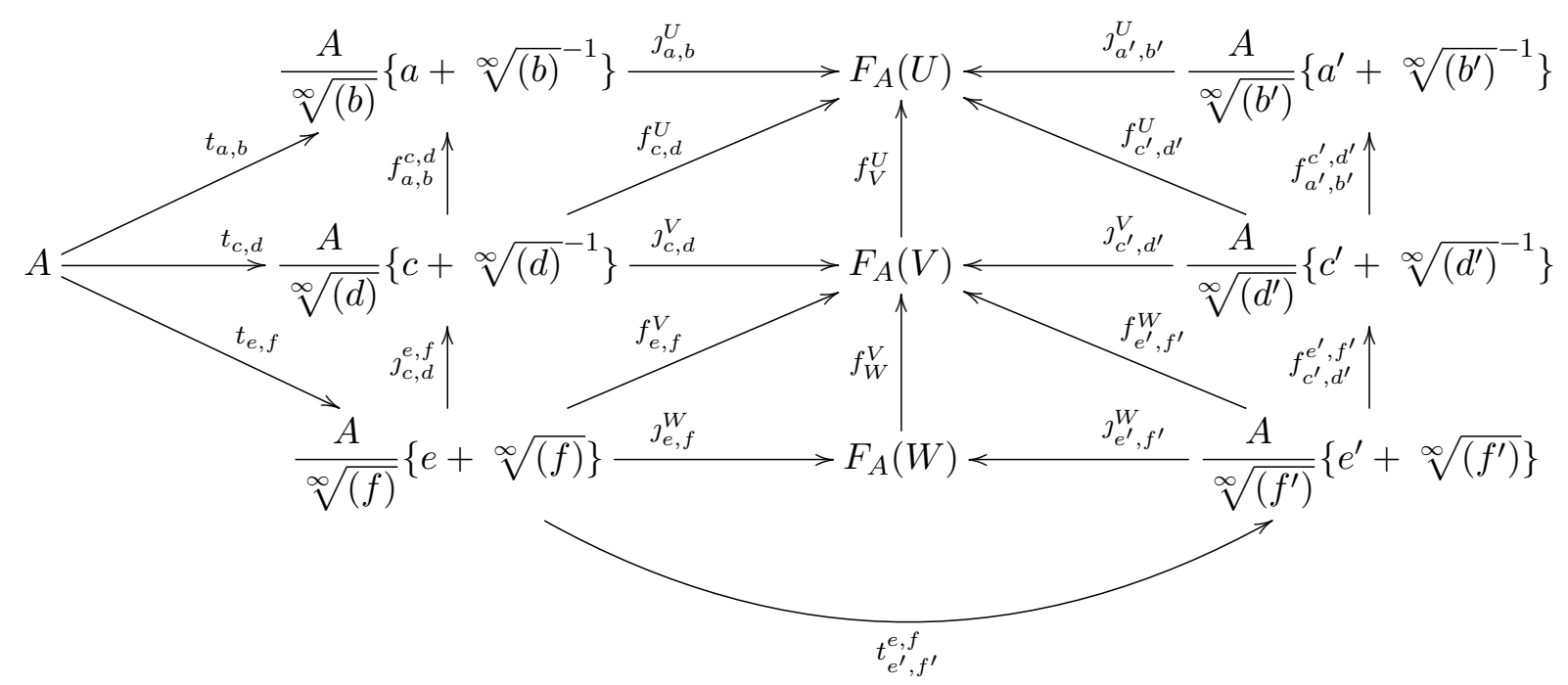

Denote $f_{e, f}^{U}:=J_{a, b}^{U} \circ f_{a, b}^{e, f}$ and $f_{e^{\prime}, f^{\prime}}^{U}:=J_{a^{\prime}, b^{\prime}}^{U} \circ f_{a^{\prime}, b^{\prime}}^{e^{\prime}, f^{\prime}}$. We have:

$$
\left(f_{V}^{U} \circ f_{W}^{V}\right) \circ \jmath_{e, f}^{W}=\left(\jmath_{a, b}^{U}\right) \circ\left(t_{a, b}^{c, d} \circ t_{c, d}^{e, f}\right)=\left(\jmath_{a, b}^{U}\right) \circ t_{a, b}^{e, f}=f_{e, f}^{U}=f_{W}^{U} \circ \jmath_{e, f}^{W},
$$

and the last equality holds because

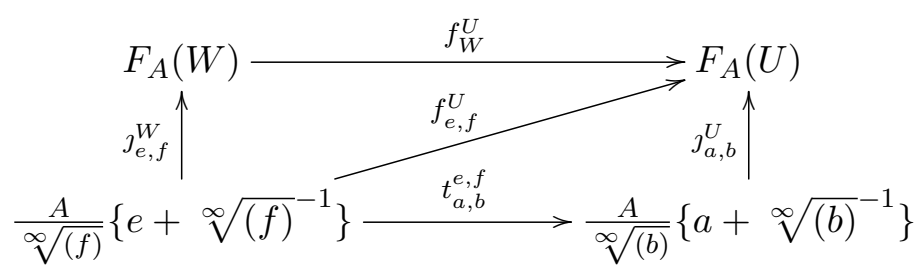


commutes

By the universal property of $F_{A}(W)$, since

$$
f_{e^{\prime}, f^{\prime}}^{U} \circ t_{e^{\prime}, f^{\prime}}^{e, f}=f_{e, f}^{U},
$$

there is a unique $\mathcal{C}^{\infty}$-homomorphism $f_{W}^{U}: F_{A}(W) \rightarrow F_{A}(U)$ such that the following diagram commutes:

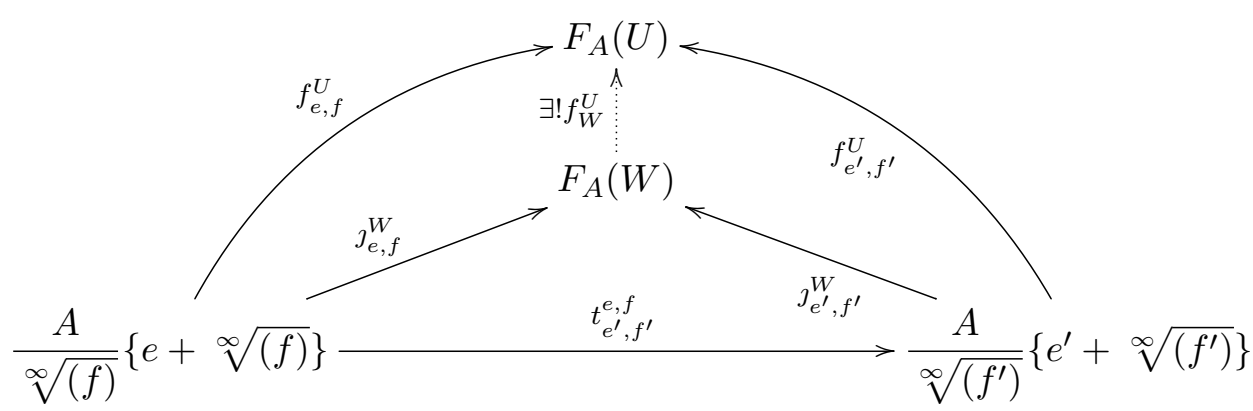

Hence, by the uniqueness of $f_{W}^{U}$, it follows that $f_{W}^{U}=f_{V}^{U} \circ f_{W}^{V}$.

Given the inclusion $\iota_{U}^{U}=\operatorname{id}_{U}: U \hookrightarrow U$, by the universal property of the colimit, there is a unique $\mathcal{C}^{\infty}$-homomorphism $f_{U}^{U}: F_{A}(U) \rightarrow F_{A}(U)$ such that the following diagram commutes for every $a, b, a^{\prime}, b^{\prime} \in$ $A$ such that $D^{\infty}(a) \cap Z^{\infty}(b)=U=D^{\infty}\left(a^{\prime}\right) \cap Z^{\infty}\left(b^{\prime}\right)$ :

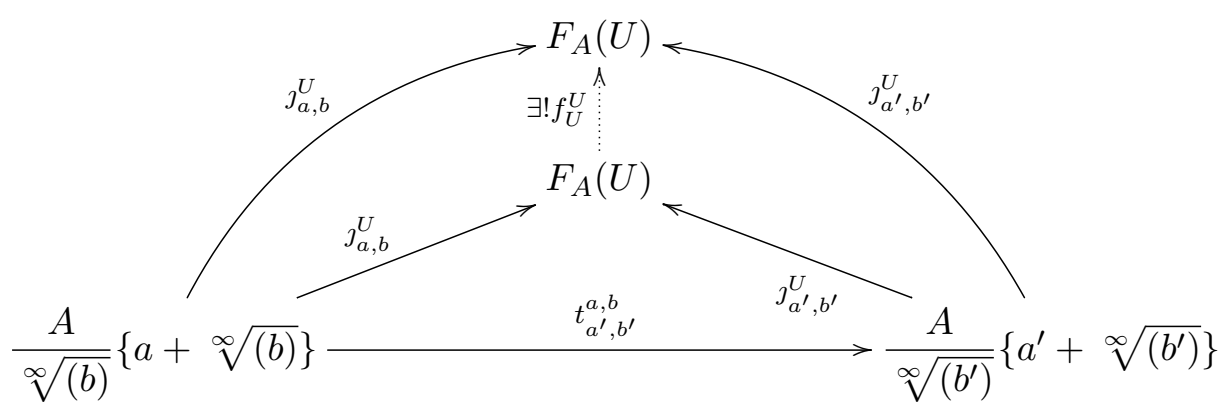

In particular, if we take $a=a^{\prime}$ and $b=b^{\prime}$, we will have $t_{a, b}^{a, b}=\operatorname{id}_{a, b}:\left(\frac{A}{\sqrt[\infty]{(b)}}\right)\{a+\sqrt[\infty]{(b)}-1\} \rightarrow$ $\left(\frac{A}{\sqrt[\infty]{(b)}}\right)\left\{a+\sqrt[\infty]{(b)}^{-1}\right\}$, so there is a unique $\mathcal{C}^{\infty}$-homomorphism $f_{U}^{U}: F_{A}(U) \rightarrow F_{A}(U)$ such that the following diagram commutes:

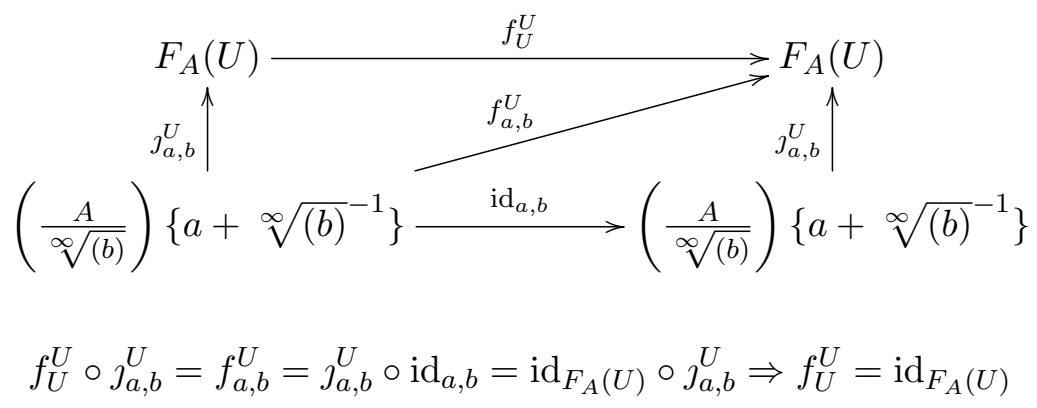

Thus, we have a functor from the category of the basic open subsets of $\operatorname{Spec}^{\infty-\text { const }}(A),\left(\mathcal{B}\left(\operatorname{Spec}^{\infty-\text { const }}\right), \subseteq\right.$ ) to the category of $\mathcal{C}^{\infty}$-rings:

$$
F_{A}:\left(\mathcal{B}\left(\operatorname{Spec}^{\infty-\text { const }}(A)\right), \subseteq\right)^{\mathrm{op}} \rightarrow \mathcal{C}^{\infty} \mathrm{Rng}
$$


Given any open set $U$ of $\operatorname{Spec}^{\infty-\text {-const }}(A)$ and any $\mathfrak{p} \in U$, there exists some basic open subset $V=D^{\infty}(a) \cap Z^{\infty}(b)$, for some $a, b \in A$, such that $\mathfrak{p} \in V \subseteq U$, so $\mathcal{B}\left(\operatorname{Spec}^{\infty-\text { const }}(A)\right)^{\text {op }}$ is a cofinal subset of Open $\left(\operatorname{Spec}^{\infty-\text { const }}(A)\right)^{\text {op }}$.

In order to describe the stalk of this pre-sheaf at $\mathfrak{p}$, it suffices to calculate the colimit over the system $\mathcal{B}\left(\operatorname{Spec}^{\infty-\text { const }}(A)\right)$ :

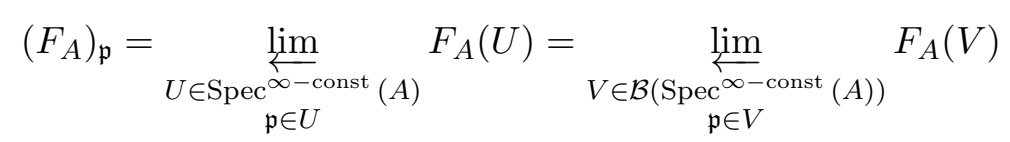

We have the following cone:

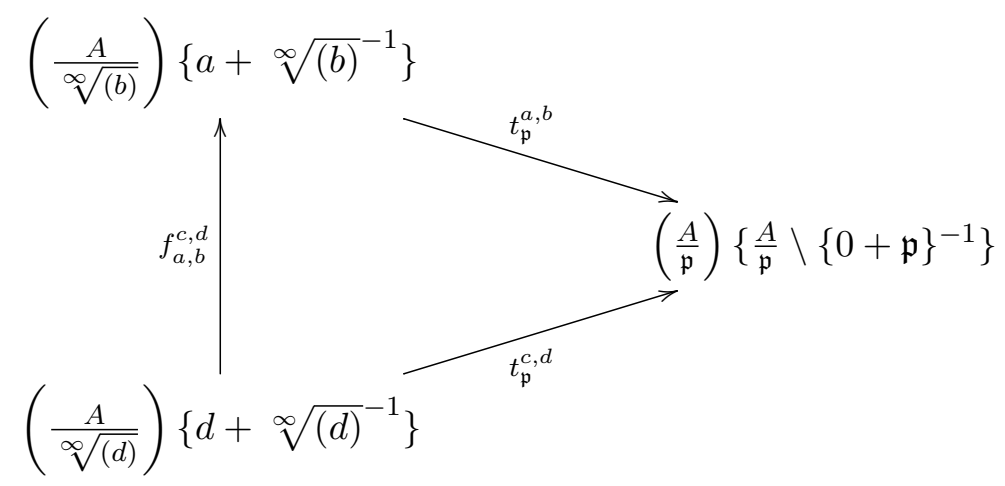

We claim that:

$$
\lim _{V \in \mathcal{B}\left(\text { Spec }^{\infty-\text { const }}(A)\right)} F_{A}(V) \cong\left(\frac{A}{\mathfrak{p}}\right)\left\{\frac{A}{\mathfrak{p}} \backslash\{0+\mathfrak{p}\}^{-1}\right\}
$$

In order to prove it, we must have:

(i) The diagram (3.4) is a commutative cone;

(ii) The family of arrows $\left\{t_{\mathfrak{p}}^{a, b}\right\}_{a, b \in U}$ is collectively surjective;

(iii) For every $x_{a, b} \in\left(\frac{A}{\sqrt[\infty]{(b)}}\right)\{a+\sqrt[\infty]{(b)}-1\}$ and $x_{a^{\prime}, b^{\prime}} \in\left(\frac{A}{\sqrt[\infty]{\left(b^{\prime}\right)}}\right)\left\{a^{\prime}+\sqrt[\infty]{\left(b^{\prime}\right)}-1\right\}$ such that:

$$
t_{\mathfrak{p}}^{a, b}\left(x_{a, b}\right)=t_{\mathfrak{p}}^{a^{\prime}, b^{\prime}}\left(x_{a^{\prime}, b^{\prime}}\right)
$$

there are $c, d \in A$ such that:

$$
D^{\infty}(c) \cap Z^{\infty}(d) \subseteq\left[D^{\infty}(a) \cap Z^{\infty}(b)\right] \cap\left[D^{\infty}\left(a^{\prime}\right) \cap Z^{\infty}\left(b^{\prime}\right)\right],
$$

i.e., there are arrows:

$$
f_{c, d}^{a, b}:\left(\frac{A}{\sqrt[\infty]{(b)}}\right)\left\{a+\sqrt[\infty]{(b)}^{-1}\right\} \rightarrow\left(\frac{A}{\sqrt[\infty]{(d)}}\right)\left\{c+\sqrt[\infty]{(d)}^{-1}\right\}
$$

and

$$
f_{c, d}^{a^{\prime}, b^{\prime}}:\left(\frac{A}{\sqrt[\infty]{\left(b^{\prime}\right)}}\right)\left\{a^{\prime}+\sqrt[\infty]{\left(b^{\prime}\right)}-1\right\} \rightarrow\left(\frac{A}{\sqrt[\infty]{(d)}}\right)\left\{c+\sqrt[\infty]{(d)}^{-1}\right\}
$$

such that: 


$$
f_{c, d}^{a, b}\left(x_{a, b}\right)=f_{c, d}^{a^{\prime}, b^{\prime}}\left(x_{a^{\prime}, b^{\prime}}\right)
$$

that is, the following diagram commutes:

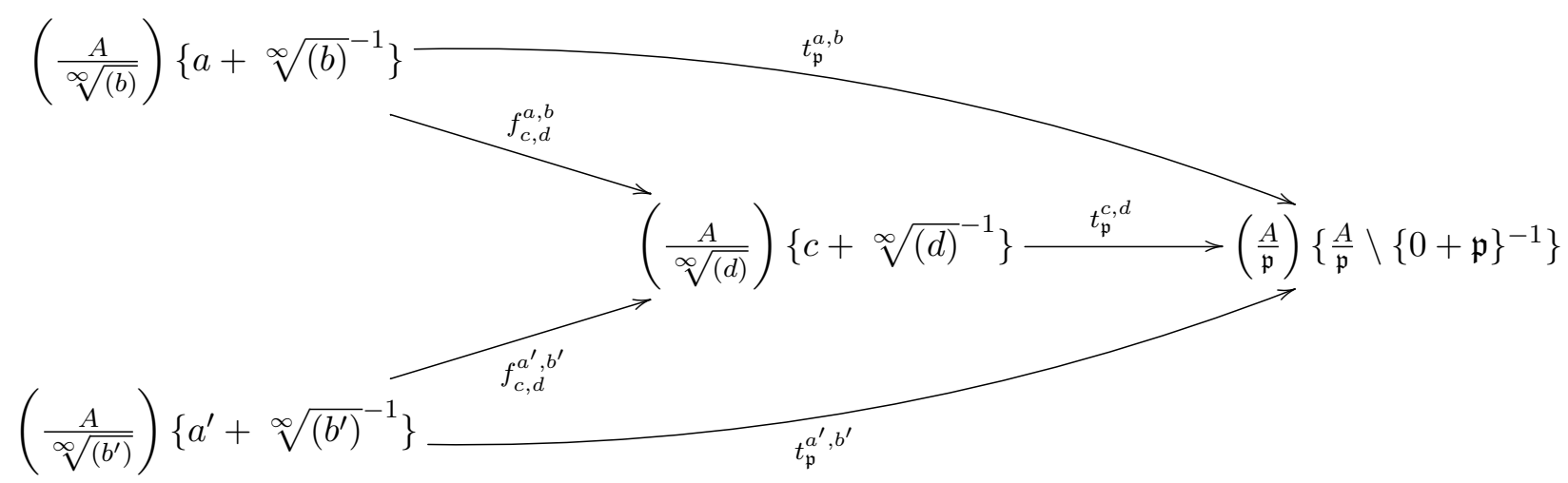

The latter condition is equivalent to saying that for every $a, b \in A$, the morphism:

$$
\begin{array}{cccc}
t_{\mathfrak{p}}^{a, b}:\left(\frac{A}{\sqrt[\infty]{(b)}}\right)\{a+\sqrt[\infty]{(b)}-1 & \rightarrow\left(\frac{A}{\mathfrak{p}}\right)\left\{\frac{A}{\mathfrak{p}} \backslash\{0+\mathfrak{p}\}^{-1}\right\} \\
\gamma & \mapsto & t_{\mathfrak{p}}^{a, b}(\gamma)
\end{array}
$$

is injective, that is, $\operatorname{ker} t_{\mathfrak{p}}^{a, b}=\{0\}$.

Since $t_{\mathfrak{p}}^{a, b}=\jmath_{a} \circ \eta_{b, \mathfrak{p}}$ and $\jmath_{a}$ is injective, $\operatorname{ker} t_{\mathfrak{p}}^{a, b}=\operatorname{ker} \eta_{b, \mathfrak{p}}$ and showing that $t_{\mathfrak{p}}^{a, b}$ is injective is equivalent to prove that ker $\eta_{b, \mathfrak{p}}\{0\}$.

Consider the following diagram:

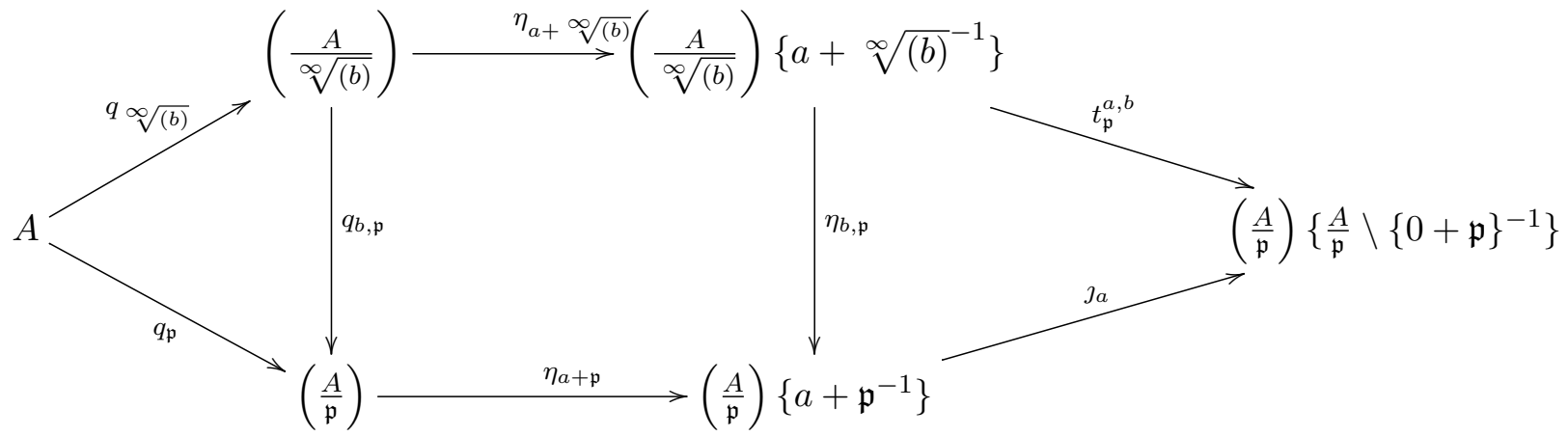

We have:

$$
t_{\mathfrak{p}}^{a, b}=\jmath_{a} \circ \eta_{b, \mathfrak{p}}
$$

so

$$
t_{\mathfrak{p}}^{a, b} \circ \eta_{a+\sqrt[\infty]{(b)}}=\jmath_{a} \circ \eta_{b, \mathfrak{p}} \circ \eta_{a+\sqrt[\infty]{(b)}}
$$

Since $\eta_{b, \mathfrak{p}} \circ \eta_{a+\sqrt[\infty]{(b)}}=\eta_{a+\mathfrak{p}} \circ q_{b, \mathfrak{p}}$, it follows that:

$$
\begin{gathered}
t_{\mathfrak{p}}^{a, b} \circ \eta_{a+\sqrt[\infty]{(b)}}=\jmath_{a} \circ \eta_{a+\mathfrak{p}} \circ q_{b, \mathfrak{p}} \\
t_{\mathfrak{p}}^{a, b} \circ \eta_{a+\sqrt[\infty]{(b)}} \circ q_{\infty} \sqrt{(b)}=\jmath_{a} \circ \eta_{a+\mathfrak{p}} \circ \underbrace{q_{b, \mathfrak{p}} \circ q_{\infty}}_{=q_{\mathfrak{p}}}
\end{gathered}
$$


hence

$$
t_{\mathfrak{p}}^{a, b} \circ \eta_{a+\infty} \sqrt{(b)} \circ q_{\infty} \sqrt{(b)}=\jmath_{a} \circ \eta_{a+\mathfrak{p}} \circ q_{\mathfrak{p}}
$$

Let $\gamma \in \operatorname{ker} t_{\mathfrak{p}}^{a, b} \subseteq\left(\frac{A}{\sqrt[\infty]{(b)}}\right)\{a+\sqrt[\infty]{(b)}-1\}$, so there are $\alpha+\sqrt[\infty]{(b)}, \in\left(\frac{A}{\sqrt[\infty]{(b)}}\right)$ and $\beta+\sqrt[\infty]{(b)} \in$ $\{a+\sqrt[\infty]{(b)}\}^{\infty-\text { sat }}$ such that $\gamma=\frac{\eta_{a+\sqrt[\infty]{(b)}}(\alpha+\sqrt[\infty]{(b)})}{\eta_{a+\sqrt[\infty]{(b)}}(\beta+\sqrt[\infty]{(b)})}$.

Now, $t_{\mathfrak{p}}^{a, b}(\gamma)=0$ if, and only if, $\left(t_{\mathfrak{p}}^{a, b} \circ \eta_{a+} \sqrt[\infty]{(b)} \circ \sqrt[\infty]{(b)}\right)\left(\alpha \cdot \beta^{-1}\right)=0$. By $[3.5)$, this last condition is equivalent to:

$$
\jmath_{a}\left(\eta_{a+\mathfrak{p}}\left(q_{\mathfrak{p}}\left(\alpha \cdot \beta^{-1}\right)\right)\right)=\jmath\left(\eta_{a+\mathfrak{p}} \circ q_{\mathfrak{p}}(\alpha) \cdot \eta_{a+\mathfrak{p}} \circ q_{\mathfrak{p}}(\beta)^{-1}\right)
$$

so

$$
\left(\eta_{a+\mathfrak{p}} \circ q_{\mathfrak{p}}\right)(\alpha) \in \operatorname{ker} \jmath_{a}=\{0+\mathfrak{p}\}
$$

and

$$
\eta_{a+\mathfrak{p}}(\alpha+\mathfrak{p})=0+\mathfrak{p}
$$

However, $\eta_{a+\mathfrak{p}}(\alpha+\mathfrak{p})=0 \Longleftrightarrow\left(\exists \delta+\mathfrak{p} \in\{a+\mathfrak{p}\}^{\infty-\mathrm{sat}}\right)((\delta+\mathfrak{p}) \cdot(\alpha+\mathfrak{p})=0+\mathfrak{p})$, equivalently, $\alpha \cdot \delta \in \mathfrak{p}$. Since $\mathfrak{p}$ is a prime ideal, $\alpha \cdot \delta \in \mathfrak{p} \rightarrow(\alpha \in \mathfrak{p}) \vee(\delta \in \mathfrak{p})$.

We claim that $\alpha \in \mathfrak{p} . \quad A b$ absurdo, suppose $\alpha \notin \mathfrak{p}$, so we must have $\delta \in \mathfrak{p}$. However, $\delta+\mathfrak{p} \in$ $\{a+\mathfrak{p}\}^{\infty-\text { sat }}$, so $\eta_{a+\mathfrak{p}}(\delta+\mathfrak{p}) \in\left(\frac{A}{\mathfrak{p}}\right)\left\{a+\mathfrak{p}^{-1}\right\}^{\times}$. But since $\delta \in \mathfrak{p}$, it would follow that $\eta_{a+\mathfrak{p}}(\delta+\mathfrak{p})=$ $\eta_{a+\mathfrak{p}}(0+\mathfrak{p}) \in\left(\frac{A}{\mathfrak{p}}\right)\left\{a+\mathfrak{p}^{-1}\right\}^{\times}$, from which would follow that $\left(\frac{A}{\mathfrak{p}}\right)\left\{a+\mathfrak{p}^{-1}\right\} \cong\{0\}$, a contradiction.

Thus we have $\alpha \in \mathfrak{p}$, so $\alpha+\sqrt[\infty]{(b)}$, so $\alpha+\sqrt[\infty]{(b)} \in q_{\infty} \sqrt{(b)}[\mathfrak{p}]$ and $q_{b, \mathfrak{p}}(\alpha+\sqrt[\infty]{(b)})=0+\mathfrak{p}$. Hence:

$$
\eta_{b, \mathfrak{p}}\left(\eta_{a+\infty} \sqrt{(b)}(\alpha+\sqrt[\infty]{(b)})\right)=\eta_{a+\mathfrak{p}}(\alpha+\mathfrak{p})=0
$$

and the result follows.

Now we are going to compute the fibers of the above presheaf.

Theorem 3.3.10. Consider the presheaf defined on the basis of the constructible topology:

$$
\begin{aligned}
& F_{A}: \mathcal{B}\left(\operatorname{Spec}^{\infty-\text { const }}(A)\right)^{\text {op }} \rightarrow \quad \rightarrow \quad \mathcal{C}^{\infty} \text { Rng } \\
& U=D^{\infty}(a) \cap Z^{\infty}(b) \mapsto F_{A}(U)=\lim _{U=D^{\infty}(a)}\left(\frac{A}{\sqrt[\infty]{(b)}}\right)\left\{(a+\sqrt[\infty]{(b)})^{-1}\right\} \\
& \imath_{U}^{V}: U \hookrightarrow V \quad \mapsto \quad \rho_{U}^{V}: F_{A}(U) \rightarrow F_{A}(V)
\end{aligned}
$$

The stalk of $F_{A}$ at a point $\mathfrak{p} \in \operatorname{Spec}^{\infty}(A)$ is isomorphic to the $\mathcal{C}^{\infty}$-field $\left(\frac{A}{\mathfrak{p}}\right)\left\{\left(\frac{A}{\mathfrak{p}} \backslash\{0+\mathfrak{p}\}^{-1}\right)\right\}$.

Proof. Let $L=\lim _{\mathfrak{p} \in U_{a, b}} \frac{A}{\sqrt[\infty]{(b)}}\left\{(a+\sqrt[\infty]{(b)})^{-1}\right\}$

Consider the following commutative diagram: 


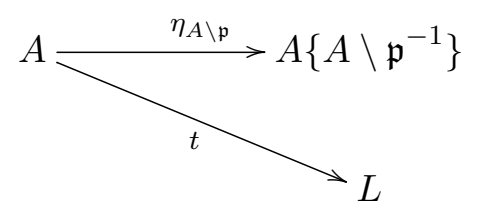

where $t=\jmath_{a, b} \circ \eta_{a+\sqrt[\infty]{(b)}} \circ q_{\infty}$.

It is clear that $(x \in A \backslash \mathfrak{p}) \rightarrow\left(t(x) \in L^{\times}\right)$. Now, since $\mathfrak{p} \in \operatorname{Spec}^{\infty}(A)$, we have $A \backslash \mathfrak{p}=A \backslash \mathfrak{p}^{\infty-\text { sat }}$, so there is a unique $\mathcal{C}^{\infty}$-homomorphism $t_{\mathfrak{p}}: A\left\{A \backslash \mathfrak{p}^{-1}\right\} \rightarrow L$ such that the following triangle commutes:

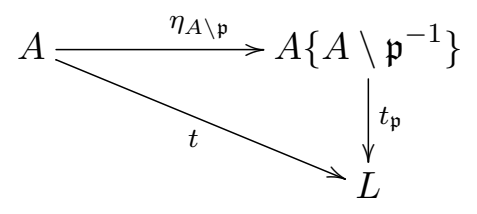

In what follows we are going to show that $t_{\mathfrak{p}}$ is a $\mathcal{C}^{\infty}$-isomorphism.

Consider the diagram:

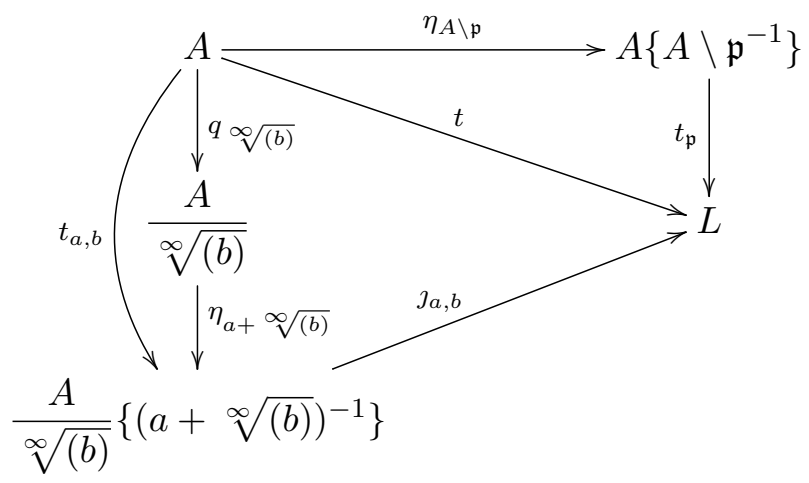

Now we claim that:

$t_{\mathfrak{p}}: A\left\{A \backslash \mathfrak{p}^{-1}\right\} \rightarrow L$ is a surjective map, i.e.,

$$
(\forall \gamma \in L)(\exists x \in A)(\exists y \in A \backslash \mathfrak{p})\left(\gamma=\frac{t(x)}{t(y)}=t_{\mathfrak{p}}\left(\frac{\eta_{\mathfrak{p}}(x)}{\eta_{\mathfrak{p}}(y)}\right)\right)
$$

Now, given $\gamma \in L$, since $\jmath_{a, b}$ is surjective there must exist $\alpha_{a, b} \in \frac{A}{\sqrt[\infty]{(b)}}\left\{(a+\sqrt[\infty]{(b)})^{-1}\right\}$ such that $\jmath_{a, b}\left(\alpha_{a, b}\right)=\gamma$.

Since $\alpha_{a, b} \in \frac{A}{\sqrt[\infty]{(b)}}\left\{(a+\sqrt[\infty]{(b)})^{-1}\right\}$, there are $c+\sqrt[\infty]{(b)} \in \frac{A}{\sqrt[\infty]{(b)}}$ and $d+\sqrt[\infty]{(b)} \in\{a+\sqrt[\infty]{(b)}\}^{\infty-\text { sat }}$ such that $\alpha_{a, b}=\frac{\eta_{a+\infty} \sqrt{(b)}(c+\sqrt[\infty]{(b)})}{\eta_{a+\sqrt[\infty]{(b)}}(d+\sqrt[\infty]{(b)})}$.

Now, $d+\sqrt[\infty]{(b)} \in\{a+\sqrt[\infty]{(b)}\}^{\infty-\text { sat }} \Longleftrightarrow(\exists z \in A)\left(\exists s \in\{a\}^{\infty-\text { sat }}\right)(s \cdot(d \cdot z-1) \in \sqrt[\infty]{(b)})$, and since $\sqrt[\infty]{(b)} \subseteq \mathfrak{p}$, we have:

$$
(\exists z \in A)\left(\exists s \in\{a\}^{\infty-\mathrm{sat}}\right)(s \cdot(d \cdot z-1) \in \mathfrak{p}) .
$$


Since $s \in\{a\}^{\infty-\text { sat }} \subseteq A \backslash \mathfrak{p}^{\infty-\text { sat }}=A \backslash \mathfrak{p}$, it follows that $s \notin \mathfrak{p}$, and since $s \cdot(d \cdot z-1) \in \sqrt[\infty]{(b)} \subseteq \mathfrak{p}$ and $\mathfrak{p}$ is a prime ideal, either $s \in \mathfrak{p}$ or $d \cdot z-1 \in \mathfrak{p}$. It is not the case that $s \in \mathfrak{p}$ so $d \cdot z-1 \in \mathfrak{p}$. However, if $d \in \mathfrak{p}$ we would have $d \cdot z-1 \in \mathfrak{p}$, hence $1 \in \mathfrak{p}$ and $\mathfrak{p}$ would not be a proper ideal, hence $d \in A \backslash \mathfrak{p}=A \backslash \mathfrak{p}^{\infty-\text { sat }}$.

It suffices to take:

$$
\alpha_{a, b}=\frac{t_{a, b}(c)}{t_{a, b}(d)}
$$

so

$$
\gamma=\jmath_{a, b}\left(\alpha_{a, b}\right)=\jmath_{a, b}\left(\frac{t_{a, b}(c)}{t_{a, b}(d)}\right)=\frac{t(c)}{t(d)}=t_{\mathfrak{p}}\left(\frac{\eta_{A \backslash \mathfrak{p}}(c)}{\eta_{A \backslash \mathfrak{p}}(d)}\right)
$$

and $t_{\mathfrak{p}}$ is surjective.

Claim: $\mathfrak{m}_{\mathfrak{p}} \subseteq \operatorname{ker} t_{\mathfrak{p}}$

Let $\frac{\eta_{A \backslash \mathfrak{p}}(x)}{\eta_{A \backslash \mathfrak{p}}(y)} \in \mathfrak{m}_{\mathfrak{p}}$, with $x \in \mathfrak{p}$ and $y \in A \backslash \mathfrak{p}$ (cf. Proposition 2.2.3. We have that $t_{\mathfrak{p}}\left(\frac{\eta_{A \backslash \mathfrak{p}}(x)}{\eta_{A \backslash \mathfrak{p}}(y)}\right)$.

Now we claim that $t(x)=0$, from what follows that:

$$
t_{\mathfrak{p}}\left(\frac{\eta_{A \backslash \mathfrak{p}}(x)}{\eta_{A \backslash \mathfrak{p}}(y)}\right)=\frac{t(x)}{t(y)}=0
$$

Since the following diagram commutes for every $a \in A \backslash \mathfrak{p}$ and $b \in \mathfrak{p}$ :

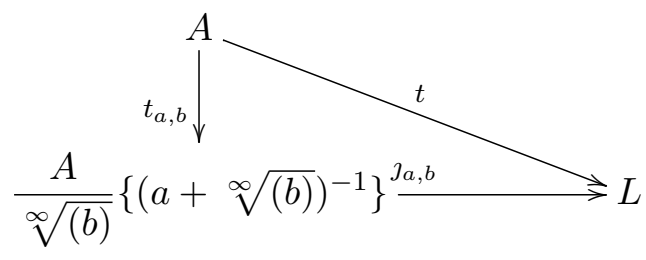

in particular, making $a=1 \in A \backslash \mathfrak{p}$ and $b=x \in \mathfrak{p}$ yields the following commutative diagram:

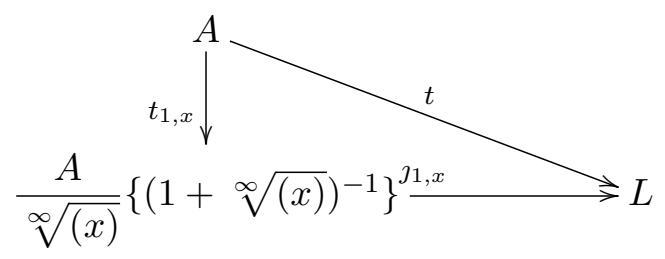

But $t(x)=\jmath_{1, x}\left(t_{1, x}(x)\right)=\jmath_{1, x}\left(\eta_{1+\sqrt[\infty]{(x)}}(0+\sqrt[\infty]{(x)})\right)=0$, hence $\frac{\eta_{A \backslash \mathfrak{p}}(x)}{\eta_{A \backslash \mathfrak{p}}(y)} \in \operatorname{ker} t_{\mathfrak{p}}$

By the Theorem of the Homomorphism, there exists a unique $\overline{t_{\mathfrak{p}}}: \frac{A_{\mathfrak{p}}}{\mathfrak{m}_{\mathfrak{p}}} \rightarrow L$ such that:

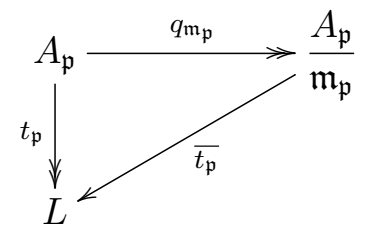


and since $t_{\mathfrak{p}}=\overline{t_{\mathfrak{p}}} \circ q_{\mathfrak{m}_{\mathfrak{p}}}$, it follows that $\overline{t_{\mathfrak{p}}}$ is surjective, hence an epimorphism.

In order to show that $\overline{t_{\mathfrak{p}}}$ is a $\mathcal{C}^{\infty}$-isomorphism, since it is already an epimorphism, it suffices to prove that it admits a section.

For every $a, b \in A$ such that $b \in \mathfrak{p}$ and $a \notin \mathfrak{p}$, consider the following diagram:

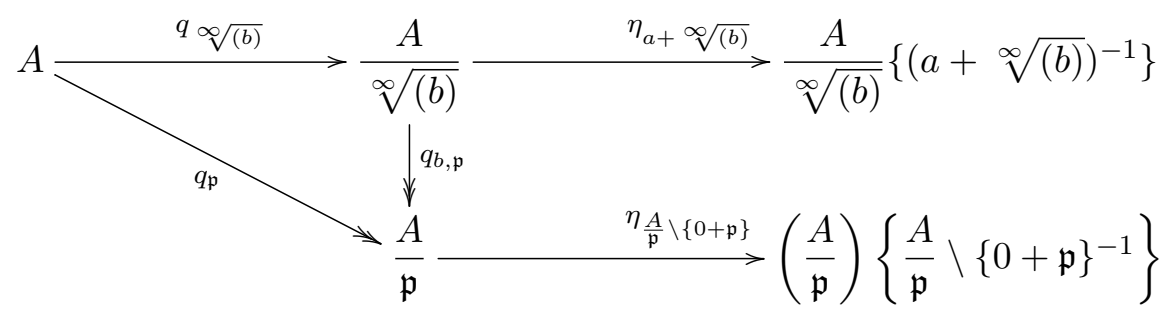

We have the following commutative diagram:

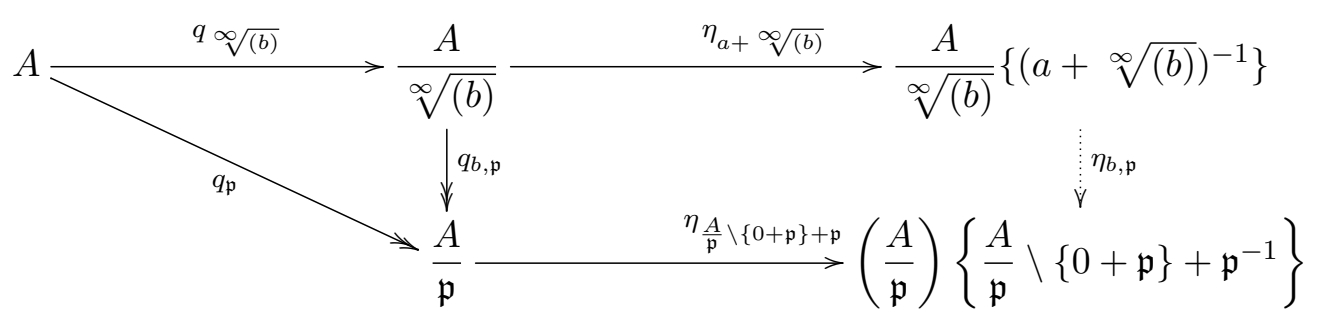

Let $a, b, c, d \in A$ be such that $U_{c, d} \subseteq U_{a, b}$, so we have the following diagram:

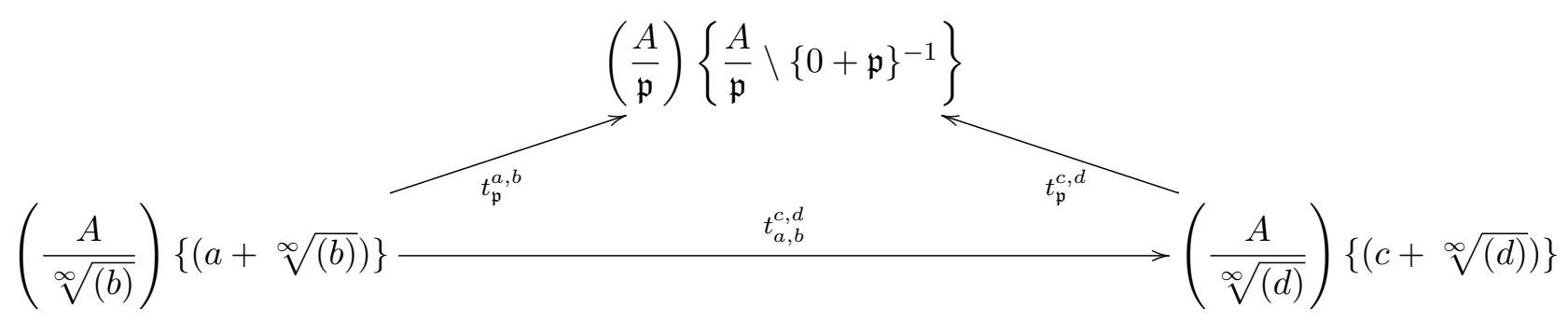

By the universal property of the inductive limit $L$, there must exist a unique

$$
\Psi_{\mathfrak{p}}: L \rightarrow\left(\frac{A}{\mathfrak{p}}\right)\left\{\frac{A}{\mathfrak{p}} \backslash\{0+\mathfrak{p}\}^{-1}\right\}
$$

such that the following diagram commutes:

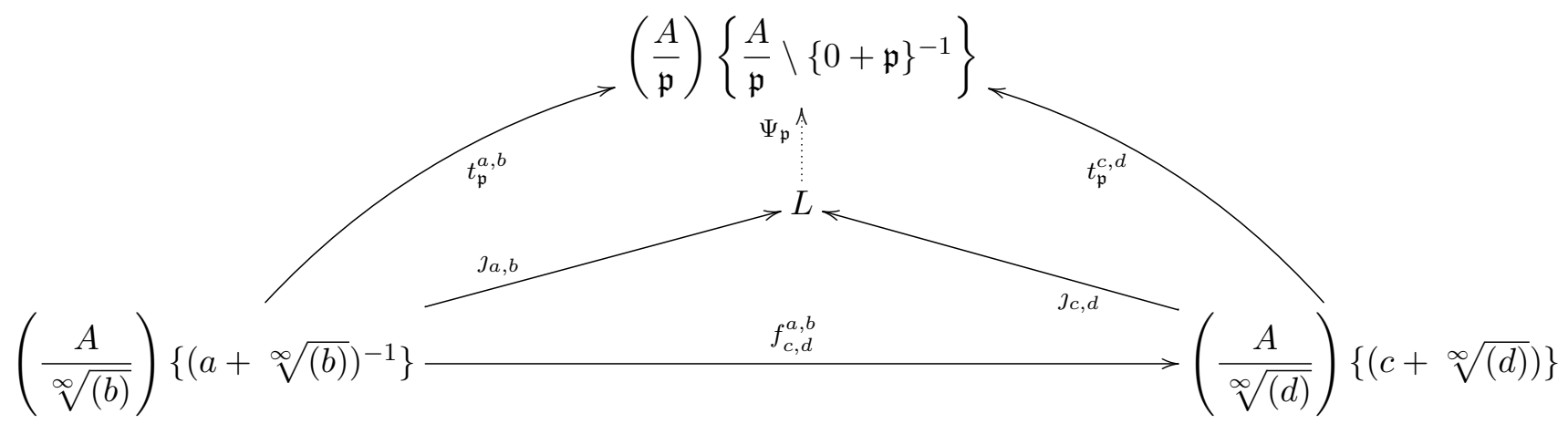

Finally we have the following diagram: 


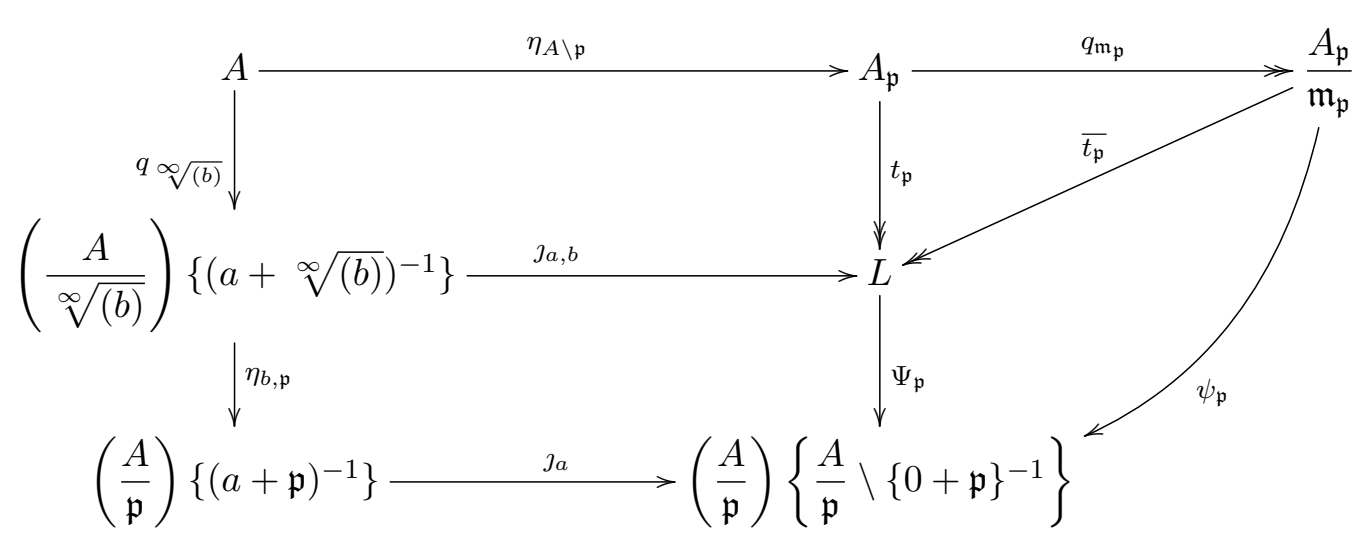

where $\psi_{\mathfrak{p}}$ is given by Theorem $\mathbf{2 . 2 . 5 3}$.

Claim: The following triangle commutes:

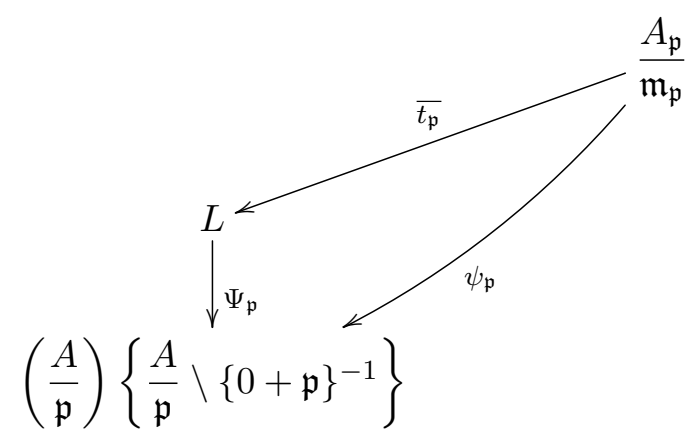

Since $q_{\mathfrak{m}_{\mathfrak{p}}}$ is an epimorphism, we have:

$$
\Psi_{\mathfrak{p}} \circ t_{\mathfrak{p}}=\Psi_{\mathfrak{p}} \circ \overline{t_{\mathfrak{p}}} \Longleftrightarrow\left(\Psi_{\mathfrak{p}} \circ t_{\mathfrak{p}}\right) \circ q_{\mathfrak{m}_{\mathfrak{p}}}=\left(\Psi_{\mathfrak{p}} \circ \overline{t_{\mathfrak{p}}}\right) \circ q_{\mathfrak{m}_{\mathfrak{p}}}
$$

Now, by the universal property of $\eta_{A \backslash \mathfrak{p}}: A \rightarrow A_{\mathfrak{p}}$, there is a unique $\mathcal{C}^{\infty}$-homomorphism $\xi: A_{\mathfrak{p}} \rightarrow$ $\left(\frac{A}{\mathfrak{p}}\right)\left\{\frac{A}{\mathfrak{p}} \backslash\{0+\mathfrak{p}\}^{-1}\right\}$ such that:

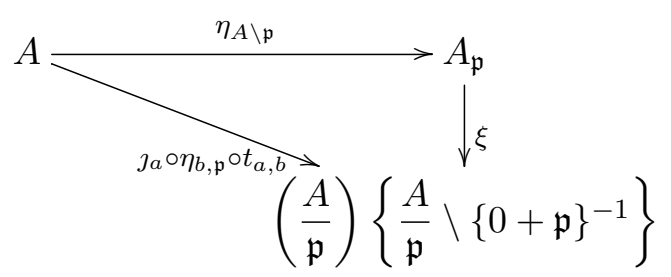

commutes, so $\left(\Psi_{\mathfrak{p}} \circ t_{\mathfrak{p}}\right) \circ q_{\mathfrak{m}_{\mathfrak{p}}}=\xi=\left(\Psi_{\mathfrak{p}} \circ \overline{t_{\mathfrak{p}}}\right) \circ q_{\mathfrak{m}_{\mathfrak{p}}}$ and the former triangle commutes.

Thus, since $\psi_{\mathfrak{p}}=\Psi_{\mathfrak{p}} \circ \overline{t_{\mathfrak{p}}}$ where $\overline{t_{\mathfrak{p}}}$ is an epimorphism which is also a section, it follows that $\overline{t_{\mathfrak{p}}}$ and $\Psi_{\mathfrak{p}}$ are both $\mathcal{C}^{\infty}$-isomorphisms, as we wanted to show.

Remark 3.3.11. If $A$ is a von Neumann regular $C^{\infty}$-ring, then for each $a, b \in V$, let e, $f$ (ditto) be the unique idempotents of $V$ such that $(a)=(e)$ and $(b)=(f)$. Then:

$$
D^{\infty}(a) \cap Z^{\infty}(b)=D^{\infty}(e) \cap Z^{\infty}(f)=D^{\infty}(e) \cap D^{\infty}(1-f)=D^{\infty}(e .(1-f))
$$

and

$$
\left(\frac{A}{\sqrt[\infty]{(b)}}\right)\left\{(a+\sqrt[\infty]{(b)})^{-1}\right\}=\left(\frac{A}{\sqrt[\infty]{(f)}}\right)\left\{(e+\sqrt[\infty]{(f)})^{-1}\right\}=
$$




$$
=\left(\frac{A}{(f)}\right)\left\{(e+(f))^{-1}\right\} \cong A\left\{(1-f)^{-1}\right\}\left\{\eta_{1-f}(e)^{-1}\right\} \cong A\left\{((1-f) \cdot e)^{-1}\right\}
$$

Thus the (pre)sheaf basis $F_{A}$ is isomorphic to the "affine" sheaf basis $\Sigma_{A}$.

Now we proceed as follows:

- For each $C^{\infty}$-ring $A, F_{A}:\left(\mathcal{B}\left(\operatorname{Spec}^{\infty-\text { const }}(A)\right), \subseteq\right)^{\text {op }} \rightarrow \mathcal{C}^{\infty} \mathbf{R n g}$ is a presheaf on the (canonical) basis of the constructible topology in $\operatorname{Spec}^{\infty}(A)$ and with the stalks $\cong k_{\mathfrak{p}}(A), \mathfrak{p} \in \operatorname{Spec}^{\infty}(A)$. Moreover there is a canonical $C^{\infty}$-homomorphism $\left.\phi_{A}: A \rightarrow F_{A}\left(\operatorname{Spec}^{\infty-\text { const }}(A)\right)\right) \cong A / \sqrt[\infty]{(0)}$, in such a way that we obtain a functor $\left.A \stackrel{\mathbb{F}}{\mapsto} F_{A}\left(\operatorname{Spec}^{\infty-\text { const }}(A)\right)\right)$ and a natural transformation $\phi: i d \rightarrow \mathbb{F}$.

- For each $C^{\infty}$-ring $A$, take $G_{A}$ the sheaf-basis functor associated to $F_{A}$ - both are defined on the basis of the constructible topology of the $C^{\infty}$-spectrum of $A$ and both share the same (up to isomorphisms) stalks. Moreover, since there is a natural transformation $F_{A} \rightarrow G_{A}$, there is a canonical $C^{\infty}$-homomorphism $\left.\gamma_{A}: A \rightarrow G_{A}\left(\operatorname{Spec}^{\infty \text {-const }}(A)\right)\right)$, in such a way that we obtain a functor $\left.A \stackrel{\mathbb{G}}{\mapsto} G_{A}\left(\operatorname{Spec}^{\infty-\text { const }}(A)\right)\right)$ and a natural transformation $\gamma: i d \rightarrow \mathbb{G}$.

- For each $C^{\infty}$-ring $A$, take $H_{A}$ the (unique up to isomorphism) sheaf extension $G_{A}$ to the basis of constructible topology into all the opens sets of this topology, thus $H_{A}\left\lceil\cong G_{A}\right.$, and both functor keeps the same stalks (up to isomorphism). Moreover, since there is a natural transformation (isomorphism) $G_{A} \rightarrow H_{A} \uparrow$, there is a canonical $C^{\infty}$-homomorphism $\left.\mu_{A}: A \rightarrow H_{A}\left(\operatorname{Spec}^{\infty \text {-const }}(A)\right)\right)$, in such a way that we obtain a functor $\left.A \stackrel{\mathbb{H}}{\mapsto} H_{A}\left(\operatorname{Spec}^{\infty \text {-const }}(A)\right)\right)$ and a natural transformation $\mu: i d \rightarrow \mathbb{H}$.

- Since $H_{A}$ is a sheaf of $C^{\infty}$-rings, defined over all the opens of a boolean space, and whose stalks are $C^{\infty}$-fields, then $\left.\mathbb{H}(A)=H_{A}\left(\operatorname{Spec}^{\infty \text {-const }}(A)\right)\right)$ is a Von Neumann regular $C^{\infty}$-ring. Thus $A \stackrel{\mathbb{H}}{\mapsto}$ $\left.H_{A}\left(\operatorname{Spec}^{\infty-\text { const }}(A)\right)\right)$ determines a functor $C^{\infty} \mathbf{R n g} \rightarrow C^{\infty} \mathbf{v N R n g}$ and we have a natural transformation $\mu: i d \rightarrow i \circ \mathbb{H}$.

We are ready to (re)state the:

Theorem 3.3.12. (bis) The inclusion functor $C^{\infty} \mathbf{v N R n g} \hookrightarrow C^{\infty} \mathbf{R n g}$ has a left adjoint functor $\mathrm{vN}$ : $C^{\infty} \mathbf{R n g} \rightarrow C^{\infty} \mathbf{v N R n g}$. In more details: for $A$ be any $\mathcal{C}^{\infty}$-ring, the pair $\left.\left.(\mathbb{H}(A)), \mu_{A}: A \rightarrow \mathbb{H}(A)\right)\right)$ is the $\mathcal{C}^{\infty}$-von Neumann regular hull of $A$, that is, for every von Neumann-regular $\mathcal{C}^{\infty}$-ring $V$ and for every $\mathcal{C}^{\infty}$-homomorphism $f: A \rightarrow V$ there is a unique $\mathcal{C}^{\infty}$-homomorphism $\widetilde{f}: A_{\omega} \rightarrow V$ such that the following diagram commutes:

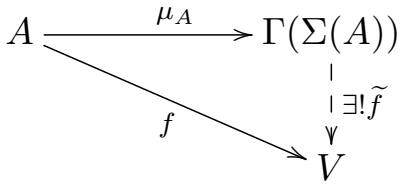

Proof. We just have observed above that all the conditions in the Theorem 3.3 .8 hold.

We finish this subsection with some general applications of the adjunction above and one of its specific "sheaf-theorectic" construction of the left adjoint vN : $C^{\infty} \mathbf{R n g} \rightarrow C^{\infty} \mathbf{v N R n g}$.

Proposition 3.3.13. The functor $\mathrm{vN}$ preserves all colimits. In particular it preserves:

- directed inductive limits;

- coproducts ( $=\infty$-tensor products in $\left.\mathbf{C}^{\infty}-\mathbf{R n g}\right)$; 
- coequalizers/quotients.

Proof. Since it is a left adjoint, vN preserves all colimits. We explain the meaning of the preservation of quotients. Consider the induced homomorphism $v N\left(q_{I}\right): \mathrm{vN}(A) \rightarrow \mathrm{vN}(A / I)$ : it is surjective since the coequalizers in $\mathbf{C}^{\infty}-\mathbf{R n g}$ and $\mathbf{C}^{\infty} \mathbf{v N R} n g$ coincide with the surjective homomorphisms. Then $\bar{I}:=$ $\operatorname{ker}\left(\mathrm{vN}\left(q_{I}\right)\right) \subseteq \mathrm{vN}(A)$ is such that $\overline{\mathrm{vN}\left(q_{I}\right)}: \mathrm{vN}(A) / \bar{I} \cong \mathrm{gN}(A / I)$ and, since $\mathrm{vN}(A) \longmapsto \prod_{\mathfrak{p} \in \operatorname{Spec}^{\infty}(A)} k_{\mathfrak{p}}(A)$, $\bar{I}$ can be identified with $\mathrm{vN}(A) \cap\left\{\vec{x}=\left(x_{\mathfrak{p}}\right)_{\mathfrak{p} \in \operatorname{Spec}^{\infty}(A)} \in \prod_{\mathfrak{p} \in \operatorname{Spec}^{\infty}(A)} k_{\mathfrak{p}}(A): \forall \mathfrak{p} \supseteq I, x_{\mathfrak{p}}=0 \in k_{\mathfrak{p}}(A)\right\}$. Note in particular, that if $I \subseteq \sqrt[\infty]{(0)}$, then $\mathrm{vN}\left(q_{I}\right): \mathrm{vN}(A) \stackrel{\cong}{\rightrightarrows} \mathrm{vN}(A / I)$, thus $\bar{I}=\{0\}$.

The following results are specific to the functor vN, i.e., they are not general consequences of it being a left adjoint functor.

Proposition 3.3.14. vN preserves localizations. More precisely, given a $\mathcal{C}^{\infty}$-ring $A$ and a subset $S \subseteq A$, denote $S^{\prime}:=\eta_{A}[S] \subseteq \mathrm{vN}(A)$ the corresponding subset and let $\eta_{A}^{S}: A\left\{S^{-1}\right\} \rightarrow \mathrm{vN}(A)\left\{S^{\prime-1}\right\}$ be the induced arrow, i.e., $\eta_{A}^{S}$ is the unique homomorphism such that $\eta_{A}^{S} \circ \sigma(A)_{S}=\sigma(\operatorname{vN}(A))_{S^{\prime}} \circ \eta_{A}$. Then $\eta_{A\left\{S^{-1}\right\}}: A\left\{S^{-1}\right\} \rightarrow \mathrm{vN}\left(A\{S\}^{-1}\right)$ thus it is isomorphic to the arrow $\eta_{A}^{S}$, through the obvious pair of inverse (iso)morphisms $\mathrm{vN}\left(A\{S\}^{-1}\right) \rightleftarrows \mathrm{vN}(A)\left\{S^{\prime}\right\}^{-1}$.

Proof. First of all, note that $\mathrm{vN}(A)\left\{S^{\prime}\right\}^{-1}$ is a vN-regular ring, cf. Proposition 2.2.68. For each vN-regular ring $V$, the bijection $\left(-\circ \eta_{A}\right): \mathcal{C}^{\infty} \mathbf{v N R n g}(\mathrm{vN}(A), V) \stackrel{\cong}{\rightarrow} \mathcal{C}^{\infty}-\mathbf{R n g}(A, V)$ restricts to the bijection:

$$
\begin{aligned}
\left(-\circ \eta_{A}\right) \Gamma:\left\{H \in \mathcal{C}^{\infty} \mathbf{v N R n g}\right. & \left.\underset{\operatorname{vN}}{ }(A), V): H\left[S^{\prime}\right] \subseteq V^{\times}\right\} \\
& \stackrel{\cong}{\rightrightarrows}\left\{h \in \mathcal{C}^{\infty}-\mathbf{R n g}(A, V): h[S] \subseteq V^{\times}\right\} .
\end{aligned}
$$

Composing the last bijection with the bijections below, obtained from the universal property of localizations,

$$
\begin{gathered}
\left(-\circ \sigma(A)_{S}\right)^{-1}:\left\{h \in \mathcal{C}^{\infty}-\mathbf{R n g}(A, V): h[S] \subseteq V^{\times}\right\} \cong \mathcal{C}^{\infty}-\mathbf{R n g}\left(A[S]^{-1}, V\right), \\
\left(-\circ \sigma(\operatorname{vN}(A))_{S^{\prime}}\right): \mathcal{C}^{\infty}-\mathbf{R n g}\left(\operatorname{vN}(A)\left\{S^{\prime-1}\right\}, V\right) \stackrel{\cong}{\rightrightarrows}\left\{H \in \mathcal{C}^{\infty}-\mathbf{R n g}(\operatorname{vN}(A), V): H\left[S^{\prime}\right] \subseteq V^{\times}\right\},
\end{gathered}
$$

we obtain, since $\mathcal{C}^{\infty} \mathbf{v N R}$ ng is a full subcategory of $\mathcal{C}^{\infty} \mathbf{R n g}$, the bijection

$$
\left(-\circ \eta_{A}^{S}\right): \mathcal{C}^{\infty} \mathbf{v N R n g}\left(\mathrm{vN}(A)\left\{S^{\prime-1}\right\}, V\right) \stackrel{\cong}{\rightarrow} \mathcal{C}^{\infty} \mathbf{R n g}\left(A\left\{S^{-1}\right\}, V\right) .
$$

Summing up, the arrow $\eta_{A}^{S}: A\left\{S^{-1}\right\} \rightarrow \mathrm{vN}(A)\left\{S^{-1}\right\}$ satisfies the universal property of vN-regular hull, thus it is isomorphic to the arrow $\eta_{A\left\{S^{-1}\right\}}: A\left\{S^{-1}\right\} \rightarrow \mathrm{vN}\left(A\left\{S^{-1}\right\}\right)$.

The following result shows us how useful is the sheaf-theoretic description of the vN-hull of a $\mathcal{C}^{\infty}$-ring.

Proposition 3.3.15. vN preserves finite products. More precisely, let $I$ be a finite set and $\left\{A_{i}: i \in\right.$ I\} any family of $\mathcal{C}^{\infty}$-rings. Denote $\pi_{j}: \prod_{i \in I} A_{i} \rightarrow A_{j}$ the projection homomorphism, $j \in I$. Then $\prod_{i \in I} \eta_{A_{i}}: \prod_{i \in I} A_{i} \rightarrow \prod_{i \in I} \mathrm{vN}\left(A_{i}\right)$ satisfies the universal property of vN-regular hull, thus it is isomorphic to the arrow $\eta_{\prod_{i \in I} A_{i}}: \prod_{i \in I} A_{i} \rightarrow \mathrm{vN}\left(\prod_{i \in I} A_{i}\right)$, through the obvious pair of inverse (iso)morphisms $\mathrm{vN}\left(\prod_{i \in I} A_{i}\right) \rightleftarrows \prod_{i \in I} \mathrm{vN}\left(A_{i}\right)$.

Proof. (Sketch) First of all, note that $\prod_{i \in I} \mathrm{vN}\left(A_{i}\right)$ is a vN-regular $\mathcal{C}^{\infty}$-ring. If $I=\emptyset$, then $\prod_{i \in I} A_{i}$, $\prod_{i \in I} \mathrm{vN}\left(A_{i}\right)$ and $\mathrm{vN}\left(\prod_{i \in I} A_{i}\right)$ are isomorphic to the trivial $\mathcal{C}^{\infty}$-ring $\{0\}$, thus the result holds in this case. have:

By induction, we only need to see that $\left(\mathrm{vN}\left(\pi_{1}\right), \mathrm{vN}\left(\pi_{2}\right)\right): \mathrm{vN}\left(A_{1} \times A_{2}\right) \stackrel{\cong}{\rightarrow} \mathrm{vN}\left(A_{1}\right) \times \mathrm{vN}\left(A_{2}\right)$. But we 
- $\left[\pi_{1}^{\star}, \pi_{2}^{\star}\right]: \operatorname{SpeC}^{\infty}\left(A_{1}\right) \sqcup \operatorname{SpeC}^{\infty}\left(A_{2}\right) \stackrel{\approx}{\rightarrow} \operatorname{Spe}^{\infty}\left(A_{1} \times A_{2}\right), \quad\left(p_{i}, i\right) \mapsto \pi_{i}^{-1}\left[p_{i}\right], i=1,2$

- $\left[\pi_{1}^{\star}, \pi_{2}^{\star}\right]: \operatorname{SpeC}^{\infty \text { const }}\left(A_{1}\right) \sqcup \operatorname{SpeC}^{\infty \text { const }}\left(A_{2}\right) \stackrel{\approx}{\rightarrow} \operatorname{SpeC}^{\infty \text { const }}\left(A_{1} \times A_{2}\right)$.

- Let $a:=\left(a_{1}, a_{2}\right) \in A_{1} \times A_{2}$ and $\bar{b}:=\left\{\left(b_{1,1}, b_{2,1}\right), \cdots,\left(b_{1, n}, b_{2, n}\right)\right\} \subseteq_{\text {fin }} A_{1} \times A_{2}$, then $\left(A_{1} \times A_{2}\right)_{a, \bar{b}} \cong$ $A_{1_{a_{1}, \bar{b}_{1}}} \times A_{2_{a_{2}, \bar{b}_{2}}}$.

- $\sqcup_{p \in \operatorname{SpeC}^{\infty}\left(A_{1} \times A_{2}\right)} k_{\mathfrak{p}}\left(A_{1} \times A_{2}\right) \approx\left(\sqcup_{\mathfrak{p}_{1} \in \operatorname{SpeC}^{\infty}\left(A_{1}\right)} k_{\mathfrak{p}_{1}}\left(A_{1}\right)\right) \sqcup\left(\sqcup_{\mathfrak{p}_{2} \in \operatorname{SpeC}^{\infty}\left(A_{1}\right)} k_{\mathfrak{p}_{2}}\left(A_{2}\right)\right)$.

- Let $U \in \operatorname{Open}\left(\operatorname{SpeC}^{\infty}\left(A_{1} \times A_{2}\right)\right)$, then $U \approx U_{1} \sqcup U_{2}$, where $U_{i}=\left(\pi_{i}^{\star}\right)^{-1}[U] \in \operatorname{Open}\left(\operatorname{SpeC}^{\infty}\left(A_{i}\right)\right)$. Then any sheaf $S$ satisfies $S(A)(U) \cong S\left(A_{1}\right)\left(U_{1}\right) \times S\left(A_{2}\right)\left(U_{2}\right)$.

Now the construction of $\mathrm{vN}$ as the global sections of of a sheaf allows to conclude $\mathrm{vN}\left(A_{1} \times A_{2}\right)=$ $S\left(A_{1} \times A_{2}\right)\left(\operatorname{SpeC}^{\infty}\left(A_{1} \times A_{2}\right)\right) \cong\left(S\left(A_{1}\right)\left(\operatorname{SpeC}^{\infty}\left(A_{1}\right)\right) \times S\left(A_{2}\right)\left(\operatorname{SpeC}^{\infty}\left(A_{2}\right)\right)\right)=\operatorname{vN}\left(A_{1}\right) \times \operatorname{vN}\left(A_{2}\right)$.

\subsection{Von Neumann-regular $\mathcal{C}^{\infty}$-Rings and Boolean Algebras}

In this section we also apply von Neumann regular $\mathcal{C}^{\infty}$-ring to naturally represent Boolean Algebras in a strong sense: i.e., not only all Boolean algebras are isomorphic to the Boolean algebra of idempotents of a von Neumann regular $\mathcal{C}^{\infty}$-ring, as every homomorphism between such boolean algebras of idempotents is induced by a $\mathcal{C}^{\infty}$-homomorphism.

We start with the following general results:

Proposition 3.4.1. Let $(L, \wedge, \vee, \leq)$ be any lattice. Then:

$$
(\forall a \in L)(\forall b \in L)(\forall c \in L)(a \wedge(b \vee c)=(a \wedge b) \vee(a \wedge c))
$$

if, and only if,

$$
(\forall a \in L)(\forall b \in L)(\forall c \in L)(a \vee(b \wedge c)=(a \vee b) \wedge(a \vee c))
$$

Proof. See, for example, Lemma 6.3 of [88].

Proposition 3.4.2. Let $(A,+, \cdot, 0,1)$ be any commutative unital ring and define $B(A):=\left\{e \in A \mid e^{2}=e\right\}$. Then $(B(A), \wedge, \vee, *, \leq, \perp, \top)$, where:

$$
\begin{array}{rlrl}
\wedge: B(A) \times B(A) & \rightarrow & B(A) \\
\left(e, e^{\prime}\right) & & \mapsto & e \cdot e^{\prime} \\
\vee: B(A) \times B(A) & & & B(A) \\
\left(e, e^{\prime}\right) & \mapsto & e+e^{\prime}-e \cdot e^{\prime} \\
*: \quad B(A) & \rightarrow & B(A) \\
e & \mapsto & 1-e \\
\leq=\left\{\left(e, e^{\prime}\right) \in B(A) \times\right. & \left.B(A) \mid e \cdot e^{\prime}=e\right\} \\
\top=1 \text { and } \perp=0
\end{array}
$$

is a bounded, distributive and complemented lattice, i.e., a Boolean Algebra. 
Proof. Claim: $\left(e \wedge e^{\prime}\right)^{2}=e \wedge e^{\prime}$, so $e \wedge e^{\prime} \in B(A)$.

$\left(e \wedge e^{\prime}\right)^{2}=\left(e \cdot e^{\prime}\right)^{2}=e \cdot e^{\prime} \cdot e \cdot e^{\prime}=e^{2} \cdot e^{\prime 2}=e \cdot e^{\prime}=e \wedge e^{\prime}$.

Claim: $\left(e \vee e^{\prime}\right)^{2}=e \vee e^{\prime}$, so $e \vee e^{\prime} \in B(A)$.

$\left(e \vee e^{\prime}\right)^{2}=\left(e+e^{\prime}-e \cdot e^{\prime}\right)^{2}=e^{2}+2 \cdot e \cdot e^{\prime}+e^{\prime 2}-2\left(e^{2} \cdot e^{\prime}+e \cdot e^{\prime 2}\right)+e \cdot e^{\prime 2}=$

$=e^{2}+e^{\prime 2}+2 \cdot e \cdot e^{\prime}-2 \cdot e \cdot e^{\prime}-2 \cdot e^{2} \cdot e^{\prime}-2 \cdot e \cdot e^{\prime 2}+e \cdot e^{\prime}=e^{2}+e^{\prime 2}-e \cdot e^{\prime}=e+e^{\prime}-e \cdot e^{\prime}=e \vee e^{\prime}$

Claim: For any $e \in B(A), e^{*}=1-e \in B(A)$.

$$
(1-e)^{2}=1-2 \cdot e+e^{2}=1-e .
$$

Note that for any $e \in B(A), e \vee e^{*}=e \vee(1-e)=e+(1-e)-e \cdot(1-e)=1$.

Claim: Given any $e, e^{\prime} \in B(A), e \vee e^{\prime}=\sup \left\{e, e^{\prime}\right\}$.

We have:

$$
e \cdot\left(e \vee e^{\prime}\right)=e \cdot\left(e+e^{\prime}-e \cdot e^{\prime}\right)=e^{2}+e \cdot e^{\prime}-e^{2} \cdot e^{\prime}=e+e \cdot e^{\prime}-e \cdot e^{\prime}=e, \text { so } e \leq\left(e \vee e^{\prime}\right)
$$

A similar reasoning shows us that $e^{\prime} \leq\left(e \vee e^{\prime}\right)$.

Let $f \in B(A)$ be such that $e \leq f$ and $e^{\prime} \leq f$. Then:

$$
e \cdot f=e \text { and } e^{\prime} \cdot f=e^{\prime},
$$

so

$$
f \cdot\left(e \vee e^{\prime}\right)=f \cdot\left(e+e^{\prime}-e \cdot e^{\prime}\right)=f \cdot e+f \cdot e^{\prime}-f \cdot e \cdot e^{\prime}=e+e^{\prime}-e \cdot e^{\prime}=e \vee e^{\prime},
$$

hence $e \vee e^{\prime}=\sup \left\{e, e^{\prime}\right\}$.

Claim: Given any $e, e^{\prime} \in B(A), e \wedge e^{\prime}=\inf \left\{e, e^{\prime}\right\}$.

We have:

$$
e \cdot e^{\prime}=e^{2} \cdot e^{\prime}=e \cdot\left(e \cdot e^{\prime}\right)=e \cdot\left(e \wedge e^{\prime}\right), \text { so } e \wedge e^{\prime} \leq e .
$$

A similar reasoning shows us that $e \wedge e^{\prime} \leq e^{\prime}$.

Let $f \in B(A)$ be such that $f \leq e$ and $f \leq e^{\prime}$, so $f \cdot e=f$ and $f \cdot e^{\prime}=f$.

We have:

$$
f \cdot\left(e \wedge e^{\prime}\right)=f \cdot\left(e \cdot e^{\prime}\right)=(f \cdot e) \cdot e^{\prime}=f \cdot e^{\prime}=f, \text { so } f \leq e \wedge e^{\prime},
$$


hence $e \wedge e^{\prime}=\inf \left\{e, e^{\prime}\right\}$.

Note that since $(\forall e \in B(A))(e \cdot 0=0)$, then $(\forall e \in B(A))(0 \leq e)$ so $0=\perp$, and since $(\forall e \in B(A))(e \cdot 1=$ $e)$, then $(\forall e \in B(A))(e \leq 1)$, so $1=\top$.

Claim: $(\forall e \in B(A))(\forall f \in B(A))(\forall g \in B(A))(e \wedge(f \vee g)=(e \wedge f) \vee(e \wedge g))$.

$$
\begin{aligned}
& e \wedge(f \vee g)=e \cdot(f \vee g)=e \cdot(f+g-f \cdot g)=e \cdot f+e \cdot g-e \cdot f \cdot g= \\
& =(e \cdot f) \vee(e \cdot g)=e \cdot f+e \cdot g-(e \cdot f) \cdot(e \cdot g)=e \cdot f+e \cdot g-e \cdot f \cdot g=e \cdot(f+g-f \cdot g)=e \wedge(f \vee g)
\end{aligned}
$$

By Proposition 3.4.1, it follows that:

$$
(\forall e \in B(A))(\forall f \in B(A))(\forall g \in B(A))(e \vee(f \wedge g)=(e \vee f) \wedge(e \vee g))
$$

It follows from these considerations that $(B(A), \wedge, \vee, *, \leq, \perp, \top)$ is a bounded, distributive and complemented lattice, hence a Boolean algebra.

Remark 3.4.3. By Stone Duality, there is an anti-equivalence of categories between the category of Boolean algebras, Bool, and the category of Boolean spaces, BoolSp.

Under this anti-equivalence, a Boolean space $(X, \tau)$ is mapped to the Boolean algebra of clopen subsets of $(X, \tau)$, Clopen $(X)$ :

$$
\begin{aligned}
& \text { Clopen : } \quad \text { BoolSp } \quad \rightarrow \quad \text { Bool } \\
& (X, \tau) \stackrel{f}{\longrightarrow}(Y, \sigma) \mapsto \text { Clopen }(Y) \stackrel{f^{-1}\lfloor}{\longrightarrow} \text { Clopen }(X)
\end{aligned}
$$

The quasi-inverse functor is given by the Stone space functor: a Boolean algebra $B$ is mapped to the Stone space of $B$, Stone $(B)=\left(\{U \subseteq B: U\right.$ is an ultrafilter in $\left.B\}, \tau_{B}\right)$, where $\tau_{B}$ is the topology whose basis is given by the image of the map $t_{B}: B \rightarrow \wp(\operatorname{Stone}(B)), b \mapsto t_{B}(b)=S_{B}(b)=\{U \in \operatorname{Stone}(B): b \in$ $U\}$.

$$
\begin{array}{cccc}
\text { Stone : } & \text { Bool } & \rightarrow & \text { BoolSp } \\
& B \stackrel{h}{\longrightarrow} B^{\prime} & \mapsto & \text { Stone }\left(B^{\prime}\right) \stackrel{h^{-1} \uparrow}{\longrightarrow} \text { Stone }(B)
\end{array}
$$

Remark 3.4.4. Let $\left(A^{\prime},+^{\prime},,^{\prime}, 0^{\prime}, 1^{\prime}\right)$ be any commutative unital ring and denote by $\wedge^{\prime}, \vee^{\prime}, *^{\prime}, \leq^{\prime}, 0^{\prime}$ and $1^{\prime}$ its respective associated Boolean algebra operations, relations and constant symbols as constructed above. Note that for any commutative unital ring homomorphism $f: A \rightarrow A^{\prime}$, the map $B(f):=f \uparrow_{B(A)}: B(A) \rightarrow$ $A^{\prime}$ is such that:

(i) $B(f)[B(A)] \subseteq B\left(A^{\prime}\right)$;

(ii) $\left(\forall e_{1} \in A\right)\left(\forall e_{2} \in A\right)\left(B(f)\left(e_{1} \wedge e_{2}\right)=f \uparrow_{B(A)}\left(e_{1} \cdot e_{2}\right)=\left(f \uparrow_{B(A)}\left(e_{1}\right)\right) \cdot\left(f \uparrow_{B(A)}\left(e_{2}\right)\right)=B(f)\left(e_{1}\right) \wedge^{\prime}\right.$ $\left.B(f)\left(e_{2}\right)\right)$

(iii) $\left(\forall e_{1} \in A\right)\left(\forall e_{2} \in A\right)\left(B(f)\left(e_{1} \vee e_{2}\right)=f \uparrow_{B(A)}\left(e_{1}+e_{2}\right)=\left(f \uparrow_{B(A)}\left(e_{1}\right)\right)+^{\prime}\left(f \uparrow_{B(A)}\left(e_{2}\right)\right)=\right.$ $\left.B(f)\left(e_{1}\right) \vee^{\prime} B(f)\left(e_{2}\right)\right)$

(iv) $(\forall e \in B(A))\left(B(f)\left(e^{*}\right)=f \uparrow_{B(A)}(1-e)=f \uparrow_{B(A)}(1)-f \uparrow_{B(A)}(e)=1^{\prime}-f \uparrow_{B(A)}(e)=B(f)(e)^{*}\right)$ 
We also have, for every ring $A, B\left(\mathrm{id}_{A}\right)=\operatorname{id}_{B(A)}$ and given any $f: A \rightarrow A^{\prime}$ and $f^{\prime}: A^{\prime} \rightarrow A^{\prime \prime}$, $B\left(f^{\prime} \circ f\right)=B\left(f^{\prime}\right) \circ B(f)$, since $B(f)=f \uparrow_{B(A)}$, so:

$$
\begin{array}{cccc}
B: \quad \text { CRing } & \rightarrow & \text { Bool } \\
A & \mapsto & B(A) \\
A \stackrel{f}{\longrightarrow} A^{\prime} & \mapsto & B(A) \stackrel{B(f)}{\longrightarrow} B\left(A^{\prime}\right)
\end{array}
$$

is a (covariant) functor.

Since we can regard any $\mathcal{C}^{\infty}$-ring $A$ as a commutative unital ring via the forgetful functor $\widetilde{U}$ : $\mathcal{C}^{\infty}$ Rng $\rightarrow$ CRing, we have a (covariant) functor:

$$
\begin{array}{rlcc}
\widetilde{B}: \quad \mathcal{C}^{\infty} \text { Rng } & \rightarrow & \text { Bool } \\
A & \mapsto & \widetilde{B}(A):=(B \circ U)(A) \\
A \stackrel{f}{\longrightarrow} A^{\prime} & \mapsto & \widetilde{B}(A) \stackrel{\widetilde{B}(f)}{\longrightarrow} \widetilde{B}\left(A^{\prime}\right)
\end{array}
$$

Now, if $A$ is any $\mathcal{C}^{\infty}$-ring, we can define the following map:

$$
\begin{array}{ccc}
\jmath_{A}: \widetilde{B}(A) & \rightarrow & \text { Clopen }\left(\operatorname{Spec}^{\infty}(A)\right) \\
e & \mapsto D^{\infty}(e)=\left\{\mathfrak{p} \in \operatorname{Spec}^{\infty}(A) \mid e \notin \mathfrak{p}\right\}
\end{array}
$$

Claim: The map defined above is a Boolean algebra homomorphism.

Note that for any $e \in B(A), D^{\infty}\left(e^{*}\right)=D^{\infty}(1-e)=\operatorname{Spec}^{\infty}(A) \backslash D^{\infty}(e)=D^{\infty}(e)^{*}$, since:

$$
D^{\infty}(e) \cap D^{\infty}(1-e)=D^{\infty}(e \cdot(1-e))=D^{\infty}(0)=\varnothing
$$

and

$D^{\infty}(e) \cup D^{\infty}(e *)=D^{\infty}(e) \cup D^{\infty}(1-e)=D^{\infty}\left(e^{2}+(1-e)^{2}\right)=D^{\infty}(e+(1-e))=D^{\infty}(1)=\operatorname{Spec}^{\infty}(A)$.

Hence $\jmath_{A}\left(e^{*}\right)=\jmath_{A}(1-e)=D^{\infty}(1-e)=\operatorname{Spec}^{\infty}(A) \backslash D^{\infty}(e)=D^{\infty}(e)^{*}=\jmath_{A}(e)^{*}$.

By the item (iii) of Lemma 1.4 of $[65], D^{\infty}\left(e \cdot e^{\prime}\right)=D^{\infty}(e) \cap D^{\infty}\left(e^{\prime}\right)$, so:

$$
\jmath_{A}\left(e \wedge e^{\prime}\right)=D^{\infty}\left(e \cdot e^{\prime}\right)=D^{\infty}(e) \cap D^{\infty}\left(e^{\prime}\right)=\jmath_{A}(e) \cap \jmath_{A}\left(e^{\prime}\right)
$$

Last,

$$
\begin{aligned}
& \jmath_{A}\left(e \vee e^{\prime}\right)=\jmath_{A}\left(e+e^{\prime}-e \cdot e^{\prime}\right)=D^{\infty}\left(e+e^{\prime}-e \cdot e^{\prime}\right)=D^{\infty}\left(e^{2}\right) \cup D^{\infty}\left(e^{\prime}-e \cdot e^{\prime}\right)= \\
& \qquad \begin{aligned}
& D^{\infty}(e) \cup D^{\infty}\left(e^{\prime} \cdot(1-e)\right)=D^{\infty}(e) \cup\left[D^{\infty}\left(e^{\prime}\right) \cap D^{\infty}(1-e)\right]= \\
&=\left[D^{\infty}(e) \cup D^{\infty}\left(e^{\prime}\right)\right] \cap\left[D^{\infty}(e) \cup D^{\infty}(1-e)\right]=\left[D^{\infty}(e) \cup D^{\infty}\left(e^{\prime}\right)\right] \cap \operatorname{Spec}^{\infty}(A)=D^{\infty}(e) \cup D^{\infty}\left(e^{\prime}\right)= \\
&=\jmath_{A}(e) \cup \jmath_{A}\left(e^{\prime}\right),
\end{aligned}
\end{aligned}
$$

and the claim is proved.

The map $\jmath_{A}: B(A) \rightarrow \operatorname{Spec}^{\infty}(A)$ suggests that the idempotent elements of the Boolean algebra $B(A)$ associated with a $\mathcal{C}^{\infty}$-ring $A$ hold a strong relationship with the canonical basis of the Zariski topology of $\operatorname{Spec}^{\infty}(A)$. In this section we are going to show that these idempotent elements, in the case of the von Neumann-regular $\mathcal{C}^{\infty}$-rings, represent all the Boolean algebras. 
Theorem 3.4.5. Let $A$ be a von Neumann regular $\mathcal{C}^{\infty}-$ ring. The map:

$$
\begin{array}{ccc}
\jmath_{A}: \widetilde{B}(A) & \rightarrow & \operatorname{Clopen}\left(\operatorname{Spec}^{\infty}(A)\right) \\
e & \mapsto & D^{\infty}(e)=\left\{\mathfrak{p} \in \operatorname{Spec}^{\infty}(A) \mid e \notin \mathfrak{p}\right\}
\end{array}
$$

is an isomorphism of Boolean algebras.

Proof. It has already been proved that $\jmath_{A}: B(A) \rightarrow \operatorname{Spec}^{\infty}(A)$ is a homomorphism of Boolean algebras, so in order to prove that $\jmath_{A}: \widetilde{B}(A) \rightarrow$ Clopen $\left(\operatorname{Spec}^{\infty}(A)\right)$ is an injective map, it suffices to show that:

$$
\jmath_{A}^{-1}\left[\operatorname{Spec}^{\infty}(A)\right]=\{1\} .
$$

(see Lemma 4.8 of $[83])$ Claim: $\left(\jmath_{A}(e)=\operatorname{Spec}^{\infty}(A)\right) \Longleftrightarrow\left(e \in A^{\times}\right)$.

Indeed,

$$
a \in A^{\times} \Rightarrow D^{\infty}(a)=\operatorname{Spec}^{\infty}(A) .
$$

On the other hand, if $a \notin A^{\times}$then there is some maximal $\mathcal{C}^{\infty}$-radical prime ideal $\mathfrak{m} \in \operatorname{Spec}^{\infty}(A)$ such that $a \in \mathfrak{m}$. If $\mathfrak{m} \notin D^{\infty}(a)$ then $\jmath_{A}(a)=D^{\infty}(a) \neq \operatorname{Spec}^{\infty}(A)$. Hence $\jmath_{A}(a)=\operatorname{Spec}^{\infty}(A) \Rightarrow a \in A^{\times}$.

Let $e \in \widetilde{B}(A)$ be such that $\jmath_{A}(e)=D^{\infty}(e)=\operatorname{Spec}^{\infty}(A)$, so $\left(\forall \mathfrak{p} \in \operatorname{Spec}^{\infty}(A)\right)(e \notin \mathfrak{p})$.

Since $0 \in \mathfrak{p}$ for every $\mathfrak{p} \in \operatorname{Spec}^{\infty}(A)$, then $(e) \neq(0)$, so $(e)=A=(1)$, and $e \in A^{\times}$and since $e$ is idempotent, $e=1$.

We already know that $\jmath_{A}$ is an injective Boolean algebras homomorphism, so it suffices to prove that it is also surjective.

Claim: Given any $a_{1}, a_{2}, \cdots, a_{n} \in A$, there is some $b \in A$ such that:

$$
D^{\infty}\left(a_{1}\right) \cup D^{\infty}\left(a_{2}\right) \cup \cdots \cup D^{\infty}\left(a_{n}\right)=D^{\infty}(b) .
$$

We are going to prove this claim by induction.

Case 1: $n=2$.

By the item (iii) of Lemma 1.4 of [67], we know that for any $a_{1}, a_{2} \in A$,

$$
D^{\infty}\left(a_{1}\right) \cup D^{\infty}\left(a_{2}\right)=D^{\infty}\left(a_{1}^{2}+a_{2}^{2}\right),
$$

so we can take $b=a_{1}^{2}+a_{2}^{2}$.

Inductive step: Suppose that given $a_{1}, \cdots, a_{n-1} \in A$ there is some $b_{n-1} \in A$ such that:

$$
D^{\infty}\left(a_{1}\right) \cup \cdots \cup D^{\infty}\left(a_{n-1}\right)=D^{\infty}\left(b_{n-1}\right) .
$$

Given $a_{1}, \cdots, a_{n}$, we know by the inductive step, that there is some $b_{n-1} \in A$ such that:

$$
D^{\infty}\left(a_{1}\right) \cup \cdots \cup D^{\infty}\left(a_{n-1}\right)=D^{\infty}\left(b_{n-1}\right) .
$$

Considering $b=b_{n-1}{ }^{2}+a_{n}^{2}$, we have: 


$$
D^{\infty}\left(a_{1}\right) \cup \cdots \cup D^{\infty}\left(a_{n-1}\right) \cup D^{\infty}\left(a_{n}\right)=D^{\infty}\left(b_{n-1}\right) \cup D^{\infty}\left(a_{n}\right)=D^{\infty}(b),
$$

which proves the claim.

Let $E \in$ Clopen $\left(\operatorname{Spec}^{\infty}(A)\right)$. Since $\left\{D^{\infty}(a) \mid a \in A\right\}$ is a basis for the topology of the compact topological space $\operatorname{Spec}^{\infty}(A)$, there are $a_{1}, a_{2}, \cdots, a_{n} \in A$ such that:

$$
E=D^{\infty}\left(a_{1}\right) \cup D^{\infty}\left(a_{2}\right) \cup \cdots \cup D^{\infty}\left(a_{n}\right)
$$

By the above claim, there is some $b \in A$ such that $E=D^{\infty}\left(a_{1}\right) \cup D^{\infty}\left(a_{2}\right) \cup \cdots \cup D^{\infty}\left(a_{n}\right)=D^{\infty}(b)$.

Now, since $A$ is a von Neumann regular $\mathcal{C}^{\infty}$-ring, there is some idempotent element $e \in A$ such that $(b)=(e)$.

We claim that $\jmath_{A}(e)=E$.

We have $\jmath_{A}(e):=D^{\infty}(e)=D^{\infty}(b)=D^{\infty}\left(a_{1}\right) \cup \cdots \cup D^{\infty}\left(a_{n}\right)=E$, and it follows that $\jmath_{A}: \widetilde{B}(A) \stackrel{\cong}{\rightarrow}$ Clopen $\left(\operatorname{Spec}^{\infty}(A)\right)$ is an isomorphism.

Theorem 3.4.6. We have the following diagram of categories, functors and natural isomorphisms:

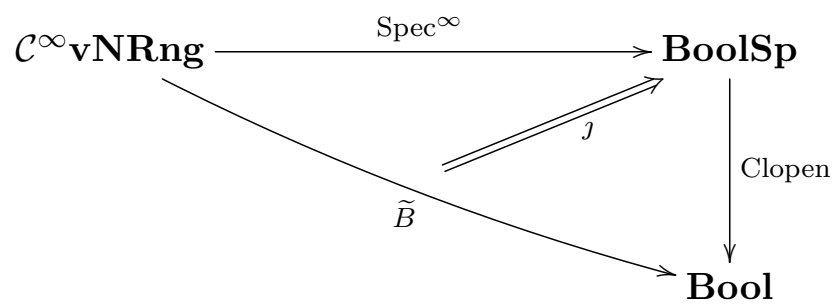

Proof. First note that since $A$ is a von Neumann-regular $\mathcal{C}^{\infty}$-ring, the set of the compact open subsets of (the Boolean space) $\operatorname{Spec}^{\infty}(A)$ equals Clopen $\left(\operatorname{Spec}^{\infty}(A)\right)$.

On the one hand, given a von Neumann regular $\mathcal{C}^{\infty}$-ring $A$, we have:

$$
\left(\operatorname{Clopen}\left(\operatorname{Spec}^{\infty}\right)\right)(A)=\operatorname{Clopen}\left(\operatorname{Spec}^{\infty}(A)\right)=\left\{D^{\infty}(e) \mid e \in \widetilde{B}(A)\right\}
$$

For every von Neumann regular $\mathcal{C}^{\infty}$-ring $A$, by Theorem 3.4.5, we have the following isomorphism of Boolean algebras:

$$
\begin{array}{rlc}
\jmath_{A}: \quad \widetilde{B}(A) & \rightarrow & \text { Clopen }\left(\operatorname{Spec}^{\infty}(A)\right) \\
e & \mapsto & D^{\infty}(e)
\end{array}
$$

It is easy to see that for every $\mathcal{C}^{\infty}$-homomorphism $f: A \rightarrow A^{\prime}$, we have the following commutative rectangle:

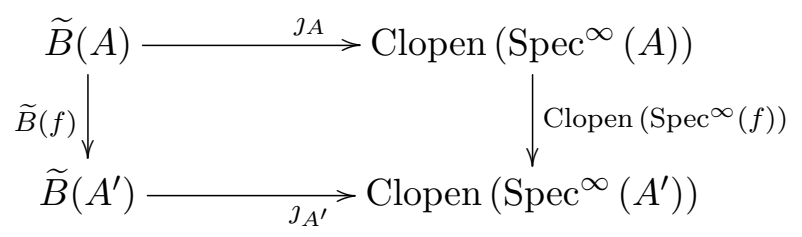


In fact, given $e \in \widetilde{B}(A)$, we have, on the one hand, $\jmath_{A}(e)=D_{A}^{\infty}(e)$ and $\operatorname{Clopen}\left(\operatorname{Spec}^{\infty}(f)\right)\left(D_{A}^{\infty}(e)\right)=$ $D_{A^{\prime}}^{\infty}(f(e))$. On the other hand, $\jmath_{A^{\prime}} \circ \widetilde{B}(f)(e)=\jmath_{A^{\prime}}\left(D_{A^{\prime}}^{\infty}(f(e))\right)$, so the rectangle commutes.

Thus $\jmath$ is a natural transformation. Thus $\jmath: \widetilde{B} \Longrightarrow$ Clopen $\circ \mathrm{Spec}^{\infty}$ is a natural isomorphism and the diagram "commutes" (up to natural isomorphism).

Lemma 3.4.7. Let $(X, \tau)$ be a Boolean topological space, and let:

$$
\mathcal{R}=\left\{R \subseteq X \times X \mid(R \text { is an equivalence relation on } X) \&\left(\frac{X}{R} \text { is a discrete compact space }\right)\right\},
$$

which is partially ordered by inclusion. Whenever $R_{i}, R_{j} \in \mathcal{R}$ are such that $R_{j} \subseteq R_{i}$, we have the continuous surjective map:

$$
\begin{aligned}
& \mu_{R_{j} R_{i}}: \frac{X}{R_{j}} \rightarrow \frac{X}{R_{i}} \\
& {[x]_{R_{j}} \mapsto[x]_{R_{j}}}
\end{aligned}
$$

so we have the inverse system $\left\{\frac{X}{R_{i}} ; \mu_{R_{j} R_{i}}: \frac{X}{R_{j}} \rightarrow \frac{X}{R_{i}}\right\}$. By definition (see, for instance, [20]),

$$
\varliminf_{R \in \mathcal{R}} \frac{X}{R}=\left\{\left([x]_{R_{i}}\right)_{R_{i} \in \mathcal{R}} \in \prod_{R \in \mathcal{R}} \frac{X}{R} \mid\left(R_{j} \subseteq R_{i} \rightarrow\left(p_{R_{i}}\left(\left([x]_{R_{i}}\right)_{R_{i} \in \mathcal{R}}\right)=\mu_{R_{j} R_{i}} \circ p_{R_{j}}\left(\left([x]_{R_{i}}\right)_{R_{i} \in \mathcal{R}}\right)\right)\right)\right\}
$$

Let $X_{\infty}$ denote $\lim _{R \in \mathcal{R}} \frac{X}{R}$, so we have the following cone:

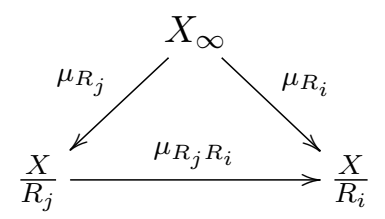

We consider $X_{\infty}$ together with the induced subspace topology of $\prod_{R \in \mathcal{R}} \frac{X}{R}$.

By the universal property of $X_{\infty}$, there is a unique continuous map map $\delta: X \rightarrow X_{\infty}$ such that the following diagram commutes:

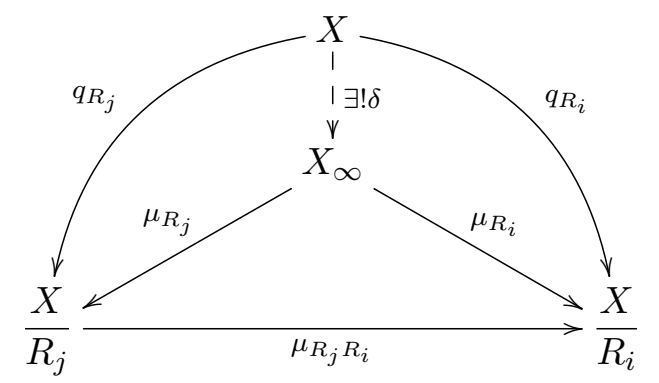

We claim that such a $\delta: X \rightarrow X_{\infty}$ is a homeomorphism, so:

$$
X \cong \lim _{\overparen{R \in \mathcal{R}}} \frac{X}{R}
$$

that is, $X$ a profinite space. 
Proof. First we observe that $\mathcal{R}$ is a downwards directed set.

Claim: Given any $R_{1}, R_{2} \in \mathcal{R}, R_{1} \cap R_{2} \in \mathcal{R}$.

Naturally, if both $R_{1}$ and $R_{2}$ are equivalence relations on $X$, then $R_{1} \cap R_{2}$ is also an equivalence relation on $X$, and it is such that $R_{1} \cap R_{2} \subseteq R_{1}$ and $R_{1} \cap R_{2} \subseteq R_{2}$. Moreover, we have an injective continuous map:

$$
\begin{aligned}
& \varphi: \frac{X}{R_{1} \cap R_{2}} \longmapsto \frac{X}{R_{1}} \times \frac{X}{R_{2}} \\
& {[x]_{R_{1} \cap R_{2}} \mapsto\left([x]_{R_{1}},[x]_{R_{2}}\right)}
\end{aligned}
$$

Claim: Given $R \in \mathcal{R}$, if $S$ is any equivalence relation on $X$ such that $R \subseteq S$, then $S \in \mathcal{R}$.

We know that each $\frac{X}{R}$ is a compact, Hausdorff and totally disconnected space, hence $\prod_{R \in \mathcal{R}} \frac{X}{R}$ is also a compact, Hausdorff and totally disconnected space, i.e., a Boolean space.

By Theorem 2.4 of 20, since each space $\frac{X}{R_{i}}$ is a Hausdorff and compact space, $X_{\infty}$ is also a Hausdorff and compact space, so it is closed in the Boolean space $\prod_{R \in \mathcal{R}} \frac{X}{R}$, hence it is a Boolean space.

Consider:

$$
\begin{array}{rlc}
\delta_{X}: \quad X & \rightarrow & X_{\infty} \\
x & \mapsto & \left([x]_{R_{i}}\right)_{R_{i} \in \mathcal{R}}
\end{array}
$$

Claim 1: $\delta_{X}$ is injective;

Let $x, x^{\prime} \in X, x \neq x^{\prime}$. Since $(X, \tau)$ is a Boolean space, there exists some clopen $U \in \tau$ such that $x \in U$ and $x^{\prime} \notin U$, that is, $x^{\prime} \notin U$.

Consider the following equivalence relation on $X$ :

$$
R=(U \times U) \cup(X \backslash U \times X \backslash U)
$$

and note that $\frac{X}{R}$ is a discrete topological space, hence $R \in \mathcal{R}$. We have $\left(x, x^{\prime}\right) \notin R$, so for every $S \in \mathcal{R}$ such that $R \supseteq S$ we have $[x]_{S} \neq\left[x^{\prime}\right]_{S}$, so $\delta_{X}(x) \neq \delta_{X}\left(x^{\prime}\right)$.

Claim 2: $\mathrm{Cl} .\left(\delta_{X}[X]\right)=X_{\infty}$.

Let $\Omega=\left(\left\{\left[a_{1}\right]_{R_{1}}\right\} \times\left\{\left[a_{2}\right]_{R_{2}}\right\} \times \cdots \times\left\{\left[a_{n}\right]_{R_{n}}\right\} \times \prod_{R \neq R_{1}, \cdots, R_{n}} \frac{X}{R}\right) \cap X_{\infty} \subset X_{\infty}$ be a basic open set of $X_{\infty}$. Let:

$$
R^{\prime}=R_{1} \cap R_{2} \cap \cdots \cap R_{n} \in \mathcal{R}
$$

Since $R_{i} \subseteq R_{j}$ implies that: 


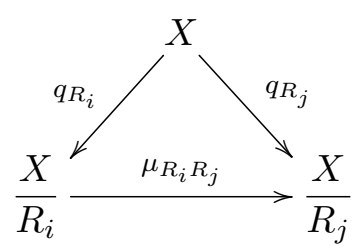

commutes, the map:

$$
\underset{R_{1} \cap R_{2} \cap \cdots \cap R_{n}}{\longrightarrow} \frac{X}{R_{1}} \times \frac{X}{R_{2}} \times \cdots \times \frac{X}{R_{n}}
$$

is injective.

Since $\mathcal{R}$ is a directed set, we can assume without lost of generality, that $\left\{R_{1}, R_{2}, \cdots, R_{n}\right\}$ has a minimum, that we shall denote by $R^{\prime}=\min \left\{R_{1}, \cdots, R_{n}\right\}$.

Now we can chose any $\left[a^{\prime}\right]_{R^{\prime}} \in \frac{X}{R^{\prime}}$. We claim that $\delta_{X}\left[\left\{a^{\prime}\right\}\right] \cap \Omega \neq \varnothing$.

We have:

$$
\delta_{X}\left(a^{\prime}\right)=\left(\left[a^{\prime}\right]_{R}\right)_{R \in \mathcal{R}}
$$

so if $R=R_{i}, i \in\{1,2, \cdots, n\}$, then $\left[a^{\prime}\right]_{R_{i}}=\mu_{R^{\prime} R_{i}}\left(\left[a^{\prime}\right]_{R^{\prime}}\right)=\left[a_{i}\right]_{R_{i}}$, hence $\delta_{X}\left(a^{\prime}\right) \in \Omega$. Thus:

$$
\delta_{X}\left(a^{\prime}\right) \in\left(\left[a^{\prime}\right]_{R}\right)_{R \in \mathcal{R}} \delta_{X}\left[\left\{a^{\prime}\right\}\right] \cap \Omega \neq \varnothing .
$$

Claim 3: $\delta_{X}: X \rightarrow X_{\infty}$ is surjective.

Since $(X, \tau)$ is a compact space, $\delta_{X}: X \rightarrow X_{\infty}$ is continuous and $X_{\infty}$ is a Hausdorff space, it follows that $\delta_{X}$ is a closed map. Hence,

$$
\delta_{X}[X]=\mathrm{Cl} .\left(\delta_{X}[X]\right)=X_{\infty}
$$

Thus $\delta_{X}: X \rightarrow X_{\infty}$ is a continuous closed bijection, hence a homeomorphism.

Let BoolSp be the category whose objects are all the Boolean spaces and whose morphisms are all the Boolean spaces homomorphisms. Given any Boolean space $(X, \tau)$, let

$$
\mathcal{R}_{X}=\{R \subseteq X \times X \mid R \text { is } \quad \text { an equivalence relation on } X \text { and }
$$

$$
\left.\frac{X}{R} \text { is discrete and compact }\right\}
$$

We are going to describe an equivalence functor between the category of the Boolean spaces and the category of profinite spaces.

First we note that given any continuous function $f: X \rightarrow X^{\prime}$ and any $R^{\prime} \in \mathcal{R}_{X^{\prime}}$,

$$
R_{f}:=(f \times f)^{-1}\left[R^{\prime}\right] \subseteq X \times X
$$

is an equivalence relation on $X$ and the following diagram: 


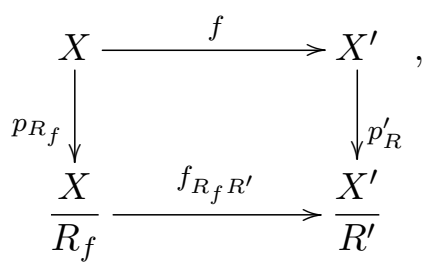

where $p_{R_{f}}: X \rightarrow \frac{X}{R_{f}}$ and $p_{R^{\prime}}: X^{\prime} \rightarrow \frac{X^{\prime}}{R^{\prime}}$ are the canonical projections, commutes.

We know, by Theorem 4.3 of 20 that $f_{R_{f} R^{\prime}}: \frac{X}{R_{f}} \rightarrow \frac{X^{\prime}}{R^{\prime}}$ is a continuous map, and it is easy to see that $f_{R_{f} R^{\prime}}$ is injective, as we are going to show.

Given any $[x]_{R_{f}},[y]_{R_{f}} \in \frac{X}{R_{f}}$ such that $[x]_{R_{f}} \neq[y]_{R_{f}}$, i.e., such that $(x, y) \notin R_{f}$, we have $(f(x), f(y)) \notin$ $R^{\prime}$, i.e., $[f(x)]_{R^{\prime}} \neq[f(y)]_{R^{\prime}}$. Thus, since:

$$
f_{R_{f} R^{\prime}}\left([x]_{R_{f}}\right)=\left(f_{R_{f} R^{\prime}} \circ p_{R_{f}}\right)(x)=\left(p_{R^{\prime}} \circ f\right)(x)=[f(x)]_{R^{\prime}}
$$

and

$$
f_{R_{f} R^{\prime}}\left([y]_{R_{f}}\right)=\left(f_{R_{f} R^{\prime}} \circ p_{R_{f}}\right)(y)=\left(p_{R^{\prime}} \circ f\right)(y)=[f(y)]_{R^{\prime}}
$$

it follows that $f_{R_{f} R^{\prime}}\left([x]_{R_{f}}\right) \neq f_{R_{f} R^{\prime}}\left([y]_{R_{f}}\right)$.

Since $f_{R_{f} R^{\prime}}: \frac{X}{R_{f}} \rightarrow \frac{X^{\prime}}{R^{\prime}}$ is an injective continuous map and $\frac{X^{\prime}}{R^{\prime}}$ is discrete, it follows that given any $\left[x^{\prime}\right]_{R^{\prime}} \in \frac{X^{\prime}}{R^{\prime}}:$

$$
f_{R_{f} R^{\prime}}^{\dashv}\left[\left\{\left[x^{\prime}\right]_{R^{\prime}}\right\}\right]=\left\{\begin{array}{l}
\varnothing, \text { if }\left[x^{\prime}\right]_{R^{\prime}} \in \operatorname{im}\left(f_{R_{f} R^{\prime}}\right), \\
\{*\}, \text { otherwise }
\end{array},\right.
$$

so every singleton of $\frac{X}{R_{f}}$ is an open subset of $\frac{X}{R_{f}}$, and $\frac{X}{R_{f}}$ is discrete. Also, since $X$ is compact, $\frac{X}{R_{f}}$ is compact, and it follows that and $R_{f} \in \mathcal{R}_{X}$.

Now, if $R_{1}^{\prime}, R_{2}^{\prime} \in \mathcal{R}_{X^{\prime}}$ are such that $R_{1}^{\prime} \subseteq R_{2}^{\prime}$, then $R_{1 f}^{\prime} \subseteq R_{2 f}^{\prime}$. In fact, given $(x, y) \in R_{1 f}^{\prime}$, we have $(f \times f)(x, y) \in R_{1}^{\prime}$, and since $R_{1}^{\prime} \subseteq R_{2}^{\prime}$, it follows that $(f \times f)(x, y) \in R_{2}^{\prime}$, so $(x, y) \in R_{2 f}^{\prime}$.

Let $X, Y, Z$ be Boolean spaces, $X \stackrel{f}{\rightarrow} Y$ and $Y \stackrel{g}{\rightarrow} Z$ be two Boolean spaces homomorphisms and $R \in \mathcal{R}_{Z}$. We have:

$$
((g \circ f) \times(g \circ f))^{-1}[R]=(f \times f)^{-1}\left[(g \times g)^{-1}[R]\right] .
$$

In fact,

$$
\begin{aligned}
\left(x, x^{\prime}\right) \in((g \circ f) \times(g \circ f))^{\dashv}[R] & \Longleftrightarrow\left(g(f(x)), g\left(f\left(x^{\prime}\right)\right)\right) \in R \Longleftrightarrow \\
& \Longleftrightarrow\left(f(x), f\left(x^{\prime}\right)\right) \in(g \times g)^{\dashv}[R] \Longleftrightarrow\left(x, x^{\prime}\right) \in(f \times f)^{\dashv}\left[(g \times g)^{\dashv}[R]\right] .
\end{aligned}
$$

Denoting $T:=(f \times f)^{-1}\left[(g \times g)^{-1}[R]\right]$ and $S:=(g \times g)^{-1}[R]$, we have the following commutative diagram: 


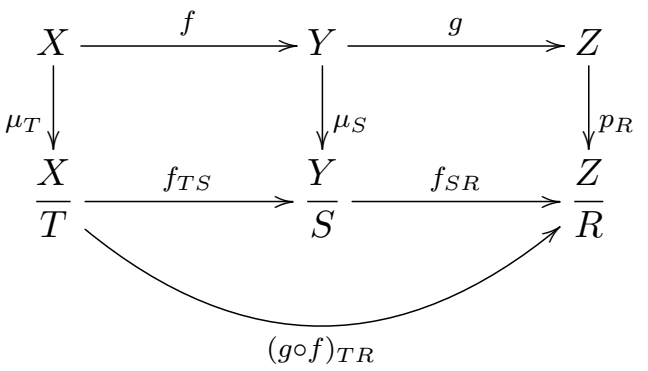

Given a continuous map between Boolean spaces, $f: X \rightarrow X^{\prime}$, we can define a map $\check{f}: \lim _{R \in \mathcal{R}_{X}} \frac{X}{R} \rightarrow$ $\lim _{\longleftarrow} R_{R^{\prime} \in \mathcal{R}_{X^{\prime}}} \frac{X^{\prime}}{R^{\prime}}$ in a functorial manner.

Let $R^{\prime}, S^{\prime} \in \mathcal{R}_{X^{\prime}}$ be any two equivalence relations such that $R^{\prime} \subseteq S^{\prime}$, so given $f: X \rightarrow X^{\prime}$ the following rectangle commutes:

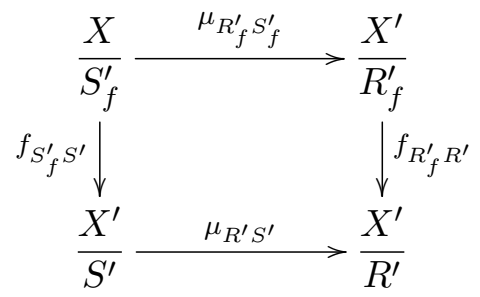

and since:

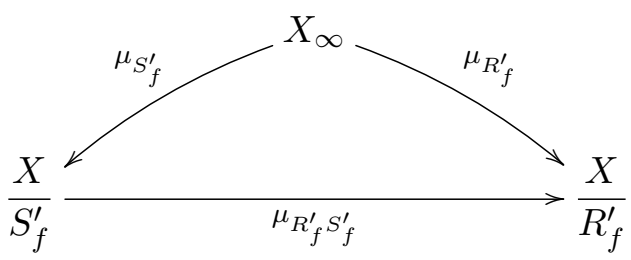

commutes, the following triangle is commutative:

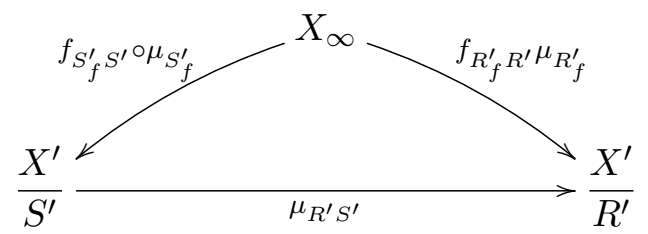

By the universal property of $X_{\infty}^{\prime}$, there is a unique $\check{f}: X_{\infty} \rightarrow X_{\infty}^{\prime}$ such that the following prism is commutative: 


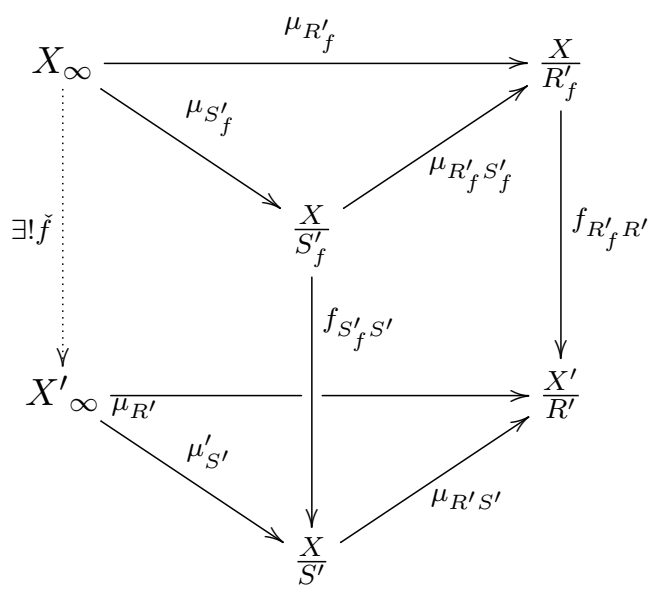

We claim that

$$
\begin{aligned}
\Delta: \quad \text { BoolSp } & \rightarrow \quad \text { ProfinSp } \\
X & \mapsto X_{\infty}={\underset{\lim }{\longleftarrow} R \in \mathcal{R}_{X}}_{X} \frac{X}{R} \\
X \stackrel{f}{\longrightarrow} X^{\prime} & \mapsto X_{\infty} \stackrel{\check{f}}{\longrightarrow} X^{\prime} \infty
\end{aligned}
$$

is a functor.

Let $R, R^{\prime} \in \mathcal{R}_{X}$ be such that $R \subseteq R^{\prime}$. Since for any $R \in \mathcal{R}_{X}$ we have $R_{\operatorname{id}_{X}}=\left(\operatorname{id}_{X} \times \operatorname{id}_{X}\right)^{-1}[R]=R$, then the following diagram commutes for every $R, R^{\prime} \in \mathcal{R}_{X}$ such that $R \subseteq R^{\prime}$ :

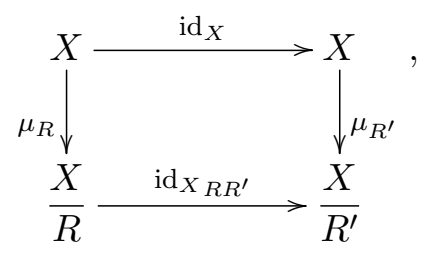

and $\operatorname{id}_{X R R^{\prime}}=\imath_{\frac{X}{R}}^{\frac{X}{R^{\prime}}}, \operatorname{since}_{\operatorname{id}_{X} R^{\prime}}\left([x]_{R}\right)=\mu_{R^{\prime}}\left(\operatorname{id}_{X}(x)\right)=[x]_{R^{\prime}}$.

We note that $\operatorname{id}_{X_{\infty}}: X_{\infty} \rightarrow X_{\infty}$ is such that the following prism commute:

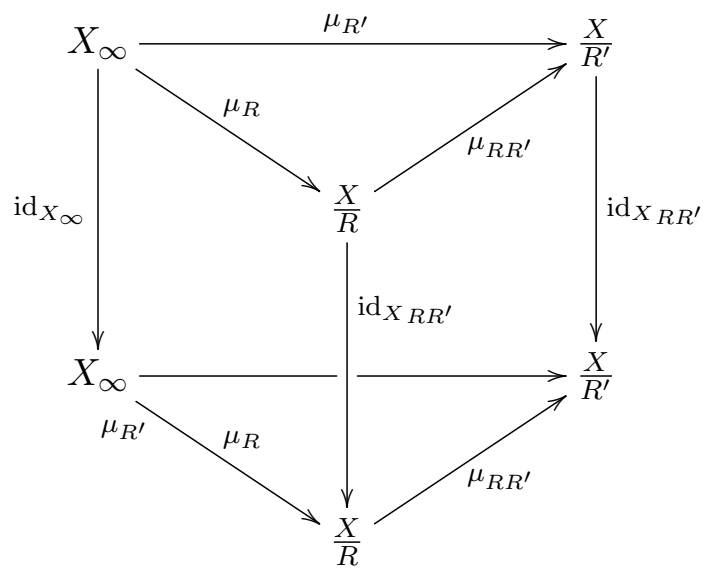

and since $\mathrm{id}_{X}: X_{\infty} \rightarrow X_{\infty}$ is the unique map with this property, then: 


$$
\Delta\left(\operatorname{id}_{X}\right)=\operatorname{id}_{X}=\operatorname{id}_{X_{\infty}}
$$

Let $X, Y, Z$ be Boolean spaces, $X \stackrel{f}{\rightarrow} Y$ and $Y \stackrel{g}{\rightarrow} Z$ be two Boolean spaces homomorphisms and $R \in \mathcal{R}_{Z}$. We have:

$$
((g \circ f) \times(g \circ f))^{-1}[R]=(f \times f)^{\dashv}\left[(g \times g)^{\dashv}[R]\right] .
$$

In fact,

$$
\begin{aligned}
\left(x, x^{\prime}\right) \in((g \circ f) \times(g \circ f))^{-1}[R] & \Longleftrightarrow\left(g(f(x)), g\left(f\left(x^{\prime}\right)\right)\right) \in R \Longleftrightarrow \\
& \Longleftrightarrow\left(f(x), f\left(x^{\prime}\right)\right) \in(g \times g)^{-1}[R] \Longleftrightarrow\left(x, x^{\prime}\right) \in(f \times f)^{-1}\left[(g \times g)^{-1}[R]\right] .
\end{aligned}
$$

Denoting $T:=(f \times f)^{-1}\left[(g \times g)^{-1}[R]\right]$ and $S:=(g \times g)^{-1}[R]$, we have the following commutative diagram:

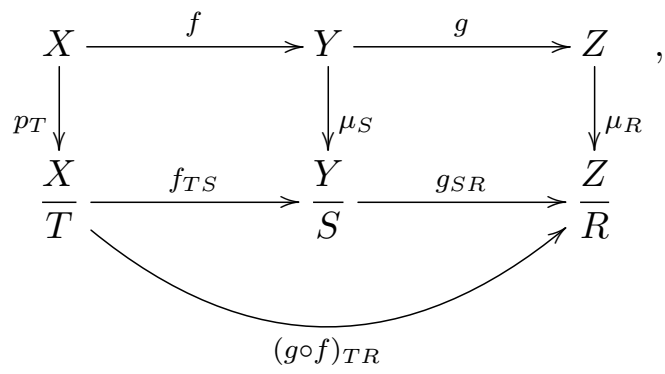

that is, $(g \circ f)_{T R}=g_{S R} \circ f_{T S}$.

Let $R^{\prime} \in \mathcal{R}_{Z}$ be such that $R \subseteq R^{\prime}$. The same argument used above proves that, denoting $T^{\prime}:=$ $(f \times f)^{-1}\left[(g \times g)^{-1}\left[R^{\prime}\right]\right]$ and $S^{\prime}:=(g \times g)^{-1}\left[R^{\prime}\right]$, we have the following commutative diagram:

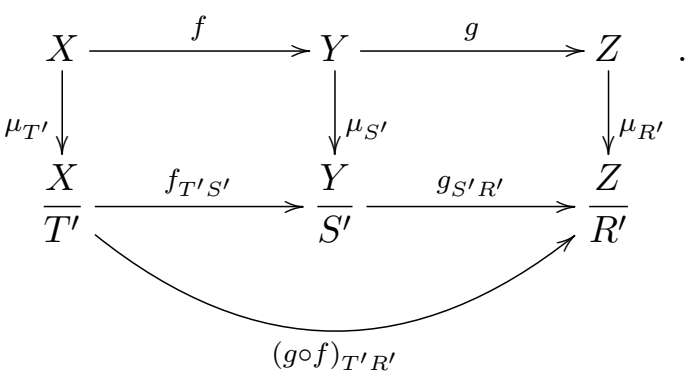

Under these circumstances, the following diagram commutes: 


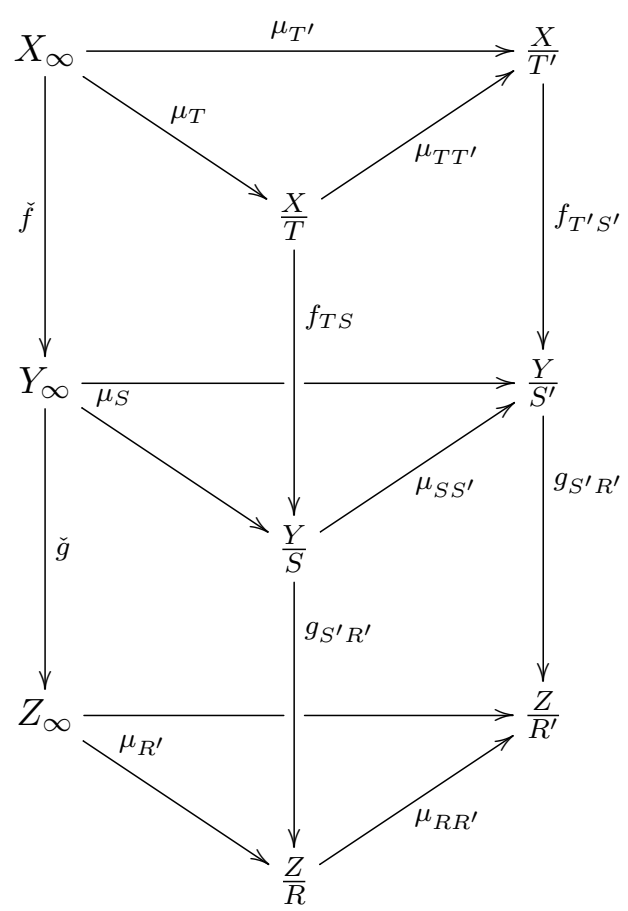

We have, then, the following commutative diagram:

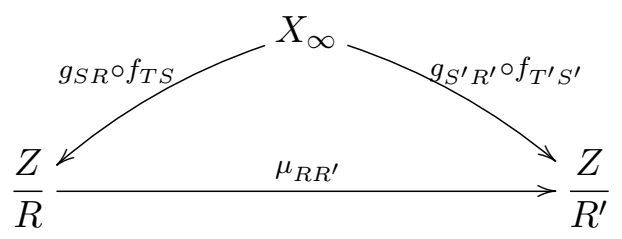

and by the universal property of $Z_{\infty}$ there is a unique $g \circ \tilde{\circ} f: X_{\infty} \rightarrow Z_{\infty}$ such that the following diagram commutes:

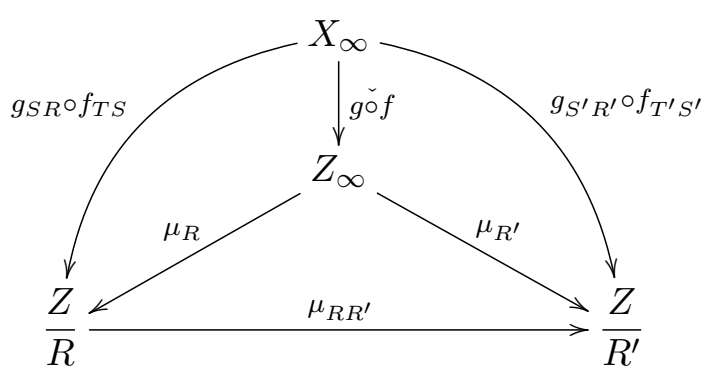

Since the following diagram commutes: 


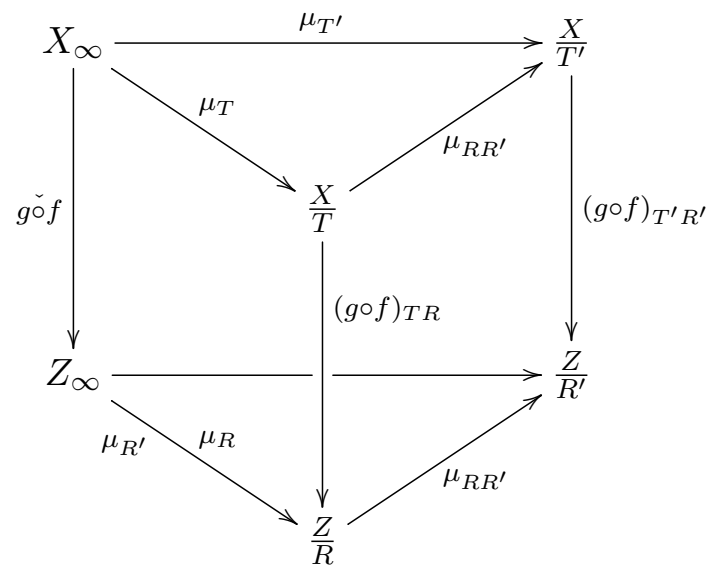

by the uniqueness of $g \check{\circ} f$, we have $g \circ \dot{f}=\check{g} \circ \check{f}$, hence

$$
\Delta(g \circ f)=g \circ \check{g}=\check{g} \circ \check{f}=\Delta(g) \circ \Delta(f) .
$$

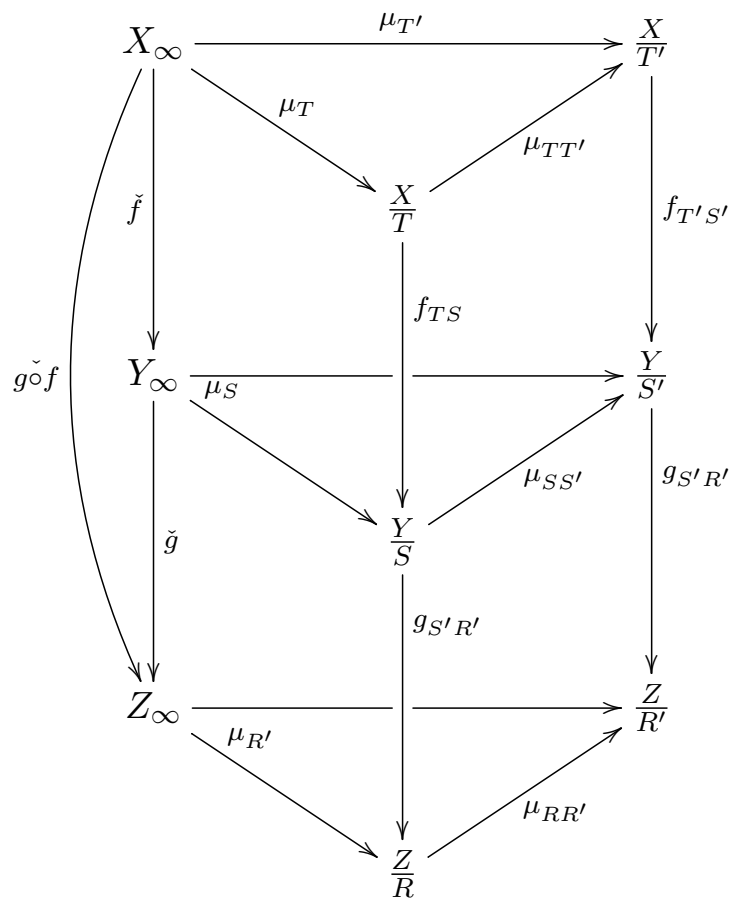

The above claims prove that

$$
\begin{aligned}
& \Delta: \text { BoolSp } \rightarrow \quad \text { BoolSp } \\
& X \quad \mapsto \delta_{X}[X]=\varliminf_{R \in \mathcal{R}_{X}} \frac{X}{R} \\
& X \stackrel{f}{\longrightarrow} Y \quad \mapsto \quad X_{\infty} \stackrel{\check{f}}{\longrightarrow} Y_{\infty}
\end{aligned}
$$

is a functor.

Theorem 3.4.8. Let $\mathbb{K}$ be a $\mathcal{C}^{\infty}$-field. Following the notation of Lemma 3.4.7, define the contravariant functor:

$$
\begin{aligned}
& \widehat{k}: \text { BoolSp } \rightarrow \quad \mathcal{C}^{\infty} \text { vNRng } \\
& (X, \tau) \mapsto R_{X}:=\lim _{R \in \mathcal{R}} \mathbb{K}^{U\left(\frac{X}{R}\right)}
\end{aligned}
$$


Then there is a natural isomorphism

$$
\epsilon: I d_{\text {BoolSp }} \cong \operatorname{SpeC}^{\infty} \circ \widehat{k}
$$

Thus the functor $\mathrm{Spec}^{\infty}: \mathcal{C}^{\infty} \mathbf{v N R n g} \rightarrow$ BoolSp is full and isomorphism-dense.

In particular: for each $(X, \tau)$ be a Boolean space, there is a von Neumann-regular $\mathcal{C}^{\infty}$-ring, $R_{X}$, such that:

$$
\operatorname{Spec}^{\infty}\left(R_{X}\right) \approx X
$$

Proof. By the Theorem $\mathbf{3 . 1 . 2 7}$.

$$
\operatorname{Spec}^{\infty}\left(R_{X}\right) \approx \lim _{R \in \mathcal{R}} \operatorname{Spec}^{\infty}\left(\mathbb{K}^{U\left(\frac{X}{R}\right)}\right)
$$

By the Theorem 3.1 .26 ,

$$
\operatorname{Spec}^{\infty}\left(\mathbb{K}^{U\left(\frac{X}{R}\right)}\right) \approx \operatorname{Discr} .\left(\frac{X}{R}\right)
$$

so

$$
\lim _{\overparen{R \in \mathcal{R}}} \operatorname{Spec}^{\infty}\left(\mathbb{K}^{U\left(\frac{X}{R}\right)}\right) \approx \lim _{\overparen{R \in \mathcal{R}}} \operatorname{Discr} .\left(\frac{X}{R}\right) \approx \lim _{\overparen{R \in \mathcal{R}}} \frac{X}{R} \approx X
$$

Since the homeomorphisms above are natural, just take $\epsilon_{X}: X \rightarrow \operatorname{Spec}^{\infty}\left(R_{X}\right)$ as the composition of the these homeomorphisms.

Hence, in particular, $\operatorname{Spec}^{\infty}\left(R_{X}\right) \approx X$.

Theorem 3.4.9. Let $\mathbb{K}$ be a $\mathcal{C}^{\infty}$-field. Define the covariant functor (composition of contravariant functors):

$$
\check{K}=\widehat{k} \circ \text { Stone }: \text { Bool } \rightarrow \mathcal{C}^{\infty} \mathbf{v N R n g}
$$

Then there is a natural isomorphism

$$
\theta: \operatorname{Id}_{\text {Bool }} \cong \widetilde{\Xi} \odot \check{K}
$$

Thus the functor $\widetilde{B}: \mathcal{C}^{\infty} \mathbf{v N R n g} \rightarrow$ Bool is full and isomorphism-dense.

In particular: given any $\mathcal{C}^{\infty}$-field $\mathbb{K}$ and any Boolean algebra $B$, there is a von Neumann regular $\mathcal{C}^{\infty}$-ring which is a $\mathbb{K}$-algebra, $\check{K}(B)$ ), such that

$$
\widetilde{B}(\check{K}(B)) \cong B
$$

Proof. This follows directly consequence by a combination of the Theorem $\mathbf{3 . 4 . 8}$ above, Stone duality (Remark 3.4.3), Theorem 3.4.6 and Theorem 3.4.5. 
The diagram below summarizes the main functorial connections stablished in this section:

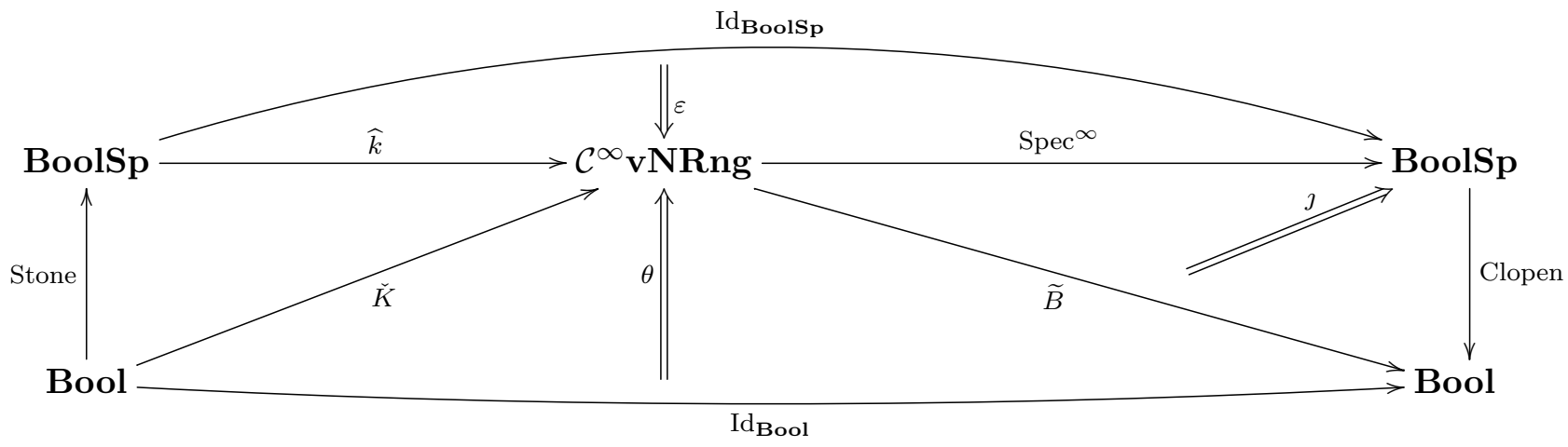




\section{Chapter 4}

\section{Some Logical Aspects of the $\mathcal{C}^{\infty}$-rings}

In the present chapter we develop the first results on the categorial-logic aspects of the theory of $\mathcal{C}^{\infty}$-rings, specifically on classifying topoi of some (geometric) theories related to $\mathcal{C}^{\infty}$-rings (the terminology will be explained in the first section). To the best of our knowledge, the first result of this kind appeared in [67], were the authors present a classifying topos for the geometric theory of Archimedean $\mathcal{C}^{\infty}$-rings. We start our contributions to the subject describing in details two results that are just stated by I. Moerdijk and G. Reyes -without any sketch of proof- in [62], page 366: the classifying topos for the theory of $\mathcal{C}^{\infty}$-rings is the pre-sheaf topos over the left-exact (essentially small category) $\mathcal{C}^{\infty} \mathbf{R n g} \mathbf{g}_{\mathrm{fp}}^{\text {op }}$ and the classifying topos for the theory of local $\mathcal{C}^{\infty}$-rings is the "smooth Zariski Topos", as described in Chapter 2. In the sequel, and finishing this chapter, we describe the classifying topos for the theory of von Neumann regular $\mathcal{C}^{\infty}$-rings as $\mathbf{S e t}^{\left(\mathcal{C}^{\infty} \mathbf{v N R n g}\right)_{\mathrm{fp}}}$, with a strictly stronger language that contains symbols for all $\mathcal{C}^{\infty}$ functions and an additional unary function symbol to represent the unique quasi-inverse of an element of a von Neumann regular $\mathcal{C}^{\infty}$-ring.

\subsection{Categorial Logic and classifying topoi}

In this section we list the main logical-categorial notions and results that we will need in the sequel of this chapter. The main references here are [50], [7], [6] and [110].

\section{(I) Sketches and its models:}

- A (small) sketch is a 4-tuple $\mathcal{S}=(G, D, P, I)([6])$, where $G$ is a (small) oriented graph; $D$ is a (set)class of small (non-commutative) diagrams over $G$; $P$ is a (set)class of (non-commutative) cones over $G ; I$ is a (set)class of (non-commutative) co-cones over $G$. $\mathcal{S}$ is a geometric sketch if $P$ is a set of cones over $G$ with finite basis. Each (small) category $C$ determines a (small) sketch: $\operatorname{sk}(\mathcal{C})=\left(|\mathcal{C}|, D_{\mathcal{C}}, P_{\mathcal{C}}, I_{\mathcal{C}}\right.$ ), where $|\mathcal{C}|$ is the underlying graph of the category, $D_{\mathcal{C}}$ is the class of all small commutative over $\mathcal{C}, P_{\mathcal{C}}$ is the class of all small limit cones over $\mathcal{C}, I_{\mathcal{C}}$ is the class of all small colimit co-cones over $\mathcal{C}$. A sketch $\mathcal{S}=(G, D, P, I)$ is called a $(\mathcal{P}, \mathcal{I})$-type if the base of all cones in $P$ are in the class $\mathcal{P}$ and if the base of all co-cones in $I$ are in the class $\mathcal{I}$.

- A morphism of sketches $\mathcal{S} \rightarrow \mathcal{S}^{\prime}$ is a homomorphism of the underlying graphs that preserves all the given structures. This determines a (very large) category SK.

- A model of a sketch $\mathcal{S}$ in a category $\mathcal{C}$ is a morphism of sketches $\mathcal{S} \rightarrow \operatorname{sk}(C)$. We will denote $\operatorname{Mod}(\mathcal{S}, \mathcal{C})$ the category whose objects are the models of $\mathcal{S}$ into the category $\mathcal{C}$ and the arrows are the natural transformations between the models (this makes sense since $\mathcal{C}$ is a category). Many usual categories of (first-order, but not necessarily finitary) mathematical structures $\mathcal{K}$ can be described as $\mathcal{K} \simeq \operatorname{Mod}(\mathcal{S}, \operatorname{Set})=\operatorname{SK}(\mathcal{S}, \operatorname{sk}($ Set $))$ for some small sketch $\mathcal{S}$; for instance: groups and their homomor- 
phisms, rings and their homomorphisms, fields and their homomorphisms, local rings and local homomorphisms, $\sigma$-Boolean algebras and their homomorphisms, Banach spaces and linear contractions etc.

- Every small sketch $\mathcal{S}$ of $(\mathcal{P}, \mathcal{I})$-type has a "canonical" $(\mathcal{P}, \mathcal{I})$-model $M: \mathcal{S} \rightarrow \operatorname{sk}(\hat{\mathcal{S}})$, where $\hat{\mathcal{S}}$ is the $\mathcal{P}$-complete and $\mathcal{I}$-cocomplete category called "the $(\mathcal{P}, \mathcal{I})$-theory of $\mathcal{S}$ ". That is, it has all limits of the type occurring. This means that for each category $\mathcal{C}$ that is $\mathcal{P}$-complete and $\mathcal{I}$-cocomplete composing with $M$ yields an equivalence of categories $\operatorname{Func}_{(\mathcal{P}, \mathcal{I})}(\hat{\mathcal{S}}, \mathcal{C}) \stackrel{\widetilde{M}}{\operatorname{Mod}}(\mathcal{S}, \mathcal{C})=\operatorname{SK}(\mathcal{S}, \operatorname{sk}(\mathcal{C}))$, where $\operatorname{Func}_{(\mathcal{P}, \mathcal{I})}(\hat{\mathcal{S}}, \mathcal{C})$ is the full subcategory of $\operatorname{Func}(\hat{\mathcal{S}}, \mathcal{C})$, of all functors that preserve $\mathcal{P}$-limits and $\mathcal{I}$-colimits. The $(\mathcal{P}, \mathcal{I})$-theory $\hat{\mathcal{S}}$ is unique up to "equivalence of categories".

\section{(II) Grothendieck Topoi and Geometric Morphisms:}

- A (small) site is a pair $(\mathcal{C}, J)$ formed by a (small) category $\mathcal{C}$ and a Grothendieck (pre)topology $J$ on $\mathcal{C}$, i.e. a map $C \in \operatorname{Obj}(\mathcal{C}) \mapsto J(C)$ where $f \in J(C)$ is a small family of $\mathcal{C}$-arrows $\mathcal{F}=\left\{f_{i}: A_{i} \rightarrow C\right\}_{i \in I}$ that satisfies: the isomorphism axiom; stability axiom and transitivity axiom $([50])$. The usual notion of covering by opens in a topological space $X$ provides a site $(\operatorname{Open}(X), J)$.

- Similar to the case of (pre)sheaves over a topological space it can be defined in general the (pre)sheaves category: $\operatorname{Sh}(\mathcal{C}, J) \hookrightarrow$ Set $^{\mathcal{C}^{\mathrm{op}}}$ and the sheafification (left adjoint) functor $a:$ Set $^{\mathcal{C}^{\mathrm{op}}} \rightarrow$ $\operatorname{Sh}(\mathcal{C}, J)$ : determines a geometric morphism.

- A Grothendieck topos $\mathcal{E}$ is a category that is equivalent to the category of sheaves over a small site $(\mathcal{C}, J), \mathcal{E} \simeq \operatorname{Sh}(\mathcal{C}, J) \hookrightarrow \operatorname{Set}^{\left(\mathcal{C}^{\text {op }}\right)}$.

- A geometric morphism between the Grothendieck topoi $\mathcal{E}, \mathcal{E}^{\prime}, f: \mathcal{E} \rightarrow \mathcal{E}^{\prime}$, is a functor $f^{*}: \mathcal{E}^{\prime} \rightarrow \mathcal{E}$ that preserves small colimits and is left exact (i.e. it preserves finite limits). Equivalently a geometric morphism $\mathcal{E} \rightarrow \mathcal{E}^{\prime}$ a is an equivalent class of adjoint functors

$$
\mathcal{E} \underset{f^{*}}{\stackrel{f_{*}}{\rightleftarrows}} \mathcal{E}^{\prime}
$$

where $f^{*}$ is left exact and left adjoint to $f_{*}$, and $\left(f^{*}, f_{*}\right) \equiv\left(g^{*}, g_{*}\right)$ if, and only if, $f^{*}=g^{*}$ ( and thus $f_{*} \cong g_{*}$ ). If $(\mathcal{C}, J)$ is a small site, the "sheafification (left adjoint) functor" $a: \operatorname{Set}^{\mathcal{C}^{\text {op }}} \rightarrow \operatorname{Sh}(\mathcal{C}, J)$ determines a geometric morphism $\operatorname{Sh}(\mathcal{C}, J) \rightarrow$ Set $^{\mathcal{C}^{\text {op }}}$.

- If $\mathcal{E}, \mathcal{F}$ are Grothendieck topoi, we denote $\operatorname{Geom}(\mathcal{F}, \mathcal{E}) \hookrightarrow \operatorname{Func}(\mathcal{E}, \mathcal{F})$ the full subcategory of the category of functors and natural transformations formed by the (left adjoint part) of geometric morphisms $\mathcal{F} \rightarrow \mathcal{E}$.

\section{(III) (Functorial) Theories:}

- A mathematical theory $T$ will be called a functorial mathematical theory, when there is a small category $\mathcal{C}_{T}$ such that the category of models of this theory in a Grothendieck topos $\mathcal{F}, \operatorname{Mod}_{\mathcal{F}}(T)$ is (naturally) equivalent to a full subcategory of $\operatorname{Hom}_{T}\left(\mathcal{C}_{T}, \mathcal{F}\right) \hookrightarrow \operatorname{Func}\left(\mathcal{C}_{T}, \mathcal{E}\right)$. This category $\mathcal{C}_{T}$ is unique up to equivalence.

- Let $\mathcal{C}$ be a small category with finite products and consider the (functorial) theory of finite product preserving functors on $\mathcal{C}$, i.e. $\mathcal{C}_{T}=C$ and $\operatorname{Mod}_{T}(\mathcal{E})=\operatorname{Hom}_{T}\left(\mathcal{C}_{T}, \mathcal{E}\right)=\operatorname{Prod}_{\text {fin }}\left(\mathcal{C}_{T}, \mathcal{E}\right) \hookrightarrow \operatorname{Func}(\mathcal{C}, \mathcal{E})$. 
- Let $\mathcal{C}$ be a small left exact category (i.e. $\mathcal{C}$ has all finite limits) and consider the (functorial) theory of left exact functors (i.e., finite limits preserving functors) on $\mathcal{C}$, i.e. $\mathcal{C}_{T}=\mathcal{C}$ and $\operatorname{Mod}_{T}(\mathcal{E})=\operatorname{Hom}_{T}\left(\mathcal{C}_{T}, \mathcal{E}\right)=$ $\operatorname{Lex}\left(\mathcal{C}_{T}, \mathcal{E}\right) \hookrightarrow \operatorname{Func}(\mathcal{C}, \mathcal{E})$

- Examples of functorial mathematical theories are given by the theories $\hat{\mathcal{S}}$ associated to small sketches $\mathcal{S}=(G, D, P, I)$ (see (I) above).

- To each geometric/coherent first-order theory in the infinitary language $L_{\infty \omega}$, one can associate a small "syntactical" category $\mathcal{C}_{T}$ in such a way to determine a functorial theory $([110])$.

\section{(IV) Classifying topoi:}

- Let $T$ be a functorial mathematical theory. $T$ admits a classifying topos when there are (i) a Grothendieck topos $\mathcal{E}(T)$; (ii) a model $M: \mathcal{C}_{T} \rightarrow \mathcal{E}(T)$; that are (2-)universal in the following sense: given a Grothendieck topos $\mathcal{F}$, composing $M$ with the left adjoint part of the geometric morphism yields an equivalence of categories $\operatorname{Geom}(\mathcal{F}, \mathcal{E}[T]) \stackrel{\simeq}{\rightarrow} \operatorname{Hom}_{T}\left(\mathcal{C}_{T}, \mathcal{F}\right)$. The topos $\mathcal{E}[T]$ is called the classifying topos of the theory $T$ and the model $M$ is called the generic model of the theory $T$.

- Each classifying topos of a functorial mathematical theory determines an equivalence of categories $\operatorname{Geom}(\mathcal{F}, \mathcal{E}[T]) \simeq \operatorname{Mod}_{F}(T)$, for each Grothendieck topos $\mathcal{F}$. When a functorial mathematical theory admits a classifying topos, it is unique up to equivalence of categories.

- Let $\mathcal{C}$ be a small left exact category, then the theory of left exact functors on $\mathcal{C}$ admits the presheaves category $\mathbf{S e t}^{\mathcal{C}^{\mathrm{op}}}$ as a classifying topos and the Yoneda embedding $Y_{\mathcal{C}}: \mathcal{C} \rightarrow \mathbf{S e t}^{\mathcal{C}^{\mathrm{op}}}$ is the generic model.

- If $(\mathcal{C}, J)$ is a small site over a left exact category $\mathcal{C}$, then the theory of left-exact (i.e. finite limit preserving) continuous (i.e. takes covering into colimits) functors is classified by the topos $\operatorname{Sh}(\mathcal{C}, J)$, where the canonical model is $\mathcal{C} \stackrel{Y}{\rightarrow} \mathbf{S e t}^{\mathcal{C} p} \stackrel{a}{\rightarrow} \operatorname{Sh}(\mathcal{C}, J)$. This includes the previous case of presheaves categories, by taking the Grothendieck topology $J(c)=\left\{\operatorname{id}_{\mathcal{C}}: \mathcal{C} \rightarrow \mathcal{C}\right\}, c \in \operatorname{Obj}(\mathcal{C})$.

- If the small category $\mathcal{C}_{T}$ that encodes a mathematical theory $T$ is freely generated by an object $u$, then the generic model $M: \mathcal{C}_{T} \rightarrow \operatorname{Sh}\left(\mathcal{C}_{T}, J\right)$ is uniquely determined (up to natural isomorphism) by $M(u)=a\left(\mathcal{C}_{T}(-, u)\right)$. In this case, $\operatorname{ev}_{u}: \operatorname{Hom}_{T}\left(\mathcal{C}_{T}, \mathcal{E}\right) \stackrel{\widetilde{ }}{\rightarrow} \operatorname{Mod}_{\mathcal{E}}(T)$ is an equivalence of categories for each Grothendieck topos $\mathcal{E}$. Such object $u$ is called the "universal" $T$-object in $\operatorname{Sh}\left(\mathcal{C}_{T}, J\right)$.

- The Mitchell-Bénabou language of a elementary/Grothendieck topos and the Kripke-Joyal semantics allows us to interpret -in particular- first-order formulas in many sorted languages $L_{\omega \omega} / L_{\infty \omega}$ in a elementary/Grothendieck topos. Every geometric theory admits a classifying topos.

- Every Grothendieck topos is the classifying topos of a small geometric sketch.

\subsection{A Classifying Topos for the Theory of $\mathcal{C}^{\infty}$-rings}

By a combination of specific results on the (algebraic) theory of $\mathcal{C}^{\infty}$-rings with the general notions and results on categorial logic stated in the previous section, we will give an explicit description of the classifying topos for the theory of $\mathcal{C}^{\infty}$-rings.

We start recalling some results on $\mathcal{C}^{\infty}$-rings, already considered in Chapter 1: 
Fact 4.2.1. (a) For each $k, n \in \mathbb{N}$, the $\operatorname{map}()^{*}: \mathcal{C}^{\infty}\left(\mathbb{R}^{n}, \mathbb{R}^{k}\right) \rightarrow \mathcal{C}^{\infty} \mathbf{R n g}\left(\mathcal{C}^{\infty}\left(\mathbb{R}^{k}\right), \mathcal{C}^{\infty}\left(\mathbb{R}^{k}\right)\right)$,

$$
\left(\mathbb{R}^{n} \stackrel{\phi}{\rightarrow} \mathbb{R}^{k}\right) \mapsto\left(\mathcal{C}^{\infty}\left(\mathbb{R}^{k}\right) \stackrel{\phi \circ-}{\rightarrow} \mathcal{C}^{\infty}\left(\mathbb{R}^{n}\right)\right.
$$

is a bijection.

(b) Since $\mathcal{C}^{\infty} \mathbf{R n g}$ is a cocomplete category and the its full subcategory of free $\mathcal{C}^{\infty}$-rings on finite generators $\mathcal{C}^{\infty} \mathbf{R n g}_{\text {freefin }} \subseteq \mathcal{C}^{\infty} \mathbf{R n g}$ is closed under finite coproducts, it follows that $\mathcal{C}^{\infty} \mathbf{R n g}_{\text {freefin }}^{\text {op }}$ has all finite products.

(c) Since $\mathcal{C}^{\infty} \mathbf{R n g}$ is a cocomplete category and its full subcategory of all finitely presentable $\mathcal{C}^{\infty}$-rings, $\mathcal{C}^{\infty} \mathbf{R n g}_{\mathrm{fp}} \subseteq \mathcal{C}^{\infty} \mathbf{R n g}$, is closed under finite colimits, it follows that $\mathcal{C}^{\infty} \mathbf{R n g}_{\mathrm{fp}}^{\mathrm{op}}$ has all finite limits.

(d) $\mathcal{C}^{\infty} \mathbf{R n g}_{\mathrm{fp}}$ is an essentially small category, i.e. it is equivalent to a small category, for instance any skeleton of $\mathcal{C}^{\infty} \mathbf{R n g}_{\mathrm{fp}}$ has size $2^{c}$, where $c=2^{\aleph_{0}}$. By simplicity We will still denote by $\mathcal{C}^{\infty} \mathbf{R n g}_{\mathrm{fp}}$ any skeleton of the original category.

Definition 4.2.2. Let $\mathcal{C}$ be a category with finite products. $A \mathcal{C}^{\infty}$-ring object in $\mathcal{C}$ is a morphism of sketches $A: \mathcal{S}_{\mathcal{C}}{ }_{\mathbf{R n g}} \rightarrow$ sk $(\mathcal{C})$, where $\mathcal{S}_{\mathcal{C}^{\infty} \mathbf{R n g}}$ is the natural "finite product"-type sketch of the algebraic theory of $\mathcal{C}^{\infty}$-rings. We will denote $\mathcal{C}^{\infty} \operatorname{Ring}(\mathcal{C}):=\operatorname{Mod}\left(\mathcal{S}_{\mathcal{C}^{\infty}} \mathbf{R n g}, s k(\mathcal{C})\right)$, the category of $\mathcal{C}^{\infty}$-ring-objects in $\mathcal{C}$ and natural transformations.

Proposition 4.2.3. Given a $\mathcal{C}^{\infty}$-ring-object $A: \mathcal{S}_{\mathcal{C}^{\infty}}$ Rng $\rightarrow \Pi_{f}(\mathcal{C})$ in $\mathcal{C}$, in the sense of the Definition 4.2.2. the object $A(|\mathbb{R}|) \in \operatorname{Obj}(\mathcal{C})$ has a natural $\mathcal{C}^{\infty}$-ring structure, $\Psi$, given by:

$$
\begin{array}{cl}
\Psi: \bigcup_{n \in \mathbb{N}} \mathcal{C}^{\infty}\left(\mathbb{R}^{n}, \mathbb{R}\right) & \rightarrow \bigcup_{n \in \mathbb{N}} \operatorname{Hom}_{\mathcal{C}}\left(A(|\mathbb{R}|)^{n}, A(|\mathbb{R}|)\right) \\
f & \mapsto A(|f|): A(|\mathbb{R}|)^{n} \rightarrow A(|\mathbb{R}|)
\end{array}
$$

Thus, we have the (universal-algebraic) $\mathcal{C}^{\infty}$-ring $(A(|\mathbb{R}|), \Psi)$ in the category $\mathcal{C}$.

Moreover, this correspondences yields an equivalence of categories.

Proof. This is an well known result, so we just present the verification that $\Psi$ satisfies the groups of axioms (E1) and (E2).

$\Psi$ preserves projections, since $A$, as a $\mathcal{C}^{\infty}$-ring object, maps the projective cones given in $\mathcal{P}$ to limit cones in $\mathcal{C}$ - that is, to products. Given $n, m_{1}, \cdots, m_{k} \in \mathbb{N}$ such that $n=m_{1}+\cdots, m_{k}$ and the projections $p_{m_{i}}^{n}: \mathbb{R}^{n} \rightarrow \mathbb{R}^{m_{i}}, i=1, \cdots, k, \Psi\left(p_{m_{i}}^{n}\right):=A\left(\left|p_{m_{i}}^{n}\right|\right): A(|\mathbb{R}|)^{n} \rightarrow A(|\mathbb{R}|)^{m_{i}}$, which must be the projections since $A$ maps the cone $\left(p_{m_{i}}^{n}: \mathbb{R}^{n} \rightarrow \mathbb{R}^{m_{i}}\right)_{i=1, \cdots, k}$ to a product in $\mathcal{C}$.

Also, for every $n \in \mathbb{N}$ and every $(n+2)$-tuple of $\mathcal{C}^{\infty}$-functions, $\left(h, g_{1}, \cdots, g_{n}, f\right)$ with $f \in \mathcal{C}^{\infty}\left(\mathbb{R}^{n}\right)$, $g_{1}, \cdots, g_{n} \in \mathcal{C}^{\infty}\left(\mathbb{R}^{k}\right)$ with:

$$
h=f \circ\left(g_{1}, \cdots, g_{n}\right)
$$

we have:

$$
\Psi\left(f \circ\left(g_{1}, \cdots, g_{n}\right)\right)=A\left(|f| \circ\left(\left|g_{1}\right|, \cdots,\left|g_{n}\right|\right)\right)=A(|f|) \circ A\left(\left(\left|g_{1}\right|, \cdots,\left|g_{n}\right|\right)\right),
$$

since $A$, as a $\mathcal{C}^{\infty}$-ring, maps the diagram:

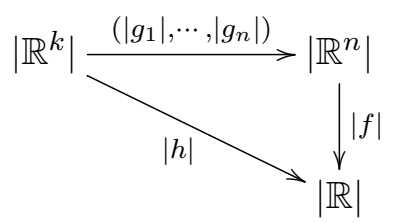

(that belongs to $\mathcal{D}$ since $h=f \circ\left(g_{1}, \cdots, g_{n}\right)$ ) to a commutative one: 


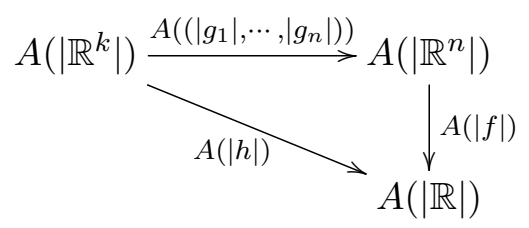

that is $A(|h|)=A(|f|) \circ A\left(\left(\left|g_{1}\right|, \cdots,\left|g_{n}\right|\right)\right)$.

Claim: $A\left(\left(\left|g_{1}\right|, \cdots,\left|g_{n}\right|\right)\right)=\left(A\left(\left|g_{1}\right|\right), \cdots, A\left(\left|g_{n}\right|\right)\right)$.

Indeed, for every $i \in\{1, \cdots, k\}$ the following diagram commutes:

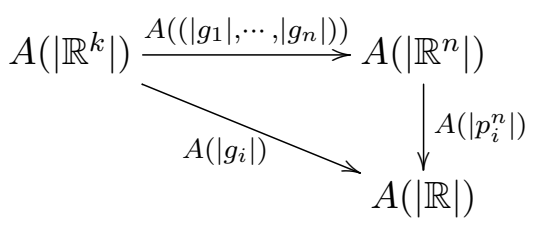

and since $A$ interprets each $p_{i}^{n}, i=1, \cdots, k$, as a projection, $A\left(\left|p_{i}^{n}\right|\right)$, it follows that:

$$
A\left(\left(\left|g_{1}\right|, \cdots,\left|g_{n}\right|\right)\right)=\left(A\left(\left|g_{1}\right|\right), \cdots, A\left(\left|g_{n}\right|\right)\right) .
$$

Thus

$$
\begin{aligned}
\Psi(h):=A(|h|)=A\left(|f| \circ\left(\left|g_{1}\right|, \cdots,\left|g_{n}\right|\right)\right) & =A(|f|) \circ A\left(\left(\left|g_{1}\right|, \cdots,\left|g_{n}\right|\right)\right)= \\
& =A(|f|) \circ\left(A\left(\left|g_{1}\right|\right), \cdots, A\left(\left|g_{n}\right|\right)\right)=\Psi(f) \circ\left(\Psi\left(g_{1}\right), \cdots, \Psi\left(g_{n}\right)\right)
\end{aligned}
$$

and $\Psi$ is a $\mathcal{C}^{\infty}$-ring structure.

Remark 4.2.4. Let $\mathcal{C}$ be a category with all finite limits. The category $\mathcal{C}^{\infty} \operatorname{Ring}(\mathcal{C})$ is not a subcategory of $\mathcal{C}$ (cf. p. 101 of $[74]$ ). However, there is a forgetful functor $U: \mathcal{C}^{\infty} \operatorname{Ring}(\mathcal{C}) \rightarrow \mathcal{C}$ which is faithfull and reflects isomorphisms (cf. Proposition 11.3.3 of [74]). It follows that $U$ reflects all the limits and colimits that it preserves and which exist in $\underline{\mathcal{C}^{\infty} \operatorname{Ring}}(\mathcal{C})$.

The following proposition gives us some properties of the category $\mathcal{C}^{\infty} \operatorname{Ring}(\mathcal{C})$ which are inherited from $\mathcal{C}$ :

Proposition 4.2.5. If a category $\mathcal{C}$ is finitely complete, then the same is true for the category $\mathcal{C}^{\infty} \operatorname{Ring}(\mathcal{C})$.

Proof. It is an immediate application of Proposition 11.5.1 of page 103 of 74 .

Proposition 4.2.6. Let $\mathcal{C}$ be a category with all finite limits. Every left-exact functor $F: \mathcal{C} \rightarrow \mathcal{C}^{\prime}$ induces a functor:

$$
F_{\underline{\mathcal{C}^{\infty}}} \text { Ring }: \underline{\mathcal{C}^{\infty} \operatorname{Ring}}(\mathcal{C}) \rightarrow \underline{\mathcal{C}^{\infty} \operatorname{Ring}}\left(\mathcal{C}^{\prime}\right)
$$

Proof. Since every functor preserves commutative diagrams, it follows that $F$ maps commutative diagrams of $\mathcal{C}$ to commutative diagrams of $\mathcal{C}^{\prime}$, so the $\mathcal{C}^{\infty}$-ring-objects of $\mathcal{C}$ are mapped to $\mathcal{C}^{\infty}$-ring-objects of $\mathcal{C}^{\prime}$.

Proposition 4.2.7. The object $\mathcal{C}^{\infty}(\mathbb{R})$ of $\mathcal{C}^{\infty} \mathbf{R n g}_{\mathrm{fp}}$ is a $\mathcal{C}^{\infty}$-ring-object in the category $\mathcal{C}^{\infty} \mathbf{R n g}_{\mathrm{fp}}$. 
Proof. Given any $f \in \mathcal{C}^{\infty}\left(\mathbb{R}^{n}, \mathbb{R}\right) \subseteq \bigcup_{k>0} \mathcal{C}^{\infty}\left(\mathbb{R}^{k}, \mathbb{R}\right)$ we define $\widehat{f}$ as the unique $\mathcal{C}^{\infty}$-homomorphism sending the identity function $\operatorname{id}_{\mathbb{R}}: \mathbb{R} \rightarrow \mathbb{R}$ to $f$, that is:

$$
\begin{array}{cc}
\widehat{f}=-\circ f: \mathcal{C}^{\infty}(\mathbb{R}) & \rightarrow \mathcal{C}^{\infty}\left(\mathbb{R}^{n}\right) \\
g & \mapsto g \circ f
\end{array}
$$

Theorem 4.2.8. The category

$$
\mathcal{C}^{\infty} \mathbf{R n g}_{\mathrm{fp}}^{\text {op }}
$$

is a category with finite limits freely generated by the $\mathcal{C}^{\infty}$-ring-object $\mathcal{C}^{\infty}(\mathbb{R})$, i.e., for any category with finite limits $\mathcal{C}$, the evaluation of a left-exact functor $F: \mathcal{C}^{\infty} \mathbf{R n g}_{\mathrm{fp}}^{\mathrm{op}} \rightarrow \mathcal{C}$ at $\mathcal{C}^{\infty}(\mathbb{R})$ yields the following equivalence of categories:

$$
\begin{aligned}
\operatorname{ev}_{\mathcal{C}^{\infty}(\mathbb{R})}: \operatorname{Lex}\left(\mathcal{C}^{\infty} \mathbf{R n g}_{\mathrm{fp}}^{\mathrm{op}}, \mathcal{C}\right) & \rightarrow \frac{\mathcal{C}^{\infty} \operatorname{Rings}(\mathcal{C})}{F\left(\mathcal{C}^{\infty}(\mathbb{R})\right)} \\
F & \mapsto
\end{aligned}
$$

Proof. First note that this correspondence is indeed a function, for if $F$ is left-exact, then it preserves $\mathcal{C}^{\infty}$-ring-objects, hence it sends the $\mathcal{C}^{\infty}$-ring object $\mathcal{C}^{\infty}(\mathbb{R})$ of $\mathcal{C}^{\infty} \mathbf{R} \mathbf{n g}_{\text {fp }}^{\text {op }}$ into a $\mathcal{C}^{\infty}$-ring object of $\mathcal{C}$.

We are going to show that this functor is full, faithful and isomorphism-dense.

- $\operatorname{ev}_{\mathcal{C}} \infty(\mathbb{R})$ is faithful;

Let $F, G \in \operatorname{Obj}\left(\operatorname{Lex}\left(\mathcal{C}^{\infty} \mathbf{R n g}_{\mathrm{fp}}^{\mathrm{op}}, \mathcal{C}\right)\right)$ and let $\eta, \theta: F \Rightarrow G$ be two natural transformations between them such that:

$$
\left(\eta_{\mathcal{C}^{\infty}(\mathbb{R})}: F\left(\mathcal{C}^{\infty}(\mathbb{R})\right) \rightarrow G\left(\mathcal{C}^{\infty}(\mathbb{R})\right)\right)=\left(\theta_{\mathcal{C}^{\infty}(\mathbb{R})}: F\left(\mathcal{C}^{\infty}(\mathbb{R})\right) \rightarrow G\left(\mathcal{C}^{\infty}(\mathbb{R})\right)\right) .
$$

We prove that given any object $A$ of $\mathcal{C}^{\infty} \mathbf{R n g}_{\mathrm{fp}}^{\text {op }}$, we have $\eta_{A}=\theta_{A}$.

First suppose $A=\mathcal{C}^{\infty}\left(\mathbb{R}^{n}\right)$, that is, $A=\mathcal{C}^{\infty}(\mathbb{R}) \otimes_{\infty} \cdots \otimes_{\infty} \mathcal{C}^{\infty}(\mathbb{R})$ (which is a product in $\mathcal{C}^{\infty} \mathbf{R n g}_{\mathrm{fp}}^{\text {op }}$ ). Since $F$ is left-exact, $F\left(\mathcal{C}^{\infty}\left(\mathbb{R}^{n}\right)\right)=F\left(\mathcal{C}^{\infty}(\mathbb{R})\right)^{n}$, and:

$$
\eta_{\mathcal{C}^{\infty}\left(\mathbb{R}^{n}\right)} \cong \eta_{\mathcal{C}^{\infty}(\mathbb{R})} \times \cdots \times \eta_{\mathcal{C}}(\mathbb{R}): F\left(\mathcal{C}^{\infty}(\mathbb{R})\right)^{n} \rightarrow G\left(\mathcal{C}^{\infty}(\mathbb{R})\right)^{n}
$$

analogously

$$
\theta_{\mathcal{C}^{\infty}\left(\mathbb{R}^{n}\right)} \cong \theta_{\mathcal{C}^{\infty}(\mathbb{R})} \times \cdots \times \theta_{\mathcal{C}^{\infty}(\mathbb{R})}: F\left(\mathcal{C}^{\infty}(\mathbb{R})\right)^{n} \rightarrow G\left(\mathcal{C}^{\infty}(\mathbb{R})\right)^{n}
$$

Since $\eta_{\mathcal{C} \infty(\mathbb{R})}=\theta_{\mathcal{C} \infty(\mathbb{R})}$, and both $\eta, \theta$ are natural transformations, it follows that $\eta_{\mathcal{C} \infty\left(\mathbb{R}^{n}\right)}=\theta_{\mathcal{C}^{\infty}\left(\mathbb{R}^{n}\right)}$.

- $\operatorname{ev}_{\mathcal{C} \infty(\mathbb{R})}$ is full;

Let $F, G \in \operatorname{Obj}\left(\operatorname{Lex}\left(\mathcal{C}^{\infty} \mathbf{R n g}_{\mathrm{fp}}^{\text {op }}, \mathcal{C}\right)\right)$ and let $\varphi: F\left(\mathcal{C}^{\infty}(\mathbb{R})\right) \rightarrow G\left(\mathcal{C}^{\infty}(\mathbb{R})\right)$ be a morphism in $\mathcal{C}^{\infty} \operatorname{Rings}(\mathcal{C})$. It suffices to take $\eta: F \Rightarrow G$ such that $\eta_{\mathcal{C}^{\infty}(\mathbb{R})}=\varphi$.

- $\operatorname{ev}_{\mathcal{C}^{\infty}(\mathbb{R})}$ is isomorphism-dense; 
Let $R$ be any object in $\underline{\mathcal{C}^{\infty} \operatorname{Rings}}(\mathcal{C})$. $R$.

Given this object $R$, we are going to construct $\phi_{R} \in \operatorname{Obj}\left(\operatorname{Lex}\left(\mathcal{C}^{\infty} \mathbf{R n g}_{\mathrm{fp}}^{\mathrm{op}}, \mathcal{C}\right)\right)$ such that $\mathrm{ev}_{\mathcal{C}^{\infty}(\mathbb{R})}\left(\phi_{R}\right) \cong$

We set $\phi_{R}\left(\mathcal{C}^{\infty}(\mathbb{R})\right)=R$.

We first define the action of $\phi_{R}$ on the free $\mathcal{C}^{\infty}$-ring objects.

Now, given a free $\mathcal{C}^{\infty}$-ring object on $n$ generators, $R^{n}$, since $\phi_{R}$ is to be left-exact, it transforms coproducts in $\mathcal{C}^{\infty} \mathbf{R n g}_{\text {fp }}$ into products of $\mathcal{C}$. Hence, since $\mathcal{C}^{\infty}\left(\mathbb{R}^{n}\right) \cong \mathcal{C}^{\infty}(\mathbb{R}) \otimes_{\infty} \cdots \otimes_{\infty} \mathcal{C}^{\infty}(\mathbb{R})$, we set:

$$
\phi_{R}\left(\mathcal{C}^{\infty}\left(\mathbb{R}^{n}\right)\right)=R^{n}
$$

which establishes the action of $\phi_{R}$ on the free objects of $\mathcal{C}^{\infty} \mathbf{R} \mathbf{n g}_{\mathrm{fp}}^{\mathrm{op}}$.

Now we shall describe the action of $\phi_{R}$ on the arrows between objects of $\mathcal{C}^{\infty} \mathbf{R} \mathbf{n g}_{\mathrm{fp}}^{\text {op }}$ :

$$
\left(\phi_{R}\right)_{1}: \operatorname{Nat}\left(\mathbf{L e x}\left(\mathcal{C}^{\infty} \mathbf{R n g}_{\mathrm{fp}}^{\text {op }}, \mathcal{C}\right)\right) \rightarrow \operatorname{Mor}\left(\underline{\mathcal{C}^{\infty} \operatorname{Rngs}}(\mathcal{C})\right)
$$

beginning with the $\mathcal{C}^{\infty}$-homomorphisms between the free objects of $\mathcal{C}^{\infty} \mathbf{R n g}_{\mathrm{fp}}^{\mathrm{op}}$.

As proved in the Lemma $\mathbf{1 . 4 . 3}$ of the section 1.4, an arrow (i.e., a $\mathcal{C}^{\infty}$-homomorphism) in $\mathcal{C}^{\infty} \mathbf{R n g}_{\mathrm{fp}}$ between free $\mathcal{C}^{\infty}$-rings is a map:

$$
\begin{aligned}
& p: \quad \mathcal{C}^{\infty}\left(\mathbb{R}^{k}\right) \quad \rightarrow \quad \mathcal{C}^{\infty}\left(\mathbb{R}^{n}\right) \\
& \left(\mathbb{R}^{k} \stackrel{g}{\longrightarrow} \mathbb{R}\right) \mapsto\left(\mathbb{R}^{n} \stackrel{\mathbf{p}(g)}{\longrightarrow} \mathbb{R}\right)
\end{aligned}
$$

given by a $k$-tuple of smooth functions, $\left(p_{1}, \cdots, p_{k}\right): \mathbb{R}^{n} \rightarrow \mathbb{R}^{k}$ :

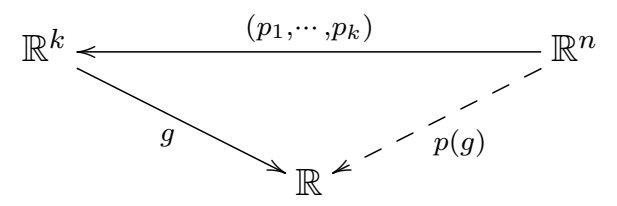

where $p_{i}=p\left(\pi_{i}\right): \mathbb{R}^{n} \rightarrow \mathbb{R}, i=1, \cdots, k$ and $\pi_{i}: \mathbb{R}^{k} \rightarrow \mathbb{R}$ is the projection on the $i$-th coordinate.

Each such smooth function $p_{i}: \mathbb{R}^{n} \rightarrow \mathbb{R}$ yields an arrow in $\mathcal{C}$ :

$$
p_{i}^{(R)}: R^{n} \rightarrow R
$$

defined from the $\mathcal{C}^{\infty}$-ring structure (defined in the Proposition 4.2 .3 , say $\Psi$, of $R \in \underline{\mathcal{C}^{\infty} \operatorname{Rings}}(\mathcal{C})$, which interprets every smooth function in $\mathcal{C}$.

We have, by the Fact 4.2.1. (a), we have a $1-1$ correspondence between $\mathcal{C}^{\infty}$-homomorphisms from $\mathcal{C}^{\infty}\left(\mathbb{R}^{k}\right)$ to $\mathcal{C}^{\infty}\left(\mathbb{R}^{n}\right)$ and $k$-tuples of smooth functions from $\mathbb{R}^{n}$ to $\mathbb{R}$ :

$$
\frac{p: \mathcal{C}^{\infty}\left(\mathbb{R}^{k}\right) \rightarrow \mathcal{C}^{\infty}\left(\mathbb{R}^{n}\right)}{\mathbb{R}^{n} \stackrel{\left(p_{1}, \cdots, p_{k}\right)}{\longrightarrow} \mathbb{R}^{k}} .
$$


The image under $\phi_{R}$ of the arrow $p: \mathcal{C}^{\infty}\left(\mathbb{R}^{k}\right) \rightarrow \mathcal{C}^{\infty}\left(\mathbb{R}^{n}\right)$ is calculated first taking the $k$-tuple of smooth functions given by the correspondence:

$$
\frac{p: \mathcal{C}^{\infty}\left(\mathbb{R}^{k}\right) \rightarrow \mathcal{C}^{\infty}\left(\mathbb{R}^{n}\right)}{\mathbb{R}^{n} \stackrel{\left(p_{1}, \cdots, p_{k}\right)}{\longrightarrow} \mathbb{R}^{k}}
$$

and then interpreting it in $R$ :

$$
\phi_{R}\left(\mathcal{C}^{\infty}\left(\mathbb{R}^{k}\right) \stackrel{p}{\longrightarrow} \mathcal{C}^{\infty}\left(\mathbb{R}^{n}\right)\right)=p^{(R)}=\left(p_{1}^{(R)}, \cdots, p_{k}^{(R)}\right): R^{n} \rightarrow R^{k}
$$

To complete the definition of the functor $\phi_{R}$ on any finitely presented $\mathcal{C}^{\infty}$-ring $\frac{\mathcal{C}^{\infty}\left(\mathbb{R}^{n}\right)}{\left\langle p_{1}, \cdots, p_{k}\right\rangle}$, we note that, by definition, this quotient fits into a coequalizer diagram:

$$
\mathcal{C}^{\infty}\left(\mathbb{R}^{k}\right) \underset{0}{\stackrel{p}{\longrightarrow}} \mathcal{C}^{\infty}\left(\mathbb{R}^{n}\right) \stackrel{q_{\left\langle p_{1}, \cdots, p_{k}\right\rangle}}{\longrightarrow} \frac{\mathcal{C}^{\infty}\left(\mathbb{R}^{n}\right)}{\left\langle p_{1}, \cdots, p_{k}\right\rangle}
$$

where $p_{i}=p\left(\pi_{i}\right)$ for $i=1, \cdots, k$ and $0\left(\pi_{i}\right)=0$ for $i=1, \cdots, k$.

The category $\mathcal{C}$, by hypothesis, has all finite limits, so the category of the $\mathcal{C}^{\infty}$-rings objects in a category $\mathcal{C}$ has equalizers, and there is an equalizer diagram:

$$
E \succ \stackrel{e}{\longrightarrow} R^{n} \underset{0^{(R)}}{\stackrel{p^{(R)}}{\longrightarrow}} R^{m}
$$

Thus we define the image under the contravariant functor $\phi_{R}$ of the finitely presented $\mathcal{C}^{\infty}$-ring $\frac{\mathcal{C}^{\infty}\left(\mathbb{R}^{n}\right)}{\left\langle p_{1}, \cdots, p_{k}\right\rangle}$ as:

$$
\phi_{R}\left(\frac{\mathcal{C}^{\infty}\left(\mathbb{R}^{n}\right)}{\left\langle p_{1}, \cdots, p_{k}\right\rangle}\right):=E
$$

that is, by the following equalizer diagram in $\mathcal{C}$ :

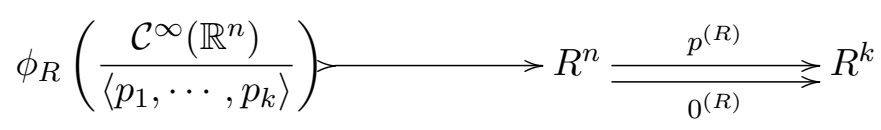

Next, we define $\phi_{R}$ on a $\mathcal{C}^{\infty}$-homomorphism $h: B \rightarrow C$ between any two finitely presented $\mathcal{C}^{\infty}$-rings. Let $\frac{\mathcal{C}^{\infty}\left(\mathbb{R}^{n}\right)}{\left\langle p_{1}, \cdots, p_{k}\right\rangle}$ and $\frac{\mathcal{C}^{\infty}\left(\mathbb{R}^{m}\right)}{\left\langle g_{1}, \cdots, g_{t}\right\rangle}$ be two finitely presented $\mathcal{C}^{\infty}$-rings and let:

$$
\frac{\mathcal{C}^{\infty}\left(\mathbb{R}^{n}\right)}{\left\langle f_{1}, \cdots, f_{k}\right\rangle} \stackrel{\Phi}{\rightarrow} \frac{\mathcal{C}^{\infty}\left(\mathbb{R}^{m}\right)}{\left\langle g_{1}, \cdots, g_{t}\right\rangle}
$$

be a $\mathcal{C}^{\infty}$-homomorphism. By Fact 4.2.1. (a), the $\mathcal{C}^{\infty}$-homomorphism $\Phi$ is determined by some $\mathcal{C}^{\infty}$-function:

$$
\begin{array}{rlc}
\varphi: \mathbb{R}^{m} & \rightarrow & \mathbb{R}^{n} \\
x & \mapsto & \left(\varphi_{1}(x), \cdots, \varphi_{n}(x)\right)
\end{array}
$$

such that $\varphi_{*}\left[\left\langle f_{1}, \cdots, f_{k}\right\rangle\right] \subseteq\left\langle g_{1}, \cdots, g_{t}\right\rangle$. Hence, the $\mathcal{C}^{\infty}$-homomorphism $\Phi$ is determined by the equivalence classes of $n \mathcal{C}^{\infty}$-functions: $\varphi_{1}, \cdots, \varphi_{n}: \mathbb{R}^{m} \rightarrow \mathbb{R}$ such that: 


$$
(\forall j \in\{1, \cdots, k\})\left(f_{j} \circ \varphi=f_{j} \circ\left(\varphi_{1}, \cdots, \varphi_{n}\right) \in\left\langle g_{1}, \cdots, g_{t}\right\rangle\right) .
$$

As in 4.2 , these $n$ smooth functions determine a $\mathcal{C}^{\infty}$-homomorhpism $\varphi^{(R)}: R^{m} \rightarrow R^{n}$. Now $\phi_{R}\left(\frac{\mathcal{C}^{\infty}\left(\overline{\left.\mathbb{R}^{n}\right)}\right.}{\left\langle f_{1}, \cdots, f_{k}\right\rangle}\right)$ and $\phi_{R}\left(\frac{\mathcal{C}^{\infty}\left(\mathbb{R}^{m}\right)}{\left\langle g_{1}, \cdots, g_{t}\right\rangle}\right)$ fit into equalizer rows:

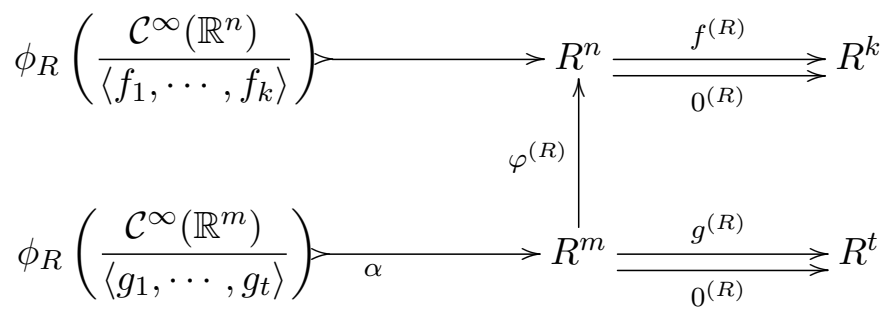

where $f^{(R)}: R^{n} \rightarrow R^{k}$ is the interpretation of $f=\left(f_{1}, \cdots, f_{k}\right): \mathbb{R}^{n} \rightarrow \mathbb{R}^{k}, g^{(R)}: R^{n} \rightarrow R^{t}$ is the interpretation of $g=\left(g_{1}, \cdots, g_{t}\right): \mathbb{R}^{m} \rightarrow \mathbb{R}^{t}$, the equalizer $\alpha$ in the lower left is determined by $m$ arrows, $\alpha=\left(\alpha_{1}, \cdots, \alpha_{m}\right)$ with

$$
\alpha_{s}: \phi_{R}\left(\frac{\mathcal{C}^{\infty}\left(\mathbb{R}^{m}\right)}{\left\langle g_{1}, \cdots, g_{t}\right\rangle}\right) \rightarrow R, s=1, \cdots, m
$$

which satisfy (by definition) $g_{\ell}\left(\alpha_{1}, \cdots, \alpha_{m}\right)=0$ for every $\ell \in\{1, \cdots, t\}$.

We have:

$$
f \circ \varphi=\left(f_{1} \circ \varphi, \cdots, f_{k} \circ \varphi\right)
$$

so

$$
f^{(R)} \circ \varphi^{(R)}=\left(f_{1}^{(R)} \circ \varphi^{(R)}, \cdots, f_{k}^{(R)} \circ \varphi^{(R)}\right) .
$$

Since for every $i=1, \cdots, k, f_{i} \circ \varphi \in\left\langle g_{1}, \cdots, g_{t}\right\rangle$, there are $\ell$ and $\mu_{1}, \cdots, \mu_{t} \in \mathcal{C}^{\infty}\left(\mathbb{R}^{m}\right)$ such that:

$$
f_{i}^{(R)} \circ \varphi^{(R)}=\sum_{\ell=1}^{t} \mu_{\ell} \cdot g_{\ell}
$$

and by (4.5), it follows that:

$$
(\forall i \in\{1, \cdots, k\})(f_{i}^{(R)} \circ \varphi^{(R)}\left(\alpha_{1}, \cdots, \alpha_{m}\right)=\sum_{\ell=1}^{t} \mu_{\ell}\left(\alpha_{1}, \cdots, \alpha_{m}\right) \cdot \underbrace{g_{\ell}\left(\alpha_{1}, \cdots, \alpha_{m}\right)}_{=0}),
$$

so

$$
f^{(R)} \circ\left(\varphi^{(R)} \circ \alpha\right)=\left(f_{1}^{(R)} \circ \varphi^{(R)}, \cdots, f_{k}^{(R)} \circ \varphi^{(R)}\right)=0^{(R)} .
$$

Hence, the composite $\varphi^{(R)} \circ \alpha$ consists of $n$ arrows to $R$ which satisfy the conditions $f \circ\left(\varphi^{(R)} \circ \alpha\right)=0$.

Therefore, by the universal property of equalizers, there is a unique arrow $\phi_{R}(h)$, indicated as follows: 


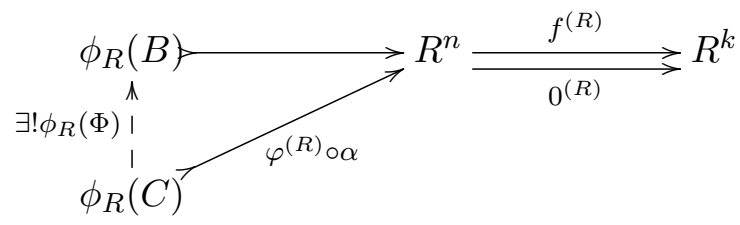

Note that $\phi_{R}(\Phi)$ is independent of the choice of $\varphi_{i}$ in their equivalence classes, and makes $\phi_{R}$ a functor, as required in 4.4).

Claim: For each $\mathcal{C}^{\infty}$-ring object $R$ in $\mathcal{C}$, the functor $\phi_{R}$ thus defined is a left-exact functor $\phi_{R}$ : $\mathcal{C}^{\infty} \mathbf{R n g}_{\mathrm{fp}} \rightarrow \mathcal{C}$

We are going to show that $\phi_{R}$ preserves terminal object, binary products and equalizers, so $\phi_{R}$ will preserve all finite limits (which are constructed from these).

In fact, $\phi_{R}\left(\mathbb{R}^{0}\right)$ is the empty product of copies of $R$ [since $\phi_{R}\left(\mathbb{R}^{0}\right)=R^{0}$ for $n=0$ ], i.e., $\phi_{R}\left(\mathbb{R}^{0}\right)=1$, so $\phi_{R}$ preserves the terminal object.

Since the product of two equalizer diagrams is again an equalizer, one easily verifies from (4.4) that $\phi_{R}$ is such that for any $\frac{\mathcal{C}^{\infty}\left(\mathbb{R}^{n}\right)}{\left\langle f_{1}, \cdots, f_{k}\right\rangle}$ and any $\frac{\mathcal{C}^{\infty}\left(\mathbb{R}^{m}\right)}{\left\langle g_{1}, \cdots, g_{t}\right\rangle}$ we have:

$$
\phi_{R}\left(\frac{\mathcal{C}^{\infty}\left(\mathbb{R}^{n}\right)}{\left\langle f_{1}, \cdots, f_{k}\right\rangle} \otimes_{\infty} \frac{\mathcal{C}^{\infty}\left(\mathbb{R}^{m}\right)}{\left\langle g_{1}, \cdots, g_{t}\right\rangle}\right) \cong \phi_{R}\left(\frac{\mathcal{C}^{\infty}\left(\mathbb{R}^{n}\right)}{\left\langle f_{1}, \cdots, f_{k}\right\rangle}\right) \times \phi_{R}\left(\frac{\mathcal{C}^{\infty}\left(\mathbb{R}^{m}\right)}{\left\langle g_{1}, \cdots, g_{t}\right\rangle}\right)
$$

that is, $\phi_{R}$ preserves binary products.

Finally, to see that $\phi_{R}$ preserves equalizers, consider a coequalizer constructed in the evident way from two arbitrary maps $s, s^{\prime}$ in the category of finitely presented $\mathcal{C}^{\infty}$-rings,

$$
\begin{aligned}
\frac{\mathcal{C}^{\infty}\left(\mathbb{R}^{m}\right)}{\left\langle p_{1}, \cdots, p_{k}\right\rangle} \underset{s^{\prime}}{\longrightarrow} \frac{\mathcal{C}^{\infty}\left(\mathbb{R}^{n}\right)}{\left\langle g_{1}, \cdots, g_{t}\right\rangle} \longrightarrow & \longrightarrow \\
& \rightarrow \frac{\mathcal{C}^{\infty}\left(\mathbb{R}^{n}\right)}{\left\langle g_{1}, \cdots, g_{t}, s \circ \pi_{1}-s^{\prime} \circ \pi_{1}, \cdots, s \circ \pi_{k}-s^{\prime} \circ \pi_{k}\right\rangle}
\end{aligned}
$$

We must show that $\phi_{R}$ sends this coequalizer 4.6 to an equalizer diagram in $\mathcal{C}$.

First of all, if 4.6 is a coequalizer, then so is the diagram:

$$
\begin{aligned}
& \mathcal{C}^{\infty}\left(\mathbb{R}^{m}\right) \underset{s^{\prime} \circ q_{I}}{\stackrel{s \circ q_{I}}{\longrightarrow}} \underset{\frac{\mathcal{C}^{\infty}\left(\mathbb{R}^{n}\right)}{\left\langle g_{1}, \cdots, g_{t}\right\rangle} \longrightarrow}{\longrightarrow} \\
& \rightarrow \frac{\mathcal{C}^{\infty}\left(\mathbb{R}^{n}\right)}{\left\langle g_{1}, \cdots, g_{t}, s \circ \pi_{1} \circ q_{I}-s^{\prime} \circ \pi_{1} \circ q_{I}, \cdots, s \circ \pi_{k} \circ q_{I}-s^{\prime} \circ \pi_{k} \circ q_{I}\right\rangle}
\end{aligned}
$$

obtained by precomposing (4.6) with the epimorphism $q_{I}: \mathcal{C}^{\infty}\left(\mathbb{R}^{m}\right) \rightarrow \frac{\mathcal{C}^{\infty}\left(\mathbb{R}^{m}\right)}{\left\langle p_{1}, \cdots, p_{k}\right\rangle}$. 
Moreover, since $\phi_{R}$ sends the latter epimorphism, $q_{I}$, to a monomorphism in $\mathcal{C}$ [in fact, to an equalizer, as in (4.4), and every equalizer is a monomorphism], $\phi_{R}$ sends 4.6 to an equalizer if, and only if, it does for (4.7). So it suffices to show that $\phi_{R}$ sends coequalizers of the special form (4.7) to equalizers in $\mathcal{C}$.

Next, since (4.7) is a coequalizer, so is

$$
\begin{aligned}
\mathcal{C}^{\infty}\left(\mathbb{R}^{m}\right) \stackrel{s \circ q_{I}-s^{\prime} \circ q_{I}}{\longrightarrow} \longrightarrow \frac{\mathcal{C}^{\infty}\left(\mathbb{R}^{n}\right)}{\left\langle g_{1}, \cdots, g_{t}\right\rangle} \longrightarrow & \longrightarrow \mathcal{C}^{\infty}\left(\mathbb{R}^{n}\right) \\
& \rightarrow \frac{\left.s^{\prime} \circ \pi_{1} \circ q_{I}, \cdots, s \circ \pi_{k} \circ q_{I}-s^{\prime} \circ \pi_{k} \circ q_{I}\right\rangle}{\left\langle g_{1}, \cdots, g_{t}, s \circ \pi_{1} \circ q_{I}-s^{\prime}\right.}
\end{aligned}
$$

and one readly checks that $\phi_{R}$ sends (4.7) to an equalizer in $\mathcal{C}$ if, and only if, it does for (4.8). So, by replacing $s$ by $s-s^{\prime}$ and $s^{\prime}$ by 0 in (4.7) we see that is suffices to show that $\phi_{R}$ sends coequalizers of the form (4.7) with $s^{\prime}=0$ to equalizers in $\mathcal{C}$.

Given a $\mathcal{C}^{\infty}$-homomorphism $p: \mathcal{C}^{\infty}\left(\mathbb{R}^{k}\right) \rightarrow \mathcal{C}^{\infty}\left(\mathbb{R}^{n}\right)$, construct the diagram:

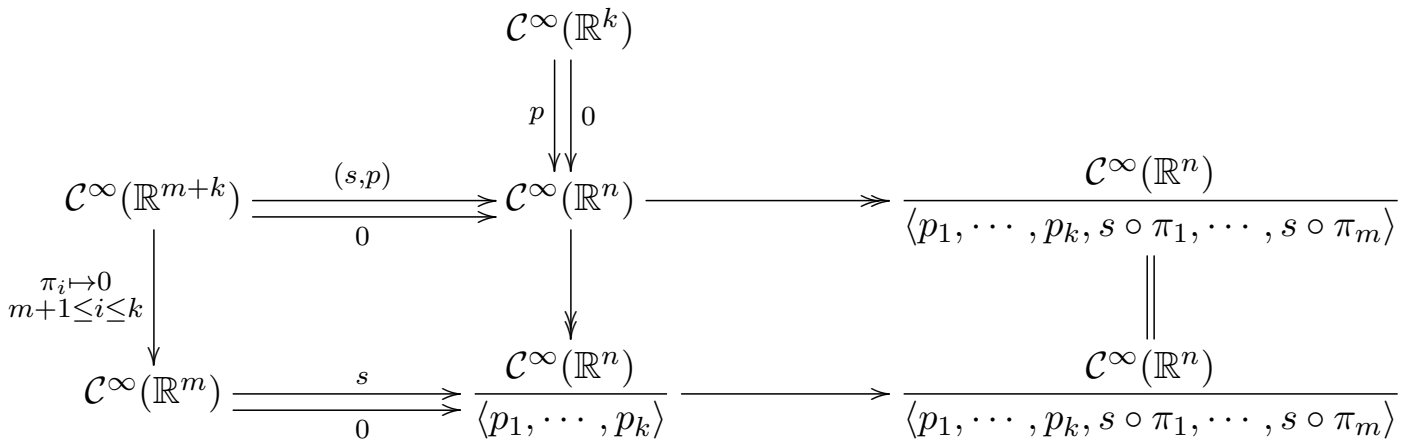

consisting of three coequalizers, two of the form (4.3). By definition (4.4), $\phi_{R}$ sends both the vertical coequalizer and the upper horizontal coequalizer to equalizers in $\mathcal{C}$. It follows, by diagram chasing that it also sends the lower horizontal coequalizer to an equalizer in $\mathcal{C}$.

This shows that $\phi_{R}$ is a left-exact functor.

By construction, $\operatorname{ev}_{\mathcal{C}^{\infty}(\mathbb{R})}\left(\phi_{R}\right)=\phi_{R}\left(\mathcal{C}^{\infty}(\mathbb{R})\right) \cong R$, so $\operatorname{ev}_{\mathcal{C}^{\infty}(\mathbb{R})}$ is a full, faithful and isomorphism-dense functor, hence determines an equivalence of categories.

Combining the results presented in this section and the ones stated in the Section 1 on classifying topoi, we obtain the following:

Theorem 4.2.9. The presheaf topos $\mathbf{S e t s}{ }^{\mathcal{C}^{\infty} \mathbf{R n g}_{\mathrm{f}}}$ is a classifying topos for $\mathcal{C}^{\infty}$-rings, and the universal

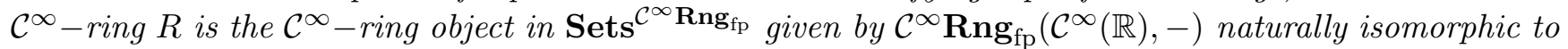
the forgetful functor from $\mathcal{C}^{\infty} \mathbf{R n g}_{\mathrm{fp}}$ to Sets. Thus, for any Grothendieck topos $\mathcal{E}$ there is an equivalence of categories, natural in $\mathcal{E}$ :

$$
\begin{array}{cl}
\operatorname{Geom}\left(\mathcal{E}, \operatorname{Sets}^{\mathcal{C}^{\infty} \mathbf{R n g}_{\mathrm{fp}}}\right) & \rightarrow \frac{\mathcal{C}^{\infty} \operatorname{Rings}}{f^{*}(R)} \\
f & \mapsto \mathcal{E})
\end{array}
$$

Proof. By the previous results, the presheaf topos $\mathbf{S e t s}^{\mathcal{C}^{\infty} \mathbf{R n g}} \mathrm{fp}_{\mathrm{p}}$ is the classifying topos for $\mathcal{C}^{\infty}$-rings, and the universal model (or just universal $\mathcal{C}^{\infty}$-ring object) is the functor $Y\left(\mathcal{C}^{\infty}(\mathbb{R})\right)=\mathcal{C}^{\infty} \mathbf{R n g}\left(\mathcal{C}^{\infty}(\mathbb{R}),-\right)$ that is (naturally isomorphic to) the inclusion functor $i: \mathcal{C}^{\infty} \mathbf{R n g}_{\mathrm{fp}} \hookrightarrow \mathcal{C}^{\infty} \mathbf{R n g}$. 


\subsection{A Classifying Topos for the Theory of Local $\mathcal{C}^{\infty}$-rings}

In this section we describe the $\mathcal{C}^{\infty}$-analog of the Zariski site, which we will verify in the next section to be the classifying topos of the theory of the $\mathcal{C}^{\infty}$-local rings.

Let $\mathcal{C}=\mathcal{C}^{\infty} \mathbf{R n g}_{\mathrm{fp}}{ }^{\text {op }}$ be the (skeleton of the) opposite category of the finitely presented $\mathcal{C}^{\infty}$-rings. In this section we are going to describe the smooth Grothendieck-Zariski pretopology on $\mathcal{C}^{\infty} \mathbf{R n g}_{\mathrm{fp}}{ }^{\text {op }}$.

Any first-order geometric mathematical theory admits a (unique up to equivalence) classifying topos, which contains a universal model of the theory.

In the following we describe the $\mathcal{C}^{\infty}$-analog of the Zariski site, which classifies the theory of the $\mathcal{C}^{\infty}$-local rings.

Let $\mathcal{C}=\mathcal{C}^{\infty} \mathbf{R n g}_{\mathrm{fp}}{ }^{\text {op }}$ be the opposite category of the finitely presented $\mathcal{C}^{\infty}$-rings. In this section we are going to describe the smooth Grothendieck-Zariski pretopology on $\mathcal{C}^{\infty} \mathbf{R n g}_{\mathrm{fp}}{ }^{\text {op }}$.

Convention: We say that a covering family of $A,\left\{g_{j}: B_{j} \rightarrow A \mid j \in J\right\} \in \operatorname{Cov}(A)$ (or a co-covering family of $\operatorname{coCov}(A))$ is generated by a family of $\mathcal{C}^{\infty}$-homomorphisms $\mathcal{F}=\left\{f_{i}: A_{i} \rightarrow A \mid i \in I\right\}$ if, and only if, $\left\{g_{j}: B_{j} \rightarrow A \mid j \in J\right\}$ consists of all the $\mathcal{C}^{\infty}$-homomorphism with codomain $A$ which are isomorphic (in the comma category $\mathcal{C}^{\infty} \mathbf{R n g}_{\mathrm{fp}} \downarrow A$ ) to some element of $\mathcal{F}$. We shall denote it by:

$$
\left\{g_{j}: B_{j} \rightarrow A \mid j \in J\right\} \doteq\left\langle\left\{f_{i}: A_{i} \rightarrow A \mid i \in I\right\}\right\rangle=\langle\mathcal{F}\rangle
$$

The covering families, in our case, will be "generated" by the dual (opposite) of the co-covering families defined as follows:

Let:

$$
\begin{array}{ccc}
\operatorname{coCov}: \operatorname{Obj}\left(\mathcal{C}^{\infty} \mathbf{R n g}_{\mathrm{fp}}\right) & \rightarrow \wp\left(\wp\left(\mathcal{C}^{\infty} \mathbf{R n g}_{\mathrm{fp}}\right)\right) \\
A & \mapsto \quad \operatorname{coCov}(A)
\end{array}
$$

For every $n$-tuple of elements of $A,\left(a_{1}, \cdots, a_{n}\right) \in A \times A \times \cdots, \times A, n \in \mathbb{N}$, such that $\left\langle a_{1}, a_{2}, \cdots, a_{n}\right\rangle=$ $A$, a family of $\mathcal{C}^{\infty}$-homomorphisms $k_{i}: A \rightarrow B_{i}$ such that:

(i) For every $i \in\{1, \cdots, n\}, k_{i}\left(a_{i}\right) \in B_{i}{ }^{\times}$;

(ii) For every $i \in\{1, \cdots, n\}$, if $k_{i}(a)=0$ for some $a \in A$, there is some $s_{i} \in\left\{a_{i}\right\}^{\infty-\text { sat }}$ such that $a \cdot s_{i}=0$

(iii) For every $b \in B_{i}$ there are $c \in\left\{a_{i}\right\}^{\infty-\text { sat }}$ and $d \in A$ such that $b \cdot k_{i}(c)=k_{i}(d)$.

will be a co-covering family of the $\mathcal{C}^{\infty}$-ring $A$, that is:

$\operatorname{coCov}(A)=\left\{\mathcal{F} \subseteq \bigcup_{B \in \operatorname{Obj}(\mathcal{C})} \operatorname{Hom}_{\mathcal{C}}{ }^{\mathbf{R n g}_{\mathrm{fp}}}(A, B) \mid \mathcal{F}=\left\{k_{i}: A \rightarrow B_{i} \mid(n \in \mathbb{N}) \&(i=1, \cdots, n) \&\right.\right.$ $\& k_{i}$ satisfies (i), (ii) and (iii) $\}$ 
In other words,

$$
\operatorname{coCov}(A)=\left\{\mathcal{F} \subseteq \bigcup_{B \in \operatorname{Obj}(\mathcal{C})} \operatorname{Hom}_{\mathcal{C}^{\infty} \mathbf{R n g}_{\mathrm{fp}}} \mid \mathcal{F}=\left\langle\eta_{a_{i}}: A \rightarrow A\left\{a_{i}{ }^{-1}\right\} \mid i \in\{1, \cdots, n\}\right\rangle\right\}
$$

In terms of diagrams, the "generators" of the co-covering families are:

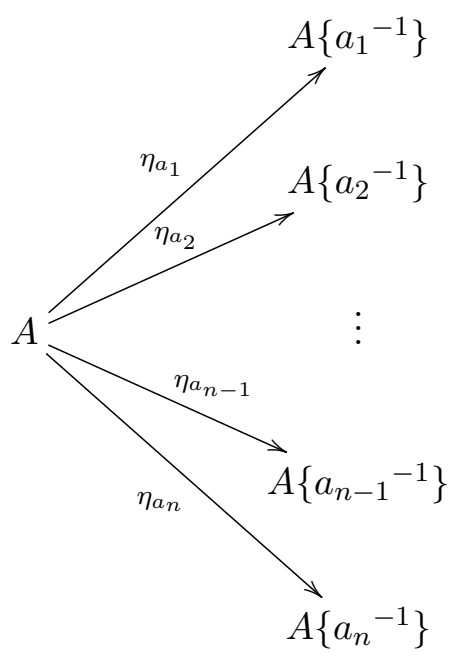

Given a finitely presented $\mathcal{C}^{\infty}$-ring, a covering family for $A$ in $\mathcal{C}^{\infty} \mathbf{R n g}$ fp is given by:

$$
\operatorname{Cov}(A)=\left\{f^{\mathrm{op}}: B \rightarrow A \mid(f: A \rightarrow B) \in \operatorname{coCov}(A)\right\}
$$

Remark 4.3.1. Note that if $A$ is a finitely presented $\mathcal{C}^{\infty}$-ring, i.e. $A \cong \frac{\mathcal{C}^{\infty}\left(\mathbb{R}^{n}\right)}{\left\langle f_{1}, \cdots, f_{k}\right\rangle}$, and $b \in A$, then $A\left\{b^{-1}\right\}$ is a finitely presented $\mathcal{C}^{\infty}$-ring:

$$
A\left\{b^{-1}\right\} \cong \frac{A\{x\}}{(b x-1)} \cong \frac{\mathcal{C}^{\infty}\left(\mathbb{R}^{n+1}\right)}{\left\langle f_{1} \circ \pi_{1}, \cdots, f_{k} \circ \pi_{1},(b x-1) \circ \pi_{2}\right\rangle}
$$

Proposition 4.3.2. Cov is a Grothendieck pretopology on $\mathcal{C}^{\infty} \mathbf{R n g}_{\mathrm{fp}}^{\mathrm{op}}$.

Proof. Let $A$ be any finitely presented $\mathcal{C}^{\infty}$-ring.

\section{Isomorphism axiom:}

Whenever $\varphi^{\mathrm{op}}: A^{\prime} \rightarrow A$ is a $\mathcal{C}^{\infty}$-isomorphism, the family $\left\{\varphi^{\mathrm{op}}: A^{\prime} \rightarrow A\right\} \in \operatorname{Cov}(A)$.

Note that $\varphi^{\mathrm{op}}: A^{\prime} \rightarrow A$ is a $\mathcal{C}^{\infty}$-isomorphism in $\mathcal{C}^{\infty} \mathbf{R n g}_{\mathrm{fp}}^{\mathrm{op}}$ if, and only if, $\varphi: A \rightarrow A^{\prime}$ is a $\mathcal{C}^{\infty}$-isomorphism in $\mathcal{C}^{\infty} \mathbf{R n g}_{\mathrm{fp}}$. Thus, we are going to show that if $\varphi: A \rightarrow A^{\prime}$ is a $\mathcal{C}^{\infty}$-isomorphism, then $\left\{\varphi: A \rightarrow A^{\prime}\right\} \in \operatorname{coCov}(A)$.

Indeed, $1_{A} \in A$ is such that $\left\langle 1_{A}\right\rangle=A$, so the one element family $\left\{\eta_{1_{A}}: A \rightarrow A\left\{1_{A}{ }^{-1}\right\}\right\} \in \operatorname{coCov}(A)$.

Since $\varphi: A \rightarrow A^{\prime}$ is a $\mathcal{C}^{\infty}$-isomorphism, $\varphi$ is, in particular, a $\mathcal{C}^{\infty}$-homomorphism, and we have, for every $s \in\left\{1_{A}\right\}^{\infty-\text { sat }}=A^{\times}, \varphi(s) \in A^{\prime \times}$. Also, if $\varphi(a)=0_{A^{\prime}}$ for some $a \in A$, since $\operatorname{ker} \varphi=\left\{0_{A}\right\}$ (for $\varphi$ is injective), $a=0_{A}$, so for every $s_{i} \in\left\{1_{A^{\prime}}\right\}^{\infty \text {-sat }}$ (in particular, there is some such $s_{i}$ ) one has $a \cdot s_{i}=0_{A}$.

Finally, given any $a^{\prime} \in A^{\prime}$, since $\varphi$ is surjective, there is some element $a \in A$ such that $\varphi(a)=a^{\prime}$. Since $1_{A} \in\left\{1_{A^{\prime}}\right\}^{\infty-\text { sat }}$ and $a=\frac{a}{1_{A}}$, we have:

$$
a^{\prime}=\varphi(a) \cdot \varphi\left(1_{A}\right)^{-1} .
$$


Since $\varphi: A \rightarrow A^{\prime}$ satisfies (i), (ii) and (iii), the one-element family $\left\{\varphi: A \rightarrow A^{\prime}\right\}$ co-covers $A$, so $\left\{\varphi^{\mathrm{op}}: A^{\prime} \rightarrow A\right\} \in \operatorname{Cov}(A)$.

\section{Stability axiom:}

Now we are going to show that our definition of Cov is stable under pullbacks, that is:

If $\left(a_{1}, \cdots, a_{n}\right) \in A \times A \times \cdots \times A$ is a $n$-tuple such that $\left\langle a_{1}, \cdots, a_{n}\right\rangle=A$ and $\left\{\eta_{a_{i}}^{\text {op }}: A\left\{a_{i}{ }^{-1}\right\} \rightarrow A\right\}_{i=1, \cdots, n}$ generates a covering family for $A$, then given a $\mathcal{C}^{\infty}$-rings homomorphism $g: A \rightarrow B$, since $\eta_{g\left(a_{i}\right)} \circ g$ is such that $\left(\eta_{g\left(a_{i}\right)} \circ g\right)\left(a_{i}\right) \in B\left\{g\left(a_{i}\right)^{-1}\right\}^{\times}$, by the universal property of $\eta_{a_{i}}: A \rightarrow A\left\{a_{i}{ }^{-1}\right\}$ there is a unique $\mathcal{C}^{\infty}$-homomorphism:

$$
g^{\prime}: A\left\{a_{i}{ }^{-1}\right\} \rightarrow B\left\{g\left(a_{i}\right)^{-1}\right\}
$$

such that the following diagram commutes:

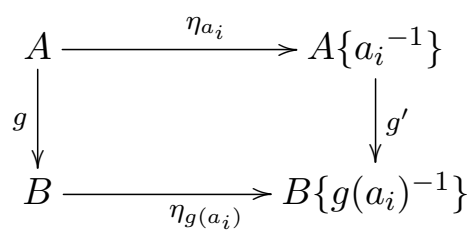

By Proposition 2.1.35, the diagram above is a pushout, so

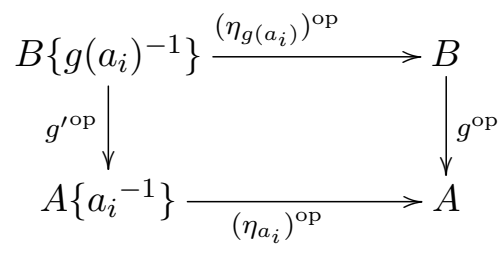

is a pullback in $\mathcal{C}^{\infty} \mathbf{R n g}_{\text {fp }}^{\text {op }}$.

In order to show that the family $\left\{\left(\eta_{g\left(a_{i}\right)}\right)^{\text {op }}: B\left\{g\left(a_{i}\right)^{-1}\right\} \rightarrow B \mid i=1, \cdots, n\right\}$ belongs to $\operatorname{Cov}(B)$, it suffices to show that $\left\{\eta_{g\left(a_{i}\right)}: B \rightarrow B\left\{g\left(a_{i}\right)^{-1}\right\} \mid i=1, \cdots, n\right\}$ belongs to $\operatorname{coCov}(B)$.

Since $a_{1}, \cdots, a_{n}$ are such that $\left\langle a_{1}, \cdots, a_{n}\right\rangle=A$, there are some $\lambda_{1}, \cdots, \lambda_{n} \in A$ such that:

$$
1_{A}=\sum_{i=1}^{n} \lambda_{i} \cdot a_{i}
$$

Since $g: A \rightarrow B$ is a $\mathcal{C}^{\infty}$-homomorphism, we have:

$$
1_{B}=g\left(1_{A}\right)=\sum_{i=1}^{n} g\left(\lambda_{i}\right) \cdot g\left(a_{i}\right),
$$

thus $\left\langle g\left(a_{1}\right), \cdots, g\left(a_{n}\right)\right\rangle=B$. Also, since for every $i=1, \cdots, n, \eta_{g\left(a_{i}\right)}: B \rightarrow B\left\{g\left(a_{i}\right)^{-1}\right\}$ is a $\mathcal{C}^{\infty}$-ring of fractions, it follows that $\left\{\eta_{g\left(a_{i}\right)}: B \rightarrow B\left\{g\left(a_{i}\right)^{-1}\right\} \mid i=1, \cdots, n\right\} \in \operatorname{coCov}(B)$, hence:

$$
\left\{\left(\eta_{g\left(a_{i}\right)}\right)^{\mathrm{op}}: B\left\{g\left(a_{i}\right)^{-1}\right\} \rightarrow B \mid i=1, \cdots, n\right\} \in \operatorname{Cov}(B)
$$

\section{Transitivity axiom:}

If $\left\{\eta_{a_{i}}^{A}: A \rightarrow A\left\{a_{i}{ }^{-1}\right\} \mid i=1, \cdots, n\right\}$ generates a co-covering family of $A$ and for each $i,\left\{\eta_{\beta_{i j}}^{A_{i}}: A\left\{a_{i}{ }^{-1}\right\} \rightarrow\right.$ 
$\left.\left(A\left\{a_{i}{ }^{-1}\right\}\right)\left\{\beta_{i j}^{-1}\right\} \mid j \in\left\{1, \cdots, n_{i}\right\}\right\}$ generates a co-covering family of $A\left\{a_{i}{ }^{-1}\right\}$, then:

$$
\left.\left\{\eta_{\left.\beta_{i j}\right)}^{A_{i}} \circ \eta_{a_{i}}^{A}: A \rightarrow\left(A\left\{a_{i}{ }^{-1}\right\}\right)\left\{\beta_{i j}\right)^{-1}\right\} \mid i \in\{1, \cdots, n\} \& j \in\left\{1, \cdots, n_{i}\right\}\right\}
$$

generates a co-covering family of $A$.

To show that the transitive axiom holds we will need the following technical result on "Smooth Commutative Algebra":

If for each $i \leq n$ and each $\beta_{i j} \in A\left\{a_{i}^{-1}\right\}, j \leq n_{i}$, we write $\beta_{i j}=\eta_{a}\left(b_{i j}\right) / \eta_{a}\left(c_{i} j\right)$, with $c_{i j} \in\left\{a_{i}\right\}^{\infty-s a t}$, then by Proposition 2.1.32, to show that:

$$
\left\{\eta_{\beta_{i j}}^{A_{i}} \circ \eta_{a_{i}}^{A}: A \rightarrow\left(A\left\{a_{i}{ }^{-1}\right\}\right)\left\{\beta_{i j}^{-1}\right\} \mid i \in\{1, \cdots, n\} \& j \in\left\{1, \cdots, n_{i}\right\}\right\}
$$

generates a co-covering family of $A$ amounts to show that:

$$
\left\{\eta_{a_{i} \cdot b_{i j}}^{A}: A \rightarrow A\left\{\left(a_{i} \cdot b_{i j}\right)^{-1}\right\} \mid(i \in\{1, \cdots n\}) \&\left(j \in\left\{1, \cdots, n_{i}\right\}\right)\right\}
$$

does.

By hypothesis, $\left.\left\{\eta_{a_{i}}^{\mathrm{op}}: A\left\{a_{i}{ }^{-1}\right\} \rightarrow A \mid i \in\{1, \cdots, n\}\right\}\right\}$ generates a covering family of $A$, so:

$$
1_{A} \in\left\langle a_{1}, \cdots, a_{n}\right\rangle
$$

or, equivalently:

$$
\operatorname{Spec}^{\infty}(A)=\bigcup_{i=1}^{n} D_{A}^{\infty}\left(a_{i}\right)
$$

Since for every $i \in\{1, \cdots, n\}$ we have a canonical homeomorphism:

$$
\begin{array}{cc}
\varphi: \operatorname{Spec}^{\infty}\left(A\left\{a_{i}{ }^{-1}\right\}\right) & \rightarrow D^{\infty}\left(a_{i}\right) \\
\mathfrak{p} & \mapsto \eta_{a_{i}}^{-1}[\mathfrak{p}]
\end{array}
$$

Also by hypothesis, for any $i \in\{1, \cdots, n\},\left\{\left(\eta_{a_{i} b_{i j}}^{A}\right)^{\text {op }}: A\left\{a_{i} \cdot b_{i j}{ }^{-1}\right\} \rightarrow A\left\{a_{i}{ }^{-1}\right\} \mid j \in\left\{1, \cdots, n_{i}\right\}\right\}$ generates a covering family of $A\left\{a_{i}{ }^{-1}\right\}$, so:

$$
\operatorname{Spec}^{\infty}\left(A\left\{a_{i}{ }^{-1}\right\}\right)=\bigcup_{j=1}^{n_{i}} D_{A\left\{a_{i}^{-} 1\right\}}^{\infty}\left(\beta_{i j}\right) \approx \bigcup_{j=1}^{n_{i}} D_{A}^{\infty}\left(a_{i}\right) \cap D_{A}^{\infty}\left(b_{i j}\right) \approx \bigcup_{j=1}^{n_{i}} D_{A}^{\infty}\left(a_{i} \cdot b_{i j}\right)
$$

Putting all together we obtain:

$$
\operatorname{Spec}^{\infty}(A)=\bigcup_{i=1}^{n} D_{A}^{\infty}\left(a_{i}\right) \approx \bigcup_{i=1}^{n} \operatorname{Spec}^{\infty}\left(A\left\{a_{i}{ }^{-1}\right\}\right) \approx \bigcup_{i=1}^{n}\left(\bigcup_{j=1}^{n_{i}} D_{A}^{\infty}\left(a_{i} \cdot b_{i j}\right)\right)
$$

thus,

$$
\operatorname{Spec}^{\infty}(A)=\bigcup_{i \leq n, j \leq n_{i}} D_{A}^{\infty}\left(a_{i} \cdot b_{i j}\right)
$$


but this is equivalent to

$$
1_{A} \in\left\langle\left\{a_{i} \cdot b_{i j}: i \leq n, j \leq n_{i}\right\}\right\rangle
$$

and the transitivity is proved.

Thus, Cov defines a Grothendieck pretopology on $\mathcal{C}^{\infty} \mathbf{R} \mathbf{n g}_{\mathrm{fp}}^{\mathrm{op}}$. We have:

$$
J_{\text {Cov }}: \operatorname{Obj}\left(\mathcal{C}^{\infty} \mathbf{R n g}_{\mathrm{fp}}\right) \rightarrow \wp\left(\wp\left(\operatorname{Mor}\left(\mathcal{C}^{\infty} \mathbf{R n g}_{\mathrm{fp}}\right)\right)\right)
$$

given by:

$$
J_{\mathrm{Cov}}(A):=\left\{\overleftarrow{S} \subseteq \bigcup_{B \in \mathrm{Obj}} \bigcup_{\left.\mathcal{C}^{\infty} \mathbf{R n g}_{\mathrm{fp}}\right)} \operatorname{Hom}_{\mathcal{C}^{\infty} \mathbf{R n g}_{\mathrm{fp}}}(B, A) \mid S \in \operatorname{Cov}(A)\right\}
$$

turning $\left(\mathcal{C}^{\infty} \mathbf{R n g}_{\mathrm{fp}}^{\mathrm{op}}, J_{\mathrm{Cov}}\right)$ into a small site.

Proposition 4.3.3. Let $I$ be a finite set (lets say $I=\{1, \cdots, n\}$ ) and let $\left\{A\left\{a_{i}^{-1}\right\} \stackrel{\eta_{a_{i}}}{\rightarrow} A \mid i \in I\right\}$ be a $\mathcal{Z}^{\infty}$-covering of $A$ in $\mathcal{C}^{\infty} \mathbf{R n g}_{\mathrm{fp}}{ }^{\text {op }}$, then the diagram below is an equalizer in the category of $\mathcal{C}^{\infty}$-rings:

(E)

$$
A \rightarrow \prod_{i \in I} A\left\{a_{i}^{-1}\right\} \rightrightarrows \prod_{i, j \in I} A\left\{\left(a_{i} . a_{j}\right)^{-1}\right\}
$$

Proof. By hypothesis, $A=\left\langle\left\{a_{i} \mid i \in I\right\}\right\rangle$, or equivalently, $\operatorname{Spec}^{\infty}(A)=D^{\infty}(1)=\bigcup_{i \in I} D^{\infty}\left(a_{i}\right)$.

Since the affine $\mathcal{C}^{\infty}$-locally ringed space of $A, \Sigma_{A}$, is in particular a sheaf of $\mathcal{C}^{\infty}$-rings, then the diagram below is an equalizer in the category of $\mathcal{C}^{\infty}$-rings.

$$
\Sigma_{A}\left(D^{\infty}(1)\right) \longrightarrow \prod_{i \in I} \Sigma_{A}\left(D^{\infty}\left(a_{i}\right)\right) \longrightarrow \prod_{i, j \in I} \Sigma_{A}\left(D^{\infty}\left(a_{i}\right) \cap D^{\infty}\left(a_{j}\right)\right)
$$

As $D^{\infty}\left(a_{i}\right) \cap D^{\infty}\left(a_{j}\right)=D^{\infty}\left(a_{i} \cdot a_{j}\right)$ and $\Sigma_{A}\left(D^{\infty}(b)\right) \cong A\left\{b^{-1}\right\}$, we have that the diagram of $\mathcal{C}^{\infty}$-rings below is an equalizer

$$
A \stackrel{\cong}{\rightrightarrows} A\left\{1^{-1}\right\} \rightarrow \prod_{i \in I} A\left\{a_{i}^{-1}\right\} \rightrightarrows \prod_{i, j \in I} A\left\{\left(a_{i} \cdot a_{j}\right)\right\}^{-1}
$$

and this finish the proof.

Remark 4.3.4. The forgetfull functor $\mathcal{O}: \mathcal{C}^{\infty} \mathbf{R n g}_{\mathrm{fp}} \rightarrow$ Sets is called the structure sheaf of the GrothendieckZariski smooth topos.

This is actually a sheaf of sets since if $\left\{\eta_{a_{i}}: A \rightarrow A\left\{a_{i}^{-1}\right\} \mid i \leq n\right\}$ is a smooth Zariski co-covering (i.e. $\left.A=\left\langle a_{1}, \cdots, a_{n}\right\rangle\right)$, then diagram of sets below must be an equalizer,

$$
A \rightarrow \prod_{i \in I} A\left\{a_{i}^{-1}\right\} \rightrightarrows \prod_{i, j \in I} A\left\{\left(a_{i} \cdot a_{j}\right)^{-1}\right\}
$$

since it is indeed an equalizer of $\mathcal{C}^{\infty}$-rings and the forgetfull functor $\mathcal{C}^{\infty} \mathbf{R n g} \rightarrow$ Sets preserves limits. 
Theorem 4.3.5. The smooth Grothendieck-Zariski topology constructed above $\left(\mathcal{C}, \mathcal{Z}^{\infty}\right)$ is subcanonical, that is, for every finitely presented $\mathcal{C}^{\infty}$-ring $B$, the representable functor:

$$
\begin{array}{cccc}
\operatorname{Hom}_{\mathcal{C}}(\bullet, B): & \mathcal{C}^{o p} & \rightarrow & \text { Set } \\
A & \mapsto & \operatorname{Hom}_{\mathcal{C}}(A, B) \\
\left(A_{1} \stackrel{f}{\longrightarrow} A_{2}\right) & \mapsto & \left(\operatorname{Hom}_{\mathcal{C}}\left(A_{2}, B\right) \stackrel{-\circ f}{\longrightarrow} \operatorname{Hom}_{\mathcal{C}}\left(A_{1}, B\right)\right)
\end{array}
$$

is a sheaf (of sets).

Proof. Let $I$ be a finite set (lets say $I=\{1, \cdots, n\}$ ) and let $\left\{A\left\{a_{i}^{-1}\right\} \stackrel{\eta_{a_{i}}}{\rightarrow} A \mid i \in I\right\}$ be a $\mathcal{Z}^{\infty}$-covering of $A$ in $\mathcal{C}$.

Recall, from Corollary 2.1.36, that for every $i, j \in I$, the following rectangle is a pushout in $\mathcal{C}^{\infty} \mathbf{R n g :}$

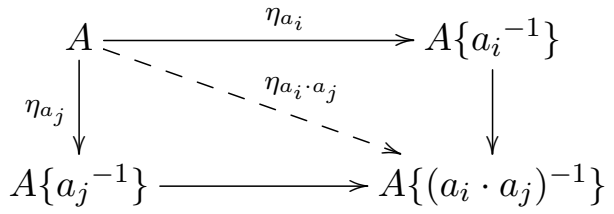

We must prove that:

(I)

$$
\mathcal{C}(A, B) \rightarrow \prod_{i \in I} \mathcal{C}\left(A\left\{a_{i}^{-1}\right\}, B\right) \rightrightarrows \prod_{i, j \in I} \mathcal{C}\left(A\left\{\left(a_{i} . a_{j}\right)^{-1}\right\}, B\right)
$$

is an equalizer diagram of sets and functions.

Since $\mathcal{C}=\mathcal{C}^{\infty} \mathbf{R n g}_{\mathrm{fp}}{ }^{\text {op }}$, this amounts to prove that

$$
\mathcal{C}^{\infty} \mathbf{R n g}_{\mathrm{fp}}(B, A) \rightarrow \prod_{i \in I} \mathcal{C}^{\infty} \mathbf{R n g}_{\mathrm{fp}}\left(B, A\left\{a_{i}^{-1}\right\}\right) \rightrightarrows \prod_{i, j \in I} \mathcal{C}^{\infty} \mathbf{R n g}_{\mathrm{fp}}\left(B, A\left\{\left(a_{i} . a_{j}\right)^{-1}\right\}\right)
$$

is an equalizer diagram of sets and functions.

As Hom functors preserve products, the diagram (II) is isomorphic to (III)

$$
\mathcal{C}^{\infty} \mathbf{R n g}_{\mathrm{fp}}(B, A) \rightarrow \mathcal{C}^{\infty} \mathbf{R n g}_{\mathrm{fp}}\left(B, \prod_{i \in I} A\left\{a_{i}^{-1}\right\}\right) \rightrightarrows \mathcal{C}^{\infty} \mathbf{R n g}_{\mathrm{fp}}\left(B, \prod_{i, j \in I} A\left\{\left(a_{i} . a_{j}\right)^{-1}\right\}\right)
$$

But this is an equalizer diagram of sets and functions since the Hom functor $\mathcal{C}^{\infty} \mathbf{R n g}_{\mathrm{fp}}(B,-)$ preserves equalizers and the diagram

(E)

$$
A \rightarrow \prod_{i \in I} A\left\{a_{i}^{-1}\right\} \rightrightarrows \prod_{i, j \in I} A\left\{\left(a_{i} \cdot a_{j}\right)^{-1}\right\}
$$

is an equalizer in the category of $\mathcal{C}^{\infty}$-rings, by Proposition 4.3.3.

Thus the Grothendieck topology $\mathcal{Z}^{\infty}$ is subcanonical.

Now we show that the topos of sheaves on the smooth Zariski site, described in the previous section, is the classifying topos of the theory of the $\mathcal{C}^{\infty}$-local rings. 
The Mitchell-Bénabou language motivates ${ }^{1}$ us to define a local $\mathcal{C}^{\infty}$-ring object in a topos $\mathcal{E}$ : it is a $\mathcal{C}^{\infty}$-ring object $R$ in $\mathcal{E}$ such that the (geometric) formula:

$$
(\forall a \in R)((\exists b \in R)(a \cdot b=1) \vee(\exists b \in R)((1-a) \cdot b=1))
$$

is valid.

By definition, this means that the union of the subobjects:

$$
\begin{gathered}
\{a \in R \mid \exists b \in R(a \cdot b=1)\} \longmapsto R, \\
\{a \in R \mid \exists b \in R((1-a) \cdot b=1)\} \longmapsto R
\end{gathered}
$$

of $R$ is all of $R$. Equivalently, consider the two subobjects of the product $R \times R$ defined by:

$$
\left\{\begin{array}{l}
U=\{(a, b) \in R \times R \mid a \cdot b=1\} \longmapsto R \times R \\
V=\{(a, b) \in R \times R \mid(1-a) \cdot b=1\} \longmapsto R \times R
\end{array}\right.
$$

The $\mathcal{C}^{\infty}$-ring object $R$ is local if, and only if, the two composites $U \longmapsto R \times R \stackrel{\pi_{1}}{\rightarrow} R$ and $V \longmapsto R \times R \stackrel{\pi_{1}}{\rightarrow} R$ form an epimorphic family in $\mathcal{E}$.

In Section 2, we have observed that there is an equivalence between $\mathcal{C}^{\infty}$-ring objects $R$ in a topos $\mathcal{E}$ and left exact functors, $\mathcal{C}^{\infty} \mathbf{R n g}_{\mathrm{fp}}^{\text {op }} \rightarrow \mathcal{E}$. Explicitly, given such a left-exact functor $F$, the corresponding $\mathcal{C}^{\infty}$-ring object $R$ in $\mathcal{E}$ is $F\left(\mathcal{C}^{\infty}(\mathbb{R})\right)$. Conversely, given a $\mathcal{C}^{\infty}$-ring $R$ in $\mathcal{E}$, the corresponding functor:

$$
\phi_{R}: \mathcal{C}^{\infty} \mathbf{R n g}_{\mathrm{fp}}^{\text {op }} \rightarrow \mathcal{E}
$$

sends the finitely presented $\mathcal{C}^{\infty}$-ring $A=\frac{\mathcal{C}^{\infty}\left(\mathbb{R}^{n}\right)}{\left\langle p_{1}, \cdots, p_{k}\right\rangle}$ to the following equalizer in $\mathcal{E}$ :

$$
\phi_{R}(A) \longmapsto R^{n} \underset{(0, \cdots, 0)}{\stackrel{\left(p_{1}, \cdots, p_{k}\right)}{\longrightarrow}} R^{k}
$$

This description yields the corresponding definition of $\phi_{R}$ on arrows.

The following lemma gives a condition for a $\mathcal{C}^{\infty}$-ring $R$ in a topos $\mathcal{E}$ to be local, phrased in terms of this corresponding functor $\phi_{R}$.

Lemma 4.3.6. Let $\mathcal{E}$ be a topos, $R$ be a $\mathcal{C}^{\infty}$-ring object in $\mathcal{E}$, and let $\phi_{R}: \mathcal{C}^{\infty} \mathbf{R n g}^{\mathrm{op}} \rightarrow \mathcal{E}$ be the corresponding left exact functor. The following are equivalent:

(i) $R$ is a local $\mathcal{C}^{\infty}$-ring in $\mathcal{E}$;

(ii) $\phi_{R}$ sends the pair of arrows in the category $\mathcal{C}^{\infty} \mathbf{R n g}_{\mathrm{fp}}$ :

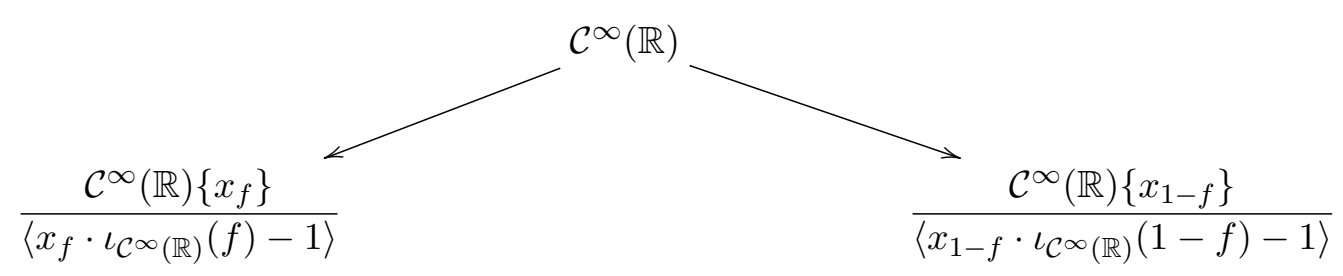

to an epimorphic family of two arrows in $\mathcal{E}$;

\footnotetext{
${ }^{1}$ although we are not going to use it
} 
(iii) For any finitely presented $\mathcal{C}^{\infty}$-ring $A$ and any elements $a_{1}, \cdots, a_{n}$ such that $\left\langle a_{1}, \cdots, a_{n}\right\rangle=A, \phi_{R}$ sends the family of arrows in $\mathcal{C}^{\infty} \mathbf{R n g}_{\mathrm{fp}}$ :

$$
\left\{A \rightarrow A\left\{a_{i}^{-1}\right\} \mid i=1, \cdots, n\right\}
$$

to an epimorphic family $\left\{\phi_{R}\left(A\left\{a_{i}{ }^{-1}\right\}\right) \rightarrow \phi_{R}(A) \mid i=1, \cdots, n\right\}$ in $\mathcal{E}$.

Proof. Ad $(i) \Longleftrightarrow(i i)$ : This follows immediately from the explicit description of the functor $\phi_{R}$. Let $A=\frac{\mathcal{C}^{\infty}(\mathbb{R})\left\{x_{f}\right\}}{\left\langle x_{f} \cdot \iota_{\mathcal{C}^{\infty}(\mathbb{R})}(f)-1\right\rangle}$ and $\left.B=\frac{\mathcal{C}^{\infty}(\mathbb{R})\left\{x_{1-f}\right\}}{\left\langle x_{1-f} \cdot \iota_{\mathcal{C}}(\mathbb{R})\right.}(1-f)-1\right\rangle$. Note that:

$$
\phi_{R}(A)=\{(a, b) \in R \times R \mid a \cdot b=1\}
$$

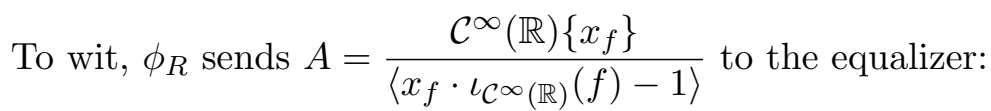

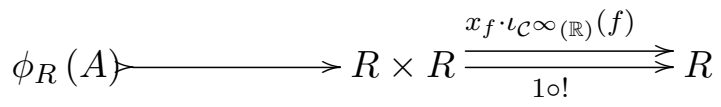

and $\phi_{R}$ sends $B$ to:

$$
\phi_{R}(B)>R \times R \frac{x_{1-f \cdot \iota_{\mathcal{C}}(\mathbb{R})}(1-f)}{1 \circ !} \longrightarrow R
$$

The arrow $\mathcal{C}^{\infty}(\mathbb{R}) \rightarrow A$ is mapped into the composite $\alpha: \phi_{R}(A) \rightarrow R$ given by $\phi_{R}(A) \longmapsto R \times R \stackrel{\pi_{1}}{\rightarrow} R$, and the arrow $\mathcal{C}^{\infty}(\mathbb{R}) \rightarrow B$, is mapped into the composite $\beta: \phi_{R}(B) \rightarrow R$, given by $\phi_{R}(B) \longmapsto R \times R \stackrel{\pi_{f}}{\rightarrow} R$.

By the definition of a local $\mathcal{C}^{\infty}$-ring, (i) is equivalent to (ii).

Ad (iii) $\Rightarrow$ (ii): is also clear, since (ii) is the special case of (iii) in which $A=\mathcal{C}^{\infty}(\mathbb{R})$ while $n=2$, $a_{1}=f$ and $a_{2}=1-f$.

Ad (ii) $\Rightarrow$ (iii): Assume that (ii) hlds, and suppose given a finitely presented $\mathcal{C}^{\infty}$-ring $A$ and elements $a_{1}, \cdots, a_{n} \in A$ with $\sum_{i=1}^{n} a_{i}=1$. This result is proved using induction. We prove the:

\section{Case $n=2$.}

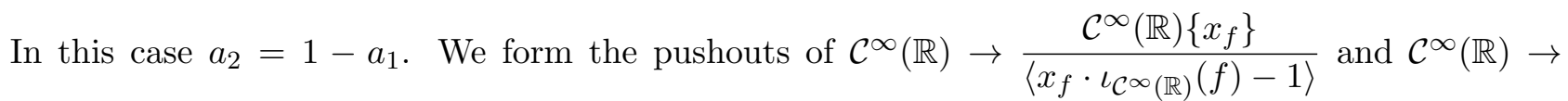

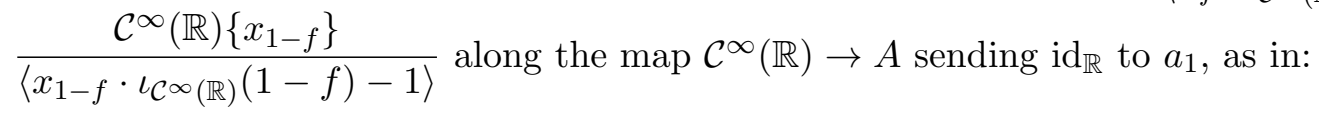

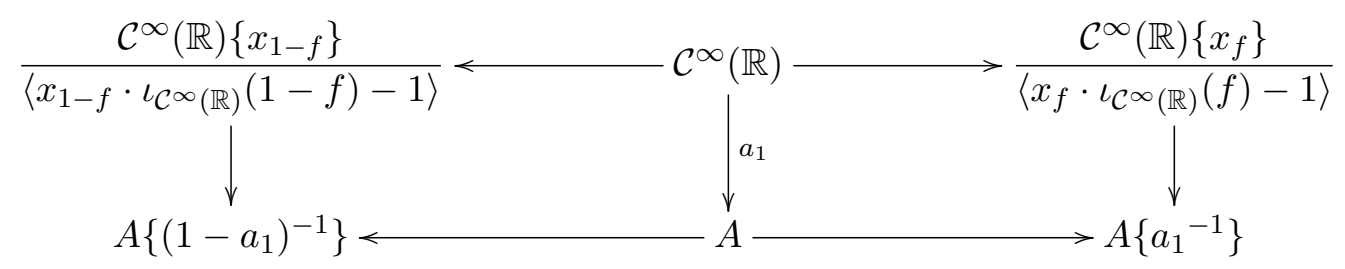

giving the indicated $\mathcal{C}^{\infty}$-rings of fractions $A\left\{\left(1-a_{1}\right)^{-1}\right\}$ or $A\left\{a_{1}^{-1}\right\}$. These squares are pullbacks in $\mathcal{C}^{\infty} \mathbf{R n g}_{\text {fp }}^{\text {op }}$, hence they are sent by the left-exact functor $\phi_{R}$ to pullbacks in $\mathcal{E}$, as in: 


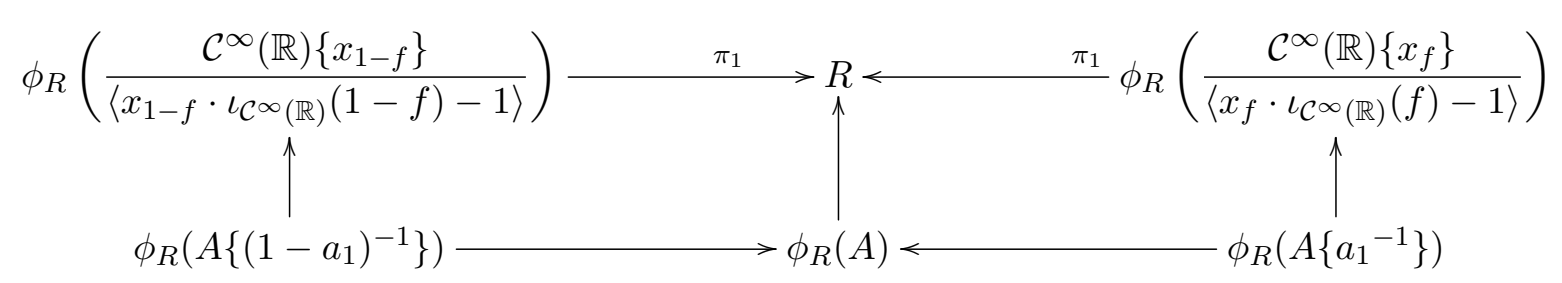

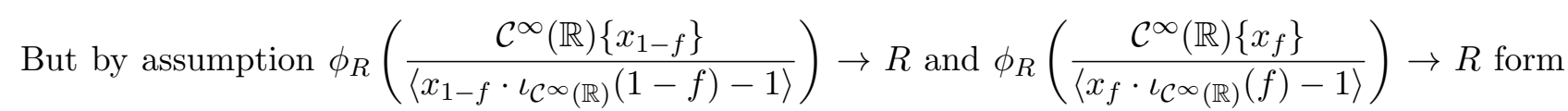
an epimorphic family in $\mathcal{E}$, and hence so does the pullback of this family. This proves (iii) for the case $n=2$.

The general case follows by induction. For instance, if $n=3$ and $a_{1}+a_{2}+a_{3}=1$, let $\beta \in A\left\{\left(a_{2}+a_{3}\right)^{-1}\right\}$ such that $\beta . \eta\left(a_{2}\right)+\beta . \eta\left(a_{3}\right)=1$. Then, again by the case $n=2, \phi_{R}$ sends the three arrows in $\mathcal{C}^{\infty} \mathbf{R n g}_{\mathrm{fp}}$

$$
\begin{gathered}
A \rightarrow A\left\{a_{1}^{-1}\right\} \\
A \rightarrow A\left\{a_{2}^{-1}\right\} \rightarrow A\left\{\left(a_{2}+a_{3}\right)^{-1}\right\}\left\{\left(\beta . \eta\left(a_{2}\right)\right)^{-1}\right\} \\
A \rightarrow A\left\{a_{3}^{-1}\right\} \rightarrow A\left\{\left(a_{2}+a_{3}\right)^{-1}\right\}\left\{\left(\beta . \eta\left(a_{3}\right)\right)^{-1}\right\}
\end{gathered}
$$

to an epimorphic family in $\mathcal{E}$. Thus $\phi_{R}$ also sends the family of canonical arrows $\left\{A \rightarrow A\left\{a_{i}^{-1}\right\}: i=1,2,3\right\}$ to an epimorphic family in $\mathcal{E}$.

Theorem 4.3.7. The smooth Grothendieck-Zariski topos $\mathcal{Z}^{\infty}=\mathrm{Sh}\left(\mathcal{C}^{\mathrm{op}}, J_{\mathrm{Cov}}\right)$, is a classifying topos for local $\mathcal{C}^{\infty}$-rings, i.e., for any Grothendieck topos $\mathcal{E}$, there is an equivalence of categories:

$$
\operatorname{Geom}\left(\mathcal{E}, \mathcal{Z}^{\infty}\right) \simeq \mathcal{C}^{\infty} \operatorname{LocRng}(\mathcal{E})
$$

where $\mathcal{C}^{\infty}$ LocRng $(\mathcal{E})$ is the category of local $\mathcal{C}^{\infty}$-ring-objects in $\mathcal{E}$.

The universal local $\mathcal{C}^{\infty}$-ring is the structure sheaf $\mathcal{O}$ of the Grothendieck-Zariski smooth topos (see Remark 4.3.4.

Proof. As a special case of the results on classifying topoi presented in the Section 1, there is an equivalence between $\operatorname{Geom}\left(\mathcal{E}, \mathcal{Z}^{\infty}\right)$ and the category of continuous left-exact functors from $\mathcal{C}^{\infty} \mathbf{R} \mathbf{n g}_{\mathrm{fp}}$ to $\mathcal{E}$.

This category is equivalent to the full subcategory $\mathcal{C}^{\infty} \operatorname{LocRng}(\mathcal{E})$ consisting of local $\mathcal{C}^{\infty}$-rings.

The identification of the universal local $\mathcal{C}^{\infty}$-ring is the object of $\mathcal{Z}^{\infty}$ represented by the object $\mathcal{C}^{\infty}(\mathbb{R})$ of the Grothendieck Zariski smooth site, this is precisely the structure sheaf (= forgetful functor) $\mathcal{O}: \mathcal{C}^{\infty} \mathbf{R n g}_{\mathrm{fp}} \rightarrow$ Sets.

\subsection{A Classifying Topos for the Theory of the von Neumann-regular $\mathcal{C}^{\infty}$-rings}

Now we turn to the problem of a classifying topos for the theory of von Neumann-regular $\mathcal{C}^{\infty}$-rings. As we have already defined, a von Neumann-regular $\mathcal{C}^{\infty}$-ring is a $\mathcal{C}^{\infty}$-rings $(A, \Phi)$ on which the first-order formula:

$$
\sigma=(\forall x \in A)(\exists ! y \in A)((x y x=x) \&(y x y=y))
$$


holds. Denoting by $\varphi(x, y):=((x y x=x) \&(y x y=y))$, we note that the formula:

$$
(\forall x \in A)(\exists ! y \in A) \varphi(x, y)
$$

defines a functional relation from $A$ to $A$, so we can define an unary functional symbol, $*$, and $\mathbb{L}^{\prime}=\mathbb{L} \cup\{*\}$

Let $\mathbb{T}_{\mathrm{vN}}$ be the theory of the von Neumann-regular $\mathcal{C}^{\infty}$-rings in the language $\mathbb{L}$ described at the beginning of the first section of the first chapter. We can define the unary functional symbol $*$ by means of the formula 4.12):

$$
\begin{aligned}
& \text { * }: A \rightarrow A \\
& x \mapsto y \text { s.t. } \varphi(x, y)
\end{aligned}
$$

in order to obtain a richer language, namely $\mathbb{L}^{\prime}=\mathbb{L} \cup\{*\}$.

Remark 4.4.1. Let $\mathbb{T}^{\prime}$ be the a theory in the language $\mathbb{L}^{\prime}=\mathbb{L} \cup\{*\}$, that contains:

- the (equational) $\mathbb{L}$-axioms for of $\mathcal{C}^{\infty}$-rings;

- the (equational) $\mathbb{L}^{\prime}$-axiom

$$
\sigma:=(\forall x)\left(\left(x x^{*} x=x\right) \&\left(x^{*} x x^{*}=x^{*}\right)\right)
$$

that is, $\mathbb{T}^{\prime}:=\mathbb{T} \cup\{\sigma\}$. By the Theorem of Extension by Definition (cf. Corollary 4.4.7 of [79]), we know that $\mathbb{T}^{\prime}$ is a conservative extension of $\mathbb{T}$.

Remark 4.4.2. (a) Note that in every von Neumann-regular $\mathcal{C}^{\infty}$-ring $V$, since $x^{*} x x^{*}=x^{*}$ holds for every $x \in V$, then $0^{*}=0$.

(b) If $F$ is a $\mathcal{C}^{\infty}$-field, thus a von Neumann-regular $\mathcal{C}^{\infty}$-ring, so

$$
F \models \sigma .
$$

Since $x x^{*} x=x$ holds for every $x \in F$, then if $x \neq 0$, we must have

$$
x^{*}=\frac{1}{x} .
$$

(c) In fact, the unary function * does not belong to the language $\mathbb{L}$, described in the first chapter.

We have seen that $\mathcal{C}^{\infty}\left(\mathbb{R}^{0}\right) \cong \mathbb{R}$, together with its canonical $\mathcal{C}^{\infty}$-structure $\Phi$, is a $\mathcal{C}^{\infty}$-field, thus a von Neumann-regular $\mathcal{C}^{\infty}$-ring, so

$$
\mathbb{R}=\sigma .
$$

Now, the function:

$$
\begin{aligned}
*: \mathbb{R} & \rightarrow \begin{cases}\frac{1}{x}, & \text { if } x \neq 0 \\
0, & \text { otherwise } .\end{cases}
\end{aligned}
$$

is defined by $\sigma$. However, there is no (continuous, and thus) smooth function $f: \mathbb{R} \rightarrow \mathbb{R}$ such that

$$
(\forall x \in \mathbb{R})\left(x^{*}=f(x)\right),
$$

that is,

$$
\left(\forall f \in \mathcal{C}^{\infty}(\mathbb{R}, \mathbb{R})\right)(\Phi(f) \neq *)
$$

so $*$ is not a symbol of the original language of $\mathcal{C}^{\infty}$-rings. 
Remark 4.4.3. (a) Since $\varphi(x, y)$ is a conjunction of two equations, the von Neumann-regular $\mathcal{C}^{\infty}$-homomorphisms preserve $*$, i.e.,

$$
(\forall x \in \mathbb{R})\left(h\left(x^{*}\right)=h(x)^{*}\right)
$$

whenever $(A, \Phi)$ and $(B, \Psi)$ are von Neumann-regular $\mathcal{C}^{\infty}$-rings and $h:(A, \Phi) \rightarrow(B, \Psi)$ is a von Neumann-regular $\mathcal{C}^{\infty}$-homomorphism.

(b) Since the $\mathbb{L}$-class of von Neumann-regular $\mathcal{C}^{\infty}$-rings is closed under quotients by $\mathcal{C}^{\infty}$-congruences and $\mathcal{C}^{\infty}$-congruences are classified by ideals, it follows from item (a) that for each von Neumann-regular $\mathcal{C}^{\infty}$-ring $V$ and any ideal $I \subseteq V$, then $x^{*}-y^{*} \in I$ whenever $x-y \in I$.

Definition 4.4.4. A finitely presented von Neumann regular $\mathcal{C}^{\infty}$-ring is a von Neumann-regular $\mathcal{C}^{\infty}$-ring $(V, \Phi)$ such that there is a finite set $X$ and an ideal $I \subseteq L(X)=\operatorname{vN}\left(\mathcal{C}^{\infty}\left(\mathbb{R}^{X}\right)\right)$ with:

$$
V \cong \frac{\mathrm{vN}\left(\mathcal{C}^{\infty}\left(\mathbb{R}^{X}\right)\right)}{I}
$$

Remark 4.4.5. $(V, \Phi)$ is a finitely presented von Neumann-regular $\mathcal{C}^{\infty}$-ring if, and only if, the representable functor:

$$
\operatorname{Hom}_{\mathcal{C}^{\infty} \mathbf{v N R n g}}(V, \bullet): \mathcal{C}^{\infty} \mathbf{v N R n g} \rightarrow \text { Set }
$$

preserves all directed colimits. That is to say that for every directed system of von Neumann-regular $\mathcal{C}^{\infty}$-rings $\left\{\left(V_{i}, \Phi_{i}\right), \nu_{i j}:\left(V_{i}, \Phi_{i}\right) \rightarrow\left(V_{j}, \Phi_{j}\right)\right\}_{i, j \in I}$ we have

\section{(cf. Proposition 3.8.14 of [6])}

$$
\operatorname{Hom}_{\mathcal{C}^{\infty}} \mathbf{v N R n g}\left(V, \underset{i \in I}{\lim _{i \in I}} V_{i}\right)=\underset{i \in I}{\lim _{i \in I}} \operatorname{Hom}_{\mathcal{C}}{ }_{\mathbf{v N R}}\left(V, V_{i}\right),
$$

Consider the category whose objects are all finitely presented von Neumann regular $\mathcal{C}^{\infty}$-ring and whose morphisms are the $\mathcal{C}^{\infty}$-homomorphisms between them, and denote it by $\mathcal{C}^{\infty} \mathbf{v N R n g}$ f.p.

Remark 4.4.6. We have:

$$
\operatorname{Obj}\left(\mathcal{C}^{\infty} \mathbf{R n g}_{\mathrm{fp}}\right) \cap \operatorname{Obj}\left(\mathcal{C}^{\infty} \mathbf{v N R n g}\right) \subseteq \mathcal{C}^{\infty} \mathbf{v N R n g}_{\mathrm{fp}} .
$$

Thus, keeping in mind the remarks above, and following the same line of the developments made in section 2 we obtain:

Theorem 4.4.7. The category

$$
\mathcal{C}^{\infty} \mathbf{v N R n g} \mathbf{f p}_{\mathrm{fp}}^{\text {op }}
$$

is a category with finite limits freely generated by the von Neumann regular $\mathcal{C}^{\infty}$-ring $\mathrm{vN}\left(\mathcal{C}^{\infty}(\mathbb{R})\right)$, i.e., for any category with finite limits $\mathcal{C}$, the evaluation of a left-exact functor $F: \mathcal{C}^{\infty} \mathbf{v N R} \mathbf{N n g}_{\mathrm{fp}}^{\mathrm{op}} \rightarrow \mathcal{C}$ at $\mathrm{vN}\left(\mathcal{C}^{\infty}(\mathbb{R})\right)$ yields the following equivalence of categories:

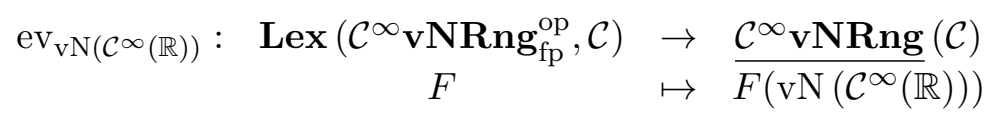

Combining the results presented in this section and the one stated in the section 1 on classifying topoi, we obtain the following:

Theorem 4.4.8. The presheaf topos $\mathbf{S e t} \mathbf{S}^{\mathcal{C}^{\infty} \mathbf{v N R n g}_{\mathrm{f}}}$ is a classifying topos for von Neumann regular $\mathcal{C}^{\infty}$-rings, and the universal von Neumann regular $\mathcal{C}^{\infty}$-ring $R$ is the von Neumann regular $\mathcal{C}^{\infty}-$ ring object in Sets $^{\mathcal{C}}{ }^{\infty} \mathbf{v R n g}_{\mathrm{fp}}$ that is given by $\mathcal{C}^{\infty} \mathbf{v N R n g}_{\mathrm{fp}}\left(\mathrm{vN}\left(\mathcal{C}^{\infty}(\mathbb{R}),-\right)\right)$, thus it is naturally isomorphic to the forgetful functor from $\mathcal{C}^{\infty} \mathbf{v N R n g}_{\mathrm{fp}}$ to Sets. Thus, for any Grothendieck topos $\mathcal{E}$ there is an equivalence of categories, natural in $\mathcal{E}$ :

$$
\begin{array}{ccc}
\operatorname{Geom}\left(\mathcal{E}, \text { Sets }^{\left.\mathcal{C}^{\infty} \mathbf{v N R n g}_{\mathrm{fp}}\right)}\right. & \rightarrow & \mathcal{C}^{\infty} \mathbf{v N R n g}(\mathcal{E}) \\
f & \mapsto & f^{*}(R)
\end{array}
$$




\section{Chapter 5}

\section{Conclusions and future works}

As already pointed out, $\mathcal{C}^{\infty}$-rings have been studied specially for their use in Singularity Theory and in order to construct topos models for Synthetic Differential Geometry. In this work, we have developed a complementary trail, deepening the knowledge about $\mathcal{C}^{\infty}$-rings through an abstract approach, making use of Category Theory and accounting them from an algebraic and logical-categorial viewpoint. Of course, we have just scratched the surface of the theme of this thesis - algebraic and logical aspects of $\mathcal{C}^{\infty}$-rings - but we modestly hope to have achieved some new results and improved some other constructions and results, providing solid basis for future works on this wide subject. In the following, we describe some possibilities and new directions that we have devised through the development of this thesis and that we intend to address in the future.

Despite being a very useful tool for many branches of Mathematics, it is difficult to find a detailed and systematic presentation of the fundamental facts about $\mathcal{C}^{\infty}$-rings (the outstanding book of I. Moerdijk and G. Reyes, [62], brings an excellent account on the main facts about $\mathcal{C}^{\infty}$-rings, but from a more sophisticated viewpoint). We have dedicated Chapter 1 of this work to describe, in an universal-algebraic fashion, the (algebraic) category of $\mathcal{C}^{\infty}$-rings, $\mathcal{C}^{\infty} \mathbf{R n g}$, and some of the constructions one can perform in it, and we state and prove many (almost folkloric) results about them, whose demonstrations could not be found, up to this point, anywhere in the literature - and in some cases, not even a sketch of the proof was pointed out. We have presented the main constructions needed in the subsequent chapters (that contain new results), providing also technical support for a beginner in this subject. As an original contribution, we describe explicitly comparisons -more precisely, pairs of adjoint functors- between (the category of) rings (respectively, $\mathbb{R}$-algebras) and (the category of) $\mathcal{C}^{\infty}$-rings.

Algebraic Geometry and Commutative Algebra have a strong interplay, to the extent that commutative rings and varieties hold many useful and important relations. The same is true for Differential Geometry and a smooth version of Commutative Algebra - the commutative algebra of $\mathcal{C}^{\infty}$-rings, that we call "Smooth Commutative Algebra". The "Algebraic Geometry" of $\mathcal{C}^{\infty}$-rings was addressed by E. Dubuc in the 1980s in [16], but only in the past five years rose again the interest on the development of this subject (but aiming mainly its applications on modern Differential Geometry) by the hand of D. Joyce $([29],[30])$. In the mid of 1980s, I. Moerdijk ang G. Reyes have developed ([66], [67]) some constructions and results on "Smooth Commutative Algebra". In our Chapter 2:

(i) we have expanded and generalized notions and results on "Smooth Commutative Algebra", presented in [66 and 67]: we analyze and study the analogous "smooth" notions to those of "ring of fractions" $\left(\mathcal{C}^{\infty}\right.$-ring of fraction), "fields/domains/local rings" $\left(\mathcal{C}^{\infty}\right.$-fields/domains/local rings), "radical ideals" $\left(\mathcal{C}^{\infty}\right.$-radical ideal), "reduced rings" $\left(\mathcal{C}^{\infty}\right.$-reduced $\mathcal{C}^{\infty}$-rings $)$, "(Zariski) prime spectrum" $\left(\mathcal{C}^{\infty}\right.$ Zariski spectrum); 
(ii) we have introduced and explored new notions $\left(\mathcal{C}^{\infty}\right.$-saturated subset, $\mathcal{C}^{\infty}$-real spectrum, $\mathcal{C}^{\infty}$-von Neumann ring) and presented new results: separation theorems in the $\mathcal{C}^{\infty}$-setting, a continuous bijection supp ${ }^{\infty}$ between the $\mathcal{C}^{\infty}$-real spectrum and $\mathcal{C}^{\infty}$-prime spectrum, and some comparisons between "Commutative Algebra" and "Smooth Commutative Algebra".

In the vein of the program of developing "Smooth Commutative Algebra", we intend to address in the future:

(I) Analyze special classes of $\mathcal{C}^{\infty}$-rings.

We intend to consider at least two relevant classes: (a) the class of Archimedean $\mathcal{C}^{\infty}$-rings (considered in [16], [67], [30]): for a local $\mathcal{C}^{\infty}$-ring it is equivalent to require that its residual $\mathcal{C}^{\infty}$-field is isomorphic to $\mathbb{R}$; (b) the class of $\mathcal{C}^{\infty}$-Gelfand rings (i.e., a $\mathcal{C}^{\infty}$-rings $A$ where for each pair of distinct maximal ideals $M_{0} \neq M_{1}$ there are $f_{i} \in M_{i}$ such that $\left.f_{0} \cdot f_{1}=0\right)$.

(II) Develop Real Algebraic Geometry and Quadratic Form Theory of $\mathcal{C}^{\infty}$-rings.

We have started a complementary program to [66], analysing order-theoretic questions on $\mathcal{C}^{\infty}$-rings by the introduction of the $\mathcal{C}^{\infty}$-real spectrum functor $\left(\mathrm{Sper}^{\infty}\right)$, getting a interesting description of it in terms of the functor $\mathrm{Spec}^{\infty}$. This is an(other) evidence that the Real Algebraic Geometry and the Quadratic Form Theory of a $\mathcal{C}^{\infty}$-rings should be interesting. We intend: (a) characterize the $\mathcal{C}^{\infty}$-rings that are Super Real Closed ([78]); (b) analyze if $\mathcal{C}^{\infty}$-rings are Faithfully Quadratic Rings $([\overline{13}])$ and, in particular, they support a natural structure of Special Group $\left([89)\right.$; (c) to study whether $\overline{\mathcal{C}}^{\infty}$-rings determine an Abstract Real Spectra $([53])$ or, dually, if they encode an interesting class of Real Semigroups $([12])$.

The central notion of a $\mathcal{C}^{\infty}$-spectrum of a $\mathcal{C}^{\infty}$-ring was introduced by I. Moerdijk and G. Reyes in [63, about which they make a more detailed exposition in subsequent papers ([67] and [65]). We have presented in Chapter 3 a explicit description of the smooth Zariski topology together with a full proof that the $\mathcal{C}^{\infty}$-spectrum is a spectral space with a distinguished property: unlike the traditional Zariski topology of Commutative algebra, the "natural basis" of the topology of $\mathcal{C}^{\infty}$-spectrum already contains all compact-open subsets. The structural sheaf of a $\mathcal{C}^{\infty}$-ring defined over its $\mathcal{C}^{\infty}$-spectrum has a behavior analogous to the "algebraic structural sheaf of a ring": each $\mathcal{C}^{\infty}$-ring is isomorphic to the global section of its structural sheaf. As an application of sheaf-theoretic ideas in $\mathcal{C}^{\infty}$-ring theory, we obtain some interesting results concerning the notion of von Neumann regular $\mathcal{C}^{\infty}$-ring - a concept introduced in the previous chapter, that has many equivalent descriptions. A deeper analysis of their properties is introduced in Chapter 3, together with a categorial and logical treatment for them. In particular, the subcategory of $\mathcal{C}^{\infty} \mathbf{R n g}$ consisting of all von Neumann regular $\mathcal{C}^{\infty}$-rings is characterized as the closure under small limits of the category of $\mathcal{C}^{\infty}$-fields, i.e., it is the smallest subcategory of $\mathcal{C}^{\infty}$ Rng which contain all $\mathcal{C}^{\infty}$-fields and is closed under small limits. Moreover, it was proven, by two different methods (with a sheaf-theoretic one), that the subcategory of von Neumann regular $\mathcal{C}^{\infty}$-rings is reflective in the category of all $\mathcal{C}^{\infty}$-rings, so each $\mathcal{C}^{\infty}$-ring as a von Neumann regular $\mathcal{C}^{\infty}$-ring hull. We also apply von Neumann regular $\mathcal{C}^{\infty}$-ring to naturally represent Boolean Algebras in a strong sense: i.e., not only all Boolean algebras are isomorphic to the Boolean algebra of idempotents of a von Neumann regular $\mathcal{C}^{\infty}$-ring, as every homomorphism between such boolean algebras of idempotents is induced by a $\mathcal{C}^{\infty}$-homomorphism.

Von Neumann $\left(\mathcal{C}^{\infty}-\right)$ rings are known as "intuitionistic $\left(\mathcal{C}^{\infty}-\right)$ fields": they are precisely the $\left(\mathcal{C}^{\infty}-\right)$ rings of global sections of a sheaf of a locally $\left(\mathcal{C}^{\infty}-\right)$ ringed space over a Boolean space, whose stalks are $\left(\mathcal{C}^{\infty}-\right)$ fields. An analogous characterization holds for the class of (algebraic) Gelfand rings: they are the global section of locally ringed space over a compact Hausdorff space. 
(III) Is there a similar characterization of $\mathcal{C}^{\infty}$-Gelfand rings?

(IV) If this is true, can we obtain from that, by sheaf theoretic methods, a $\left(\mathcal{C}^{\infty}-\right)$ Gelfand hull for each $\left(\mathcal{C}^{\infty}-\right)$ ring?

(V) Can we apply these ideas and methods to the case of sheaf of $\mathcal{C}^{\infty}$-rings whose stalks are Archimedean local rings (see [67, 29])?

(VI) And in the vein of Grothendieck topologies, are there natural and useful versions of étale ([23]) and/or real-étale $\left([\overline{73]})\right.$ sites in the setting of $\mathcal{C}^{\infty}$-rings?

These are questions that we intend to address as a sequel of the present work.

In Chapter 4 we have developed the first results on the categorial-logic aspects of the theory of $\mathcal{C}^{\infty}$-rings. We have started providing a compendium of the main categorial-logical notions needed in the chapter. In the sequel we have described in details two results that are just stated (without any sketch of proof) in 62] : the classifying topos for the theory of $\mathcal{C}^{\infty}$-rings is the presheaf topos over the left-exact (essentially small category) $\left(\mathcal{C}^{\infty} \mathbf{R n g}\right)_{\mathrm{fp}}$ and the classifying topos for the theory of local $\mathcal{C}^{\infty}$-rings is the "smooth Zariski Topos", the category of sheaves of a (subcanonical, Zariski) Grothendieck topology on the dual category of all finitely presentable $\mathcal{C}^{\infty}$-rings. Finishing this work, we have described the

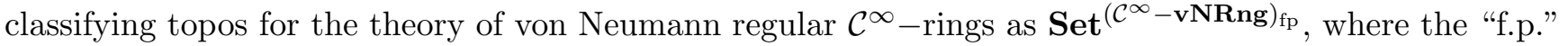
here refers to the strictly stronger language that contains symbols for all $\mathcal{C}^{\infty}$ functions and an additional unary function symbol to represent the unique quasi-inverse of an element of a von Neumann regular $\mathcal{C}^{\infty}$-ring.

In 67 the authors present classifying topos for the geometric theory of Archimedean $\mathcal{C}^{\infty}$-ring. This reinforce the questions:

(VII) Are there other sensible descriptions of classifying toposes for other distinguished classes of $\mathcal{C}^{\infty}$-rings?

(VIII) In particular, is there a nice description of the theory of von Neumann regular $\mathcal{C}^{\infty}$-rings in the language of $\mathcal{C}^{\infty}$-rings?

Another logical aspect of the theory of $\mathcal{C}^{\infty}$-rings should be considered in the future: the traditional first-order model-theoretical analysis. We believe that are nice clues that this is well behaved and will provide a better understanding (besides new results) on the subject. We briefly sketch the main lines that we have devised during the development of the this thesis:

In 66] it is proven that each $\mathcal{C}^{\infty}$-field is real closed (Theorem 2.10) and, moreover, they satisfy a stronger property of being $\mathcal{C}^{\infty}$-real closed (Theorem 2.10').

(IX) It is natural to ask if the class of $\mathcal{C}^{\infty}$-fields is model-complete in the language of $\mathcal{C}^{\infty}$-rings or even admits elimination of quantifiers (possibly in the language expanded by a unary predicate for the positive cone of a ordering). If the former holds, then the relation $\mathcal{R}$ between pairs of morphism with the same source and target $\mathcal{C}^{\infty}$-fields, considered in Section $\mathbf{5}$ of Chapter 2, that encodes $\mathrm{Sper}^{\infty}$, is already a transitive relation (as occurs in the algebraic case). If the latter holds, then it is possible to adapt the definition and results provided in [72] on "Model-theoretic Spectra" and describe "logically" the spectral topological spaces $\operatorname{Spec}^{\infty}(A)$ and/or $\operatorname{Sper}^{\infty}(A)$ as certain classes of equivalence of homomorphisms of $A$ 
into models of a nice theory $T$. Moreover, since the techniques in this paper provide structural sheaves of "definable functions", we could compare them with others previously defined and determine other new natural model-theoretic spectra in $\mathcal{C}^{\infty}$-structures.

(X) After a good understanding of the model-theory of distinguished classes of $\mathcal{C}^{\infty}$-rings, it is natural consider model-theoretic aspects of simple constructions/structures related to the $\mathcal{C}^{\infty}$ setting. In particular, the class of modules over $\mathcal{C}^{\infty}$-rings, that has been considered by D. Joyce in the recent development of the Algebraic Geometry of $\mathcal{C}^{\infty}$-rings $([30],[29])$, should be better studied. It will be interesting to start the model-theoretic analysis of Modules over Finitely Generated $\mathcal{C}^{\infty}$-rings in the vein of [33]: by defining a 2 sorted language that contains axioms for a $\mathcal{C}^{\infty}$-ring part and a module of it and (at least) symbols for the Grassmannians to express linear (in)dependence without the need of quantifiers.

(XI) Finally there is also a mathematical aspect of $\mathcal{C}^{\infty}$-rings that seems to have been only laterally considered: since each free $\mathcal{C}^{\infty}$-ring $\mathcal{C}^{\infty}\left(\mathbb{R}^{X}\right)$ encodes many possible $\mathbb{R}$-derivations, then every "smooth polynomial ring" $A\{X\}$ in the set of variables $X$ with coefficients over a $\mathcal{C}^{\infty}$ - ring $A$ admits many $A$ derivations. Thus classes of $\mathcal{C}^{\infty}$-rings endowed with derivations should be interesting and deserve a systematic study under many aspects, including the model-theoretic one. 


\section{Compendium}

For the reader's convenience we record here some results on Smooth Analysis that were used along this work, together with their proofs. In each case we indicate references in which one finds them.

The first result we present is Hadamard's Lemma, which allows us to prove that the $\mathcal{C}^{\infty}$-congruences of a $\mathcal{C}^{\infty}$-ring are classified by their ring-theoretic ideals.

Theorem 5.0.1. (Hadamard's Lemma)For every smooth function $f \in \mathcal{C}^{\infty}\left(\mathbb{R}^{n}\right)$ there are smooth functions $g_{1}, \cdots, g_{n} \in \mathcal{C}^{\infty}\left(\mathbb{R}^{2 n}\right)$ such that $\forall\left(x_{1}, \cdots, x_{n}\right),\left(y_{1}, \cdots, y_{n}\right) \in \mathbb{R}^{n}$ :

$$
f\left(x_{1}, \cdots, x_{n}\right)-f\left(y_{1}, \cdots, y_{n}\right)=\sum_{i=1}^{n}\left(x_{i}-y_{i}\right) \cdot g_{i}\left(x_{1}, \cdots, x_{n}, y_{1}, \cdots, y_{n}\right)
$$

Proof. Holding $\left(v_{1}, \cdots, v_{n}\right)=\vec{v} \in \mathbb{R}^{n}$ fixed, we define:

$$
h(t)=f(\vec{y}+t \cdot(\vec{x}-\vec{y})) .
$$

We have:

$$
\left.f(\vec{x})-f(\vec{y})=\int_{0}^{1} h^{\prime}(t) d t=\int_{0}^{1} \sum_{i=1}^{n} \frac{\partial f}{\partial v_{i}}(\vec{y}+t \cdot(\vec{x}-\vec{y}))\right) \cdot\left(x_{i}-y_{i}\right) d t,
$$

where the second equality uses the chain rule.

The result follows by putting:

$$
g_{i}(\vec{x}, \vec{y})=\int_{0}^{1} \frac{\partial f}{\partial v_{i}}(\vec{y}+t \cdot(\vec{x}-\vec{y})) d t
$$

The following lemma grants us the existence of convenient bump functions, that are used to prove the next theorem.

Lemma 5.0.2. Given any $\varepsilon>0$, there is a smooth function, $r_{\varepsilon}: \mathbb{R} \rightarrow[0, \infty[$ such that:

$$
\begin{cases}0, & \text { if }|x| \geq \varepsilon \\ >0, & \text { otherwise. }\end{cases}
$$

Proof. It suffices to consider:

$$
\begin{aligned}
r_{\varepsilon}: \mathbb{R} & \rightarrow \begin{cases}e^{-\frac{1}{1-\left(\frac{x}{\varepsilon}\right)^{2}}}, & \text { if }|x|<\varepsilon \\
0, & \text { otherwise. }\end{cases}
\end{aligned}
$$


We find the following important result, together with its proof, in 28 .

Theorem 5.0.3. Let $\varphi \in \mathcal{C}^{\infty}\left(\mathbb{R}^{n}\right)$ and $U_{\varphi}=\left\{x \in \mathbb{R}^{n} \mid \varphi(x) \neq 0\right\}=\operatorname{Coz}(\varphi)$. Then:

$$
\mathcal{C}^{\infty}\left(U_{\varphi}\right) \cong \frac{\mathcal{C}^{\infty}\left(\mathbb{R}^{n+1}\right)}{\langle\{y \cdot \varphi(x)-1\}\rangle}
$$

Proof. Let:

$$
\begin{aligned}
& \rho: \mathcal{C}^{\infty}\left(\mathbb{R}^{n+1}\right) \rightarrow \quad \mathcal{C}^{\infty}\left(U_{\varphi}\right) \\
& \begin{aligned}
f \quad \mapsto(f): U_{\varphi} & \rightarrow \\
x & \mapsto f\left(x, \frac{1}{\varphi(x)}\right)
\end{aligned}
\end{aligned}
$$

We claim that $\rho$ is surjective, i.e., any $h \in \mathcal{C}^{\infty}\left(U_{\varphi}\right)$ may be lifted to some $f \in \mathcal{C}^{\infty}\left(\mathbb{R}^{n+1}\right)$.

Let $h \in \mathcal{C}^{\infty}\left(U_{\varphi}\right)$. Define, for $\varepsilon>0$ :

$$
\begin{aligned}
f: \mathbb{R}^{n+1} & \rightarrow \\
(x, y) & \mapsto \begin{cases}r_{\varepsilon}\left(y-\frac{1}{\varphi(x)}\right) \cdot h(x), & \text { if } x \in U_{\varphi} \\
0, & \text { if }|y \cdot \varphi(x)-1|>\varepsilon \cdot|\varphi(x)| .\end{cases}
\end{aligned}
$$

Using the fact that $\mathbb{R}$ is an ordered local ring, one easily checks that $f \in \mathcal{C}^{\infty}\left(\mathbb{R}^{n+1}\right)$. Indeed, either $|\varphi(x)|<\frac{1}{\varepsilon+|y|}$ and hence $|y \cdot \varphi(x)-1|>\varepsilon \cdot|\varphi(x)|$ or $|\varphi(x)|>\frac{1}{2(\varepsilon+|y|)}$, which implies that $\varphi(x)$ is invertible. Clearly,

$$
\left(\forall x \in U_{\varphi}\right)\left(h(x)=f\left(x, \frac{1}{\varphi(x)}\right)\right)
$$

Assume now that $f \in \operatorname{ker}(\rho)$. Using Hadamard's Lemma for $f \in \mathcal{C}^{\infty}\left(\mathbb{R}^{n} \times \mathbb{R}, \mathbb{R}\right)$, we have:

$$
f(x, t)-f(x, s)=(t-s) \cdot \int_{0}^{1} \frac{\partial f}{\partial t}(x, s+(t-s) \cdot u) d u
$$

we conclude the existence of some $f_{1} \in \mathcal{C}^{\infty}\left(\mathbb{R}^{n+2}\right)$ such that:

$$
f(x, y)-f\left(x, \frac{1}{\varphi(x)}\right)=\left(y-\frac{1}{\varphi(x)}\right) \cdot f_{i}\left(x, y, \frac{1}{\varphi(x)}\right) .
$$

Define:

$$
\begin{aligned}
\nu: \mathbb{R}^{n+1} & \rightarrow \begin{cases}\frac{f(x, y)}{y \cdot \varphi(x)-1}, & \text { if } y \cdot \varphi(x)-1 \neq 0 \\
\frac{f_{1}\left(x, y, \frac{1}{\varphi(x)}\right)}{\varphi(x)}, & \text { if } \varphi(x) \neq 0 .\end{cases}
\end{aligned}
$$

Once again, it is easily checked that $\nu \in \mathcal{C}^{\infty}\left(\mathbb{R}^{n+1}\right)$. Therefore, $f(x, y)=\nu(x, y) \cdot(y \cdot \varphi(x)-1) \in$ $\langle\{y \cdot \varphi(x)-1\}\rangle$. 
Lemma 5.0.4. Let $F \subseteq U \subseteq \mathbb{R}^{n}$ with $F$ closed and $U$ open. Then there exists a smooth function $\psi: U \rightarrow \mathbb{R}$ such that $F=\psi^{-1}[\{0\}]$.

Proof. Since $\mathbb{R}^{n}$ is a Lindelöf space and since the open balls are a basis for the topology of $\mathbb{R}^{n}$, we can write the open set $A \backslash F$ as a countable union of open balls, $A \backslash F=\bigcup_{i=1}^{\infty} B\left(q_{i}, r_{i}\right)$. We construct smooth functions $\psi_{i}: U \rightarrow[0, \infty[$ such that:

(a) $\left(\forall x \in B\left(q_{i}, r_{i}\right)\right)\left(\psi_{i}(x)>0\right)$

(b) $\psi_{i}$ and all its derivatives up to order $i$ are uniformly bounded by $\frac{1}{2^{i}}$, that is:

$$
(\forall m \leq i)\left(\left\|\psi_{i}^{(m)}\right\|_{\infty} \leq \frac{1}{2^{i}}\right)
$$

Consider the function:

$$
\begin{aligned}
F: \mathbb{R} & \rightarrow \begin{array}{ll}
e^{-\frac{1}{(x-1)^{2}}} \cdot e^{-\frac{1}{(x+1)^{2}}}, & \text { if }|x|<1 \\
0, & \text { otherwise. }
\end{array}
\end{aligned}
$$

and define:

$$
\begin{array}{rllc}
\psi_{i}: & U & \rightarrow & {[0, \infty[} \\
x & \mapsto & F\left(\frac{\left|x-q_{i}\right|}{r_{i}}\right)
\end{array}
$$

Since $\psi_{i}$ and all its derivatives are bounded, the condition (b) can be enforced by multiplying each $\psi_{i}$ by a sufficiently small positive number. We now write:

$$
\begin{array}{rlrc}
\psi: & \rightarrow & {[0, \infty[} \\
x & \mapsto & \sum_{i=1}^{n} \psi_{i}(x)
\end{array}
$$

In view of:

$$
(\forall m \leq i)\left(\left\|\psi_{i}^{(m)}\right\|_{\infty} \leq \frac{1}{2^{i}}\right)
$$

we have:

$$
\left|\sum_{i=1}^{\infty} \psi_{i}^{(m)}\right|=\left|\sum_{i=1}^{m-1} \psi_{i}^{(m)}+\sum_{i=m}^{\infty} \psi_{i}^{(m)}\right| \leq\left|\sum_{i=1}^{m-1} \psi_{i}^{(m)}\right|+\left|\sum_{i=m}^{\infty} \psi_{i}^{(m)}\right| \leq\left|\sum_{i=1}^{m-1} \psi_{i}^{(m)}\right|+\sum_{i=m}^{\infty} \frac{1}{2^{i}}
$$

thus the sum converges uniformly in $U$, so $\psi$ is a smooth function. Also, in view of (a), we have $\psi(x)>0 \Longleftrightarrow x \in B\left(q_{i}, r_{i}\right)$ for some $i$, thus $\psi(x)>0$ if $x \notin F$.

The following theorem was first proved by H. Whitney (usually known as Whitney's Theorem), and it plays an important role in this work. The proof we present is an adaptation of the one we have found in [113].

Theorem 5.0.5. (Whitney's Theorem) Let $n \in \mathbb{N}$ and let $F \subseteq \mathbb{R}^{n}$ be a closed set. Then there is some $f \in \mathcal{C}^{\infty}\left(\mathbb{R}^{n}, \mathbb{R}\right)$ such that:

$$
F=Z(f)=f^{\dashv}[\{0\}] .
$$

Dually, every open subset $U \subseteq \mathbb{R}^{n}$ admits a "smooth characteristic function":

$$
U=\operatorname{Coz}(f)=f^{-1}\left[\mathbb{R}^{n} \backslash\{0\}\right] .
$$


Proof. Since $\mathbb{R}^{n}$ is a Lindelöf space, we can choose a locally finite open cover, $\mathcal{U}=\left\{U_{i} \mid i \in I\right\}$ for $\mathbb{R}^{n}$. Let $\left(\lambda_{i}\right)_{i \in I}$ be a partition of unit such that:

$$
(\forall i \in I)\left(\operatorname{supp}\left(\lambda_{i}\right) \subseteq U_{i}\right)
$$

Then $F \cap \operatorname{supp}\left(\lambda_{i}\right)$ is closed in $U_{i}$. By Lemma 5.0.4 there exists, for each $i \in I$, a function $\psi_{i}: U_{i} \rightarrow\left[0, \infty\left[\right.\right.$ such that $\psi_{i}(x)=0 \Longleftrightarrow x \in F \cap \operatorname{supp}\left(\lambda_{i}\right)$. We extend $\psi_{i}$ by declaring it to be zero if $x \in \mathbb{R}^{n} \backslash U_{i}$. We define:

$$
\begin{array}{rlc}
f: \mathbb{R}^{n} & \rightarrow & {[0, \infty[} \\
x & \mapsto & \sum_{i \in I} \lambda_{i}(x) \cdot \psi_{i}(x)
\end{array}
$$

and this is well defined since the partition is locally finite. If $x \in F$, then $\psi_{i}(x)=0$ for every $i \in I$, thus $f(x)=0$. If $x \notin F$, then $\psi_{i_{0}}(x)>0$ for some $i_{0} \in I$, so $x \notin F \cap \operatorname{supp}\left(\psi_{i}\right)$. Thus, $\psi_{i}(x)>0$ and $f(x) \geq \lambda_{i_{0}}(x) \cdot \psi_{i_{0}}(x)>0$.

The following result (Theorem of Ortega \& Muñoz), together with its proof can be found in [66].

Theorem 5.0.6. (Ortega \& Muñoz)Let $U \subseteq \mathbb{R}^{n}$ be open, and $g \in \mathcal{C}^{\infty}(U)$. Then there are $h, k \in$ $\mathcal{C}^{\infty}\left(\mathbb{R}^{n}\right)$ with $\operatorname{Coz}(k)=U_{k}=U$ and $g \cdot k \uparrow_{U}=h \uparrow_{U}$.

Proof. Let $\left(\alpha_{n}\right)_{n \in \mathbb{N}}$ be a sequence of smooth functions such that Cl. $\left(U_{\alpha_{n}}\right) \subseteq U$ for each $n \in \mathbb{N}$ and such that $U=\bigcup_{n \in \mathbb{N}} U_{\alpha_{n}}$.

Define

$$
\begin{aligned}
g_{n}: \mathbb{R}^{n} & \rightarrow \mathbb{R} \\
x & \mapsto \begin{cases}\alpha_{n}(x) \cdot g(x), & \text { if } x \notin U \\
0, & \text { if } x \in U .\end{cases}
\end{aligned}
$$

Now, if $\left(p_{n}\right)_{n \in \mathbb{N}}$ is an increasing sequence of seminorms defining the Fréchet topology on $\mathcal{C}^{\infty}\left(\mathbb{R}^{n}\right)$, we can put:

$$
h(x)=\sum_{n=0}^{\infty} \frac{g_{n}(x)}{2^{n} \cdot\left(1+p_{n}\left(\alpha_{n}\right)\right) \cdot\left(1+p_{n}\left(g_{n}\right)\right)}
$$

and

$$
k(x)=\sum_{n=0}^{\infty} \frac{\alpha_{n}(x)}{2^{n} \cdot\left(1+p_{n}\left(\alpha_{n}\right)\right) \cdot\left(1+p_{n}\left(g_{n}\right)\right)} .
$$




\section{Bibliography}

[1] M. A. Arbib, E. G. Manes, Arrows, structures, and functors - The Categorical Imperative, Academic Press, 1975.

[2] P. Arndt, H. L. Mariano, The von Neumann-regular Hull of (preordered) rings and quadratic forms, South American Journal of Logic, Vol. 2, n. 2, 2016, pp. 201-244.

[3] M. F. Atiyah, I. G. MacDonald, Introduction to Commutative Algebra, Addison-Wesley Series in Mathematics, Westview Press, 1969.

[4] L. Belair, G. Reyes, Calcul Infinitesimal en Geométrie Différentielle Synthétique, Sydney Category Seminar Reports, 1982, 2pp.

[5] F. Borceux, Handbook of Categorical Algebra, 1, Basic Category Theory, Cambridge University Press, 1994.

[6] F. Borceux, Handbook of Categorical Algebra, 2, Categories and Structures, Cambridge University Press, 1994.

[7] F. Borceux, Handbook of Categorical Algebra, 3, Categories of Sheaves., Cambridge University Press, 1994.

[8] M. Bunge, Synthetic Aspects of $\mathcal{C}^{\infty}$-mappings, Journal of Pure and Applied Algebra 28 (1983) 41-63, North-Holland Publishing Company 41.

[9] M. Bunge, E. Dubuc, Archimedian Local $\mathcal{C}^{\infty}$-rings and Models of SDG Cahiers de Topologie et Géometrie Différentielle Catégoriques, vol 27(3), 1986, 3-22.

[10] M. Bunge, E. Dubuc, Local concepts in synthetic differential geometry and germ representability, in "Mathematical Logic and Theoretical Computer Sciences", D. Kueher, E.G.K. Lopez-Escobar and C. Smith eds., Dekker, New York, 1987, pp. 39-158.

[11] M. Bunge, F. Gago, Synthetic aspects of $\mathcal{C}^{\infty}$-mappings II: Mather's theorem for infinitesimally represented germs, J. Pure Appl. Algebra 55 (1988), 213-250.

[12] M. Dickmann, A. Petrovich, Real Semigroups and Abstract Real Spectra, Contemporary Mathematics AMS 344 (2004), 99-119.

[13] M. Dickmann, F. Miraglia, Faithfully Quadratic Rings, Memoirs of the American Mathematical Society, vol. 238, nov. 2015

[14] M. Dickmann, F. Miraglia, Quadratic Form Theory over preordered von Neumann-regular rings, Journal of Algebra 319(4) (2008), 946-971.

[15] Druck, I., Un modèle de filtres pour l'analyse réelle synthétique Cahiers de Topologie et Géométrie Différentielle Catégoriques, Volume 34 (1993) no. 2 , p. 83-120.

[16] E. Dubuc, $\mathcal{C}^{\infty}$-schemes,American Jounal Math. vol 103, n4, 683-690, 1981

[17] E. Dubuc, Sur les Modèles de la Géométrie Différentielle Synthétique, Cahiers de Topologie et Géométrie Différentielle Catégoriques, Volume 20 (1979) no. 3 , p. 231-279. Zbl 0473.18008 - MR 557083

[18] E. Dubuc, Open Covers and Infinitary Operations in $\mathcal{C}^{\infty}$-rings, Cahiers de Topologie et Géométrie Différentielle Catégoriques, vol 22(3), 1981, 287-300.

[19] E. Dubuc, G. Reyes, Subtoposes of the ring classifier, in [35], 101-122.

[20] J. Dugundu, Topology, Allyn and Bacon, Inc.; 1st edition (1966).

[21] C. Ehresmann, Oeuvres completes et commentées 1.1 et 1.2, Supplements 1 et 2 au vol. XXIV des Cahiers de Topologie et Geométrie Differentielle, (1983).

[22] Y. Felix, R. Lavendhome, On De Rham's theorem in synthetic differential geometry, J. Pure Appl. Algebra 69 (1990),21- 31.

[23] A. Grothendieck, J. Dieudonné, Élements de Géométrie Algebrique, Inst. Hautes Et. Sc., publ. math. nOS 4, 8, 11, 17,20, 24, 28, 32 (de 1960 a 1967).

[24] M. Hochster, Prime Ideal Structure in Commutative Rings, Trans. Amer. Math. Soc. 142 (1969), 43-60, August 5 1968.

[25] A. Javanpeykar, Locally Ringed Spaces and Affinne Schemes, Leiden University, February 282011.

[26] P. Johnstone, Topos theory, Academic Press, 1977. 
[27] P. Johnstone, Rings, Fields and Spectra, Journal of Algebra 49, 1977.

[28] A. Joyal, G. Reyes, Separably Closed Local Rings, Journal of Pure and Applied Algebra 43 (1986) $271-279$.

[29] D. Joyce, Algebraic Geometry over $\mathcal{C}^{\infty}$-rings, 2016. arXiv:1001.0023v7[math.AG] 1 Nov 2016, http://arxiv.org/ pdf/1001.0023.pdf

[30] D. JoyCE, An introduction to $\mathcal{C}^{\infty}$-schemes and $\mathcal{C}^{\infty}$-algebraic geometry, arXiv:1104.4951v2 [math.DG], 16 Nov 2012.

[31] G. Kainz, A. KriedL, P. Michor , $\mathcal{C}^{\infty}$-algebras from the functional analytic viewpoint, Journal of Pure and Applied Algebra 46 (1987) 89-107, 1973, North Holland.

[32] E. Kalashnikov, $\mathcal{C}^{\infty}$-Algebraic Geometry, Linacre College University of Oxford. A thesis submitted for the degree of MSc in Mathematics and the Foundations of Computer Science, Sept 1, 2014.

[33] M. Kamensky, The Model Completion of the Theory of Modules over Finitely Generated Commutative Algebras, The Journal of Symbolic Logic, Volume 74, Number 3, Sept. 2009, 734-750, arXiv:math/0607418v2 [math.LO], 20 Mar 2007.

[34] J. F. Kennison, Integral Domain Type Representations in Sheaves and Other Topoi, Math. Z. 151 (1976), 35-56.

[35] A. Kock (ED), Topos theoretic Methods in Geometry, Various Publications Series naO, Aarhus, 1979.

[36] A. Kock, Synthetic Differential Geometry, Cambridge University Press, 2nd edition, 2006.

[37] A. Kock, Synthetic Characterization of Reduced Algebras, Journal of Pure and Applied Algebra 36 (1985) 273 - 279, North Holland.

[38] A. Kock (ED), Category theoretic Methods in Geometry, edited by A.Kock, Various Publications Series n35, Aarhus, 1983.

[39] A. Kock, Taylor Series calculus for ring objects of line type, Journ. Pure Appl. Alg. 18 (1978), 271-293.

[40] A. Kock, Differential forms in synthetic differential geometry, Aarhus Preprint Series n281978/79), llpp.

[41] A. Kock, Properties of well adapted models for synthetic differential geometry, Journ. Pure Appl. Alg. 20 (1981), 55-70.

[42] A. Kock, G. Reyes, Models for synthetic integration theory, Math. Scand. 48 (1981), 145-152.

[43] A. Kock, G. Reyes, B. Veit, Forms and integration in synthetic differential geometry, Aarhus Preprint Series, n31 $(1979 / 80)$.

[44] A. Kock, Synthetic Geometry of Manifolds-Cambridge University Press (2009), Cambridge Tracts in Mathematics Vol. 180, Cambridge University Press.

[45] R. P. Kostecki, Differential Geometry in Toposes, http://www.fuw.edu.pl/ kostecki/sdg.pdf.

[46] R. Lavendhome, Basic Concepts of Synthetic Differential Geometry, Kluver Texts in Math Science, vol $13,1996$.

[47] R. Lavendhomme, Leçons de Géométrie Différentielle Synthétique Nä̈ve, Monographies de mathematiques' 3, Ciaco, Louvain-la-Neuve, (1987),204pp.

[48] F. LaWvere, Categorical Dynamics, em 35], 1-28.

[49] S. MacLane, Categories for the Working Mathematician, Springer Verlag, 1998.

[50] I. Moerdiuk, S. MacLane, Sheaves in Logic and Geometry - a First Introduction to Topos Theory, Springer Verlag, 1992.

[51] C. MACLARTy, Local and some global results in synthetic differential geometry in ([38]), 226-256.

[52] B. Malgrange, Ideals of differentiable functions, Tata Institute of Fundamental Research in Mathematics. Studies in mathematics, Oxford University Press, 1966.

[53] M. A. Marshall, Spaces of Orderings and Abstract Real Spectra, Lecture Notes in Mathematics 1636, Springer-Verlag, Berlin, Germany, 1996.

[54] H. MerklenEstructuras Algebraicas V (Teoria de Cuerpos), monografía no. 22, série de matemática, Secretaria General de la Organización de los Estados Americanos, Programa Regional de Desarollo Científico y Tecnológico, 1979.

[55] P. Michor, J. Vanzura, Characterizing Algebras of Smooth Functions on Manifolds, Comm. Math. Univ. Carolina, arXiv:math/9404228v1 [math.GT], 1 Apr 1994.

[56] P. Milman The Malgrange-Mather Division Theorem Topology Vol. 16, pp. 395-401 Pergamon Press. 1977. Printed m Great Britain (Received 19 July 1976).

[57] M. Minguez, Cálculo diferencial sintético y su interpretación en modelos de prehaces, PhD. Thesis, 1985.

[58] F. Miraglia, An Introduction to Partially Ordered Structures and Sheaves, Polimetrica S.a.s. International Scientific Publisher, 2006.

[59] I. Moerdijk, G. Reyes, Cohomology theories in synthetic differential geometry, in ([38]), 1-67.

[60] I. MoerdiJk, G. Reyes, De Rham's theorem in a smooth topos, Report 83-20, Amsterdam (1983), 22pp.

[61] I. MoERDiJK, On the embedding of manifolds into smooth Zariski topos, Mathematical Logic and Theoretical Computer Science 1987. Lecture Notes in Pure and Applied Mathematics 106, 261-276. 
[62] I. MoerdiJk, G. Reyes, Models for Smooth Infinitesimal Analysis, Springer-Verlag, New York, 1991.

[63] I. MoerdiJk, G. Reyes, Smooth Spaces versus Continuous Spaces in Models for Synthetic Differential Geometry, Journal of Pure and Applied Algebra 32 (1984) 143-176.

[64] I. Moerdijk, G. Reyes, The tangent functor category revisited by leke Moerdijk and Gonzalo E. Reyes, 2 Article in Indagationes Mathematicae Proceedings A 89 (4), 1986, 449-454.

[65] I. Moerdijk, G. Reyes, A Smooth Version of the Zariski Topos, Advances in Mathematics 65, 229-253 (1987).

[66] I. Moerdijk, G. Reyes. Rings of Smooth Functions and Their Localizations, I, Journal of Algebra 9, 324-336 (1986).

[67] I. Moerdijk, N. van Quê, G. E. Reyes, Rings of smooth functions and their localizations II, Mathematical Logic and Theoretical Computer Science 1987. Lecture Notes in Pure and Applied Mathematics 106, 277-300.

[68] M. Müger, An Introduction to Differential Topology, de Rham Theory and Morse Theory, , accessed in November 20th, 2018 .

[69] J. Penon, De l'infinitesimal au local, State PhD. Thesis, Paris, 1985, 191pp.

[70] R. S. Pierce, Introduction to the Theory of Abstract Algebras, Dover Books on Mathematics, 2014.

[71] G. Reyes, G. Wraith, A note on tangent bundle in a category with a ring object, Math. Scand. 42 (1978), 53-63.

[72] R. O. Robson, Model Theory and Spectra, Journal of Pure and Applied Algebra 63 (1990) 301-327, North-Holland 301.

[73] C. Scheiderer, Real and Étale Cohomology, Lecture Notes in Mathematics 1588, Springer-Verlag, Berlin, Germany, 1994.

[74] H. Schubert. Categories, translated from the German by Eva Gray, Springer-Verlag Berlin Heidelberg New York 1972.

[75] M. SpIVAK, A Comprehensive Introduction to Differential Geometry, Publisher Perish, Berkeley, vol. I-V, 1979.

[76] E. Tengan, H. Borges, Álgebra Comutativa em Quatro Movimentos, Projeto Euclides, IMPA, 2015.

[77] J. Tougeron, Idéaux de fonctions différentiables, I. Annales de l'Institut Fourier, (1968), tome XVIII. Numdam — Zbl 0188.45102

[78] M. Tressl, Super Real Closed Rings, Fundamenta Mathematicae 194 (2), 2007, 121-177, www.maths.manchester.ac. uk/raag/preprints/0177.ps.gz

[79] D. van Dalen, Logic and Structure, 4th edition. Springer Verlag Berlin Heidelberg 2004. ISSN 0172-5939.

[80] A. WeIL, Théorie des points prôches sur les variétés différentiables,in Conference Geom. Diff., Strasbourg, 1953, took up again in "Oeuvres scientifiques, collected papers", vol. II, Springer 1979, 103- 109.

\section{Complementary References}

[81] L. Belair, Calcul Infinitesimal en Géométrie Différentielle Synthétique, MSc Thesis, Montreal, 1981.

[82] J. L. BeLL, Two approaches to modelling the universe: Synthtetic Differential Geometry and Frame-Valued Sets.

[83] J. L Bell, A. B. Slomson, Models and Ultraproducts - an Introduction, Dover Publications, Inc. Mineola, New York, 2006. ISBN-10: 0-486-44979-3

[84] F. Bergeron, Objet infinitesimalement lineaire dans un modèle bien adapté de G.D.S., in Geometrie Differentielle synthetique, Section 2, Analysis in smooth topos, edited by G.E. REYES, Research report, Montreal, (1980),67-76.

[85] U. Bunke, T. Nikolausyand, M. Volklz, Differential Cohomology Theories as Sheaves of Spectra, arXiv:1311. 3188v1 [math.KT], 13 Nov 2013

[86] M. Bunge, P. SAwyer, On connections, geodesics and sprays in synthetic differential geometry, Cahier Top. Geom. Diff., 25 (1984), 221-258 (preprinted in ([26])).

[87] H. CARTAN, Notions d'algèbres différentielles; applications aux groupes de Lie et aux variétes où opère un groupe de Lie, Colloque de Topologie, Bruxelles, 1951, 15-27.

[88] B.A. Davey, H. A. Priestley, Introduction to Lattices and Order, Cambridge University Press, 1992

[89] M. Dickmann, F. Miraglia, Special Groups: Boolean-Theoretic Methods in the Theory of Quadratic Forms, Memoirs of the AMS 689, American Mathematical Society, Providence, USA, 2000.

[90] E. Dubuc, Germ Representability and Local Integration of Vector Fields in a Well Adapted Model of SDG, Journal of Pure and Applied Algebra 64 (1990) 131-144, North-Holland 131.

[91] E. Dubuc, J. Penon, Objects compacts dans les topos, J. Austr. Math. Soc., Ser. A, 40 (1986), 203-217.

[92] M. Enshov, WebPage do Curso "Survey of Algebra" http://people.virginia.edu/ mve2x/7752

[93] F. Gago, Morse Germs in SDG, in "Categorial Algebra and its Applications" , F. Borceux ed., Springer Lecture Notes 1348 (1988), 125-129

[94] F. GaGo, Singularités dans la géométrie différentielle synthétique, Bull. Soc. Math. Belgique, Ser. A, 41 (1989), $279-287$.

[95] C. Godbillon, Géométrie diffeéentielle et mecanique analytique, Paris, Hermann, 1969. 
[96] D. Joyce, D-manifolds and d-orbifolds: a theory of derived differential geometry, 2012, The Mathematical Institute, Oxford University. Semi-final version, December 2012. Updates will be posted on http://people.maths.ox.ac.uk/\$ sim\$joyce/dmanifolds.html.

[97] S. Kobayashi, K. Nomizu, Foundations of differential geometry, Interscience, vol. I 1963, vol. II, 1969.

[98] A. Kock, A simple axiomatic for differentiation, Math. Scand. 40 (1977), 183-193.

[99] J. F. Kennison, Integral Domain Type Representations in Sheaves and Other Topoi, Math. Z. 151 (1976), 35-36

[100] A. Kock, On the synthetic theory of vector fields, in 35],139-157.

[101] A. Kock, Differential forms with values in groups, Bull. Australian Math. Soc. 25 (1982), 357-386.

[102] A. Kock, A combinatorial theory of connections, Contemporary Mathematics, AMS, 30 (1984), 132-144.

[103] A. Kock, R. LAVEndhome, Strong infinitesimal linearity, with applications to strong difference and affine connections, Cahiers Top. Geom. diff. 25 (1984), 311-324.

[104] A. Kock, G. ReYes, Connections in formal differential geometry, in [35], 158-195.

[105] J.-L. Koszul Homologie et cohomologie des algèbres de Lie Bull. Soc. Math. France 78 (1950), 65-127.

[106] R. Lavendhomme, Notes sur l'algèbre de Lie d'un groupe de Lie en géométrie différentielle synthétique, Sem. Math. pure ni 11, Louvain-la-Neuve, (1981), 19pp.

[107] R. Lavendhomme, Objets de Lie, Bull Soc. Math. Belgique, Ser. B, 43 (1991), 83-112.

[108] R. Lavendhomme, Algèbres de Lie et Groupes microlinéaires, Cahiers Top. et Geom. Diff.Cat.,bf 35-1 (1994) 29-47.

[109] P. Libermann, C.-M. Marle, Symplectic Geometry and Analytical Mechanics, Reidel, 1987.

[110] M. Makkai, G. Reyes, First Order Categorical Logic: Model-Theoretical Methods in the Theory of Topoi and Related Categories, Lecture Notes in Mathematics 611, Springer-Verlag, Berlin, Germany, 1977.

[111] M. Minguez, Wedge product of forms in synthetic differential geometry, Cahiers Top. Geom. Diff. Cat. 29 (1988), 59-66.

[112] M. Minguez, Some combinatorial calculus on Lie derivative, Cahiers Top. Geom. Diff. Cat. 29 (1988), 241-247.

[113] M. MüGer, An Introduction to Differential Topology, de Rham Theory and Morse Theory, available in www.math.ru. nl/ mueger/diff_notes.pdf, 2005.

[114] C. J. Mulvey, A Generalisation of Gelfand Duality, Journal of Algebra 56 (1979), 499-505, Academic Press.

[115] D. PIERCE, Model-theory of Vector-Spaces over Unespecified Fields, D. Arch. Math. Logic (2009) 48:421-436, Pierce, D.

[116] R. S. Pierce, Modules over Commutative Regular Rings, Memoirs of the AMS 70, American Mathematical Society, Providence, USA, 1967. 


\section{Index}

$R$-algebra, 54

$\mathcal{C}^{\infty}$-ring object, 252

$\mathcal{C}^{\infty}$-von Neumann-regular hull, 206

$\mathcal{C}^{\infty}$-congruence, 11

$\mathcal{C}^{\infty}$-domain, 128

$\mathcal{C}^{\infty}$-field, 128

$\mathcal{C}^{\infty}$-ordering, 173

$\mathcal{C}^{\infty}$-polynomial, 171

$\mathcal{C}^{\infty}$-radical, 124

$\mathcal{C}^{\infty}$-real closed, 171

$\mathcal{C}^{\infty}$-real spectrum of $A, 173$

$\mathcal{C}^{\infty}$-reduced, 130

$\mathcal{C}^{\infty}$-ring, 6

finitely generated, 57

finitely presented $\mathcal{C}^{\infty}$-ring, 57

morphism of $\mathcal{C}^{\infty}$-rings, 7

$\mathcal{C}^{\infty}$-ring of fractions, 84

$\mathcal{C}^{\infty}$-ringed space, 196

morphism of $\mathcal{C}^{\infty}$-ringed spaces, 196

$\mathcal{C}^{\infty}$-structure, 5

morphism of $\mathcal{C}^{\infty}$-structures, 5

$\mathcal{C}^{\infty}$-subring, 8

$\mathcal{C}^{\infty}$-subring generated by a subset, 9

$\mathcal{C}^{\infty}$-support, 173

Boolean space, 165

Boolean spaces, 183

classifying topos, 251

closure operation, 9

closure operator, 137

complete Heyting algebra, 143

conservative extension, 269

constructible topology, 196

coproduct, 53

directed family, 10

free $\mathcal{C}^{\infty}$-ring, 24

functorial mathematical theory, 251

functorial theory, 250

Fundamental Theorem of the $\mathcal{C}^{\infty}$-Homomorphism,

15

Galois connection, 134

generic model, 251. generic point, 187

Grothendieck (pre)topology, 250

Grothendieck topos, 251

Grothendieck-Zariski smooth topos, 264

irreducible set, 187

left exact category, 251

local $\mathcal{C}^{\infty}$-ring, 128

Mitchell-Bénabou, 251

pre-order, 169

prime filter, 128

product, 11

quotient $\mathcal{C}^{\infty}$-ring, 13

real closed field, 171

Separation Theorems, 145

site, 250

sketch, 249

smooth Grothendieck-Zariski pretopology, 260

smooth Harrison topology, 174

smooth saturation, 88

smooth Zariski spectrum, 142

smooth Zariski topology, 186

spectral space, 188

structure sheaf, 162

Theorem of Ortega \& Muñoz, 278

theory of local $\mathcal{C}^{\infty}$-rings, 260

universal model, 260

von Neumann regular $\mathcal{C}^{\infty}$-ring, 159

finitely presented von Neumann regular $\mathcal{C}^{\infty}$-ring, 270

von Neumann-regular $\mathcal{C}^{\infty}$-ring

theory of, 268

Whitney's Theorem, 276, 277 